\title{
Carbon Monoxide Concentrations and Carboxyhemoglobin Profiles from Portable Generators with a CO Safety Shutoff Operating in a Test House
}

Steven J. Emmerich Stephen M. Zimmerman

Steven J. Nabinger

Matthew J. Brookman

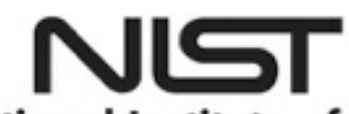




\title{
Carbon Monoxide Concentrations and Carboxyhemoglobin Profiles from Portable Generators with a CO Safety Shutoff Operating in a Test House
}

\author{
Steven J. Emmerich \\ Stephen M. Zimmerman \\ Steven J. Nabinger \\ Energy and Environment Division \\ Engineering Laboratory \\ Matthew J. Brookman \\ U.S. Consumer Product Safety Commission
}

This publication is available free of charge from:

https://doi.org/10.6028/NIST.TN.2049

June 2019

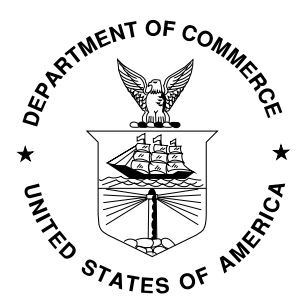

U.S. Department of Commerce Wilbur L. Ross, Jr., Secretary

National Institute of Standards and Technology Walter Copan, NIST Director and Under Secretary of Commerce for Standards and Technology 
Certain commercial entities, equipment, or materials may be identified in this document in order to describe an experimental procedure or concept adequately. Such identification is not intended to imply recommendation or endorsement by the National Institute of Standards and Technology, nor is it intended to imply that the entities, materials, or equipment are necessarily the best available for the purpose.

This technical note, coauthored by the National Institute of Standards and Technology and the U.S. Consumer Product Safety Commission staff, has not been reviewed or approved by, and may not necessarily reflect the views of, the Commission.

National Institute of Standards and Technology Technical Note 2049

Natl. Inst. Stand. Technol. Tech. Note 2049, 258 pages (June 2019)

CODEN: NTNOEF

This publication is available free of charge from:

https://doi.org/10.6028/NIST.TN.2049 


\section{Abstract}

The U.S. Consumer Product Safety Commission (CPSC) is focused on addressing the hazard of acute carbon monoxide (CO) poisoning of consumers from portable generators that can result in death or serious and/or lasting adverse health effects in exposed individuals. Under an interagency agreement with CPSC (CPSC-I-17-0023), the National Institute of Standards and Technology (NIST) conducted a series of tests to characterize the indoor $\mathrm{CO}$ concentrations and the associated calculated carboxyhemoglobin $(\mathrm{COHb})$ profiles on simulated occupants resulting from portable generators operating in or near a test house. The tests are intended to provide information to enable CPSC staff to make comparisons of residential CO exposures reflecting operation of current designs of portable engine-driven electric generators, inside or near homes or in attached garages, versus operation of portable generators equipped with a CO safety shutoff device. This report presents measured $\mathrm{CO}$ data and calculated $\mathrm{COHb}$ levels based on that $\mathrm{CO}$ data from tests conducted with five different generators under various operational and environmental conditions. Those data were then used to evaluate how well the indoor air quality model CONTAM can predict CO levels in the garage and the house in support of a planned study that CPSC staff will use to evaluate the impact of CO mitigation requirements in two recently-published voluntary standards.

\section{Keywords}

Generator; carbon monoxide; carboxyhemoglobin; CONTAM; exposure; indoor air quality; health; measurements; multizone airflow model; simulation 


\section{Table of Contents}

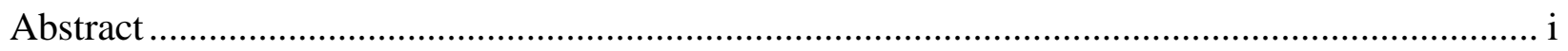

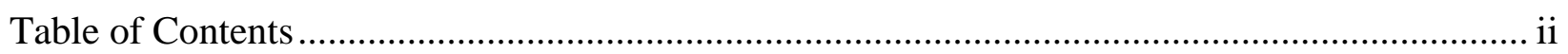

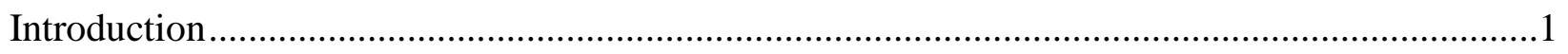

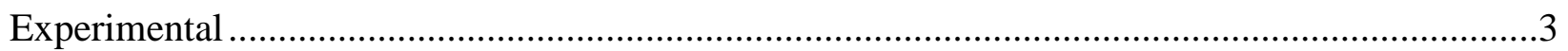

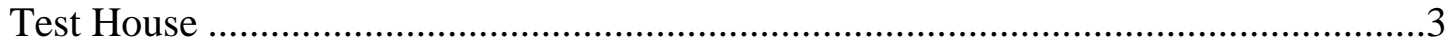

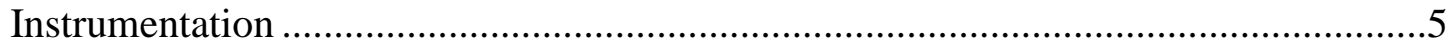

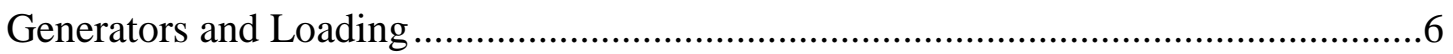

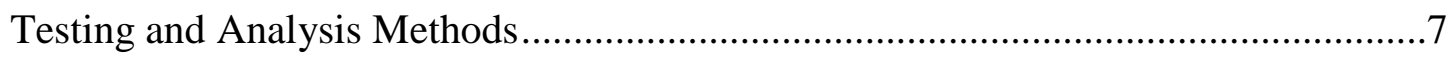

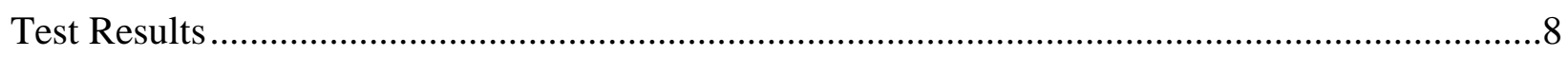

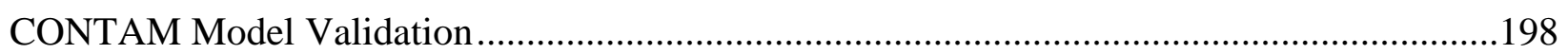

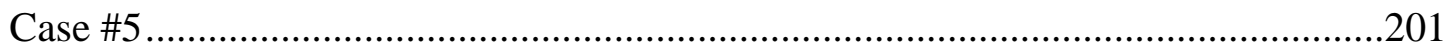

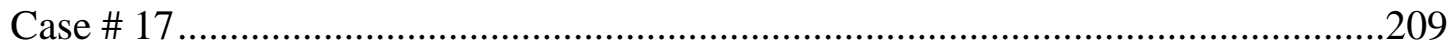

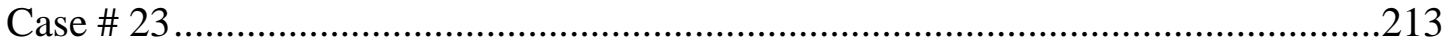

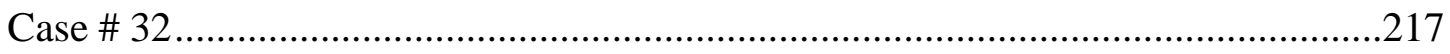

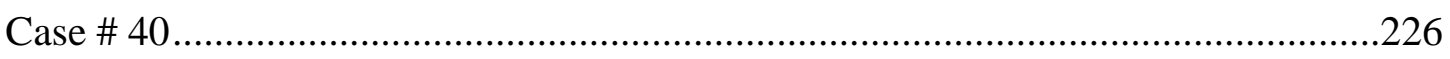

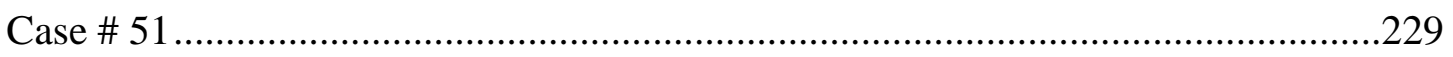

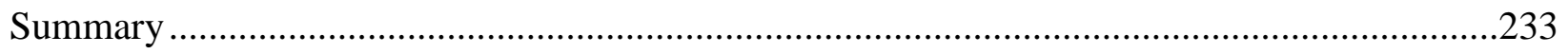

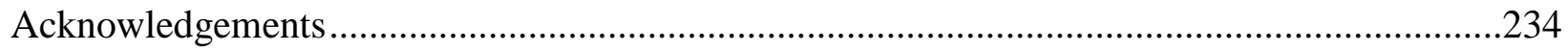

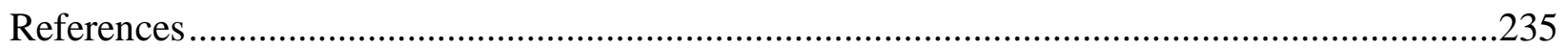

Appendix 1 - CPSC Letter to NIST on Portable Generator Characterization for Evaluation of

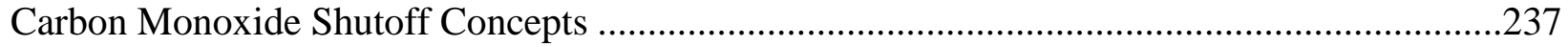

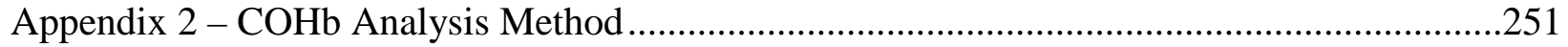




\section{Introduction}

The U.S. Consumer Product Safety Commission (CPSC, Commission) is focused on addressing the hazard of acute carbon monoxide (CO) poisonings of consumers from portable generators that can result in death or serious and/or lasting adverse health effects in exposed individuals. As of June 27, 2018, CPSC databases contain records of at least 943 (880 from generator use alone, 63 from generator use in conjunction with another $\mathrm{CO}$-producing consumer product) consumer deaths from CO poisoning associated with non-work-related use of generators in the period of 2005 through 2017 (Hnatov 2018). In addition, the percentage of estimated non-fire, consumer productrelated CO poisoning deaths specifically associated with generators for CPSC's four most recent years of data are 26 \% (2010), 39 \% (2011), 42 \% (2012), and 38 \% (2013) (Hnatov 2017). Typically, these deaths occur when consumers use a generator in an enclosed or partially enclosed space or outdoors near an open door, window or vent, and they often occur after severe weather events such as hurricanes and ice or snow storms.

The health impact of CO is caused by anoxia: deprivation of oxygen supply. When inhaled, CO preferentially binds with the oxygen carrier in the red blood cells, hemoglobin (Hb), to form carboxyhemoglobin (COHb), which causes the anoxia (Stewart 1975). The COHb level reflects the percentage of the body's total hemoglobin pool occupied by CO. In considering CO exposure, it serves as a useful measure of expected poisoning severity.

In previous work, documented in National Institute of Standards and Technology (NIST) Technical Note (TN) 1925, a computer simulation study was conducted to provide CPSC staff with information to support model-based estimates of residential $\mathrm{CO}$ exposures reflecting operation of current designs of portable engine-driven electric generators, both inside homes and in attached garages. These results were compared to simulated operation of reduced CO emission generators by CPSC staff to estimate the effectiveness of the reduced CO emission rates in preventing deaths that occurred with current generators. CPSC staff then recommended specific reduced $\mathrm{CO}$ emission rates as performance requirements to the Commission in a briefing package for a notice of proposed rulemaking (NPR) as the means to address the CO hazard associated with portable generators. The Commission subsequently voted to approve the NPR. (Proposed Safety Standard for Portable Generators, Federal Register, 81 FR 83556, November 21, 2016.). These simulations employed the multizone airflow and contaminant transport model CONTAM (Dols and Polidoro 2015), which was applied to a set of 40 buildings (37 houses and 3 detached garages, considered representative of fatal CO poisoning incidents reported in CPSC databases) that are based on a collection of models representative of the U.S. housing stock (Persily et. al. 2006). CONTAM assumes that the CO concentration is uniform within each building zone, which was a reasonable assumption for that modeling effort given that the generator run time was dependent on the fuel consumption and capacity rather than the $\mathrm{CO}$ concentration at a single point corresponding to the potential location of a shutoff sensor.

After CPSC issued the NPR, two industry voluntary standards were published in 2018 to address portable generator CO safety: ANSI/PGMA G300-2018, Safety and Performance of Portable Generators (referred to as PGMA G300) and ANSI/UL 2201-2018, Carbon Monoxide (CO) Emission Rate of Portable Generators (referred to as UL 2201).

PGMA G300 includes a requirement for generators to be equipped with an onboard CO sensor. This sensor, when tested to the requirements in the standard, must shut the generator off before the 
CO concentration measured at a location 1 inch to 2 inches above the approximate center of the portable generator's top surface exceeds either a rolling 10 -minute average of $400 \mathrm{ppmv}$ or an instantaneous reading of 800 ppmv. PGMA G300 also requires notification after a shutoff event, which is required to be a red indication. The standard also requires that this notification remain active for a minimum of 5 minutes after shutoff occurs unless the generator is restarted. Furthermore, PGMA G300 requires a label about the automatic shutoff in close proximity to the indicator, instructing the consumer about moving the generator to an outdoor area and seeking medical help if feeling sick.

UL 2201 includes a requirement for maximum weighted CO emission rate of $150 \mathrm{~g} / \mathrm{h}$ and an additional requirement for generators to shut off when the $\mathrm{CO}$ concentration one foot above the centerline of the top of the generator registers either an average of $150 \mathrm{ppmv}$ of CO for a 10-minute period or an instantaneous reading of 400 ppmv. UL 2201 does not contain a requirement for notification after a shutoff event.

In simulating the impact of these requirements on indoor CO concentrations, CONTAM's assumption of uniform concentration in a building zone may not be valid. CO concentration variations in a zone are important because a generator-mounted shutoff system will shut the generator off based on the concentration of $\mathrm{CO}$ near the generator or at the generator-mounted sensor, not the room average concentration. Therefore, the effectiveness of a shutoff system could depend on multiple factors, including where the generator is located within the space, how the exhaust is oriented relative to surfaces that the exhaust stream comes into contact with and how close that surface is to the generator, and the velocity and temperature at which the exhaust jet exits the tailpipe. In order to understand the impacts of generator shutoff strategies on CO exposure, testing was performed using a variety of generators with a shutoff device under various loads, operating in and near a test house and its attached garage to characterize the rate at which the $\mathrm{CO}$ emitted from the generator accumulated in, transported within, and left the test house.

This report presents the measured $\mathrm{CO}$ data and calculated $\mathrm{COHb}$ profiles of potential occupants from tests conducted with five different generators under various use and environmental conditions. Those data are then compared to CONTAM predictions of CO levels and COHb profiles calculated from CONTAM CO predictions. As part of this comparison, NIST examined modeling options to reduce the uncertainty in the CO levels predicted by CONTAM, and these options are discussed. The overall goal of this modeling exercise is to demonstrate that CONTAM predictions can be relied upon to estimate $\mathrm{COHb}$ profiles for a planned analysis by CPSC staff to evaluate the impact of the requirements in these voluntary standards on indoor CO exposure.

This report is not intended to evaluate the overall effectiveness of either voluntary standard or of shutoff systems, but rather reports on a series of tests designed to evaluate the impacts of CO safety shutoff on CO exposure as well as whether CONTAM modeling could be useful in estimating CO exposures under the conditions of interest. The results in this Technical Note are limited to one house, specific weather situations, and the five tested generators, which is one reason this report cannot solely be used to determine the exposure impacts of the requirements in either standard under other circumstances. 


\section{Experimental}

This section describes the experimental work performed including the test house in which the measurements were made, the instrumentation employed in the test, the generators that were tested, and testing and analysis methods used.

\section{Test House}

The test house used in this study was a manufactured house located on the NIST campus, which was erected in 2002 (Nabinger and Persily 2008, Nabinger et al. 2010). An aerial view and floorplan of the house are shown in Figures 1 and 2. The house includes three bedrooms (MBd, B2, and B3), two bathrooms (MBa and HB), a living room (LR), a dining room (DR), a kitchen (Kit), a family room (FR), a utility room (UR) and an attached garage (Gar). The house has a floor area of $140 \mathrm{~m}^{2}$ and a volume of $340 \mathrm{~m}^{3}$. The attached garage has a floor area of $36.5 \mathrm{~m}^{2}$ and a volume of $90 \mathrm{~m}^{3}$, and was built as an addition to the house in 2007. The interior of the garage, including the ceiling, is finished with painted gypsum board. As part of the garage construction, the underlayment and siding of the exterior west wall of the house were removed and replaced with $3 / 4$ inch gypsum board on studs with fibrous glass batt insulation in the wall cavity. Figure 4 shows the location of $\mathrm{CO}$ sensors, thermocouples and generators, and the generator exhaust direction.

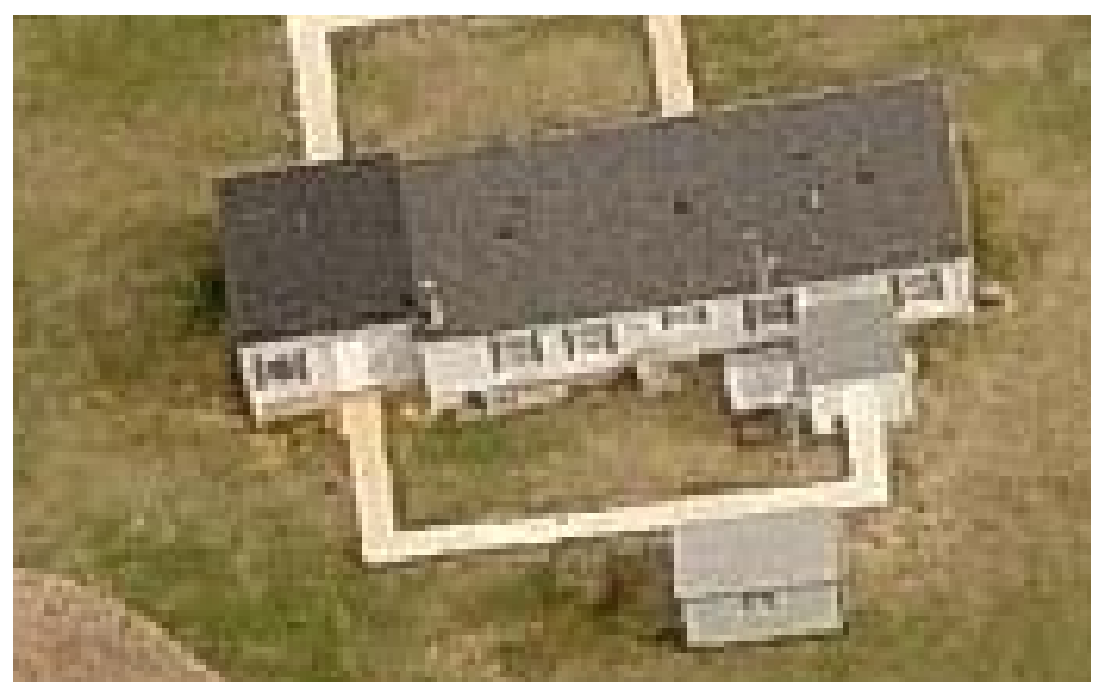

Figure 1 Aerial view of NIST manufactured test house

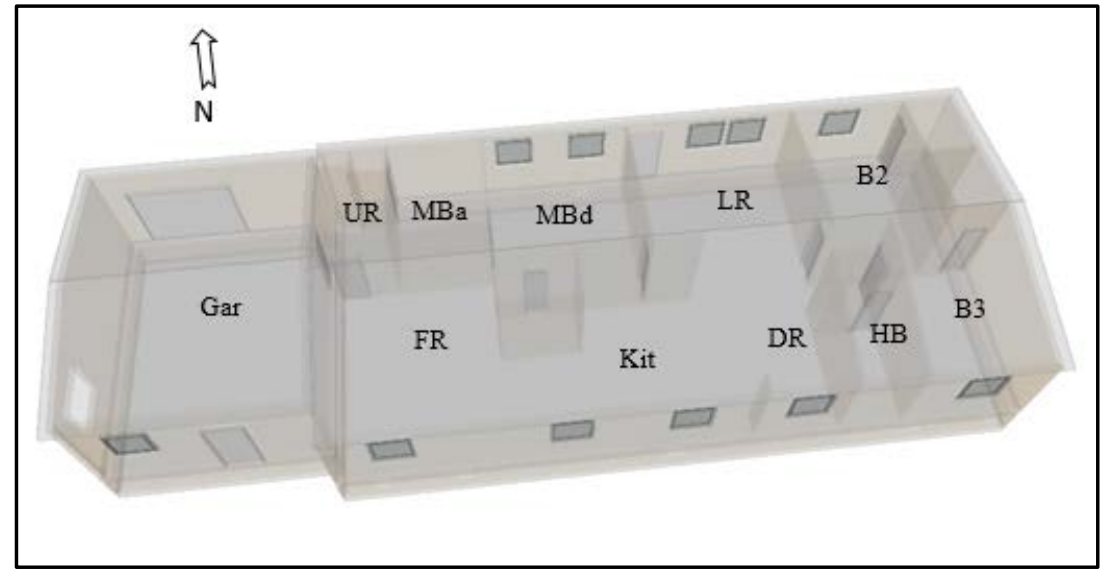

Figure 2 Floorplan of NIST manufactured test house 


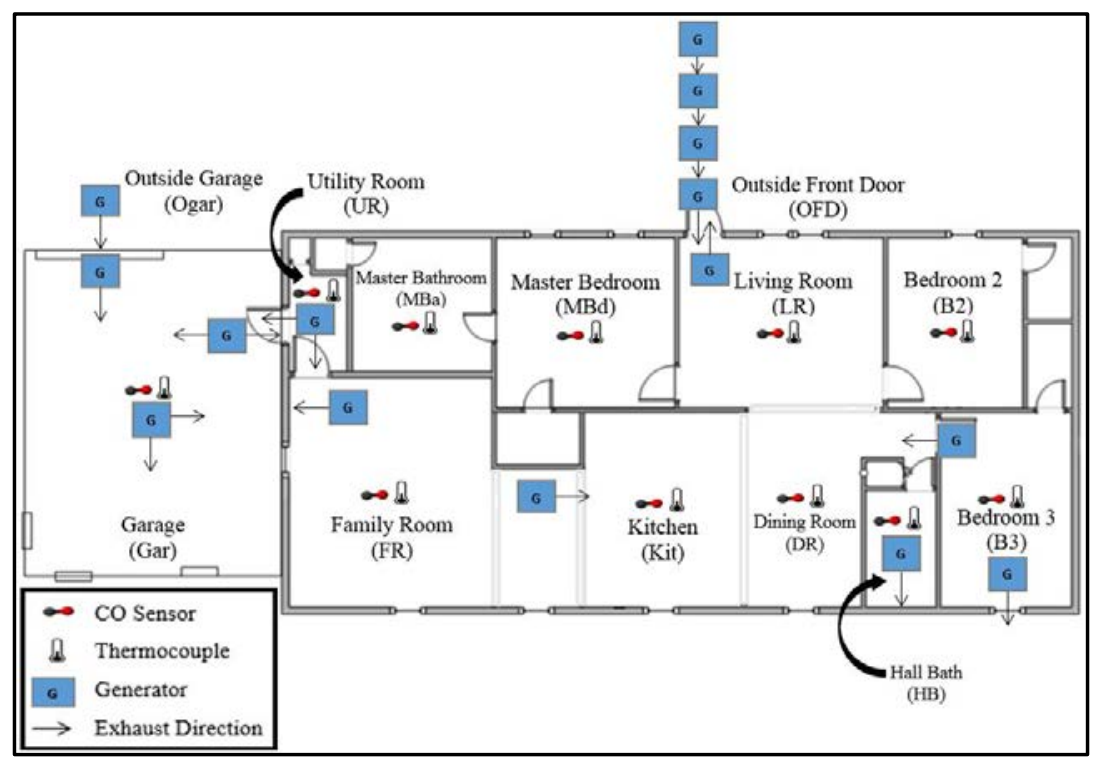

Figure 3 NIST manufactured test house layout showing location of CO sensors, thermocouples, and generator placement with exhaust direction.

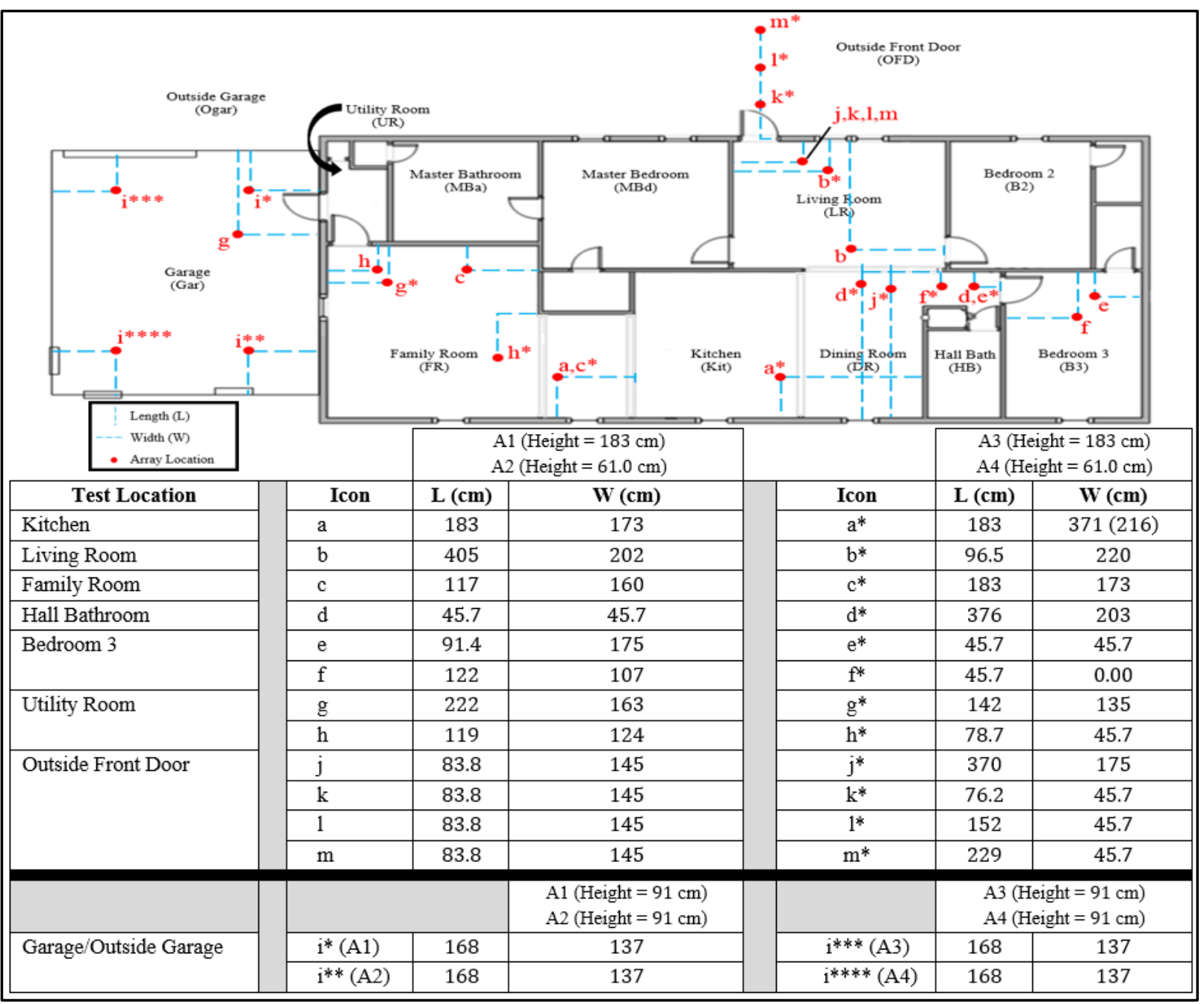

Figure 4 NIST manufactured test house layout showing positions of the four-point array for measuring CO concentration at each test location. 


\section{Instrumentation}

Gas concentrations in the test house were measured using two multi-gas analyzers and arrays of electrochemical sensors. The multi-gas analyzers used non-dispersive infrared (NDIR) technology to measure the $\mathrm{CO}$ concentration and each analyzer contained two $\mathrm{CO}$ channels. Of the four channels used to measure $\mathrm{CO}$, one was used to simulate a sensor onboard the generator and the other three were used to measure concentrations throughout the house. The location of the generator sensor channel was chosen to represent a possible location for a sensor on an actual production generator equipped with a CO safety shutoff device. Typically, the sample location was near the electrical panel of the generator. Ultra-pure $\mathrm{CO}$ with a nitrogen balance was used to span the analyzers at the beginning of each test day. The maximum ranges for these channels are described below (all 4 channels were not used in every test):

- Channel 1 (generator shutoff channel): 1,000 ppmv

- Channel 2: 2,000 ppmv

- Channel 3: 2,000 ppmv

- Channel 4: 10,000 ppmv

The electrochemical sensors were used to measure CO concentrations in each room of the test house. A four-point sensor array was also used to measure concentrations near the generator. Two ranges (a low range and a high range) of sensors were placed at each measurement point. The low range sensor had a range of $800 \mathrm{ppmv}$ with integrated temperature correction, and the high range sensor had a range up to $5000 \mathrm{ppmv}$ without integrated temperature correction. A low range sensor and high range sensor were placed in the center of each room, $0.84 \mathrm{~m}$ above the floor. The position of the four-point sensor array used to measure concentrations around the generator was dependent on the test case (Figure 4). Sensor locations for different tests are indicated with letters and letters with asterisks in Figure 4. For example, when the test location was in the Kitchen, the A1 and A2 sensors for the four-point array were located at position a in Figure 4 and the A3 and A4 sensors were located at position $\mathrm{a}^{*}$. Since the high range electrochemical sensors were not equipped with integrated temperature correction, type $\mathrm{J}$ thermocouples were placed near each of these sensors. Temperature correction was then performed in real time by the data acquisition program.

The electrochemical sensor modules were calibrated against a NDIR gas analyzer in CPSC's Combustion Lab. First the analyzer was calibrated with NIST traceable primary standard gas across a range up to 1000 ppmv for the low range sensors and 5000 ppmv for the high range sensors. The sensors were tested in batches inside a sealed conditioning chamber with a high degree of mixing. A sample was withdrawn from the center of the chamber near the center of the set of sensors being calibrated and directed to the NDIR analyzer. The same power supply and data acquisition hardware used in field testing were used to power and read the NDIR analyzer and sensor outputs during these calibrations. The sensors were calibrated by gradually increasing the concentration of $\mathrm{CO}$ in the chamber through a series of steps, then gradually decreasing the concentration back to zero. The time series data from the sensors and the analyzer were sampled at $1 \mathrm{~Hz}$. The measurement from each sensor was then compared to the measurement from the analyzer to determine a calibration factor for the sensors. Both the low- and high-range sensors were within $+/-4 \%$ of the concentrations reported by the analyzer. This results in an expanded 
uncertainty of $8 \%$ with a coverage factor of $\mathrm{k}=2$. A single calibration factor was applied in the data acquisition system for each low range and high range sensor. The sensors were not found to drift significantly during testing based on calibrations performed before and after the test program, as well as random sensor verification during testing.

The data acquisition system consisted of hardware and software with a custom program to record CO concentrations with temperature correction, test start and stop time, generator load, and weather conditions, all at a frequency of $1 \mathrm{~Hz}$. The program also included an algorithm that shut the generator down when the $\mathrm{CO}$ measured by the channel that simulated the CO shutoff device on the generator reached specified criteria for shutoff. The shutoff criteria were selected prior to testing and included the G300 requirements and UL 2201 requirements per these standards. Analog to digital converters with a minimum resolution of 16 bit were used for the CO channels and integrated cold junction correction for the thermocouples.

Air change rates were measured using the tracer gas decay method (ASTM 2011). A pulse of sulfur hexafluoride $\left(\mathrm{SF}_{6}\right)$ was injected and allowed to mix before the tracer gas concentrations were measured with a gas chromatograph with an electron capture detector. A local weather station located about $6 \mathrm{~m}$ behind the house was used to measure ambient conditions including temperature, humidity, wind speed and wind direction. An additional portable weather station was used to record weather conditions near the generators when operated outside. All gas concentration, air temperature, and humidity data were recorded by an automated data acquisition system. Nabinger and Persily (2008) provide more details on the $\mathrm{SF}_{6}$, temperature, humidity, and ambient weather condition measurements including uncertainties.

\section{Generators and Loading}

Five generators were selected for testing to represent the range of power outputs from commercially-available portable generators. CPSC staff characterized the generator operation with various applied loads to develop potential inputs for validation modeling purposes (Brookman 2018, included in Appendix 1 of this report). The generator operating characteristics reported include $\mathrm{CO}$ emission rate, exhaust temperature and velocity, and block temperature. These generators are identified throughout this report as G2, G2P, G6, G7a, G7b, and G15.

\section{G2: $2 \mathrm{~kW}$ gasoline-powered generator \\ G2P: $2 \mathrm{~kW}$ propane-powered generator \\ G6: $6 \mathrm{~kW}$ gasoline-powered generator \\ G7a/b: $7 \mathrm{~kW}$ gasoline-powered generator \\ G15: $15 \mathrm{~kW}$ gasoline-powered generator}

There are no restrictions on emission rates in PGMA G300, therefore all generators selected for testing may be used with the G300 shutoff criteria. In addition to testing with the G300 shutoff criteria, the G2, G2P, and G7 generators were also tested with the UL 2201 shutoff criteria because they were characterized at loads that produced modal emission rates expected to be within the weighted emission rate limits of UL 2201. The G7b generator was used during the restart and relocation phase of testing, as described below. Both G7a and G7b are the same make and model and exhibited similar $\mathrm{CO}$ emission rates during generator characterization. 
The generators were operated using either reformulated gasoline with $10 \%$ ethanol obtained from the NIST motor pool, which is purchased to the same specification year-round, or with consumergrade propane. The generators were cold-started for all tests in this report except as specifically noted.

A portable alternating current (AC) resistive load bank connected to the generator's 120-volt or 240-volt receptacle was used to draw electrical power, and thereby act as a surrogate for consumer appliance loads. The load bank has manual switches for specific loads and a variable transformer for infinite load step resolution with a maximum setting of $20 \mathrm{~kW}$.

\section{Testing and Analysis Methods}

To characterize the emission and transport of $\mathrm{CO}$ and the resulting $\mathrm{COHb}$ profiles when operating a portable generator equipped with a simulated $\mathrm{CO}$ shutoff device, the generators were operated until the specified shutoff criteria was measured at the shutoff sensor. Ventilation conditions were specified for each scenario, including any changes during the tests as described in the results section. Exterior doors and windows were closed and interior doors were fully open except as specifically noted. Upon activation of the shutoff algorithm, the generator was either left in place not running, restarted, or relocated. These options were designed to mimic the actions a consumer may take after the generator shuts down. For restart scenarios, the generator was restarted approximately 10 minutes after initial activation of the shutoff system. The test load was then applied immediately following restart. For relocation scenarios, movement of the generator was simulated by waiting approximately 10 minutes from the time of shutoff, then fully opening an exterior door for approximately 1 minute. The exterior door was then left open $10 \mathrm{~cm}$ to simulate the passage of cords through the doorway. Other ventilation conditions were not changed unless specifically noted. Relocation scenarios assumed that the generator was moved to a safe location, far enough away to prevent CO from entering the home. Therefore, for the purposes of testing, it was not necessary to restart the generator after relocation. The relocation scenarios were designed to change the ventilation during the scenario based on possible actions taken by the consumer, which only affected the decay in CO concentration in the house after shutoff occurred..

Estimated $\mathrm{COHb}$ profiles were calculated from the $\mathrm{CO}$ measurements using the Coburn-ForsterKane (CFK) non-linear differential equation (Peterson and Stewart 1975, Coburn et al. 1965) and input values determined in consultation with CPSC. These input values include a respiratory minute volume (RMV) value of $10 \mathrm{~L} / \mathrm{min}$ (representing a time-weighted average 24-hour value for males and females 16 to 80 years old, for residential indoor activity). COHb levels were calculated assuming an individual remained in the same room for the duration of the test. Calculations were performed for all rooms except the bathrooms, utility room and the garage; however, $\mathrm{COHb}$ calculations were done for the garage when the generator was in or outside of the garage. Additional details on the $\mathrm{COHb}$ calculation are included in Appendix 2 of this report. 


\section{Test Results}

As described above, NIST conducted a series of tests to provide empirical CO data to characterize the emission and transport of CO in NISTs manufactured test house, as well as the resulting calculated $\mathrm{COHb}$ profiles, when operating portable generators equipped with a simulated $\mathrm{CO}$ shutoff device in the living space, attached garage and outside the house. The resulting data, presented in this section, are organized by the location of the generator. The tests performed at each location are summarized in Tables 1 through 9. The tables include columns listing the generator location, the generator tested, the load (as a \% of the maximum generator capacity), whether the test included a restart or relocation after shutoff, the case and test number, the shutoff criteria used, the average outdoor temperature, the average wind speed, the peak CO concentration in the house or garage, the calculated shutoff ratio, the range of peak $\mathrm{COHb}$ values reached, the ventilation settings of the house, and notes. The column labeled "Peak CO in House and Garage" is the maximum $\mathrm{CO}$ concentration measured by any sensor located within the test house and garage (i.e. four-point array and centrally-located room sensors) during the test. The "Shutoff Ratio" column presents the ratio of the average source room concentration to the generator shutoff sensor at the time of shutoff. The average source room concentration is determined by averaging the values at the time of shutoff from the centrally located room sensor and any sensors from the sensor array that were in the source room. Note that these results apply to the specific generators tested and that other generators, houses and test conditions may produce different results. Also note that the combinations of generator location, load, exhaust direction, and door/window positions were selected to evaluate the shutoff algorithms under a wide range of possible conditions and with consideration of reported incidents. Note that not all scenarios were intended to represent specific reported incidents. Additionally, note that Cases 5, 17, 23, 32, 40 and 51 were used as CONTAM model validation cases; those comparisons are found in the Model Validation Section of this report immediately following this Test Results Section.

The following sections contain the individual test results organized by generator location. In each of these sections, four to five figures are presented for each test, designated as Figure $\mathrm{Xa}, \mathrm{Xb}, \mathrm{Xc}$, $\mathrm{Xd}$ and Xe. Figure Xa shows the position of the generator, generator exhaust direction, and the position of the $\mathrm{CO}$ sensor array used to measure $\mathrm{CO}$ around the generator. Figure $\mathrm{Xb}$ shows the $\mathrm{CO}$ concentrations measured by the shutoff sensor and the sensor array. Figure Xc shows the CO concentrations measured by the centrally located room sensors in each room. Figure Xd shows the CO concentrations averaged over a 1-minute time step measured by the centrally located room sensors in the areas of the home expected to be occupied. This figure also presents projected CO concentrations after the test was ended. These estimates are obtained for each room by applying the equation $\mathrm{C}=\mathrm{C}_{0} \mathrm{e}^{-\mathrm{rt}}$, where $\mathrm{C}$ is the concentration of $\mathrm{CO}$ in the room at time $\mathrm{t}, \mathrm{C}_{0}$ is the last $\mathrm{CO}$ measurement taken from the room (the initial CO concentration for projected values) and is the exponential decay constant of the room (calculated by an exponential fit to the measured data typically over the last hour of the test). These data, displayed each in Figure Xd, were used to develop the $\mathrm{COHb}$ profiles in the corresponding Figure Xe. 


\section{Generator Location: Kitchen}

Table 1 summarizes the results for all tests performed with a generator located in the test house kitchen. The details are discussed below and shown in Figures 5 through 30.

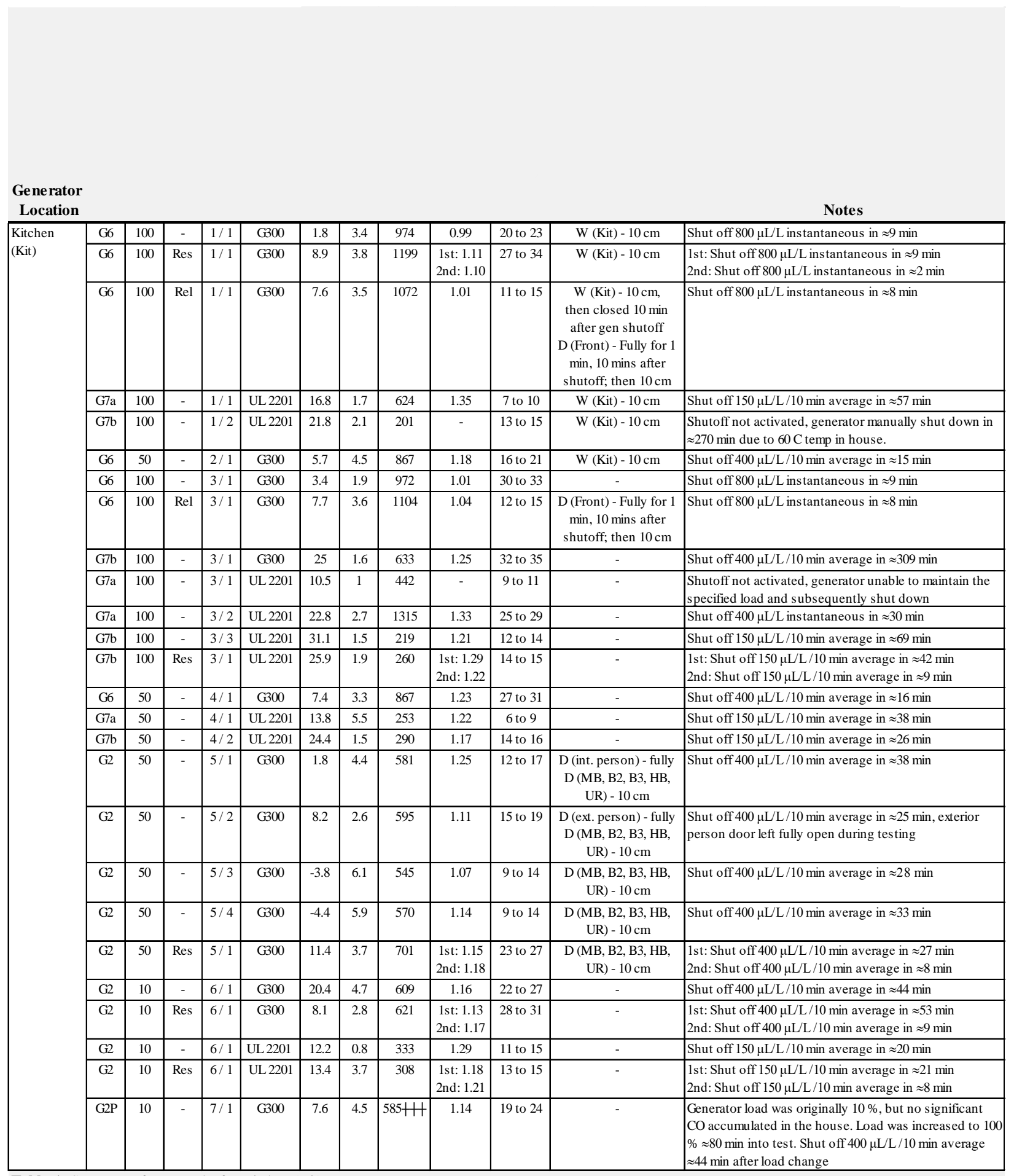

Table 1. Summary of test results for Kitchen (Kit) tests.

+Peak COHb Values are for house zones only.

HVentilation Note: Unless specified otherwise, during the test - all exterior doors are closed, all interior doors are fully open, and all windows are closed.

HThe max CO with a $10 \%$ load was $59 \mu \mathrm{L} / \mathrm{L}$ - the max CO was $585 \mu \mathrm{L} / \mathrm{L}$ after the load was increased to $100 \%$. 


\section{Case 1 Test 1 - G6 G300}

The following figures illustrate the results for Case 1 Test 1 applying the G300 shutoff criteria ( $>800$ ppmv instantaneous or $\geq 400$ ppmv 10 min average). The G6 generator was fully fueled, positioned in the kitchen (30 cm from the master bedroom closet wall with the exhaust facing the dining room), and tested at $100 \%$ load $(6,000 \mathrm{~W})$. The kitchen window nearest to the generator was open $10 \mathrm{~cm}$. The CO sensing channel simulating the CO shutoff device (referred to as the "shutoff sensor" throughout the Test Results section) activated the algorithm to shut off the generator after approximately 9 min due to an instantaneous sensor measurement of $800 \mu \mathrm{L} / \mathrm{L}$. Figure 5a shows the test house layout with generator location, generator exhaust direction, and sensor array locations. Figure $\mathbf{5 b}$ shows the $\mathrm{CO}$ concentrations measured in the kitchen (by the dedicated room sensor), by two sensors located on the kitchen-family room boundary (A1: height $183 \mathrm{~cm}, \mathrm{A2}$ : height $61 \mathrm{~cm}$ ), by the other two sensors located on the kitchen-dining room boundary (A3: height $183 \mathrm{~cm}, \mathrm{~A} 4$ : height $61 \mathrm{~cm}$ ), and by the shutoff sensor located on the generator near its electrical panel. The timeframe of the figure has been selected to show the degree of CO uniformity in the area around the generator between the shutoff sensor and the peak CO concentrations measured elsewhere in the kitchen near the time of shutoff. Figure $5 \mathbf{c}$ shows the CO concentration measured in each room of the house over the test. Figure 5d shows the CO concentration in the house, with each room represented by a single line composed of both a 'solid-line' pattern (showing measured CO concentration) and a 'dotted-line with a symbol' pattern (showing projected $\mathrm{CO}$ concentration). Figure 5e shows the calculated $\mathrm{COHb}$ profiles of simulated house occupants in each room of the house.

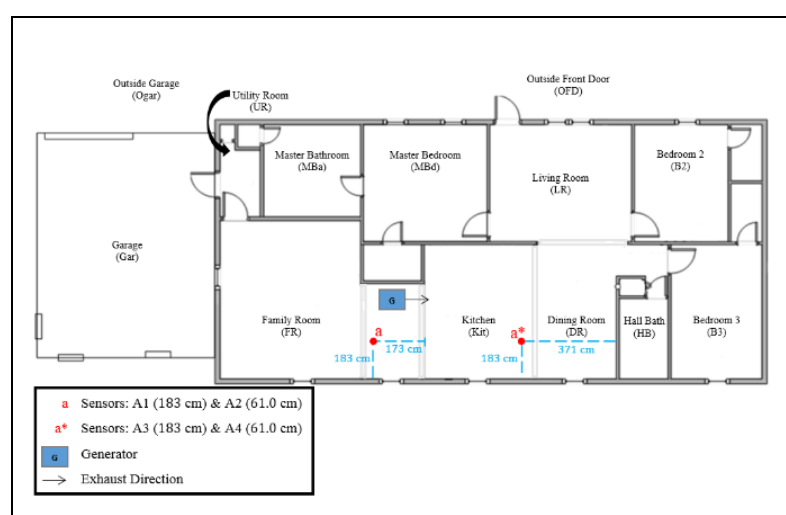

Figure 5a. Generator, exhaust direction, and sensor array layout during Case 1 Test 1 - G6 G300.

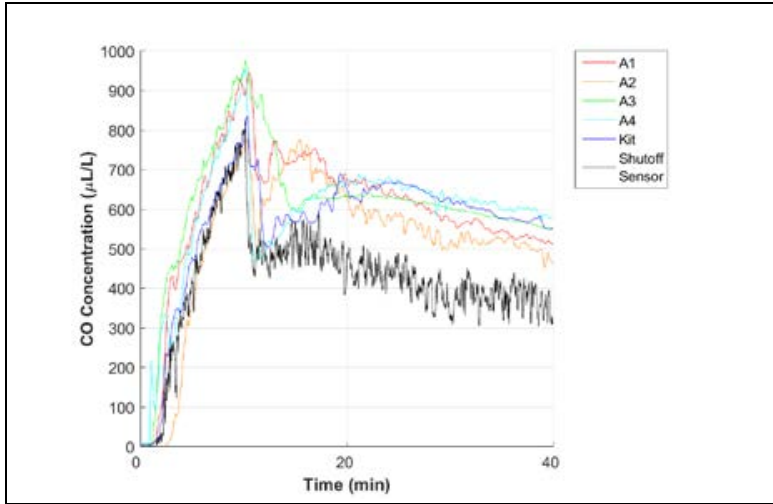

Figure 5b. CO concentration measured by the sensor array and the designated kitchen sensor during Case 1 Test 1 - G6 G300. 


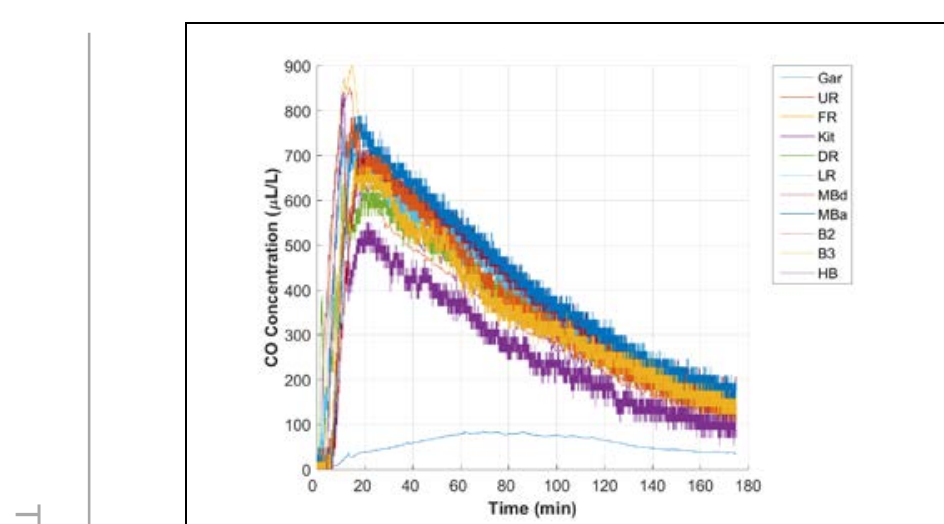

Figure 5c. CO concentration measured in each room of the house during Case 1 Test 1 - G6 G300.

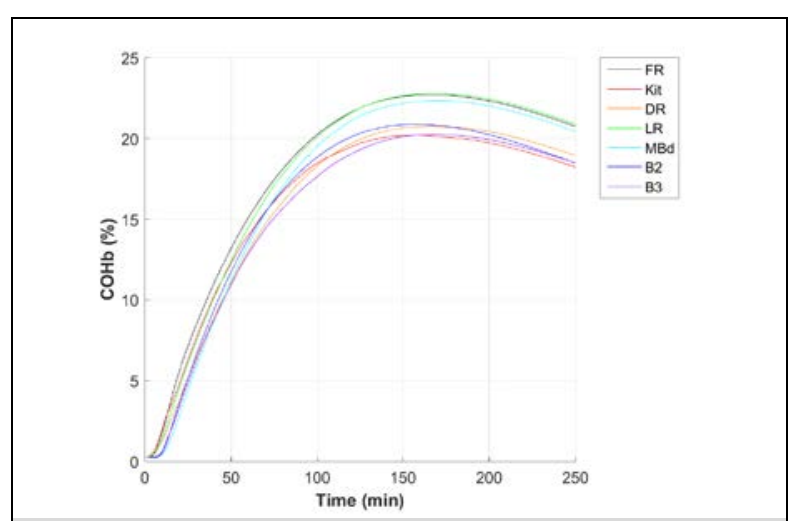

Figure 5e. $\mathrm{COHb}$ of house occupants during Case 1 Test 1 - G6 G300.

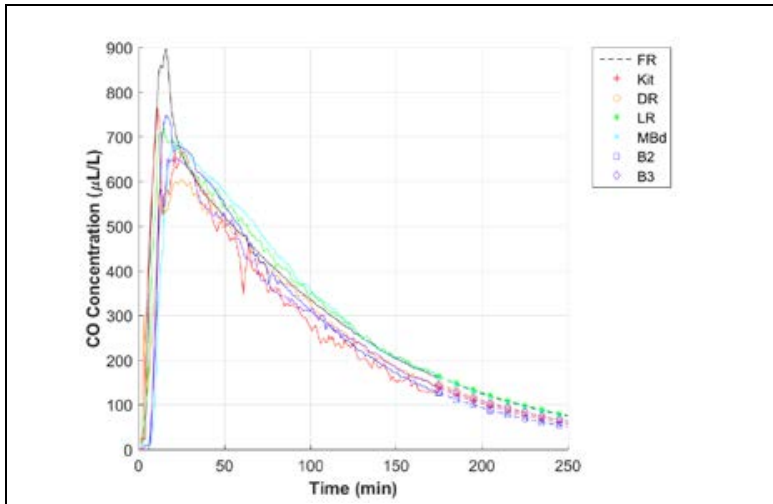

Figure 5d. CO concentration (measured \& projected) in the house during Case 1 Test 1 - G6 G300. 


\section{Case 1 Test 1 - G6 G300 Restart}

The following figures illustrate the results for Restart Case 1 Test 1 applying the G300 shutoff criteria. The G6 generator was fully fueled, positioned in the kitchen (30 cm from the master bedroom closet wall with the exhaust facing the dining room), and tested at $100 \%$ load (6,000 W). The kitchen window nearest to the generator was open $10 \mathrm{~cm}$. For the first generator run, the shutoff sensor activated the algorithm to shut off the generator after approximately 9 min due to an instantaneous sensor measurement of $800 \mu \mathrm{L} / \mathrm{L}$. The generator was restarted and the test load restored approximately $10 \mathrm{~min}$ after the first instance of shutoff initiated by the shutoff sensor. For the second generator run, the shutoff sensor activated the algorithm to shut off the generator after approximately 2 min due to an instantaneous sensor measurement of $800 \mu \mathrm{L} / \mathrm{L}$. Figure 6a shows the test house layout with generator location, generator exhaust direction, and sensor array location. Figure 6b shows the CO concentration measured in the kitchen (by the dedicated room sensor), by two sensors located on the kitchen-family room boundary (A1: height $183 \mathrm{~cm}, \mathrm{A2}$ : height $61 \mathrm{~cm}$ ), by the other two sensors located on the kitchen-dining room boundary (A3: height $183 \mathrm{~cm}$, A4: height $61 \mathrm{~cm}$ ), and by the shutoff sensor located on the generator near the generator's electrical panel. The timeframe of the figure has been selected to show the degree of CO uniformity in the area around the generator between the shutoff sensor and the peak CO concentrations measured elsewhere in the kitchen near the time of shutoff. Figure 6c shows the CO concentration measured in each room of the house over the test. Figure 6d shows the CO concentration in the house with each room represented by a single line, which is composed of both a 'solid-line' pattern (showing measured CO concentration) and a 'dotted-line with a symbol' pattern (showing projected $\mathrm{CO}$ concentration). Figure 6e shows the calculated $\mathrm{COHb}$ profiles of simulated house occupants in each room of the house.

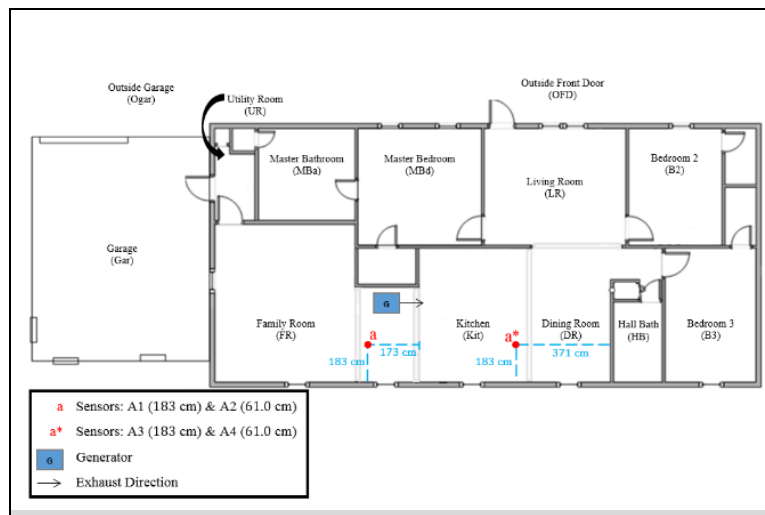

Figure 6a. Generator, exhaust direction, and sensor array layout during Restart Case 1 Test 1 - G6 G300.

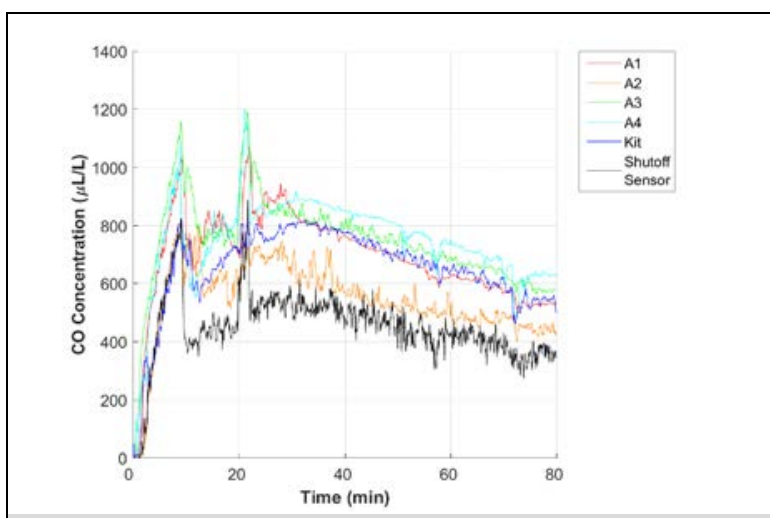

Figure 6b. CO concentration measured by the sensor array and the designated kitchen sensor during Restart Case 1 Test 1 - G6 G300. 

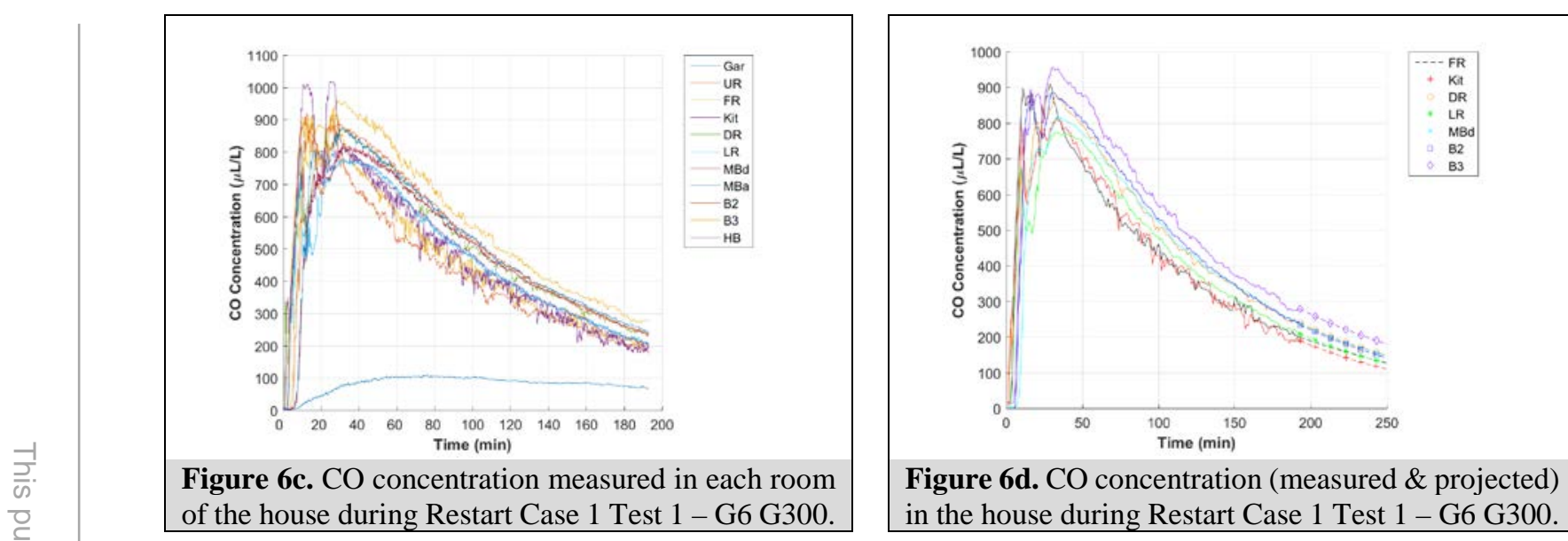
of the house during Restart Case 1 Test 1 - G6 G300.

Figure 6d. CO concentration (measured \& projected) in the house during Restart Case 1 Test 1 - G6 G300.

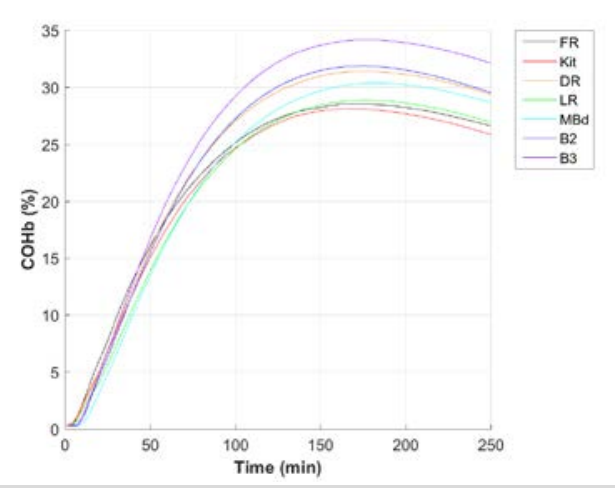

Figure 6e. $\mathrm{COHb}$ of house occupants during Restart Case 1 Test 1 - G6 G300. 


\section{Case 1 Test 1 - G6 G300 Relocate}

The following figures illustrate the results for Relocate Case 1 Test 1 applying the G300 shutoff criteria. The G6 generator was fully fueled, positioned in the kitchen (30 cm from the master bedroom closet wall with the exhaust facing the dining room), and tested at $100 \%$ load (6,000 W). The kitchen window nearest to the generator was initially open $10 \mathrm{~cm}$ while the generator was running. The shutoff sensor activated the algorithm to shut off the generator after approximately 8 min due to an instantaneous sensor measurement of $800 \mu \mathrm{L} / \mathrm{L}$. Ten min after the generator shutoff, the kitchen window was closed and the front door, which was initially closed, was then opened fully for $1 \mathrm{~min}$ and then, it was then set to $10 \mathrm{~cm}$ open for the remainder of the test. Figure 7a shows the test house layout with generator location, generator exhaust direction, and sensor array location. Figure $\mathbf{7 b}$ shows the CO concentration measured in the kitchen (by the dedicated room sensor), by two sensors located on the kitchen-family room boundary (A1: height $183 \mathrm{~cm}, \mathrm{~A} 2$ : height $61 \mathrm{~cm}$ ), by the other two sensors located on the kitchen-dining room boundary (A3: height $183 \mathrm{~cm}$, A4: height $61 \mathrm{~cm}$ ), and by the shutoff sensor located on the generator near the generator's electrical panel. The timeframe of the figure has been selected to show the degree of CO uniformity in the area around the generator between the shutoff sensor and the peak CO concentrations measured elsewhere in the kitchen near the time of shutoff. Figure 7c shows the CO concentration measured in each room of the house over the test. Figure 7d shows the CO concentration in the house with each room represented by a single line, which is composed of both a 'solid-line' pattern (showing measured CO concentration) and a 'dotted-line with a symbol' pattern (showing projected CO concentration). Figure 7e shows the calculated $\mathrm{COHb}$ profiles of simulated house occupants in each room of the house.

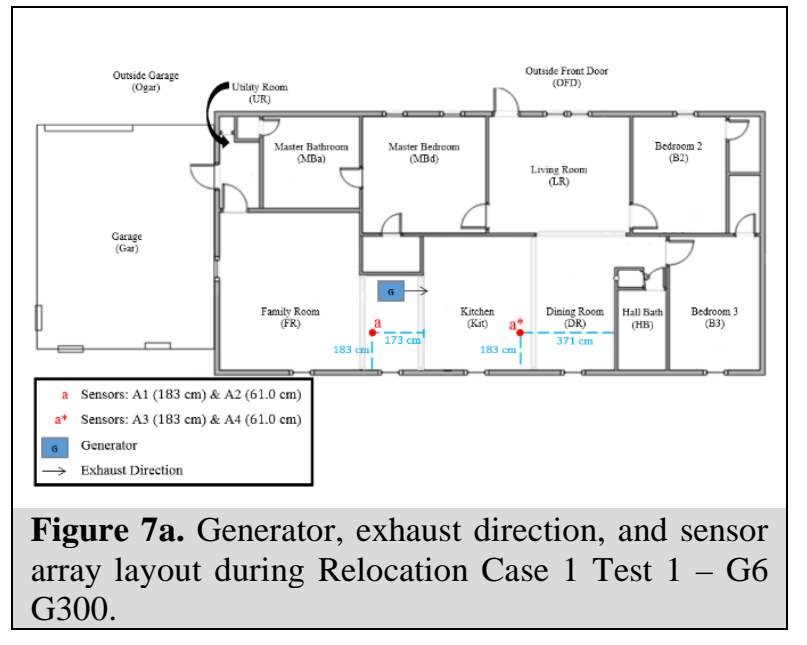

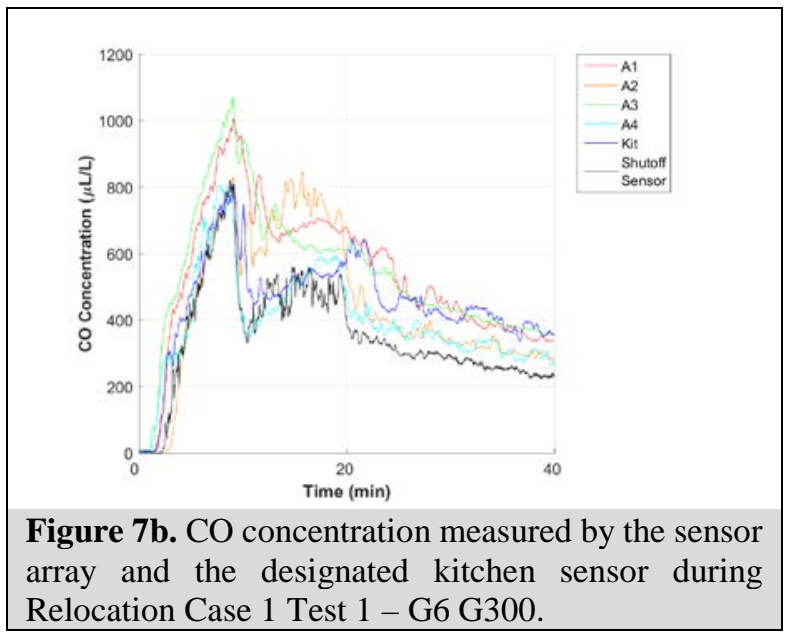




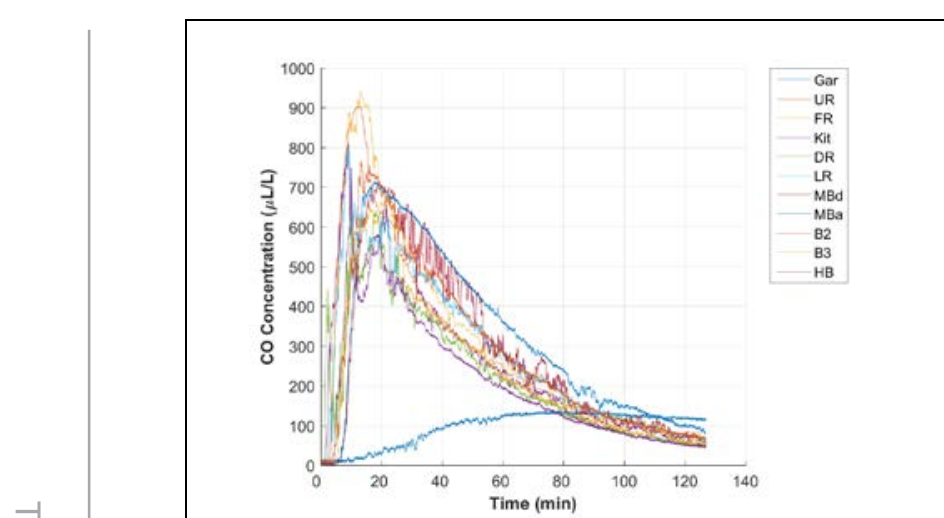

Figure 7c. CO concentration measured in each room of the house during Relocation Case 1 Test 1 - G6 G300.

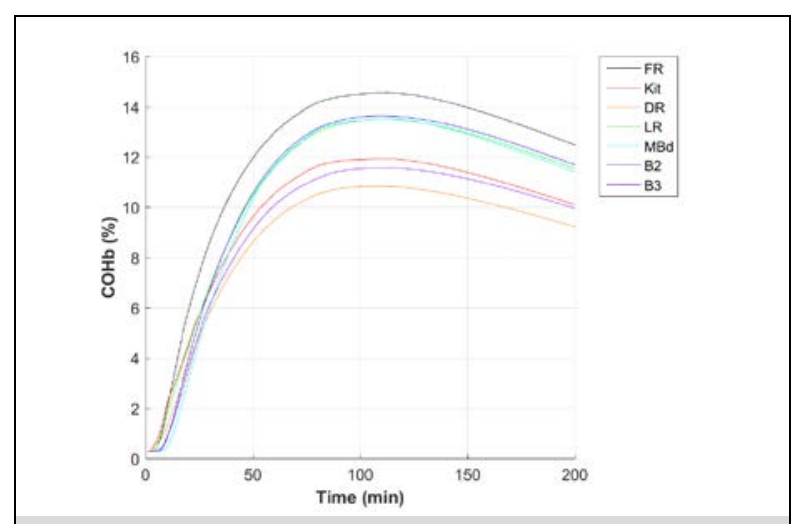

Figure 7e. $\mathrm{COHb}$ of house occupants during Relocation Case 1 Test 1 - G6 G300.

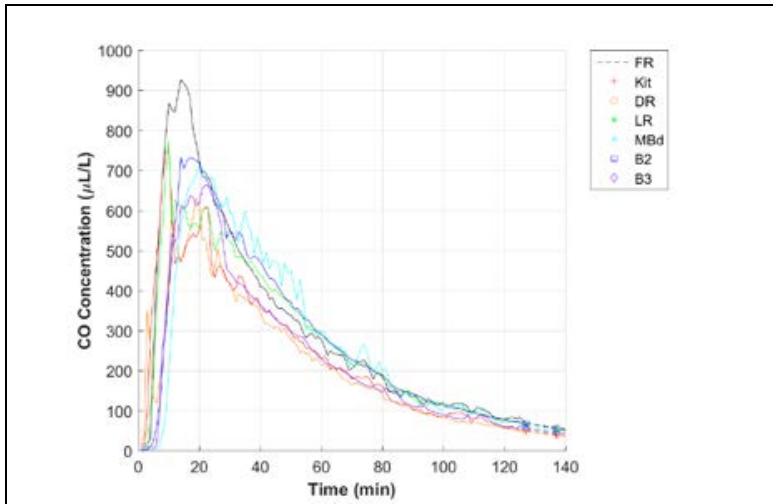

Figure 7d. CO concentration (measured \& projected) in the house during Relocation Case 1 Test 1 - G6 G300. 


\section{Case 1 Test 1 - G7a UL2201}

The following figures illustrate the results for Case 1 Test 1 applying the UL 2201 shutoff criteria ( $>400$ ppmv instantaneous or $>150$ ppmv 10 min average). The G7a generator was fully fueled, positioned in the kitchen (30 $\mathrm{cm}$ from the master bedroom closet wall with the exhaust facing the dining room), and tested at $100 \%$ load $(7,000 \mathrm{~W})$. The kitchen window nearest to the generator was open $10 \mathrm{~cm}$. The shutoff sensor activated the algorithm to shut off the generator after approximately $57 \mathrm{~min}$ due to an average measurement of $150 \mu \mathrm{L} / \mathrm{L}$ for $10 \mathrm{~min}$. Figure 8a shows the test house layout with generator location, generator exhaust direction, and sensor array locations. Figure $\mathbf{8 b}$ shows the CO concentrations measured in the kitchen (by the dedicated room sensor), by two sensors located on the kitchen-family room boundary (A1: height $183 \mathrm{~cm}, \mathrm{~A} 2$ : height $61 \mathrm{~cm}$ ), by the other two sensors located on the kitchen-dining room boundary (A3: height $183 \mathrm{~cm}$, A4: height $61 \mathrm{~cm}$ ), and by the shutoff sensor located on the generator near the generator's electrical panel. The timeframe of the figure has been selected to show the degree of CO uniformity in the area around the generator between the shutoff sensor and the peak CO concentrations measured elsewhere in the kitchen near the time of shutoff. Figure 8c shows the CO concentration measured in each room of the house over the test. Figure 8d shows the CO concentration in the house, with each room represented by a single line composed of both a 'solid-line' pattern (showing measured CO concentration) and a 'dotted-line with a symbol' pattern (showing projected CO concentration). Figure 8e shows the calculated COHb profiles of simulated house occupants in each room of the house.

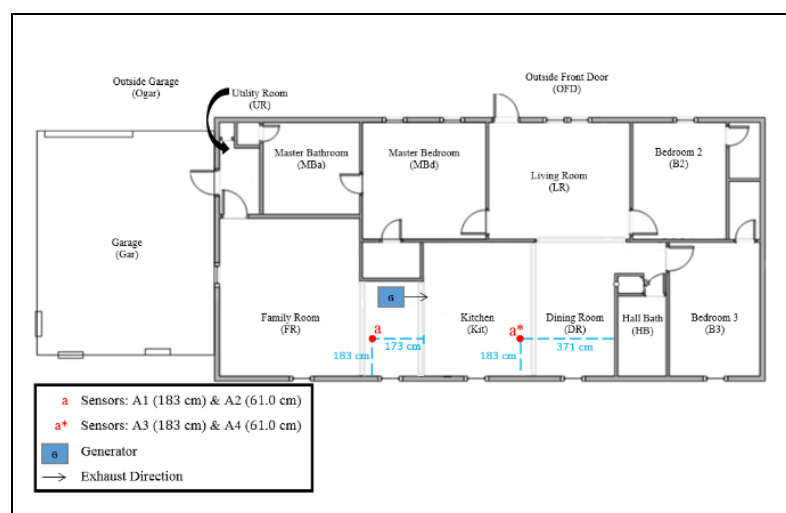

Figure 8a. Generator, exhaust direction, and sensor array layout during Case 1 Test 1 - G7a UL 2201.

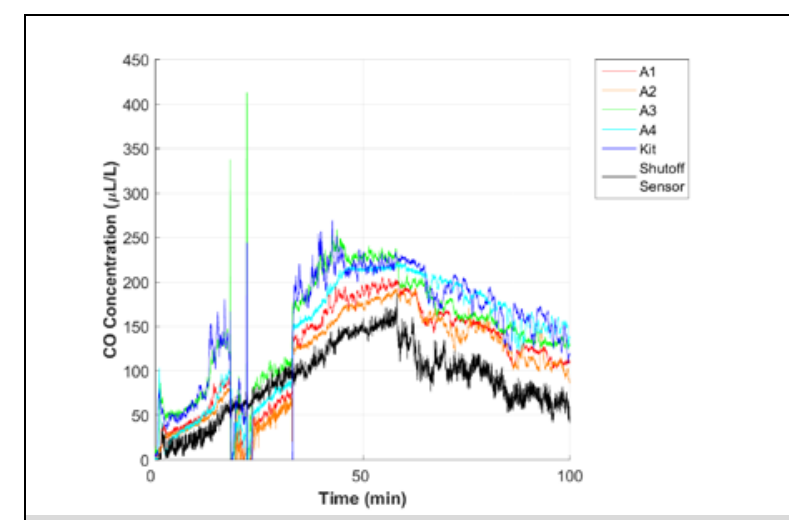

Figure 8b. CO concentration measured by the sensor array and the designated kitchen sensor during Case 1 Test 1 - G7a UL 2201. 


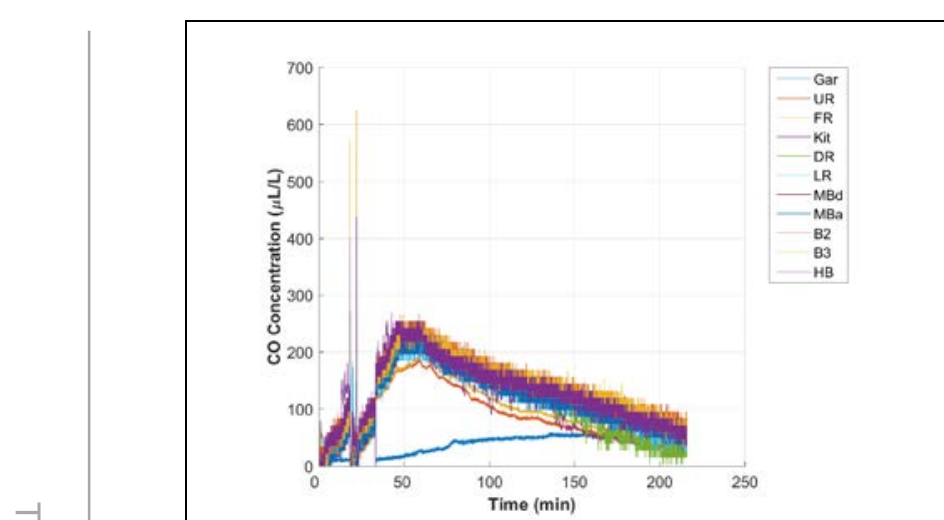

Figure 8c. CO concentration measured in each room of the house during Case 1 Test 1 - G7a UL 2201.

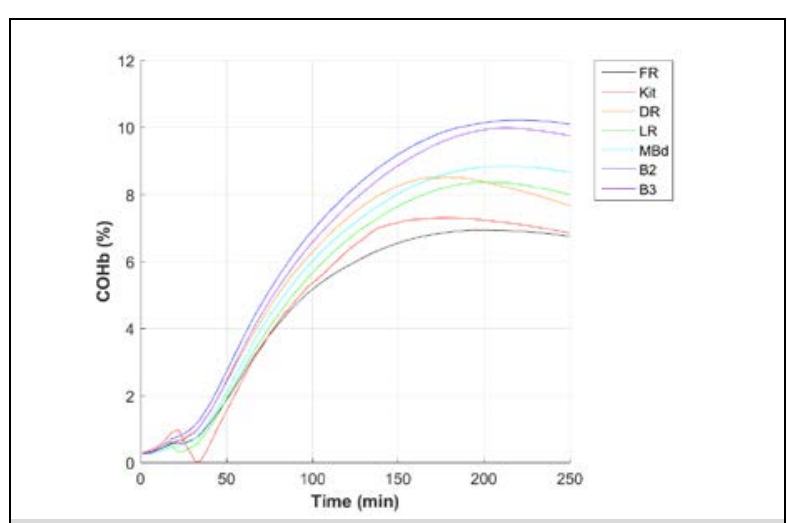

Figure 8e. COHb of house occupants during Case 1 Test 1 - G7a UL 2201.

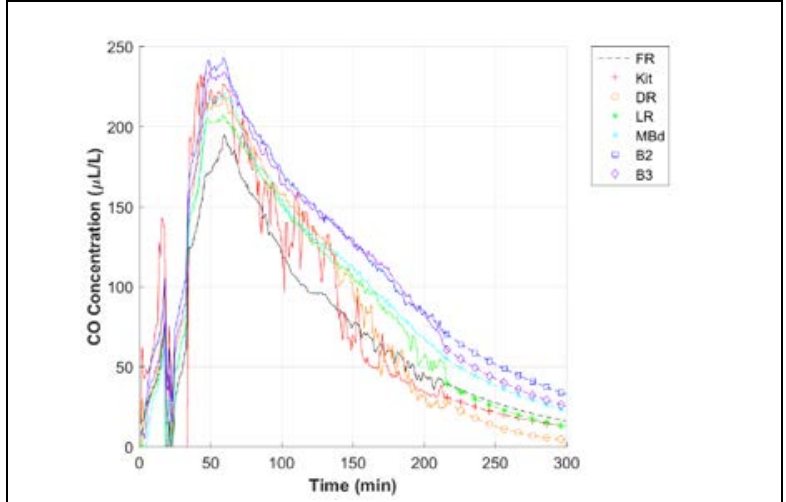

Figure 8d. CO concentration (measured \& projected) in the house during Case 1 Test 1 - G7a UL 2201. 


\section{Case 1 Test 2 - G7b UL2201}

The following figures illustrate the results for Case 1 Test 2 applying the UL 2201 shutoff criteria. The G7b generator was fully fueled, positioned in the kitchen (30 $\mathrm{cm}$ from the master bedroom closet wall with the exhaust facing the dining room), and tested at $100 \%$ load $(7,000 \mathrm{~W})$. The kitchen window nearest to the generator was open $10 \mathrm{~cm}$. The shutoff sensor did not activate the algorithm to shut off the generator. The generator was manually shut down after approximately 270 min due to a house temperature exceeding $60^{\circ} \mathrm{C}$. Figure 9a shows the test house layout with generator location, generator exhaust direction, and sensor array locations. Figure $\mathbf{9 b}$ shows the CO concentrations measured in the kitchen (by the dedicated room sensor), by a sensor located on the kitchen-dining room boundary (A3: height $183 \mathrm{~cm}$ ), and by the shutoff sensor located on the generator near the generator's electrical panel. Three sensors have not been included due to signal interference (A1: height $183 \mathrm{~cm}, \mathrm{A2}$ : height $61 \mathrm{~cm}$, A4: height $61 \mathrm{~cm}$ ). The timeframe of the figure has been selected to show the degree of CO uniformity in the area around the generator between the shutoff sensor and the peak CO concentrations measured elsewhere in the kitchen near the time of shutoff. Figure 9c shows the CO concentration measured in each room of the house over the test. Figure 9d shows the CO concentration in the house, with each room represented by a single line composed of both a 'solid-line' pattern (showing measured CO concentration) and a 'dottedline with a symbol' pattern (showing projected CO concentration). Figure 9e shows the calculated $\mathrm{COHb}$ profiles of simulated house occupants in each room of the house.

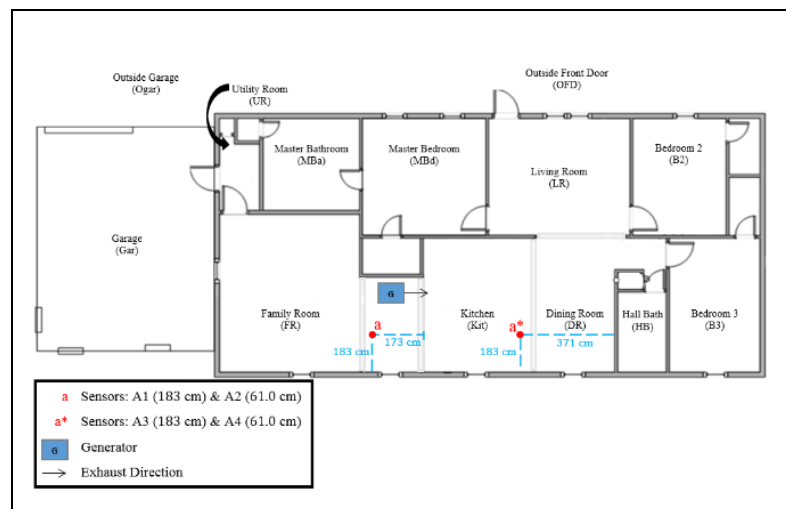

Figure 9a. Generator, exhaust direction, and sensor array layout during Case 1 Test 2 - G7b UL 2201.

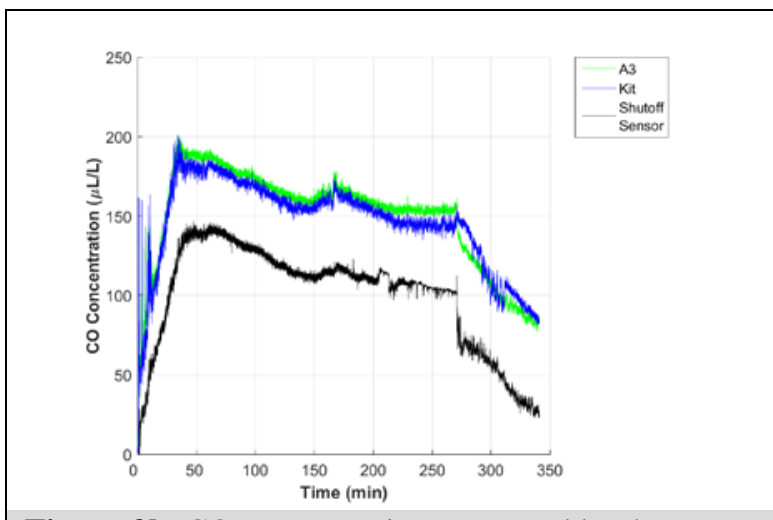

Figure 9b. CO concentration measured by the sensor array and the designated kitchen sensor during Case 1 Test 2 - G7b UL 2201. 


$$
\frac{D}{L}
$$




\section{Case 2 Test 1 - G6 G300}

The following figures illustrate the results for Case 2 Test 1 applying the G300 shutoff criteria. The G6 generator was fully fueled, positioned in the kitchen (30 cm from the master bedroom closet wall with the exhaust facing the dining room), and tested at $50 \%$ load (3,000 W). The kitchen window nearest to the generator was open $10 \mathrm{~cm}$. The shutoff sensor activated the algorithm to shut off the generator after approximately 15 min due to a sensor measurement of 400 $\mu \mathrm{L} / \mathrm{L}$ for a $10 \mathrm{~min}$ average. Figure 10a shows the test house layout with generator location, generator exhaust direction, and sensor array location. Figure 10b shows the CO concentration measured in the kitchen (by the dedicated room sensor), by two sensors located on the kitchenfamily room boundary (A1: height $183 \mathrm{~cm}, \mathrm{A2}$ : height $61 \mathrm{~cm}$ ), by the other two sensors located on the kitchen-dining room boundary (A3: height $183 \mathrm{~cm}$, A4: height $61 \mathrm{~cm}$ ), and by the shutoff sensor located on the generator near the generator's electrical panel. The timeframe of the figure has been selected to show the degree of CO uniformity in the area around the generator between the shutoff sensor and the peak CO concentrations measured elsewhere in the kitchen near the time of shutoff. Figure 10c shows the $\mathrm{CO}$ concentration measured in each room of the house over the test. Figure 10d shows the CO concentration in the house, with each room represented by a single line, which is composed of both a 'solid-line' pattern (showing measured CO concentration) and a 'dotted-line with a symbol' pattern (showing projected CO concentration). Figure 10e shows the $\mathrm{COHb}$ profiles of simulated house occupants in each room of the house.

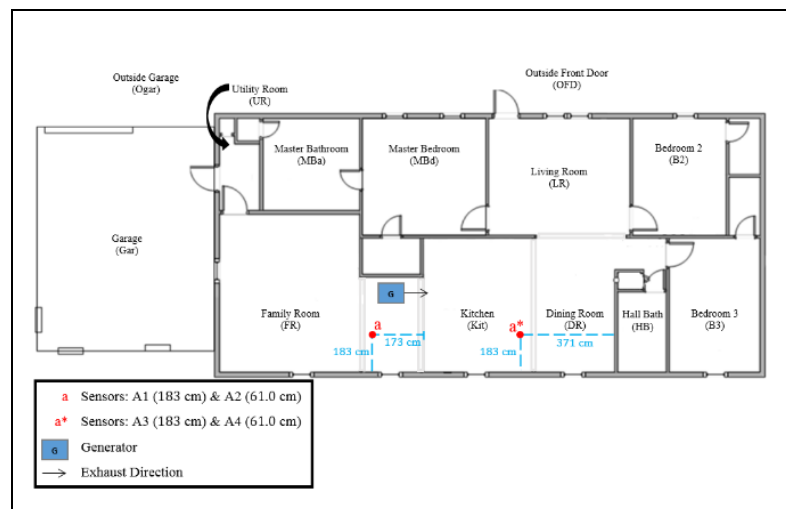

Figure 10a. Generator, exhaust direction, and sensor array layout during Case 2 Test 1 - G6 G300.

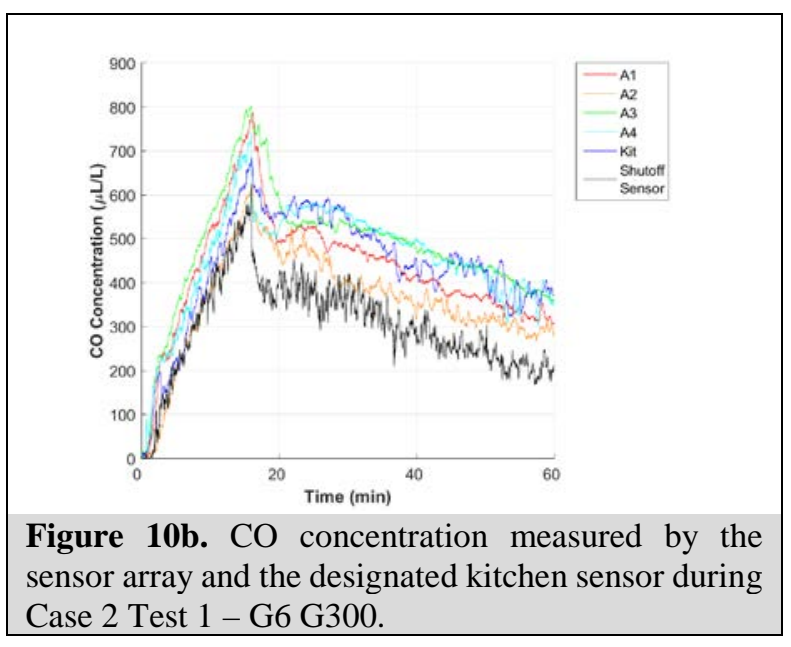




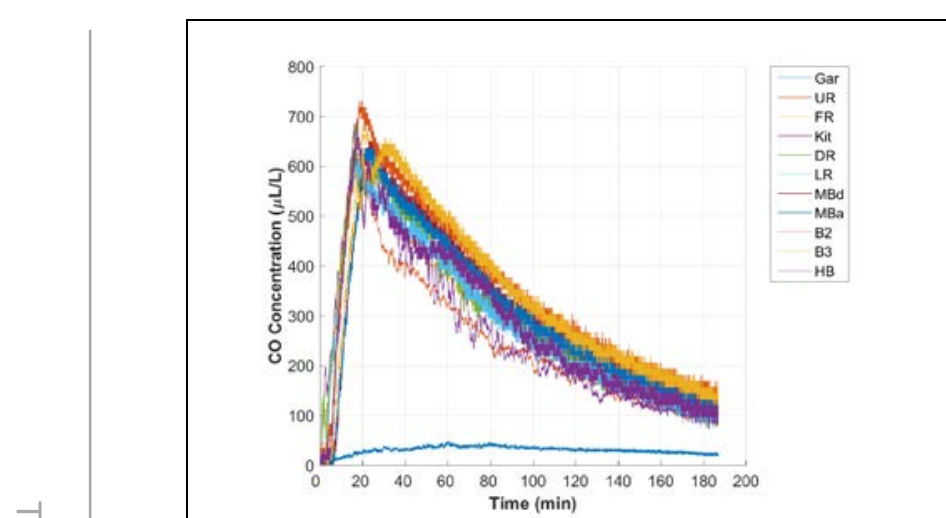

Figure 10c. CO concentration measured in each room of the house during Case 2 Test 1 - G6 G300.

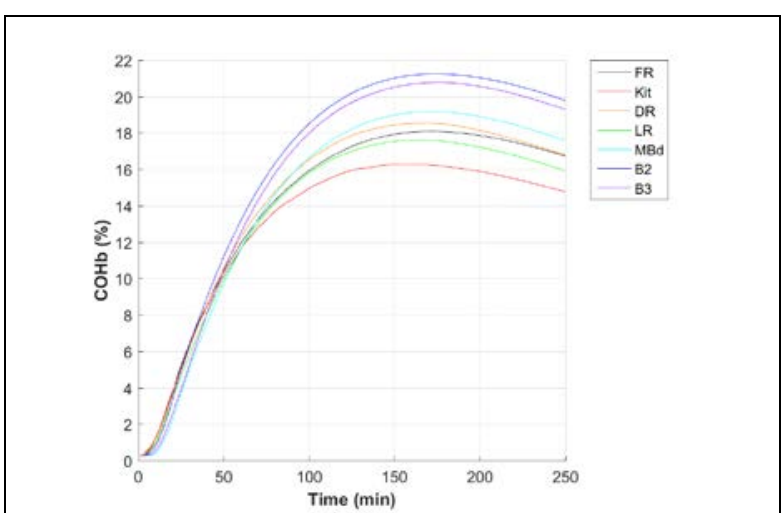

Figure 10e. $\mathrm{COHb}$ of house occupants during Case 2 Test 1 - G6 G300.

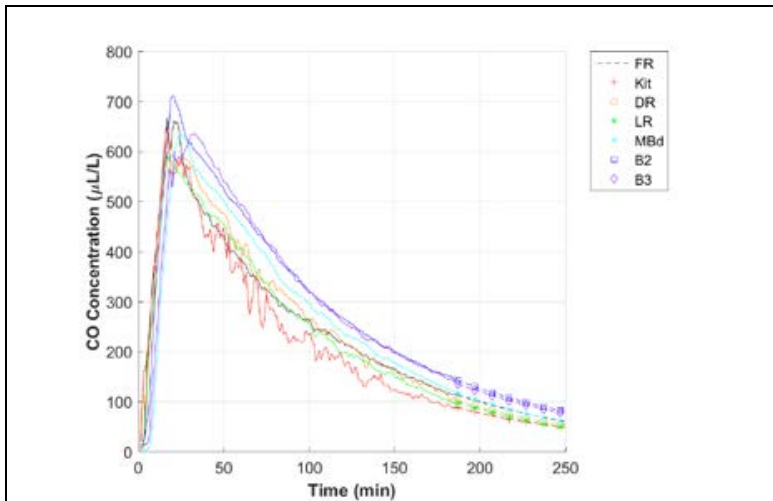

Figure 10d. CO concentration (measured \& projected) in the house during Case 2 Test 1 - G6 G300. 


\section{Case 3 Test 1 - G6 G300}

The following figures illustrate the results for Case 3 Test 1 applying the G300 shutoff criteria. The G6 generator was fully fueled, positioned in the kitchen (30 cm from the master bedroom closet wall with the exhaust facing the dining room), and tested at $100 \%$ load $(6,000 \mathrm{~W})$. The kitchen window nearest to the generator was closed during the test. The shutoff sensor activated the algorithm to shut off the generator after approximately 9 min due to an instantaneous sensor measurement of $800 \mu \mathrm{L} / \mathrm{L}$. Figure 11a shows the test house layout with generator location, exhaust direction, and sensor array location. Figure 11b shows the CO concentration measured in the kitchen (by the dedicated room sensor), by two sensors located on the kitchen-family room boundary (A1: height $183 \mathrm{~cm}, \mathrm{A2}$ : height $61 \mathrm{~cm}$ ), by the other two sensors located on the kitchendining room boundary (A3: height $183 \mathrm{~cm}, \mathrm{~A} 4$ : height $61 \mathrm{~cm}$ ), and by the shutoff sensor located on the generator near the generator's electrical panel. The timeframe of the figure has been selected to show the degree of CO uniformity in the area around the generator between the shutoff sensor and the peak CO concentrations measured elsewhere in the kitchen near the time of shutoff. Figure 11c shows the $\mathrm{CO}$ concentration measured in each room of the house over the test. Figure 11d shows the $\mathrm{CO}$ concentration in the house with each room represented by a single line, which is composed of both a 'solid-line' pattern (showing measured CO concentration) and a 'dotted-line with a symbol' pattern (showing projected CO concentration). Figure 11e shows the COHb profiles of house occupants in each room of the house.

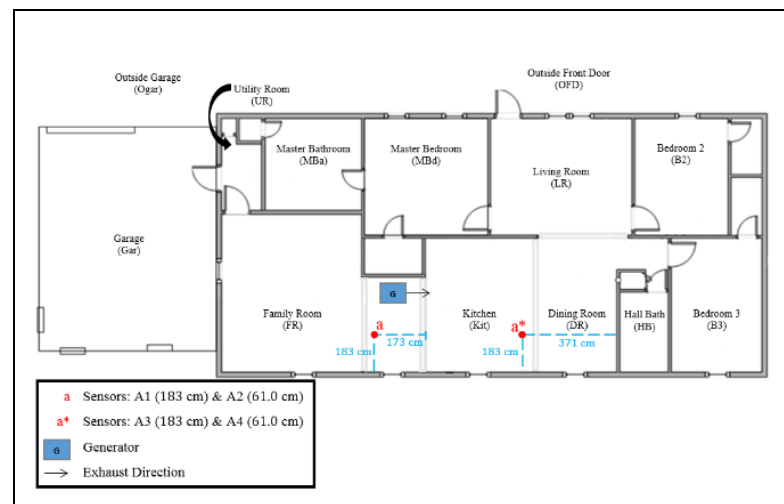

Figure 11a. Generator, exhaust direction, and sensor array layout during Case 3 Test 1 - G6 G300.

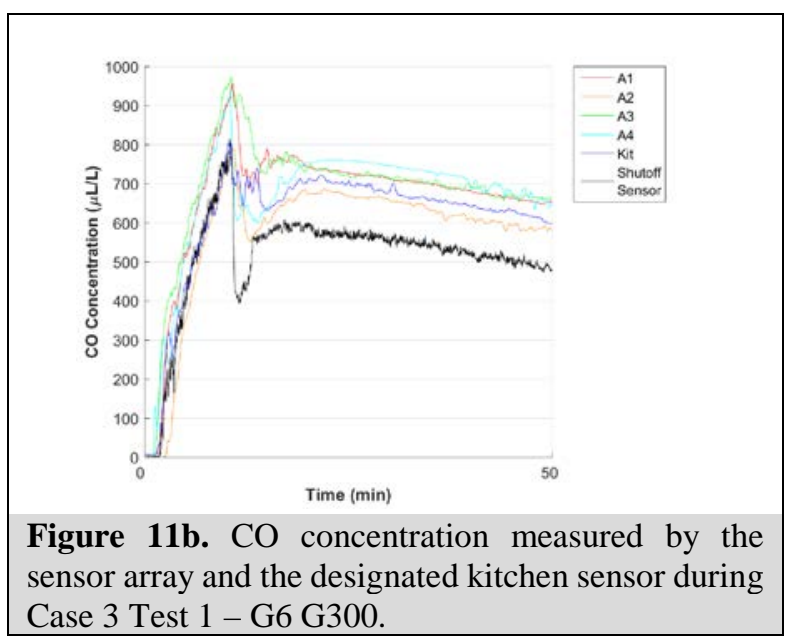




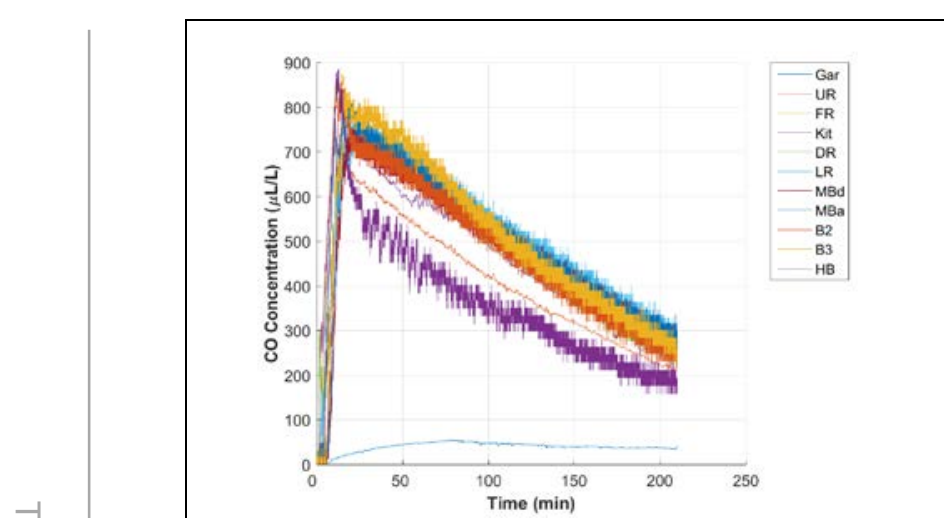

Figure 11c. $\mathrm{CO}$ concentration measured in each room of the house during Case 3 Test 1 - G6 G300.

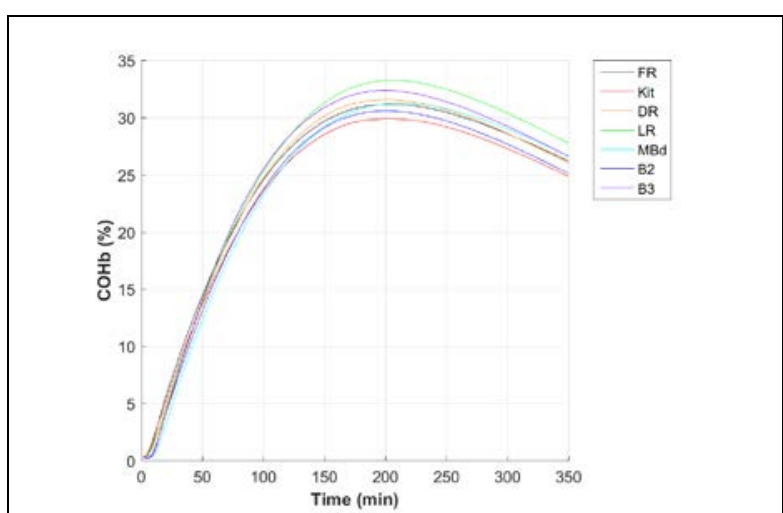

Figure 11e. $\mathrm{COHb}$ of house occupants during Case 3 Test 1 - G6 G300.

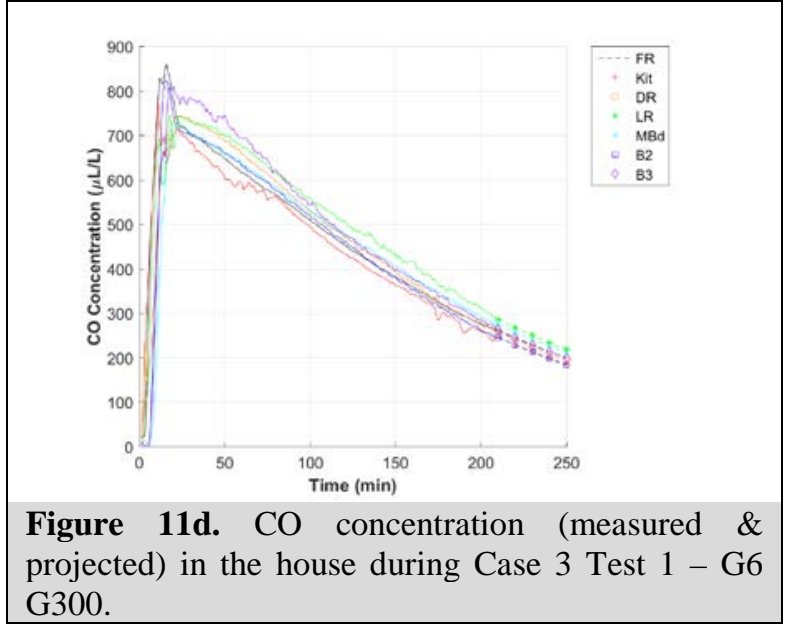

Figure 11d. CO concentration (measured \& projected) in the house during Case 3 Test 1 - G6 


\section{Case 3 Test 1 - G6 G300 Relocate}

The following figures illustrate the results for Relocate Case 3 Test 1 applying the G300 shutoff criteria. The G6 generator was fully fueled, positioned in the kitchen (30 cm from the master bedroom closet wall with the exhaust facing the dining room), and tested at $100 \%$ load $(6,000 \mathrm{~W})$. The kitchen window nearest to the generator was closed during the test. The shutoff sensor activated the algorithm to shut off the generator after approximately 8 min due to an instantaneous sensor measurement of $800 \mu \mathrm{L} / \mathrm{L}$. The front door was initially closed, but $10 \mathrm{~min}$ after the generator shut off, it was opened fully for $1 \mathrm{~min}$, and then it was set to $10 \mathrm{~cm}$ for the remainder of the test. Figure 12a shows the test house layout with generator location, exhaust direction, and sensor array location. Figure 12b shows the $\mathrm{CO}$ concentration measured in the kitchen (by the dedicated room sensor), by two sensors located on the kitchen-family room boundary (A1: height $183 \mathrm{~cm}, \mathrm{~A} 2$ : height $61 \mathrm{~cm}$ ), by the other two sensors located on the kitchen-dining room boundary (A3: height $183 \mathrm{~cm}$, A4: height $61 \mathrm{~cm}$ ), and by the shutoff sensor located on the generator near the generator's electrical panel. The timeframe of the figure has been selected to show the degree of CO uniformity in the area around the generator between the shutoff sensor and the peak CO concentrations measured elsewhere in the kitchen near the time of shutoff. Figure 12c shows the CO concentration measured in each room of the house over the test. Figure 12d shows the CO concentration in the house with each room represented by a single line, which is composed of both a 'solid-line' pattern (showing measured CO concentration) and a 'dotted-line with a symbol' pattern (showing projected CO concentration). Figure 12e shows the COHb profiles of house occupants in each room of the house.

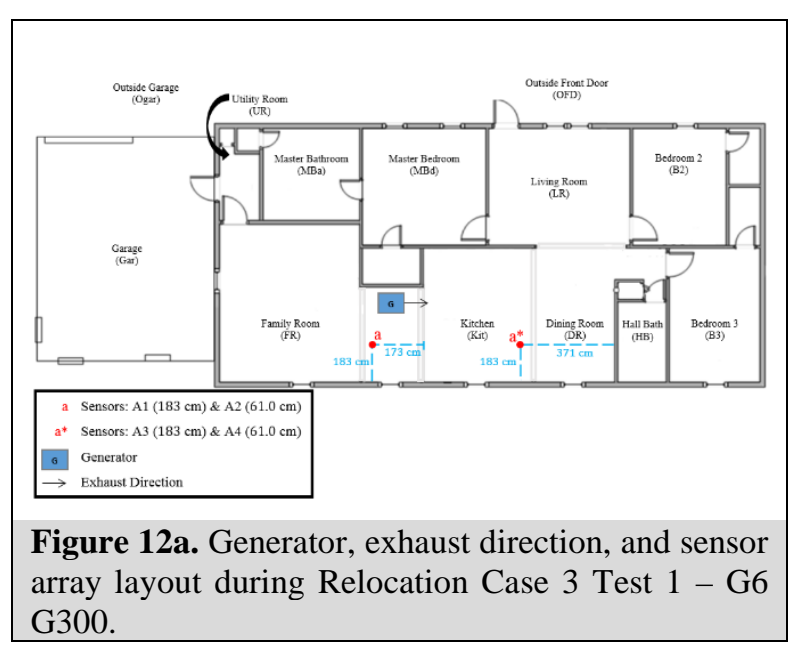

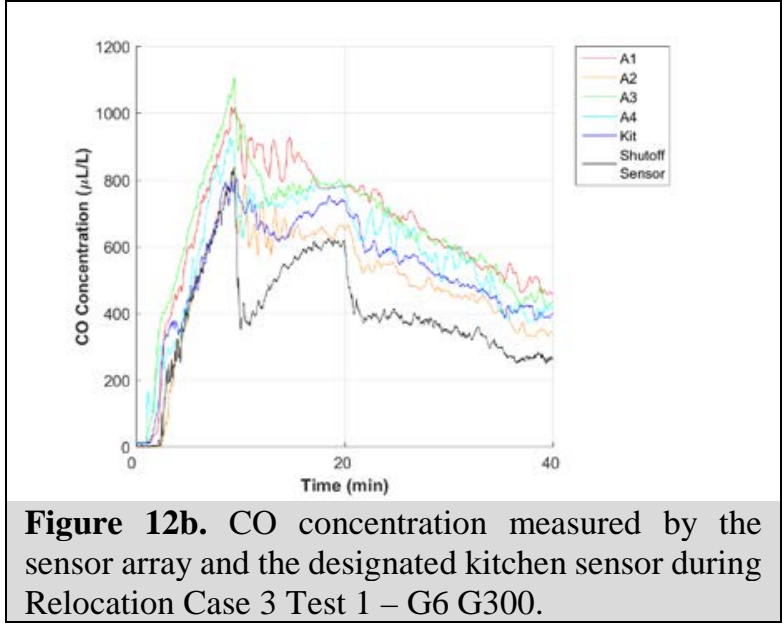



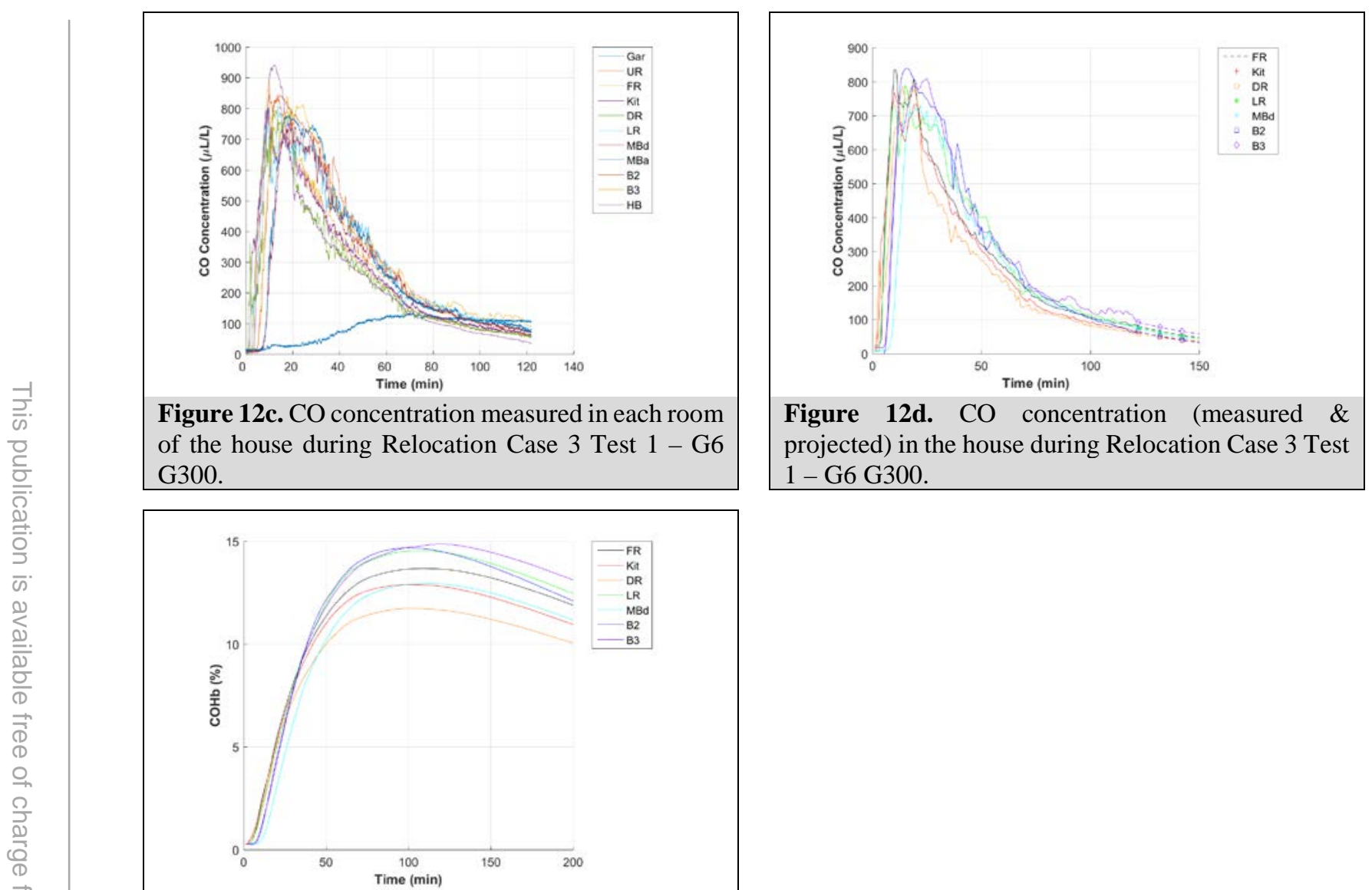

Figure 12e. $\mathrm{COHb}$ of house occupants during Relocation Case 3 Test 1 - G6 G300. 


\section{Case 3 Test 1 - G7b G300}

The following figures illustrate the results for Case 3 Test 1 applying the G300 shutoff criteria. The G7b generator was fully fueled, positioned in the kitchen (30 cm from the master bedroom closet wall with the exhaust facing the dining room), and tested at $100 \%$ load $(7,000 \mathrm{~W})$. The kitchen window nearest to the generator was closed during the test. The shutoff sensor activated the algorithm to shut off the generator after approximately 309 min due to an average sensor measurement of $400 \mu \mathrm{L} / \mathrm{L}$ for $10 \mathrm{~min}$. The generator began to drop the load entirely after about two hours and the kitchen temperature exceeded $60{ }^{\circ} \mathrm{C}$ at the end of the test. Figure 13a shows the test house layout with generator location, exhaust direction, and sensor array location. Figure 13b shows the $\mathrm{CO}$ concentration measured in the kitchen (by the dedicated room sensor), by two sensors located on the kitchen-family room boundary (A1: height $183 \mathrm{~cm}$, A2: height $61.0 \mathrm{~cm})$, by the other two sensors located on the kitchen-dining room boundary (A3: height $183 \mathrm{~cm}$, A4: height $61.0 \mathrm{~cm}$ ), and by the shutoff sensor located on the generator near the generator's electrical panel. The timeframe of the figure has been selected to show the degree of CO uniformity in the area around the generator between the shutoff sensor and the peak $\mathrm{CO}$ concentrations measured elsewhere in the kitchen near the time of shutoff. Figure 13c shows the CO concentration measured in each room of the house over the test. Figure 13d shows the CO concentration in the house with each room represented by a single line, which is composed of both a 'solid-line' pattern (showing measured CO concentration) and a 'dotted-line with a symbol' pattern (showing projected CO concentration). Figure 13e shows the COHb profiles of house occupants in each room of the house.

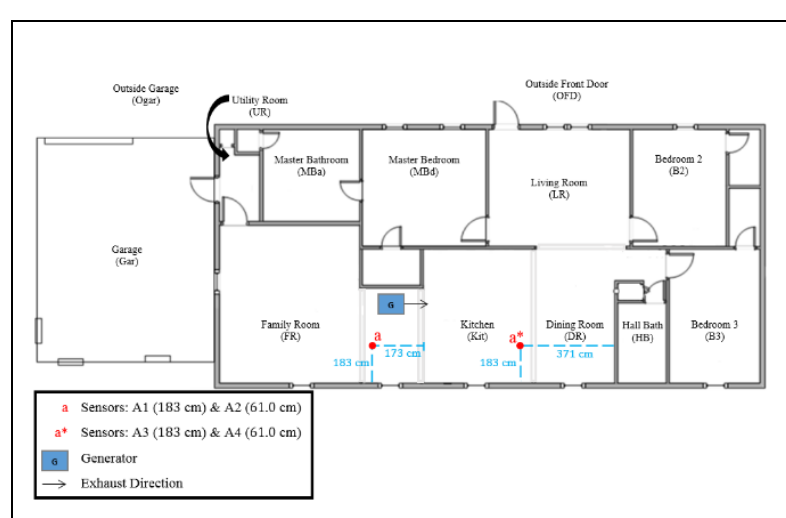

Figure 13a. Generator, exhaust direction, and sensor array layout during Case 3 Test 1 - G7b G300.

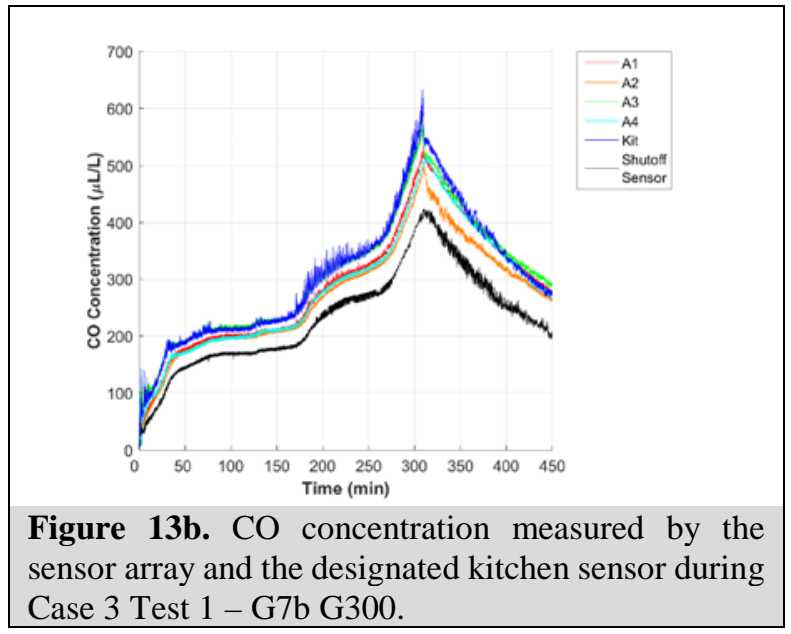

Figure 13b. CO concentration measured by the Case 3 Test 1 - G7b G300. 


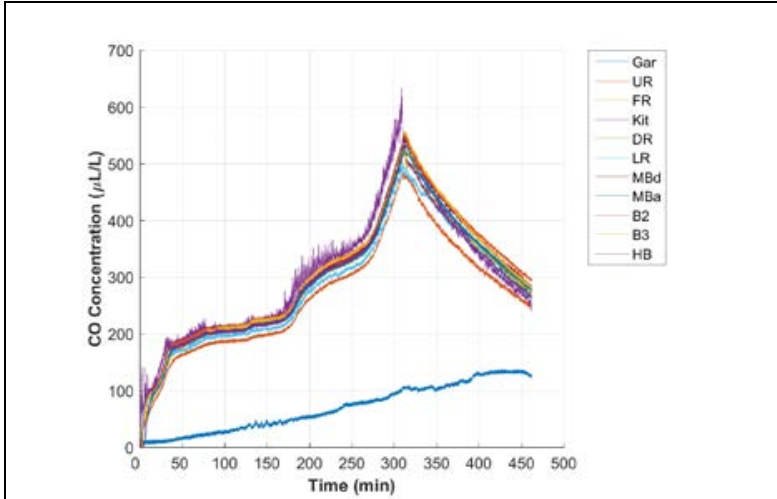

Figure 13c. CO concentration measured in each room of the house during Case 3 Test 1 - G7b G300.

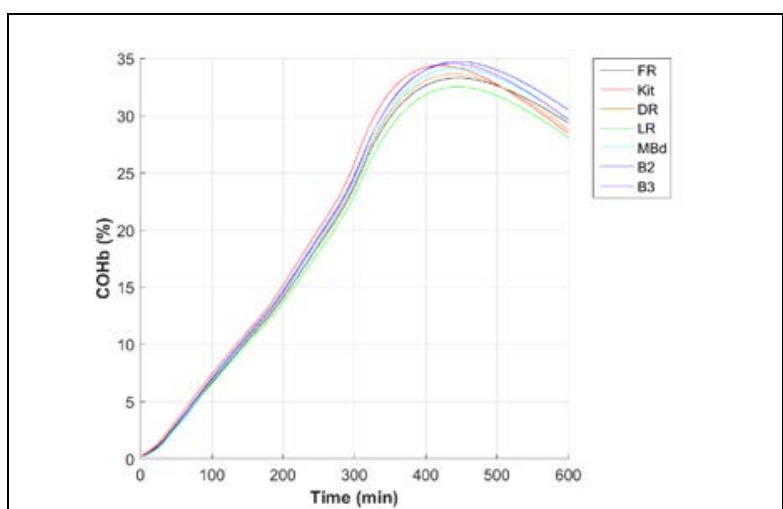

Figure 13e. $\mathrm{COHb}$ of house occupants during Case 3 Test 1 - G7b G300.

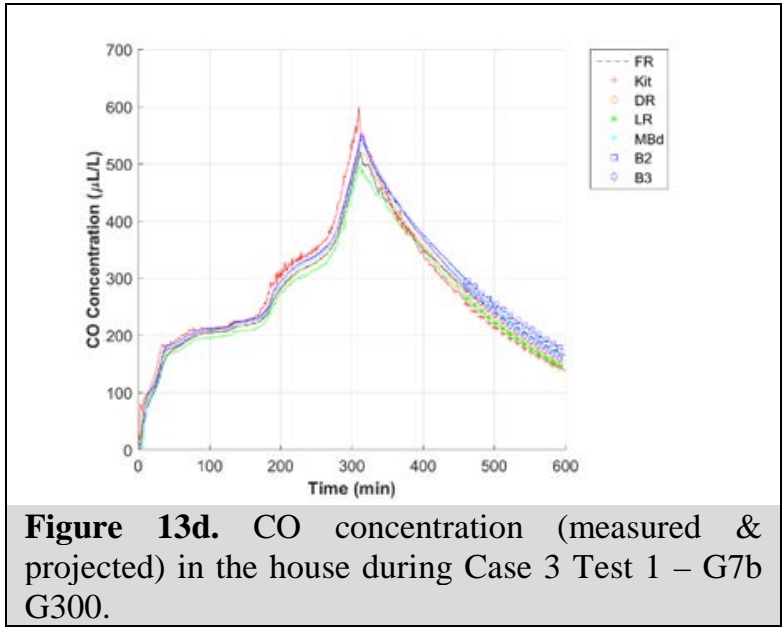

Figure 13d. CO concentration (measured \& projected) in the house during Case 3 Test $1-\mathrm{G} 7 \mathrm{~b}$ 


\section{Case 3 Test 1 - G7a UL 2201}

The following figures illustrate the results for Case 3 Test 1 applying the UL 2201 shutoff criteria. The G7a generator was fully fueled, positioned in the kitchen $(30 \mathrm{~cm}$ from the master bedroom closet wall with the exhaust facing the dining room), and tested at $100 \% \operatorname{load}(7,000 \mathrm{~W})$. The kitchen window nearest to the generator was closed during the test. During the test, the generator was unable to maintain the specified load and subsequently shut down without activating the CO shutoff system. CO concentrations measured by the shutoff sensor were approximately $130 \mathrm{ppmv}$ and rising at the time the generator shut off. Figure 14a shows the test house layout with generator location, generator exhaust direction, and sensor array location. Figure 14b shows the CO concentration measured in the kitchen (by the dedicated room sensor), by two sensors located on the kitchen-family room boundary (A1: height $183 \mathrm{~cm}, \mathrm{A2}$ : height $61 \mathrm{~cm}$ ), by the other two sensors located on the kitchen-dining room boundary (A3: height $183 \mathrm{~cm}$, A4: height $61 \mathrm{~cm}$ ), and by the shutoff sensor located on the generator near the generator's electrical panel. The timeframe of the figure has been selected to show the degree of CO uniformity in the area around the generator between the shutoff sensor and the peak CO concentrations measured elsewhere in the kitchen near the time of shutoff. Figure 14c shows the $\mathrm{CO}$ concentration measured in each room of the house over the test. Figure 14d shows the CO concentration in the house with each room represented by a single line, which is composed of both a 'solid-line' pattern (showing measured CO concentration) and a 'dotted-line with a symbol' pattern (showing projected CO concentration). Figure 14e shows the calculated $\mathrm{COHb}$ profiles of simulated house occupants in each room of the house.

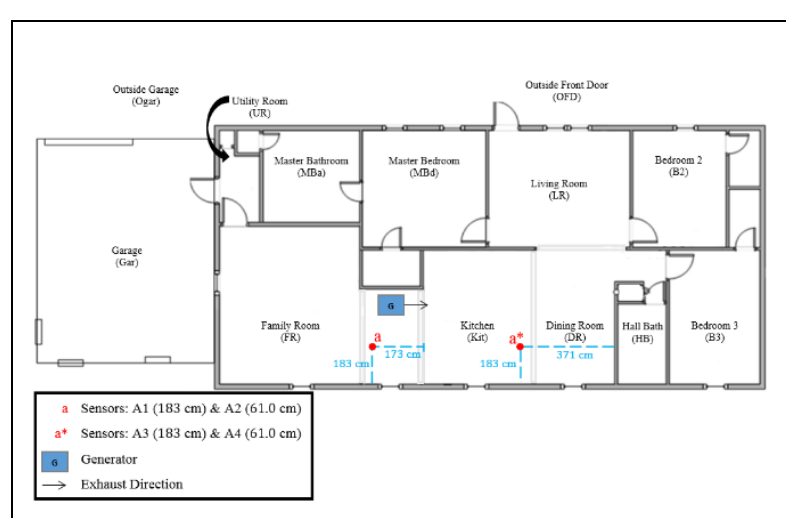

Figure 14a. Generator, exhaust direction, and sensor array layout during Case 3 Test 1 - G7a UL 2201.

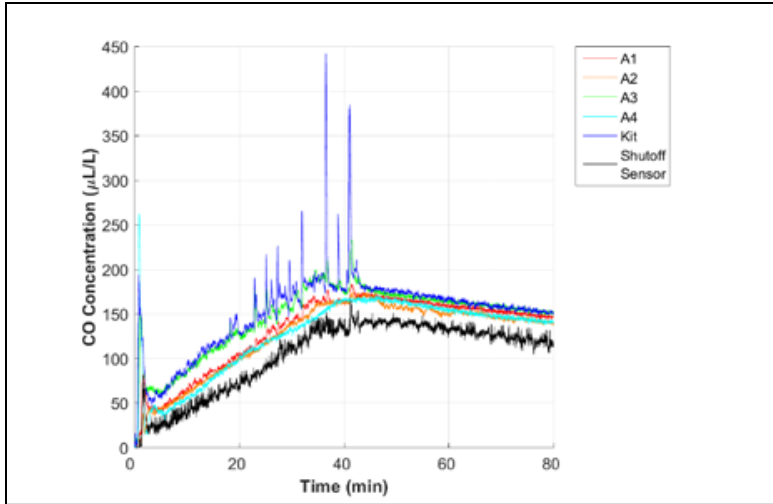

Figure 14b. CO concentration measured by the sensor array and the designated kitchen sensor during Case 3 Test 1 - G7a UL 2201. 


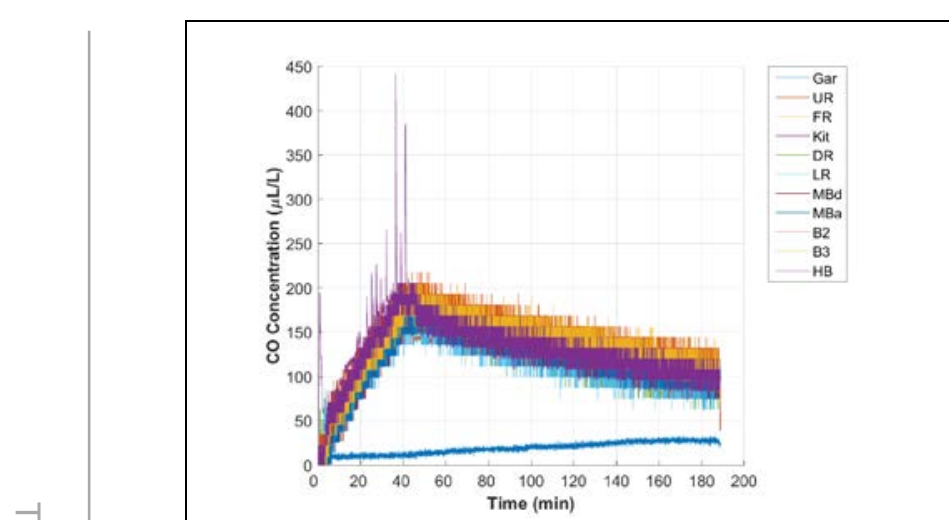

Figure 14c. CO concentration measured in each room of the house during Case 3 Test 1 - G7a UL 2201.

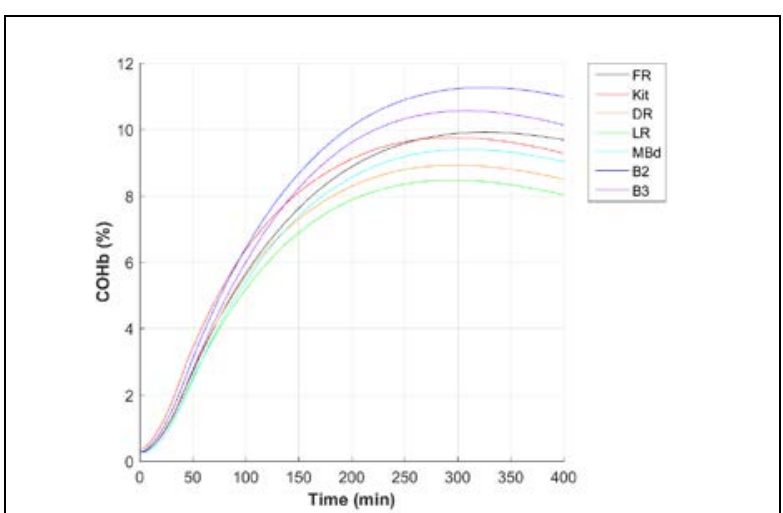

Figure 14e. $\mathrm{COHb}$ of house occupants during Case 3 Test 1 - G7a UL 2201.

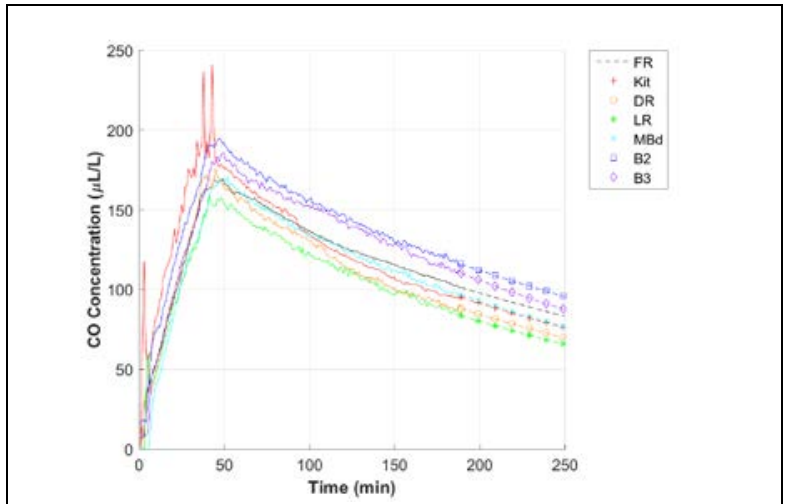

Figure 14d. CO concentration (measured \& projected) in the house during Case 3 Test $1-\mathrm{G} 7 \mathrm{a}$ UL 2201. 


\section{Case 3 Test 2 - G7a UL 2201}

The following figures illustrate the results for Case 3 Test 2 applying the UL 2201 shutoff criteria. The G7a generator was fully fueled, positioned in the kitchen (30 $\mathrm{cm}$ from the master bedroom closet wall with the exhaust facing the dining room), and tested at $100 \% \operatorname{load}(7,000 \mathrm{~W})$. The kitchen window nearest to the generator was closed during the test. The shutoff sensor activated the algorithm to shut off the generator after approximately 30 min due to an instantaneous sensor measurement of $400 \mu \mathrm{L} / \mathrm{L}$. Approximately 25 min after generator startup, there was an unusual large surge in CO. Figure 15a shows the test house layout with generator location, generator exhaust direction, and sensor array location. Figure 15b shows the CO concentration measured in the kitchen (by the dedicated room sensor), by two sensors located on the kitchen-family room boundary (A1: height $183 \mathrm{~cm}, \mathrm{A2}$ : height $61 \mathrm{~cm}$ ), by the other two sensors located on the kitchendining room boundary (A3: height $183 \mathrm{~cm}$, A4: height $61 \mathrm{~cm}$ ), and by the shutoff sensor located on the generator near the generator's electrical panel. The timeframe of the figure has been selected to show the degree of CO uniformity in the area around the generator between the shutoff sensor and the peak $\mathrm{CO}$ concentrations measured elsewhere in the kitchen near the time of shutoff. Figure 15c shows the CO concentration measured in each room of the house over the test. Figure 15d shows the $\mathrm{CO}$ concentration in the house with each room represented by a single line, which is composed of both a 'solid-line' pattern (showing measured CO concentration) and a 'dotted-line with a symbol' pattern (showing projected CO concentration). Figure 15e shows the calculated $\mathrm{COHb}$ profiles of simulated house occupants in each room of the house.

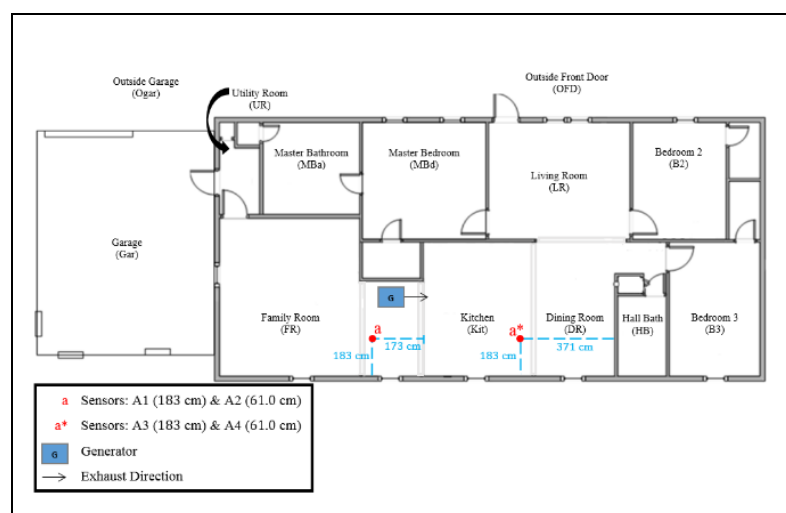

Figure 15a. Generator, exhaust direction, and sensor array layout during Case 3 Test 2 - G7a UL 2201.

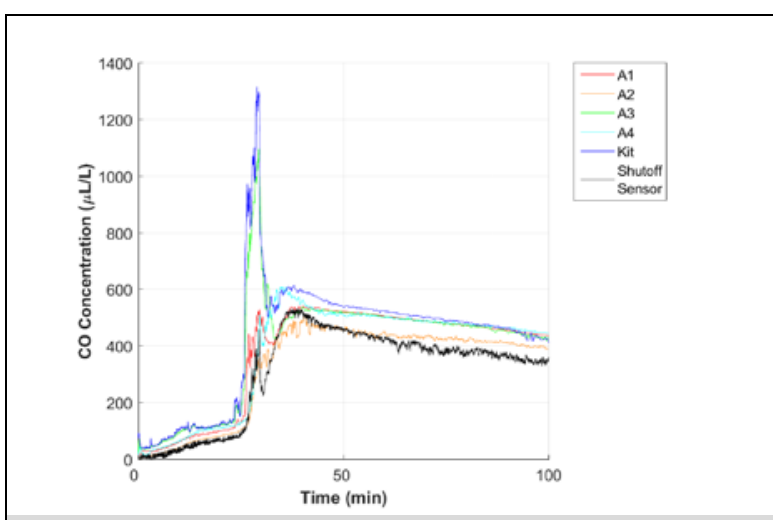

Figure 15b. CO concentration measured by the sensor array and the designated kitchen sensor during Case 3 Test 2 - G7a UL 2201. 


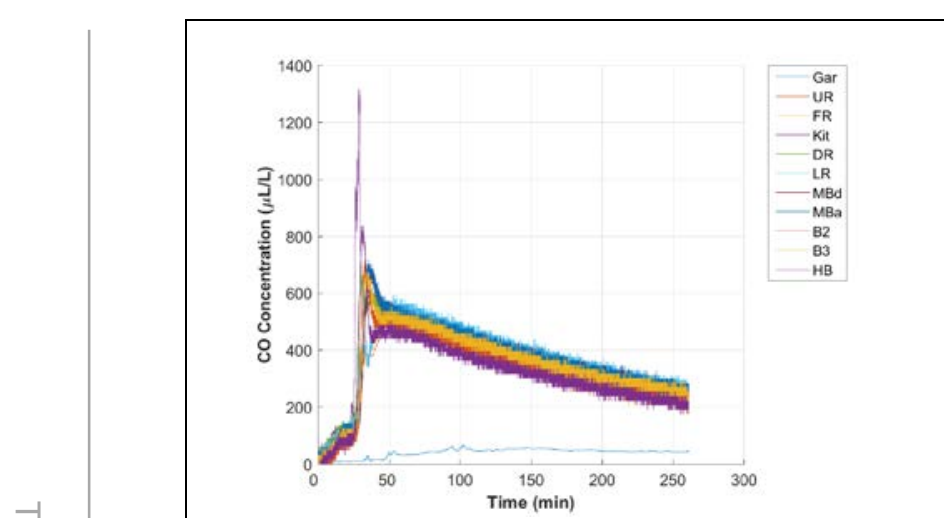

Figure 15c. CO concentration measured in each room of the house during Case 3 Test 2 - G7a UL 2201.

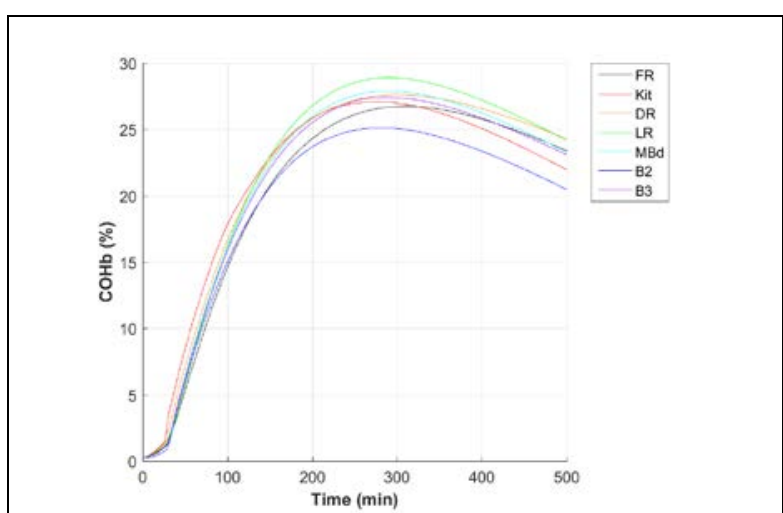

Figure 15e. $\mathrm{COHb}$ of house occupants during Case 3 Test 2 - G7a UL 2201.

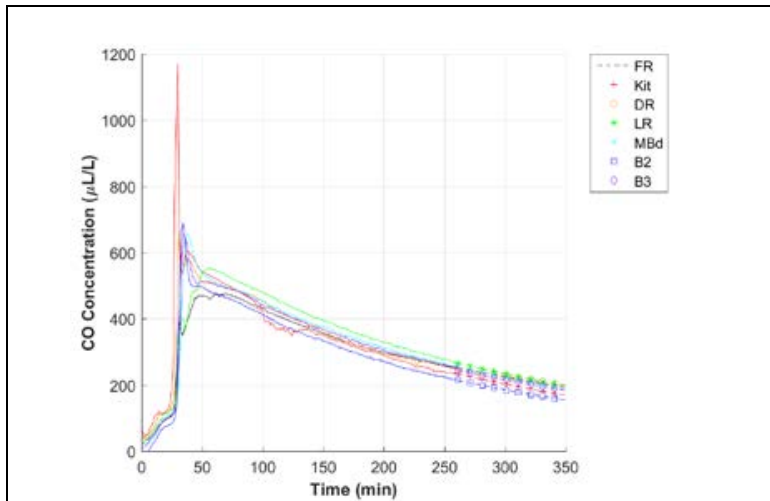

Figure 15d. CO concentration (measured \& projected) in the house during Case 3 Test 2 - G7a UL 2201. 


\section{Case 3 Test 3 - G7b UL2201}

The following figures illustrate the results for Case 3 Test 3 applying the UL 2201 shutoff criteria. The G7b generator was fully fueled, positioned in the kitchen (30 $\mathrm{cm}$ from the master bedroom closet wall with the exhaust facing the dining room), and tested at $100 \%$ load $(7,000 \mathrm{~W})$. The kitchen window nearest to the generator was closed during the test. The shutoff sensor activated the algorithm to shut off the generator after approximately 69 min due to a sensor measurement of $150 \mu \mathrm{L} / \mathrm{L}$ for a $10 \mathrm{~min}$ average. Figure 16a shows the test house layout with generator location, generator exhaust direction, and sensor array location. Figure 16b shows the CO concentrations measured in the kitchen (by the dedicated room sensor), by a sensor located on the kitchen-dining room boundary (A3: height $183 \mathrm{~cm}$ ), and by the shutoff sensor located on the generator near the generator's electrical panel. Three sensors have not been included due to signal interference (A1: height $183 \mathrm{~cm}, \mathrm{~A} 2$ : height $61 \mathrm{~cm}$, A4: height $61 \mathrm{~cm})$. The timeframe of the figure has been selected to show the degree of $\mathrm{CO}$ uniformity in the area around the generator between the shutoff sensor and the peak $\mathrm{CO}$ concentrations measured elsewhere in the kitchen near the time of shutoff. Figure 16c shows the CO concentration measured in each room of the house over the test. Figure 16d shows the $\mathrm{CO}$ concentration in the house with each room represented by a single line, which is composed of both a 'solid-line' pattern (showing measured CO concentration) and a 'dotted-line with a symbol' pattern (showing projected CO concentration). Figure 16e shows the calculated $\mathrm{COHb}$ profiles of simulated house occupants in each room of the house.

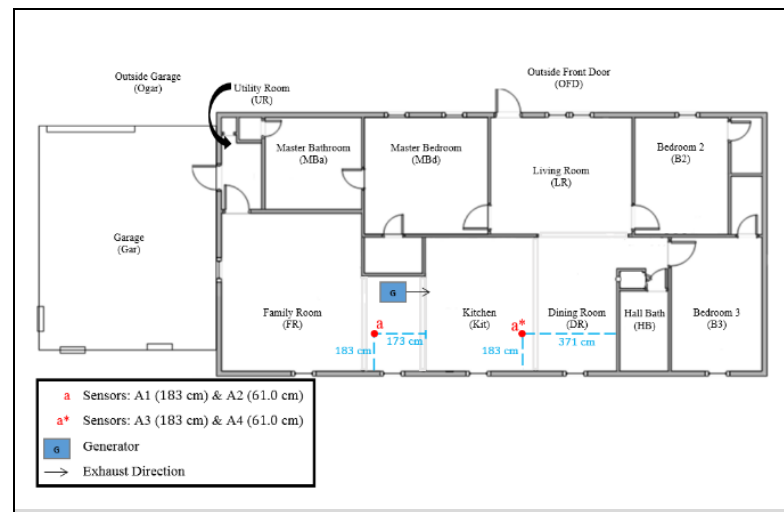

Figure 16a. Generator, exhaust direction, and sensor array layout during Case 3 Test 3 - G7b UL 2201.

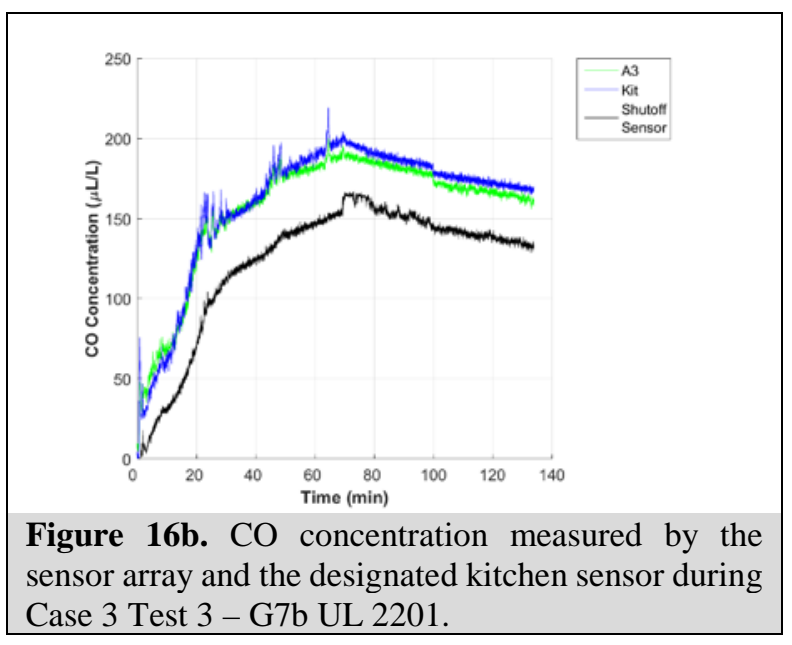




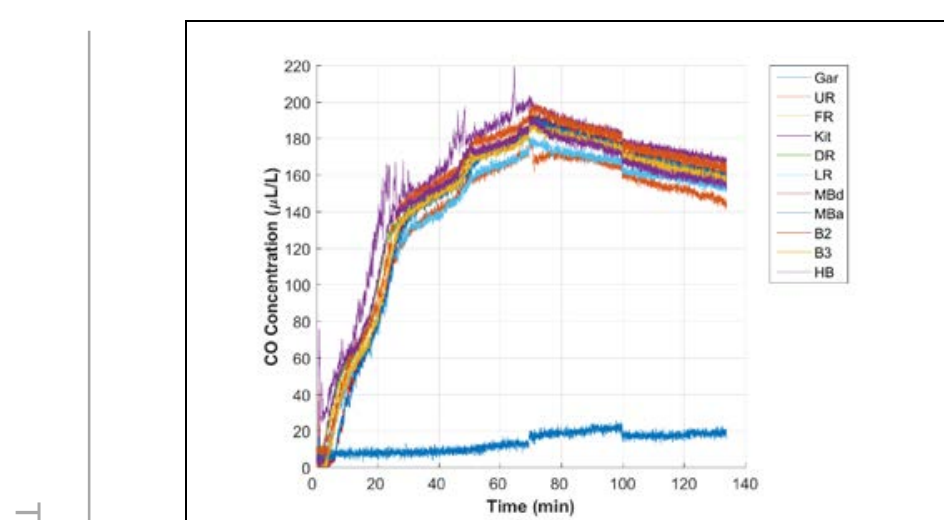

Figure 16c. CO concentration measured in each room of the house during Case 3 Test 3-G7b UL 2201.

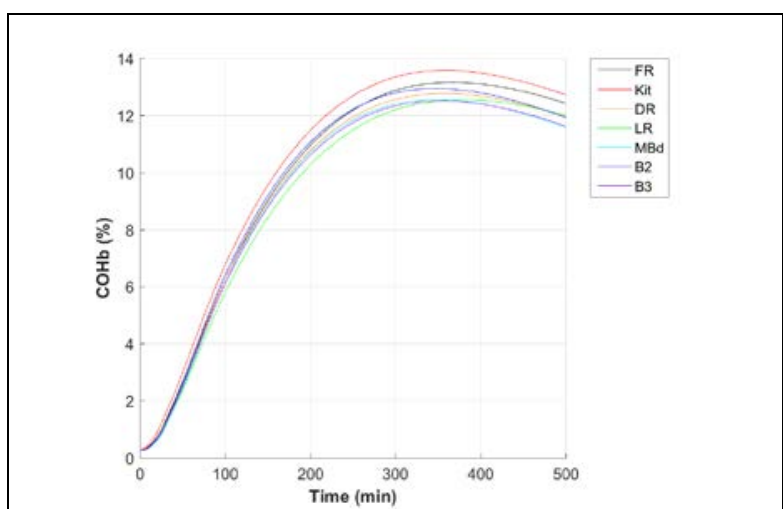

Figure 16e. $\mathrm{COHb}$ of house occupants during Case 3 Test 3-G7b UL 2201.

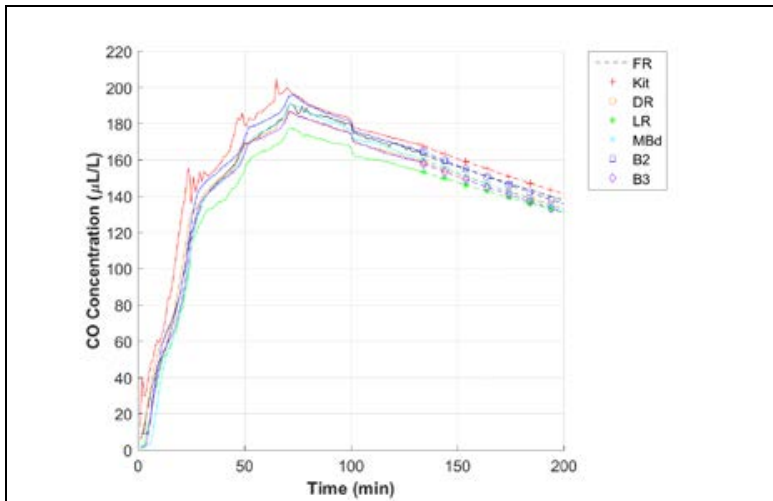

Figure 16d. CO concentration (measured \& projected) in the house during Case 3 Test 3 - G7b UL 2201. 


\section{Case 3 Test 1 - G7b UL 2201 Restart}

The following figures illustrate the results for Restart Case 3 Test 1 applying the UL 2201 shutoff criteria. The G7b generator was fully fueled, positioned in the kitchen $(30 \mathrm{~cm}$ from the master bedroom closet wall with the exhaust facing the dining room), and tested at $100 \%$ load (7,000 W). The kitchen window nearest to the generator was closed during the test. For the first generator run, the shutoff sensor activated the algorithm to shut off the generator after approximately 42 min due to a sensor measurement of $150 \mu \mathrm{L} / \mathrm{L}$ for a $10 \mathrm{~min}$ average. The generator was restarted and the test load restored approximately $10 \mathrm{~min}$ after the first instance of shutoff initiated by the shutoff sensor. For the second generator run, the shutoff sensor activated the algorithm to shut off the generator after approximately 9 min due to a sensor measurement of $150 \mu \mathrm{L} / \mathrm{L}$ for a $10 \mathrm{~min}$ average. Figure 17a shows the test house layout with generator location, generator exhaust direction, and sensor array location. Figure 17b shows the CO concentration measured in the kitchen (by the dedicated room sensor), by two sensors located on the kitchen-family room boundary (A1: height $183 \mathrm{~cm}, \mathrm{~A} 2$ : height $61 \mathrm{~cm}$ ), by the other two sensors located on the kitchendining room boundary (A3: height $183 \mathrm{~cm}, \mathrm{~A} 4$ : height $61 \mathrm{~cm}$ ), and by the shutoff sensor located on the generator near the generator's electrical panel. The timeframe of the figure has been selected to show the degree of $\mathrm{CO}$ uniformity in the area around the generator between the shutoff sensor and the peak CO concentrations measured elsewhere in the kitchen near the time of shutoff. Figure 17c shows the CO concentration measured in each room of the house over the test. Figure 17d shows the $\mathrm{CO}$ concentration in the house with each room represented by a single line, which is composed of both a 'solid-line' pattern (showing measured CO concentration) and a 'dotted-line with a symbol' pattern (showing projected CO concentration). Figure 17e shows the calculated $\mathrm{COHb}$ profiles of simulated house occupants in each room of the house.

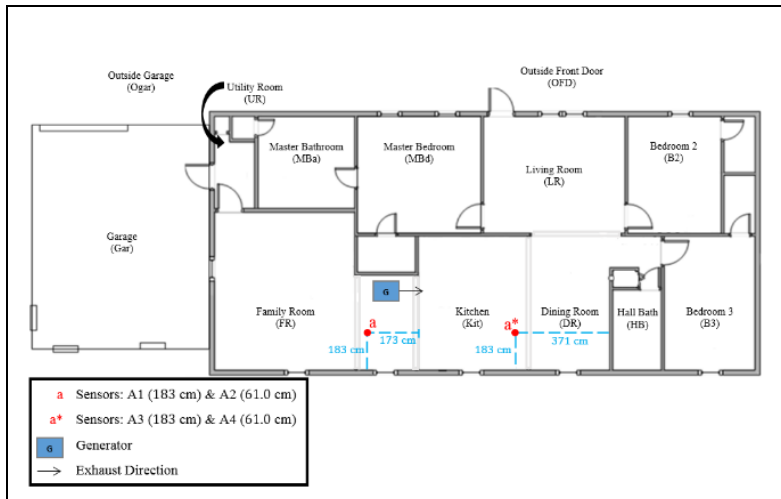

Figure 17a. Generator, exhaust direction, and sensor array layout during Restart Case 3 Test 1 - G7b UL 2201.

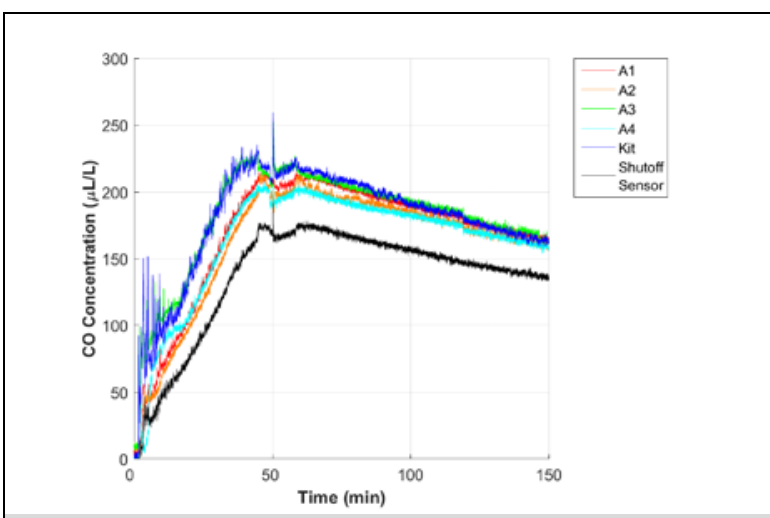

Figure 17b. CO concentration measured by the sensor array and the designated kitchen sensor during Restart Case 3 Test 1 - G7b UL 2201. 


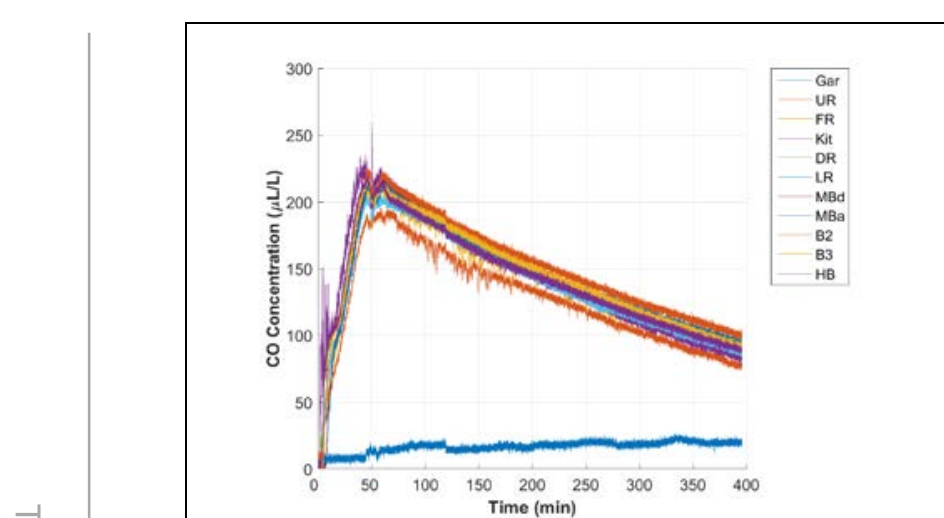

Figure 17c. CO concentration measured in each room of the house during Restart Case 3 Test 1 - G7b UL 2201.

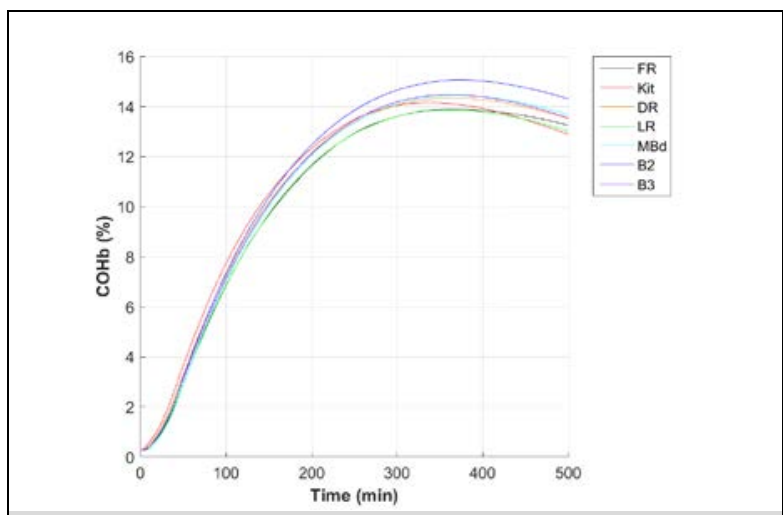

Figure 17e. COHb of house occupants during Restart Case 3 Test 1 - G7b UL 2201.

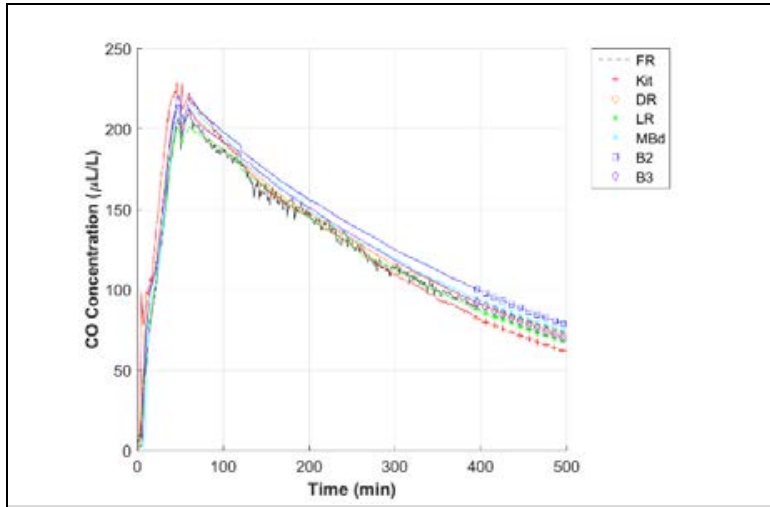

Figure 17d. CO concentration (measured \& projected) in the house during Restart Case 3 Test 1 G7b UL 2201. 


\section{Case 4 Test 1 - G6 G300}

The following figures illustrate the results for Case 4 Test 1 applying the G300 shutoff criteria. The G6 generator was fully fueled, positioned in the kitchen (30 cm from the master bedroom closet wall with the exhaust facing the dining room), and tested at $50 \%$ load (3,000 W). The kitchen window nearest to the generator was closed during the test. The shutoff sensor activated the algorithm to shut off the generator after approximately 16 min due to a sensor measurement of $400 \mu \mathrm{L} / \mathrm{L}$ for a 10 min average. Figure 18a shows the test house layout with generator location, exhaust direction, and sensor array location. Figure 18b shows the CO concentration measured in the kitchen (by the dedicated room sensor), by two sensors located on the kitchen-family room boundary (A1: height $183 \mathrm{~cm}, \mathrm{A2}$ : height $61 \mathrm{~cm}$ ), by the other two sensors located on the kitchendining room boundary (A3: height $183 \mathrm{~cm}$, A4: height $61 \mathrm{~cm}$ ), and by the shutoff sensor located on the generator near the generator's electrical panel. The timeframe of the figure has been selected to show the degree of CO uniformity in the area around the generator between the shutoff sensor and the peak CO concentrations measured elsewhere in the kitchen near the time of shutoff. Figure 18c shows the $\mathrm{CO}$ concentration measured in each room of the house over the test. Figure 18d shows the $\mathrm{CO}$ concentration in the house with each room represented by a single line, which is composed of both a 'solid-line' pattern (showing measured CO concentration) and a 'dotted-line with a symbol' pattern (showing projected CO concentration). Figure 18e shows the calculated $\mathrm{COHb}$ profiles of house occupants in each room of the house.

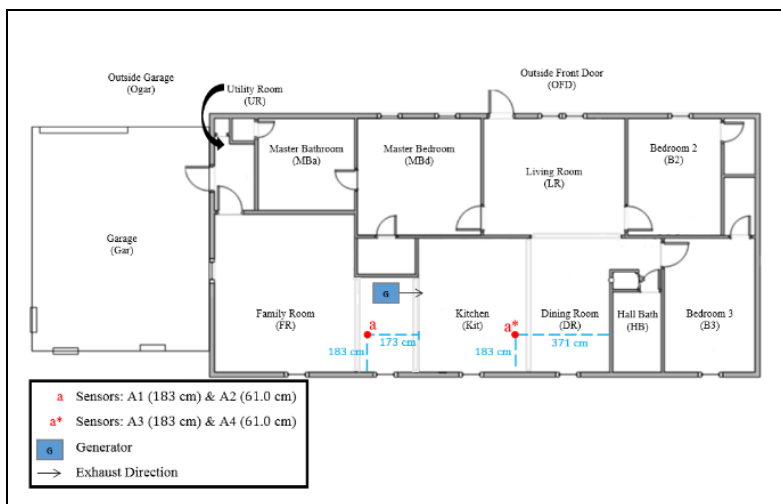

Figure 18a. Generator, exhaust direction, and sensor array layout during Case 4 Test 1 - G6 G300.

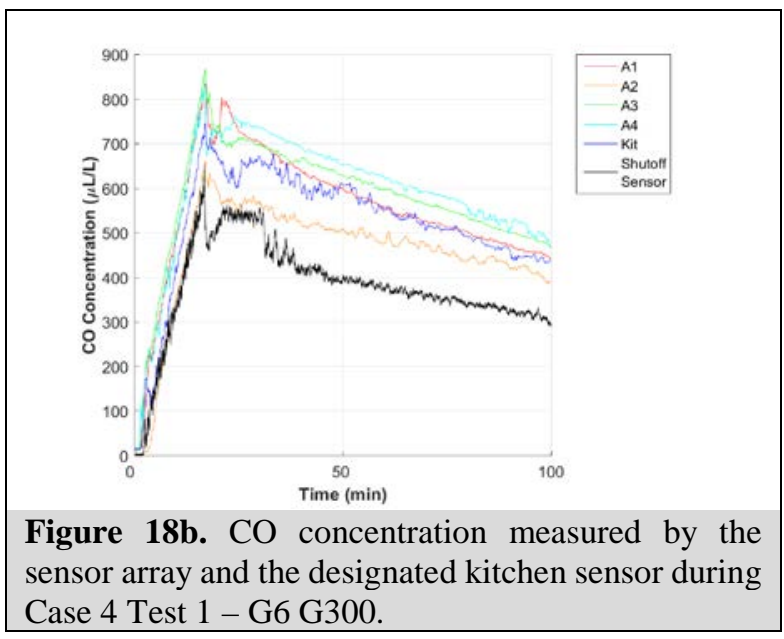




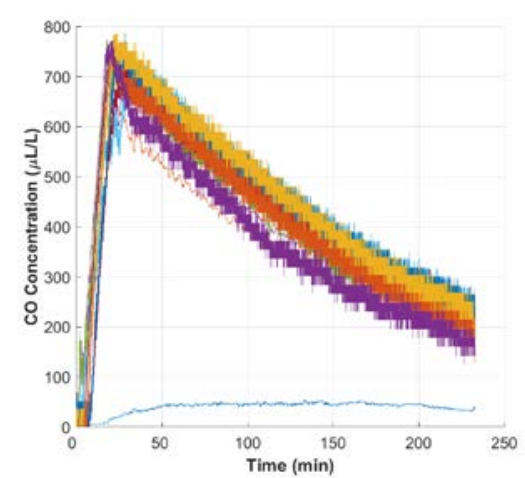

Figure 18c. CO concentration measured in each room of the house during Case 4 Test 1 - G6 G300.

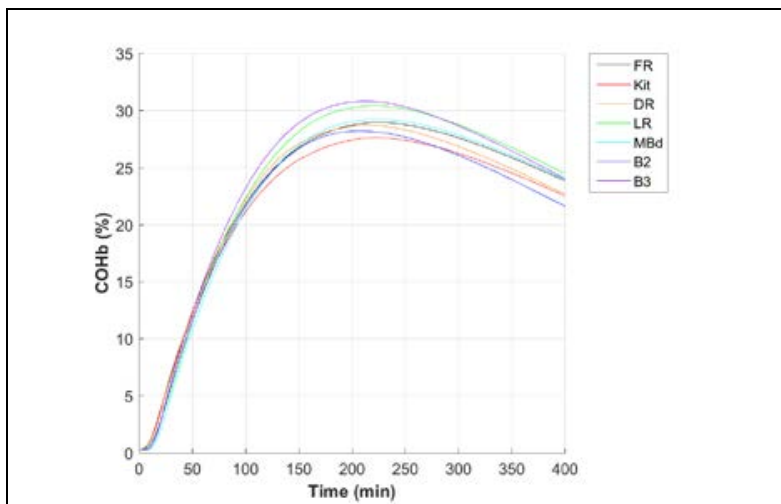

Figure 18e. $\mathrm{COHb}$ of house occupants during Case 4 Test 1 - G6 G300.

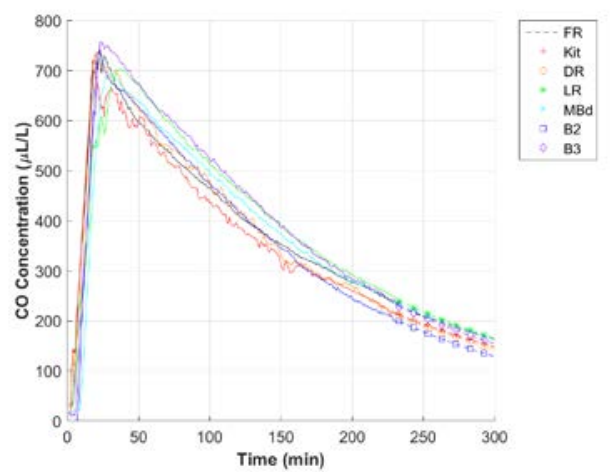

Figure 18d. CO concentration (measured \& projected) in the house during Case 4 Test 1 - G6 G300. 


\section{Case 4 Test 1 - G7a UL2201}

The following figures illustrate the results for Case 4 Test 1 applying the UL 2201 shutoff criteria. The G7a generator was fully fueled, positioned in the kitchen $(30 \mathrm{~cm}$ from the master bedroom closet wall with the exhaust facing the dining room), and tested at $50 \%$ load $(3,500 \mathrm{~W})$. The kitchen window nearest to the generator was closed during the test. The shutoff sensor activated the algorithm to shut off the generator after approximately 38 min due to a sensor measurement of $150 \mu \mathrm{L} / \mathrm{L}$ for a $10 \mathrm{~min}$ average. Figure 19a shows the test house layout with generator location, generator exhaust direction, and sensor array location. Figure 19b shows the CO concentration measured in the kitchen (by the dedicated room sensor), by two sensors located on the kitchenfamily room boundary (A1: height $183 \mathrm{~cm}, \mathrm{~A} 2$ : height $61 \mathrm{~cm}$ ), by the other two sensors located on the kitchen-dining room boundary (A3: height $183 \mathrm{~cm}$, A4: height $61 \mathrm{~cm}$ ), and by the shutoff sensor located on the generator near the generator's electrical panel. The timeframe of the figure has been selected to show the degree of CO uniformity in the area around the generator between the shutoff sensor and the peak CO concentrations measured elsewhere in the kitchen near the time of shutoff. Figure 19c shows the CO concentration measured in each room of the house over the test. Figure 19d shows the CO concentration in the house with each room represented by a single line, which is composed of both a 'solid-line' pattern (showing measured CO concentration) and a 'dotted-line with a symbol' pattern (showing projected CO concentration). Figure 19e shows the calculated $\mathrm{COHb}$ profiles of simulated house occupants in each room of the house.

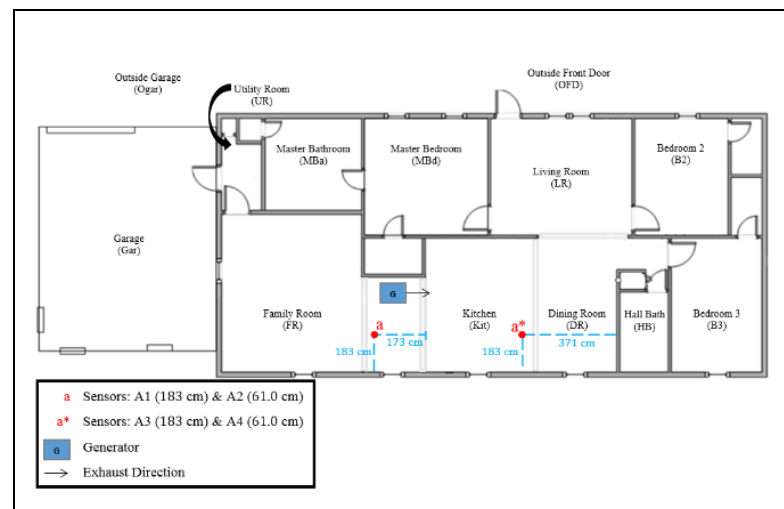

Figure 19a. Generator, exhaust direction, and sensor array layout during Case 4 Test 1 - G7a UL 2201.

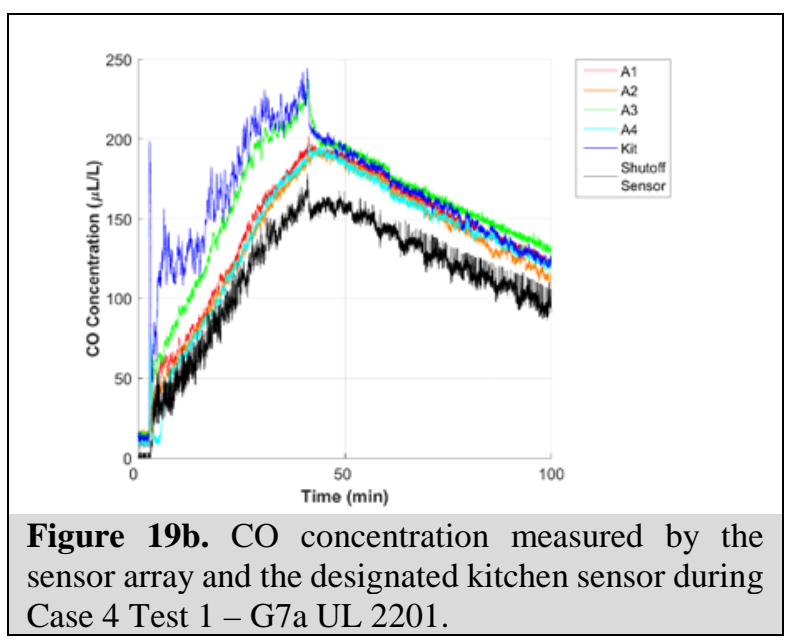




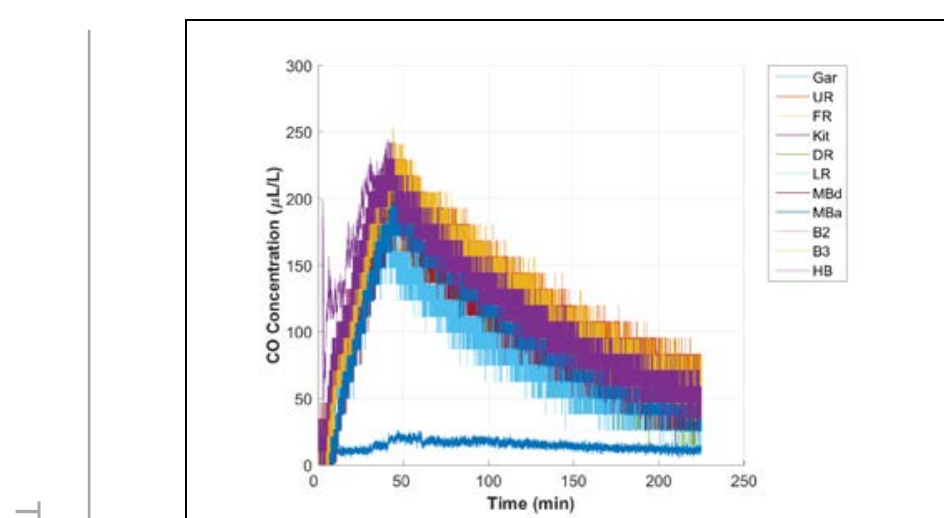

Figure 19c. CO concentration measured in each room of the house during Case 4 Test 1 - G7a UL 2201.

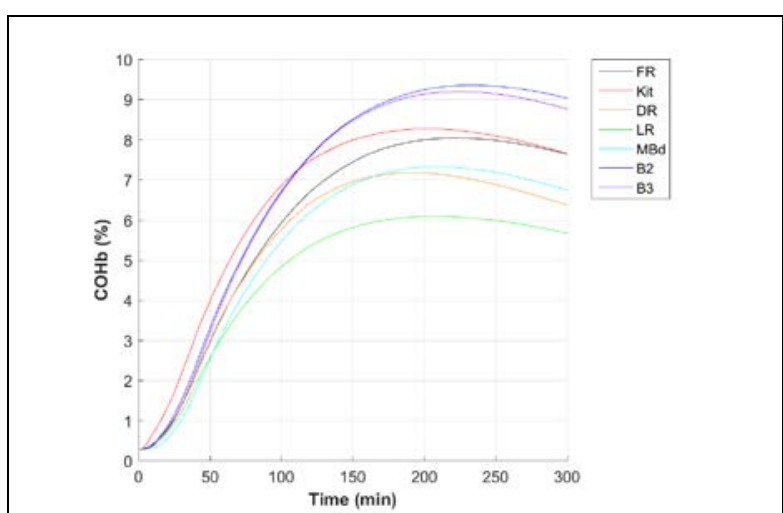

Figure 19e. $\mathrm{COHb}$ of house occupants during Case 4 Test 1 - G7a UL 2201.

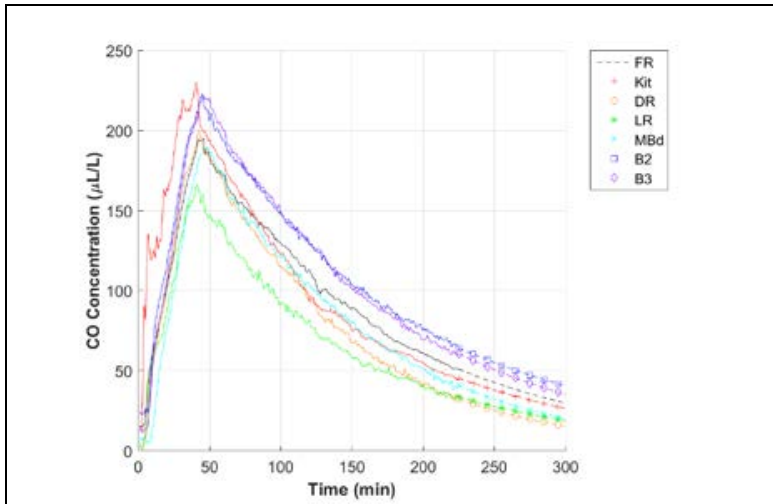

Figure 19d. CO concentration (measured \& projected) in the house during Case 4 Test 1 - G7a UL 2201. 


\section{Case 4 Test 2 - G7b UL 2201}

The following figures illustrate the results for Case 4 Test 2 applying the UL 2201 shutoff criteria. The G7b generator was fully fueled, positioned in the kitchen $(30 \mathrm{~cm}$ from the master bedroom closet wall with the exhaust facing the dining room), and tested at $50 \%$ load (3,000 W). The kitchen window nearest to the generator was closed during the test. The sample pump for the shutoff sensor was inadvertently not switched on until 7 min after the generator was started, however, this did not impact the test results. The shutoff sensor activated the algorithm to shut off the generator after approximately $26 \mathrm{~min}$ due to a sensor measurement of $150 \mu \mathrm{L} / \mathrm{L}$ for a $10 \mathrm{~min}$ average. Figure 20a shows the test house layout with generator location, exhaust direction, and sensor array location. Figure 20b shows the $\mathrm{CO}$ concentration measured in the kitchen (by the dedicated room sensor), by two sensors located on the kitchen-family room boundary (A1: height $183 \mathrm{~cm}, \mathrm{A2}$ : height $61 \mathrm{~cm}$ ), by the other two sensors located on the kitchen-dining room boundary (A3: height $183 \mathrm{~cm}, \mathrm{~A} 4$ : height $61 \mathrm{~cm}$ ), and by the shutoff sensor located on the generator near the generator's electrical panel. The timeframe of the figure has been selected to show the degree of CO uniformity in the area around the generator between the shutoff sensor and the peak CO concentrations measured elsewhere in the kitchen near the time of shutoff. Figure 20c shows the $\mathrm{CO}$ concentration measured in each room of the house over the test. Figure 20d shows the CO concentration in the house with each room represented by a single line, which is composed of both a 'solid-line' pattern (showing measured CO concentration) and a 'dotted-line with a symbol' pattern (showing projected CO concentration). Figure 20e shows the $\mathrm{COHb}$ profiles of house occupants in each room of the house.

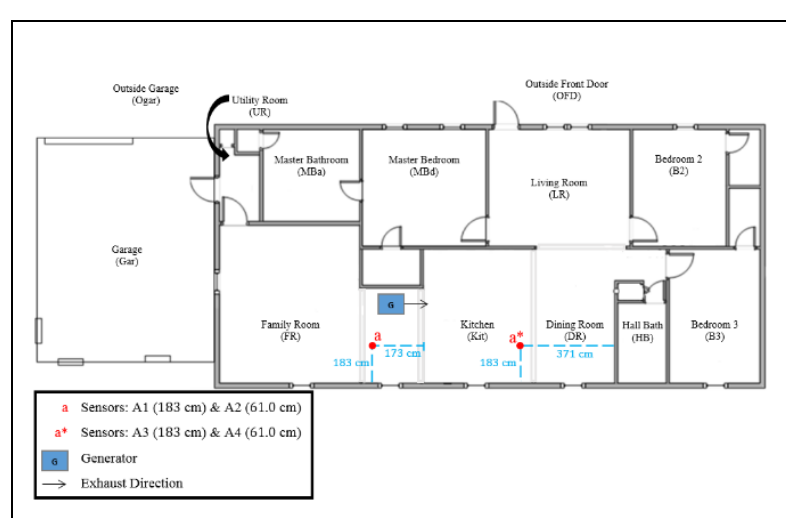

Figure 20a. Generator, exhaust direction, and sensor array layout during Case 4 Test 2 - G7b UL 2201.

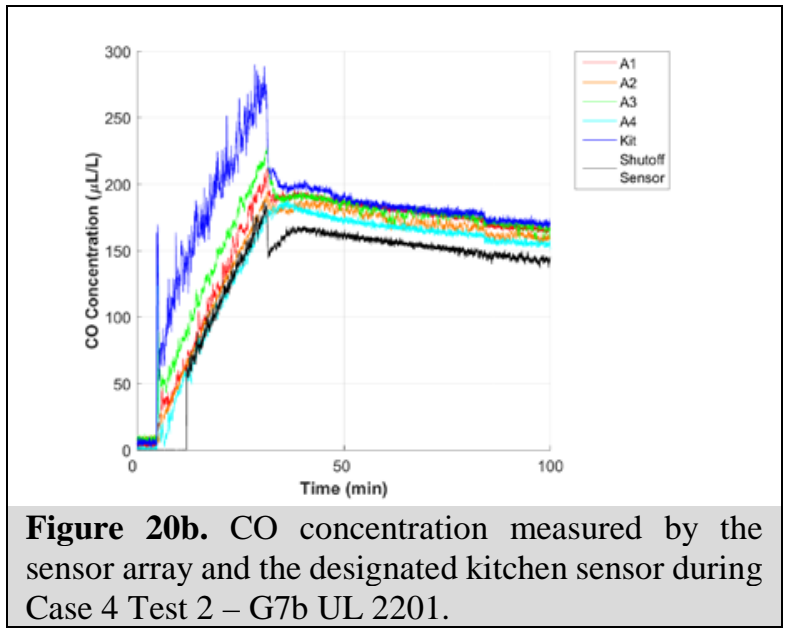

Figure 20b. CO concentration measured by the Case 4 Test 2 - G7b UL 2201. 


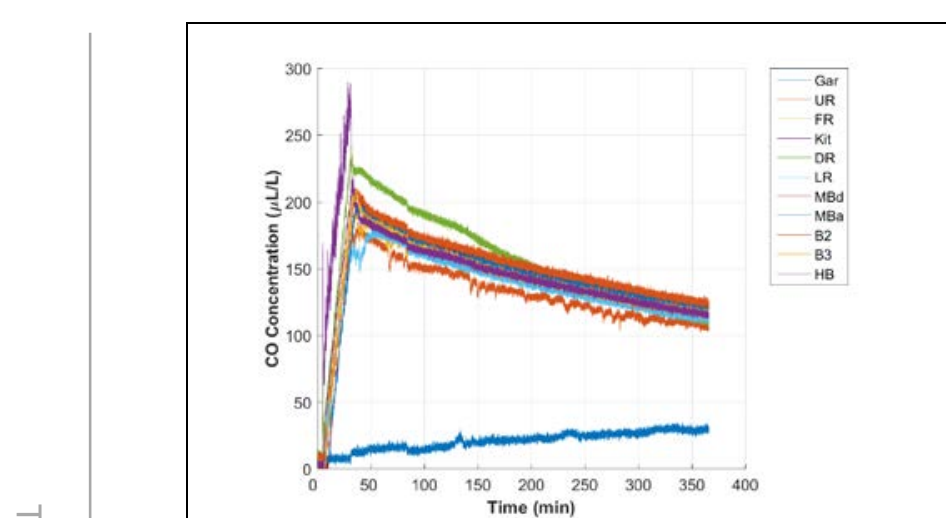

Figure 20c. CO concentration measured in each room of the house during Case 4 Test 2 - G7b UL 2201.

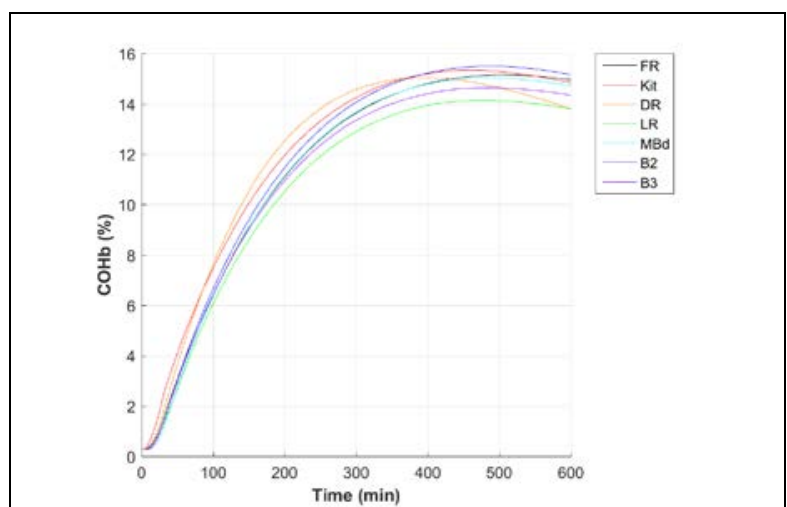

Figure 20e. $\mathrm{COHb}$ of house occupants during Case 4 Test 2 - G7b UL 2201.

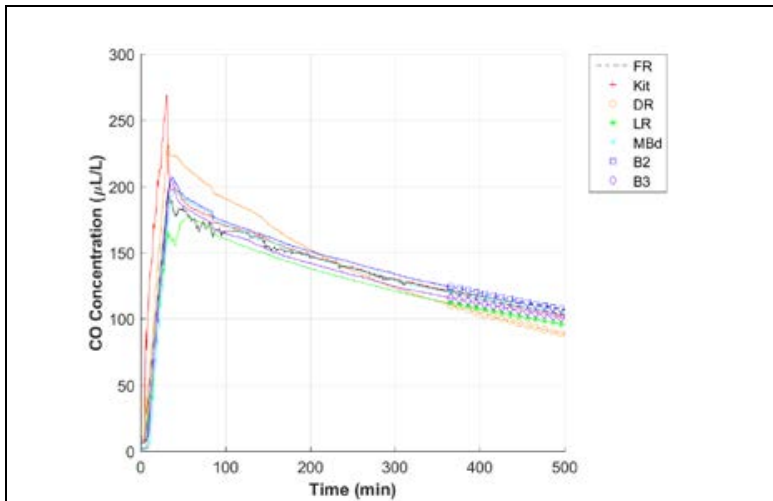

Figure 20d. CO concentration (measured \& projected) in the house during Case 4 Test $2-$ G7b UL 2201. 


\section{Case 5 Test 1 - G2 G300}

The following figures illustrate the results for Case 5 Test 1 applying the G300 shutoff criteria. The G2 generator was fully fueled, positioned in the kitchen (30 cm from the master bedroom closet wall with the exhaust facing the dining room), and tested at $50 \%$ load (800 W). The kitchen window nearest to the generator was closed during the test. The interior doors (MB, B2, B3, HB, UR) were open $10 \mathrm{~cm}$. The shutoff sensor activated the algorithm to shut off the generator after approximately $38 \mathrm{~min}$ due to a sensor measurement of $400 \mu \mathrm{L} / \mathrm{L}$ for a $10 \mathrm{~min}$ average. The interior person door (between the garage and utility room) was inadvertently left open thus invalidating it as an intended repeat of the validation test (see Case 5 Tests 2, 3, and 4 in the model validation section). However, the data is reported here for completeness. Figure 21a shows the test house layout with generator location, exhaust direction, and sensor array location. Figure 21b shows the CO concentration measured in the kitchen (by the dedicated room sensor), by two sensors located on the kitchen-family room boundary (A1: height $183 \mathrm{~cm}$, A2: height $61 \mathrm{~cm}$ ), by the other two sensors located on the kitchen-dining room boundary (A3: height $183 \mathrm{~cm}, \mathrm{~A} 4$ : height $61 \mathrm{~cm}$ ), and by the shutoff sensor located on the generator near the generator's electrical panel. The timeframe of the figure has been selected to show the degree of $\mathrm{CO}$ uniformity in the area around the generator between the shutoff sensor and the peak $\mathrm{CO}$ concentrations measured elsewhere in the kitchen near the time of shutoff. Figure 21c shows the CO concentration measured in each room of the house over the test. Figure 21d shows the CO concentration in the house with each room represented by a single line, which is composed of both a 'solid-line' pattern (showing measured CO concentration) and a 'dotted-line with a symbol' pattern (showing projected CO concentration). Figure 21e shows the calculated $\mathrm{COHb}$ profiles of simulated house occupants in each room of the house.

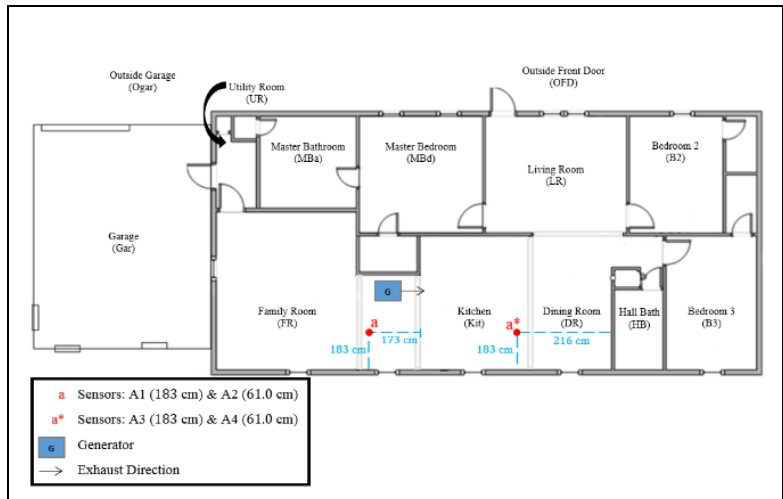

Figure 21a. Generator, exhaust direction, and sensor array layout during Case 5 Test 1 - G2 G300.

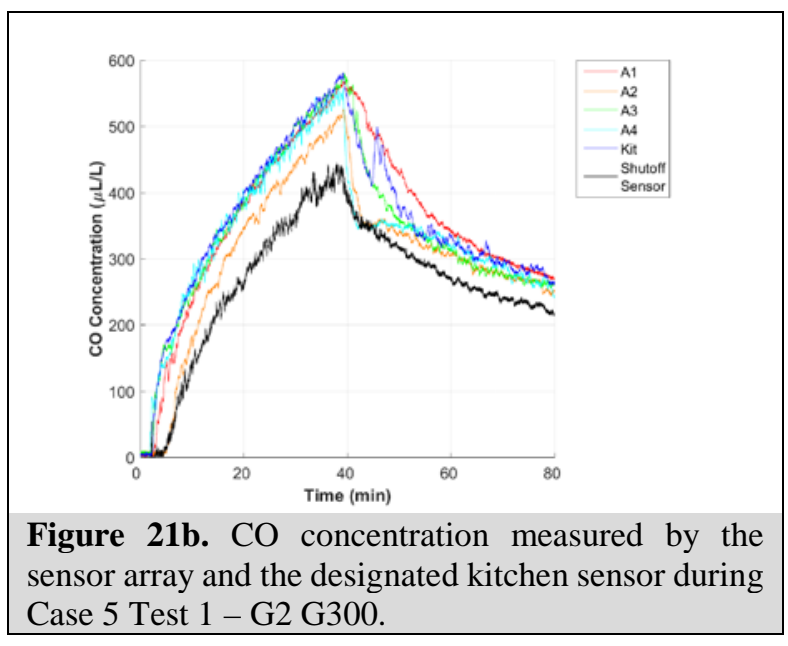




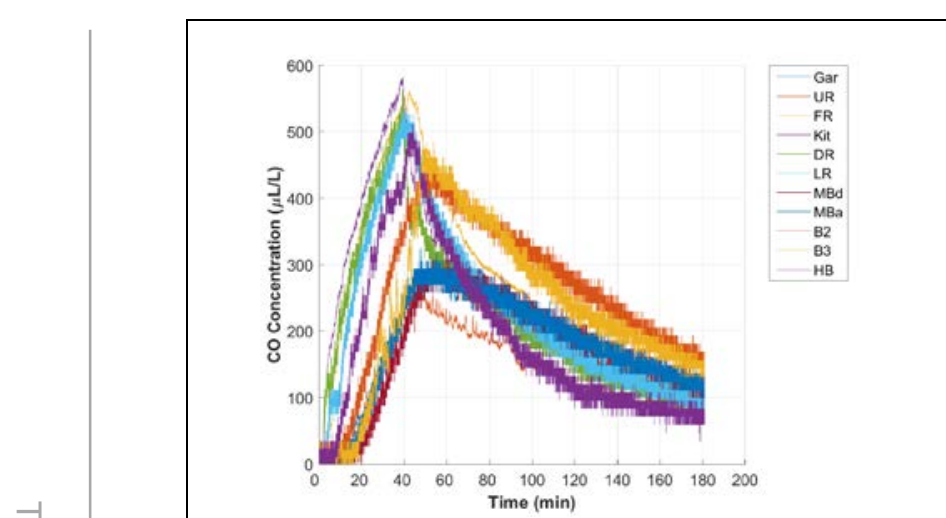

Figure 21c. CO concentration measured in each room of the house during Case 5 Test 1 - G2 G300.

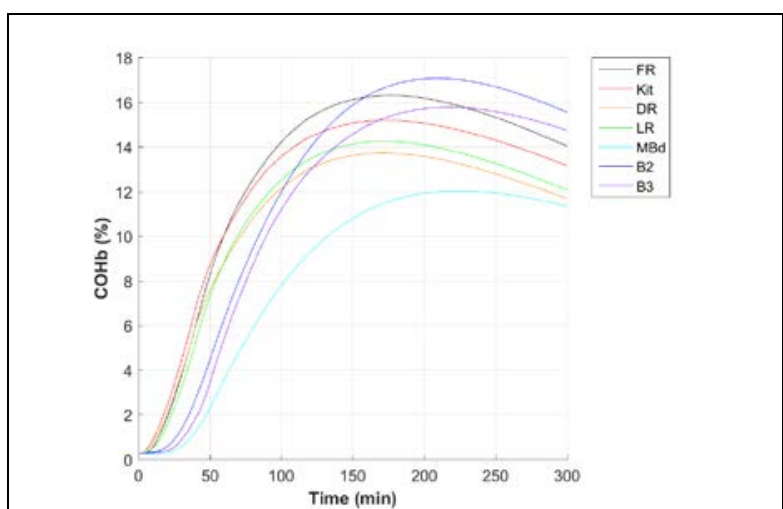

Figure 21e. $\mathrm{COHb}$ of house occupants during Case 5 Test $1-$ G2 G300.

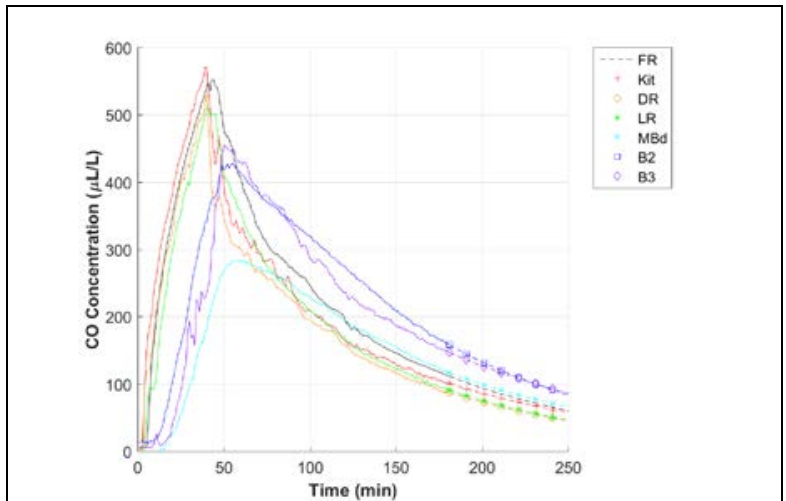

Figure 21d. CO concentration (measured \& projected) in the house during Case 5 Test 1 - G2 G300. 


\section{Case 5 Test 2 - G2 G300}

The following figures illustrate the results for Case 5 Test 2 applying the G300 shutoff criteria. The G2 generator was fully fueled, positioned in the kitchen (30 cm from the master bedroom closet wall with the exhaust facing the dining room), and tested at $50 \%$ load (800 W). The kitchen window nearest to the generator was closed during the test. The interior doors (MB, B2, B3, HB, UR) were open $10 \mathrm{~cm}$. The shutoff sensor activated the algorithm to shut off the generator after approximately $25 \mathrm{~min}$ due to a sensor measurement of $400 \mu \mathrm{L} / \mathrm{L}$ for a $10 \mathrm{~min}$ average. Although the exterior person door (from garage to backyard) was inadvertently left fully open for the duration of the test, this would be expected to have only a small impact on test house living space concentrations so this test was included as a model validation case. Figure 22a shows the test house layout with generator location, exhaust direction, and sensor array location. Figure 22b shows the CO concentration measured in the kitchen (by the dedicated room sensor), by two sensors located on the kitchen-family room boundary (A1: height $183 \mathrm{~cm}, \mathrm{A2}$ : height $61 \mathrm{~cm}$ ), by the other two sensors located on the kitchen-dining room boundary (A3: height $183 \mathrm{~cm}$, A4: height $61 \mathrm{~cm})$, and by the shutoff sensor located on the generator near the generator's electrical panel. The timeframe of the figure has been selected to show the degree of CO uniformity in the area around the generator between the shutoff sensor and the peak $\mathrm{CO}$ concentrations measured elsewhere in the kitchen near the time of shutoff. Figure 22c shows the CO concentration measured in each room of the house over the test. Figure 22d shows the CO concentration in the house with each room represented by a single line, which is composed of both a 'solid-line' pattern (showing measured CO concentration) and a 'dotted-line with a symbol' pattern (showing projected CO concentration). Figure 22e shows the calculated $\mathrm{COHb}$ profiles of simulated house occupants in each room of the house.

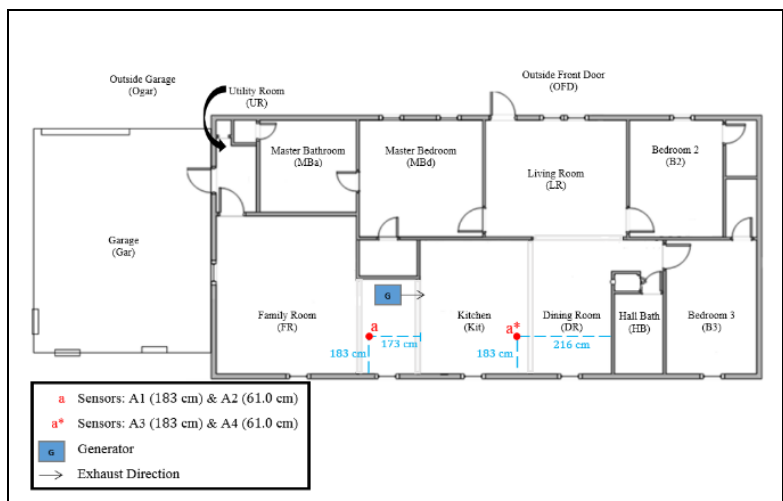

Figure 22a. Generator, exhaust direction, and sensor array layout during Case 5 Test 2 - G2 G300.

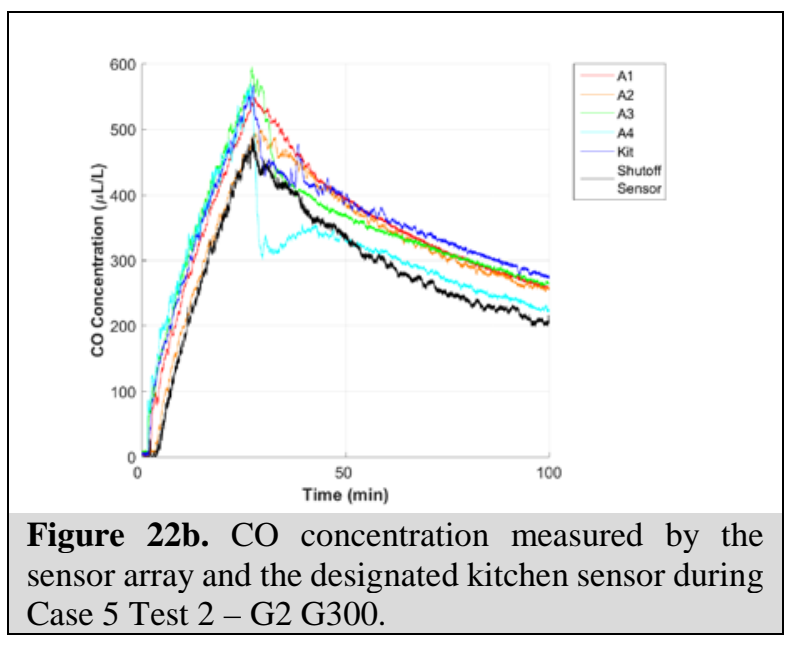




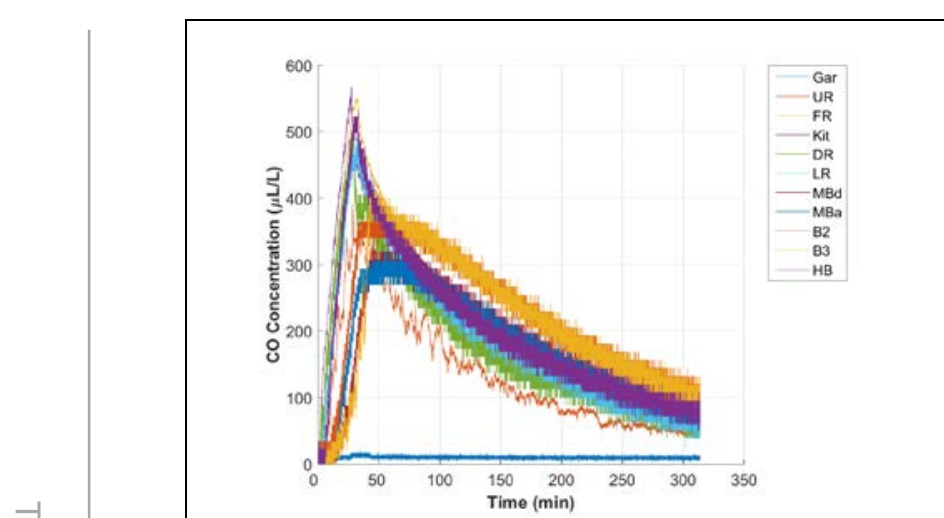

Figure 22c. $\mathrm{CO}$ concentration measured in each room of the house during Case 5 Test 2 - G2 G300.

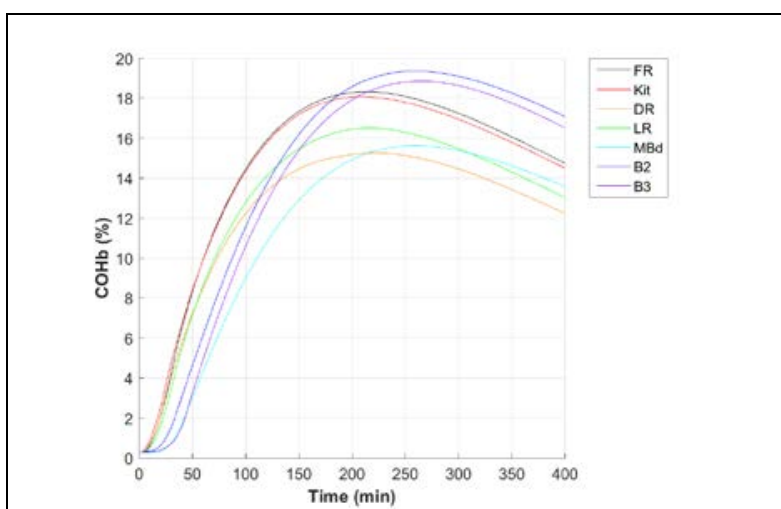

Figure 22e. $\mathrm{COHb}$ of house occupants during Case 5 Test $2-$ G2 G300.

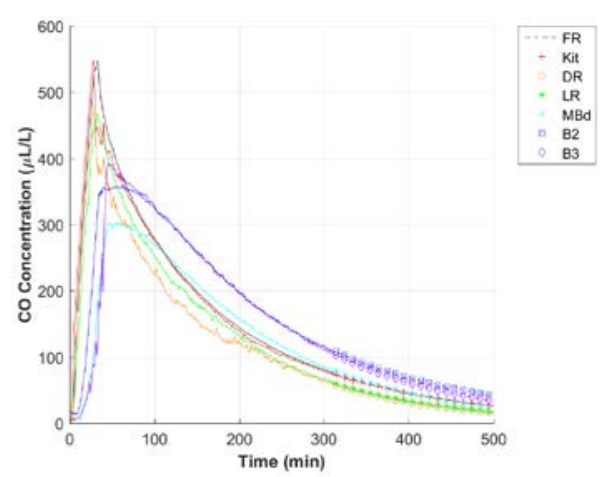

Figure 22d. CO concentration (measured \& projected) in the house during Case 5 Test 2 - G2 G300. 


\section{Case 5 Test 3 - G2 G300}

The following figures illustrate the results for Case 5 Test 3 applying the G300 shutoff criteria. The G2 generator was fully fueled, positioned in the kitchen (30 cm from the master bedroom closet wall with the exhaust facing the dining room), and tested at $50 \%$ load (800 W). The kitchen window nearest to the generator was closed during the test. The interior doors (MB, B2, B3, HB, UR) were open $10 \mathrm{~cm}$. The shutoff sensor activated the algorithm to shut off the generator after approximately $28 \mathrm{~min}$ due to a sensor measurement of $400 \mu \mathrm{L} / \mathrm{L}$ for a $10 \mathrm{~min}$ average. Figure 23a shows the test house layout with generator location, exhaust direction, and sensor array location. Figure 23b shows the CO concentration measured in the kitchen (by the dedicated room sensor), by two sensors located on the kitchen-family room boundary (A1: height $183 \mathrm{~cm}, \mathrm{~A} 2$ : height 61.0 $\mathrm{cm}$ ), by the other two sensors located on the kitchen-dining room boundary (A3: height $183 \mathrm{~cm}$, A4: height $61.0 \mathrm{~cm}$ ), and by the shutoff sensor located on the generator near the generator's electrical panel. The timeframe of the figure has been selected to show the degree of CO uniformity in the area around the generator between the shutoff sensor and the peak CO concentrations measured elsewhere in the kitchen near the time of shutoff. Figure 23c shows the CO concentration measured in each room of the house over the test. Figure 23d shows the CO concentration in the house with each room represented by a single line, which is composed of both a 'solid-line' pattern (showing measured CO concentration) and a 'dotted-line with a symbol' pattern (showing projected CO concentration). Figure 23e shows the calculated COHb profiles of simulated house occupants in each room of the house.

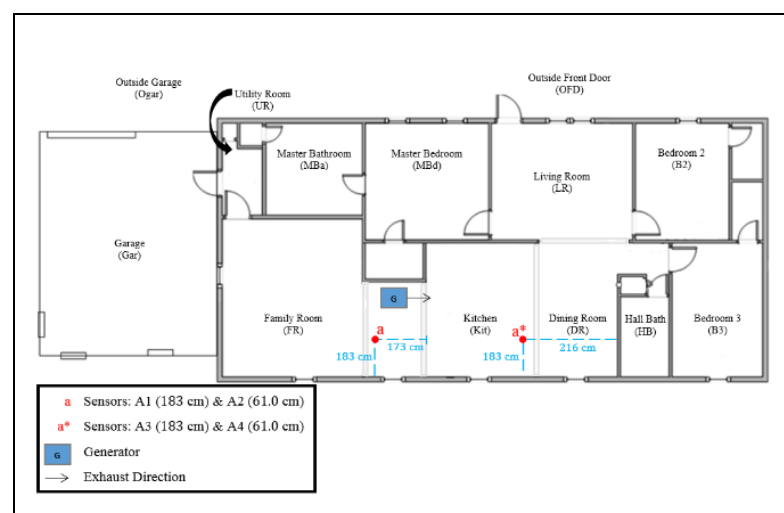

Figure 23a. Generator, exhaust direction, and sensor array layout during Case 5 Test 3 - G2 G300.

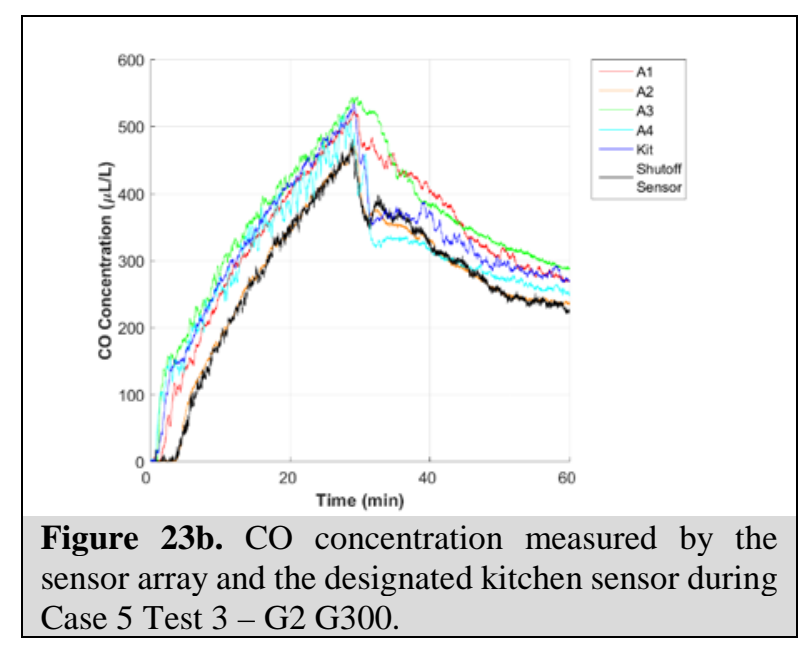




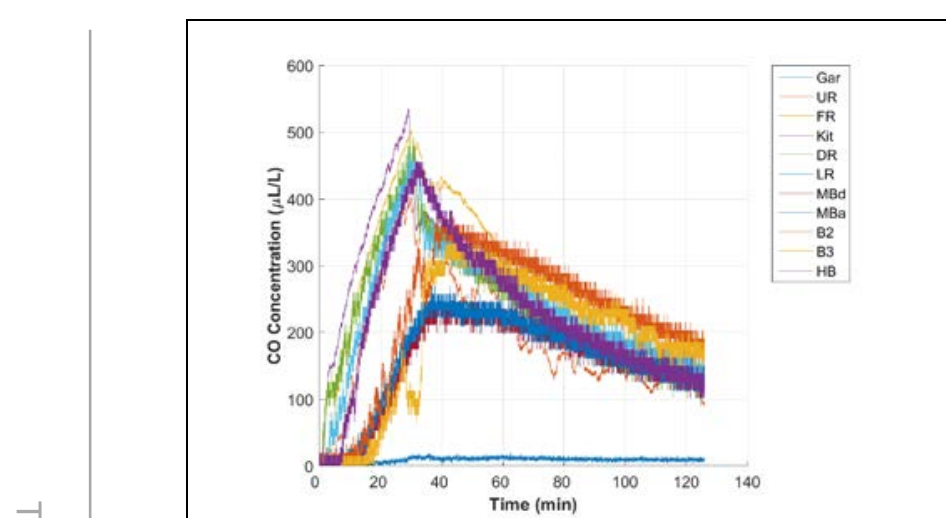

Figure 23c. CO concentration measured in each room of the house during Case 5 Test 3 - G2 G300.

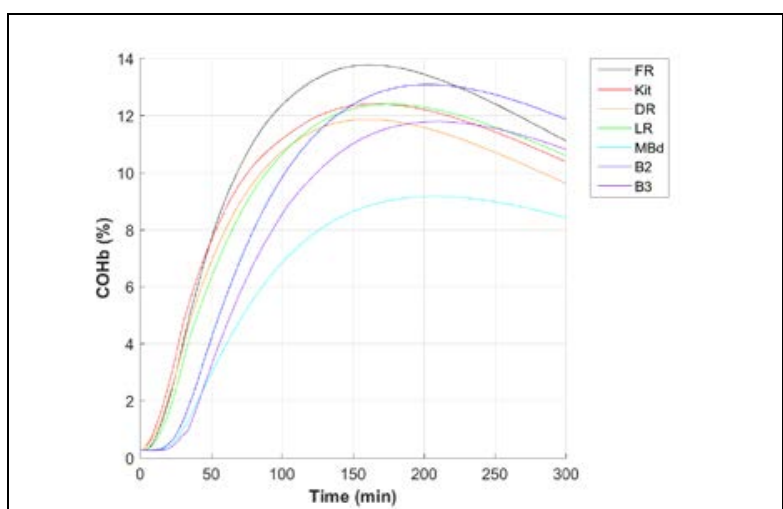

Figure 23e. $\mathrm{COHb}$ of house occupants during Case 5 Test $3-$ G2 G300.

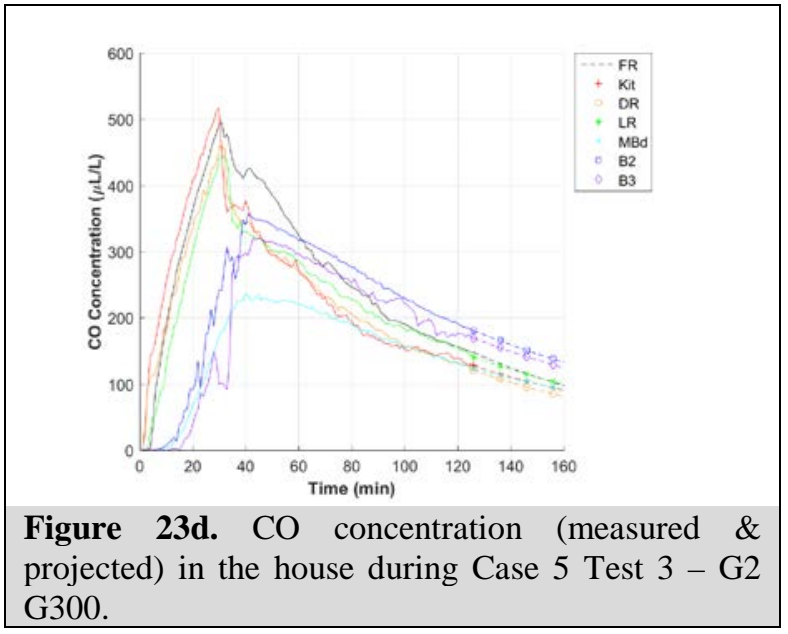

Figure 23d. CO concentration (measured \& projected) in the house during Case 5 Test 3 - G2 G300. 


\section{Case 5 Test 4 - G2 G300}

The following figures illustrate the results for Case 5 Test 4 applying the G300 shutoff criteria. The G2 generator was fully fueled, positioned in the kitchen (30 cm from the master bedroom closet wall with the exhaust facing the dining room), and tested at $50 \%$ load (800 W). The kitchen window nearest to the generator was closed during the test. The interior doors (MB, B2, B3, HB, UR) were opened $10 \mathrm{~cm}$. The shutoff sensor activated the algorithm to shut off the generator after approximately $33 \mathrm{~min}$ due to a sensor measurement of $400 \mu \mathrm{L} / \mathrm{L}$ for a $10 \mathrm{~min}$ average. Figure 24a shows the test house layout with generator location, exhaust direction, and sensor array location. Figure 24b shows the CO concentration measured in the kitchen (by the dedicated room sensor), by two sensors located on the kitchen-family room boundary (A1: height $183 \mathrm{~cm}, \mathrm{~A} 2$ : height 61.0 $\mathrm{cm}$ ), by the other two sensors located on the kitchen-dining room boundary (A3: height $183 \mathrm{~cm}$, A4: height $61.0 \mathrm{~cm}$ ), and by the shutoff sensor located on the generator near the generator's electrical panel. The timeframe of the figure has been selected to show the degree of CO uniformity in the area around the generator between the shutoff sensor and the peak CO concentrations measured elsewhere in the kitchen near the time of shutoff. Figure 24c shows the CO concentration measured in each room of the house over the test. Figure 24d shows the CO concentration in the house with each room represented by a single line, which is composed of both a 'solid-line' pattern (showing measured CO concentration) and a 'dotted-line with a symbol' pattern (showing projected CO concentration). Figure 24e shows the calculated COHb profiles of simulated house occupants in each room of the house.

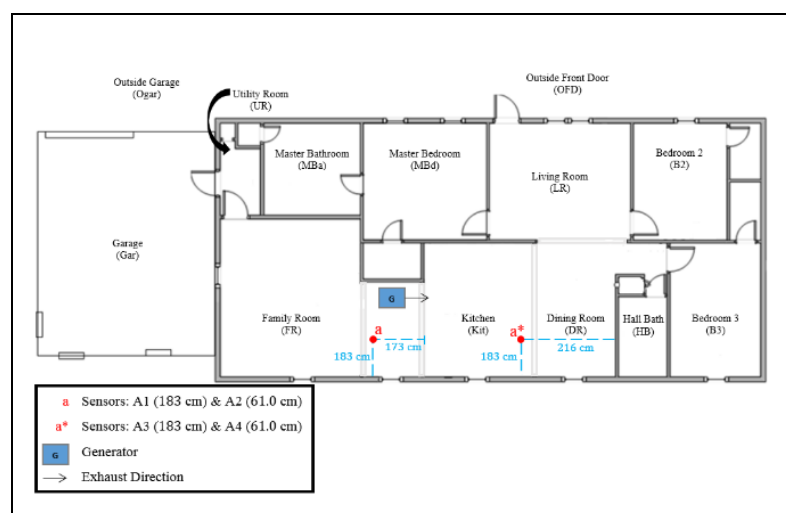

Figure 24a. Generator, exhaust direction, and sensor array layout during Case 5 Test 4 - G2 G300.

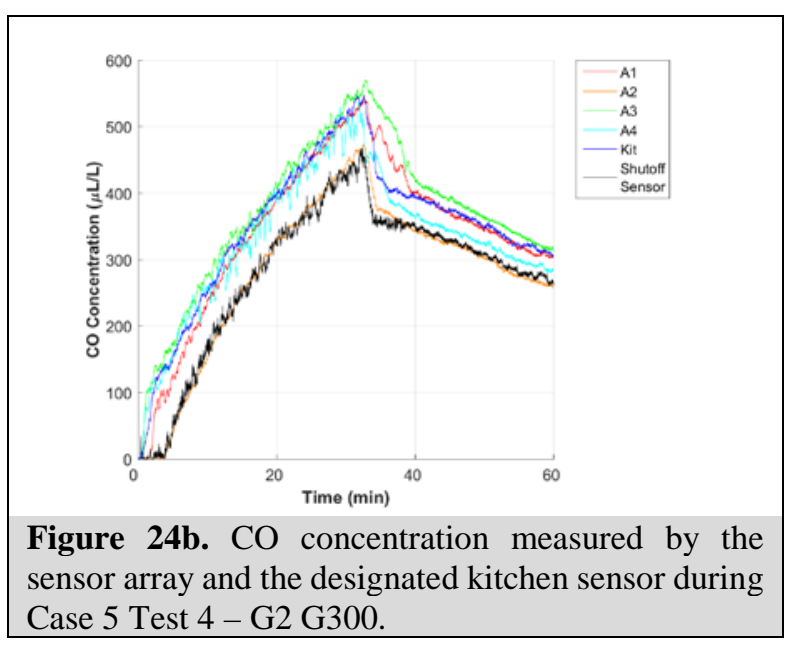

Figure 24b. CO concentration measured by the sensor array and the designated kitchen sensor during Case 5 Test 4 - G2 G300. 


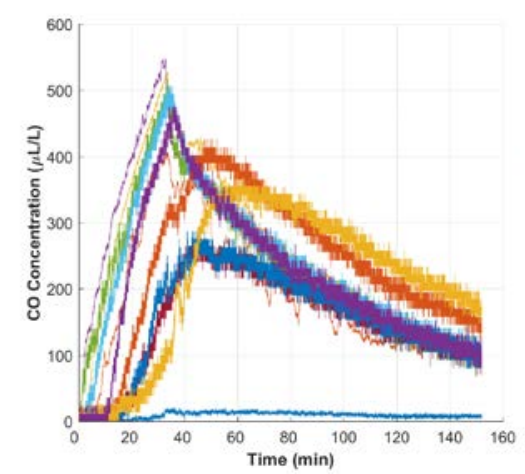

Figure 24c. CO concentration measured in each room of the house during Case 5 Test 4 - G2 G300.

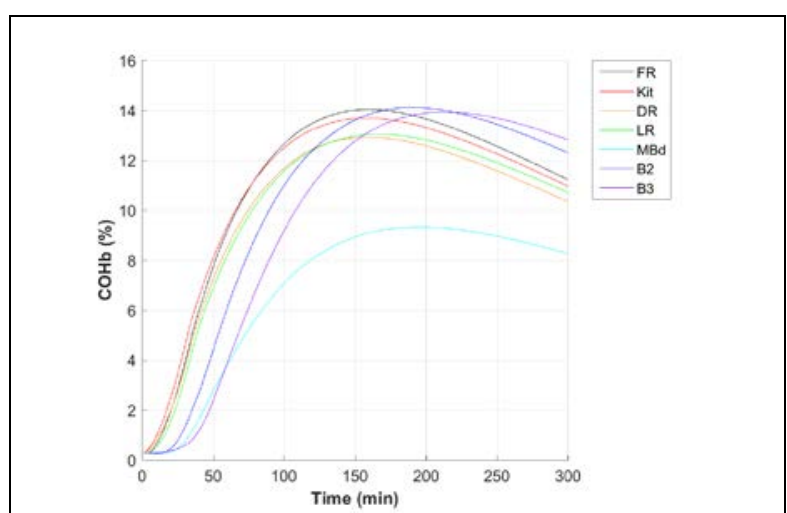

Figure 24e. $\mathrm{COHb}$ of house occupants during Case 5 Test $4-$ G2 G300.

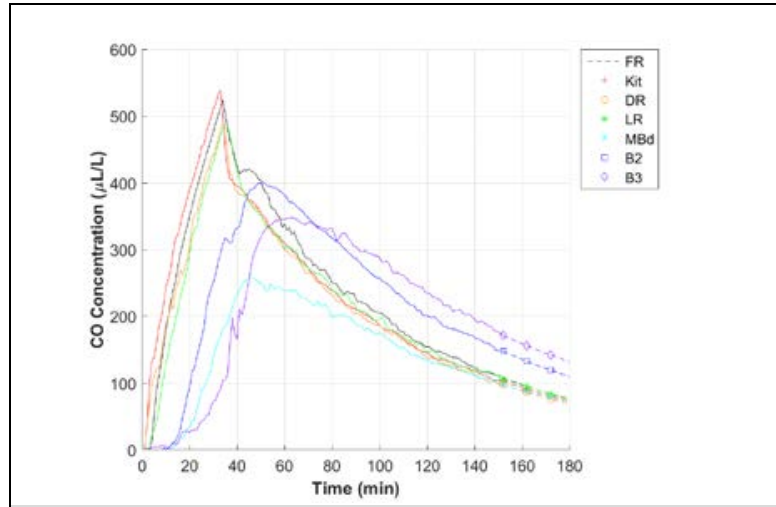

Figure 24d. CO concentration (measured \& projected) in the house during Case 5 Test $4-\mathrm{G} 2$ G300.

For the Case 5 tests that were run in triplicate (Tests 2, 3, and 4), in which the tests differed only by the environmental conditions, the results are very similar. Test 3 and 4 had nearly identical outdoor air temperatures and wind speed, and the generator shutoff both times nominally 30 minutes after start-up. The resulting $\mathrm{COHb}$ profiles are strikingly similar, within approximately 2 $\% \mathrm{COHb}$ of each other over the course of the tests. For Test 2, the warmer, less breezy weather resulted, as expected, in a quicker build-up and slower decay of CO causing the generator to shut off 5 minutes quicker than tests 3 and 4 and a slightly higher COHb profile compared to tests 3 and 4. Overall, one can conclude that the results were very repeatable. 


\section{Case 5 Test 1 - G2 G300 Restart}

The following figures illustrate the results for Restart Case 5 Test 1 applying the G300 shutoff criteria. The G2 generator was fully fueled, positioned in the kitchen (30 cm from the master bedroom closet wall with the exhaust facing the dining room), and tested at $50 \%$ load (800 W). The kitchen window nearest to the generator was closed during the test. The interior doors (MB, B2, B3, HB, UR) were open $10 \mathrm{~cm}$. For the first generator run, the shutoff sensor activated the algorithm to shut off the generator after approximately 27 min due to a sensor measurement of 400 $\mu \mathrm{L} / \mathrm{L}$ for a $10 \mathrm{~min}$ average. The generator was restarted and the test load restored approximately 10 min after the first instance of shutoff initiated by the shutoff sensor. For the second generator run, the shutoff sensor activated the algorithm to shut off the generator after approximately 8 min due to a sensor measurement of $400 \mu \mathrm{L} / \mathrm{L}$ for a 10 min average. Figure 25a shows the test house layout with generator location, exhaust direction, and sensor array location. Figure 25b shows the CO concentration measured in the kitchen (by the dedicated room sensor), by two sensors located on the kitchen-family room boundary (A1: height $183 \mathrm{~cm}$, A2: height $61 \mathrm{~cm}$ ), by the other two sensors located on the kitchen-dining room boundary (A3: height $183 \mathrm{~cm}, \mathrm{~A} 4$ : height $61 \mathrm{~cm}$ ), and by the shutoff sensor located on the generator near the generator's electrical panel. The timeframe of the figure has been selected to show the degree of $\mathrm{CO}$ uniformity in the area around the generator between the shutoff sensor and the peak CO concentrations measured elsewhere in the kitchen near the time of shutoff. Figure 25c shows the $\mathrm{CO}$ concentration measured in each room of the house over the test. Figure 25d shows the $\mathrm{CO}$ concentration in the house with each room represented by a single line, which is composed of both a 'solid-line' pattern (showing measured CO concentration) and a 'dotted-line with a symbol' pattern (showing projected CO concentration). Figure 25e shows the calculated $\mathrm{COHb}$ profiles of simulated house occupants in each room of the house.

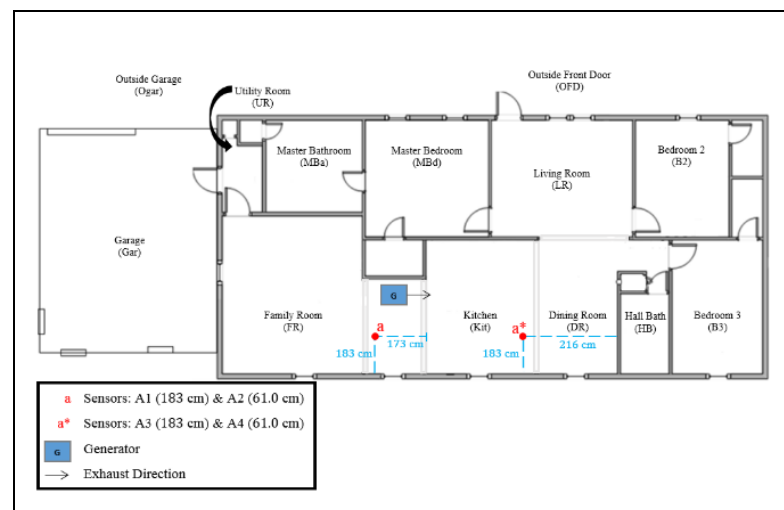

Figure 25a. Generator, exhaust direction, and sensor array layout during Restart Case 5 Test 1 - G2 G300.

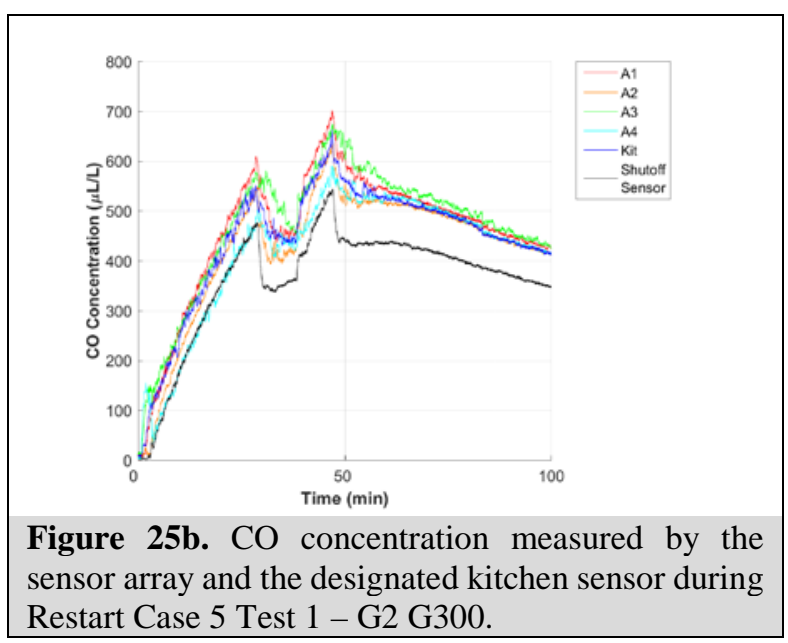

Figure 25b. CO concentration measured by the sensor array and the designated kitchen sensor during Restart Case 5 Test 1 - G2 G300. 


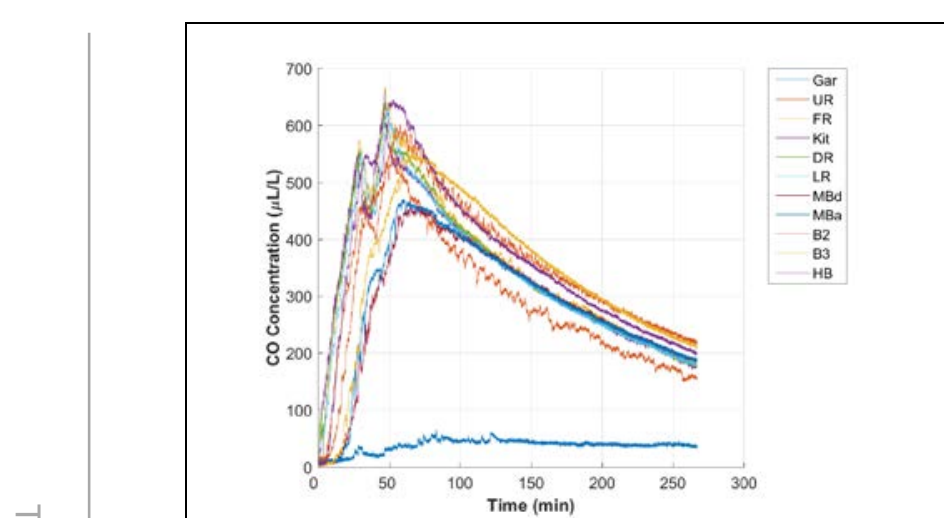

Figure 25c. CO concentration measured in each room of the house during Restart Case 5 Test 1 - G2 G300.

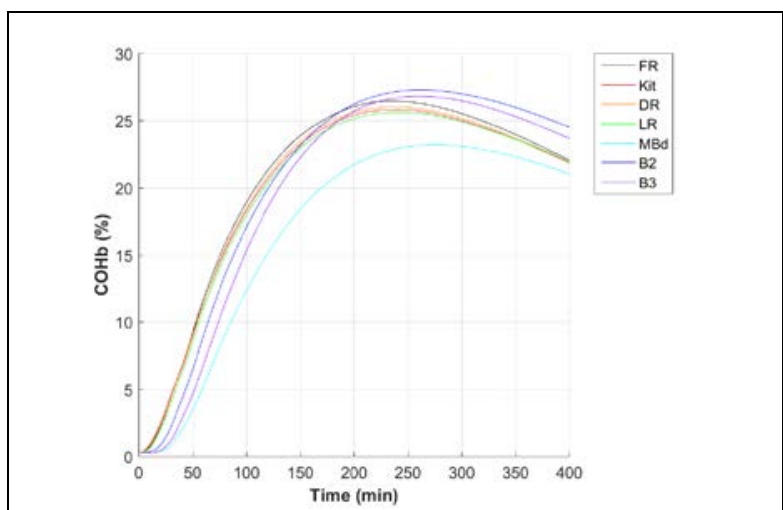

Figure 25e. COHb of house occupants during Restart Case 5 Test 1 - G2 G300.

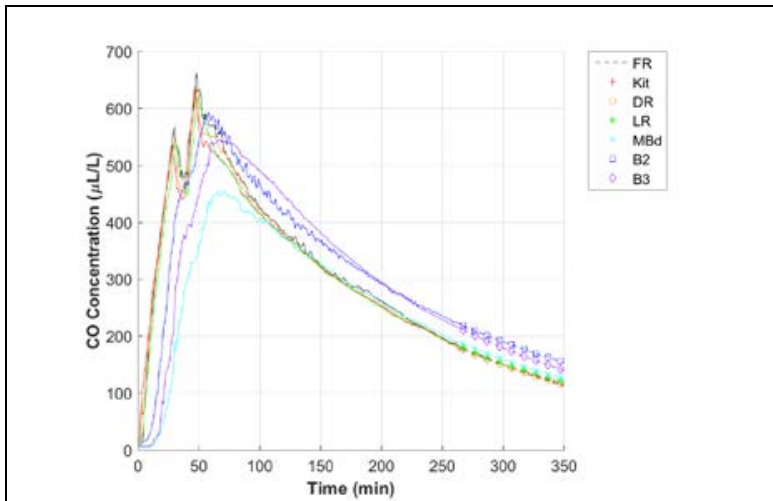

Figure 25d. CO concentration (measured \& projected) in the house during Restart Case 5 Test 1 G2 G300. 


\section{Case 6 Test 1 - G2 G300}

The following figures illustrate the results for Case 6 Test 1 applying the G300 shutoff criteria. The G2 generator was fully fueled, positioned in the kitchen (30 cm from the master bedroom closet wall with the exhaust facing the dining room), and tested at $10 \%$ load (160 W). The kitchen window nearest to the generator was closed during the test. The shutoff sensor activated the algorithm to shut off the generator after approximately 44 min due to a sensor measurement of 400 $\mu \mathrm{L} / \mathrm{L}$ for a $10 \mathrm{~min}$ average. Figure 26a shows the test house layout with generator location, exhaust direction, and sensor array location. Figure 26b shows the CO concentration measured in the kitchen (by the dedicated room sensor), by two sensors located on the kitchen-family room boundary (A1: height $183 \mathrm{~cm}, \mathrm{A2}$ : height $61 \mathrm{~cm}$ ), by the other two sensors located on the kitchendining room boundary (A3: height $183 \mathrm{~cm}, \mathrm{~A} 4$ : height $61 \mathrm{~cm}$ ), and by the shutoff sensor located on the generator near the generator's electrical panel. The timeframe of the figure has been selected to show the degree of CO uniformity in the area around the generator between the shutoff sensor and the peak CO concentrations measured elsewhere in the kitchen near the time of shutoff. Figure 26c shows the CO concentration measured in each room of the house over the test. Figure 26d shows the $\mathrm{CO}$ concentration in the house with each room represented by a single line, which is composed of both a 'solid-line' pattern (showing measured CO concentration) and a 'dotted-line with a symbol' pattern (showing projected CO concentration). Figure 26e shows the calculated $\mathrm{COHb}$ profiles of simulated house occupants in each room of the house.

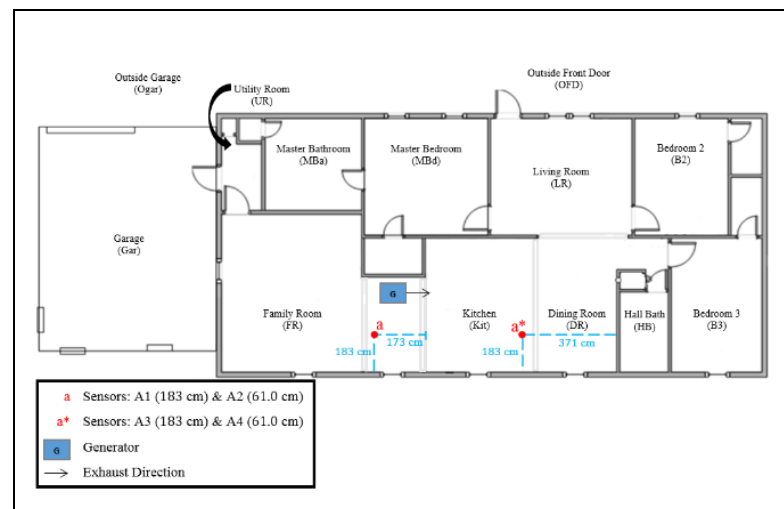

Figure 26a. Generator, exhaust direction, and sensor array layout during Case 6 Test 1 - G2 G300.

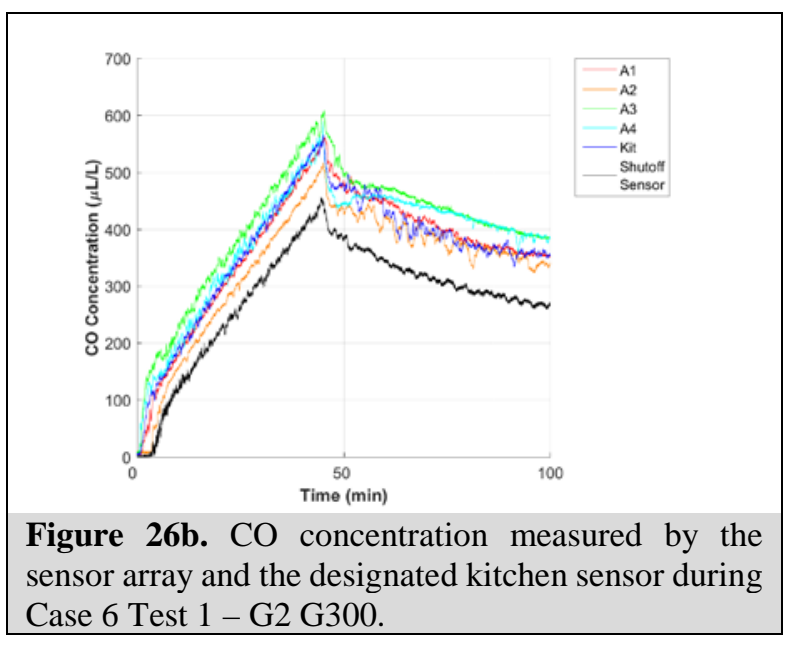




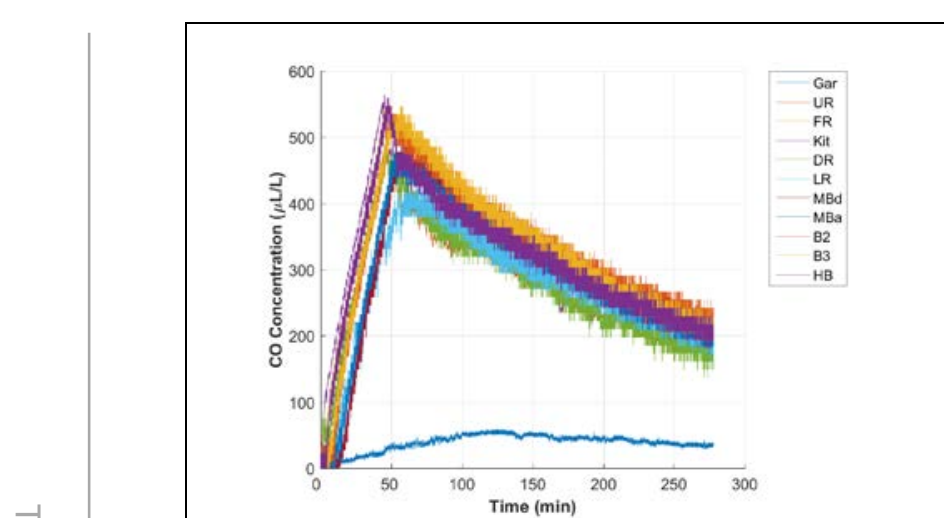

Figure 26c. $\mathrm{CO}$ concentration measured in each room of the house during Case 6 Test 1-G2 G300.

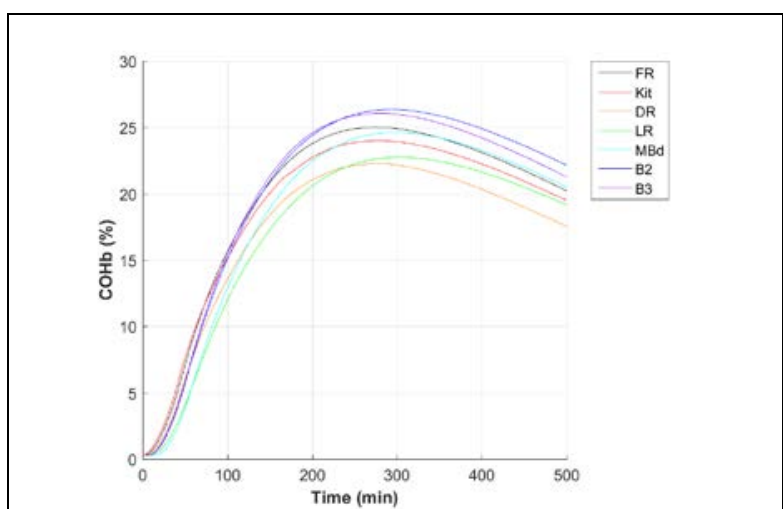

Figure 26e. $\mathrm{COHb}$ of house occupants during Case 6 Test 1 - G2 G300.

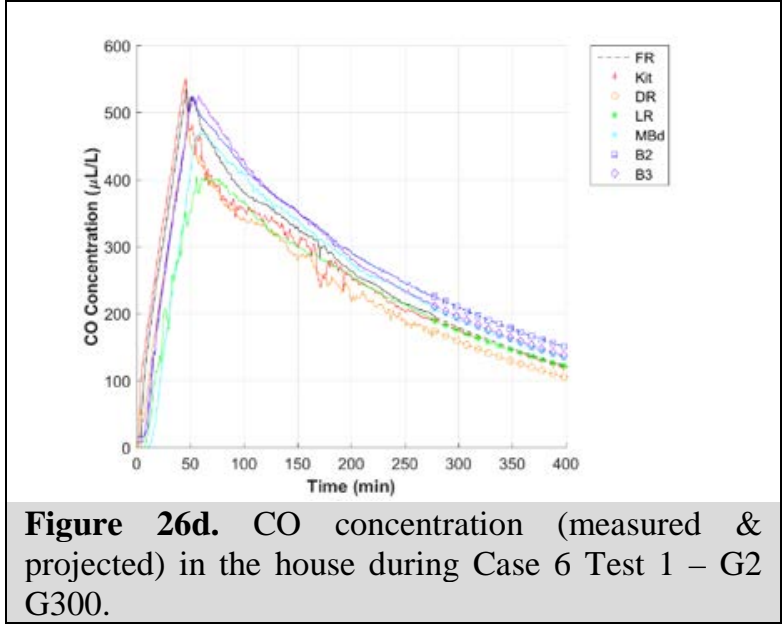

Figure 26d. CO concentration (measured \& projected) in the house during Case 6 Test 1 - G2 


\section{Case 6 Test 1 - G2 G300 Restart}

The following figures illustrate the results for Restart Case 6 Test 1 applying the G300 shutoff criteria. The G2 generator was fully fueled, positioned in the kitchen (30 cm from the master bedroom closet wall with the exhaust facing the dining room), and tested at $10 \%$ load (160 W). The kitchen window nearest to the generator was closed during the test. For the first generator run, the shutoff sensor activated the algorithm to shut off the generator after approximately 53 min due to a sensor measurement of $400 \mu \mathrm{L} / \mathrm{L}$ for a $10 \mathrm{~min}$ average. The generator was restarted and the test load restored approximately $10 \mathrm{~min}$ after the first instance of shutoff initiated by the shutoff sensor. For the second generator run, the shutoff sensor activated the algorithm to shut off the generator after approximately 9 min due to a sensor measurement of $400 \mu \mathrm{L} / \mathrm{L}$ for a $10 \mathrm{~min}$ average. Figure 27a shows the test house layout with generator location, exhaust direction, and sensor array location. Figure 27b shows the CO concentration measured in the kitchen (by the dedicated room sensor), by two sensors located on the kitchen-family room boundary (A1: height $183 \mathrm{~cm}, \mathrm{~A} 2$ : height $61 \mathrm{~cm}$ ), by the other two sensors located on the kitchen-dining room boundary (A3: height $183 \mathrm{~cm}, \mathrm{~A} 4$ : height $61 \mathrm{~cm}$ ), and by the shutoff sensor located on the generator near the generator's electrical panel. The timeframe of the figure has been selected to show the degree of $\mathrm{CO}$ uniformity in the area around the generator between the shutoff sensor and the peak CO concentrations measured elsewhere in the kitchen near the time of shutoff. Figure 27c shows the CO concentration measured in each room of the house over the test. Figure 27d shows the CO concentration in the house with each room represented by a single line, which is composed of both a 'solid-line' pattern (showing measured CO concentration) and a 'dotted-line with a symbol' pattern (showing projected CO concentration). Figure 27e shows the calculated COHb profiles of simulated house occupants in each room of the house.

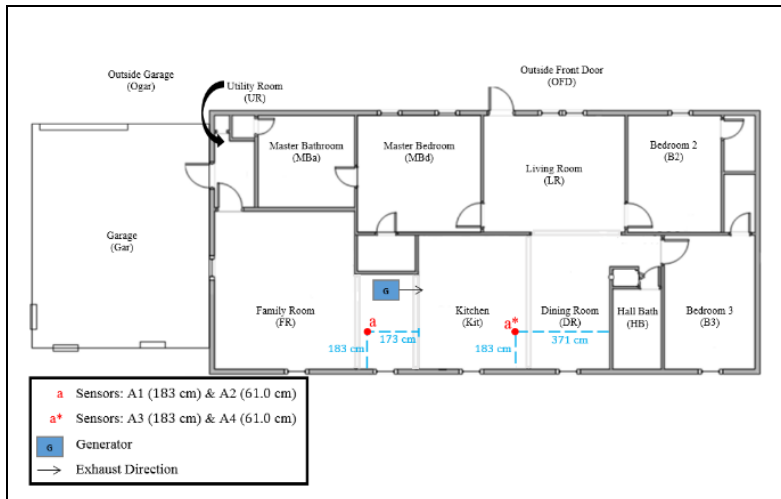

Figure 27a. Generator, exhaust direction, and sensor array layout during Restart Case 6 Test 1 - G2 G300.

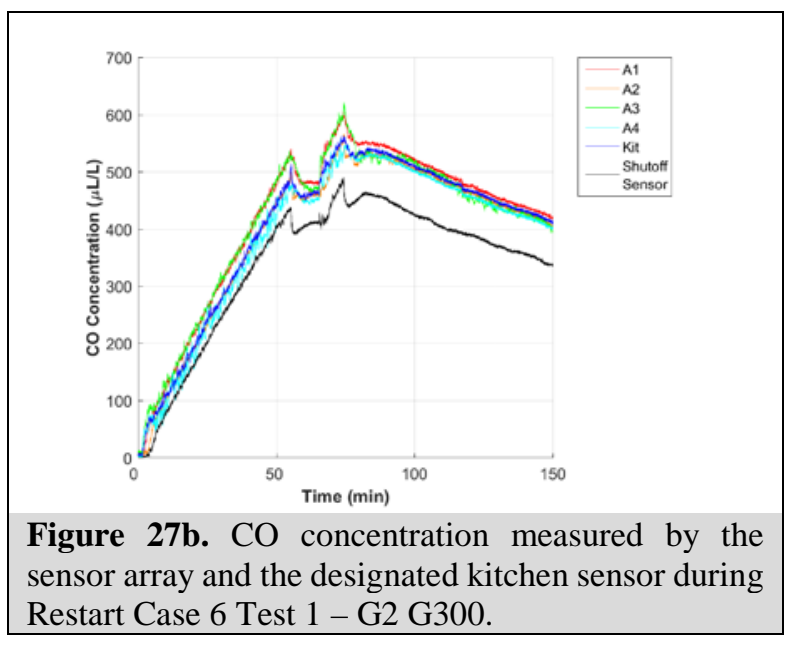




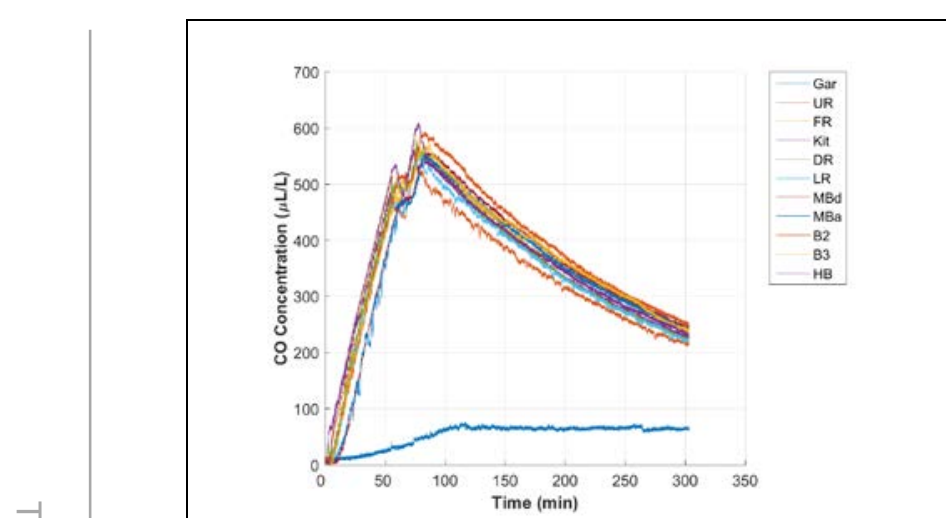

Figure 27c. CO concentration measured in each room of the house during Restart Case 6 Test 1 - G2 G300.

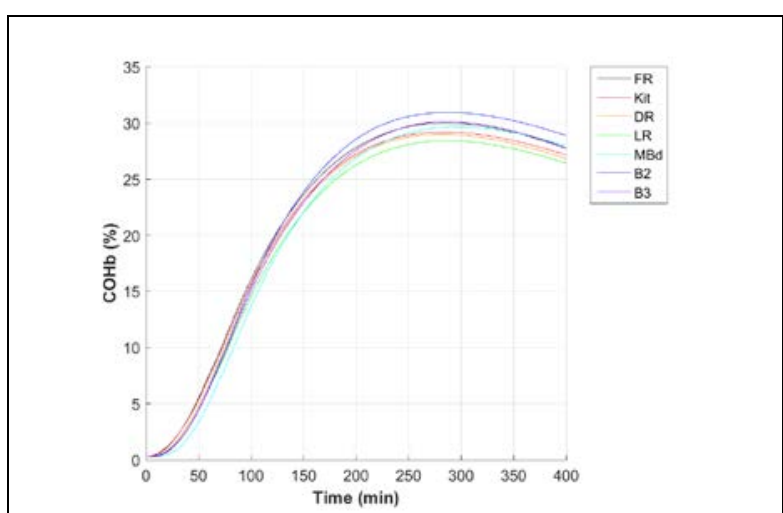

Figure 27e. $\mathrm{COHb}$ of house occupants during Restart Case 6 Test 1 - G2 G300.

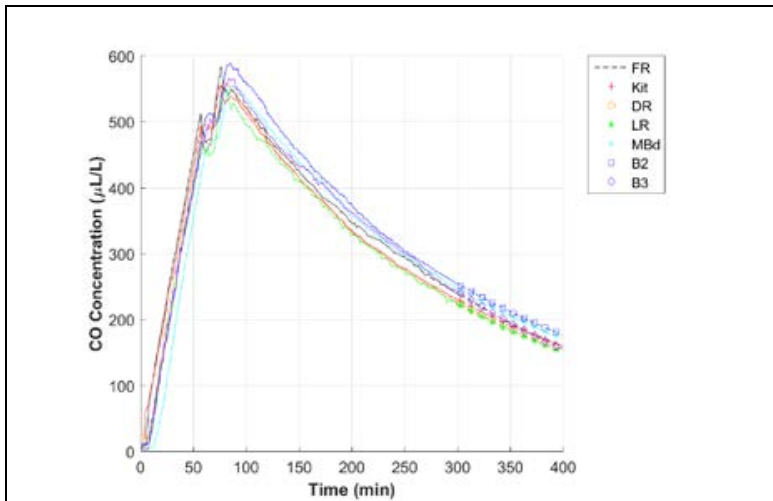

Figure 27d. CO concentration (measured \& projected) in the house during Restart Case 6 Test 1 G2 G300. 


\section{Case 6 Test 1 - G2 UL 2201}

The following figures illustrate the results for Case 6 Test 1 applying the UL 2201 shutoff criteria. The G2 generator was fully fueled, positioned in the kitchen (30 cm from the master bedroom closet wall with the exhaust facing the dining room), and tested at $10 \%$ load (160 W). The kitchen window nearest to the generator was closed during the test. The shutoff sensor activated the algorithm to shut off the generator after approximately 20 min due to a sensor measurement of 150 $\mu \mathrm{L} / \mathrm{L}$ for a $10 \mathrm{~min}$ average. Figure 28a shows the test house layout with generator location, exhaust direction, and sensor array location. Figure 28b shows the CO concentration measured in the kitchen (by the dedicated room sensor), by two sensors located on the kitchen-family room boundary (A1: height $183 \mathrm{~cm}, \mathrm{A2}$ : height $61 \mathrm{~cm}$ ), by the other two sensors located on the kitchendining room boundary (A3: height $183 \mathrm{~cm}, \mathrm{~A} 4$ : height $61 \mathrm{~cm}$ ), and by the shutoff sensor located on the generator near the generator's electrical panel. The timeframe of the figure has been selected to show the degree of CO uniformity in the area around the generator between the shutoff sensor and the peak CO concentrations measured elsewhere in the kitchen near the time of shutoff. Figure 28c shows the CO concentration measured in each room of the house over the test. Figure 28d shows the $\mathrm{CO}$ concentration in the house with each room represented by a single line, which is composed of both a 'solid-line' pattern (showing measured CO concentration) and a 'dotted-line with a symbol' pattern (showing projected CO concentration). Figure 28e shows the calculated $\mathrm{COHb}$ profiles of simulated house occupants in each room of the house.

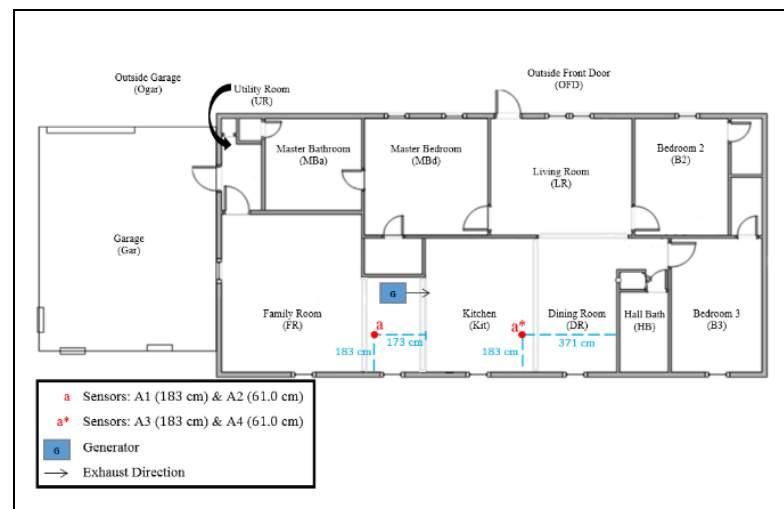

Figure 28a. Generator, exhaust direction, and sensor array layout during Case 6 Test 1 - G2 UL 2201.

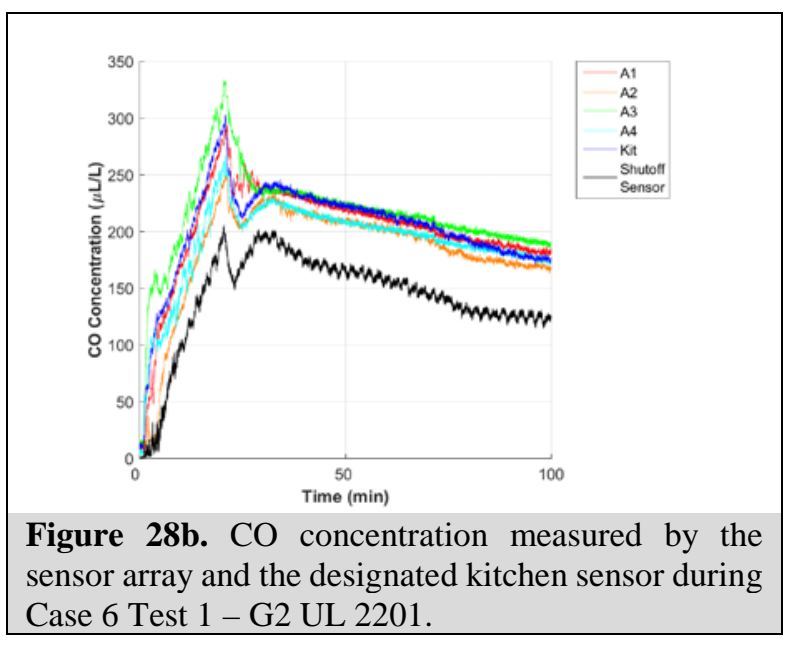




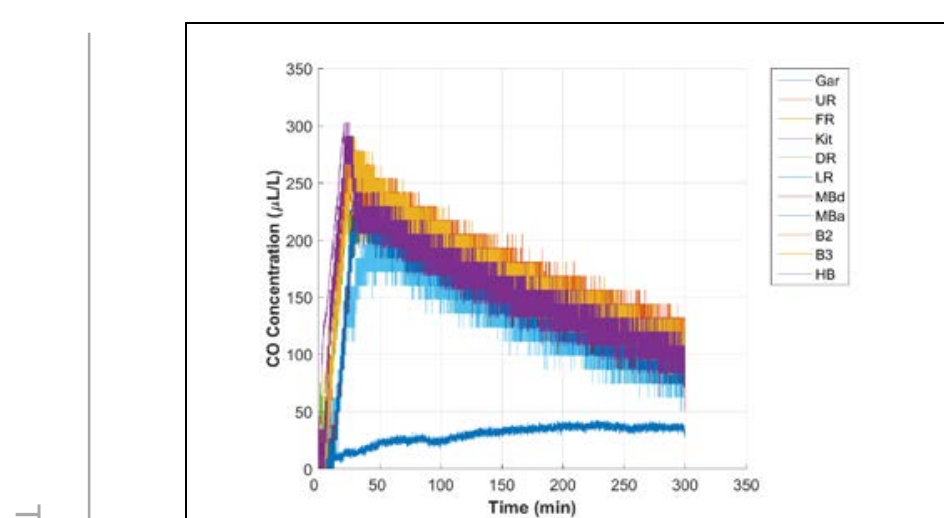

Figure 28c. CO concentration measured in each room of the house during Case 6 Test 1-G2 UL 2201.

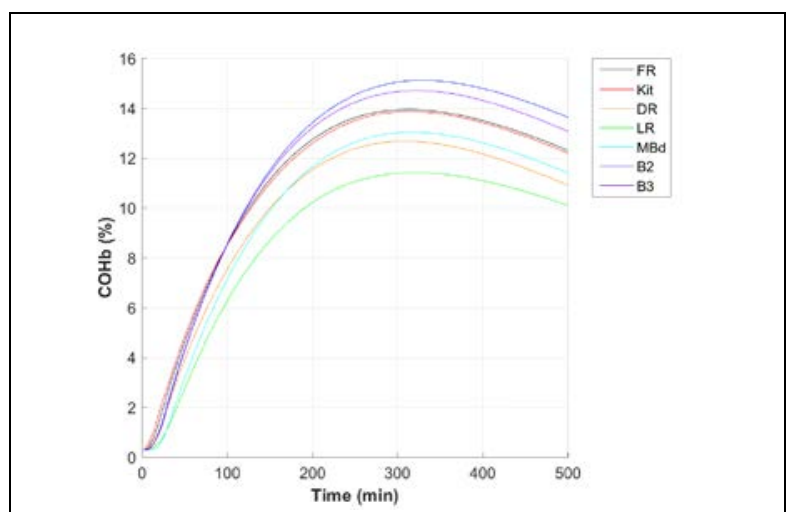

Figure 28e. $\mathrm{COHb}$ of house occupants during Case 6 Test 1 - G2 UL 2201.

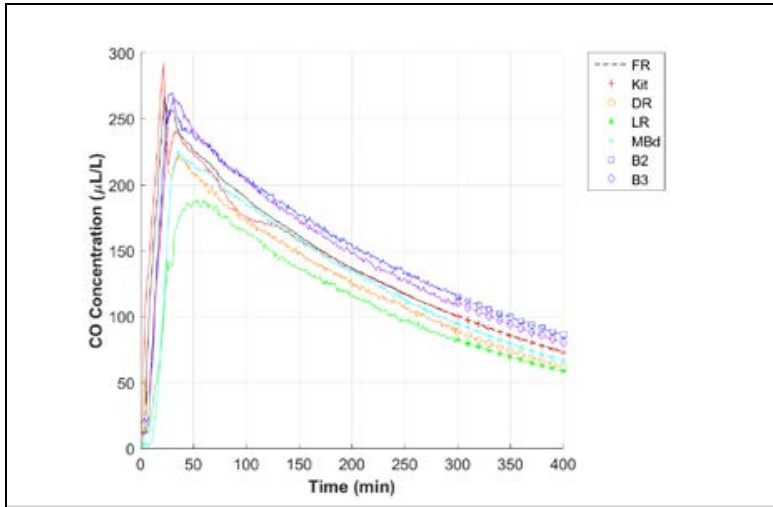

Figure 28d. CO concentration (measured \& projected) in the house during Case 6 Test 1 - G2 UL 2201. 


\section{Case 6 Test 1 - G2 UL 2201 Restart}

The following figures illustrate the results for Restart Case 6 Test 1 applying the UL 2201 shutoff criteria. The G2 generator was fully fueled, positioned in the kitchen (30 cm from the master bedroom closet wall with the exhaust facing the dining room), and tested at $10 \%$ load (160 W). The kitchen window nearest to the generator was closed during the test. For the first generator run, the shutoff sensor activated the algorithm to shut off the generator after approximately 21 min due to a sensor measurement of $150 \mu \mathrm{L} / \mathrm{L}$ for a $10 \mathrm{~min}$ average. The generator was restarted and the test load restored approximately $10 \mathrm{~min}$ after the first instance of shutoff initiated by the shutoff sensor. For the second generator run, the shutoff sensor activated the algorithm to shut off the generator after approximately 8 min due to a sensor measurement of $150 \mu \mathrm{L} / \mathrm{L}$ for a $10 \mathrm{~min}$ average. Figure 29a shows the test house layout with generator location, exhaust direction, and sensor array location. Figure 29b shows the CO concentration measured in the kitchen (by the dedicated room sensor), by two sensors located on the kitchen-family room boundary (A1: height $183 \mathrm{~cm}, \mathrm{~A} 2$ : height $61 \mathrm{~cm}$ ), by the other two sensors located on the kitchen-dining room boundary (A3: height $183 \mathrm{~cm}, \mathrm{~A} 4$ : height $61 \mathrm{~cm}$ ), and by the shutoff sensor located on the generator near the generator's electrical panel. The timeframe of the figure has been selected to show the degree of $\mathrm{CO}$ uniformity in the area around the generator between the shutoff sensor and the peak CO concentrations measured elsewhere in the kitchen near the time of shutoff. Figure 29c shows the $\mathrm{CO}$ concentration measured in each room of the house over the test. Figure 29d shows the CO concentration in the house with each room represented by a single line, which is composed of both a 'solid-line' pattern (showing measured CO concentration) and a 'dotted-line with a symbol' pattern (showing projected CO concentration). Figure 29e shows the calculated COHb profiles of simulated house occupants in each room of the house.

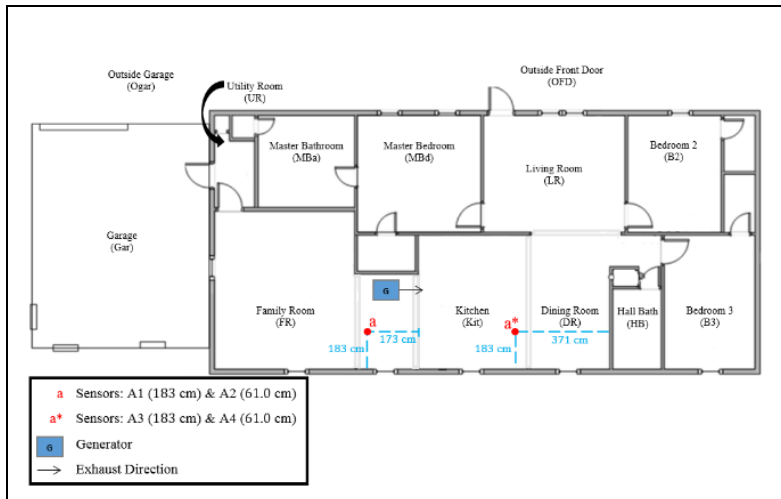

Figure 29a. Generator, exhaust direction, and sensor array layout during Restart Case 6 Test 1 - G2 UL 2201.

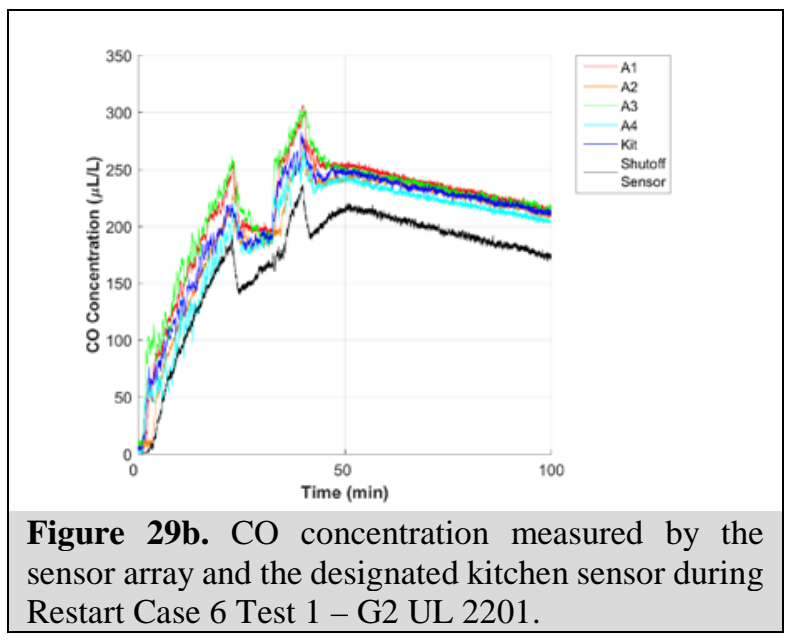




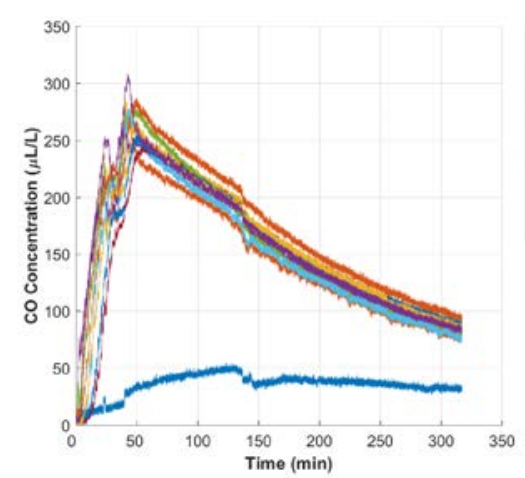

Figure 29c. CO concentration measured in each room of the house during Restart Case 6 Test 1 - G2 UL 2201.

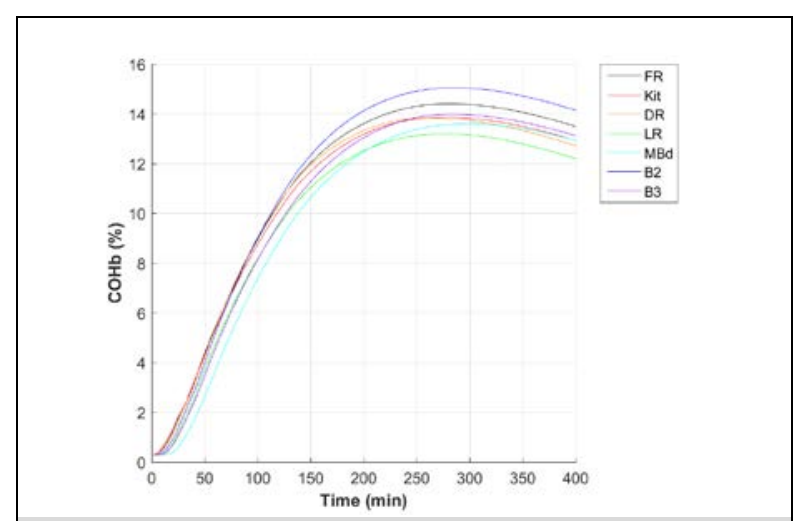

Figure 29e. $\mathrm{COHb}$ of house occupants during Restart Case 6 Test 1 - G2 UL 2201.

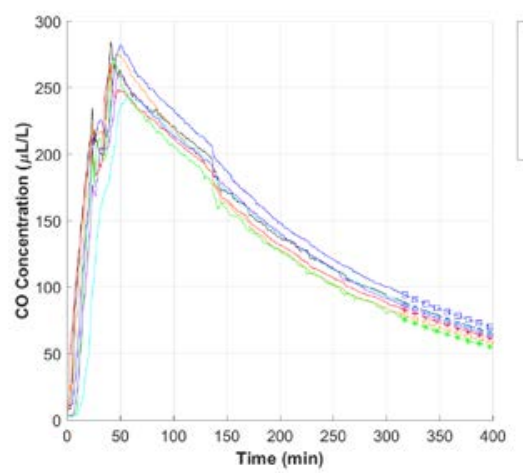

Figure 29d. CO concentration (measured \& projected) in the house during Restart Case 6 Test 1 G2 UL 2201. 


\section{Case 7 Test 1 - G2P G300}

The following figures illustrate the results for Case 7 Test 1 applying the G300 shutoff criteria. The G2P generator was connected to a tank of HD-5 consumer grade propane, positioned in the kitchen (30 cm from the master bedroom closet wall with the exhaust facing the dining room), and tested at $10 \%$ load $(160 \mathrm{~W})$. The kitchen window nearest to the generator was closed during the test. At $10 \%$ load, no significant CO accumulated in the house (approximately $80 \mathrm{~min}$ ); therefore, the load was increased to $100 \%$. The shutoff sensor activated the algorithm to shut off the generator approximately $44 \mathrm{~min}$ after increasing the load due to a sensor measurement of $400 \mu \mathrm{L} / \mathrm{L}$ for a $10 \mathrm{~min}$ average. Figure 30a shows the test house layout with generator location, exhaust direction, and sensor array location. Figure 30b shows the $\mathrm{CO}$ concentration measured in the kitchen (by the dedicated room sensor), by two sensors located on the kitchen-family room boundary (A1: height $183 \mathrm{~cm}, \mathrm{A2}$ : height $61 \mathrm{~cm}$ ), by the other two sensors located on the kitchendining room boundary (A3: height $183 \mathrm{~cm}, \mathrm{~A} 4$ : height $61 \mathrm{~cm}$ ), and by the shutoff sensor located on the generator near the generator's electrical panel. The timeframe of the figure has been selected to show the degree of $\mathrm{CO}$ uniformity in the area around the generator between the shutoff sensor and the peak $\mathrm{CO}$ concentrations measured elsewhere in the kitchen near the time of shutoff. Figure 30c shows the CO concentration measured in each room of the house over the test. Figure 30d shows the $\mathrm{CO}$ concentration in the house with each room represented by a single line, which is composed of both a 'solid-line' pattern (showing measured CO concentration) and a 'dotted-line with a symbol' pattern (showing projected CO concentration). Figure 30e shows the calculated $\mathrm{COHb}$ profiles of simulated house occupants in each room of the house.

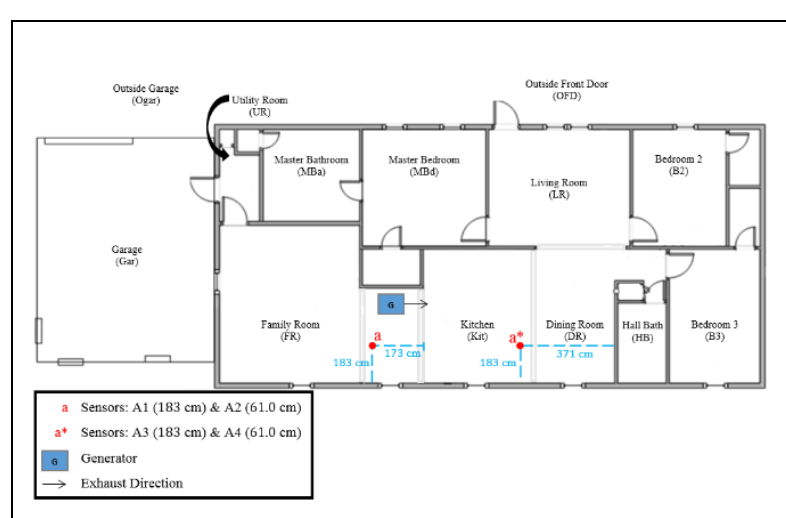

Figure 30a. Generator, exhaust direction, and sensor array layout during Case 7 Test 1 - G2P G300.

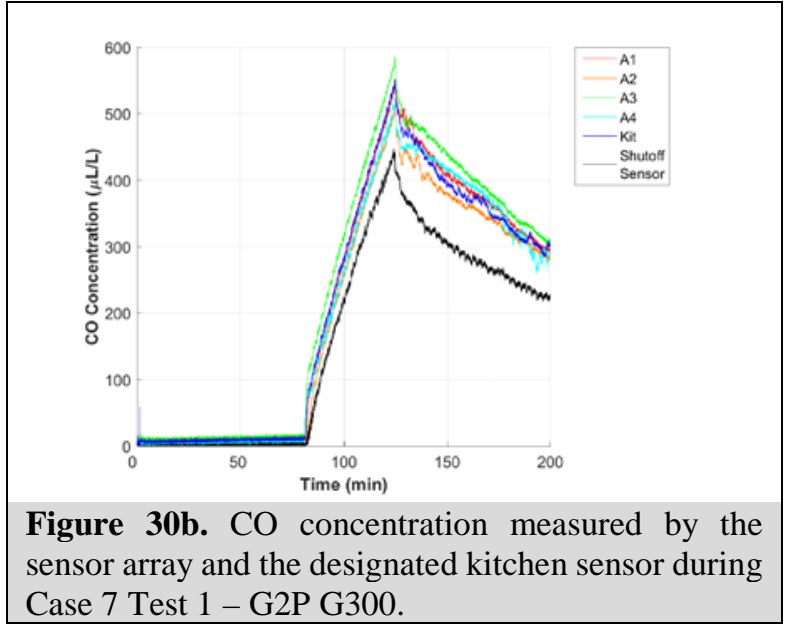

Figure 30b. CO concentration measured by the Case 7 Test 1 - G2P G300. 


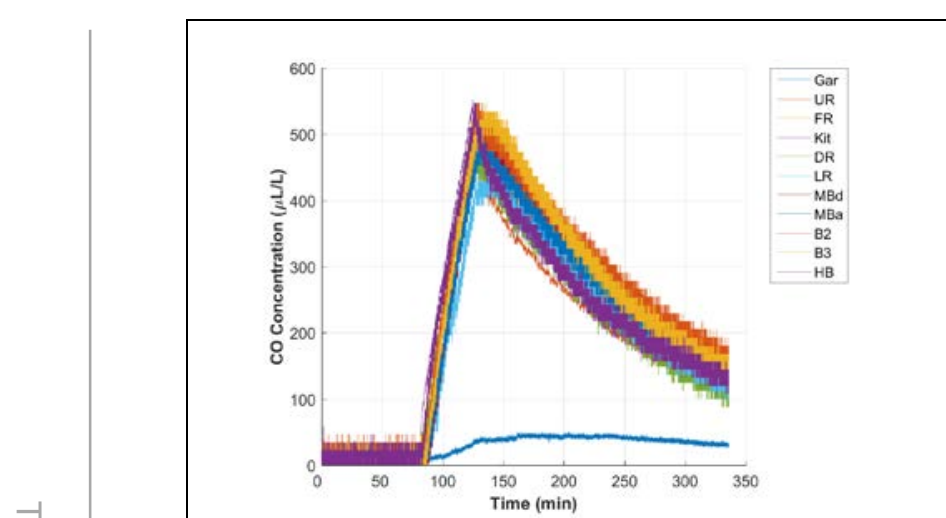

Figure 30c. CO concentration measured in each room of the house during Case 7 Test 1 - G2P G300.

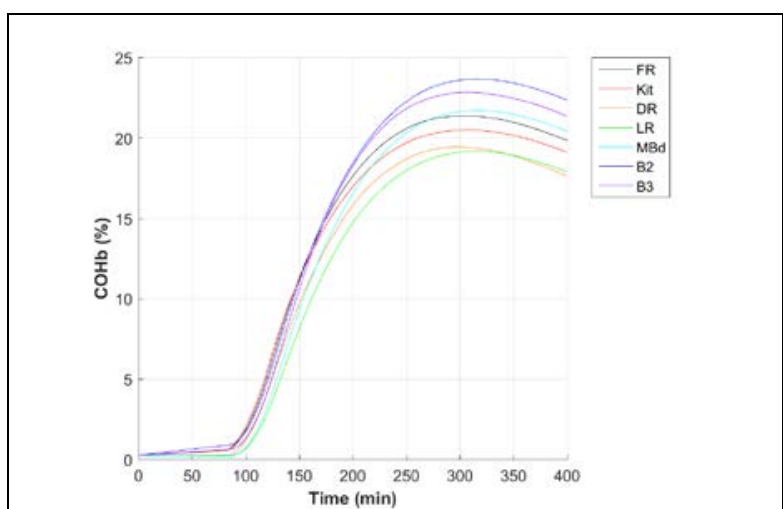

Figure 30e. $\mathrm{COHb}$ of house occupants during Case 7 Test 1 - G2P G300.

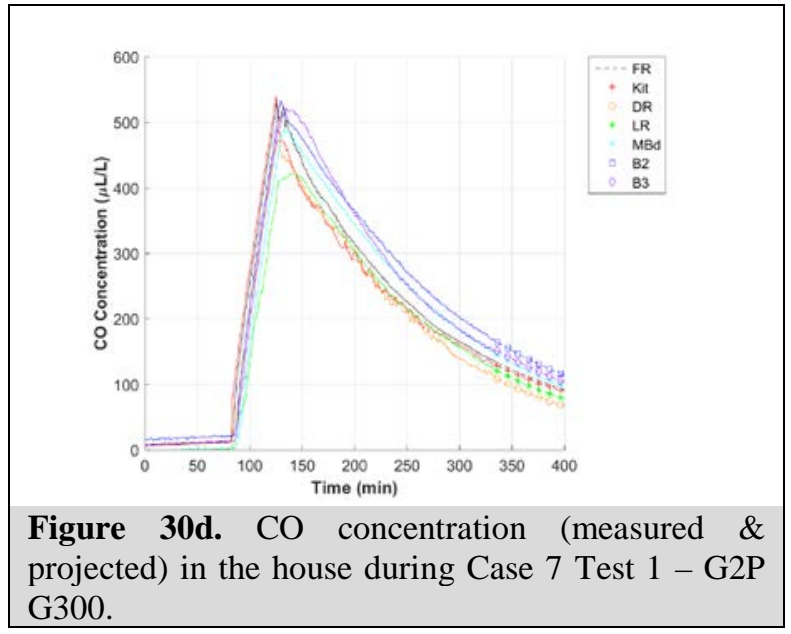

G300. 


\section{Generator Location: Living Room}

Table 2 summarizes the results for all tests performed with a generator located in the test house living room. The details are discussed below and shown in Figures 31 through 33.

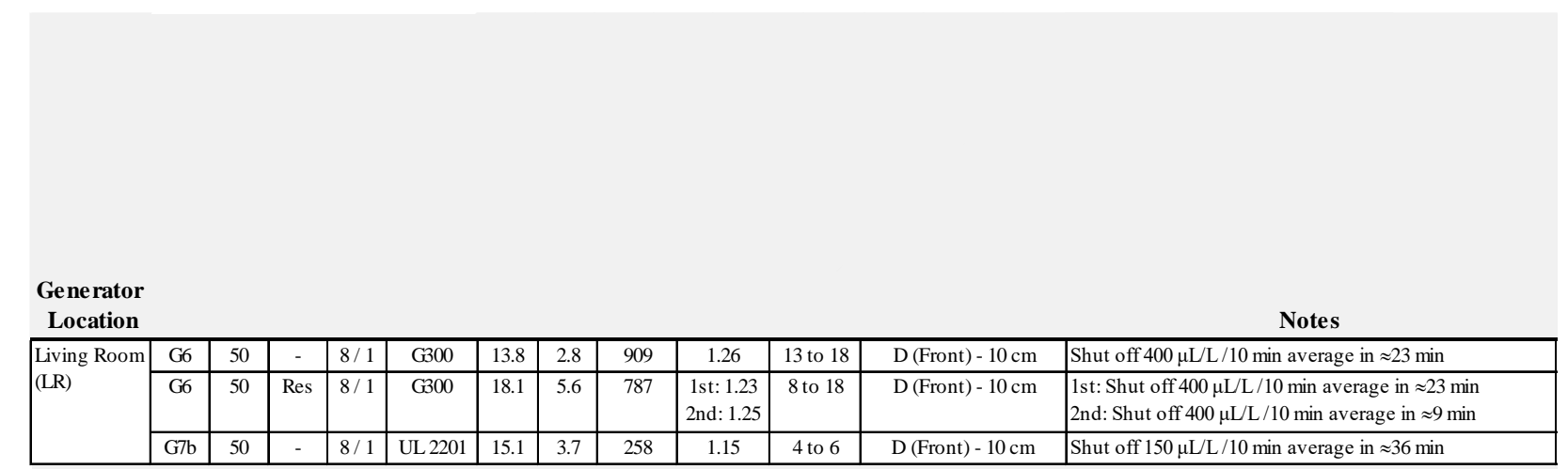

Table 2. Summary of test results for Living Room (LR) tests.

+Peak COHb Values are for house zones only.

+Ventilation Note: Unless specified otherwise, during the test - all exterior doors are closed, all interior doors are fully open, and all windows are closed. 


\section{Case 8 Test 1 - G6 G300}

The following figures illustrate the results for Case 8 Test 1 applying the G300 shutoff criteria. The G6 generator was fully fueled, positioned in the living room (near the front door with the exhaust facing the front door), and tested at $50 \%$ load $(3,000 \mathrm{~W})$. The front door was open $10 \mathrm{~cm}$. The shutoff sensor activated the algorithm to shut off the generator after approximately 23 min due to a sensor measurement of $400 \mu \mathrm{L} / \mathrm{L}$ for a 10 min average. Figure 31a shows the test house layout with generator location, exhaust direction, and sensor array location. Figure 31b shows the CO concentration measured in the living room (by the dedicated room sensor), the two sensor arrays located in the living room as shown in Figure 31a (A1: height $183 \mathrm{~cm}$, A2: height $61 \mathrm{~cm}$, A3: height $183 \mathrm{~cm}$, A4: height $61 \mathrm{~cm}$ ), and by the shutoff sensor located on the generator near the generator's electrical panel. The timeframe of the figure has been selected to show the degree of $\mathrm{CO}$ uniformity in the area around the generator between the shutoff sensor and the peak CO concentrations measured elsewhere in the living room near the time of shutoff. Figure 31c shows the $\mathrm{CO}$ concentration measured in each room of the house over the test. Figure 31d shows the CO concentration in the house with each room represented by a single line, which is composed of both a 'solid-line' pattern (showing measured CO concentration) and a 'dotted-line with a symbol' pattern (showing projected CO concentration). Figure 31e shows the calculated COHb profiles of simulated house occupants in each room of the house.

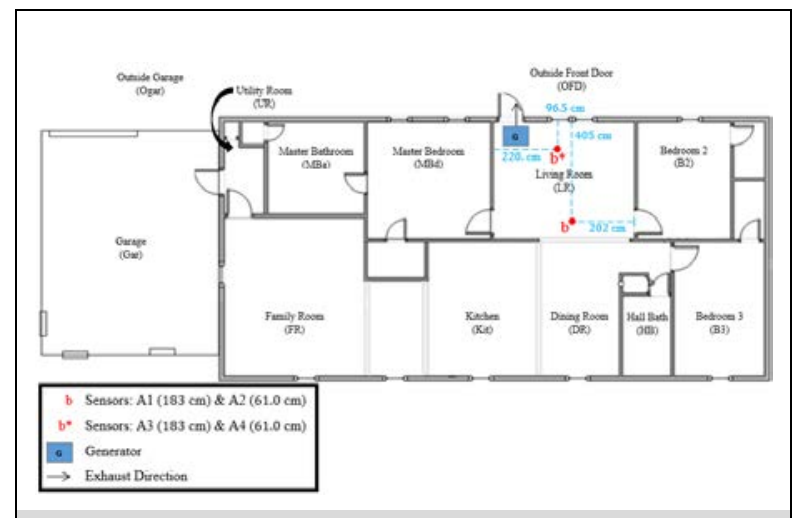

Figure 31a. Generator, exhaust direction, and sensor array layout during Case 8 Test 1 - G6 G300.

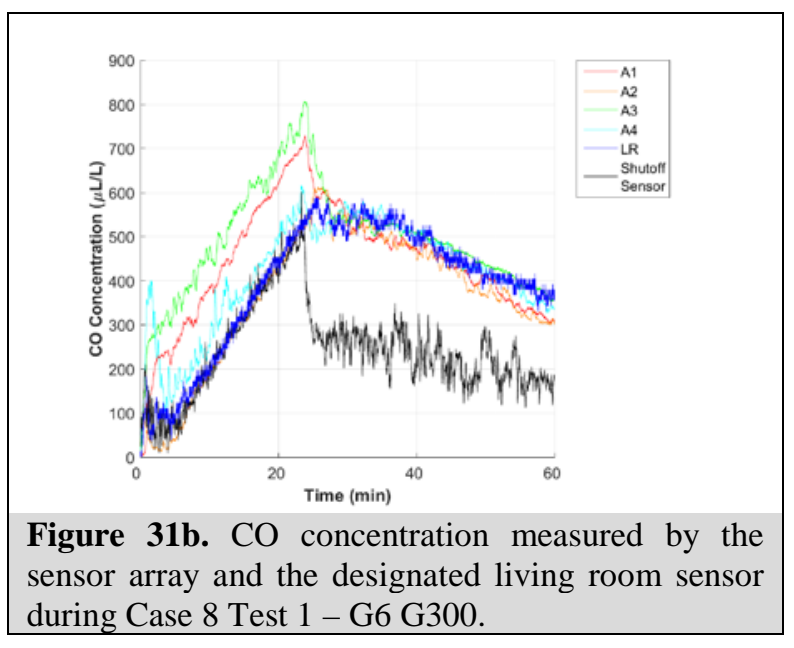




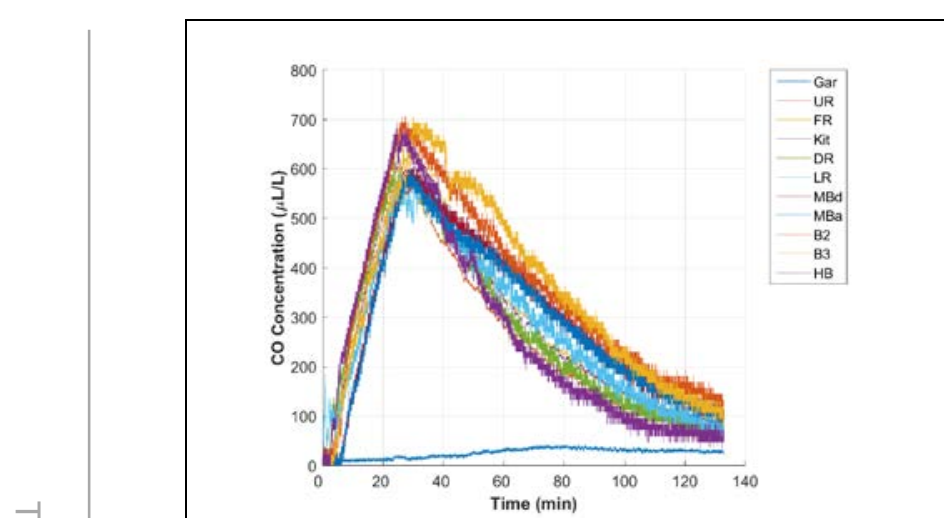

Figure 31c. CO concentration measured in each room of the house during Case 8 Test 1 - G6 G300.

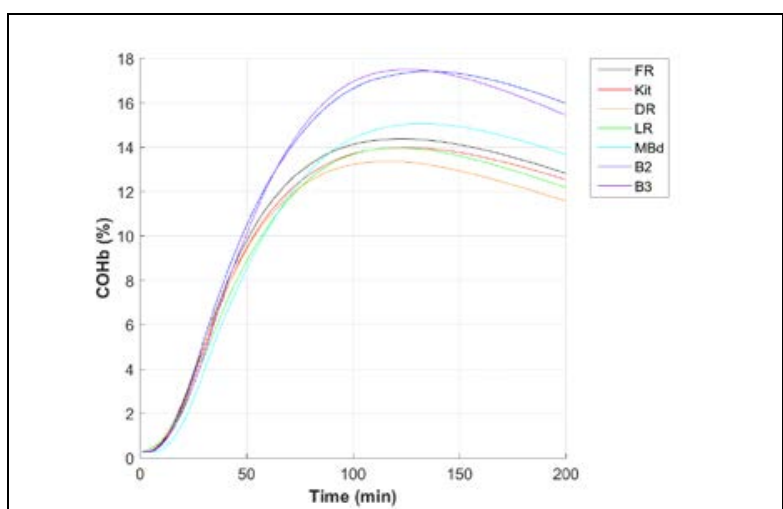

Figure 31e. $\mathrm{COHb}$ of house occupants during Case 8 Test 1 - G6 G300.

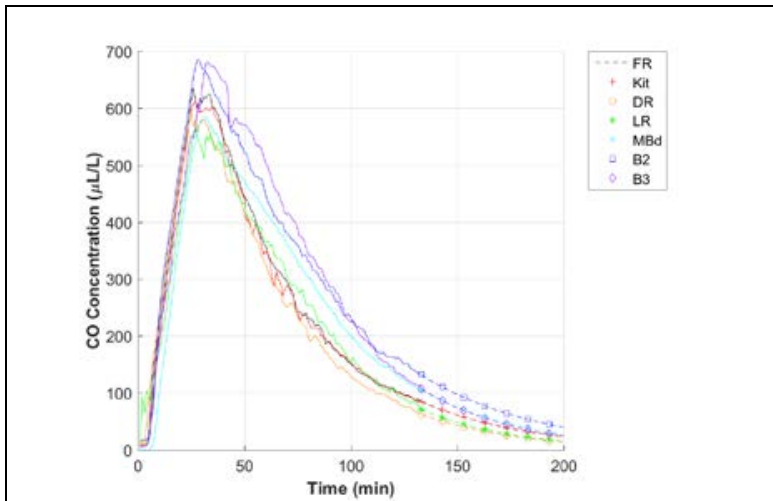

Figure 31d. CO concentration (measured \& projected) in the house during Case 8 Test 1 - G6 G300. 


\section{Case 8 Test 1 - G6 G300 Restart}

The following figures illustrate the results for Restart Case 8 Test 1 applying the G300 shutoff criteria. The G6 generator was fully fueled, positioned in the living room (near the front door with the exhaust facing the front door), and tested at $50 \%$ load $(3,000 \mathrm{~W})$. The front door was open 10 $\mathrm{cm}$. For the first generator run, the shutoff sensor activated the algorithm to shut off the generator after approximately $23 \mathrm{~min}$ due to a sensor measurement of $400 \mu \mathrm{L} / \mathrm{L}$ for a $10 \mathrm{~min}$ average. The generator was restarted and the test load restored approximately $10 \mathrm{~min}$ after the first instance of shutoff initiated by the shutoff sensor. For the second generator run, the shutoff sensor activated the algorithm to shut off the generator after approximately 9 min due to a sensor measurement of $400 \mu \mathrm{L} / \mathrm{L}$ for a $10 \mathrm{~min}$ average. Figure 32a shows the test house layout with generator location, exhaust direction, and sensor array location. Figure 32b shows the CO concentration measured in the living room (by the dedicated room sensor), the two sensor arrays located in the living room as shown in Figure 32a (A1: height $183 \mathrm{~cm}$, A2: height $61 \mathrm{~cm}$, A3: height $183 \mathrm{~cm}$, A4: height 61 $\mathrm{cm})$, and by the shutoff sensor located on the generator near the generator's electrical panel. The timeframe of the figure has been selected to show the degree of CO uniformity in the area around the generator between the shutoff sensor and the peak CO concentrations measured elsewhere in the living room near the time of shutoff. Figure 32c shows the CO concentration measured in each room of the house over the test. Figure 32d shows the CO concentration in the house with each room represented by a single line, which is composed of both a 'solid-line' pattern (showing measured CO concentration) and a 'dotted-line with a symbol' pattern (showing projected CO concentration). Figure 32e shows the calculated $\mathrm{COHb}$ profiles of simulated house occupants in each room of the house.

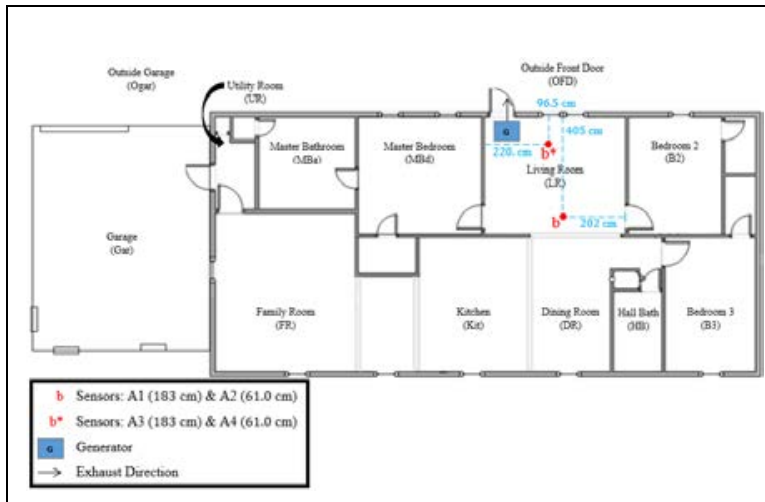

Figure 32a. Generator, exhaust direction, and sensor array layout during Restart Case 8 Test 1 - G6 G300.

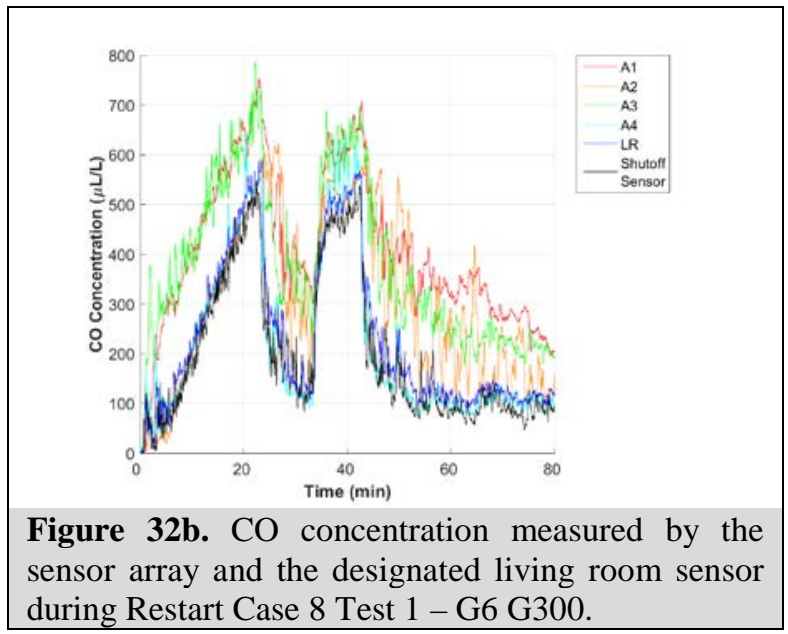

Figure 32b. CO concentration measured by the during Restart Case 8 Test 1 - G6 G300. 


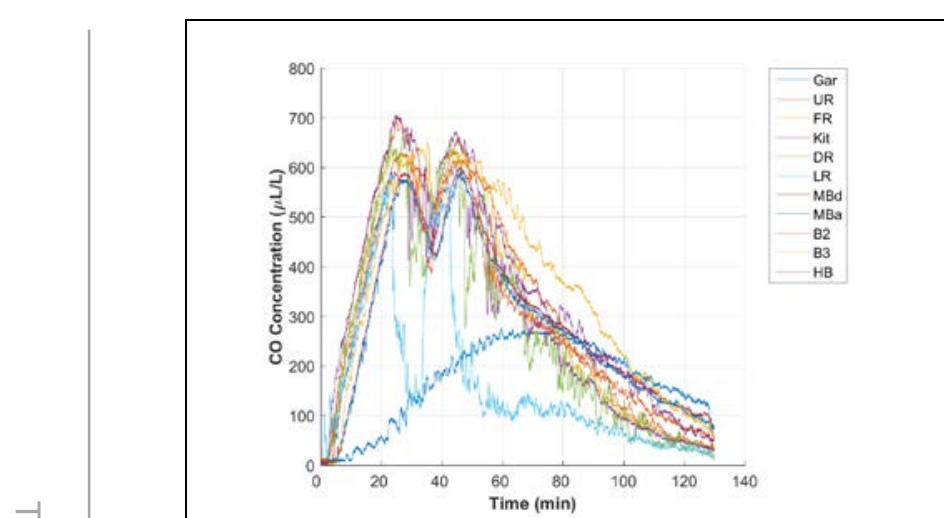

Figure 32c. CO concentration measured in each room of the house during Restart Case 8 Test 1 - G6 G300.

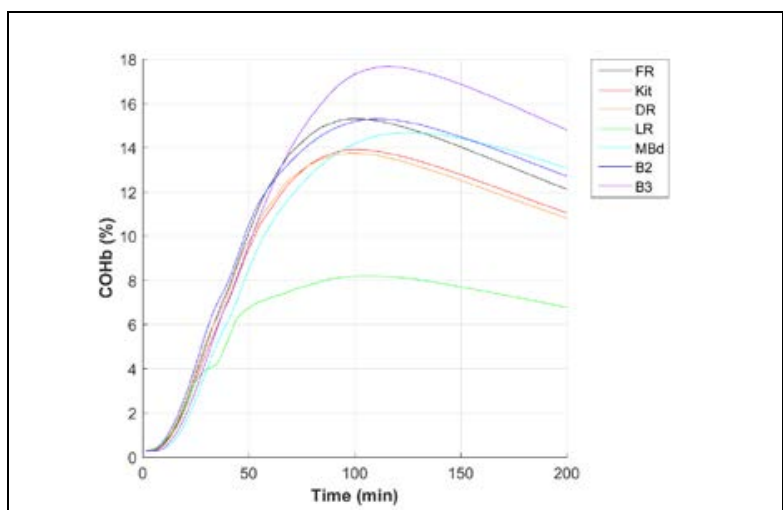

Figure 32e. COHb of house occupants during Restart Case 8 Test 1 - G6 G300.

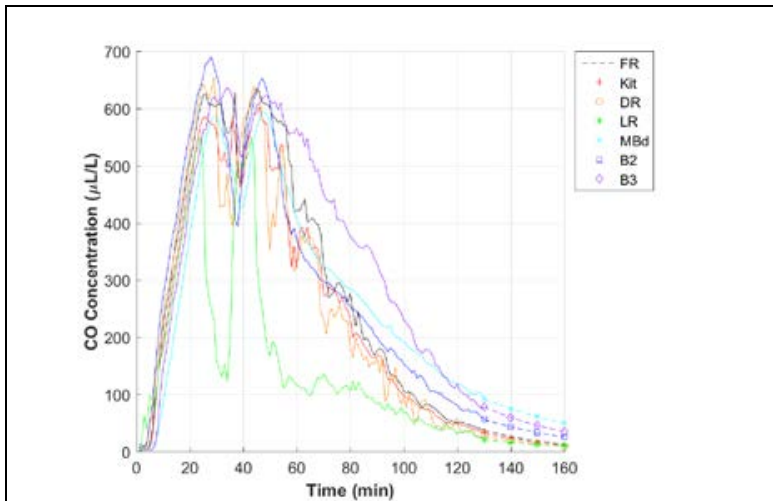

Figure 32d. CO concentration (measured \& projected) in the house during Restart Case 8 Test 1 G6 G300. 


\section{Case 8 Test 1 - G7b UL 2201}

The following figures illustrate the results for Case 8 Test 1 applying the UL 2201 shutoff criteria. The G7b generator was fully fueled, positioned in the living room (near the front door with the exhaust facing the front door), and tested at $50 \%$ load $(3,000 \mathrm{~W})$. The front door was open $10 \mathrm{~cm}$. The shutoff sensor activated the algorithm to shut off the generator after approximately 36 min due to a sensor measurement of $150 \mu \mathrm{L} / \mathrm{L}$ for a 10 min average. Figure 33a shows the test house layout with generator location, exhaust direction, and sensor array location. Figure 33b shows the CO concentration measured in the living room (by the dedicated room sensor), the two sensor arrays located in the living room as shown in Figure 33a (A1: height $183 \mathrm{~cm}, \mathrm{~A} 2$ : height $61 \mathrm{~cm}$, A3: height $183 \mathrm{~cm}$, A4: height $61 \mathrm{~cm}$ ), and by the shutoff sensor located on the generator near the generator's electrical panel. The timeframe of the figure has been selected to show the degree of $\mathrm{CO}$ uniformity in the area around the generator between the shutoff sensor and the peak CO concentrations measured elsewhere in the living room near the time of shutoff. Figure 33c shows the CO concentration measured in each room of the house over the test. Figure 33d shows the CO concentration in the house with each room represented by a single line, which is composed of both a 'solid-line' pattern (showing measured CO concentration) and a 'dotted-line with a symbol' pattern (showing projected CO concentration). Figure 33e shows the calculated COHb profiles of simulated house occupants in each room of the house.

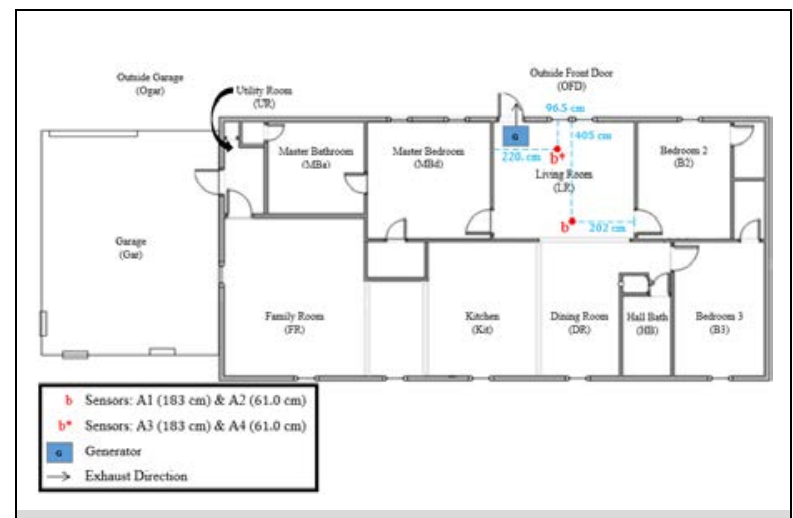

Figure 33a. Generator, exhaust direction, and sensor array layout during Case 8 Test 1 - G7b UL 2201.

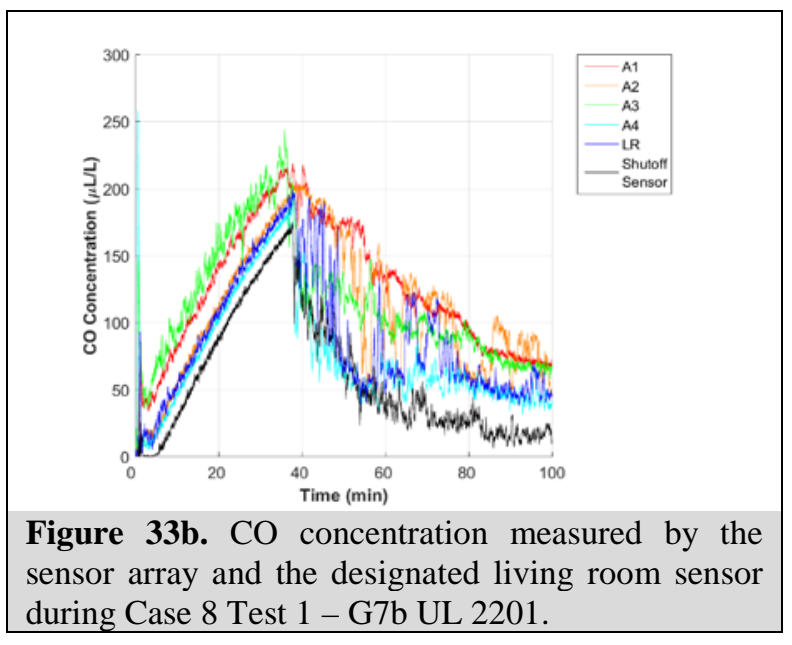




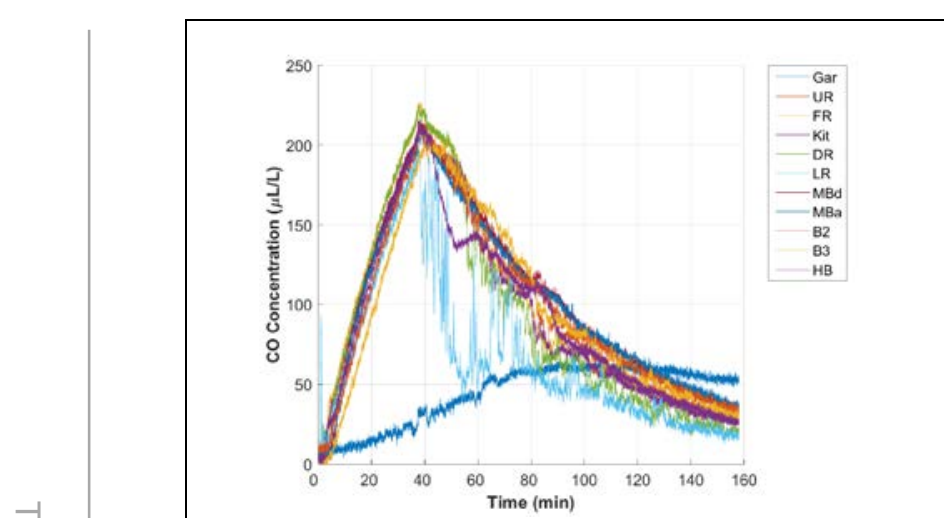

Figure 33c. CO concentration measured in each room of the house during Case 8 Test 1 - G7b UL 2201.

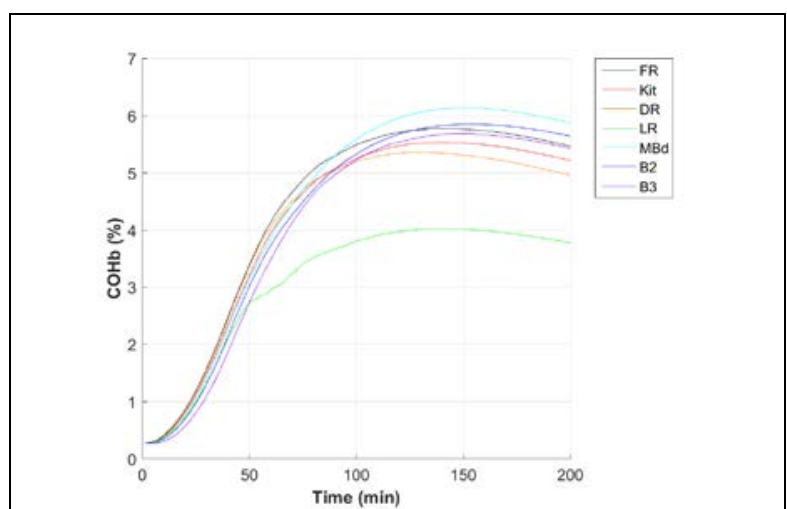

Figure 33e. $\mathrm{COHb}$ of house occupants during Case 8 Test 1 - G7b UL 2201.

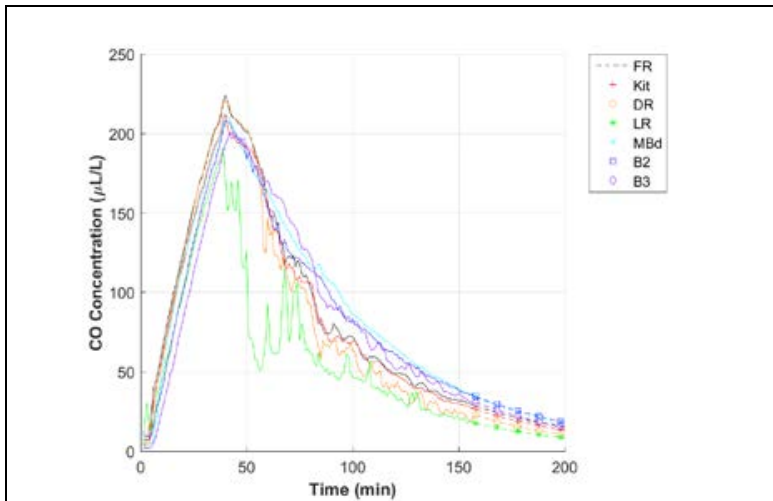

Figure 33d. CO concentration (measured \& projected) in the house during Case 8 Test $1-$ G7b UL 2201. 


\section{Generator Location: Family Room}

Table 3 summarizes the results for all tests performed with a generator located in the test house family room. The details are discussed below and shown in Figures 34 through 41.

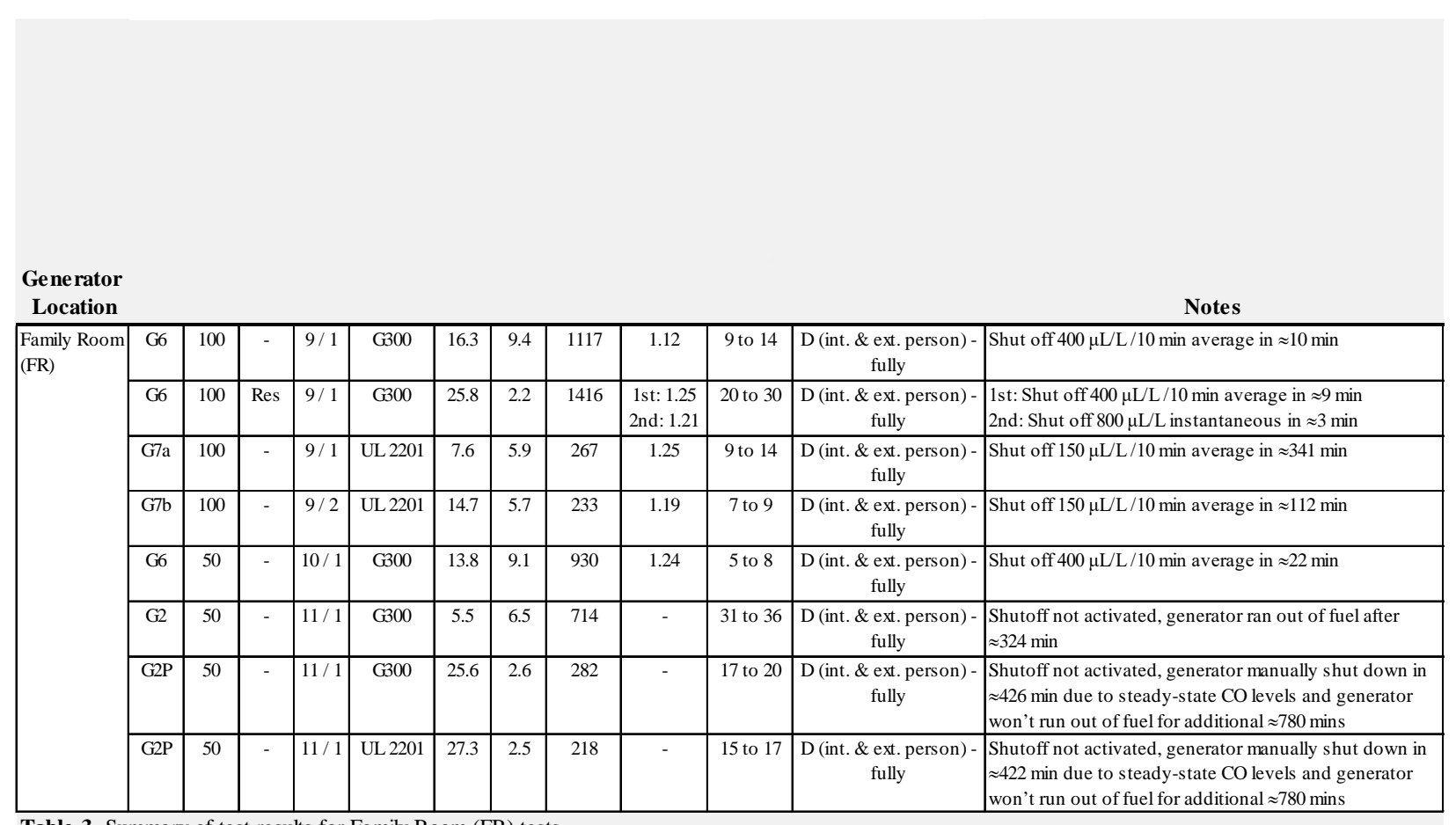

Table 3. Summary of test results for Family Room (FR) tests.

+Peak COHb Values are for house zones only.

HVentilation Note: Unless specified otherwise, during the test - all exterior doors are closed, all interior doors are fully open, and all windows are closed. 


\section{Case 9 Test 1 - G6 G300}

The following figures illustrate the results for Case 9 Test 1 applying the G300 shutoff criteria. The G6 generator was fully fueled, positioned in the family room (near the door leading to the utility room with the exhaust facing the garage wall), and tested at $100 \%$ load $(6,000 \mathrm{~W})$. The door leading from the family room to the utility room, the interior person door (from garage to utility room), and the exterior person door (from garage to backyard) were all fully open; the garage bay door was closed during the test. The shutoff sensor activated the algorithm to shut off the generator after approximately $10 \mathrm{~min}$ due to a sensor measurement of $400 \mu \mathrm{L} / \mathrm{L}$ for a $10 \mathrm{~min}$ average. Figure 34a shows the test house layout with generator location, exhaust direction, and sensor array location. Figure 34b shows the CO concentration measured in the family room (by the dedicated room sensor), by the two sensor arrays located in the family room as shown in Figure 34a (A1: height $183 \mathrm{~cm}, \mathrm{A2}$ : height $61 \mathrm{~cm}, \mathrm{~A} 3$ : height $183 \mathrm{~cm}, \mathrm{~A} 4$ : height $61 \mathrm{~cm}$ ), and by the shutoff sensor located on the generator near the generator's electrical panel. The timeframe of the figure has been selected to show the degree of CO uniformity in the area around the generator between the shutoff sensor and the peak $\mathrm{CO}$ concentrations measured elsewhere in the family room near the time of shutoff. Figure 34c shows the $\mathrm{CO}$ concentration measured in each room of the house over the test. Figure 34d shows the CO concentration in the house with each room represented by a single line, which is composed of both a 'solid-line' pattern (showing measured CO concentration) and a 'dotted-line with a symbol' pattern (showing projected CO concentration). Figure 34e shows the calculated $\mathrm{COHb}$ profiles of simulated house occupants in each room of the house.

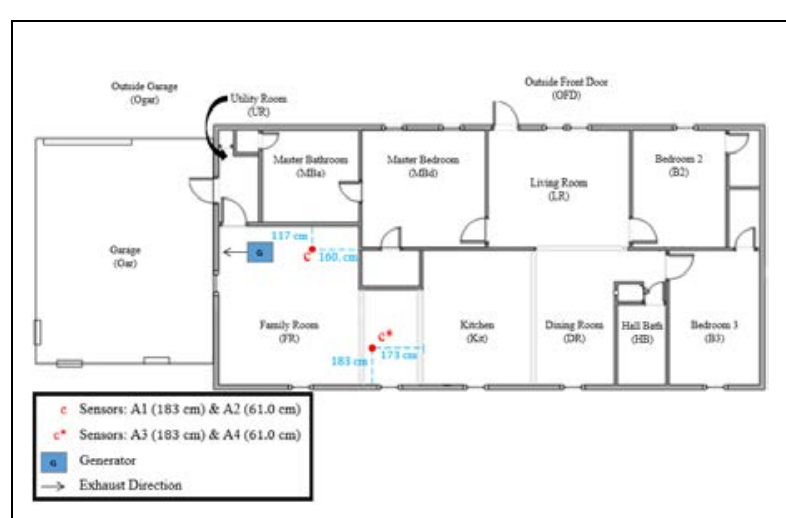

Figure 34a. Generator, exhaust direction, and sensor array layout during Case 9 Test 1 - G6 G300.

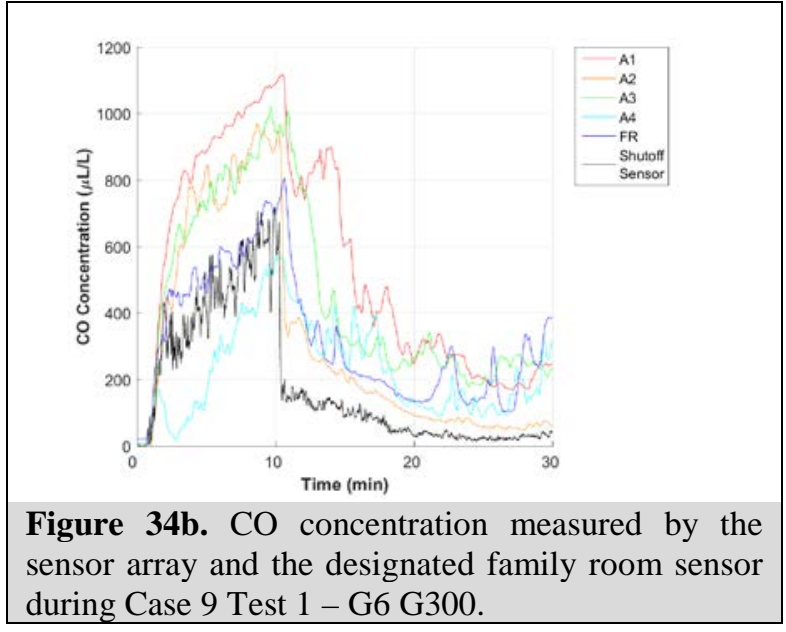

Figure 34b. CO concentration measured by the during Case 9 Test 1 - G6 G300. 


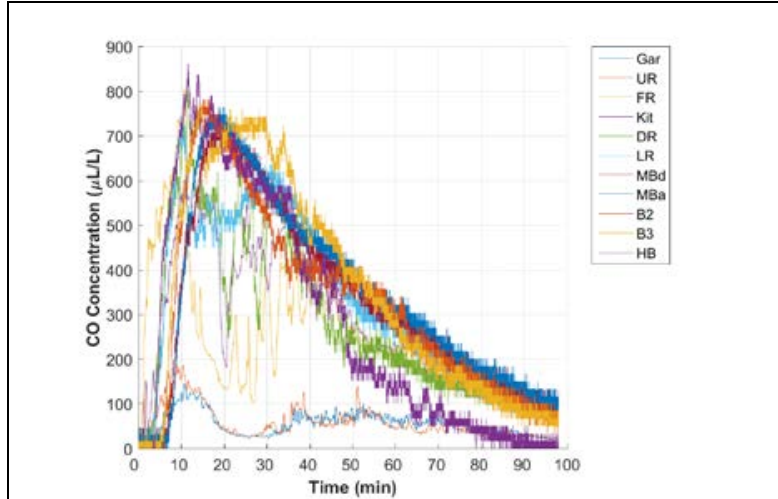

Figure 34c. CO concentration measured in each room of the house during Case 9 Test 1 - G6 G300.

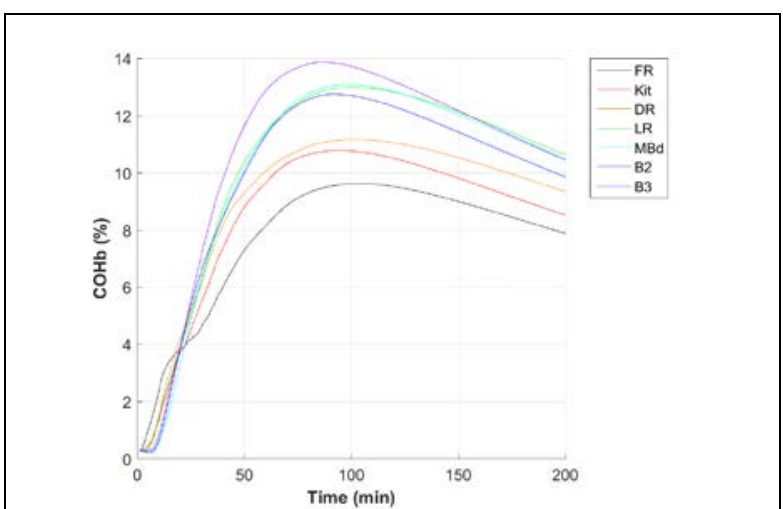

Figure 34e. $\mathrm{COHb}$ of house occupants during Case 9 Test 1 - G6 G300.

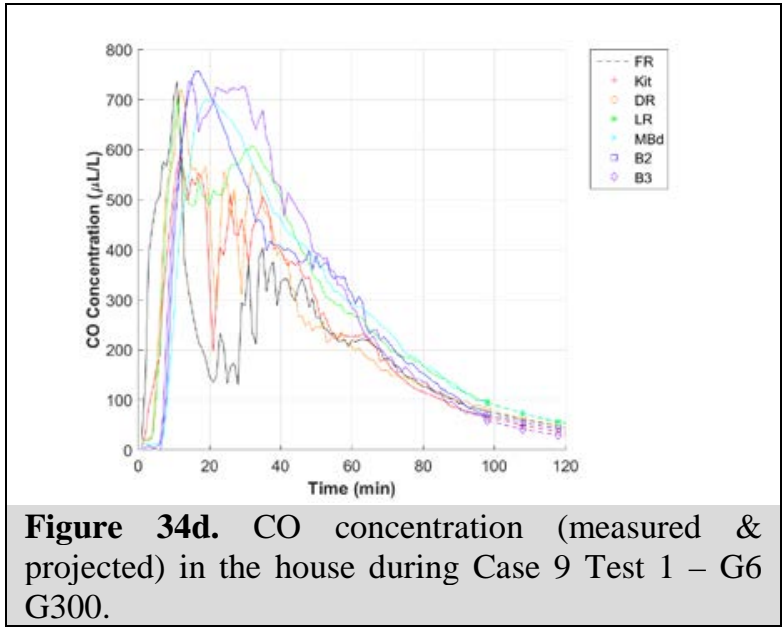

Figure 34d. CO concentration (measured \& projected) in the house during Case 9 Test 1 - G6 


\section{Case 9 Test 1 - G6 G300 Restart}

The following figures illustrate the results for Restart Case 9 Test 1 applying the G300 shutoff criteria. The G6 generator was fully fueled, positioned in the family room (near the door leading to the utility room with the exhaust facing the garage wall), and tested at $100 \%$ load $(6,000 \mathrm{~W})$. The door leading from the family room to the utility room, the interior person door (from garage to utility room), and the exterior person door (from garage to backyard) were all fully open; the garage bay door was closed during the test. For the first generator run, the shutoff sensor activated the algorithm to shut off the generator after approximately 9 min due to a sensor measurement of $400 \mu \mathrm{L} / \mathrm{L}$ for a $10 \mathrm{~min}$ average. The generator was restarted and the test load restored approximately $10 \mathrm{~min}$ after the first instance of shutoff initiated by the shutoff sensor. For the second generator run, the shutoff sensor activated the algorithm to shut off the generator after approximately 3 min due to an instantaneous sensor measurement of $800 \mu \mathrm{L} / \mathrm{L}$. Figure 35a shows the test house layout with generator location, exhaust direction, and sensor array location. Figure 35b shows the CO concentration measured in the family room (by the dedicated room sensor), by the two sensor arrays located in the family room as shown in Figure 35a (A1: height $183 \mathrm{~cm}, \mathrm{~A} 2$ : height $61 \mathrm{~cm}$, A3: height $183 \mathrm{~cm}, \mathrm{~A} 4$ : height $61 \mathrm{~cm}$ ), and by the shutoff sensor located on the generator near the generator's electrical panel. The timeframe of the figure has been selected to show the degree of CO uniformity in the area around the generator between the shutoff sensor and the peak CO concentrations measured elsewhere in the family room near the time of shutoff. Figure 35c shows the $\mathrm{CO}$ concentration measured in each room of the house over the test. Figure 35d shows the $\mathrm{CO}$ concentration in the house with each room represented by a single line, which is composed of both a 'solid-line' pattern (showing measured CO concentration) and a 'dottedline with a symbol' pattern (showing projected CO concentration). Figure 35e shows the calculated $\mathrm{COHb}$ profiles of simulated house occupants in each room of the house.

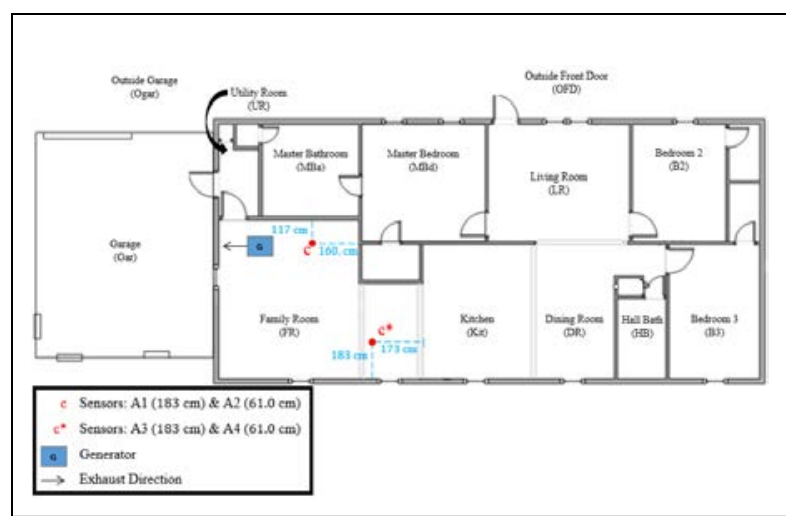

Figure 35a. Generator, exhaust direction, and sensor array layout during Restart Case 9 Test 1 - G6 G300.

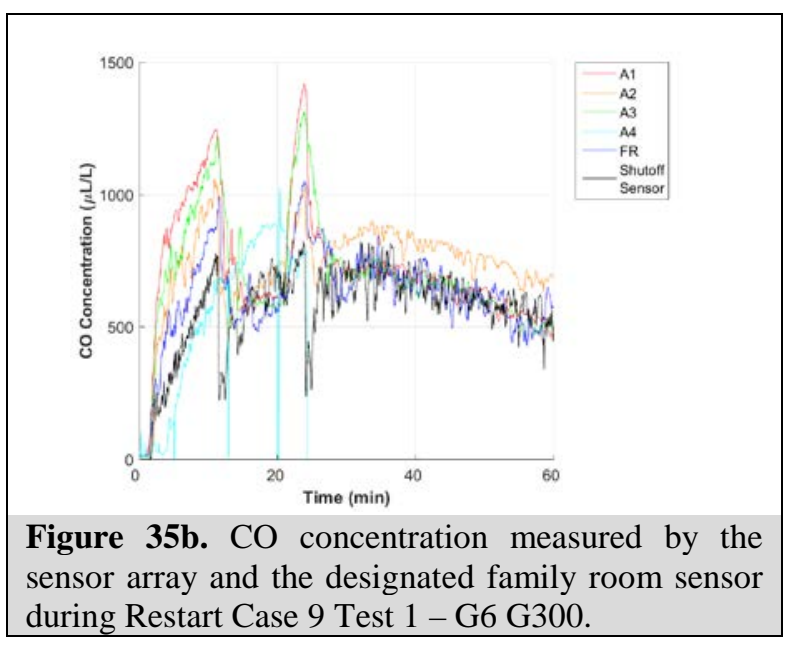




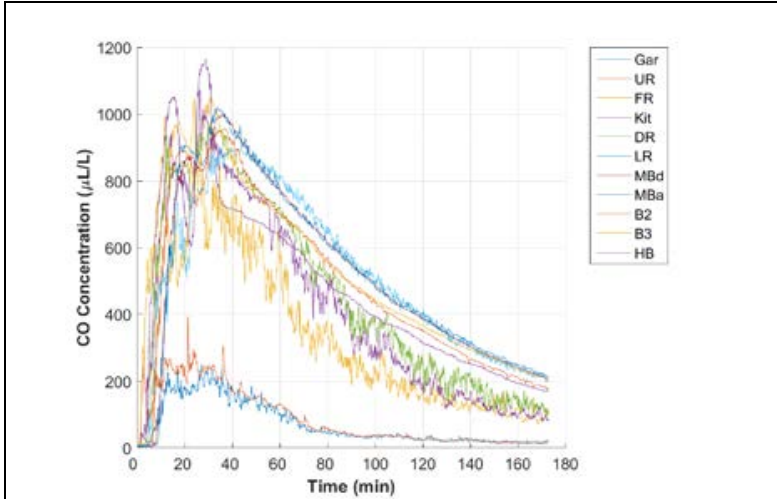

Figure 35c. CO concentration measured in each room of the house during Restart Case 9 Test 1 - G6 G300.

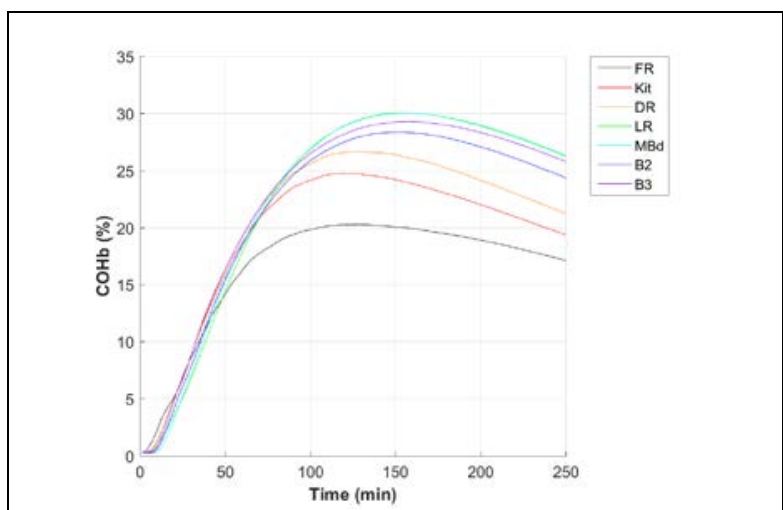

Figure 35e. $\mathrm{COHb}$ of house occupants during Restart Case 9 Test 1 - G6 G300.

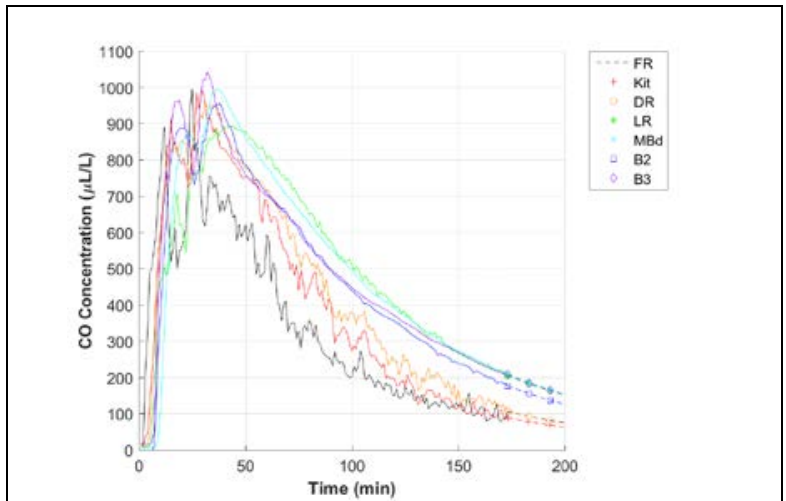

Figure 35d. CO concentration (measured \& projected) in the house during Restart Case 9 Test 1 G6 G300. 


\section{Case 9 Test 1 - G7a UL 2201}

The following figures illustrate the results for Case 9 Test 1 applying the UL 2201 shutoff criteria. The G7a generator was fully fueled, positioned in the family room (near the utility room door with the exhaust facing the wall shared with the garage), and tested at $100 \%$ load $(7,000 \mathrm{~W})$. The door leading from the family room to the utility room, the interior person door (from garage to utility room), and the exterior person door (from garage to backyard) were all fully open; the garage bay door was closed during the test. The shutoff sensor activated the algorithm to shut off the generator after approximately $341 \mathrm{~min}$ due to a sensor measurement of $150 \mu \mathrm{L} / \mathrm{L}$ for a $10 \mathrm{~min}$ average. Figure 36a shows the test house layout with generator location, generator exhaust direction, and sensor array location. Figure 36b shows the CO concentration measured in the family room (by the dedicated room sensor), by two sensors located in the family room (A1: height $183 \mathrm{~cm}, \mathrm{~A} 2$ : height $61 \mathrm{~cm}$ ), by the other two sensors located on the kitchen-family room boundary (A3: height $183 \mathrm{~cm}$, A4: height $61 \mathrm{~cm}$ ), and by the shutoff sensor located on the generator near the generator's electrical panel. The timeframe of the figure has been selected to show the degree of CO uniformity in the area around the generator between the shutoff sensor and the peak CO concentrations measured elsewhere in the family room near the time of shutoff. Figure 36c shows the CO concentration measured in each room of the house over the test. Figure 36d shows the CO concentration in the house with each room represented by a single line, which is composed of both a 'solid-line' pattern (showing measured CO concentration) and a 'dotted-line with a symbol' pattern (showing projected CO concentration). Figure 36e shows the calculated COHb profiles of simulated house occupants in each room of the house.

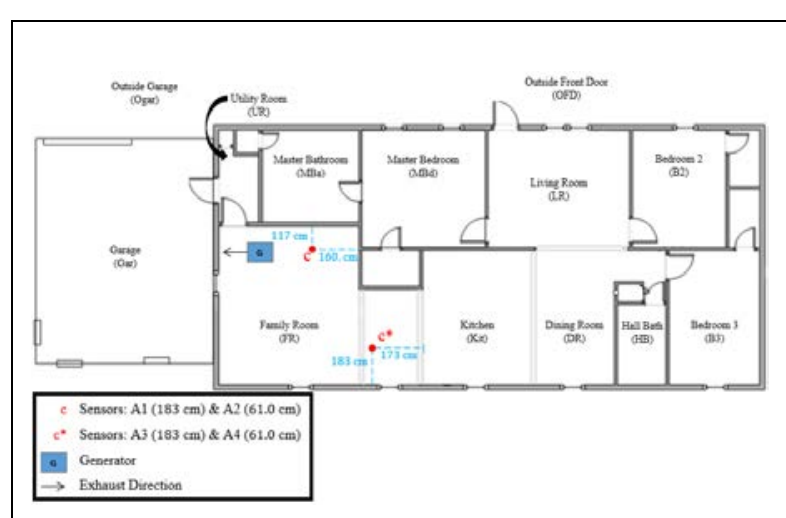

Figure 36a. Generator, exhaust direction, and sensor array layout during Case 9 Test 1 - G7a UL 2201.

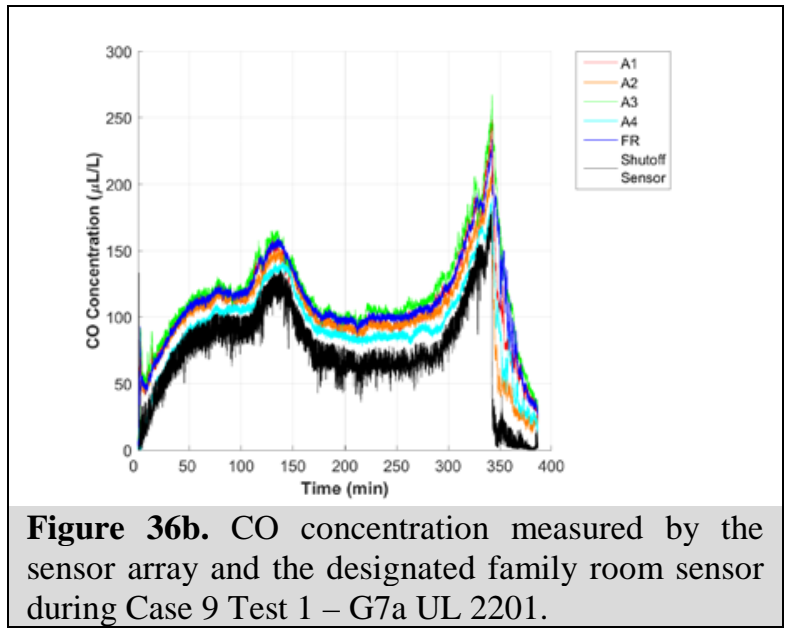
during Case 9 Test 1 - G7a UL 2201. 

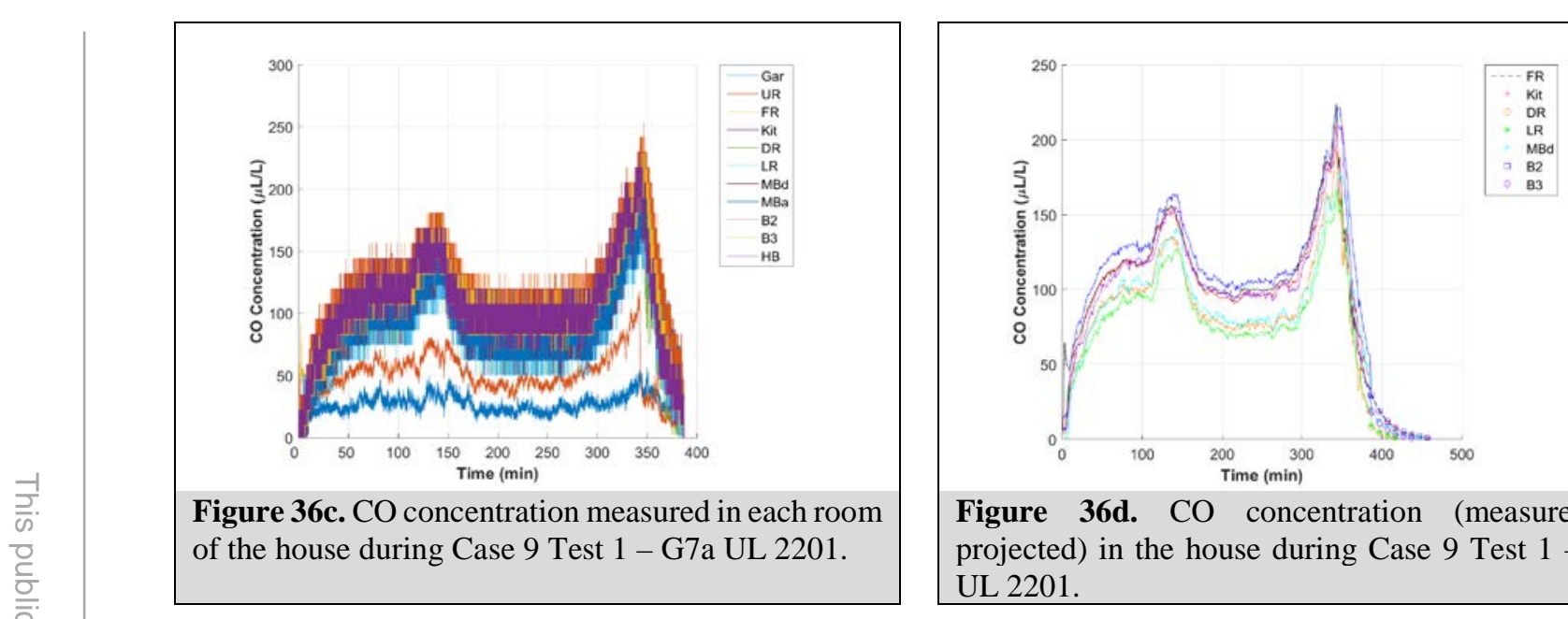

Figure 36d. CO concentration (measured \& projected) in the house during Case 9 Test $1-$ GPa UL 2201.

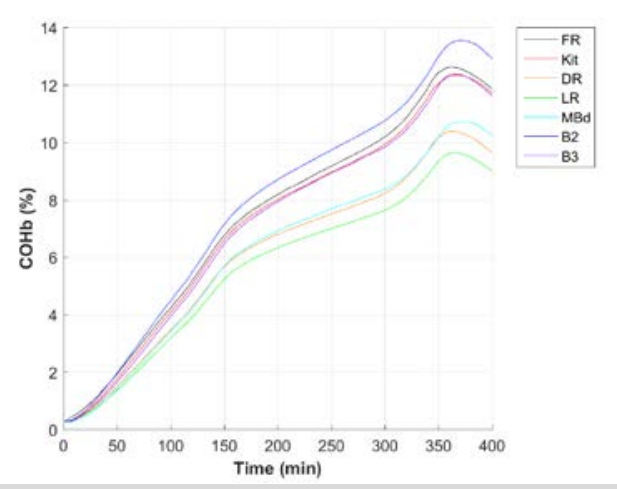

Figure 36e. $\mathrm{COHb}$ of house occupants during Case 9 Test 1 - G7a UL 2201.

75 


\section{Case 9 Test 2 - G7b UL 2201}

The following figures illustrate the results for Case 9 Test 2 applying the UL 2201 shutoff criteria. The G7b generator was fully fueled, positioned in the family room (near the utility room door with the exhaust facing the wall shared with the garage), and tested at $100 \%$ load $(7,000 \mathrm{~W})$. The door leading from the family room to the utility room, the interior person door (from garage to utility room), and the exterior person door (from garage to backyard) were all fully open; the garage bay door was closed during the test. The shutoff sensor activated the algorithm to shut off the generator after approximately $112 \mathrm{~min}$ due to a sensor measurement of $150 \mu \mathrm{L} / \mathrm{L}$ for a $10 \mathrm{~min}$ average. Figure 37a shows the test house layout with generator location, generator exhaust direction, and sensor array location. Figure 37b shows the CO concentration measured in the family room (by the dedicated room sensor), by two sensors located in the family room (A1: height $183 \mathrm{~cm}, \mathrm{~A} 2$ : height $61 \mathrm{~cm}$ ), by the other two sensors located on the kitchen-family room boundary (A3: height $183 \mathrm{~cm}$, A4: height $61 \mathrm{~cm}$ ), and by the shutoff sensor located on the generator near the generator's electrical panel. The timeframe of the figure has been selected to show the degree of CO uniformity in the area around the generator between the shutoff sensor and the peak CO concentrations measured elsewhere in the family room near the time of shutoff. Figure 37c shows the CO concentration measured in each room of the house over the test. Figure 37d shows the CO concentration in the house with each room represented by a single line, which is composed of both a 'solid-line' pattern (showing measured CO concentration) and a 'dotted-line with a symbol' pattern (showing projected CO concentration). Figure 37e shows the calculated COHb profiles of simulated house occupants in each room of the house.

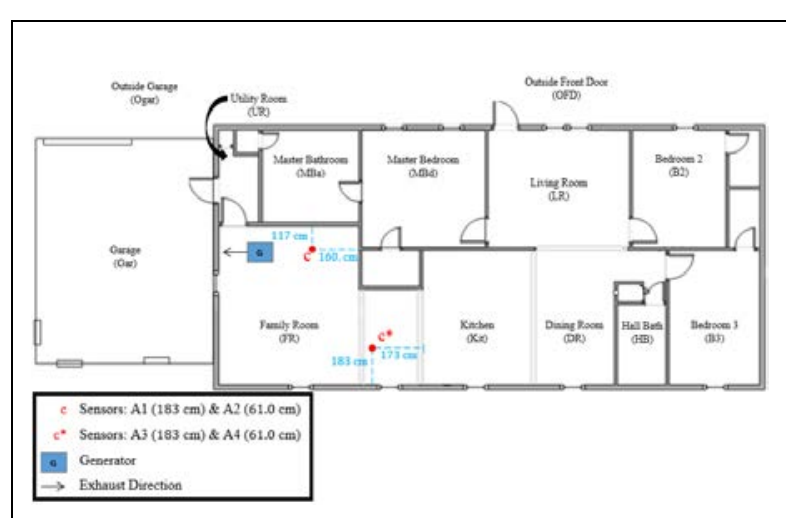

Figure 37a. Generator, exhaust direction, and sensor array layout during Case 9 Test 2 - G7b UL 2201.

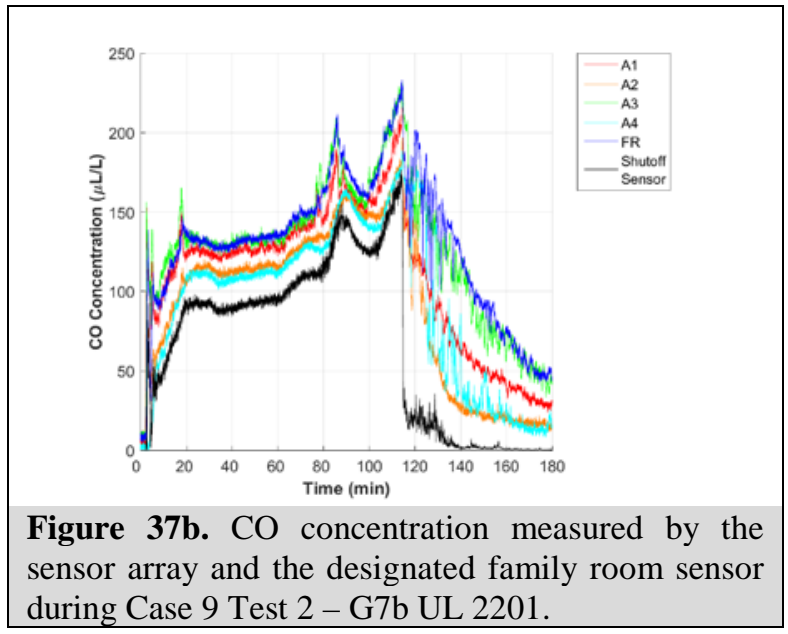

Figure 37b. CO concentration measured by the during Case 9 Test 2 - G7b UL 2201. 


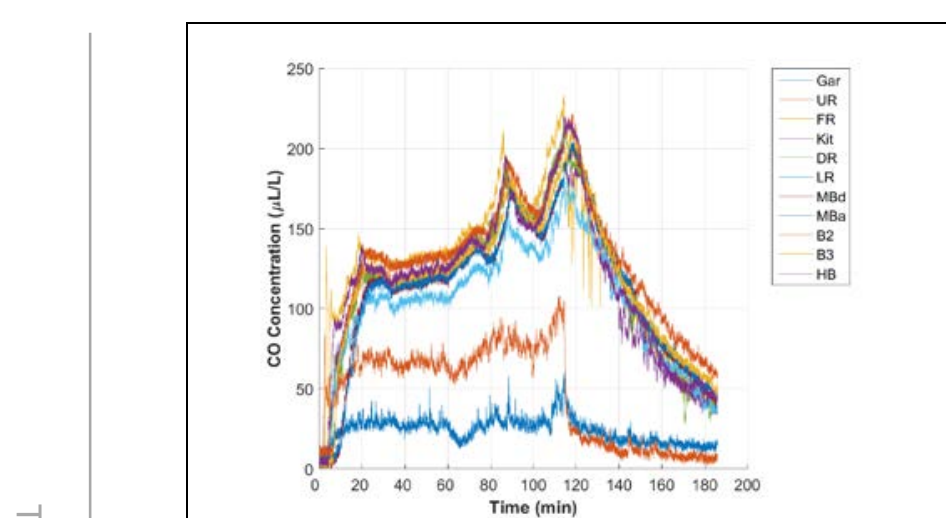

Figure 37c. CO concentration measured in each room of the house during Case 9 Test 2 - G7b UL 2201.

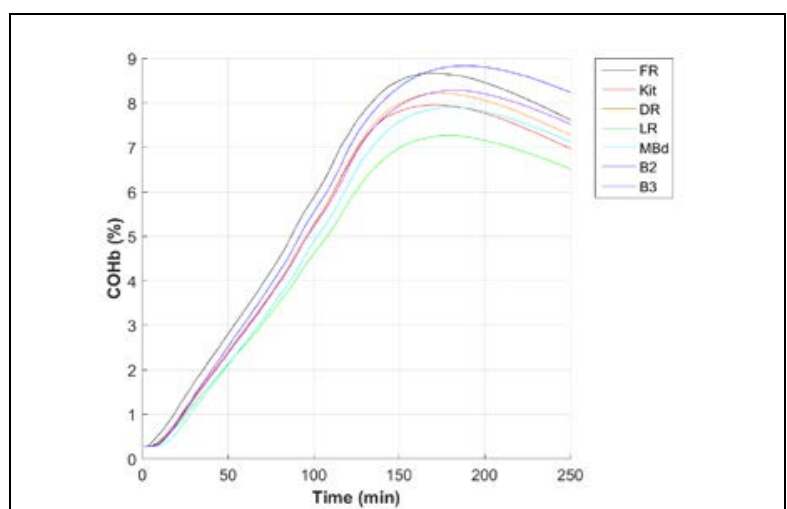

Figure 37e. $\mathrm{COHb}$ of house occupants during Case 9 Test 2 - G7b UL 2201.

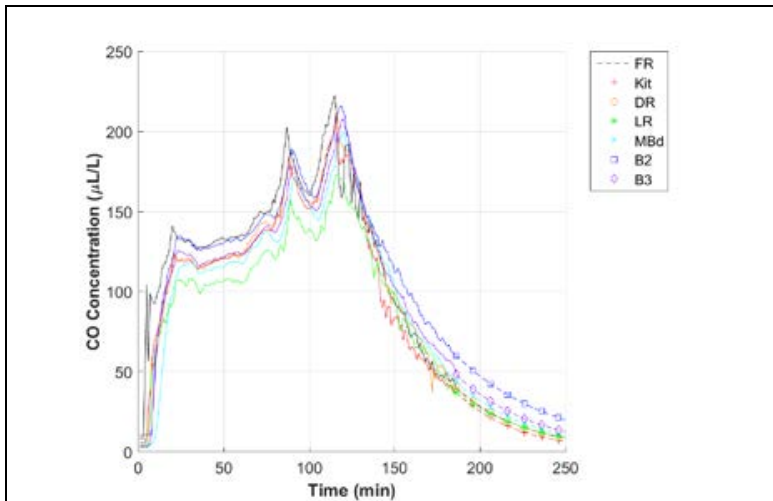

Figure 37d. CO concentration (measured \& projected) in the house during Case 9 Test $2-$ G7b UL 2201. 


\section{Case 10 Test 1 - G6 G300}

The following figures illustrate the results for Case 10 Test 1 applying the G300 shutoff criteria. The G6 generator was fully fueled, positioned in the family room (near the door leading to the utility room with the exhaust facing the garage wall), and tested at $50 \%$ load (3,000 W). The door leading from the family room to the utility room, the interior person door (from garage to utility room), and the exterior person door (from garage to backyard) were all fully open; the garage bay door was closed during the test. The shutoff sensor activated the algorithm to shut off the generator after approximately $22 \mathrm{~min}$ due to a sensor measurement of $400 \mu \mathrm{L} / \mathrm{L}$ for a $10 \mathrm{~min}$ average. Figure 38a shows the test house layout with generator location, exhaust direction, and sensor array location. Figure 38b shows the $\mathrm{CO}$ concentration measured in the family room (by the dedicated room sensor), by the two sensor arrays located in the family room as shown in Figure 38a (A1: height $183 \mathrm{~cm}, \mathrm{~A} 2$ : height $61 \mathrm{~cm}, \mathrm{A3}$ : height $183 \mathrm{~cm}, \mathrm{~A} 4$ : height $61 \mathrm{~cm}$ ), and by the shutoff sensor located on the generator near the generator's electrical panel. The timeframe of the figure has been selected to show the degree of CO uniformity in the area around the generator between the shutoff sensor and the peak CO concentrations measured elsewhere in the family room near the time of shutoff. Figure 38c shows the CO concentration measured in each room of the house over the test. Figure 38d shows the $\mathrm{CO}$ concentration in the house with each room represented by a single line, which is composed of both a 'solid-line' pattern (showing measured CO concentration) and a 'dotted-line with a symbol' pattern (showing projected CO concentration). Figure 38e shows the calculated COHb profiles of simulated house occupants in each room of the house.

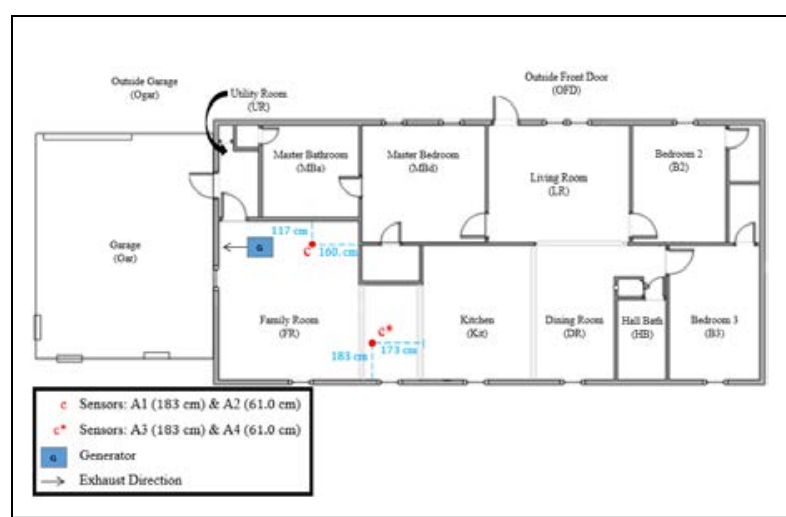

Figure 38a. Generator, exhaust direction, and sensor array layout during Case 10 Test 1 - G6 G300.

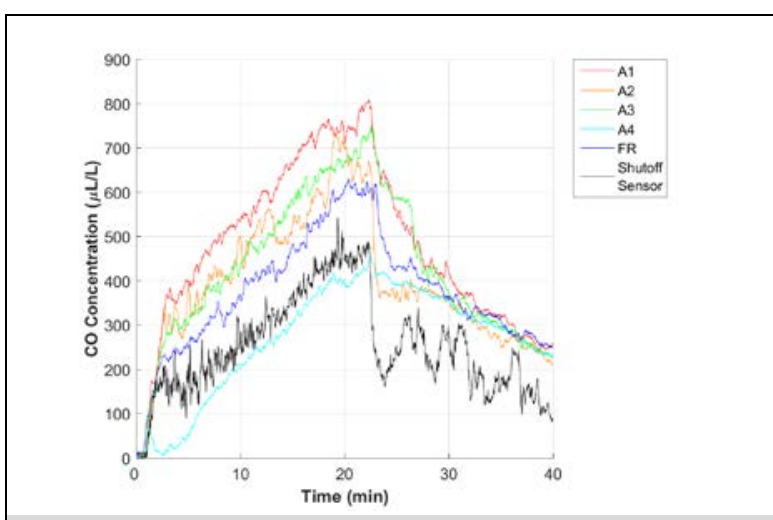

Figure 38b. CO concentration measured by the sensor array and the designated family room sensor during Case 10 Test 1 - G6 G300. 


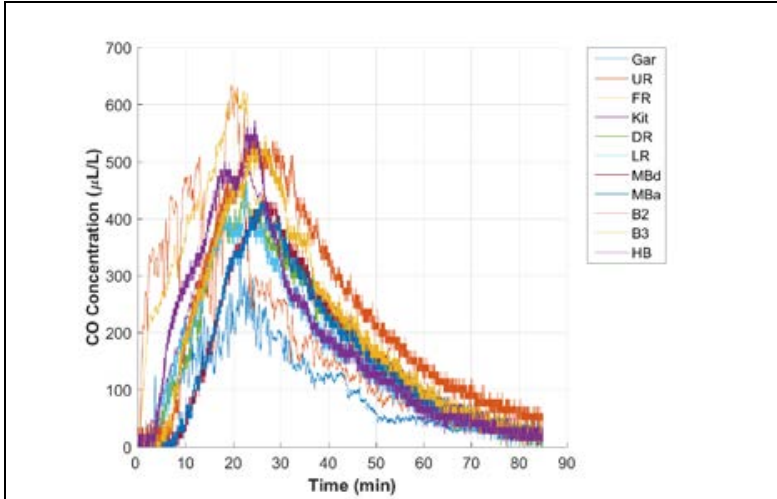

Figure 38c. CO concentration measured in each room of the house during Case 10 Test 1 - G6 G300.

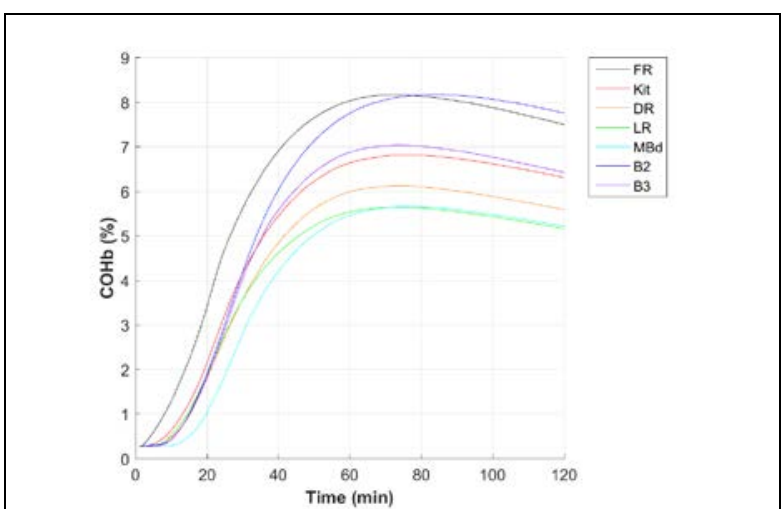

Figure 38e. $\mathrm{COHb}$ of house occupants during Case 10 Test 1 - G6 G300.

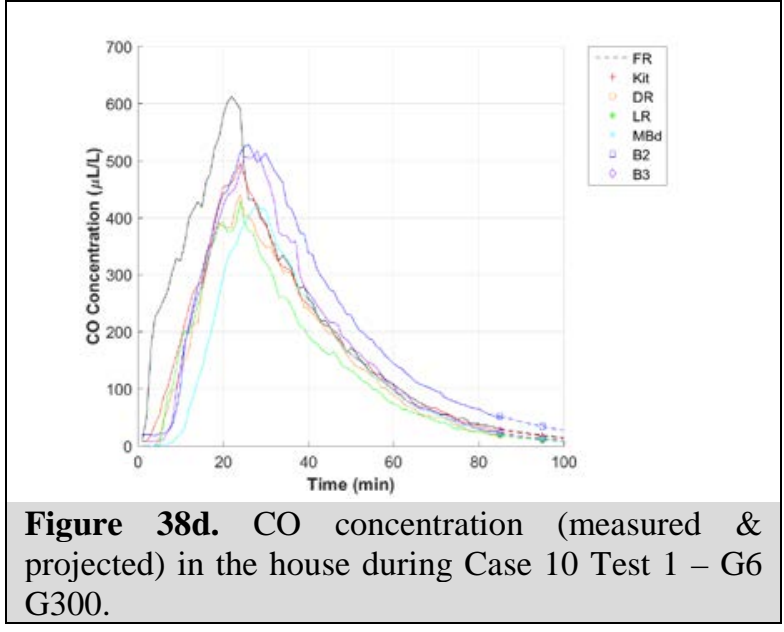

Figure 38d. CO concentration (measured \& G300. 


\section{Case 11 Test 1 - G2 G300}

The following figures illustrate the results for Case 11 Test 1 applying the G300 shutoff criteria. The G2 generator was fully fueled, positioned in the family room (near the door leading to the utility room with the exhaust facing the garage wall), and tested at $50 \%$ load (800 W). The door leading from the family room to the utility room, the interior person door (from garage to utility room), and the exterior person door (from garage to backyard) were all fully open; the garage bay door was closed during the test. The shutoff criteria were inadvertently set to UL 2201 specifications initially, which led to a shutoff after approximately $20 \mathrm{~min}$. Within $2 \mathrm{~min}$ of this shutdown event, the generator was restarted with the shutoff criteria changed to G300 specifications. After the shutoff criteria was switched to G300 specifications the shutoff sensor was not activated and the generator ran out of fuel after approximately $324 \mathrm{~min}$. Figure 39a shows the test house layout with generator location, exhaust direction, and sensor array location. Figure 39b shows the CO concentration measured in the family room (by the dedicated room sensor), by the two sensor arrays located in the family room as shown in Figure 39a (A1: height 183 cm, A2: height $61 \mathrm{~cm}$, A3: height $183 \mathrm{~cm}, \mathrm{~A} 4$ : height $61 \mathrm{~cm}$ ), and by the shutoff sensor located on the generator near the generator's electrical panel. The figure shows the degree of CO uniformity in the area around the generator between the shutoff sensor and the peak $\mathrm{CO}$ concentrations measured elsewhere in the family room. Figure 39c shows the CO concentration measured in each room of the house over the test. Figure 39d shows the CO concentration in the house with each room represented by a single line, which is composed of both a 'solid-line' pattern (showing measured $\mathrm{CO}$ concentration) and a 'dotted-line with a symbol' pattern (showing projected CO concentration). Figure 39e shows the calculated $\mathrm{COHb}$ profiles of simulated house occupants in each room of the house.

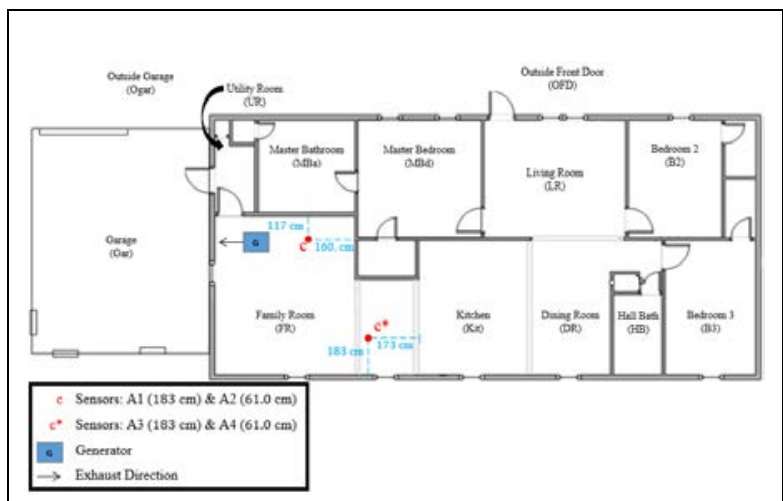

Figure 39a. Generator, exhaust direction, and sensor array layout during Case 11 Test 1 - G2 G300.

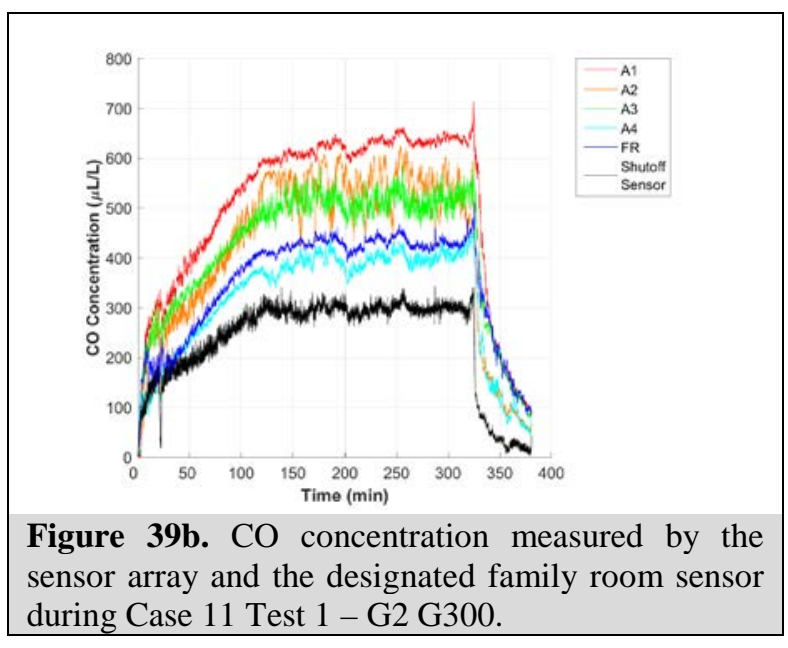




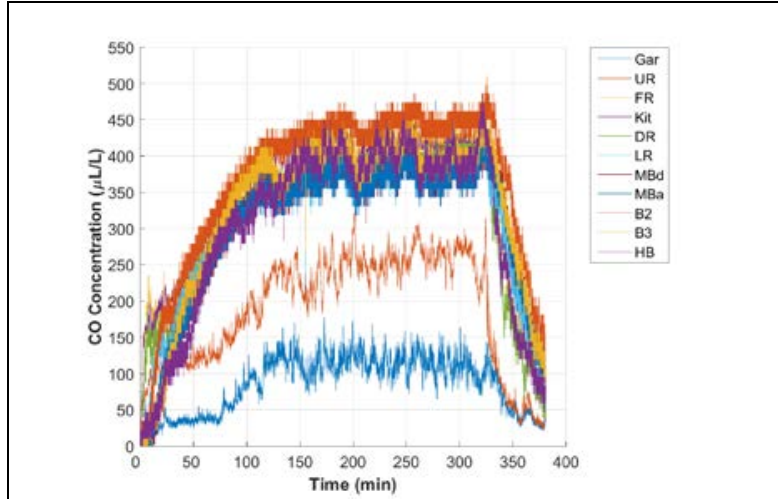

Figure 39c. CO concentration measured in each room of the house during Case 11 Test 1 - G2 G300.

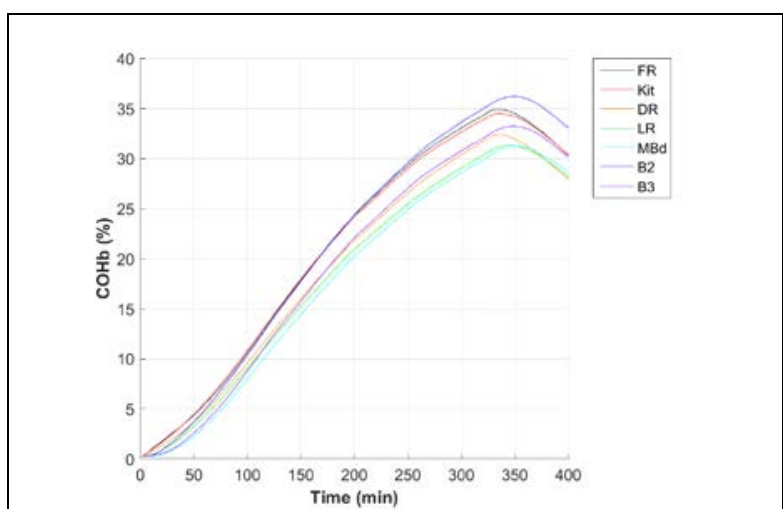

Figure 39e. $\mathrm{COHb}$ of house occupants during Case 11 Test $1-$ G2 G300.

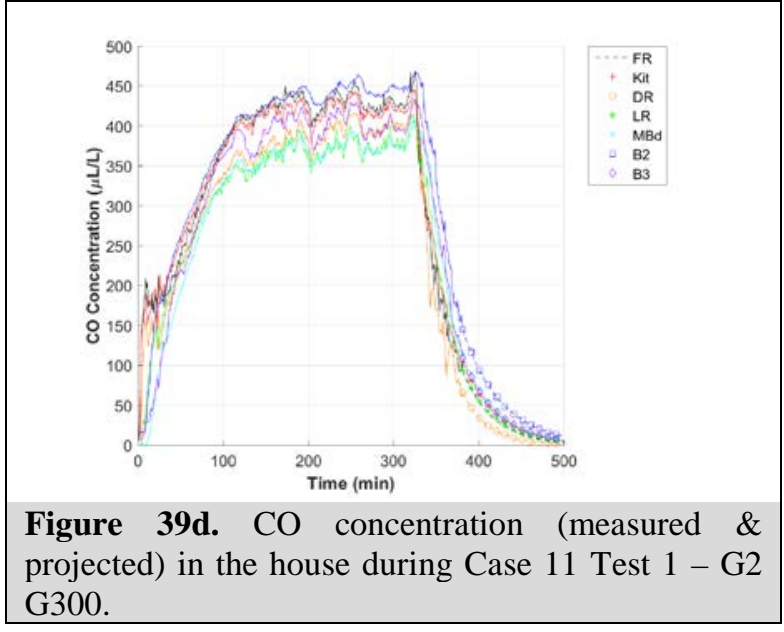

Figure 39d. CO concentration (measured \& G300. 


\section{Case 11 Test 1 - G2P G300}

The following figures illustrate the results for Case 11 Test 1 applying the G300 shutoff criteria. The G2P generator, with a full propane tank connected, was positioned in the family room (near the door leading to the utility room with the exhaust facing the garage wall) and tested at $50 \%$ load $(800 \mathrm{~W})$. The door leading from the family room to the utility room, the interior person door (from garage to utility room), and the exterior person door (from garage to backyard) were all fully open; the garage bay door was closed during the test. The shutoff sensor was not activated, and the generator manually shut off after approximately $426 \mathrm{~min}$ and CO concentrations were then allowed to decay naturally for around $60 \mathrm{~min}$. Figure 40a shows the test house layout with generator location, exhaust direction, and sensor array location. Figure 40b shows the CO concentration measured in the family room (by the dedicated room sensor), by the two sensor arrays located in the family room as shown in Figure 40a (A1: height $183 \mathrm{~cm}, \mathrm{A2}$ : height $61 \mathrm{~cm}$, A3: height $183 \mathrm{~cm}, \mathrm{~A} 4$ : height $61 \mathrm{~cm}$ ), and by the shutoff sensor located on the generator near the generator's electrical panel. The figure shows the degree of CO uniformity in the area around the generator between the shutoff sensor and the peak $\mathrm{CO}$ concentrations measured elsewhere in the family room. Figure 40c shows the $\mathrm{CO}$ concentration measured in each room of the house over the test. Figure 40d shows the calculated $\mathrm{COHb}$ profiles of simulated house occupants in each room of the house.

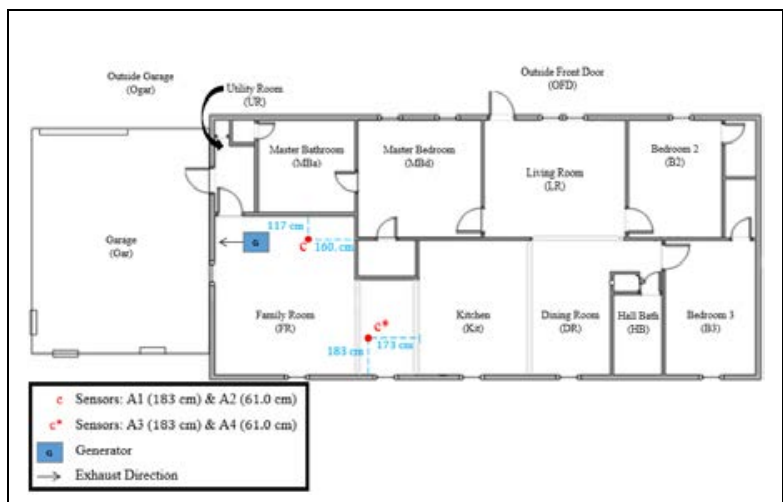

Figure 40a. Generator, exhaust direction, and sensor array layout during Case 11 Test 1 - G2P G300.

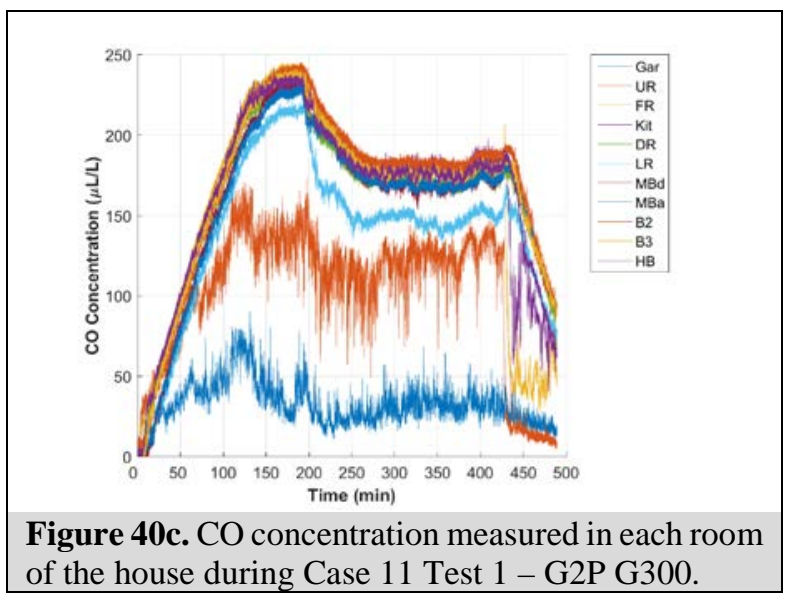

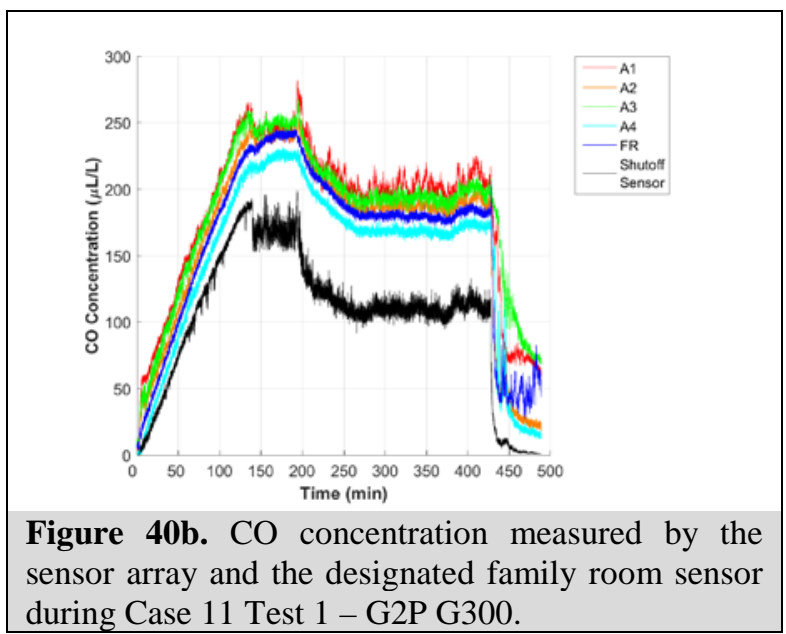

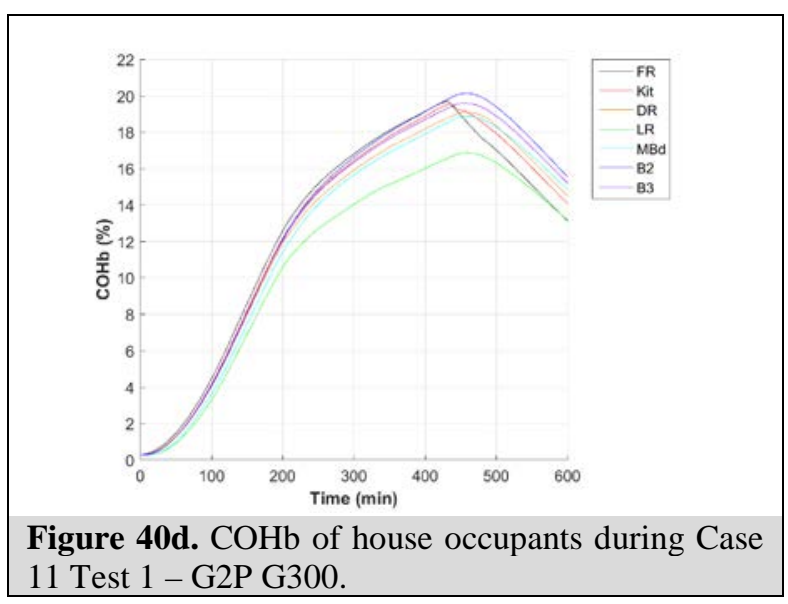




\section{Case 11 Test 1 - G2P UL 2201}

The following figures illustrate the results for Case 11 Test 1 applying the UL 2201 shutoff criteria. The G2P generator, with a full propane tank connected, was positioned in the family room (near the door leading to the utility room with the exhaust facing the garage wall) and tested at $50 \%$ load $(800 \mathrm{~W})$. The door leading from the family room to the utility room, the interior person door (from garage to utility room), and the exterior person door (from garage to backyard) were all fully open; the garage bay door was closed during the test. The shutoff sensor was not activated, and the generator manually shut off after approximately 422 min and CO concentrations were then allowed to decay naturally for around $60 \mathrm{~min}$. Figure 41a shows the test house layout with generator location, exhaust direction, and sensor array location. Figure 41b shows the CO concentration measured in the family room (by the dedicated room sensor), by the two sensor arrays located in the family room as shown in Figure 41a (A1: height $183 \mathrm{~cm}, \mathrm{A2}$ : height $61 \mathrm{~cm}$, A3: height $183 \mathrm{~cm}, \mathrm{~A} 4$ : height $61 \mathrm{~cm}$ ), and by the shutoff sensor located on the generator near the generator's electrical panel. The figure shows the degree of CO uniformity in the area around the generator between the shutoff sensor and the peak $\mathrm{CO}$ concentrations measured elsewhere in the family room. Figure 41c shows the $\mathrm{CO}$ concentration measured in each room of the house over the test. Figure 41d shows the calculated $\mathrm{COHb}$ profiles of simulated house occupants in each room of the house.

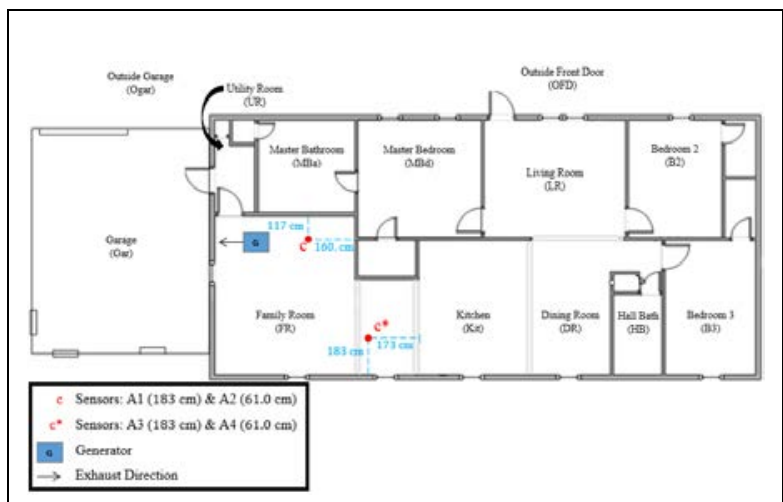

Figure 41a. Generator, exhaust direction, and sensor array layout during Case 11 Test 1 - G2P UL 2201.

Figure 41c. CO concentration measured in each room of the house during Case 11 Test 1 - G2P UL 2201.
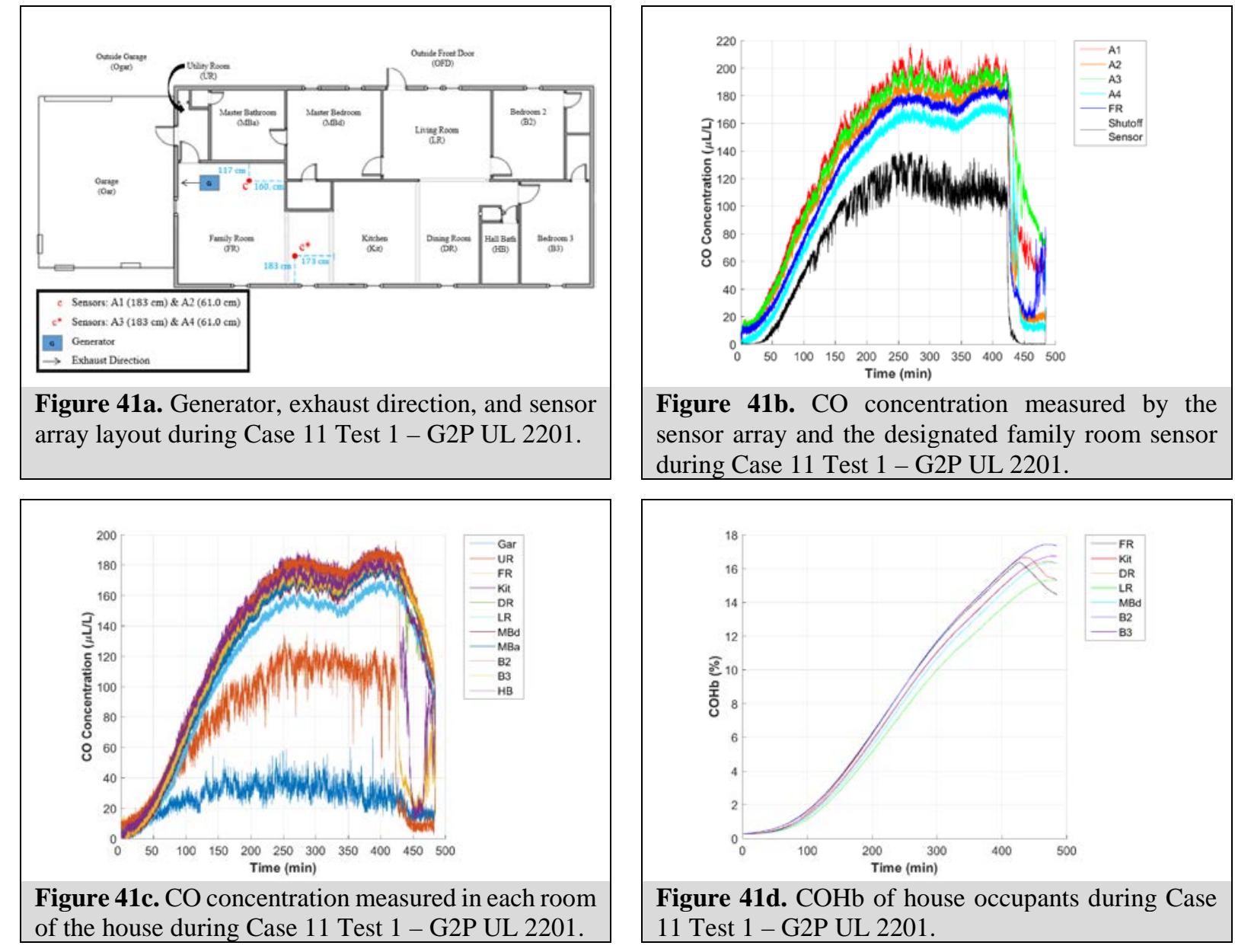


\section{Generator Location: Hall Bath}

Table 4 summarizes the results for all tests performed with a generator located in the test house hall bath. The details are discussed below and shown in Figures 42 through 44.

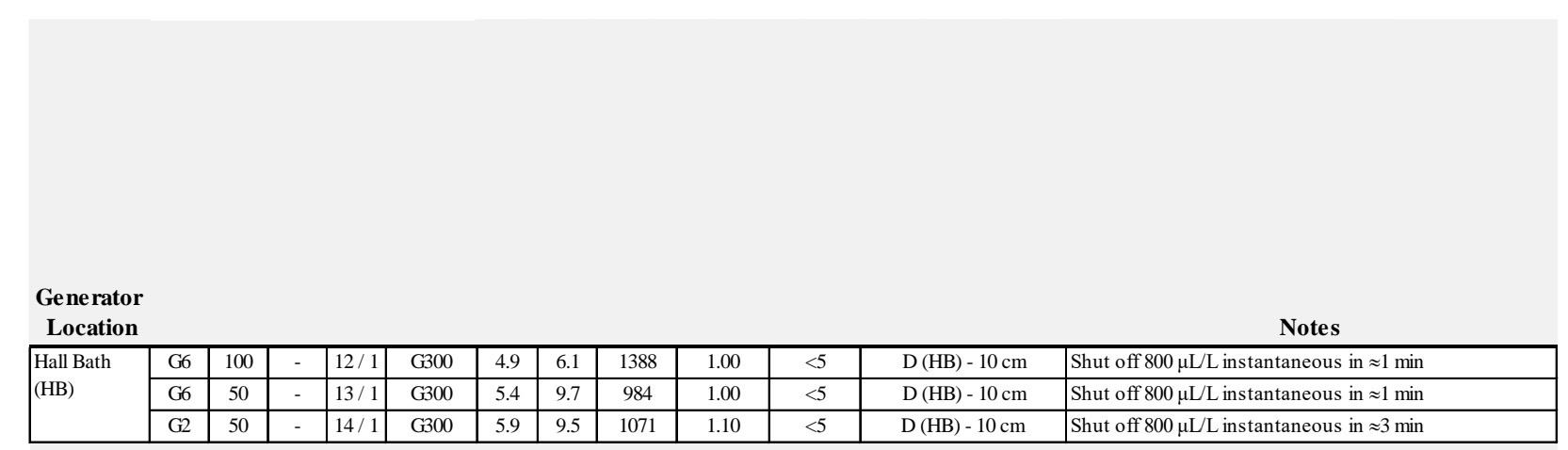

Table 4. Summary of test results for Hall Bath (HB) tests.

+Peak COHb Values are for house zones only.

+Ventilation Note: Unless specified otherwise, during the test - all exterior doors are closed, all interior doors are fully open, and all windows are closed. 


\section{Case 12 Test 1 - G6 G300}

The following figures illustrate the results for Case 12 Test 1 applying the G300 shutoff criteria. The G6 generator was fully fueled, positioned in the hall bathroom (centered, exhaust facing the back wall), and tested at $100 \%$ load $(6,000 \mathrm{~W})$. The hall bathroom door was open $10 \mathrm{~cm}$. The shutoff sensor activated the algorithm to shut off the generator after approximately 1 min due to an instantaneous sensor measurement of $800 \mu \mathrm{L} / \mathrm{L}$. Figure 42a shows the test house layout with generator location, exhaust direction, and sensor array location. Figure 42b shows the CO concentration measured in the hall bathroom (by the dedicated room sensor), the sensor array placed in the hallway outside the bathroom located as shown in Figure 42a (A1: height $183 \mathrm{~cm}$, A2: height $61 \mathrm{~cm}$ ), the sensor array placed on the dining-living room border as shown in Figure 42a (A3: height $183 \mathrm{~cm}$, A4: height $61 \mathrm{~cm}$ ), and by the shutoff sensor located on the generator near the generator's electrical panel. The timeframe of the figure has been selected to show the degree of CO uniformity in the area around the generator between the shutoff sensor and the peak CO concentrations measured elsewhere in and near the hall bathroom near the time of shutoff. Also illustrated in this figure, the $\mathrm{CO}$ concentration near the shutoff sensor exceeded the range of the sensor. Figure 42c shows the CO concentration measured in each room of the house over the test. Figure 42d shows the calculated $\mathrm{COHb}$ profiles of simulated house occupants in each room of the house. Due to the low CO concentration at the end of the test, the CO concentration was not projected beyond the test time and the calculated COHb for simulated house occupants did not reach a peak and did not exceed $5 \%$ during the test.

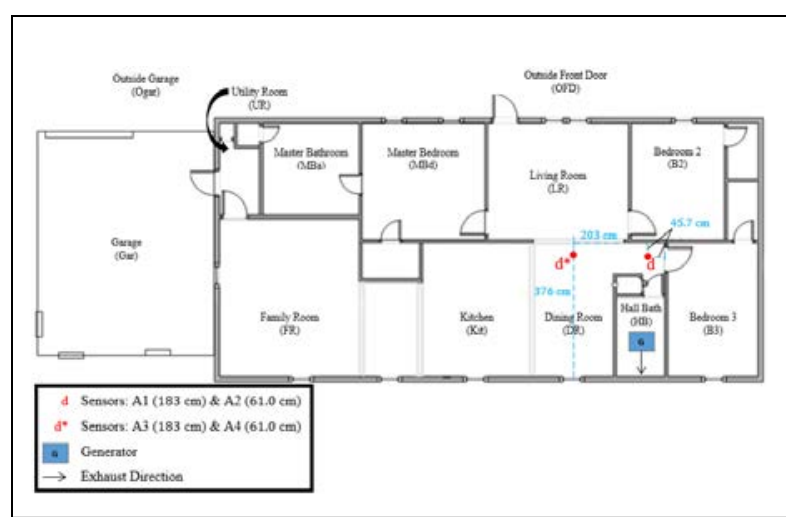

Figure 42a. Generator, exhaust direction, and sensor array layout during Case 12 Test 1 - G6 G300.

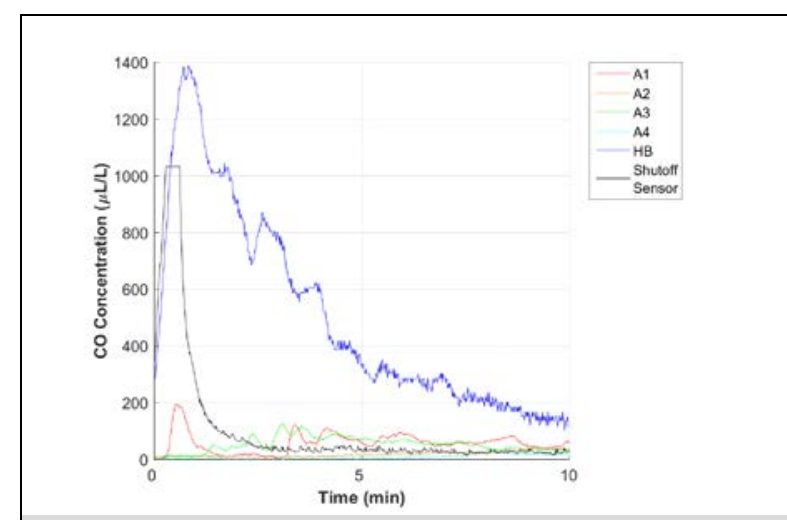

Figure 42b. CO concentration measured by the sensor array and the designated hall bath sensor during Case 12 Test 1 - G6 G300. 


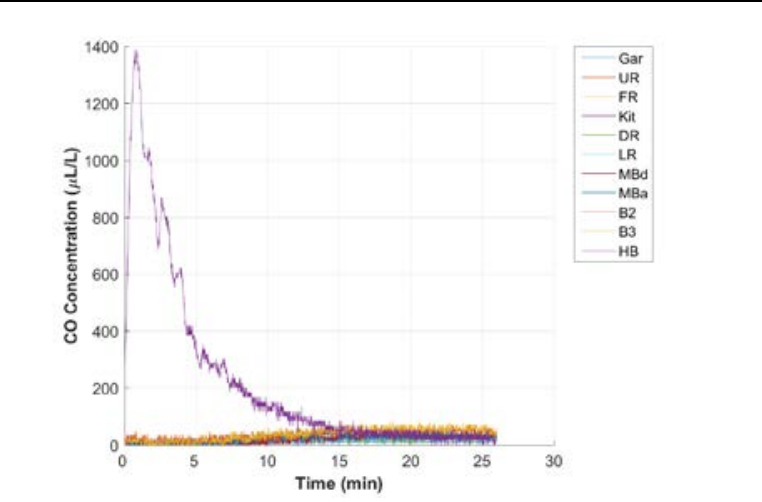

Figure 42c. $\mathrm{CO}$ concentration measured in each room of the house during Case 12 Test 1 - G6 G300.

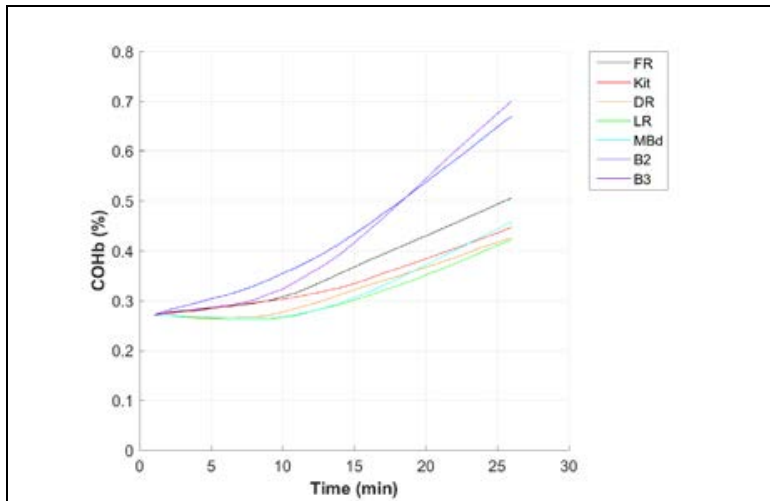

Figure 42d. $\mathrm{COHb}$ of house occupants during Case 12 Test 1 - G6 G300. 


\section{Case 13 Test 1 - G6 G300}

The following figures illustrate the results for Case 13 Test 1 applying the G300 shutoff criteria. The G6 generator was fully fueled, positioned in the hall bathroom (centered, exhaust facing the back wall), and tested at $50 \%$ load $(3,000 \mathrm{~W})$. The hall bathroom door was open $10 \mathrm{~cm}$. This test immediately followed Case 12 Test 1 and was not verified as a cold start. The shutoff sensor activated the algorithm to shut off the generator after approximately 1 min due to an instantaneous sensor measurement of $800 \mu \mathrm{L} / \mathrm{L}$. Figure 43a shows the test house layout with generator location, exhaust direction, and sensor array location. Figure 43b shows the CO concentration measured in the hall bathroom (by the dedicated room sensor), the sensor array placed in the hallway outside the bathroom located as shown in Figure 43a (A1: height $183 \mathrm{~cm}$, A2: height $61 \mathrm{~cm})$, the sensor array placed on the dining-living room border as shown in Figure 43a (A3: height $183 \mathrm{~cm}, \mathrm{~A} 4$ : height $61 \mathrm{~cm}$ ), and by the shutoff sensor located on the generator near the generator's electrical panel. The timeframe of the figure has been selected to show the degree of CO uniformity in the area around the generator between the shutoff sensor and the peak CO concentrations measured elsewhere in and near the hall bathroom near the time of shutoff. Figure 43c shows the CO concentration measured in each room of the house over the test. Figure 43d shows the calculated $\mathrm{COHb}$ profiles of simulated house occupants in each room of the house. Due to the low CO concentration at the end of the test, the CO concentration was not projected beyond the test time and the calculated $\mathrm{COHb}$ for simulated house occupants did not exceed $5 \%$ during the test.

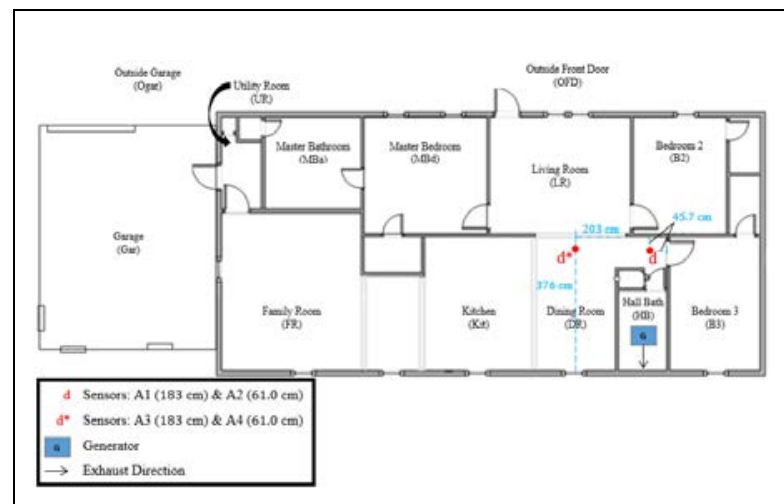

Figure 43a. Generator, exhaust direction, and sensor array layout during Case 13 Test 1 - G6 G300.

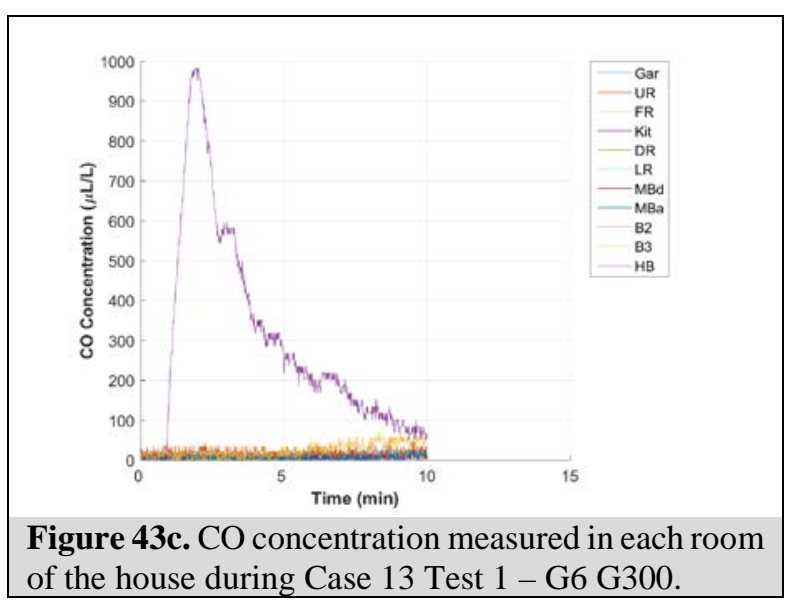

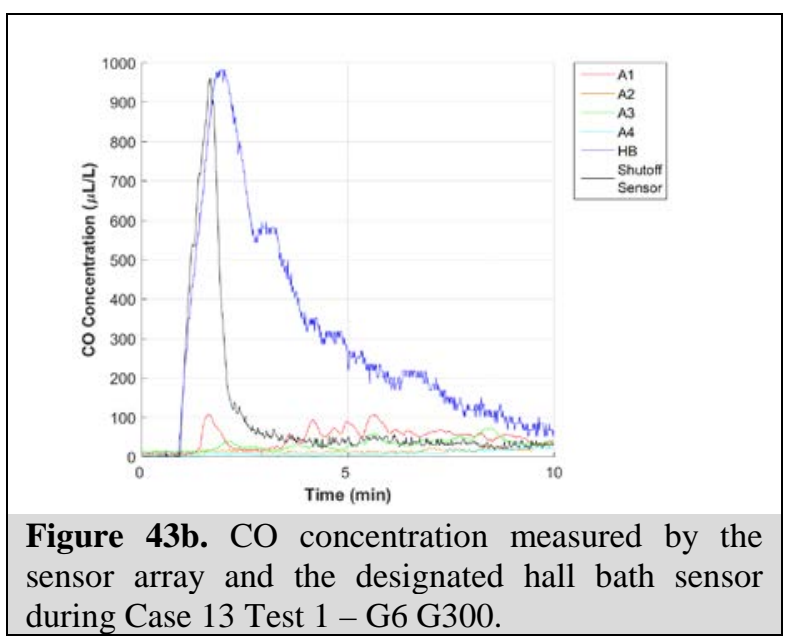

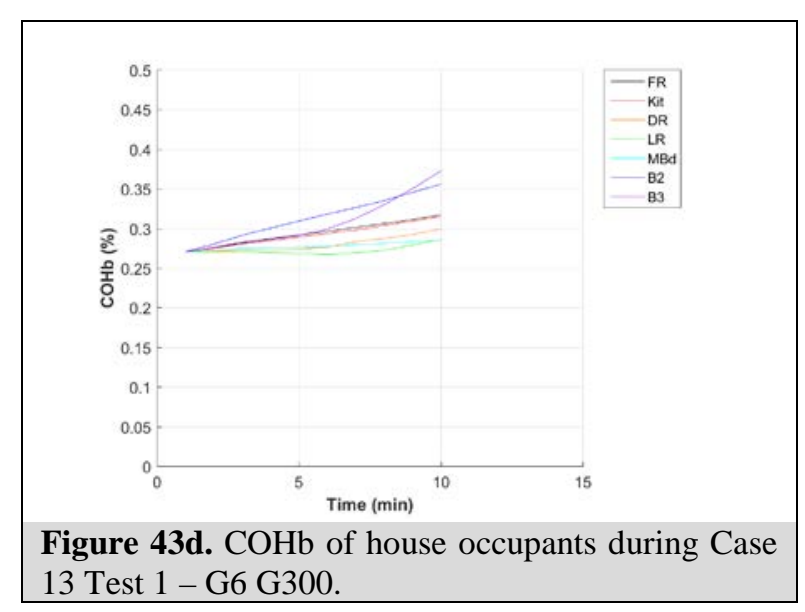




\section{Case 14 Test 1 - G2 G300}

The following figures illustrate the results for Case 14 Test 1 applying the G300 shutoff criteria. The G2 generator was fully fueled, positioned in the hall bathroom (centered, exhaust facing the back wall), and tested at $50 \%$ load $(800 \mathrm{~W})$. The hall bathroom door was open $10 \mathrm{~cm}$. The shutoff sensor activated the algorithm to shut off the generator after approximately 3 min due to an instantaneous sensor measurement of $800 \mu \mathrm{L} / \mathrm{L}$. Figure 44a shows the test house layout with generator location, exhaust direction, and sensor array location. Figure 44b shows the CO concentration measured in the hall bathroom (by the dedicated room sensor), the sensor array placed in the hallway outside the bathroom located as shown in Figure 44a (A1: height $183 \mathrm{~cm}$, A2: height $61 \mathrm{~cm}$ ), the sensor array placed on the dining-living room border as shown in Figure 44a (A3: height $183 \mathrm{~cm}, \mathrm{~A} 4$ : height $61 \mathrm{~cm}$ ), and by the shutoff sensor located on the generator near the generator's electrical panel. The timeframe of the figure has been selected to show the degree of CO uniformity in the area around the generator between the shutoff sensor and the peak CO concentrations measured elsewhere in and near the hall bathroom near the time of shutoff. Figure 44c shows the $\mathrm{CO}$ concentration measured in each room of the house over the test. Figure 44d shows the calculated $\mathrm{COHb}$ profiles of simulated house occupants in each room of the house. Due to the low CO concentration at the end of the test, the CO concentration was not projected beyond the test time and the calculated $\mathrm{COHb}$ for simulated house occupants did not exceed $5 \%$ during the test.

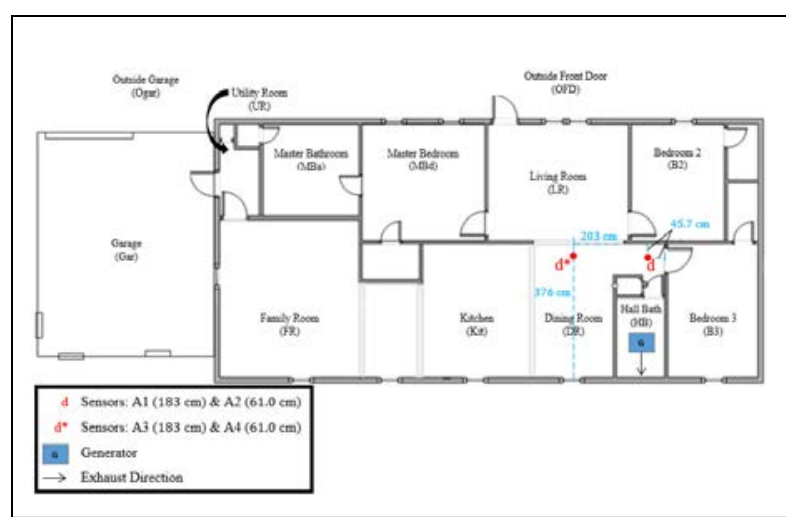

Figure 44a. Generator, exhaust direction, and sensor array layout during Case 14 Test 1 - G2 G300.

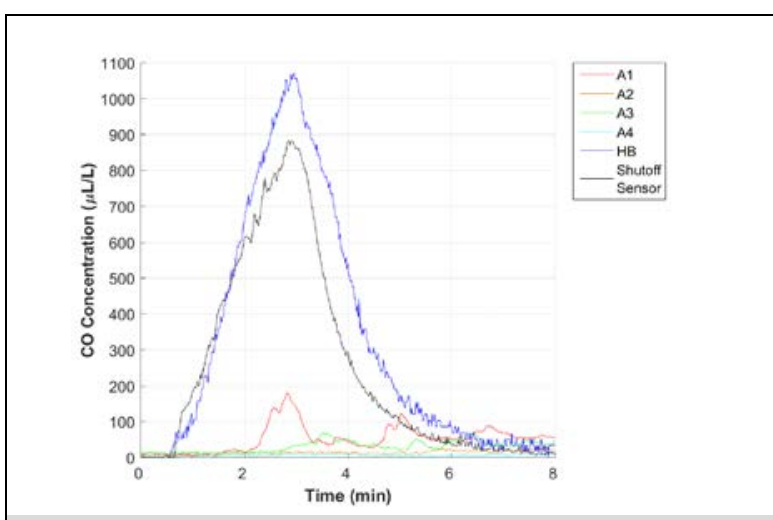

Figure 44b. CO concentration measured by the sensor array and the designated hall bath sensor during Case 14 Test 1 - G2 G300. 


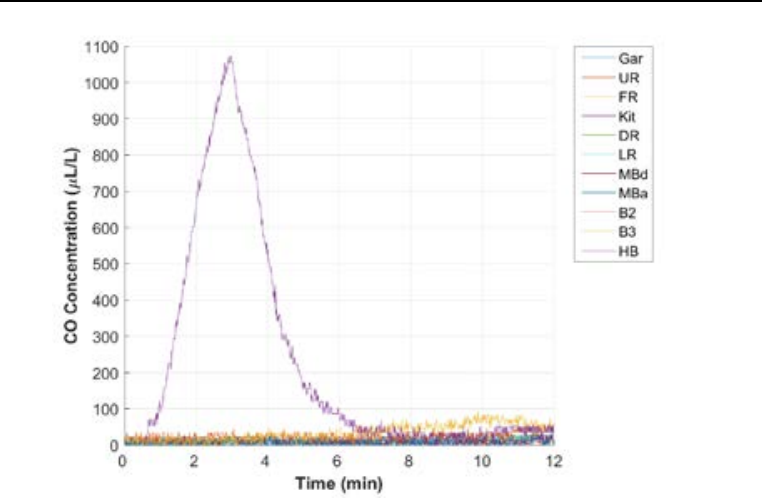

Figure 44c. $\mathrm{CO}$ concentration measured in each room of the house during Case 14 Test 1-G2 G300.

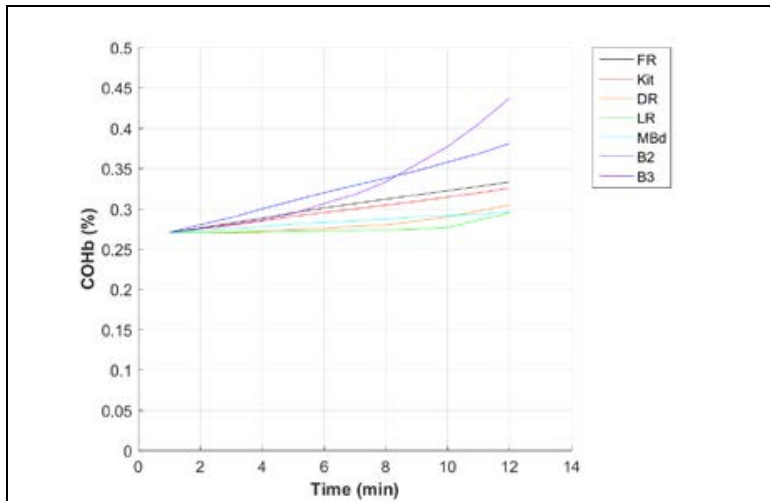

Figure 44d. $\mathrm{COHb}$ of house occupants during Case 14 Test 1 - G2 G300. 


\section{Generator Location: Bedroom 3}

Table 5 summarizes the results for all tests performed with a generator located in the test house bedroom 3. The details are discussed below and shown in Figures 45 through 57.

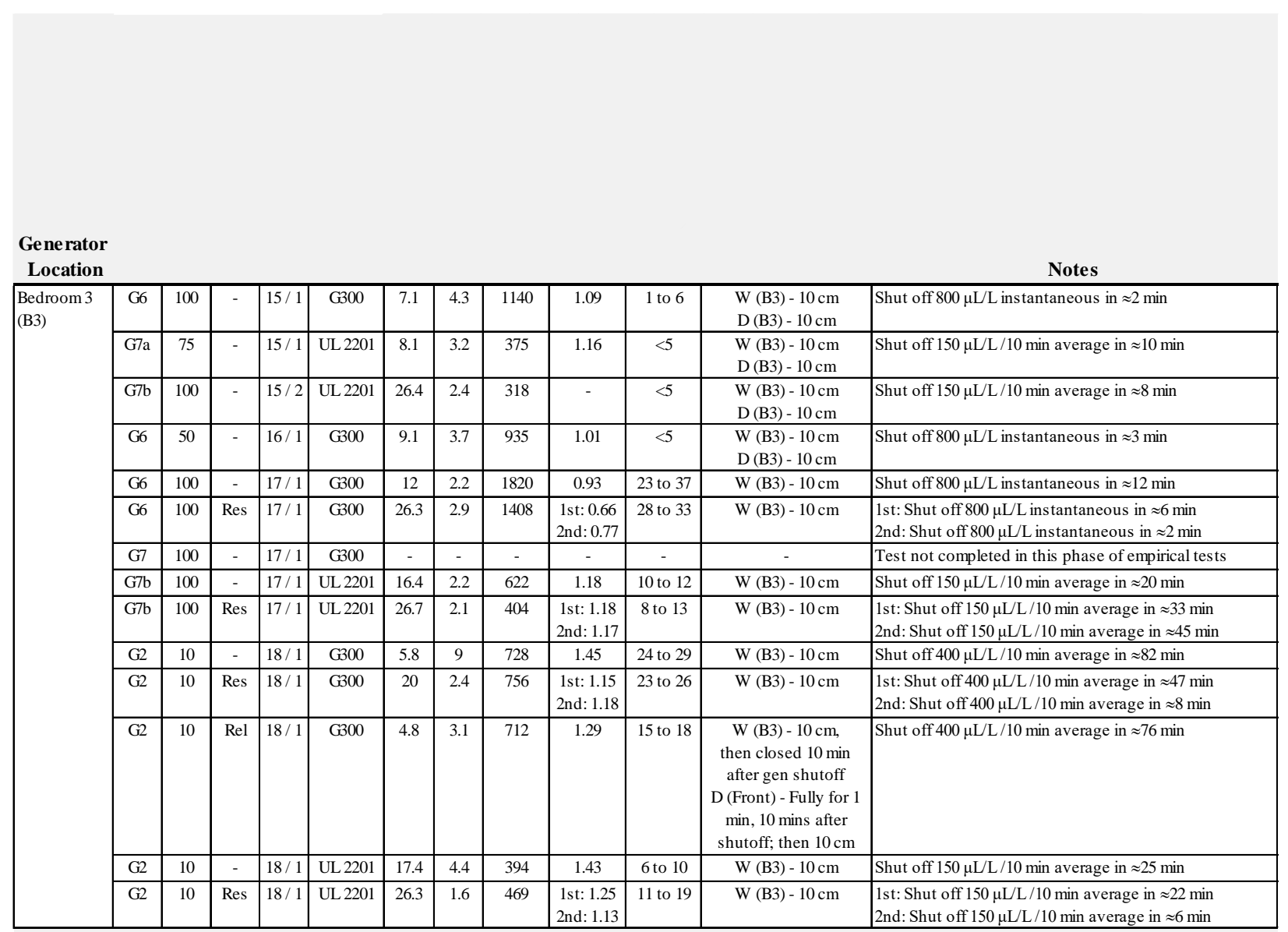

Table 5. Summary of test results for Bedroom 3 (B3) tests.

+Peak COHb Values are for house zones only.

HVentilation Note: Unless specified otherwise, during the test - all exterior doors are closed, all interior doors are fully open, and all windows are closed. 


\section{Case 15 Test 1 - G6 G300}

The following figures illustrate the results for Case 15 Test 1 applying the G300 shutoff criteria. The G6 generator was fully fueled, positioned in Bedroom 3 (30 cm from the window with the exhaust facing the window), and tested at $100 \%$ load $(6,000 \mathrm{~W})$. The Bedroom 3 window and door were open $10 \mathrm{~cm}$ during the test. The shutoff sensor activated the algorithm to shut off the generator after approximately 2 min due to an instantaneous sensor measurement of $800 \mu \mathrm{L} / \mathrm{L}$. Figure 45a shows the test house layout with generator location, exhaust direction, and sensor array location. Figure 45b shows the CO concentration measured in Bedroom 3 (by the dedicated room sensor), by the sensor array placed in Bedroom 3 located as shown in Figure 45a (A1: height 183 $\mathrm{cm}, \mathrm{A2}$ : height $61 \mathrm{~cm}$ ), the sensor array placed in the hall outside Bedroom 3 as shown in Figure 45a (A3: height $183 \mathrm{~cm}, \mathrm{~A} 4$ : height $61 \mathrm{~cm}$ ), and by the shutoff sensor located on the generator near the generator's electrical panel. The timeframe of the figure has been selected to show the degree of $\mathrm{CO}$ uniformity in the area around the generator between the shutoff sensor and the peak $\mathrm{CO}$ concentrations measured elsewhere in and near Bedroom 3 near the time of shutoff. Figure 45c shows the CO concentration measured in each room of the house over the test. Figure 45d shows the calculated $\mathrm{COHb}$ profiles of simulated house occupants in each room of the house. Due to the quick decay and low CO concentration at the end of the test, the $\mathrm{CO}$ concentration was not projected beyond the test time and the calculated COHb for simulated house occupants did not exceed $10 \%$ during the test.

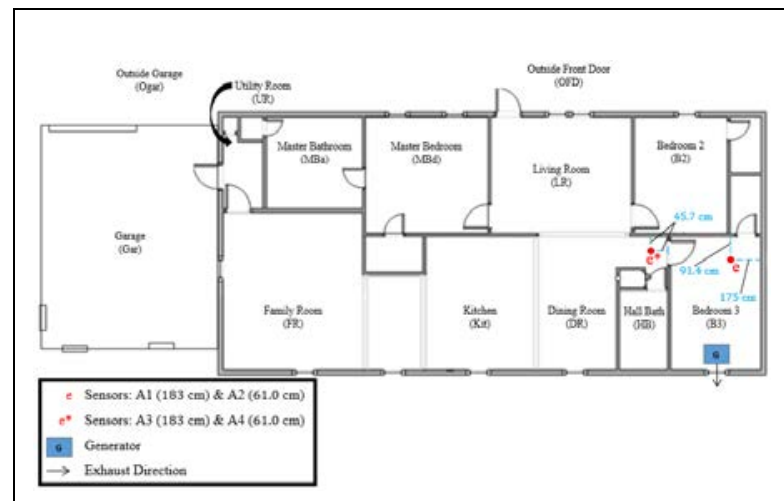

Figure 45a. Generator, exhaust direction, and sensor array layout during Case 15 Test 1 - G6 G300.

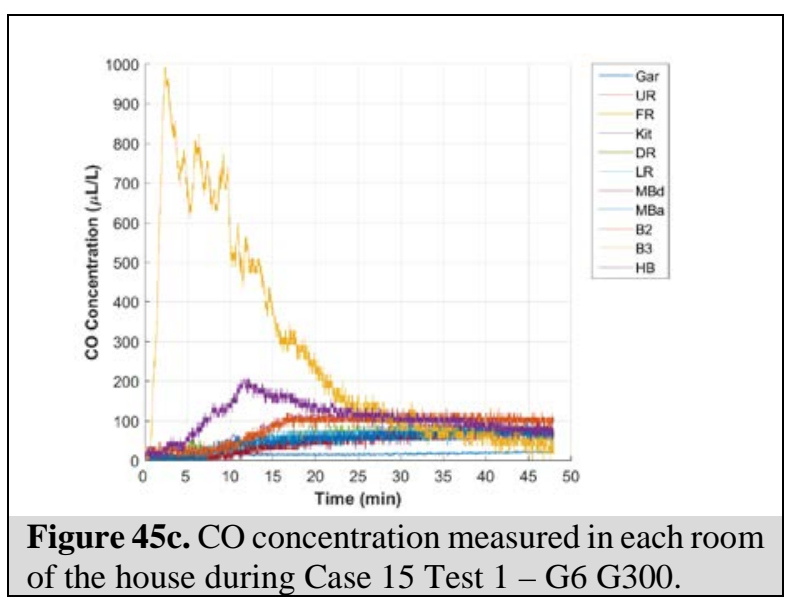

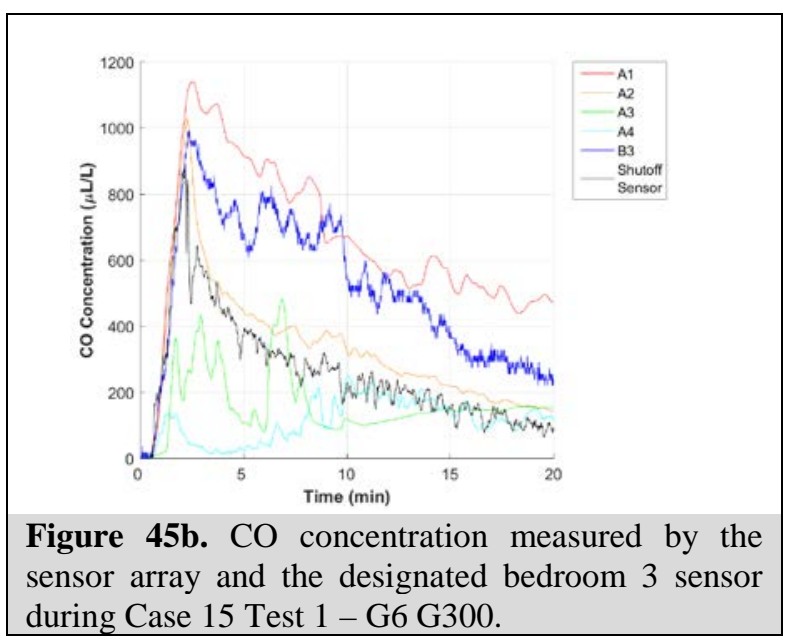

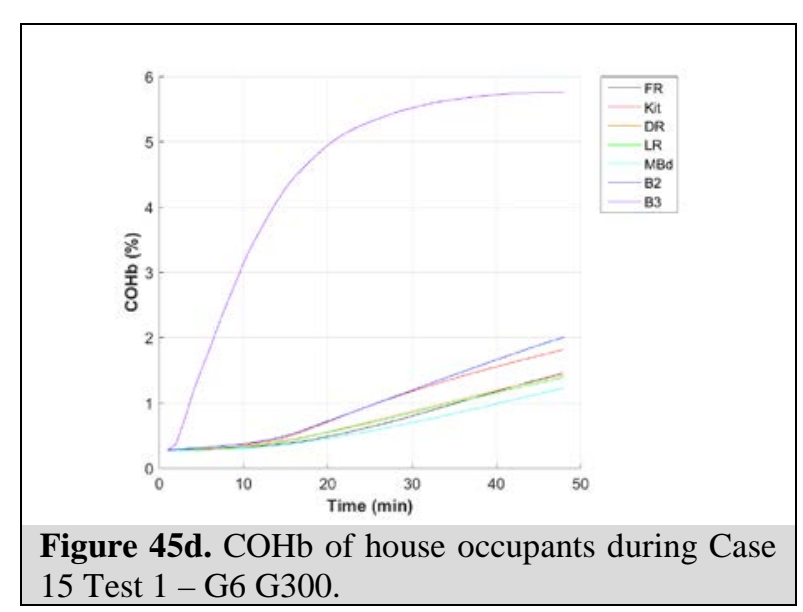




\section{Case 15 Test 1 - G7a UL2201}

The following figures illustrate the results for Case 15 Test 1 applying the UL 2201 shutoff criteria. The G7a generator was fully fueled, positioned in the third bedroom (near the window with the exhaust facing the window), and tested at $75 \%$ load $(5,250 \mathrm{~W})$. A full load was not applied due to issues at the time of this test maintaining full load on this generator. The Bedroom 3 window and door were open $10 \mathrm{~cm}$ during the test. The shutoff sensor activated the algorithm to shut off the generator after approximately $10 \mathrm{~min}$ due to a sensor measurement of $150 \mu \mathrm{L} / \mathrm{L}$ for a $10 \mathrm{~min}$ average. Figure 46a shows the test house layout with generator location, generator exhaust direction, and sensor array location. Figure 46b shows the $\mathrm{CO}$ concentration measured in the third bedroom (by the dedicated room sensor), by two sensors located in the third bedroom (A1: height $183 \mathrm{~cm}, \mathrm{A2}$ : height $61 \mathrm{~cm}$ ), by the other two sensors located in the hallway outside the third bedroom (A3: height $183 \mathrm{~cm}$, A4: height $61 \mathrm{~cm}$ ), and by the shutoff sensor located on the generator near the generator's electrical panel. The timeframe of the figure has been selected to show the degree of CO uniformity in the area around the generator between the shutoff sensor and the peak CO concentrations measured elsewhere in the third bedroom and hallway near the time of shutoff. Figure 46c shows the $\mathrm{CO}$ concentration measured in each room of the house over the test. Figure 46d shows the $\mathrm{CO}$ concentration in the house with each room represented by a single line, which is composed of both a 'solid-line' pattern (showing measured CO concentration) and a 'dottedline with a symbol' pattern (showing projected CO concentration). Figure 46e shows the calculated $\mathrm{COHb}$ profiles of simulated house occupants in each room of the house.

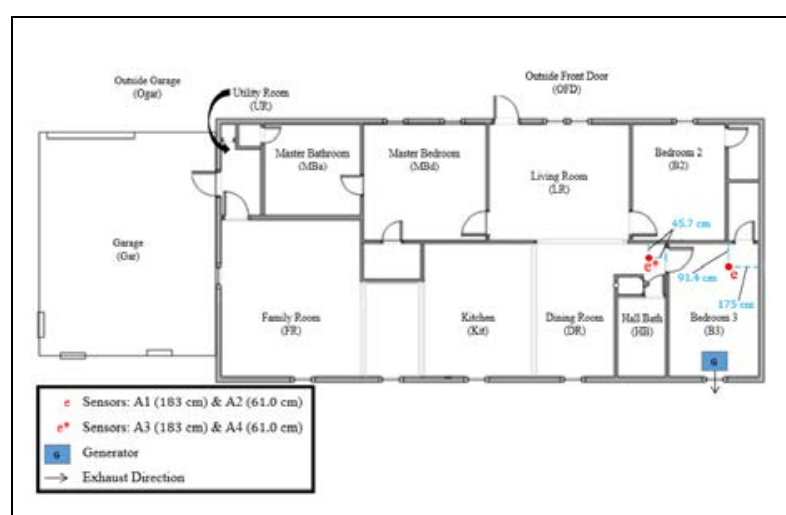

Figure 46a. Generator, exhaust direction, and sensor array layout during Case 15 Test 1 - G7a UL 2201.

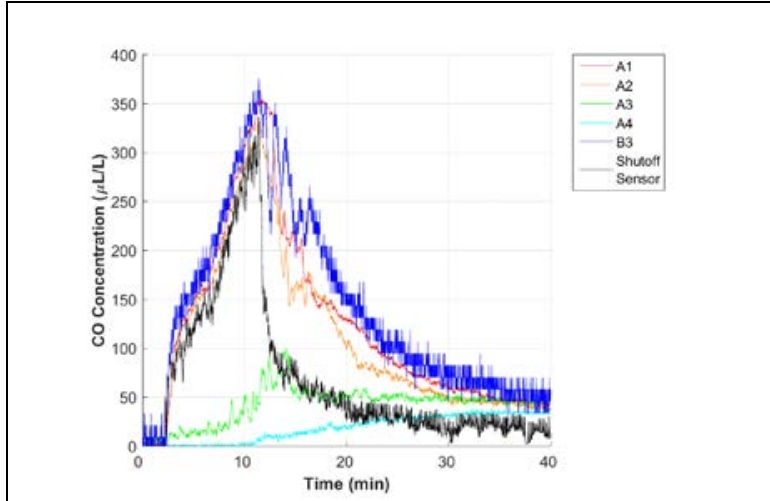

Figure 46b. CO concentration measured by the sensor array and the designated bedroom 3 sensor during Case 15 Test 1 - G7a UL 2201. 


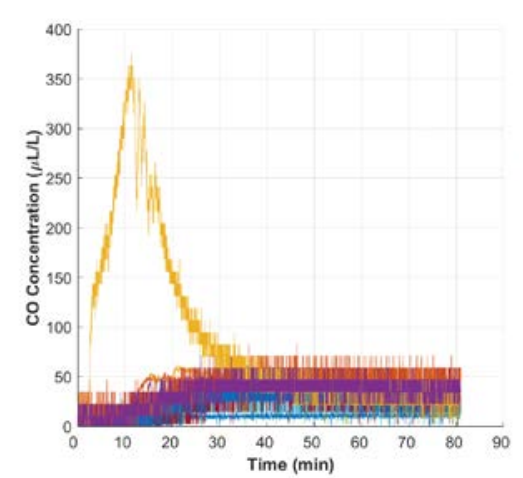

Figure 46c. CO concentration measured in each room of the house during Case 15 Test 1 - G7a UL 2201.

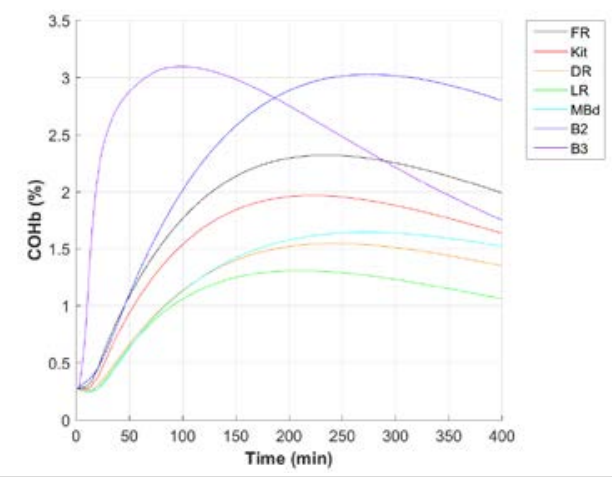

Figure 46e. $\mathrm{COHb}$ of house occupants during Case 15 Test 1 - G7a UL 2201.

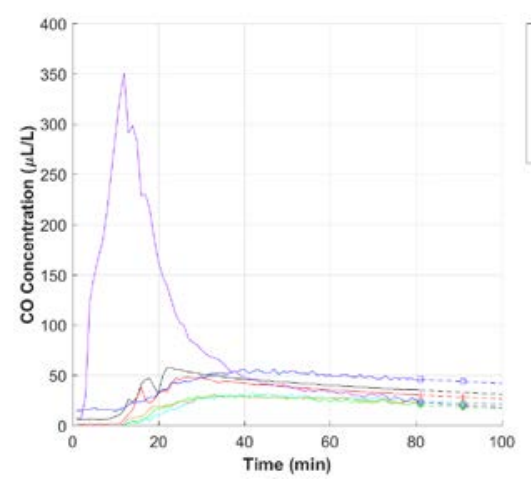

Figure 46d. CO concentration (measured \& projected) in the house during Case 15 Test 1 - G7a UL 2201. 


\section{Case 15 Test 2 - G7b UL 2201}

The following figures illustrate the results for Case 15 Test 2 applying the UL 2201 shutoff criteria (the data acquisition program did not record data for the first 3 min of the test). The G7b generator was fully fueled, positioned in the third bedroom (near the window with the exhaust facing the window), and tested at $100 \%$ load $(7,000 \mathrm{~W})$. The Bedroom 3 window and door were open $10 \mathrm{~cm}$ during the test. The shutoff sensor activated the algorithm to shut off the generator after approximately $8 \mathrm{~min}$ due to a sensor measurement of $150 \mu \mathrm{L} / \mathrm{L}$ for a $10 \mathrm{~min}$ average. Figure 47a shows the test house layout with generator location, generator exhaust direction, and sensor array location. Figure 47b shows the CO concentration measured in the third bedroom (by the dedicated room sensor), by two sensors located in the third bedroom (A1: height $183 \mathrm{~cm}, \mathrm{~A} 2$ : height 61.0 $\mathrm{cm}$ ), by the other two sensors located in the hallway outside the third bedroom (A3: height 183 $\mathrm{cm}$, A4: height $61.0 \mathrm{~cm}$ ), and by the shutoff sensor located on the generator near the generator's electrical panel. The timeframe of the figure has been selected to show the degree of CO uniformity in the area around the generator between the shutoff sensor and the peak CO concentrations measured elsewhere in the third bedroom and hallway near the time of shutoff. Figure 47c shows the $\mathrm{CO}$ concentration measured in each room of the house over the test. Figure 47d shows the CO concentrations in the house with each room represented by a single line, which is composed of both a 'solid-line' pattern (showing measured CO concentration) and a 'dotted-line with a symbol' pattern (showing projected CO concentration). Figure 47e shows the calculated COHb profiles of simulated house occupants in each room of the house.

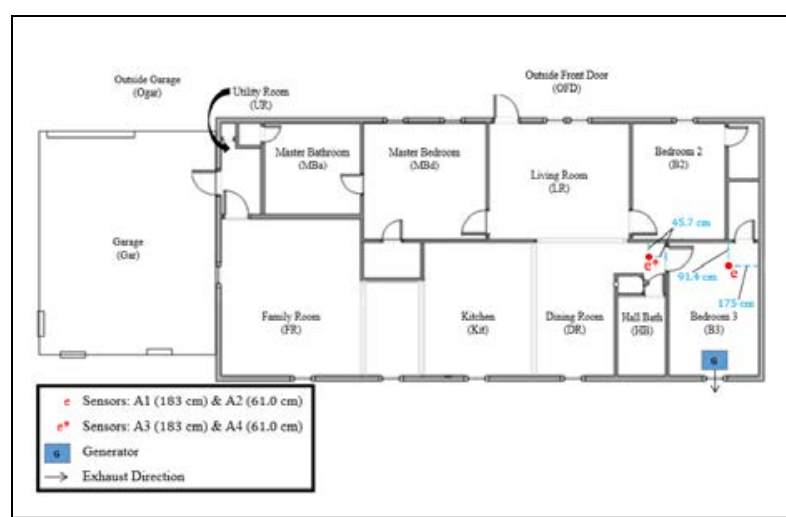

Figure 47a. Generator, exhaust direction, and sensor array layout during Case 15 Test 2 - G7b UL 2201.

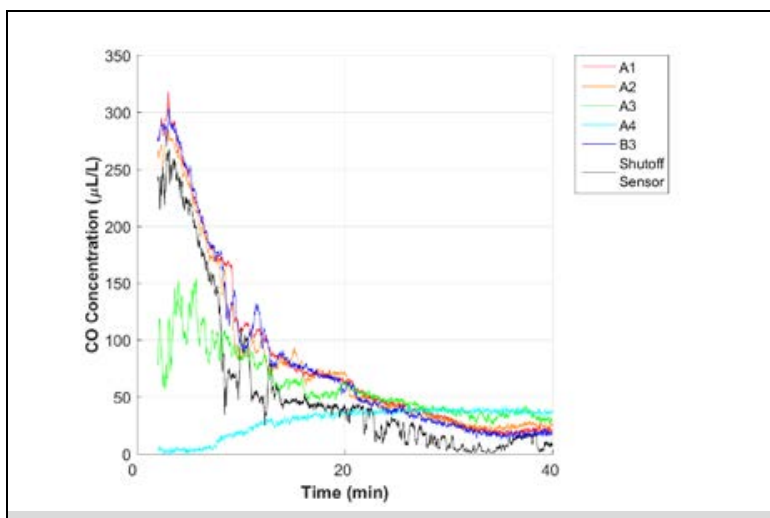

Figure 47b. CO concentration measured by the sensor array and the designated bedroom 3 sensor during Case 15 Test 2 - G7b UL 2201. 


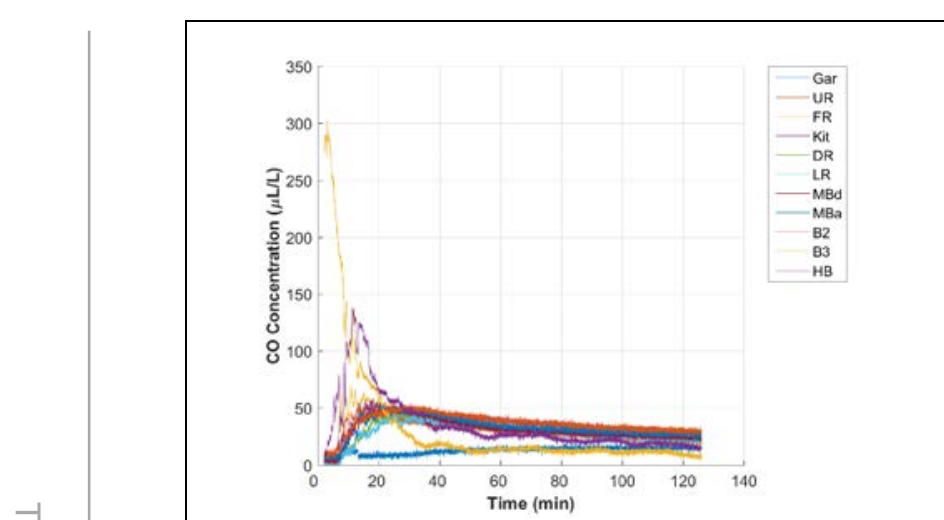

Figure 47c. CO concentration measured in each room of the house during Case 15 Test 2 - G7b UL 2201.

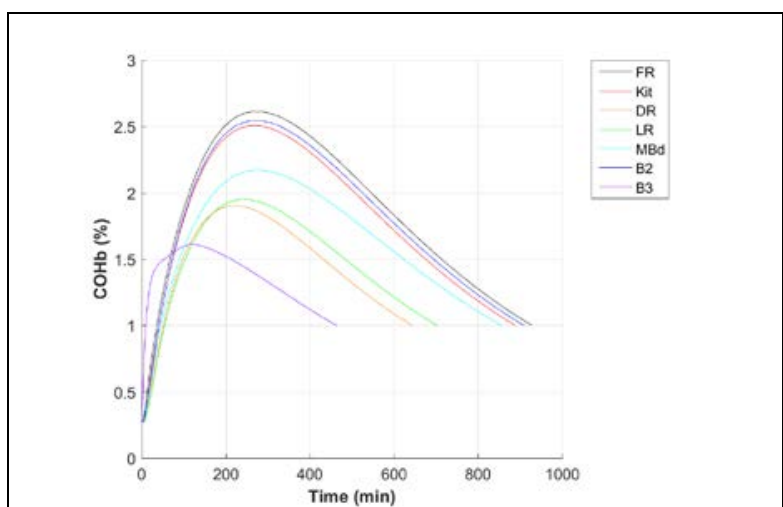

Figure 47e. $\mathrm{COHb}$ of house occupants during Case 15 Test 2 - G7b UL 2201.

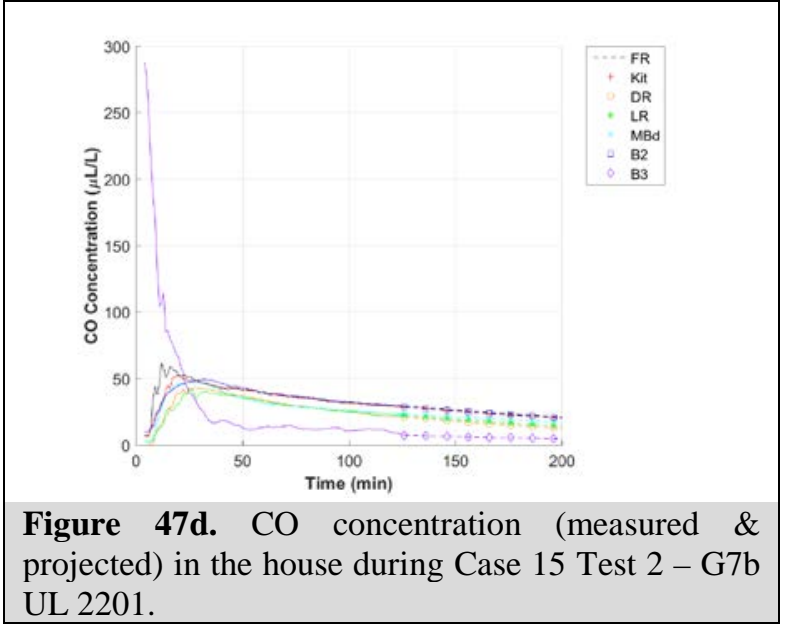




\section{Case 16 Test 1 - G6 G300}

The following figures illustrate the results for Case 16 Test 1 applying the G300 shutoff criteria. The G6 generator was fully fueled, positioned in Bedroom 3 (30 cm from the window with the exhaust facing the window), and tested at $50 \%$ load $(3,000 \mathrm{~W})$. The Bedroom 3 window and door were open $10 \mathrm{~cm}$ during the test. The shutoff sensor activated the algorithm to shut off the generator after approximately 3 min due to an instantaneous sensor measurement of $800 \mu \mathrm{L} / \mathrm{L}$. Figure 48a shows the test house layout with generator location, exhaust direction, and sensor array location. Figure 48b shows the CO concentration measured in Bedroom 3 (by the dedicated room sensor), by the sensor array placed in Bedroom 3 located as shown in Figure 48a (A1: height 183 $\mathrm{cm}$, A2: height $61 \mathrm{~cm}$ ), the sensor array placed in the hall outside Bedroom 3 as shown in Figure 48a (A3: height $183 \mathrm{~cm}$, A4: height $61 \mathrm{~cm}$ ), and by the shutoff sensor located on the generator near the generator's electrical panel. The timeframe of the figure has been selected to show the degree of $\mathrm{CO}$ uniformity in the area around the generator between the shutoff sensor and the peak $\mathrm{CO}$ concentrations measured elsewhere in and near Bedroom 3 near the time of shutoff. Figure 48c shows the CO concentration measured in each room of the house over the test. Figure 48d shows the calculated $\mathrm{COHb}$ profiles of simulated house occupants in each room of the house. Due to the quick decay and low CO concentration at the end of the test, the $\mathrm{CO}$ concentration was not projected beyond the test time and the calculated COHb for simulated house occupants did not exceed $5 \%$ during the test.

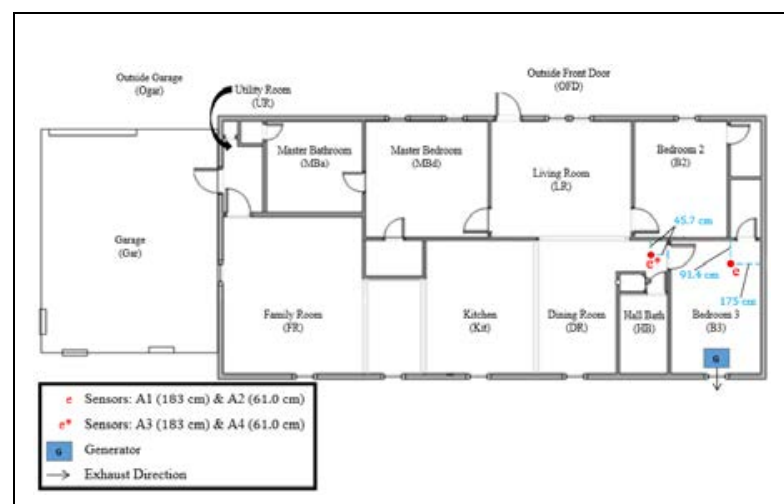

Figure 48a. Generator, exhaust direction, and sensor array layout during Case 16 Test 1 - G6 G300.

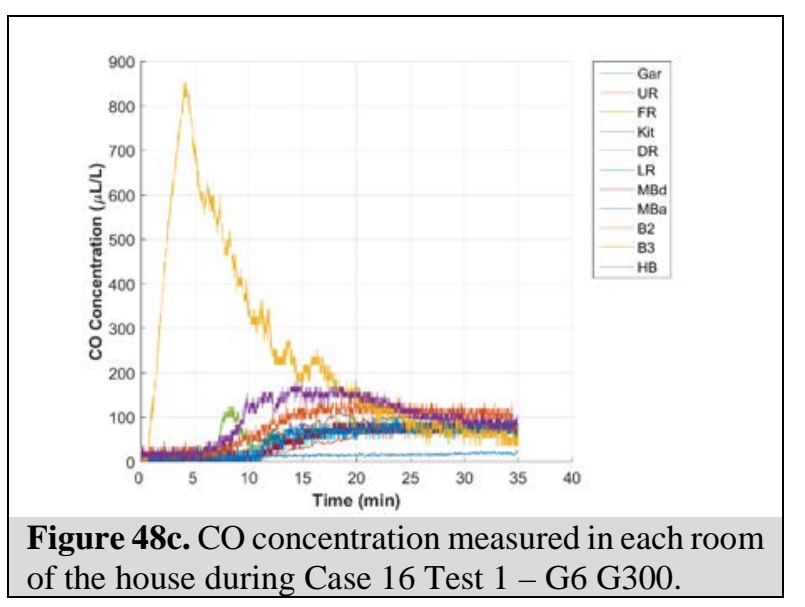

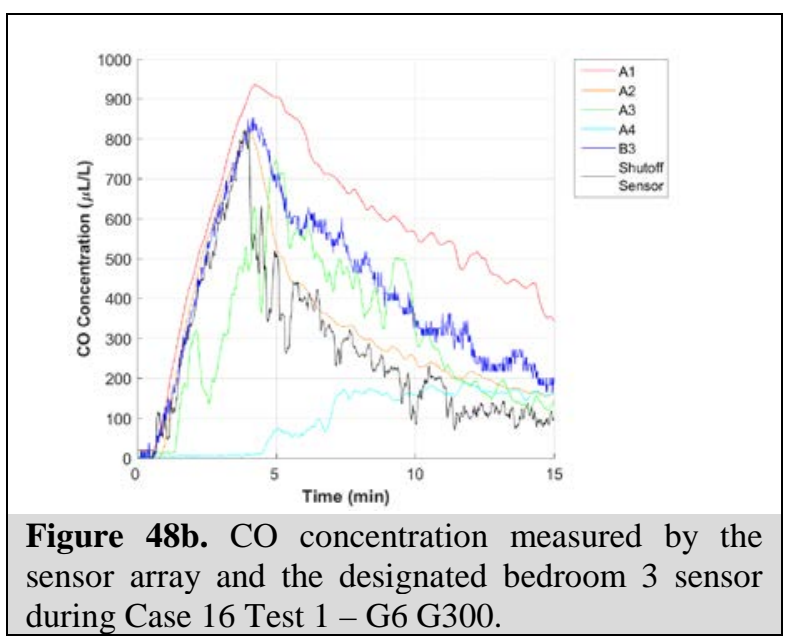

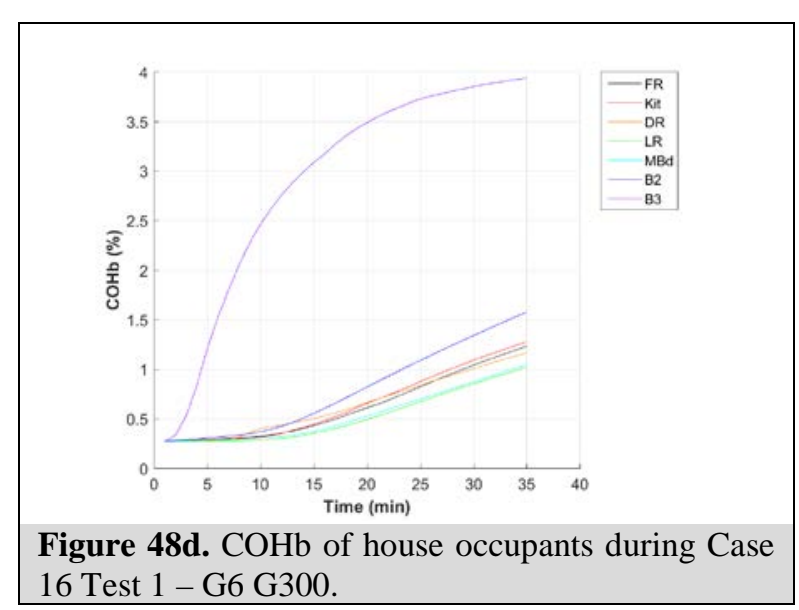




\section{Case 17 Test 1 - G6 G300}

The following figures illustrate the results for Case 17 Test 1 applying the G300 shutoff criteria. The G6 generator was fully fueled, positioned in Bedroom 3 (near the door with the exhaust facing the hallway and in the plane of the door), and tested at $100 \%$ load $(6,000 \mathrm{~W})$. The Bedroom 3 door was fully open; the Bedroom 3 window was open $10 \mathrm{~cm}$. The shutoff sensor activated the algorithm to shut off the generator after approximately 12 min due to an instantaneous sensor measurement of $800 \mu \mathrm{L} / \mathrm{L}$. Figure 49a shows the test house layout with generator location, exhaust direction, and sensor array location. Figure 49b shows the $\mathrm{CO}$ concentration measured in Bedroom 3 (by the dedicated room sensor), by the sensor array placed in bedroom 3 located as shown in Figure 49a (A1: height $183 \mathrm{~cm}$, A2: height $61 \mathrm{~cm}$ ), the sensor array placed in the hall outside Bedroom 3 as shown in Figure 49a (A3: height $183 \mathrm{~cm}$, A4: height $61 \mathrm{~cm}$ ), and by the shutoff sensor located on the generator near the generator's electrical panel. The timeframe of the figure has been selected to show the degree of CO uniformity in the area around the generator between the shutoff sensor and the peak CO concentrations measured elsewhere in and near Bedroom 3 near the time of shutoff. Figure 49c shows the CO concentration measured in each room of the house over the test. Figure 49d shows the CO concentration in the house - each room is represented by a single line, which is composed of both a 'solid-line' pattern (showing measured CO concentration) and a 'dotted-line with a symbol' pattern (showing projected CO concentration). Figure 49e shows the calculated $\mathrm{COHb}$ profiles of simulated house occupants in each room of the house.

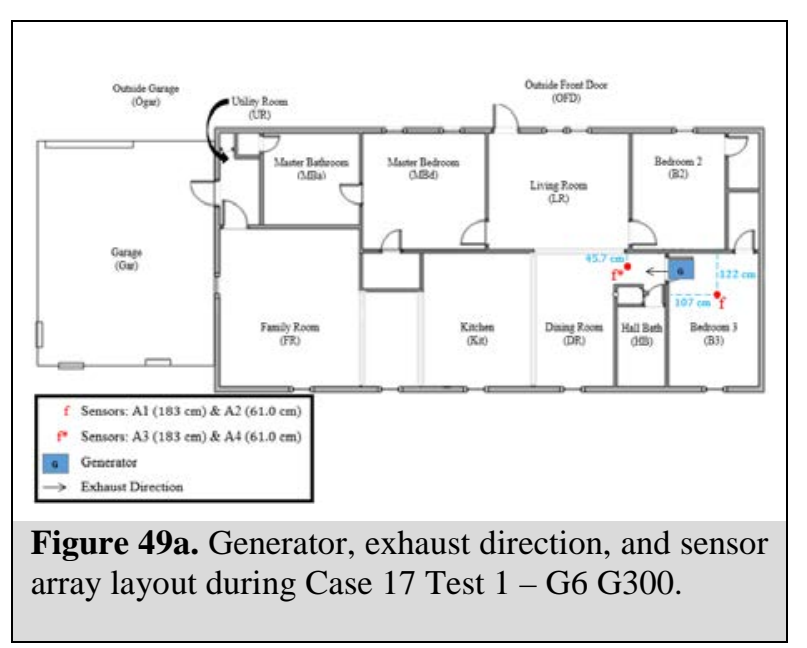

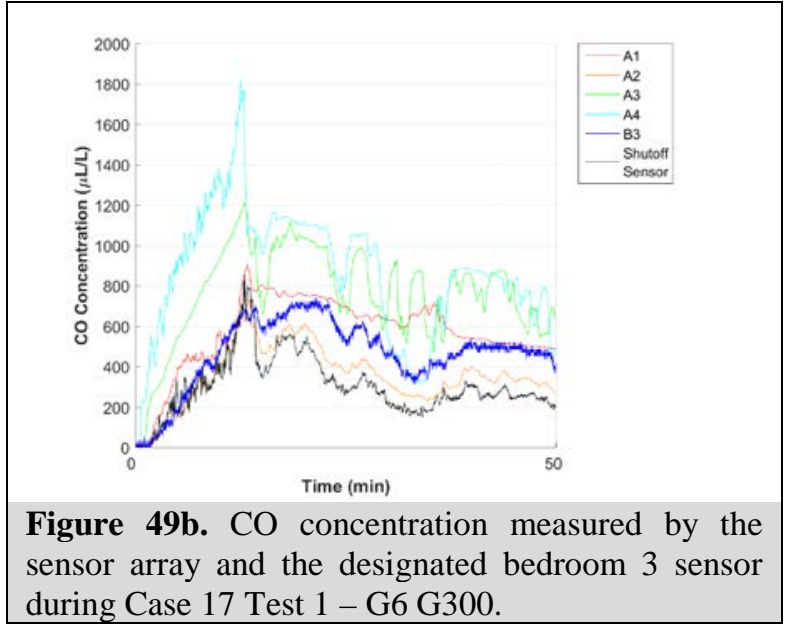




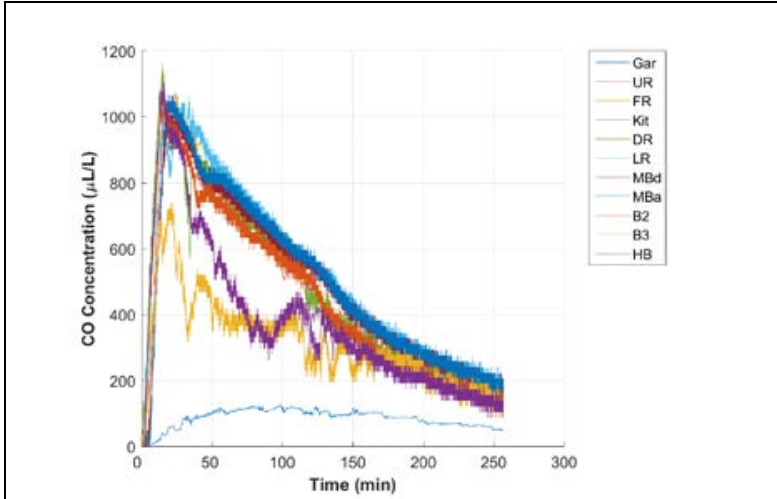

Figure 49c. CO concentration measured in each room of the house during Case 17 Test 1 - G6 G300.

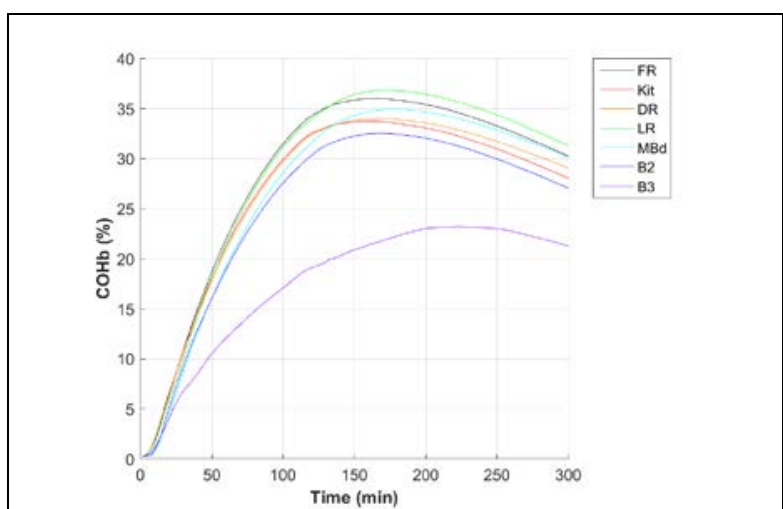

Figure 49e. $\mathrm{COHb}$ of house occupants during Case 17 Test 1 - G6 G300.

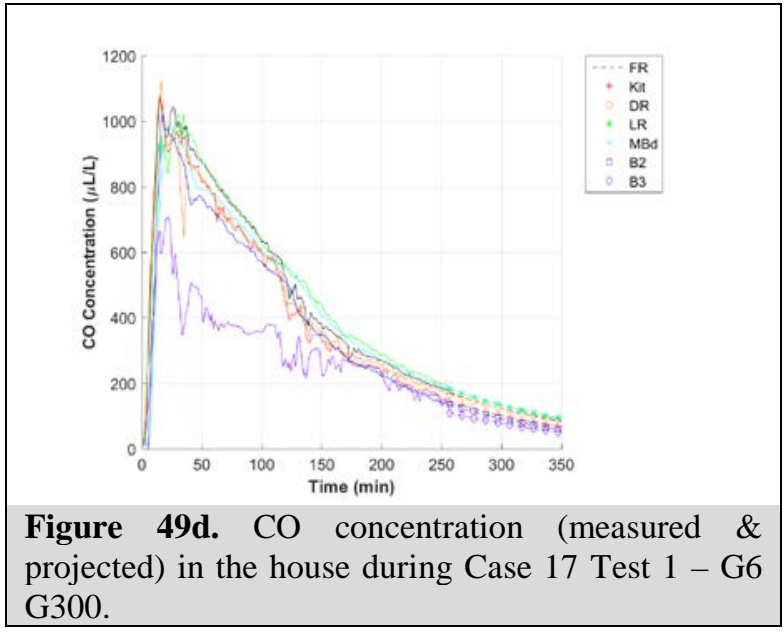

Figure 49d. CO concentration (measured \& projected) in the house during Case 17 Test 1 - G6 


\section{Case 17 Test 1 - G6 G300 Restart}

The following figures illustrate the results for Restart Case 17 Test 1 applying the G300 shutoff criteria. The G6 generator was fully fueled, positioned in Bedroom 3 (near the door with the exhaust facing the hallway and in the plane of the door), and tested at $100 \%$ load $(6,000 \mathrm{~W})$. The Bedroom 3 door was fully open; the Bedroom 3 window was open $10 \mathrm{~cm}$. For the first generator run, the shutoff sensor activated the algorithm to shut off the generator after approximately 6 min due to an instantaneous sensor measurement of $800 \mu \mathrm{L} / \mathrm{L}$. The generator was restarted and the test load restored approximately $10 \mathrm{~min}$ after the first instance of shutoff initiated by the shutoff sensor. For the second generator run, the shutoff sensor activated the algorithm to shut off the generator after approximately 2 min due to an instantaneous sensor measurement of $800 \mu \mathrm{L} / \mathrm{L}$. Figure 50a shows the test house layout with generator location, exhaust direction, and sensor array location. Figure 50b shows the CO concentration measured in Bedroom 3 (by the dedicated room sensor), by the sensor array placed in bedroom 3 located as shown in Figure 50a (A1: height 183 cm, A2: height $61 \mathrm{~cm}$ ), the sensor array placed in the hall outside Bedroom 3 as shown in Figure 50a (A3: height $183 \mathrm{~cm}$, A4: height $61 \mathrm{~cm}$ ), and by the shutoff sensor located on the generator near the generator's electrical panel. The timeframe of the figure has been selected to show the degree of $\mathrm{CO}$ uniformity in the area around the generator between the shutoff sensor and the peak CO concentrations measured elsewhere in and near Bedroom 3 near the time of shutoff. Figure 50c shows the $\mathrm{CO}$ concentration measured in each room of the house over the test. Figure 50d shows the CO concentration in the house - each room is represented by a single line, which is composed of both a 'solid-line' pattern (showing measured CO concentration) and a 'dotted-line with a symbol' pattern (showing projected CO concentration). Figure 50e shows the calculated COHb profiles of simulated house occupants in each room of the house.

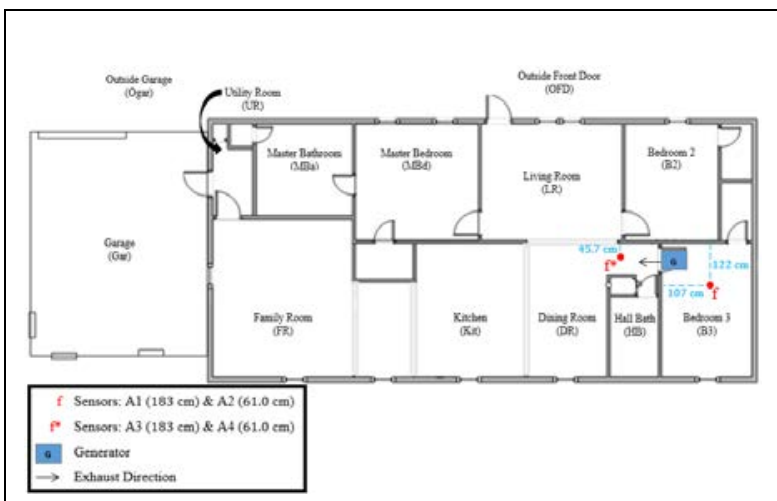

Figure 50a. Generator, exhaust direction, and sensor array layout during Restart Case 17 Test 1 - G6 G300.

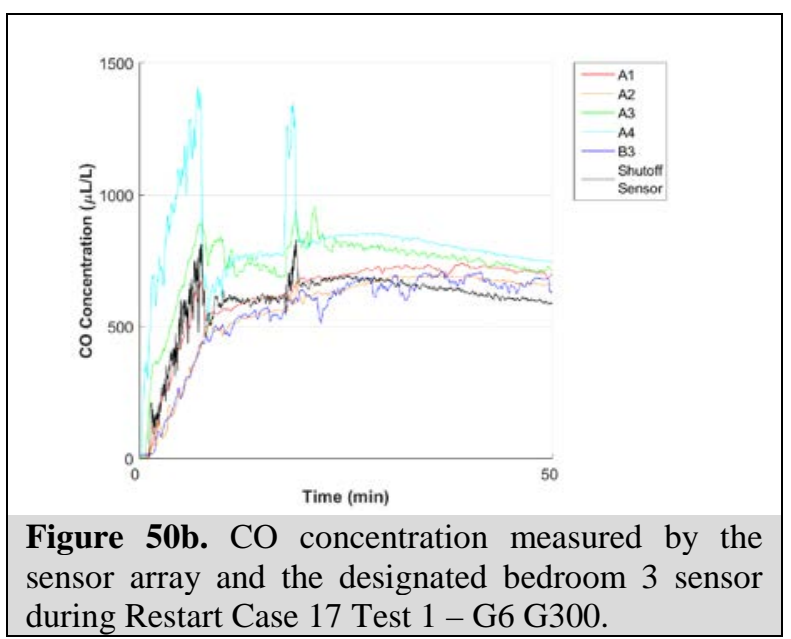




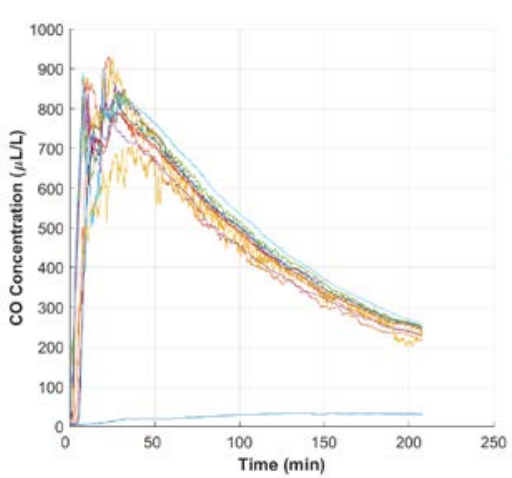

Figure 50c. CO concentration measured in each room of the house during Restart Case 17 Test 1 - G6 G300.

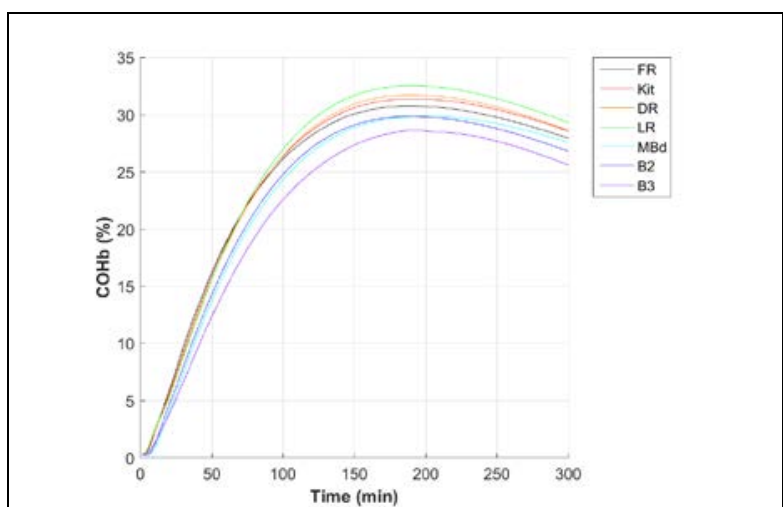

Figure 50e. COHb of house occupants during Restart Case 17 Test 1 - G6 G300.

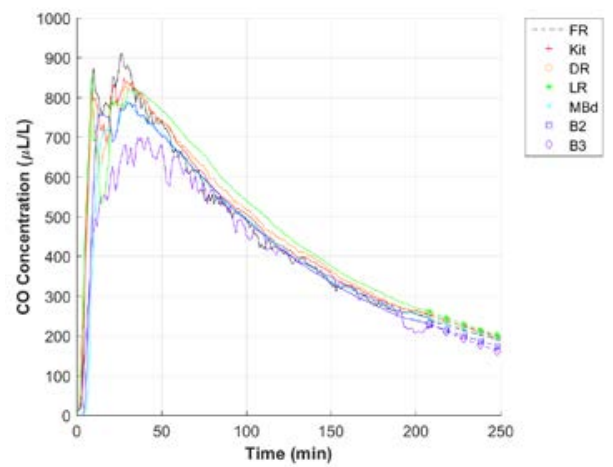

Figure 50d. CO concentration (measured \& projected) in the house during Restart Case 17 Test 1 - G6 G300. 


\section{Case 17 Test 1 - G7b UL2201}

The following figures illustrate the results for Case 17 Test 1 applying the UL 2201 shutoff criteria. The G7b generator was fully fueled, positioned in Bedroom 3 (near the door with the exhaust facing the hallway and in the plane of the door), and tested at $100 \%$ load $(7,000 \mathrm{~W})$. The door leading from the bedroom to the hallway was fully open; the bedroom window was open $10 \mathrm{~cm}$. The shutoff sensor activated the algorithm to shut off the generator after approximately 20 min due to an average sensor measurement of $150 \mu \mathrm{L} / \mathrm{L}$ for $10 \mathrm{~min}$. Figure 51a shows the test house layout with generator location, exhaust direction, and sensor array location. Figure 51b shows the CO concentration measured in Bedroom 3 (by the dedicated room sensor), by the sensor array placed in bedroom 3 located as shown in Figure 51a (A1: height $183 \mathrm{~cm}$, A2: height $61 \mathrm{~cm}$ ), the sensor array placed in the hall outside Bedroom 3 as shown in Figure 51a (A3: height $183 \mathrm{~cm}$, A4: height $61 \mathrm{~cm}$ ), and by the shutoff sensor located on the generator near the generator's electrical panel. The timeframe of the figure has been selected to show the degree of CO uniformity in the area around the generator between the shutoff sensor and the peak CO concentrations measured elsewhere in and near Bedroom 3 near the time of shutoff. Figure 51c shows the CO concentration measured in each room of the house over the test. Figure 51d shows the CO concentration in the house - each room is represented by a single line, which is composed of both a 'solid-line' pattern (showing measured CO concentration) and a 'dotted-line with a symbol' pattern (showing projected CO concentration). Figure 51e shows the calculated COHb profiles of simulated house occupants in each room of the house.

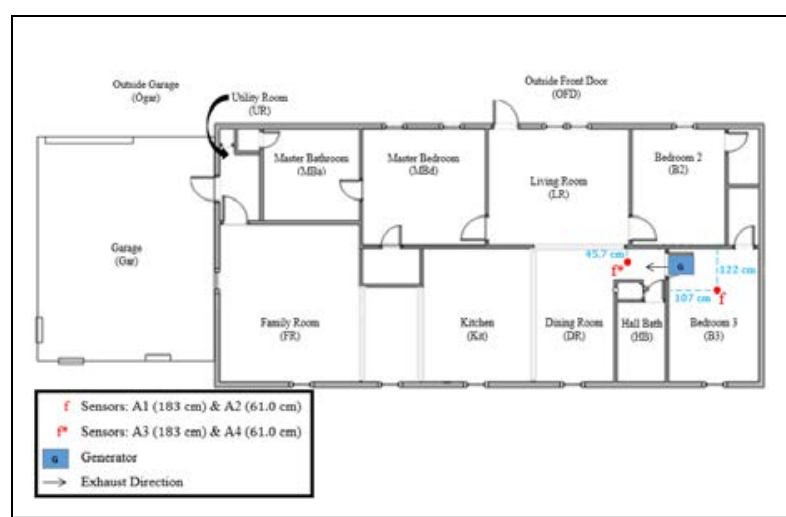

Figure 51a. Generator, exhaust direction, and sensor array layout during Case 17 Test 1 - G7b UL 2201.

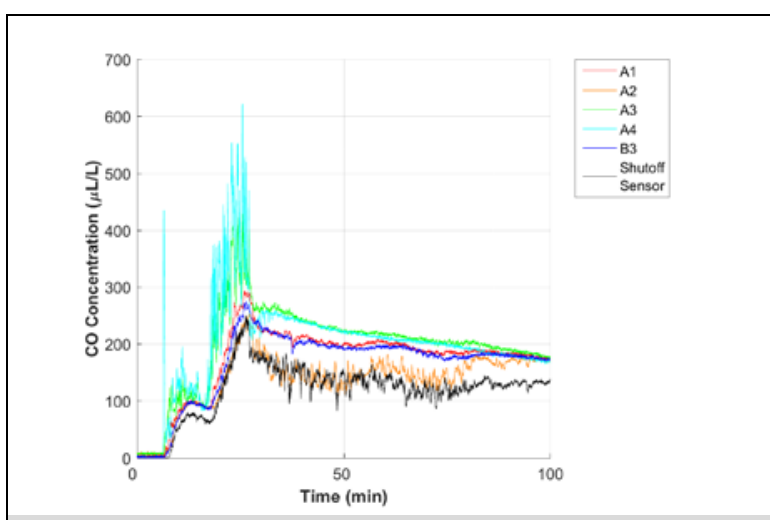

Figure 51b. CO concentration measured by the sensor array and the designated bedroom 3 sensor during Case 17 Test 1 - G7b UL 2201. 


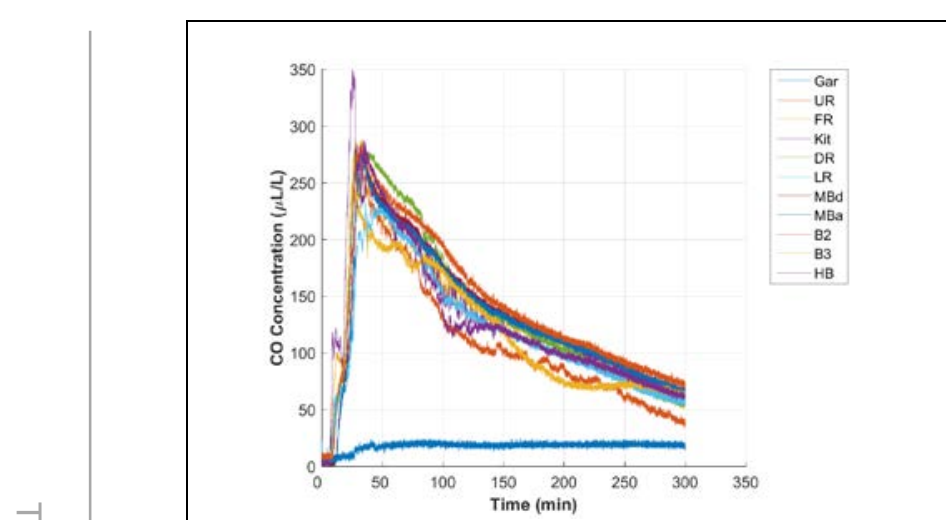

Figure 51c. CO concentration measured in each room of the house during Case 17 Test 1 - G7b UL 2201.

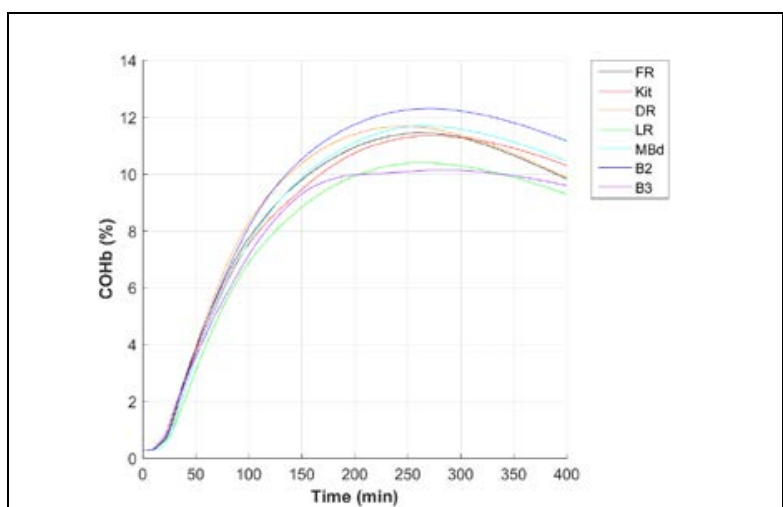

Figure 51e. $\mathrm{COHb}$ of house occupants during Case 17 Test 1 - G7b UL 2201.

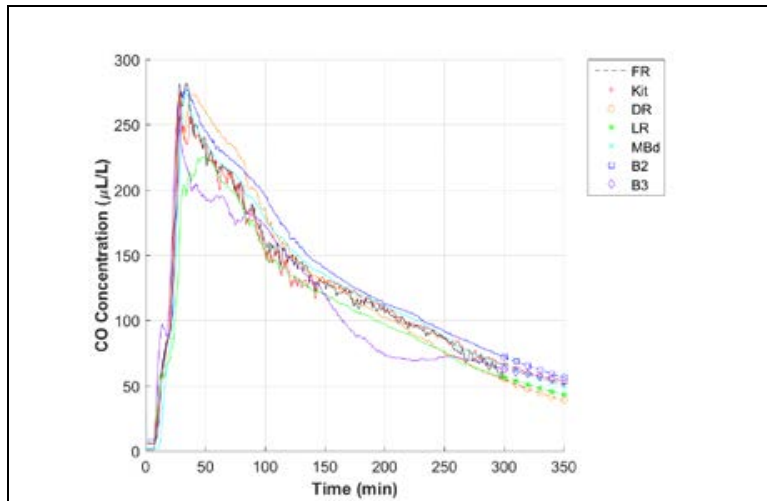

Figure 51d. CO concentration (measured \& projected) in the house during Case 17 Test 1 - G7b UL 2201. 


\section{Case 17 Test 1 - G7b UL 2201 Restart}

The following figures illustrate the results for Restart Case 17 Test 1 applying the UL 2201 shutoff criteria. The G7b generator was fully fueled, positioned in Bedroom 3 (near the door with the exhaust facing the hallway and in the plane of the door), and tested at $100 \%$ load $(7,000 \mathrm{~W})$. The Bedroom 3 door was fully open; the Bedroom 3 window was open $10 \mathrm{~cm}$. For the first generator run, the shutoff sensor activated the algorithm to shut off the generator after approximately 33 min due to a sensor measurement of $150 \mu \mathrm{L} / \mathrm{L}$ for a $10 \mathrm{~min}$ average. The generator was restarted and the test load restored approximately $10 \mathrm{~min}$ after the first instance of shutoff initiated by the shutoff sensor. For the second generator run, the shutoff sensor activated the algorithm to shut off the generator after approximately 45 min due to a sensor measurement of $150 \mu \mathrm{L} / \mathrm{L}$ for a $10 \mathrm{~min}$ average. Figure 52a shows the test house layout with generator location, exhaust direction, and sensor array location. Figure 52b shows the CO concentration measured in Bedroom 3 (by the dedicated room sensor), by the sensor array placed in bedroom 3 located as shown in Figure 52a (A1: height $183 \mathrm{~cm}, \mathrm{~A} 2$ : height $61 \mathrm{~cm}$ ), the sensor array placed in the hall outside Bedroom 3 as shown in Figure 52a (A3: height $183 \mathrm{~cm}$, A4: height $61 \mathrm{~cm}$ ), and by the shutoff sensor located on the generator near the generator's electrical panel. The timeframe of the figure has been selected to show the degree of $\mathrm{CO}$ uniformity in the area around the generator between the shutoff sensor and the peak $\mathrm{CO}$ concentrations measured elsewhere in and near Bedroom 3 near the time of shutoff. Figure 52c shows the CO concentration measured in each room of the house over the test. Figure 52d shows the CO concentration in the house - each room is represented by a single line, which is composed of both a 'solid-line' pattern (showing measured CO concentration) and a 'dotted-line with a symbol' pattern (showing projected CO concentration). Figure 52e shows the calculated $\mathrm{COHb}$ profiles of simulated house occupants in each room of the house.

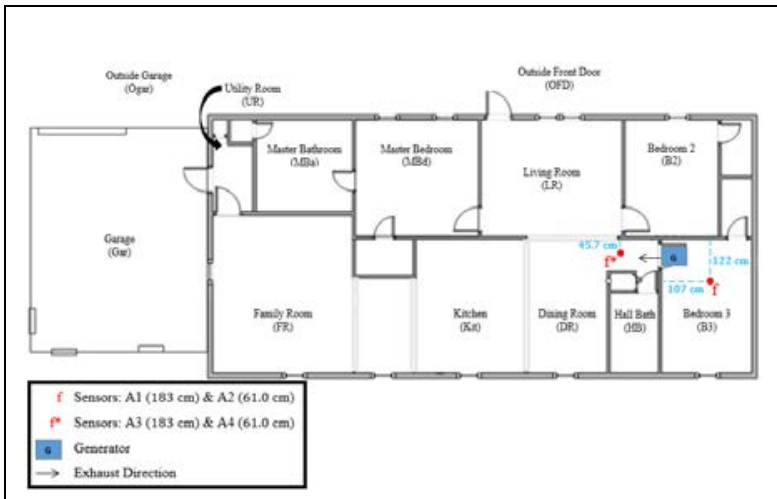

Figure 52a. Generator, exhaust direction, and sensor array layout during Restart Case 17 Test 1 - G7b UL 2201.

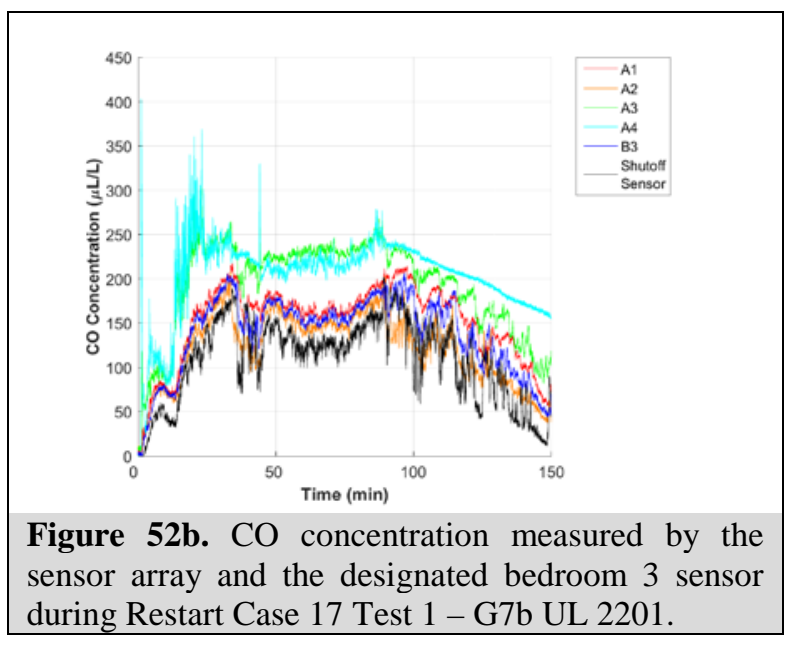




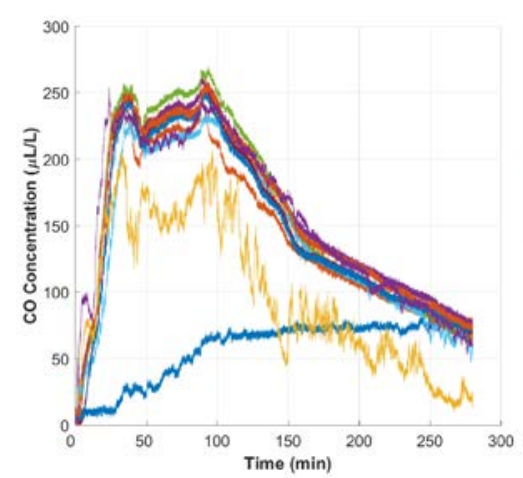

Figure 52c. CO concentration measured in each room of the house during Restart Case 17 Test 1 - G7b UL 2201.

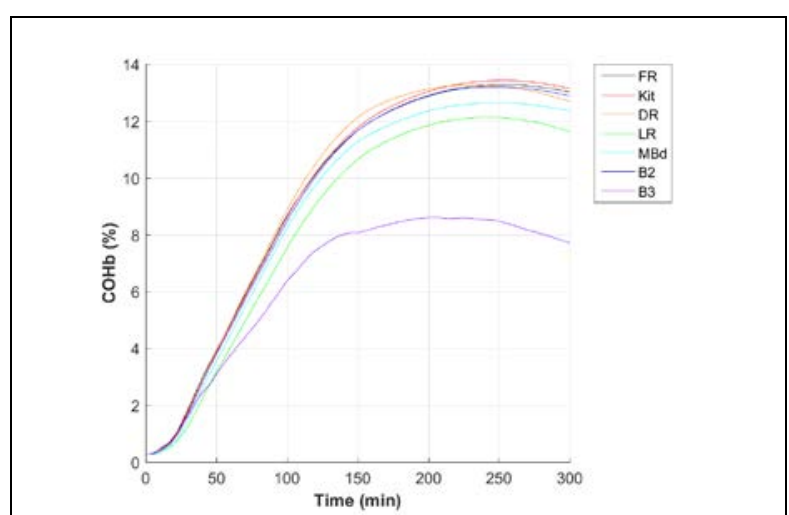

Figure 52e. $\mathrm{COHb}$ of house occupants during Restart Case 17 Test 1 - G7b UL 2201.

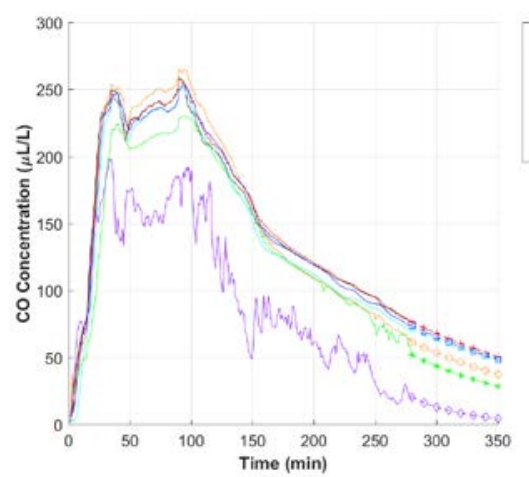

Figure 52d. CO concentration (measured \& projected) in the house during Restart Case 17 Test 1 - G7b UL 2201. 


\section{Case 18 Test 1 - G2 G300}

The following figures illustrate the results for Case 18 Test 1 applying the G300 shutoff criteria. The G2 generator was fully fueled, positioned in Bedroom 3 (near the door with the exhaust facing the hallway and in the plane of the door), and tested at $10 \%$ load $(160 \mathrm{~W})$. The door leading from the bedroom to the hallway was fully open; the bedroom window was open $10 \mathrm{~cm}$. The shutoff sensor activated the algorithm to shut off the generator after approximately 82 min due to a sensor measurement of $400 \mu \mathrm{L} / \mathrm{L}$ for a $10 \mathrm{~min}$ average. Figure 53a shows the test house layout with generator location, exhaust direction, and sensor array location. Figure 53b shows the CO concentration measured in Bedroom 3 (by the dedicated room sensor), by the sensor array placed in bedroom 3 located as shown in Figure 53a (A1: height $183 \mathrm{~cm}$, A2: height $61.0 \mathrm{~cm})$, the sensor array placed in the hall outside Bedroom 3 as shown in Figure 53a (A3: height $183 \mathrm{~cm}$, A4: height $61.0 \mathrm{~cm}$ ), and by the shutoff sensor located on the generator near the generator's electrical panel. The timeframe of the figure has been selected to show the degree of CO uniformity in the area around the generator between the shutoff sensor and the peak CO concentrations measured elsewhere in and near Bedroom 3 near the time of shutoff. Figure 53c shows the CO concentration measured in each room of the house over the test. Figure 53d shows the CO concentration in the house - each room is represented by a single line, which is composed of both a 'solid-line' pattern (showing measured CO concentration) and a 'dotted-line with a symbol' pattern (showing projected CO concentration). Figure 53e shows the calculated COHb profiles of simulated house occupants in each room of the house.

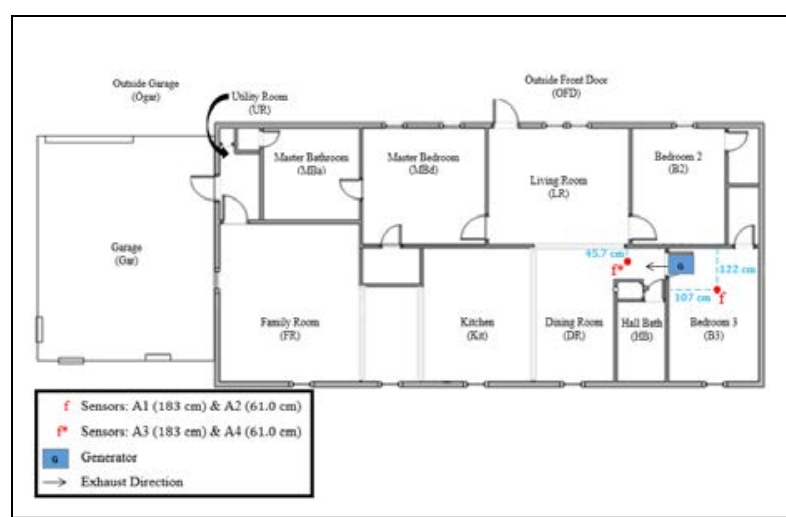

Figure 53a. Generator, exhaust direction, and sensor array layout during Case 18 Test 1 - G2 G300.

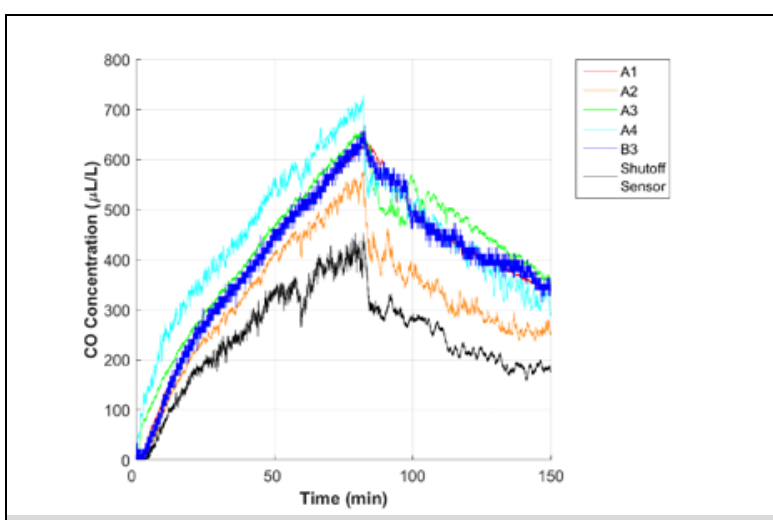

Figure 53b. CO concentration measured by the sensor array and the designated bedroom 3 sensor during Case 18 Test 1 - G2 G300. 

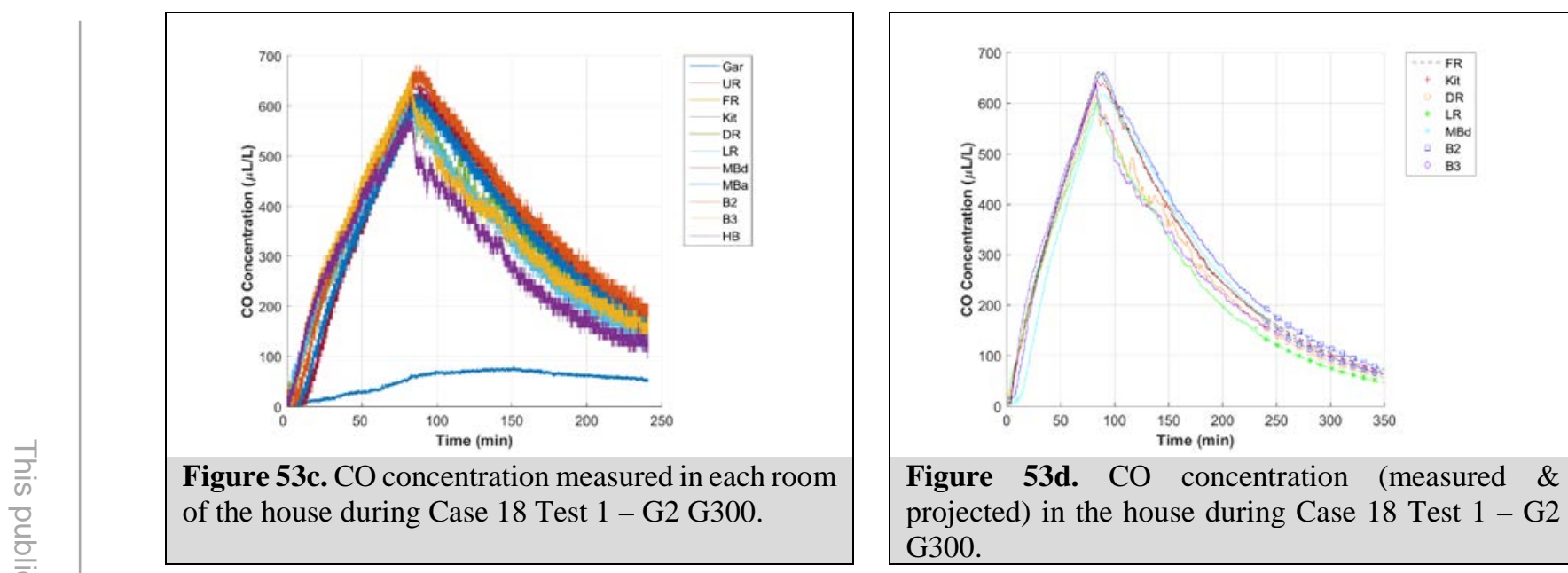

Figure 53d. CO concentration (measured \& projected) in the house during Case 18 Test 1 - G2 G300.

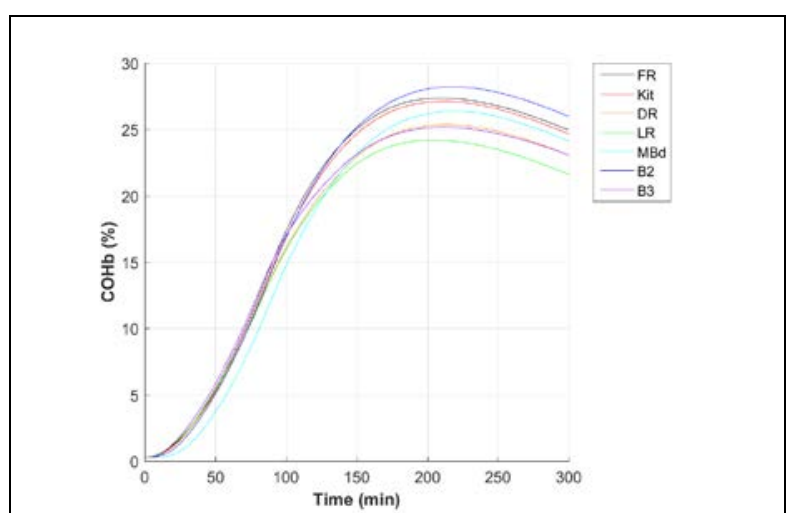

Figure 53e. $\mathrm{COHb}$ of house occupants during Case 18 Test $1-$ G2 G300. 


\section{Case 18 Test 1 - G2 G300 Restart}

The following figures illustrate the results for Restart Case 18 Test 1 applying the G300 shutoff criteria. The G2 generator was fully fueled, positioned in Bedroom 3 (near the door with the exhaust facing the hallway and in the plane of the door), and tested at $10 \%$ load (160 W). The Bedroom 3 door was fully open; the Bedroom 3 window was open $10 \mathrm{~cm}$. For the first generator run, the shutoff sensor activated the algorithm to shut off the generator after approximately 47 min due to a sensor measurement of $400 \mu \mathrm{L} / \mathrm{L}$ for a $10 \mathrm{~min}$ average. The generator was restarted and the test load restored approximately $10 \mathrm{~min}$ after the first instance of shutoff initiated by the shutoff sensor. For the second generator run, the shutoff sensor activated the algorithm to shut off the generator after approximately 8 min due to a sensor measurement of $400 \mu \mathrm{L} / \mathrm{L}$ for a $10 \mathrm{~min}$ average. Figure 54a shows the test house layout with generator location, exhaust direction, and sensor array location. Figure 54b shows the CO concentration measured in Bedroom 3 (by the dedicated room sensor), by the sensor array placed in bedroom 3 located as shown in Figure 54a (A1: height $183 \mathrm{~cm}, \mathrm{A2}$ : height $61 \mathrm{~cm}$ ), the sensor array placed in the hall outside Bedroom 3 as shown in Figure 54a (A3: height $183 \mathrm{~cm}$, A4: height $61 \mathrm{~cm}$ ), and by the shutoff sensor located on the generator near the generator's electrical panel. The timeframe of the figure has been selected to show the degree of $\mathrm{CO}$ uniformity in the area around the generator between the shutoff sensor and the peak CO concentrations measured elsewhere in and near Bedroom 3 near the time of shutoff. Figure 54c shows the CO concentration measured in each room of the house over the test. Figure 54d shows the CO concentration in the house - each room is represented by a single line, which is composed of both a 'solid-line' pattern (showing measured CO concentration) and a 'dotted-line with a symbol' pattern (showing projected CO concentration). Figure 54e shows the calculated $\mathrm{COHb}$ profiles of simulated house occupants in each room of the house.

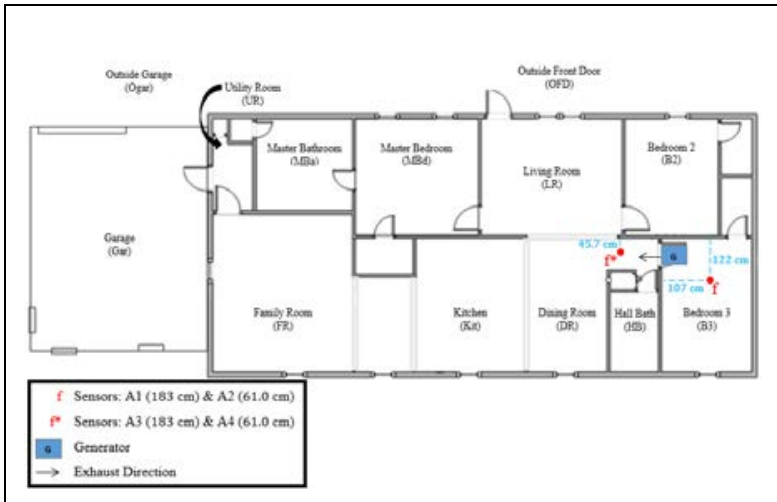

Figure 54a. Generator, exhaust direction, and sensor array layout during Restart Case 18 Test 1 - G2 G300.

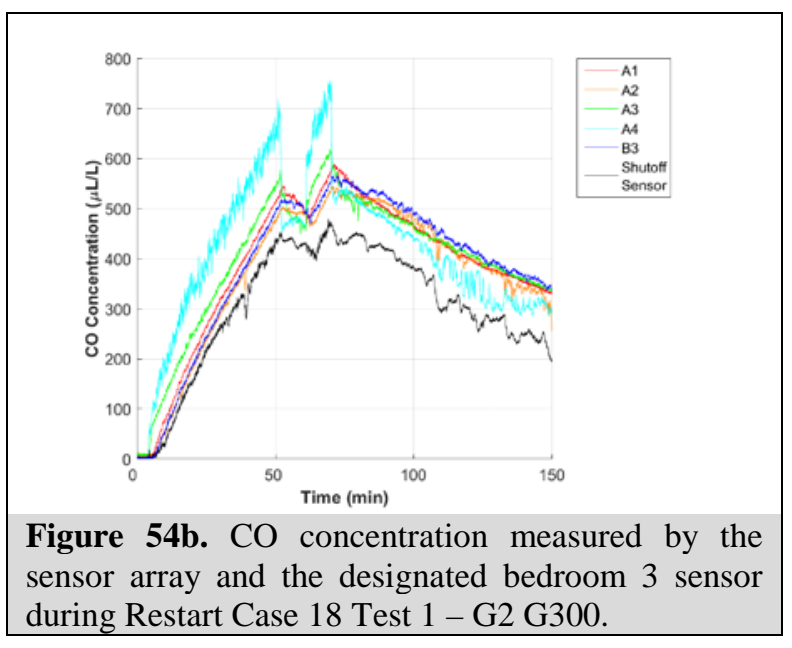

Figure 54b. CO concentration measured by the during Restart Case 18 Test 1 - G2 G300. 

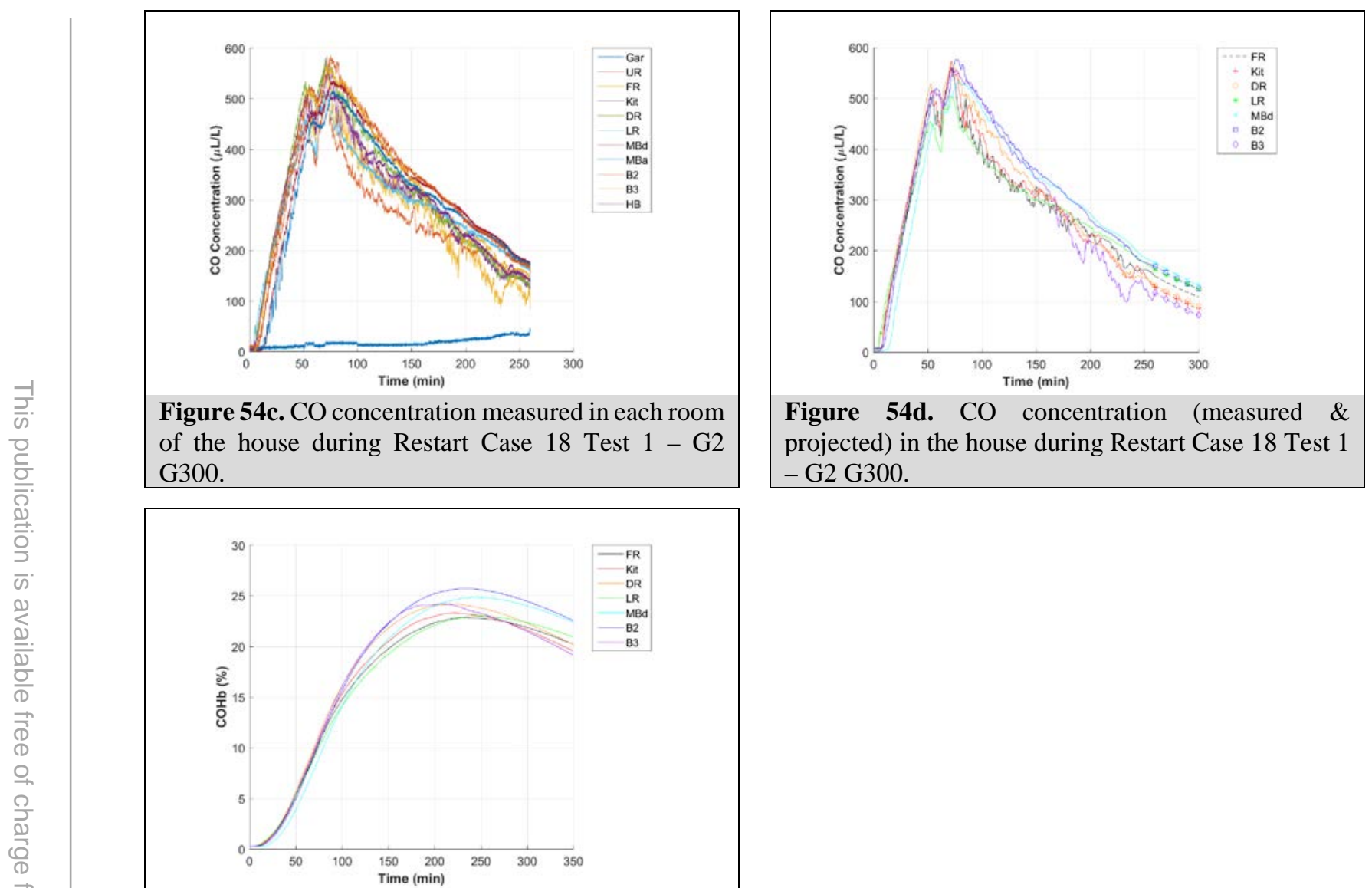

Figure 54e. $\mathrm{COHb}$ of house occupants during Restart Case 18 Test 1 - G2 G300. 


\section{Case 18 Test 1 - G2 G300 Relocate}

The following figures illustrate the results for Relocate Case 18 Test 1 applying the G300 shutoff criteria. The G2 generator was fully fueled, positioned in Bedroom 3 (near the door with the exhaust facing the hallway and in the plane of the door), and tested at $10 \%$ load (160 W). The Bedroom 3 door was fully open. The Bedroom 3 window was initially open $10 \mathrm{~cm}$. The shutoff sensor activated the algorithm to shut off the generator after approximately 76 min due to a sensor measurement of $400 \mu \mathrm{L} / \mathrm{L}$ for a $10 \mathrm{~min}$ average. Ten min after generator shutoff, the bedroom window was closed and the front door, which was initially closed, was opened fully for 1 min and then set to $10 \mathrm{~cm}$ for the remainder of the test. Figure 55a shows the test house layout with generator location, exhaust direction, and sensor array location. Figure 55b shows the CO concentration measured in Bedroom 3 (by the dedicated room sensor), by the sensor array placed in bedroom 3 located as shown in Figure 55a (A1: height $183 \mathrm{~cm}, \mathrm{A2}$ : height $61 \mathrm{~cm})$, the sensor array placed in the hall outside Bedroom 3 as shown in Figure 55a (A3: height $183 \mathrm{~cm}$, A4: height $61 \mathrm{~cm})$, and by the shutoff sensor located on the generator near the generator's electrical panel. The timeframe of the figure has been selected to show the degree of CO uniformity in the area around the generator between the shutoff sensor and the peak $\mathrm{CO}$ concentrations measured elsewhere in and near Bedroom 3 near the time of shutoff. Figure 55c shows the CO concentration measured in each room of the house over the test. Figure 55d shows the CO concentration in the house - each room is represented by a single line, which is composed of both a 'solid-line' pattern (showing measured CO concentration) and a 'dotted-line with a symbol' pattern (showing projected $\mathrm{CO}$ concentration). Figure 55e shows the calculated $\mathrm{COHb}$ profiles of simulated house occupants in each room of the house.

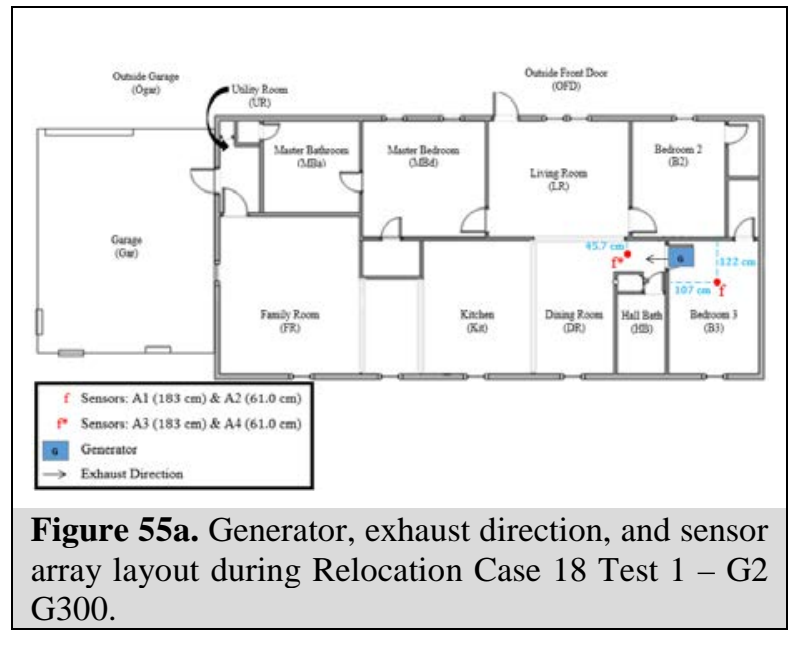

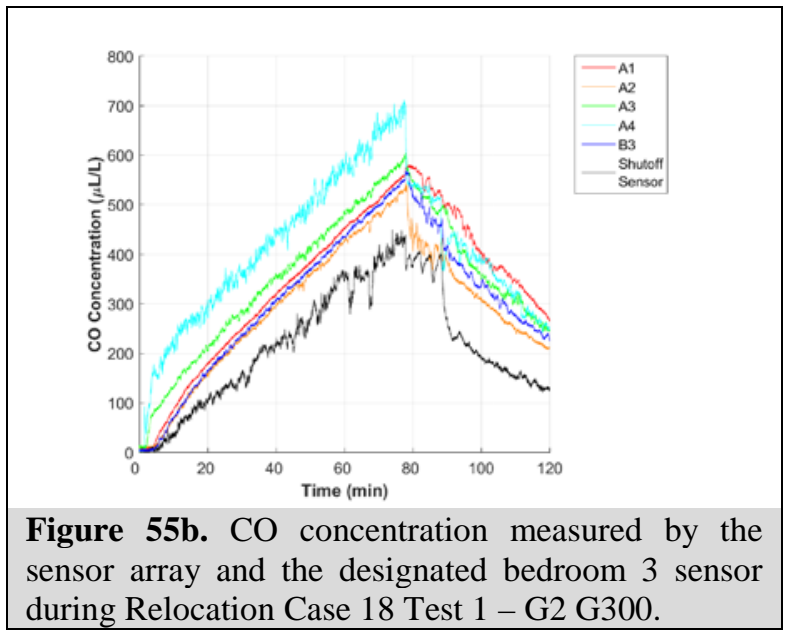



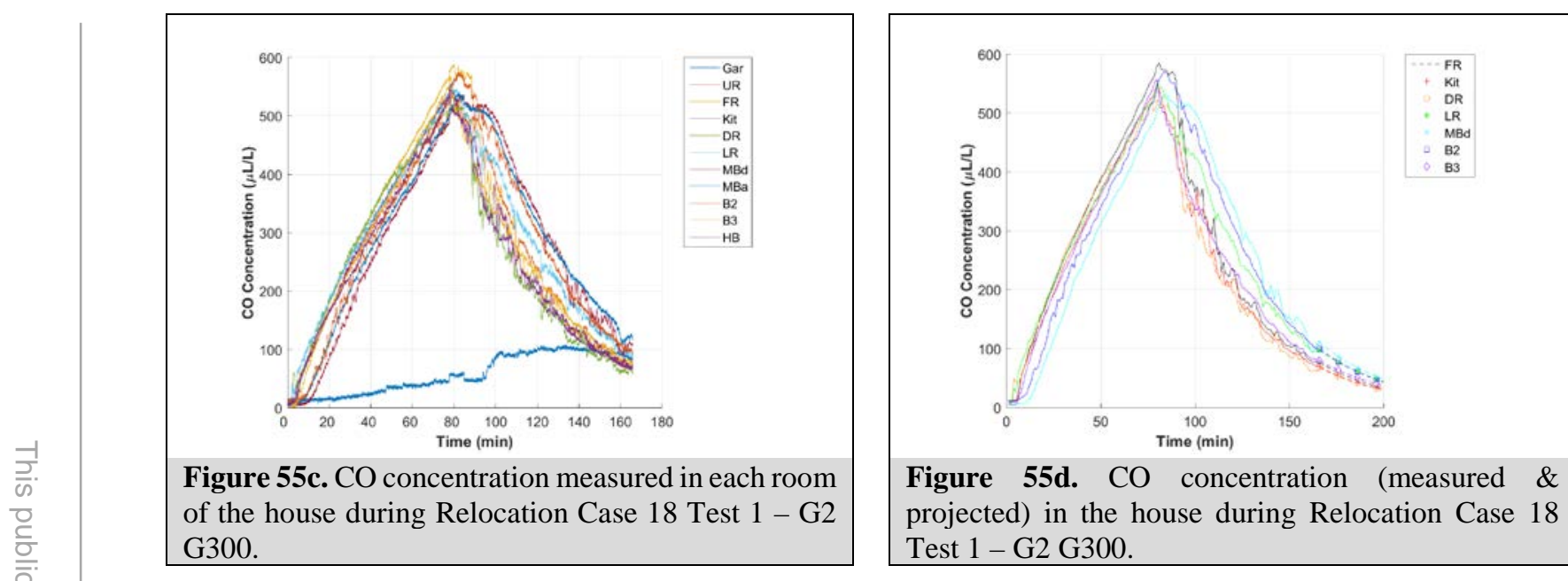

Figure 55d. CO concentration (measured \& projected) in the house during Relocation Case 18 Test 1 - G2 G300.

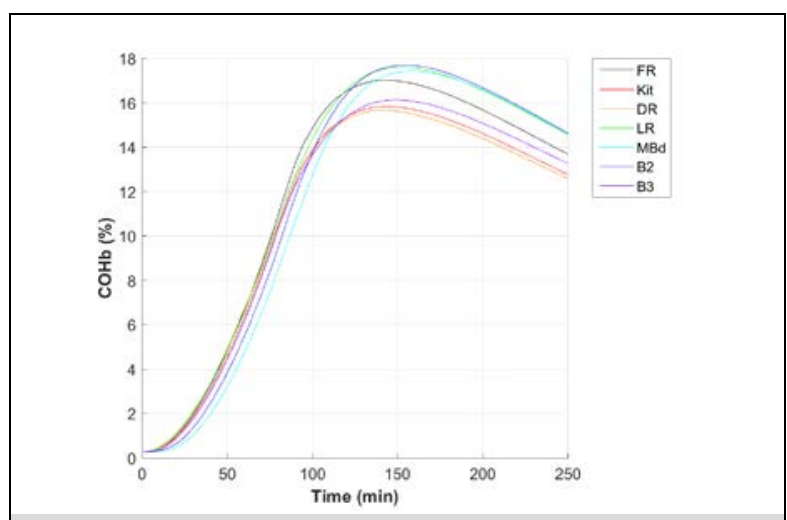

Figure 55e. $\mathrm{COHb}$ of house occupants during Relocation Case 18 Test 1 - G2 G300. 


\section{Case 18 Test 1 - G2 UL 2201}

The following figures illustrate the results for Case 18 Test 1 applying the UL 2201 shutoff criteria. The G2 generator was fully fueled, positioned in Bedroom 3 (near the door with the exhaust facing the hallway and in the plane of the door), and tested at $10 \%$ load (160 W). The door leading from the bedroom to the hallway was fully open; the bedroom window was open $10 \mathrm{~cm}$. The shutoff sensor activated the algorithm to shut off the generator after approximately 25 min due to a sensor measurement of $150 \mu \mathrm{L} / \mathrm{L}$ for a $10 \mathrm{~min}$ average. Figure 56a shows the test house layout with generator location, exhaust direction, and sensor array location. Figure $\mathbf{5 6 b}$ shows the CO concentration measured in Bedroom 3 (by the dedicated room sensor), by the sensor array placed in Bedroom 3 located as shown in Figure 56a (A1: height $183 \mathrm{~cm}, \mathrm{A2}$ : height $61 \mathrm{~cm})$, the sensor array placed in the hall outside Bedroom 3 as shown in Figure 56a (A3: height $183 \mathrm{~cm}$, A4: height $61 \mathrm{~cm})$, and by the shutoff sensor located on the generator near the generator's electrical panel. The timeframe of the figure has been selected to show the degree of CO uniformity in the area around the generator between the shutoff sensor and the peak CO concentrations measured elsewhere in and near Bedroom 3 near the time of shutoff. Figure 56c shows the CO concentration measured in each room of the house over the test. Figure 56d shows the CO concentration in the house - each room is represented by a single line, which is composed of both a 'solid-line' pattern (showing measured CO concentration) and a 'dotted-line with a symbol' pattern (showing projected CO concentration). Figure 56e shows the calculated COHb profiles of simulated house occupants in each room of the house.

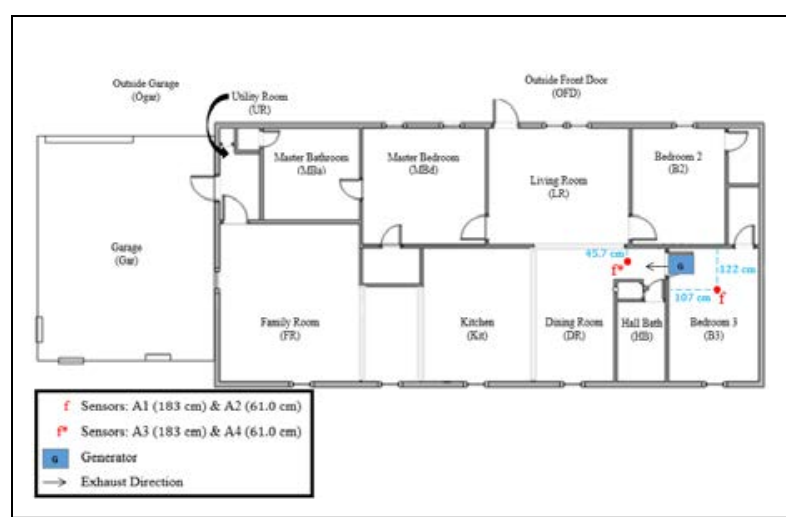

Figure 56a. Generator, exhaust direction, and sensor array layout during Case 18 Test 1 - G2 UL 2201.

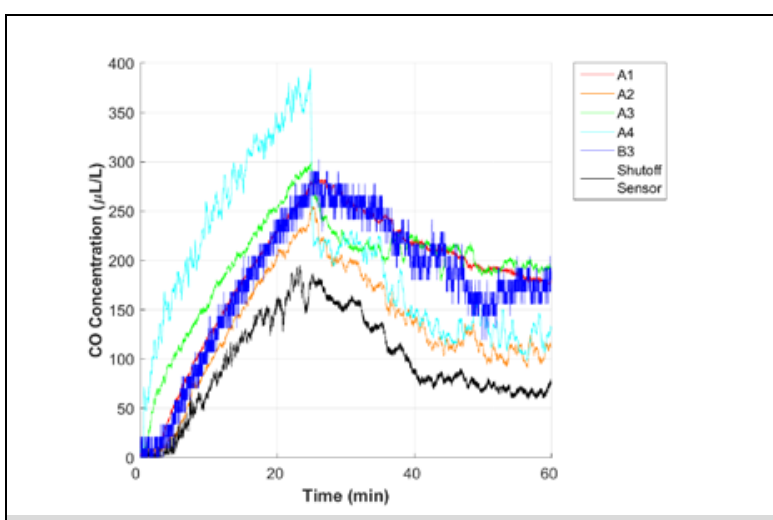

Figure 56b. CO concentration measured by the sensor array and the designated bedroom 3 sensor during Case 18 Test 1 - G2 UL 2201. 


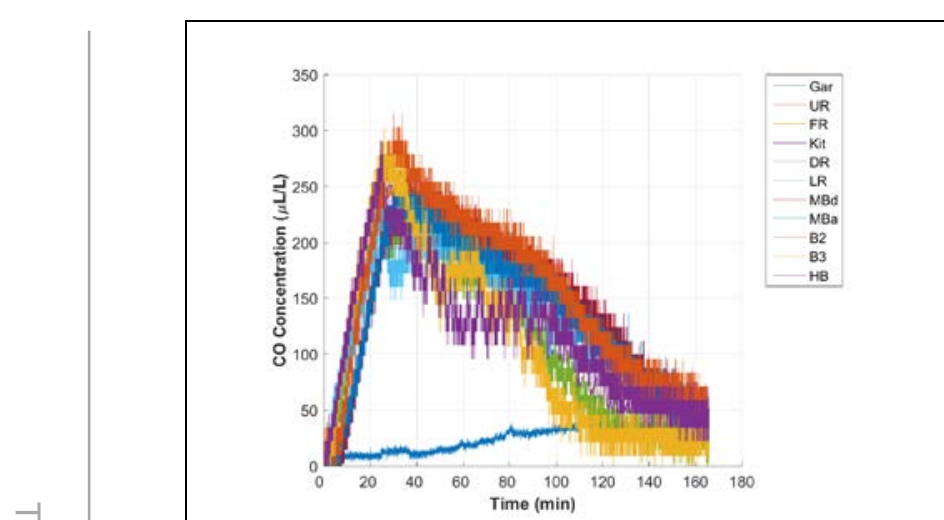

Figure 56c. CO concentration measured in each room of the house during Case 18 Test 1 - G2 UL 2201.

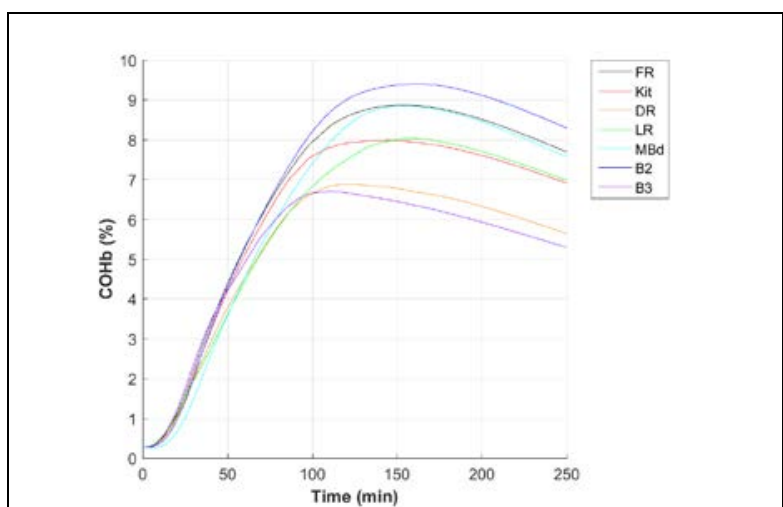

Figure 56e. $\mathrm{COHb}$ of house occupants during Case 18 Test 1 - G2 UL 2201.

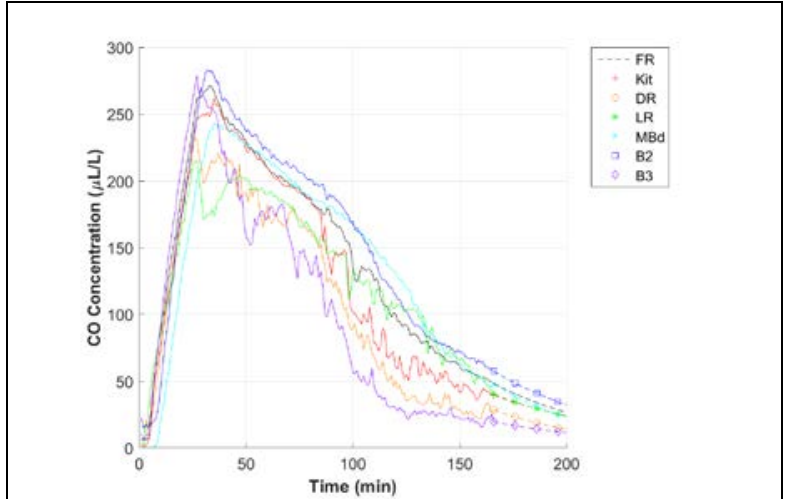

Figure 56d. CO concentration (measured \& projected) in the house during Case 18 Test 1 - G2 UL 2201. 


\section{Case 18 Test 1 - G2 UL 2201 Restart}

The following figures illustrate the results for Restart Case 18 Test 1 applying the UL 2201 shutoff criteria. The G2 generator was fully fueled, positioned in Bedroom 3 (near the door with the exhaust facing the hallway and in the plane of the door), and tested at $10 \%$ load (160 W). The Bedroom 3 door was fully open; the Bedroom 3 window was open $10 \mathrm{~cm}$. For the first generator run, the shutoff sensor activated the algorithm to shut off the generator after approximately 22 min due to a sensor measurement of $150 \mu \mathrm{L} / \mathrm{L}$ for a $10 \mathrm{~min}$ average. The generator was restarted and the test load restored approximately $10 \mathrm{~min}$ after the first instance of shutoff initiated by the shutoff sensor. For the second generator run, the shutoff sensor activated the algorithm to shut off the generator after approximately 6 min due to a sensor measurement of $150 \mu \mathrm{L} / \mathrm{L}$ for a $10 \mathrm{~min}$ average. Figure 57a shows the test house layout with generator location, exhaust direction, and sensor array location. Figure 57b shows the CO concentration measured in Bedroom 3 (by the dedicated room sensor), by the sensor array placed in Bedroom 3 located as shown in Figure 57a (A1: height $183 \mathrm{~cm}, \mathrm{~A} 2$ : height $61.0 \mathrm{~cm}$ ), the sensor array placed in the hall outside Bedroom 3 as shown in Figure 57a (A3: height $183 \mathrm{~cm}$, A4: height $61.0 \mathrm{~cm}$ ), and by the shutoff sensor located on the generator near the generator's electrical panel. The timeframe of the figure has been selected to show the degree of $\mathrm{CO}$ uniformity in the area around the generator between the shutoff sensor and the peak $\mathrm{CO}$ concentrations measured elsewhere in and near Bedroom 3 near the time of shutoff. Figure 57c shows the CO concentration measured in each room of the house over the test. Figure 57d shows the CO concentration in the house - each room is represented by a single line, which is composed of both a 'solid-line' pattern (showing measured CO concentration) and a 'dotted-line with a symbol' pattern (showing projected CO concentration). Figure 57e shows the calculated $\mathrm{COHb}$ profiles of simulated house occupants in each room of the house.

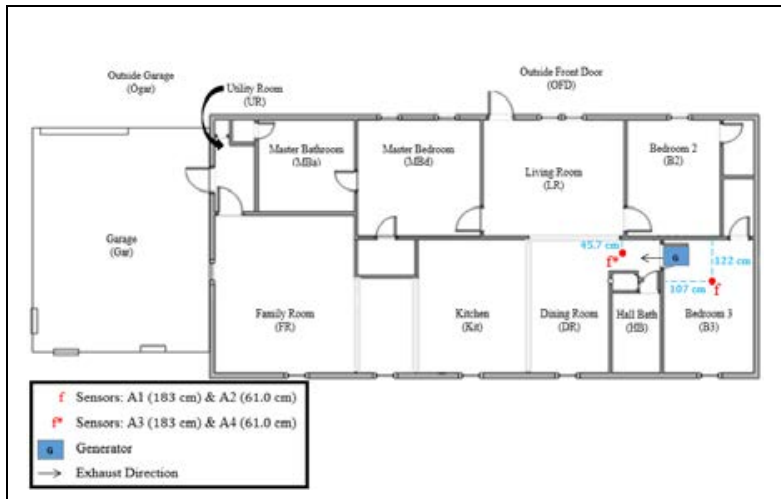

Figure 57a. Generator, exhaust direction, and sensor array layout during Restart Case 18 Test 1 - G2 UL 2201.

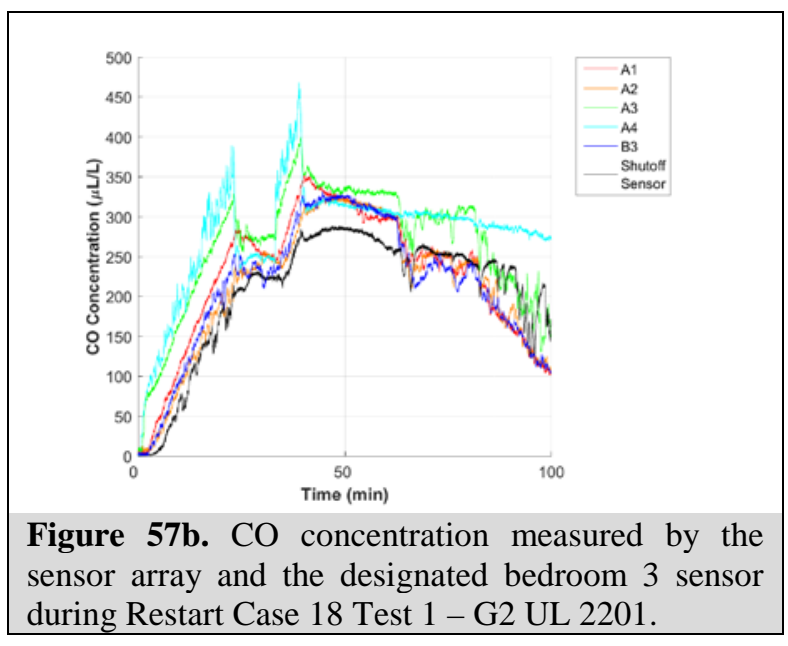



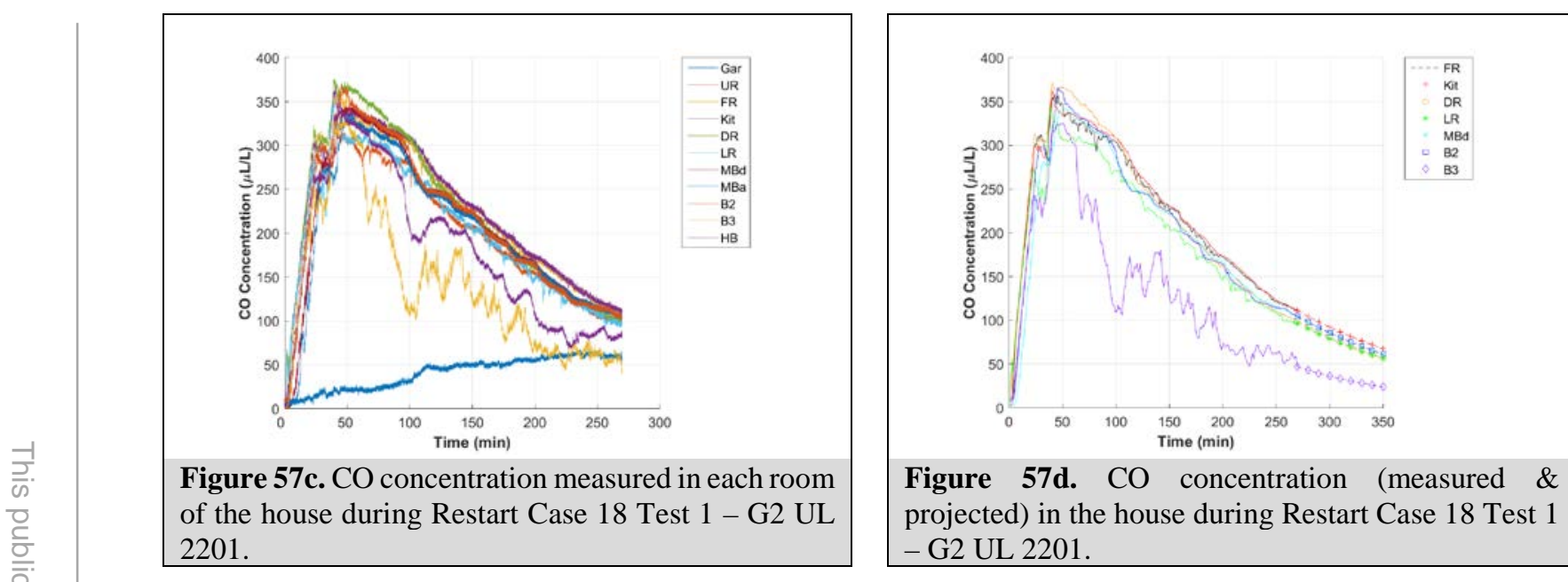

Figure 57d. CO concentration (measured \& projected) in the house during Restart Case 18 Test 1 - G2 UL 2201.

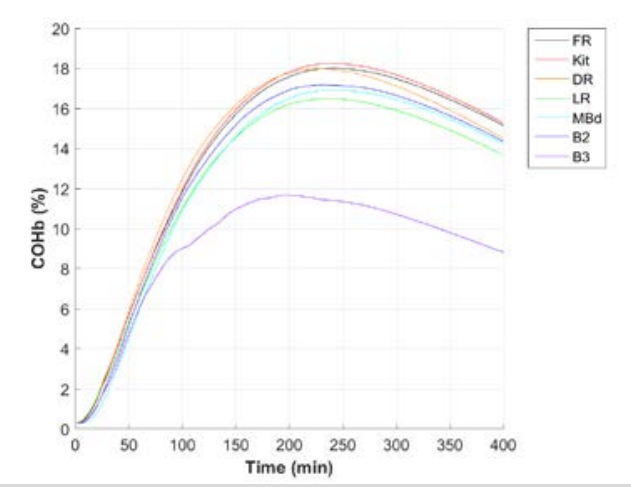

Figure 57e. $\mathrm{COHb}$ of house occupants during Restart Case 18 Test 1 - G2 UL 2201. 


\section{Generator Location: Utility Room}

Table 6 summarizes the results for all tests performed with a generator located in the test house utility room. The details are discussed below and shown in Figures 58 through 65.

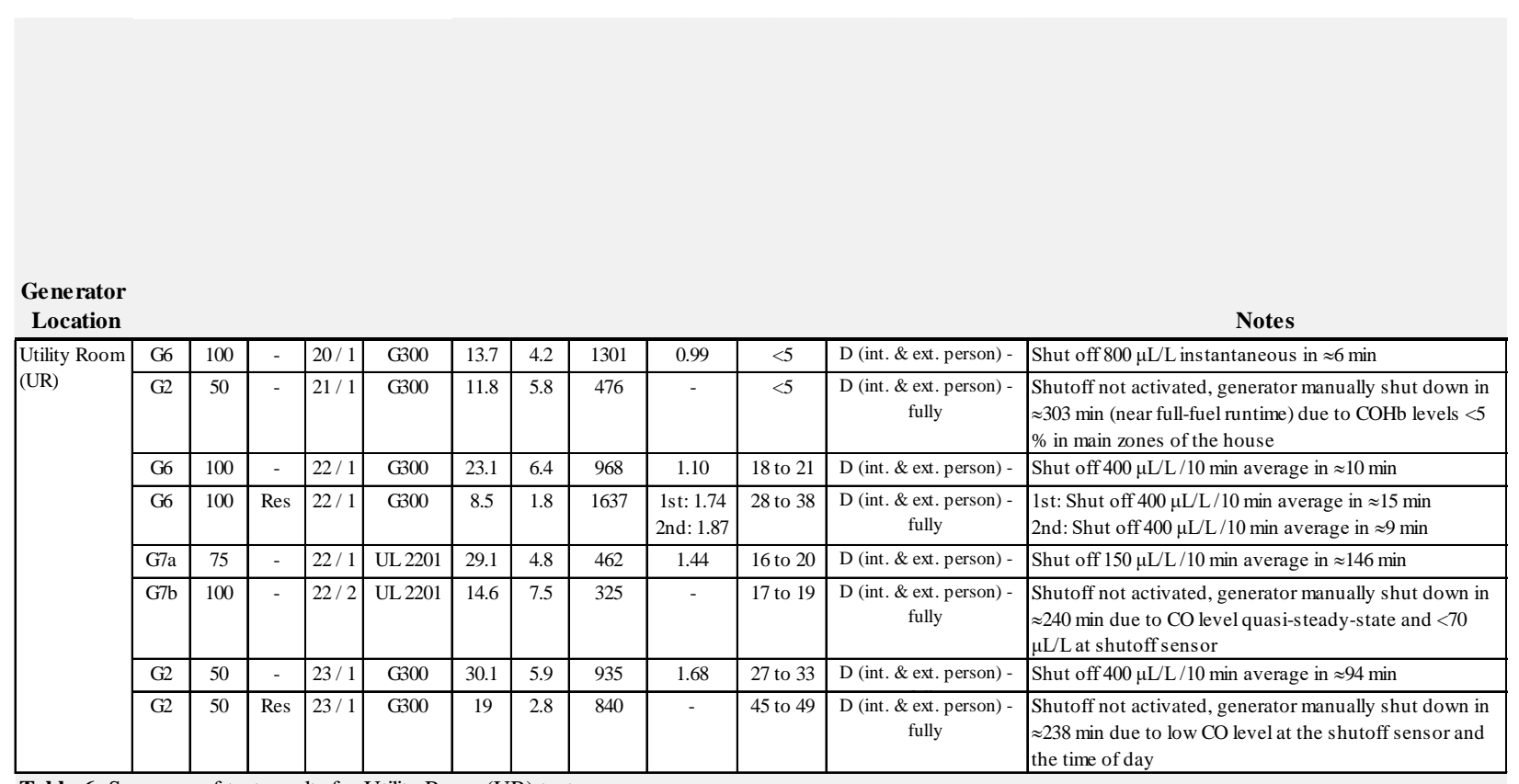

Table 6. Summary of test results for Utility Room (UR) tests.

+Peak COHb Values are for house zones only.

HVentilation Note: Unless specified otherwise, during the test - all exterior doors are closed, all interior doors are fully open, and all windows are closed. 


\section{Case 20 Test 1 - G6 G300}

The following figures illustrate the results for Case 20 Test 1 applying the G300 shutoff criteria. The G6 generator was fully fueled, positioned in the utility room (centered, with the exhaust facing out to the garage), and tested at $100 \%$ load $(6,000 \mathrm{~W})$. The interior person door (from garage to utility room) and exterior person door (from garage to backyard) were fully open; the garage bay door was closed. The shutoff sensor activated the algorithm to shut off the generator after approximately 6 min due to an instantaneous sensor measurement of $800 \mu \mathrm{L} / \mathrm{L}$. Figure 58a shows the test house layout with generator location, exhaust direction, and sensor array location. Figure 58b shows the CO concentration measured in the utility room (by the dedicated room sensor), by the sensor array placed in the garage located as shown in Figure 58a (A1: height $183 \mathrm{~cm}$, A2: height $61 \mathrm{~cm}$ ), by the sensor array placed in the family room located as shown in Figure 58a (A3: height $183 \mathrm{~cm}$, A4: height $61 \mathrm{~cm}$ ), and by the shutoff sensor located on the generator near the generator's electrical panel. The timeframe of the figure has been selected to show the degree of $\mathrm{CO}$ uniformity in the area around the generator between the shutoff sensor and the peak CO concentrations measured elsewhere in and near the utility room near the time of shutoff. Figure 58c shows the CO concentration measured in each room of the house over the test. Figure 58d shows the calculated $\mathrm{COHb}$ profiles of simulated house occupants in each room of the house.

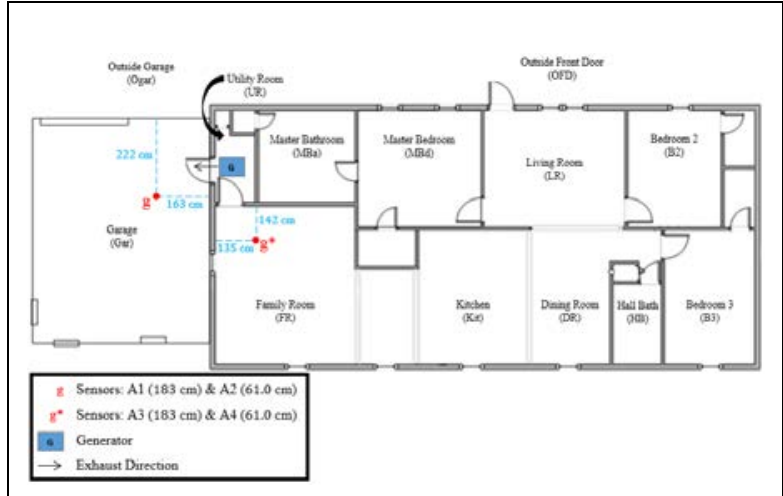

Figure 58a. Generator, exhaust direction, and sensor array layout during Case 20 Test 1 - G6 G300.

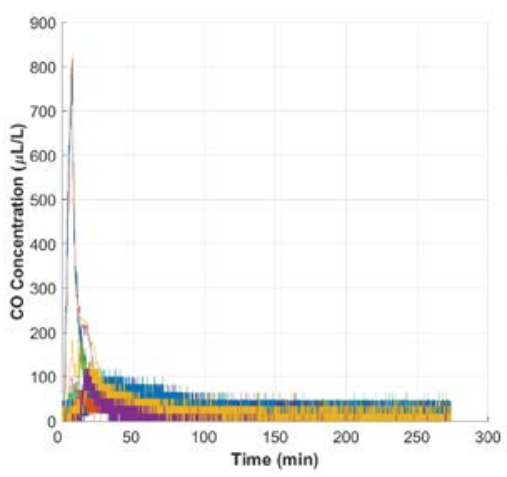

Figure 58c. CO concentration measured in each room of the house during Case 20 Test 1 - G6 G300.

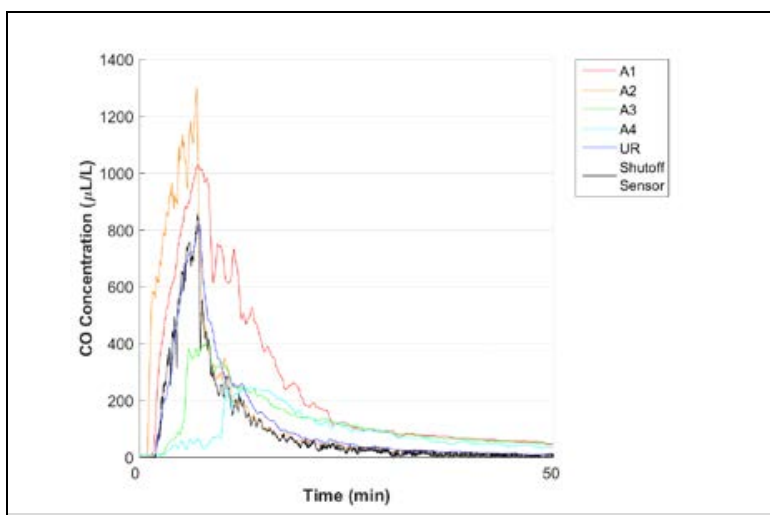

Figure 58b. CO concentration measured by the sensor array and the designated utility room sensor during Case 20 Test 1 - G6 G300.

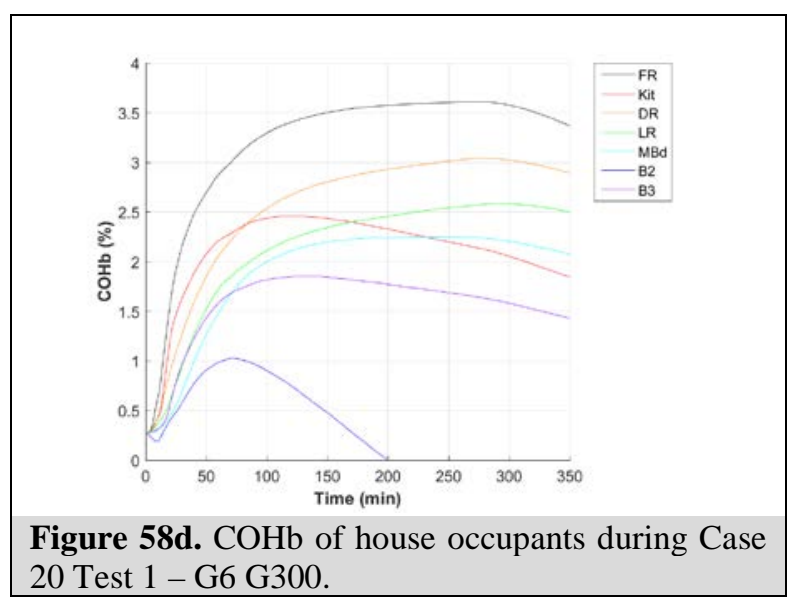




\section{Case 21 Test 1 - G2 G300}

The following figures illustrate the results for Case 21 Test 1 applying the G300 shutoff criteria. The G2 generator was fully fueled, positioned in the utility room (centered, exhaust facing out to the garage), and tested at $50 \%$ load $(800 \mathrm{~W}$ ). Both the interior person door (from garage to utility room) and exterior person door (from garage to backyard) were fully open and the garage bay door was closed. The generator was shut down manually after approximately $303 \mathrm{~min}$, which is the approximate runtime for the generator's fuel capacity, due to COHb levels $<5 \%$ in main zones of the house. Figure 59a shows the test house layout with generator location, exhaust direction, and sensor array location. Figure 59b shows the CO concentration measured in the utility room (by the dedicated room sensor), by the sensor array placed in the garage located as shown in Figure 59a (A1: height $183 \mathrm{~cm}$, A2: height $61 \mathrm{~cm}$ ), by the sensor array placed in the family room located as shown in Figure 59a (A3: height $183 \mathrm{~cm}$, A4: height $61 \mathrm{~cm}$ ), and by the shutoff sensor located on the generator near the generator's electrical panel. The figure shows the degree of CO uniformity in the area around the generator between the shutoff sensor and the peak CO concentrations measured elsewhere in and near the utility room. Figure 59c shows the CO concentration measured in each room of the house over the test. Figure 59d shows the calculated $\mathrm{COHb}$ profiles of simulated house occupants in each room of the house. The calculated $\mathrm{COHb}$ for simulated house occupants did not exceed $5 \%$ during the test.

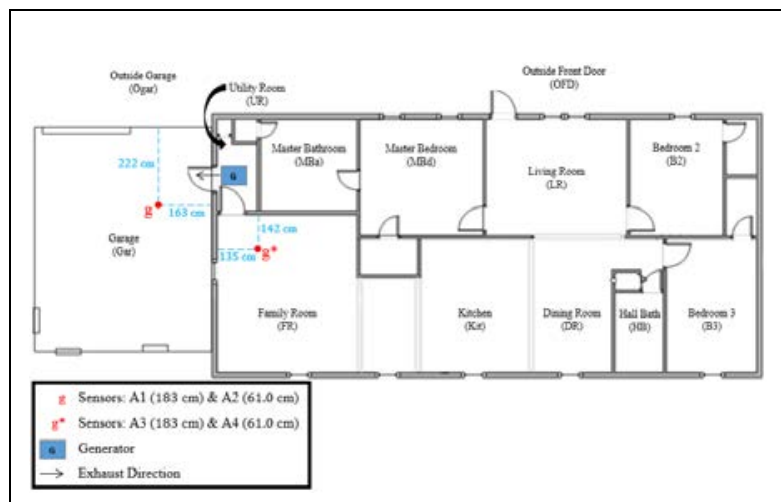

Figure 59a. Generator, exhaust direction, and sensor array layout during Case 21 Test 1 - G2 G300.

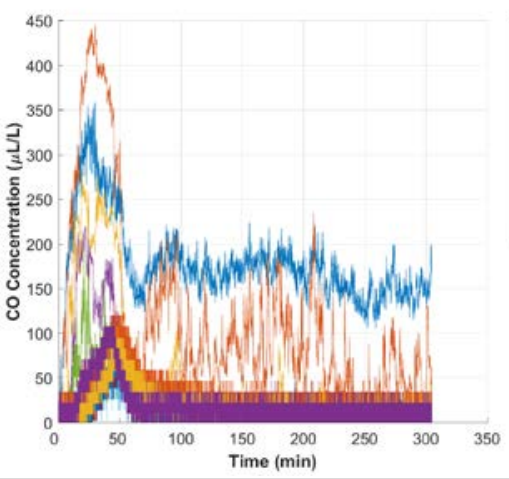

Figure 59c. CO concentration measured in each room of the house during Case 21 Test 1 - G2 G300.

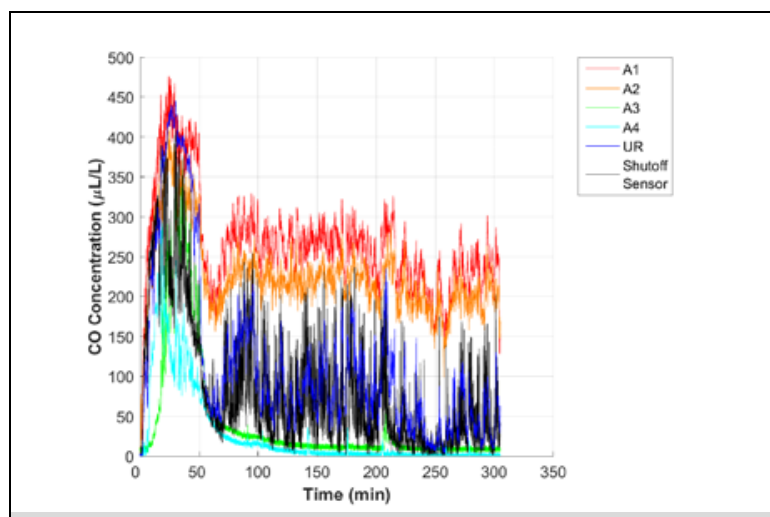

Figure 59b. CO concentration measured by the sensor array and the designated utility room sensor during Case 21 Test 1 - G2 G300.

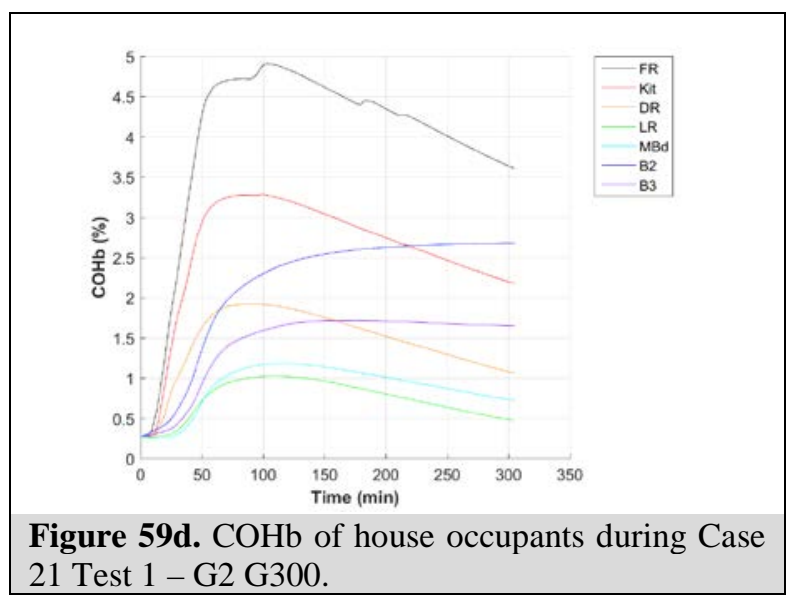




\section{Case 22 Test 1 - G6 G300}

The following figures illustrate the results for Case 22 Test 1 applying the G300 shutoff criteria. The G6 generator was fully fueled, positioned in the utility room (centered, with the exhaust facing into the family room), and tested at $100 \%$ load $(6,000 \mathrm{~W})$. The interior person door (from garage to utility room) and exterior person door (from garage to backyard) were fully open and the garage bay door was closed. The shutoff sensor activated the algorithm to shut off the generator after approximately $10 \mathrm{~min}$ due to a sensor measurement of $400 \mu \mathrm{L} / \mathrm{L}$ for a $10 \mathrm{~min}$ average. Figure 60a shows the test house layout with generator location, exhaust direction, and sensor array location. Figure 60b shows the $\mathrm{CO}$ concentration measured in the utility room (by the dedicated room sensor), by the two sensor arrays placed in the family room located as shown in Figure 60a (A1: height $183 \mathrm{~cm}$, A2: height $61 \mathrm{~cm}$, A3: height $183 \mathrm{~cm}$, A4: height $61 \mathrm{~cm}$ ), and by the shutoff sensor located on the generator near the generator's electrical panel. The timeframe of the figure has been selected to show the degree of CO uniformity in the area around the generator between the shutoff sensor and the peak CO concentrations measured elsewhere in and near the utility room near the time of shutoff. Figure 60c shows the $\mathrm{CO}$ concentration measured in each room of the house over the test. Figure 60d shows the CO concentration in the house with each room represented by a single line, which is composed of both a 'solid-line' pattern (showing measured CO concentration) and a 'dotted-line with a symbol' pattern (showing projected CO concentration). Figure 60e shows the calculated $\mathrm{COHb}$ profiles of simulated house occupants in each room of the house.

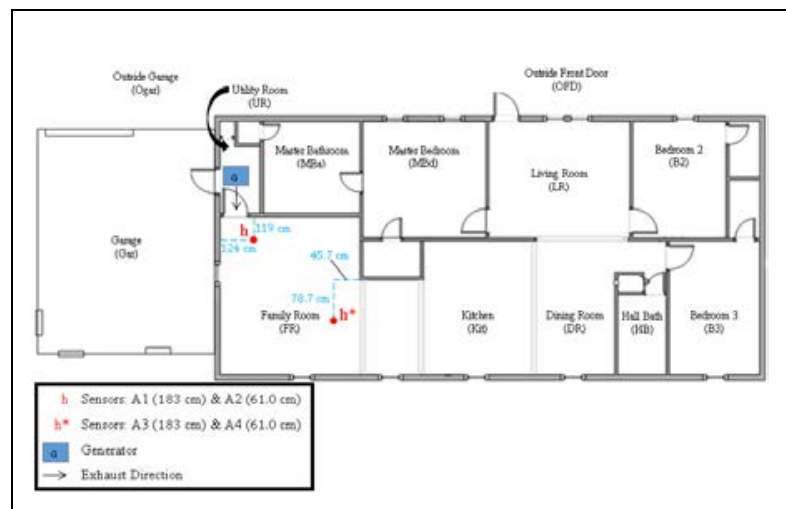

Figure 60a. Generator, exhaust direction, and sensor array layout during Case 22 Test 1 - G6 G300.

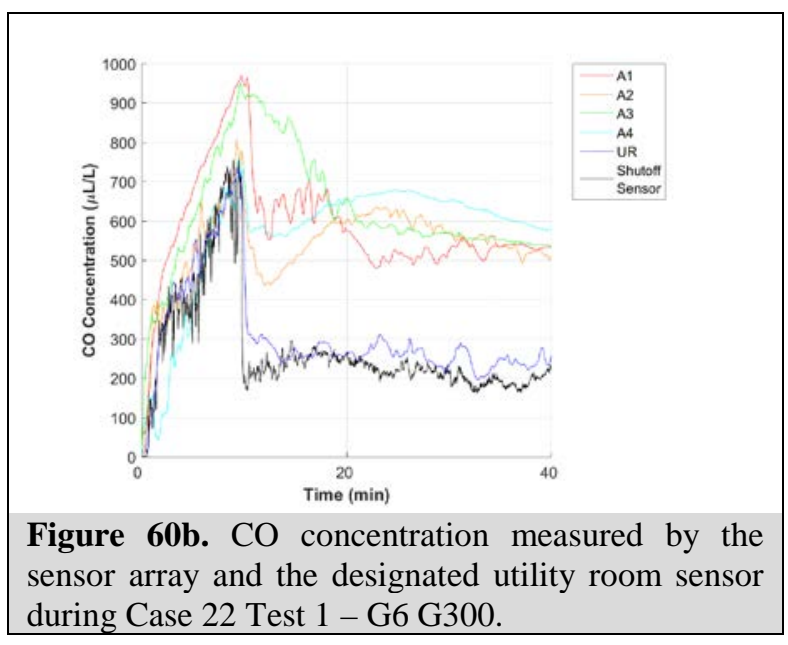

Figure 60b. $\mathrm{CO}$ concentration measured by the sensor array and the designated utility room sensor during Case 22 Test 1 - G6 G300. 


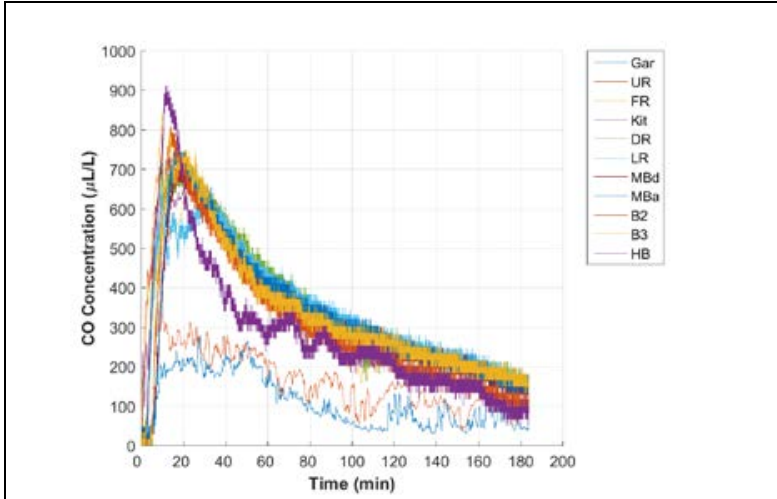

Figure 60c. CO concentration measured in each room of the house during Case 22 Test 1 - G6 G300.

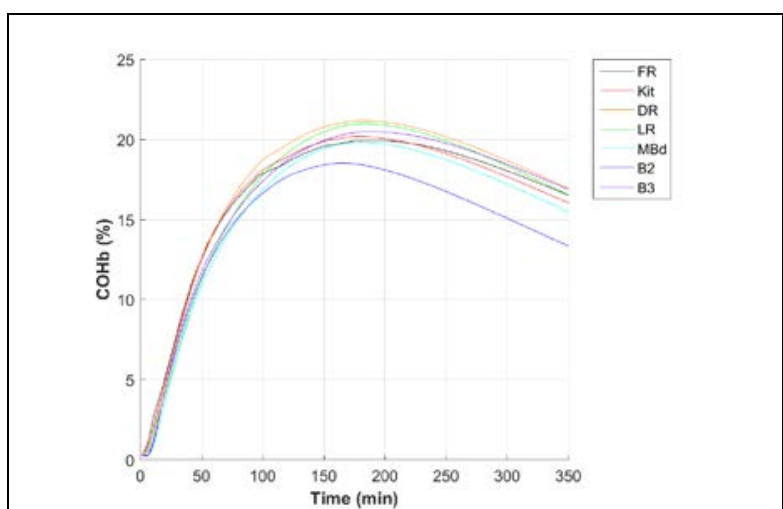

Figure 60e. $\mathrm{COHb}$ of house occupants during Case 22 Test 1 - G6 G300.

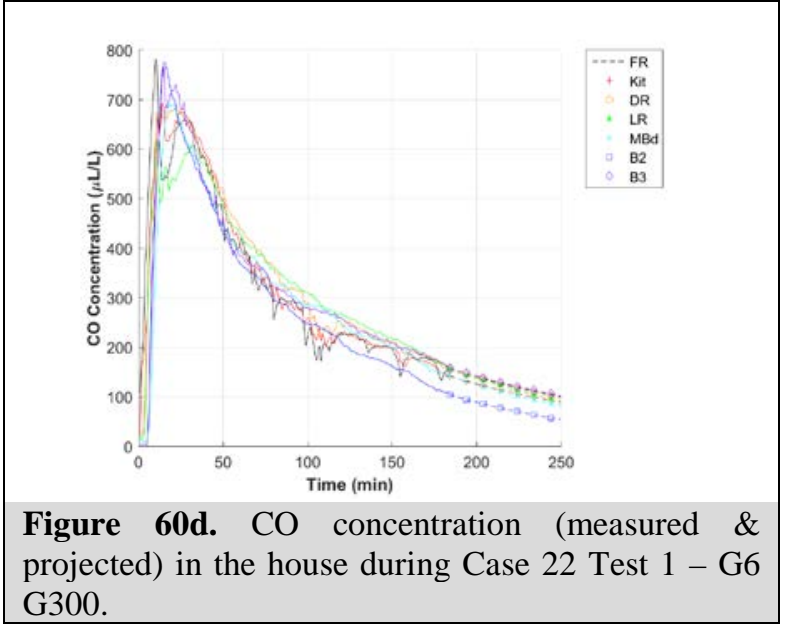

Figure 60d. CO concentration (measured \& G300. 


\section{Case 22 Test 1 - G6 G300 Restart}

The following figures illustrate the results for Restart Case 22 Test 1 applying the G300 shutoff criteria. The G6 generator was fully fueled, positioned in the utility room (centered, with the exhaust facing into the family room), and tested at $100 \%$ load $(6,000 \mathrm{~W})$. The interior person door (from garage to utility room) and exterior person door (from garage to backyard) were fully open; the garage bay door was closed. For the first generator run, the shutoff sensor activated the algorithm to shut off the generator after approximately 15 min due to a sensor measurement of 400 $\mu \mathrm{L} / \mathrm{L}$ for a $10 \mathrm{~min}$ average. The generator was restarted and the test load restored approximately 10 min after the first instance of shutoff initiated by the shutoff sensor. For the second generator run, the shutoff sensor activated the algorithm to shut off the generator after approximately 9 min due to a sensor measurement of $400 \mu \mathrm{L} / \mathrm{L}$ for a $10 \mathrm{~min}$ average. Figure 61a shows the test house layout with generator location, exhaust direction, and sensor array location. Figure 61b shows the CO concentration measured in the utility room (by the dedicated room sensor), by the two sensor arrays placed in the family room located as shown in Figure 61a (A1: height $183 \mathrm{~cm}$, A2: height $61 \mathrm{~cm}$, A3: height $183 \mathrm{~cm}$, A4: height $61 \mathrm{~cm}$ ), and by the shutoff sensor located on the generator near the generator's electrical panel. The timeframe of the figure has been selected to show the degree of CO uniformity in the area around the generator between the shutoff sensor and the peak CO concentrations measured elsewhere in and near the utility room near the time of shutoff. Figure 61c shows the $\mathrm{CO}$ concentration measured in each room of the house over the test. Figure 61d shows the $\mathrm{CO}$ concentration in the house with each room represented by a single line, which is composed of both a 'solid-line' pattern (showing measured CO concentration) and a 'dotted-line with a symbol' pattern (showing projected CO concentration). Figure 61e shows the calculated $\mathrm{COHb}$ profiles of simulated house occupants in each room of the house.

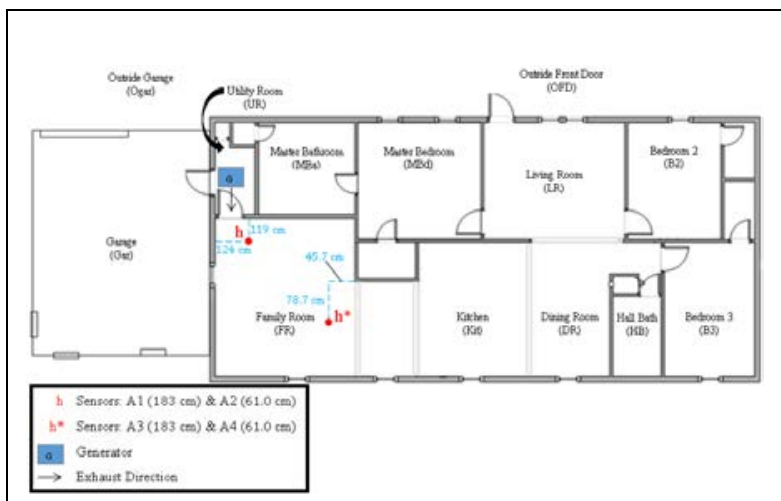

Figure 61a. Generator, exhaust direction, and sensor array layout during Restart Case 22 Test 1 - G6 G300.

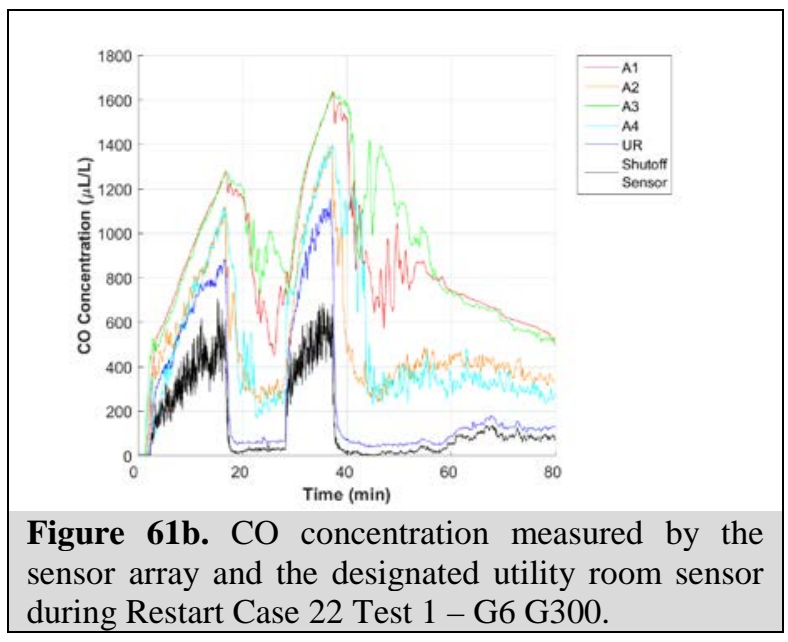



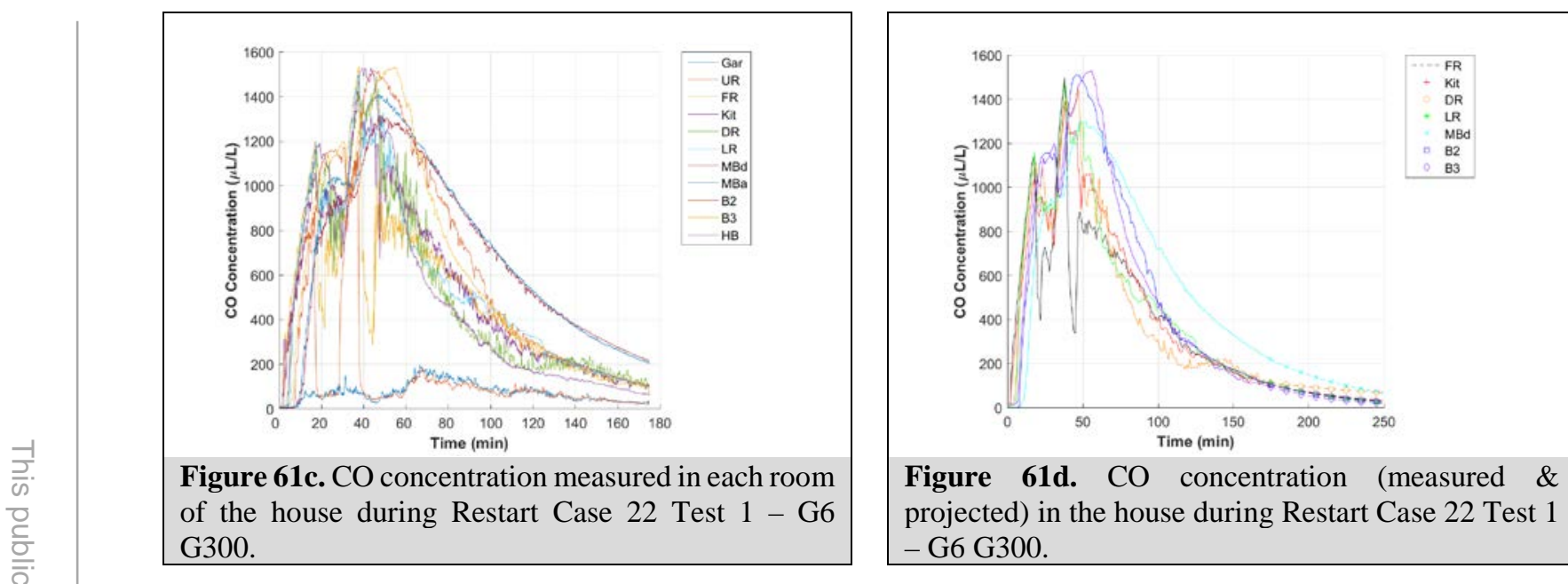

Figure 61d. CO concentration (measured \& projected) in the house during Restart Case 22 Test 1 - G6 G300.

Figure 61e. $\mathrm{COHb}$ of house occupants during Restart Case 22 Test 1 - G6 G300. 


\section{Case 22 Test 1 - G7a UL 2201}

The following figures illustrate the results for Case 22 Test 1 applying the UL 2201 shutoff criteria. The G7a generator was fully fueled, positioned in the utility room (near the center of the room with the exhaust facing the family room), and tested at $75 \%$ load (5,250 W). The interior and exterior person doors were fully open during the test. The shutoff sensor activated the algorithm to shut off the generator after approximately $146 \mathrm{~min}$ due to a sensor measurement of $150 \mu \mathrm{L} / \mathrm{L}$ for a 10 min average. Figure 62a shows the test house layout with generator location, generator exhaust direction, and sensor array location. Figure 62b shows the CO concentration measured in the utility room (by the dedicated room sensor), by two sensors located in the family room near the utility room (A1: height $183 \mathrm{~cm}, \mathrm{~A} 2$ : height $61.0 \mathrm{~cm}$ ), by the other two sensors located on the kitchen-family room boundary (A3: height $183 \mathrm{~cm}$, A4: height $61.0 \mathrm{~cm}$ ), and by the shutoff sensor located on the generator near the generator's electrical panel. The timeframe of the figure has been selected to show the degree of CO uniformity in the area around the generator between the shutoff sensor and the peak CO concentrations measured elsewhere in the utility room and family room near the time of shutoff. Figure 62c shows the $\mathrm{CO}$ concentration measured in each room of the house over the test. Figure 62d shows the $\mathrm{CO}$ concentration in the house with each room represented by a single line, which is composed of both a 'solid-line' pattern (showing measured CO concentration) and a 'dotted-line with a symbol' pattern (showing projected CO concentration). Figure 62e shows the calculated $\mathrm{COHb}$ profiles of simulated house occupants in each room of the house.

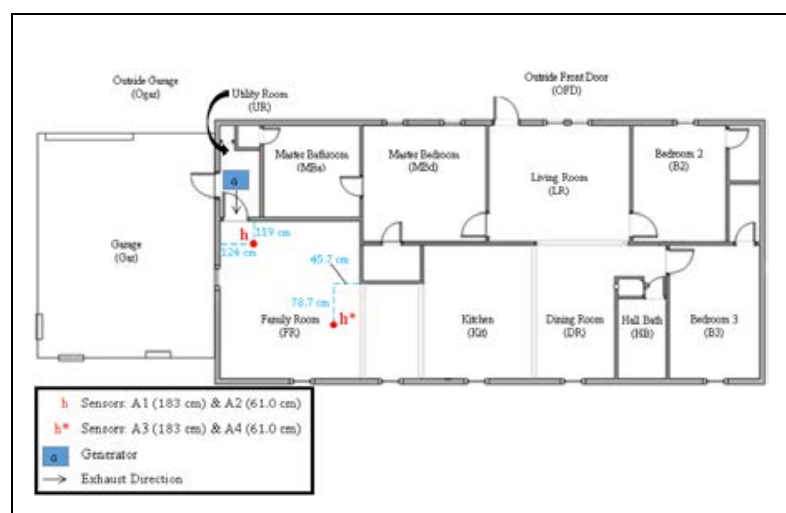

Figure 62a. Generator, exhaust direction, and sensor array layout during Case 22 Test 1 - G7a UL 2201.

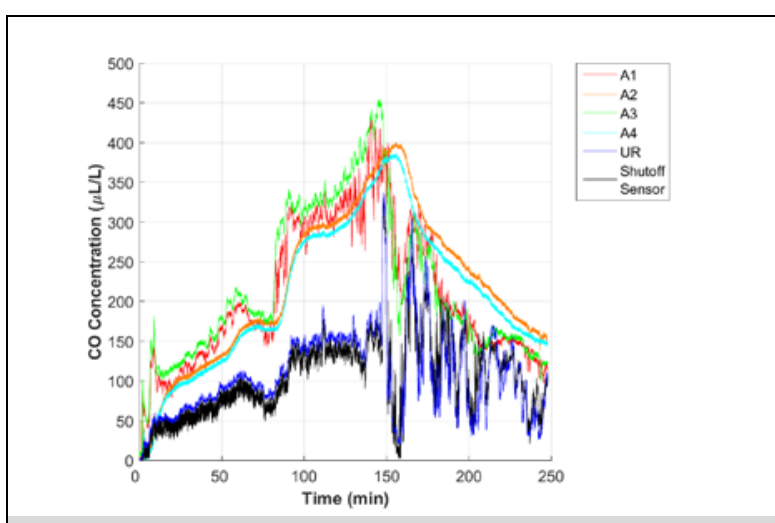

Figure 62b. CO concentration measured by the sensor array and the designated utility room sensor during Case 22 Test 1 - G7a UL 2201. 


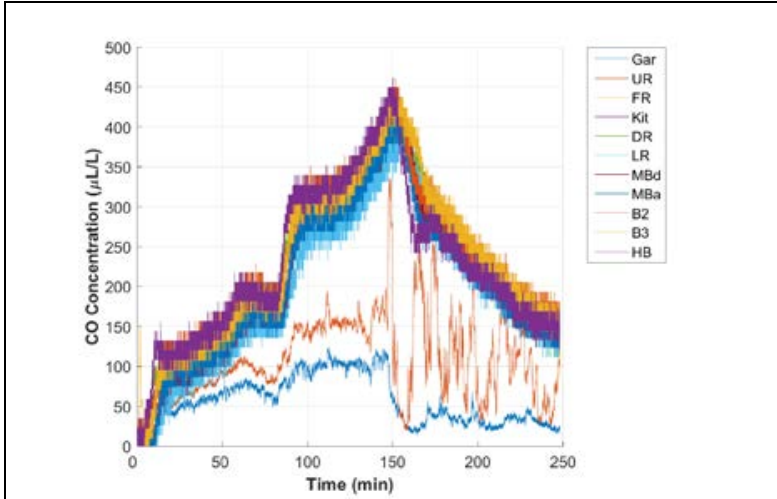

Figure 62c. CO concentration measured in each room of the house during Case 22 Test 1 - G7a UL 2201.

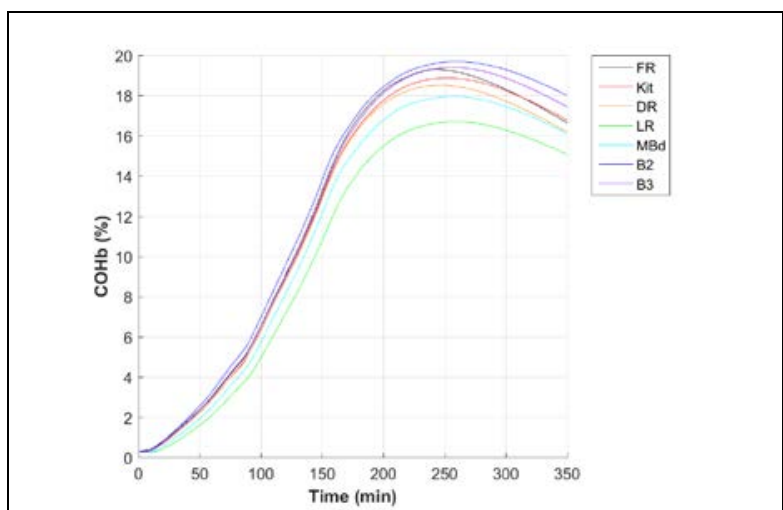

Figure 62e. $\mathrm{COHb}$ of house occupants during Case 22 Test 1 - G7a UL 2201.

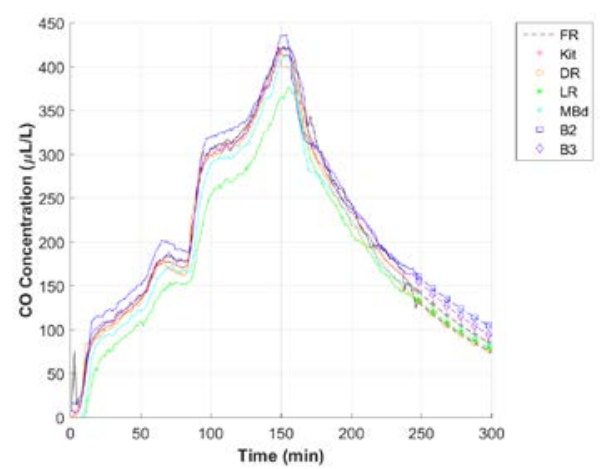

Figure 62d. CO concentration (measured \& projected) in the house during Case 22 Test 1 - G7a UL 2201. 


\section{Case 22 Test 2 - G7b UL 2201}

The following figures illustrate the results for Case 22 Test 2 applying the UL 2201 shutoff criteria. The G7b generator was fully fueled, positioned in the utility room (near the center of the room with the exhaust facing the family room), and tested at $100 \%$ load (7,000 W). The interior and exterior person doors were fully open during the test. The generator was shut down manually after approximately 240 min due to quasi-steady-state CO levels in the main zones of the house and a $\mathrm{CO}$ concentration $<70 \mu \mathrm{L} / \mathrm{L}$ at the shutoff sensor. Figure 63a shows the test house layout with generator location, generator exhaust direction, and sensor array location. Figure 63b shows the $\mathrm{CO}$ concentration measured in the utility room (by the dedicated room sensor), by two sensors located in the family room near the utility room (A1: height $183 \mathrm{~cm}$, A2: height $61.0 \mathrm{~cm})$, by the other two sensors located on the kitchen-family room boundary (A3: height $183 \mathrm{~cm}$, A4: height $61.0 \mathrm{~cm}$ ), and by the shutoff sensor located on the generator near the generator's electrical panel. The timeframe of the figure has been selected to show the degree of CO uniformity in the area around the generator between the shutoff sensor and the peak $\mathrm{CO}$ concentrations measured elsewhere in the utility room and family room near the time of shutoff. Figure 63c shows the CO concentration measured in each room of the house over the test. Figure 63d shows the calculated $\mathrm{COHb}$ profiles of simulated house occupants in each room of the house which did not peak during the test.

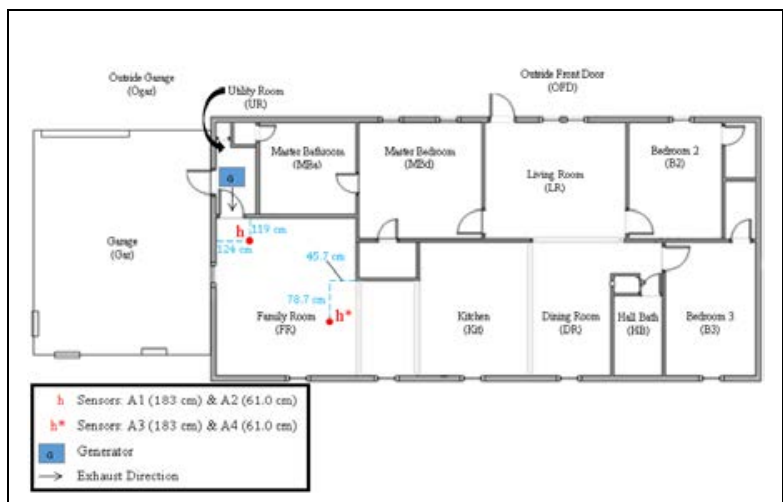

Figure 63a. Generator, exhaust direction, and sensor array layout during Case 22 Test 2 - G7b UL 2201.

Figure 63c. CO concentration measured in each room of the house during Case 22 Test 2 - G7b UL 2201.
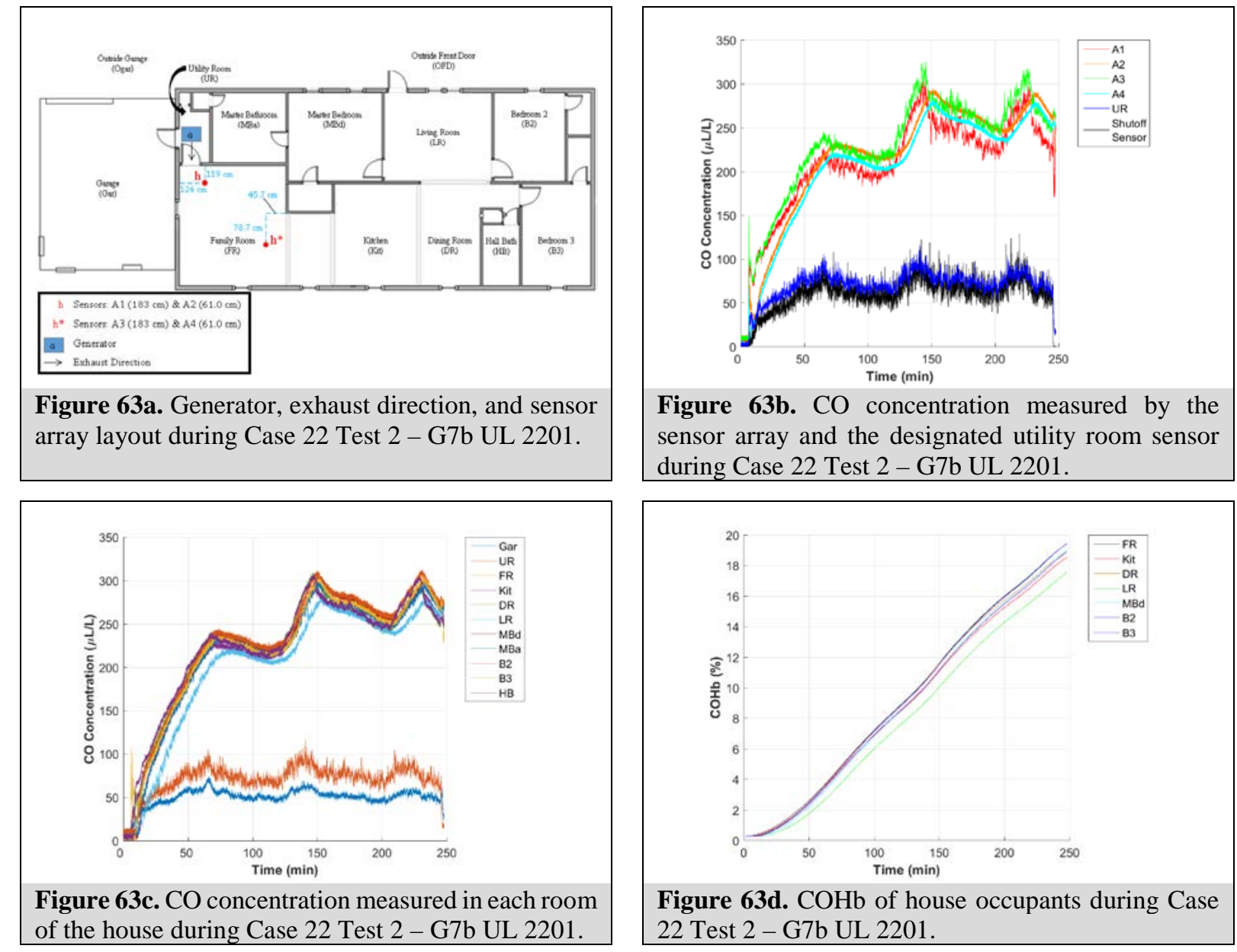


\section{Case 23 Test 1 - G2 G300}

The following figures illustrate the results for Case 23 Test 1 applying the G300 shutoff criteria. The G2 generator was fully fueled, positioned in the utility room (centered, with the exhaust facing into the family room), and tested at $50 \%$ load $(800 \mathrm{~W})$. The interior person door (from garage to utility room) and exterior person door (from garage to backyard) were fully open; the garage bay door was closed. The shutoff sensor activated the algorithm to shut off the generator after approximately 94 min due to a sensor measurement of $400 \mu \mathrm{L} / \mathrm{L}$ for a $10 \mathrm{~min}$ average. Figure 64a shows the test house layout with generator location, exhaust direction, and sensor array location. Figure 64b shows the $\mathrm{CO}$ concentration measured in the utility room (by the dedicated room sensor), by the two sensor arrays placed in the family room located as shown in Figure 64a (A1: height $183 \mathrm{~cm}$, A2: height $61 \mathrm{~cm}$, A3: height $183 \mathrm{~cm}$, A4: height $61 \mathrm{~cm}$ ), and by the shutoff sensor located on the generator near the generator's electrical panel. The timeframe of the figure has been selected to show the degree of CO uniformity in the area around the generator between the shutoff sensor and the peak CO concentrations measured elsewhere in and near the utility room near the time of shutoff. Figure 64c shows the CO concentration measured in each room of the house over the test. Figure 64d shows the CO concentration in the house with each room represented by a single line, which is composed of both a 'solid-line' pattern (showing measured CO concentration) and a 'dotted-line with a symbol' pattern (showing projected CO concentration). Figure 64e shows the calculated $\mathrm{COHb}$ profiles of simulated house occupants in each room of the house.

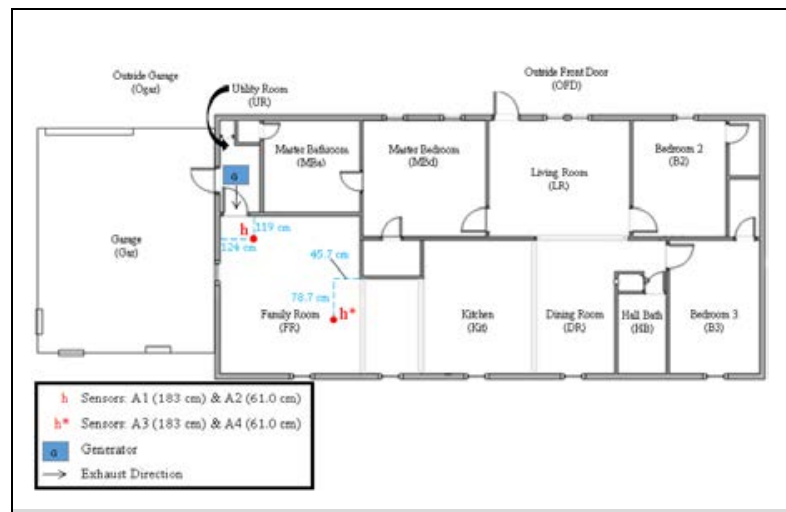

Figure 64a. Generator, exhaust direction, and sensor array layout during Case 23 Test 1 - G2 G300.

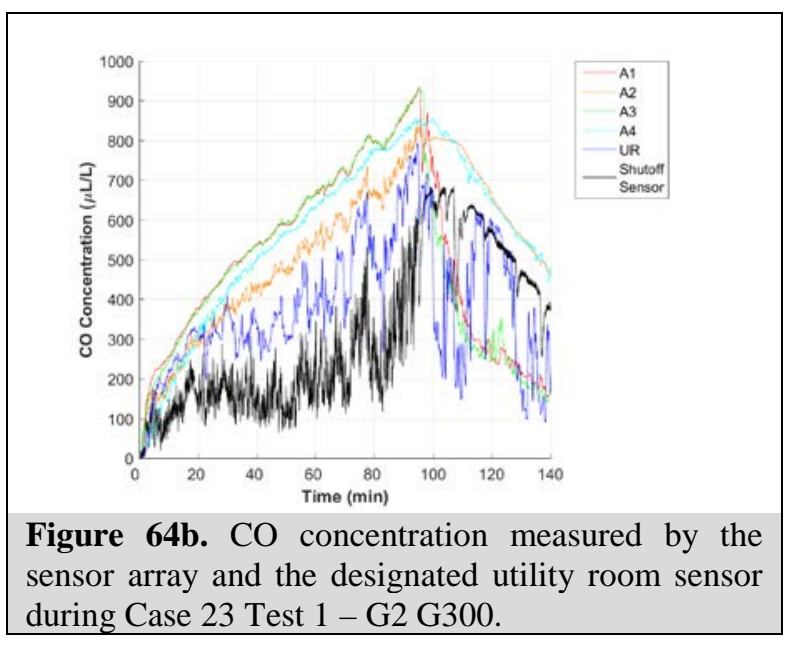

Figure 64b. $\mathrm{CO}$ concentration measured by the sensor array and the designated utility room sensor during Case 23 Test 1 - G2 G300. 


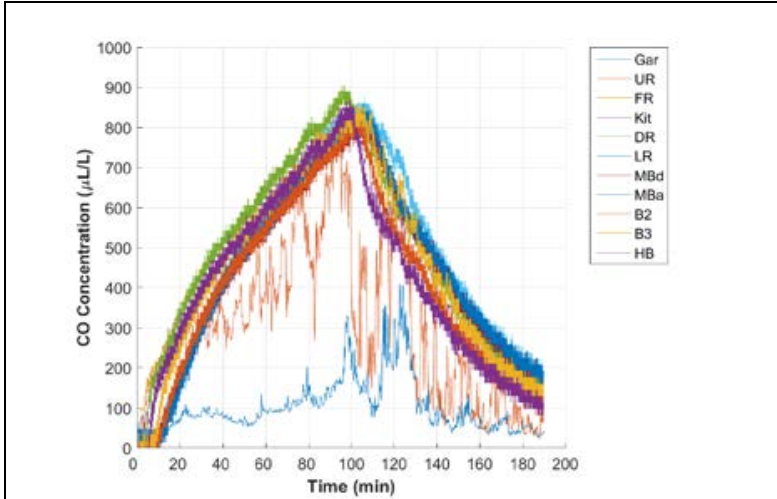

Figure 64c. CO concentration measured in each room of the house during Case 23 Test 1 - G2 G300.

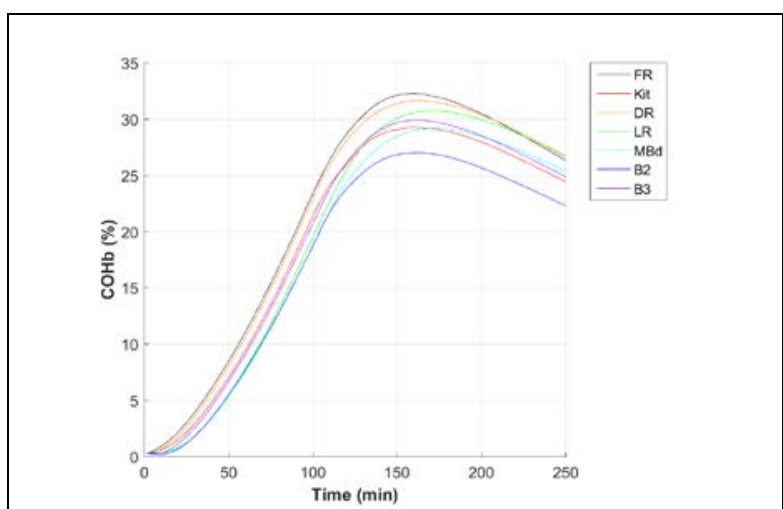

Figure 64e. $\mathrm{COHb}$ of house occupants during Case 23 Test $1-$ G2 G300.

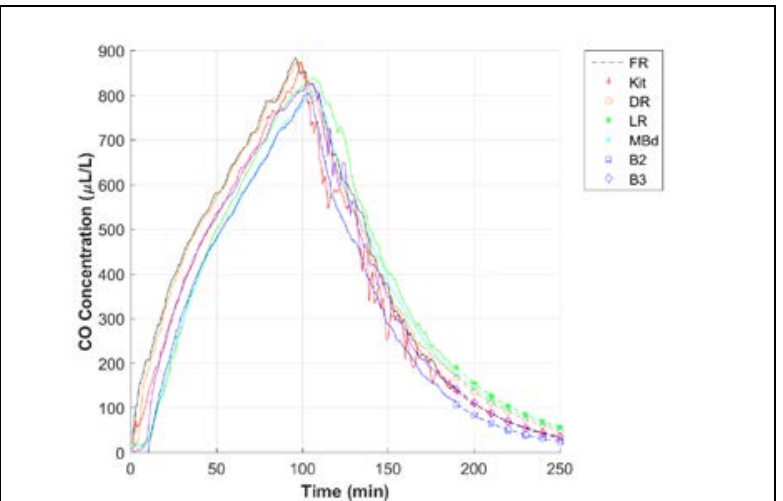

Figure 64d. CO concentration (measured \& projected) in the house during Case 23 Test 1 - G2 G300. 


\section{Case 23 Test 1 - G2 G300 Restart}

The following figures illustrate the results for Restart Case 23 Test 1 applying the G300 shutoff criteria. The G2 generator was fully fueled, positioned in the utility room (centered, with the exhaust facing into the family room), and tested at $50 \%$ load (800 W). The interior person door (from garage to utility room) and exterior person door (from garage to backyard) were fully open; the garage bay door was closed. The generator was shut down manually after approximately 238 min due to low CO level at the shutoff sensor and the time of day. Figure 65a shows the test house layout with generator location, exhaust direction, and sensor array location. Figure 65b shows the CO concentration measured in the utility room (by the dedicated room sensor), by the two sensor arrays placed in the family room located as shown in Figure 65a (A1: height $183 \mathrm{~cm}$, A2: height $61.0 \mathrm{~cm}$, A3: height $183 \mathrm{~cm}$, A4: height $61.0 \mathrm{~cm}$ ), and by the shutoff sensor located on the generator near the generator's electrical panel. The timeframe of the figure has been selected to show the degree of CO uniformity in the area around the generator between the shutoff sensor and the peak CO concentrations measured elsewhere in and near the utility room near the time of shutoff. Figure 65c shows the CO concentration measured in each room of the house over the test. Figure 65d shows the calculated $\mathrm{COHb}$ profiles of simulated house occupants in each room of the house which did not peak during the test.

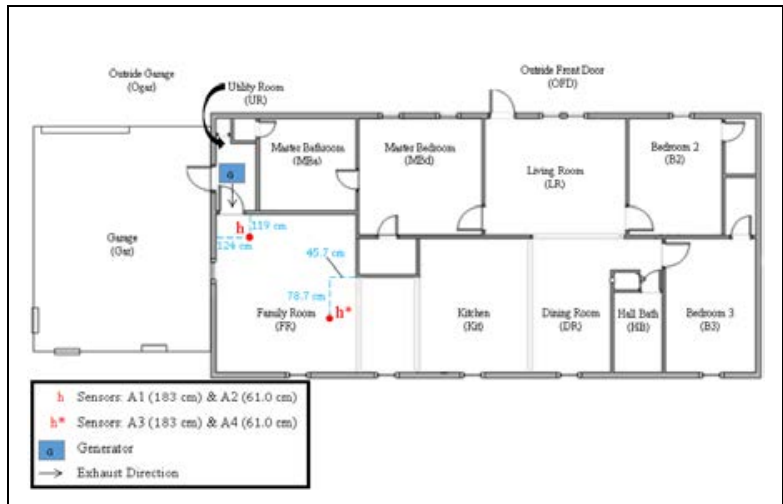

Figure 65a. Generator, exhaust direction, and sensor array layout during Restart Case 23 Test 1 - G2 G300.

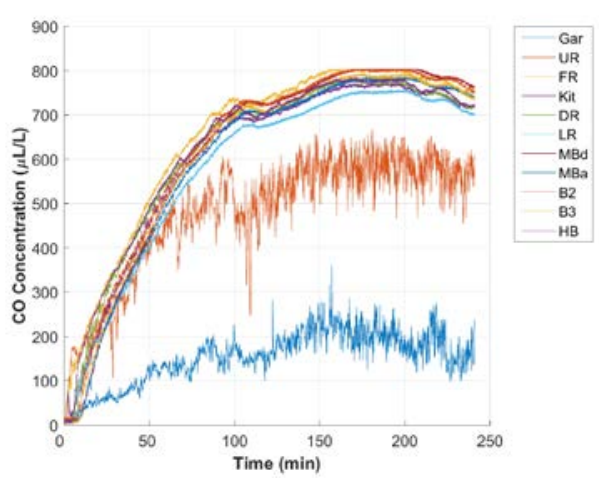

Figure 65c. CO concentration measured in each room of the house during Restart Case 23 Test 1 - G2 G300.

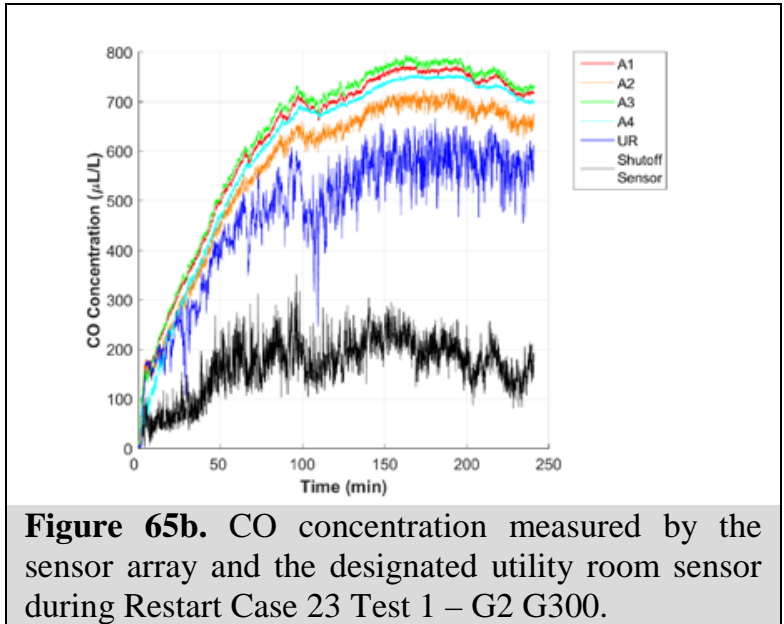
during Restart Case 23 Test 1 - G2 G300.

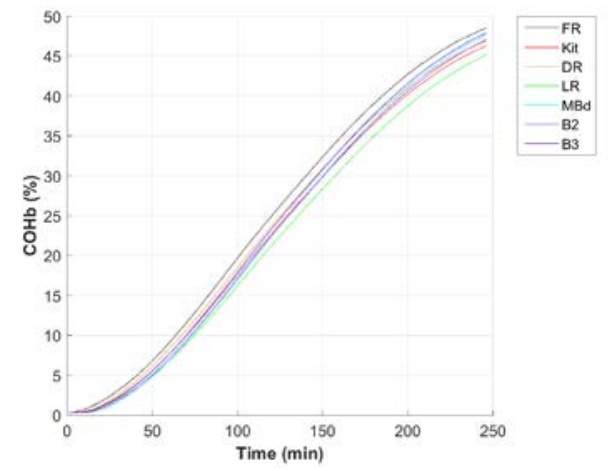

Figure 65d. COHb of house occupants during Restart Case 23 Test 1 - G2 G300. 


\section{Generator Location: Garage}

Table 7 summarizes the results for all tests performed with a generator located in the test house garage. The details are discussed below and shown in Figures 66 through 91.

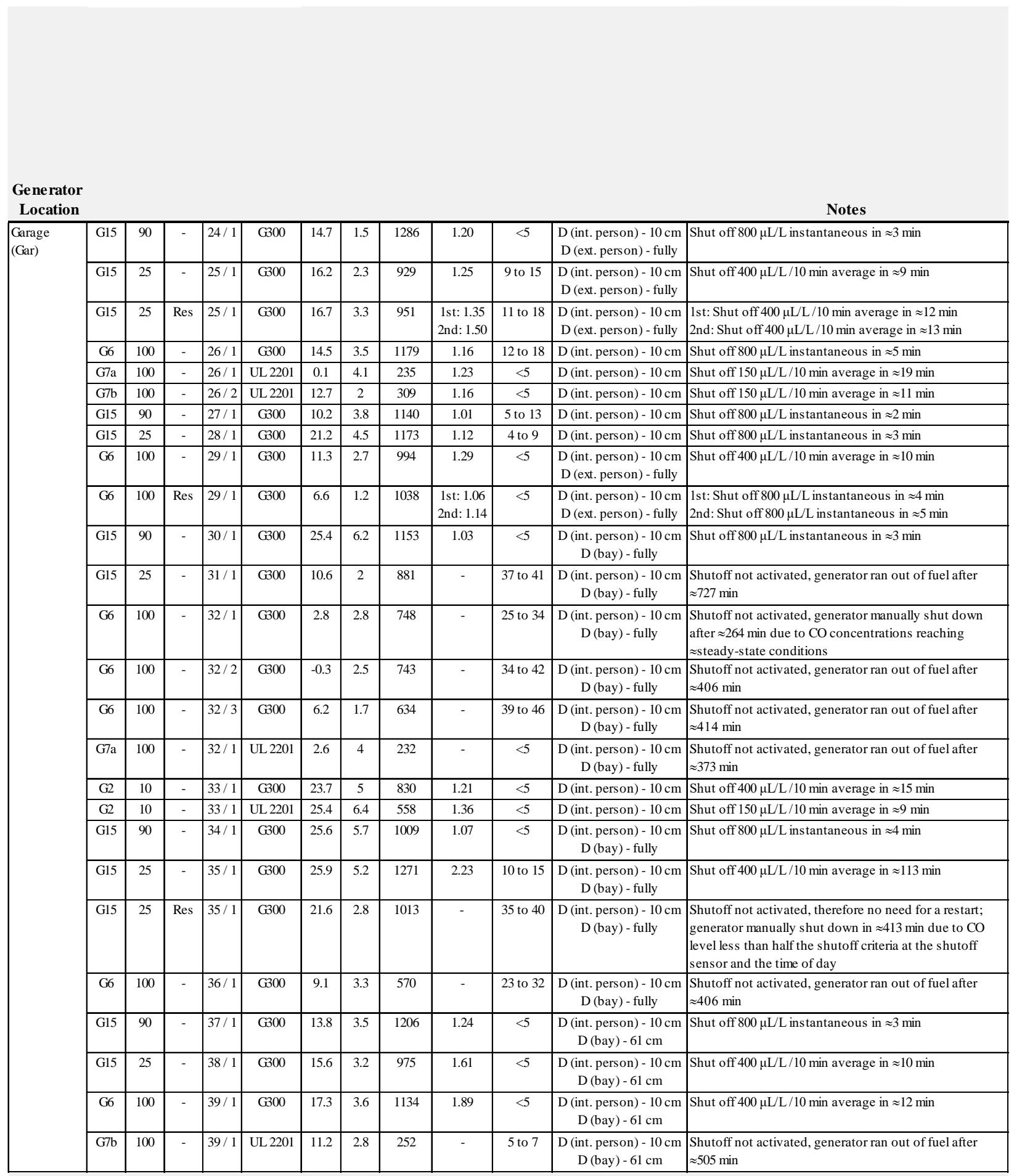

Table 7. Summary of test results for Garage (Gar) tests.

+Peak COHb Values are for house zones only.

+Ventilation Note: Unless specified otherwise, during the test - all exterior doors are closed, all interior doors are fully open, and all windows are closed. 


\section{Case 24 Test 1 - G15 G300}

The following figures illustrate the results for Case 24 Test 1 applying the G300 shutoff criteria. The G15 generator was fully fueled, positioned in the garage (30 $\mathrm{cm}$ from the interior person door from the garage to the utility room with the exhaust facing toward the utility room), and tested at $90 \%$ load $(13,500 \mathrm{~W})$. The interior person door (from garage to utility room) was open $10 \mathrm{~cm}$ and exterior person door (from garage to backyard) was fully open; the garage bay door was closed. The shutoff sensor activated the algorithm to shut off the generator after approximately 3 min due to an instantaneous sensor measurement of $800 \mu \mathrm{L} / \mathrm{L}$. Figure 66a shows the test house layout with generator location, exhaust direction, and sensor array location. Figure 66b shows the CO concentration measured in the garage (by the dedicated room sensor), by the sensor array placed in the garage located as shown in Figure 66a (A1: height $91 \mathrm{~cm}, \mathrm{A2}$ : height $91 \mathrm{~cm}$, A3: height 91 $\mathrm{cm}$, A4: height $91 \mathrm{~cm}$ ), and by the shutoff sensor located on the generator near the generator's electrical panel. The timeframe of the figure has been selected to show the degree of CO uniformity in the area around the generator between the shutoff sensor and the peak CO concentrations measured elsewhere in the garage near the time of shutoff. Figure 66c shows the CO concentration measured in each room of the house over the test. Figure 66d shows the calculated COHb profiles of simulated house occupants in each room of the house. The calculated $\mathrm{COHb}$ for simulated house occupants did not exceed $5 \%$ during the test.

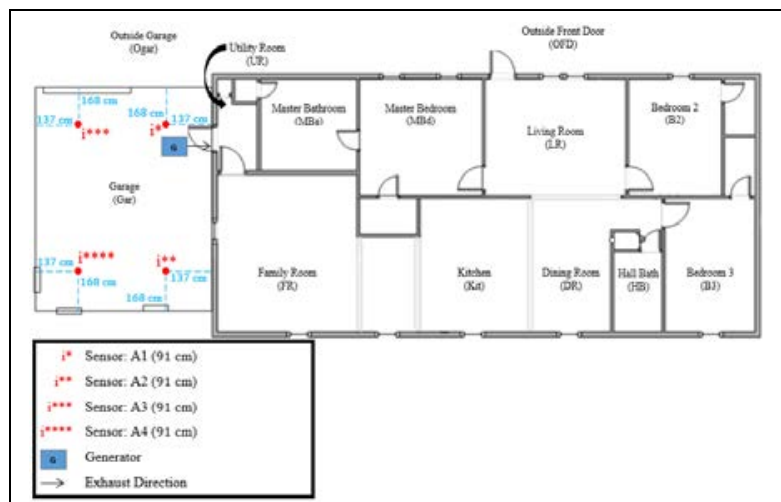

Figure 66a. Generator, exhaust direction, and sensor array layout during Case 24 Test 1 - G15 G300.

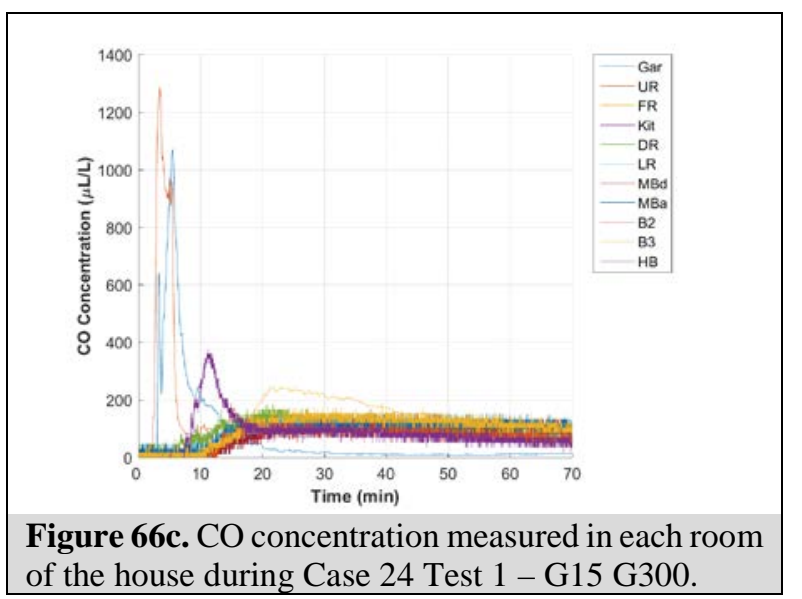

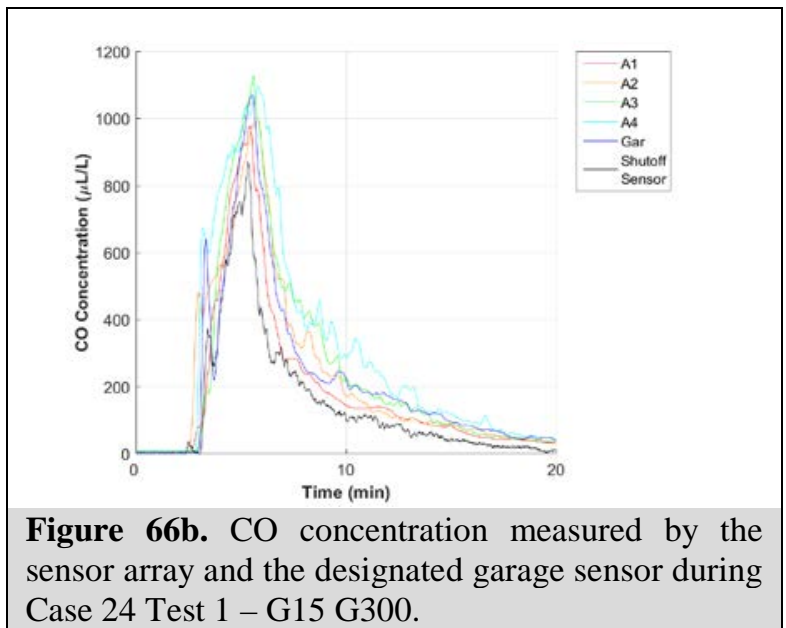

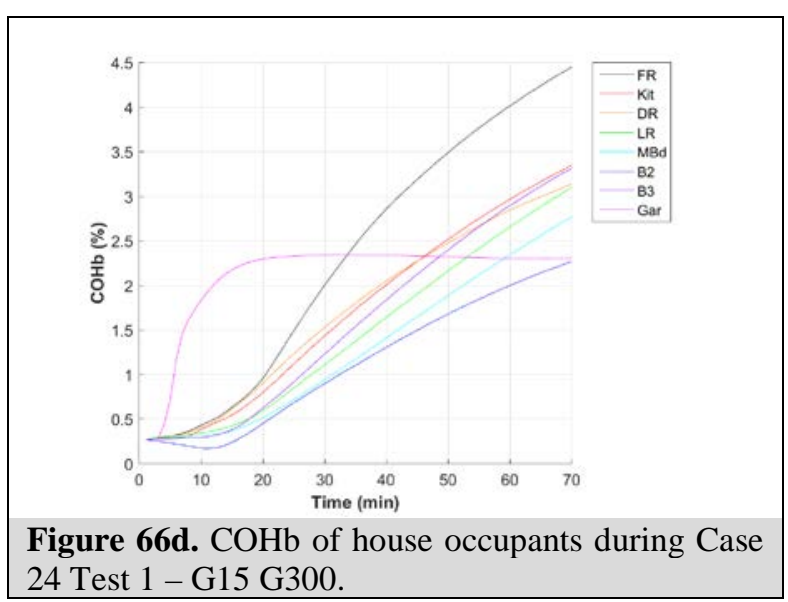




\section{Case 25 Test 1 - G15 G300}

The following figures illustrate the results for Case 25 Test 1 applying the G300 shutoff criteria. The G15 generator was fully fueled, positioned in the garage (30 $\mathrm{cm}$ from interior person door from the garage to the utility room with the exhaust facing toward the utility room), and tested at $25 \%$ load $(3,750 \mathrm{~W})$. The interior person door (from garage to utility room) was open $10 \mathrm{~cm}$ and exterior person door (from garage to backyard) was fully open; the garage bay door was closed. The shutoff sensor activated the algorithm to shut off the generator after approximately 9 min due to a sensor measurement of $400 \mu \mathrm{L} / \mathrm{L}$ for a 10 min average. Figure 67a shows the test house layout with generator location, exhaust direction, and sensor array location. Figure 67b shows the CO concentration measured in the garage (by the dedicated room sensor), by the sensor array placed in the garage located as shown in Figure 67a (A1: height $91 \mathrm{~cm}, \mathrm{A2}$ : height $91 \mathrm{~cm}$, A3: height 91 $\mathrm{cm}$, A4: height $91 \mathrm{~cm}$ ), and by the shutoff sensor located on the generator near the generator's electrical panel. The timeframe of the figure has been selected to show the degree of CO uniformity in the area around the generator between the shutoff sensor and the peak CO concentrations measured elsewhere in the garage near the time of shutoff. Figure 67c shows the CO concentration measured in each room of the house over the test. Figure 67d shows the CO concentration in the house with each room represented by a single line, which is composed of both a 'solid-line' pattern (showing measured CO concentration) and a 'dotted-line with a symbol' pattern (showing projected CO concentration). Figure 67e shows the calculated COHb profiles of simulated house occupants in each room of the house.

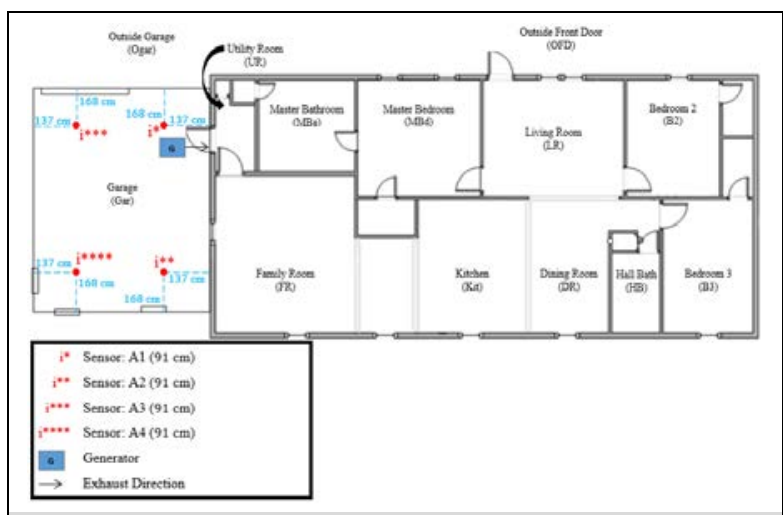

Figure 67a. Generator, exhaust direction, and sensor array layout during Case 25 Test 1 - G15 G300.

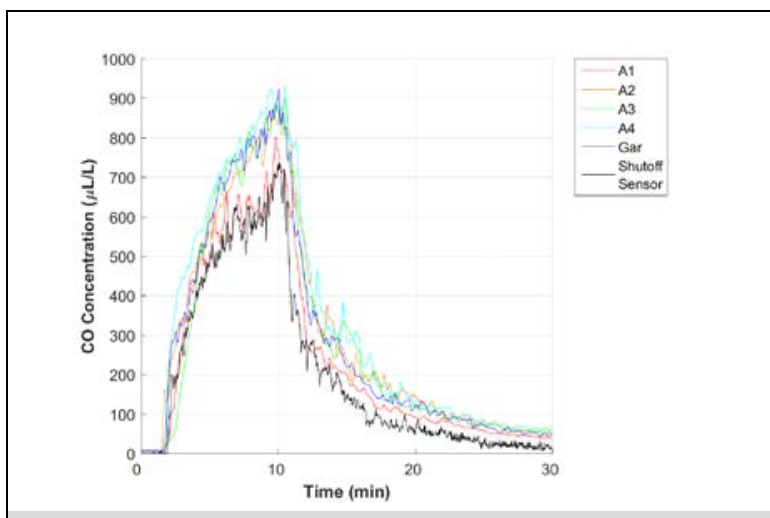

Figure 67b. CO concentration measured by the sensor array and the designated garage sensor during Case 25 Test 1 - G15 G300. 


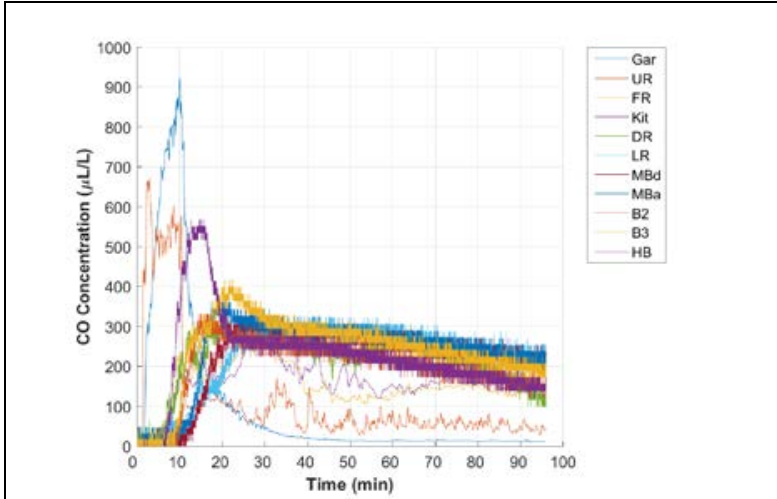

Figure 67c. CO concentration measured in each room of the house during Case 25 Test 1 - G15 G300.

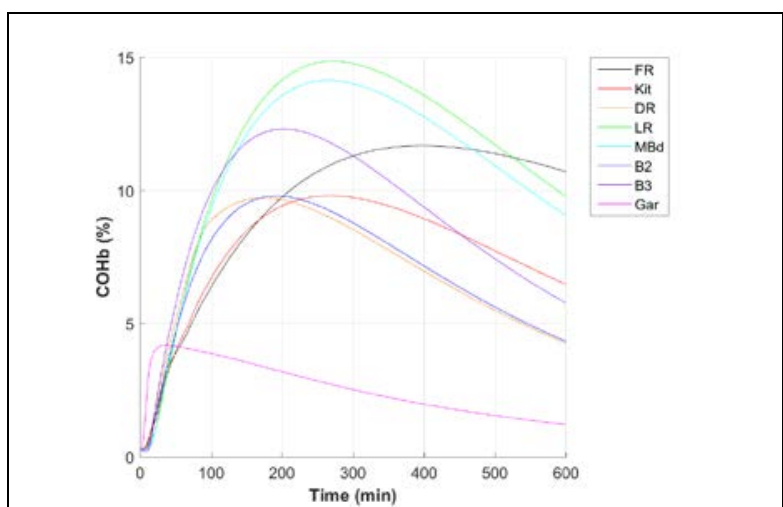

Figure 67e. $\mathrm{COHb}$ of house occupants during Case 25 Test 1 - G15 G300.

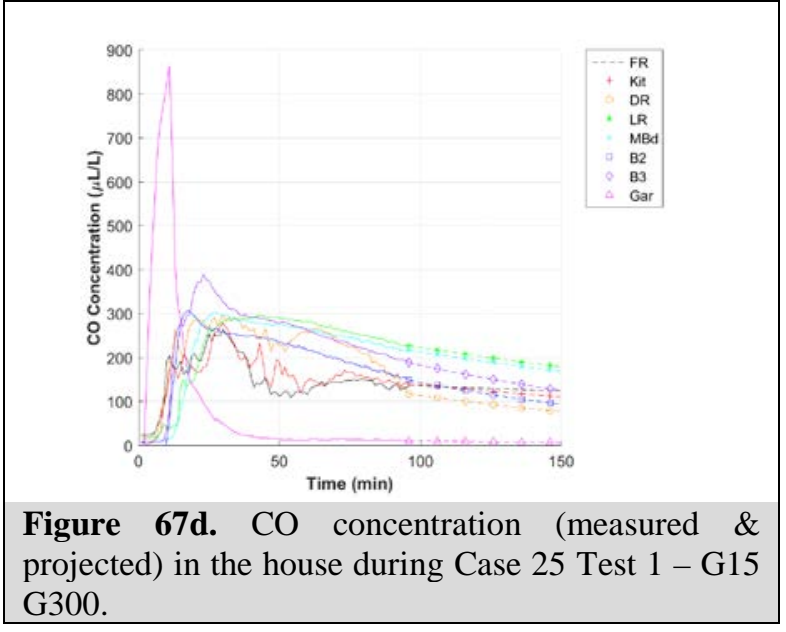

Figure 67d. CO concentration (measured \& projected) in the house during Case 25 Test $1-$ G15 


\section{Case 25 Test 1 - G15 G300 Restart}

The following figures illustrate the results for Restart Case 25 Test 1 applying the G300 shutoff criteria. The G15 generator was fully fueled, positioned in the garage (30 cm from interior person door from the garage to the utility room with the exhaust facing toward the utility room), and tested at $25 \%$ load $(3,750 \mathrm{~W})$. The interior person door (from garage to utility room) was open $10 \mathrm{~cm}$ and exterior person door (from garage to backyard) was fully open; the garage bay door was closed. For the first generator run, the shutoff sensor activated the algorithm to shut off the generator after approximately $12 \mathrm{~min}$ due to a sensor measurement of $400 \mu \mathrm{L} / \mathrm{L}$ for a $10 \mathrm{~min}$ average. The generator was restarted and the test load restored approximately $10 \mathrm{~min}$ after the first instance of shutoff initiated by the shutoff sensor. For the second generator run, the shutoff sensor activated the algorithm to shut off the generator after approximately 13 min due to a sensor measurement of $400 \mu \mathrm{L} / \mathrm{L}$ for a $10 \mathrm{~min}$ average. Figure 68a shows the test house layout with generator location, exhaust direction, and sensor array location. Figure 68b shows the CO concentration measured in the garage (by the dedicated room sensor), by the sensor array placed in the garage located as shown in Figure 68a (A1: height $91 \mathrm{~cm}, \mathrm{A2}$ : height $91 \mathrm{~cm}, \mathrm{A3}$ : height $91 \mathrm{~cm}, \mathrm{~A} 4$ : height $91 \mathrm{~cm}$ ), and by the shutoff sensor located on the generator near the generator's electrical panel. The timeframe of the figure has been selected to show the degree of $\mathrm{CO}$ uniformity in the area around the generator between the shutoff sensor and the peak CO concentrations measured elsewhere in the garage near the time of shutoff. Figure 68c shows the $\mathrm{CO}$ concentration measured in each room of the house over the test. Figure 68d shows the CO concentration in the house with each room represented by a single line, which is composed of both a 'solid-line' pattern (showing measured CO concentration) and a 'dotted-line with a symbol' pattern (showing projected CO concentration). Figure 68e shows the calculated $\mathrm{COHb}$ profiles of simulated house occupants in each room of the house.

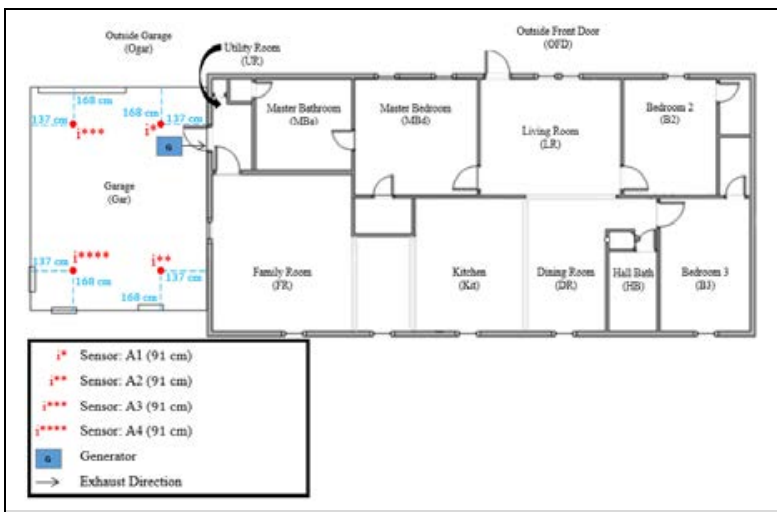

Figure 68a. Generator, exhaust direction, and sensor array layout during Restart Case 25 Test 1 - G15 G300.

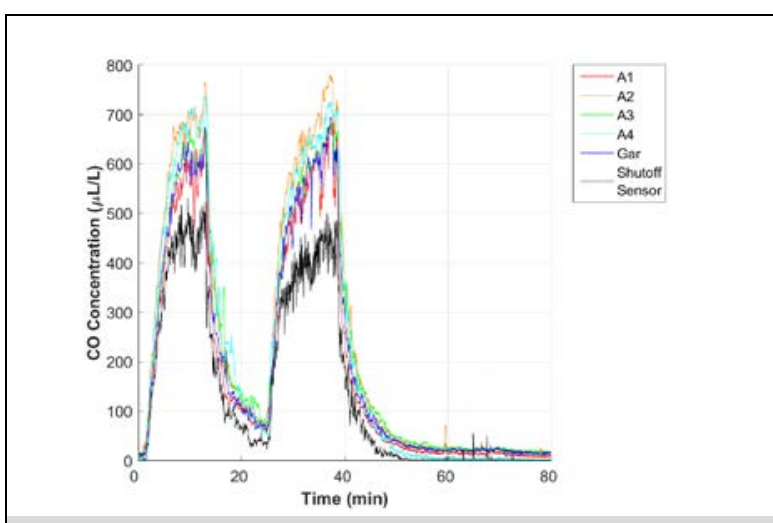

Figure 68b. CO concentration measured by the sensor array and the designated garage sensor during Restart Case 25 Test 1 - G15 G300. 

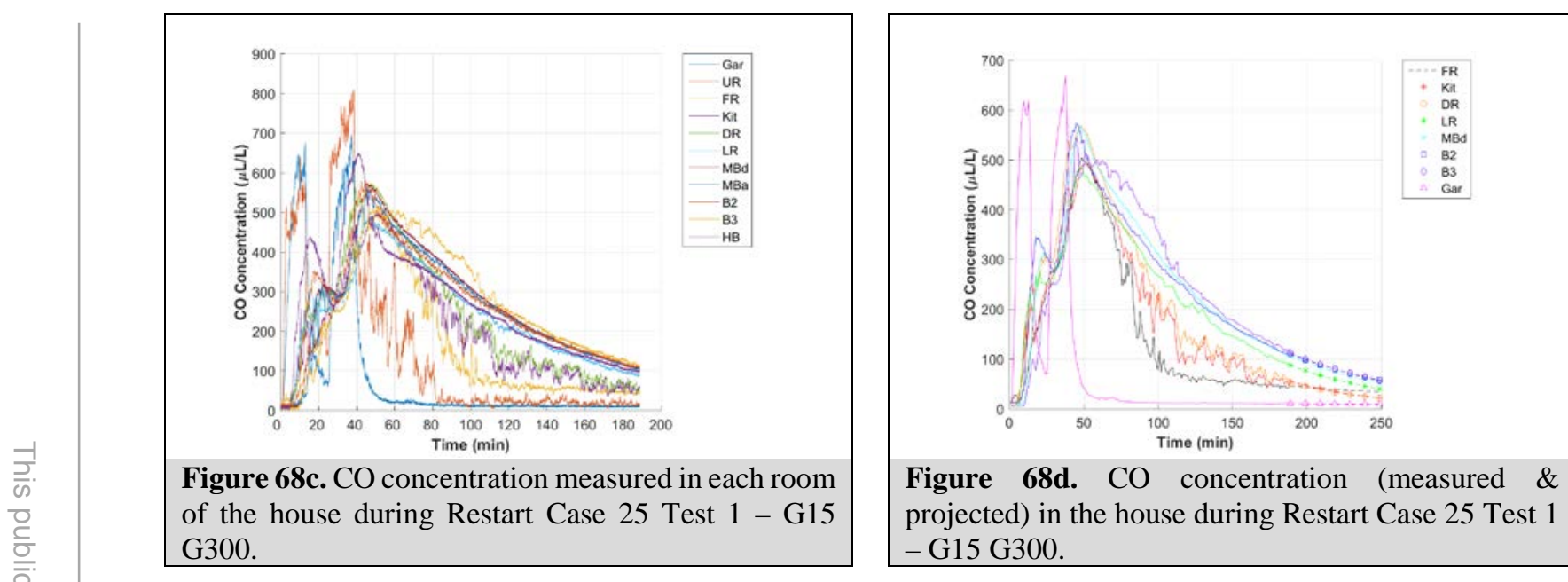

Figure 68d. CO concentration (measured \& projected) in the house during Restart Case 25 Test 1 - G15 G300.

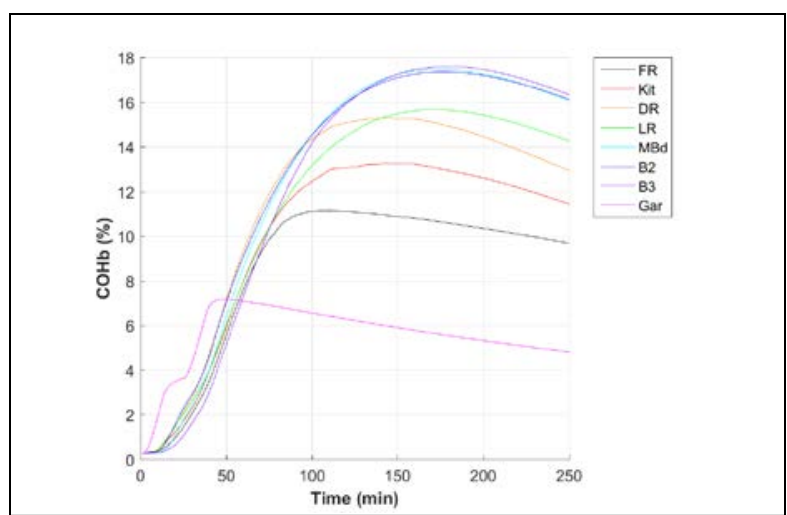

Figure 68e. $\mathrm{COHb}$ of house occupants during Restart Case 25 Test 1 - G15 G300. 


\section{Case 26 Test 1 - G6 G300}

The following figures illustrate the results for Case 26 Test 1 applying the G300 shutoff criteria. The G6 generator was fully fueled, positioned in the garage (30 cm from interior person door from the garage to the utility room with the exhaust facing toward the utility room), and tested at $100 \%$ load $(6,000 \mathrm{~W})$. The interior person door (from garage to utility room) was open $10 \mathrm{~cm}$; the exterior person door (from garage to backyard) and the garage bay door were closed. The shutoff sensor activated the algorithm to shut off the generator after approximately $5 \mathrm{~min}$ due to an instantaneous sensor measurement of $800 \mu \mathrm{L} / \mathrm{L}$. Figure 69a shows the test house layout with generator location, exhaust direction, and sensor array location. Figure 69b shows the CO concentration measured in the garage (by the dedicated room sensor), by the sensor array placed in the garage located as shown in Figure 69a (A1: height $91 \mathrm{~cm}$, A2: height $91 \mathrm{~cm}$, A3: height $91 \mathrm{~cm}$, A4: height $91 \mathrm{~cm}$ ), and by the shutoff sensor located on the generator near the generator's electrical panel. The timeframe of the figure has been selected to show the degree of CO uniformity in the area around the generator between the shutoff sensor and the peak CO concentrations measured elsewhere in the garage near the time of shutoff. Figure 69c shows the $\mathrm{CO}$ concentration measured in each room of the house over the test. Figure 69d shows the CO concentration in the house with each room represented by a single line, which is composed of both a 'solid-line' pattern (showing measured CO concentration) and a 'dotted-line with a symbol' pattern (showing projected CO concentration). Figure 69e shows the calculated $\mathrm{COHb}$ profiles of simulated house occupants in each room of the house.

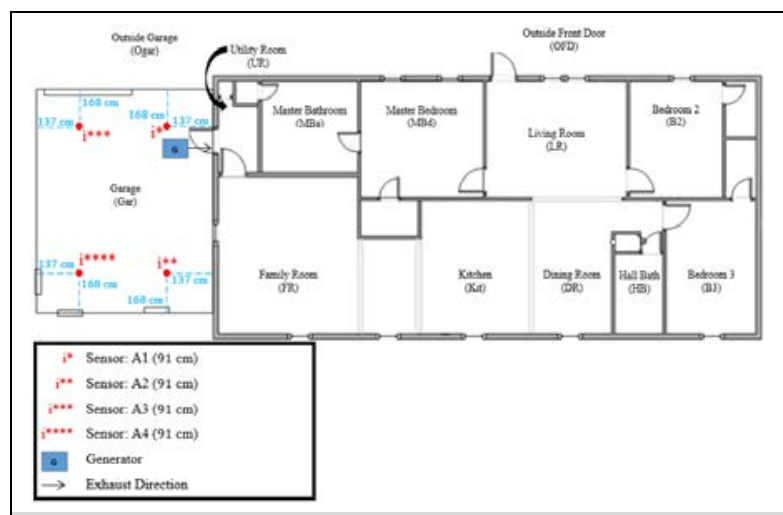

Figure 69a. Generator, exhaust direction, and sensor array layout during Case 26 Test 1 - G6 G300.

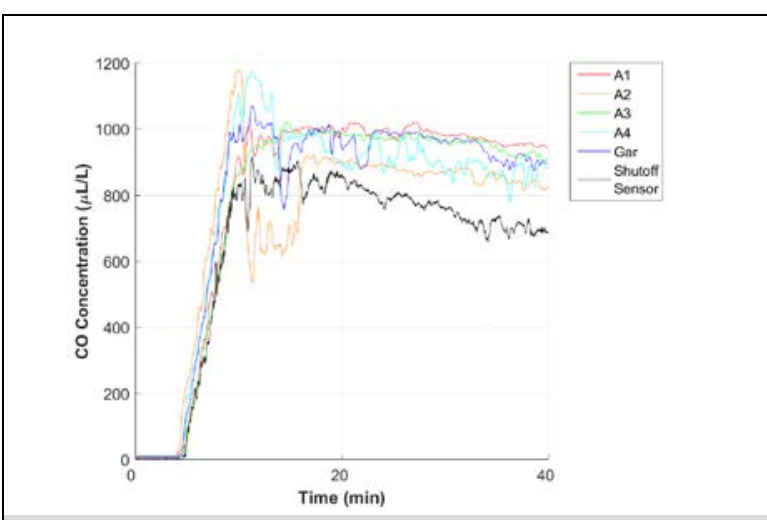

Figure 69b. CO concentration measured by the sensor array and the designated garage sensor during Case 26 Test 1 - G6 G300. 


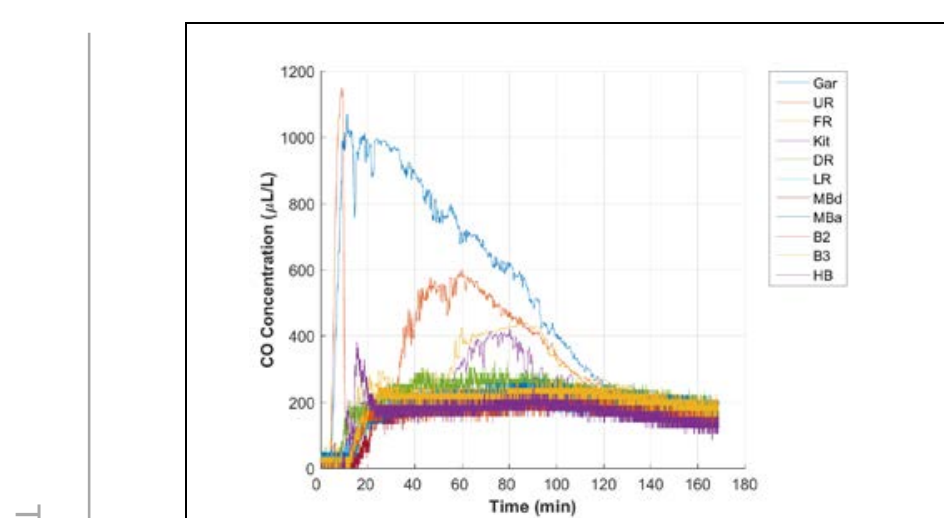

Figure 69c. $\mathrm{CO}$ concentration measured in each room of the house during Case 26 Test 1 - G6 G300.

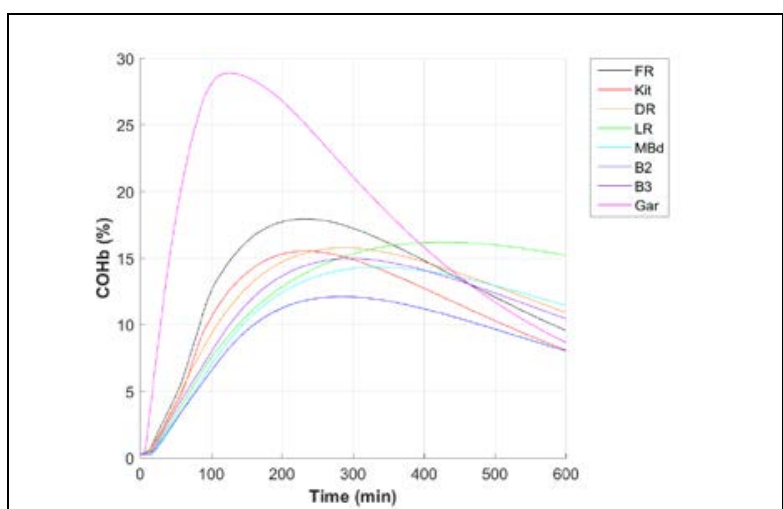

Figure 69e. $\mathrm{COHb}$ of house occupants during Case 26 Test 1 - G6 G300.

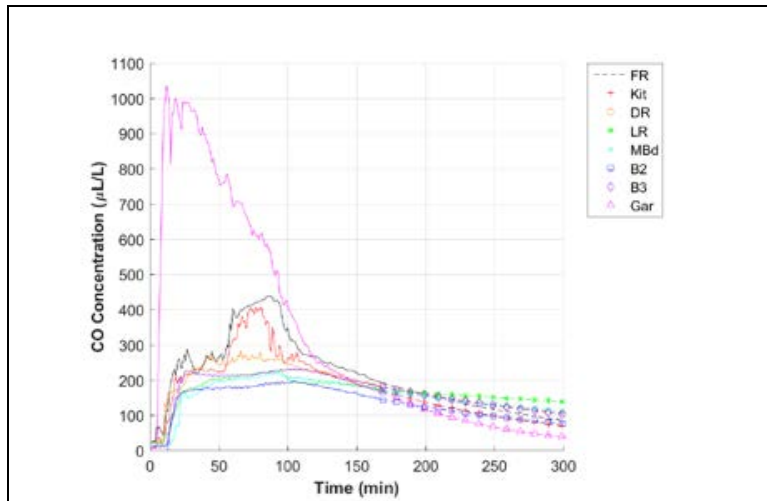

Figure 69d. CO concentration (measured \& projected) in the house during Case 26 Test 1 - G6 G300. 


\section{Case 26 Test 1 - G7a UL 2201}

The following figures illustrate the results for Case 26 Test 1 applying the UL 2201 shutoff criteria. The G7a generator was fully fueled, positioned in the garage (near the interior person door from the garage to the utility room with the exhaust facing toward the utility room), and tested at $100 \%$ load $(7,000 \mathrm{~W})$. The interior person door was open $10 \mathrm{~cm}$ during the test. The shutoff sensor activated the algorithm to shut off the generator after approximately 19 min due to a sensor measurement of $150 \mu \mathrm{L} / \mathrm{L}$ for a $10 \mathrm{~min}$ average. Figure 70a shows the test house layout with generator location, generator exhaust direction, and sensor array location. Figure 70b shows the $\mathrm{CO}$ concentration measured in the garage (by the dedicated room sensor), by four sensors located in the garage (A1, A2, A3, and A4: height $91.0 \mathrm{~cm}$ ), and by the shutoff sensor located on the generator near the generator's electrical panel. The timeframe of the figure has been selected to show the degree of $\mathrm{CO}$ uniformity in the area around the generator between the shutoff sensor and the peak $\mathrm{CO}$ concentrations measured elsewhere in the garage near the time of shutoff. Figure 70c shows the $\mathrm{CO}$ concentration measured in each room of the house over the test. Figure 70d shows the calculated $\mathrm{COHb}$ profiles of simulated house occupants in each room of the house.

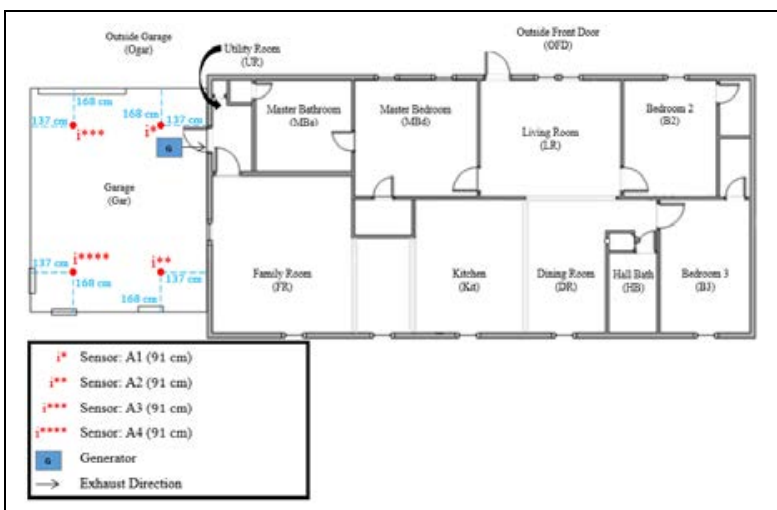

Figure 70a. Generator, exhaust direction, and sensor array layout during Case 26 Test 1 - G7a UL 2201.

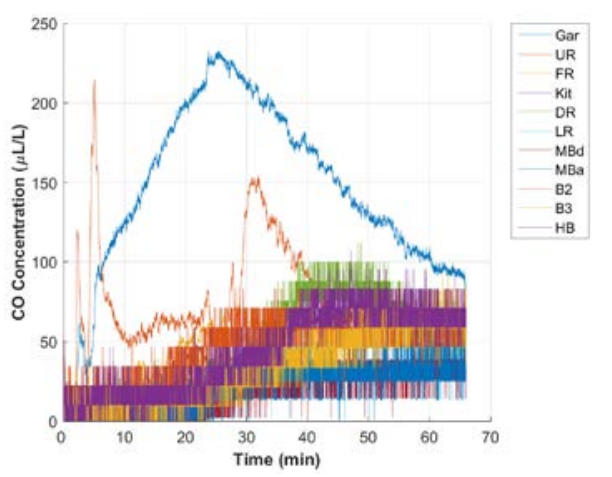

Figure 70c. CO concentration measured in each room of the house during Case 26 Test 1 - G7a UL 2201.

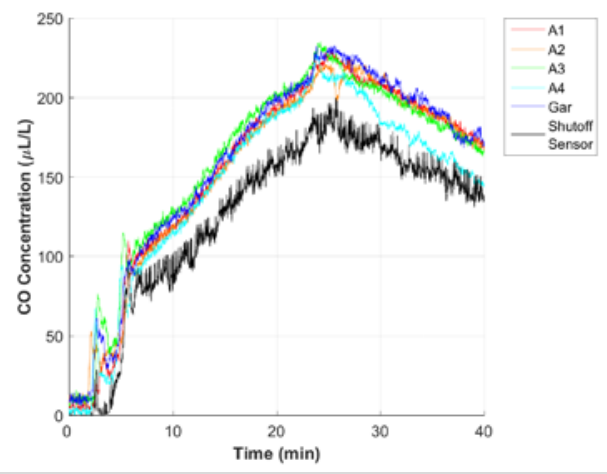

Figure 70b. CO concentration measured by the sensor array and the designated garage sensor during Case 26 Test 1 - G7a UL 2201.

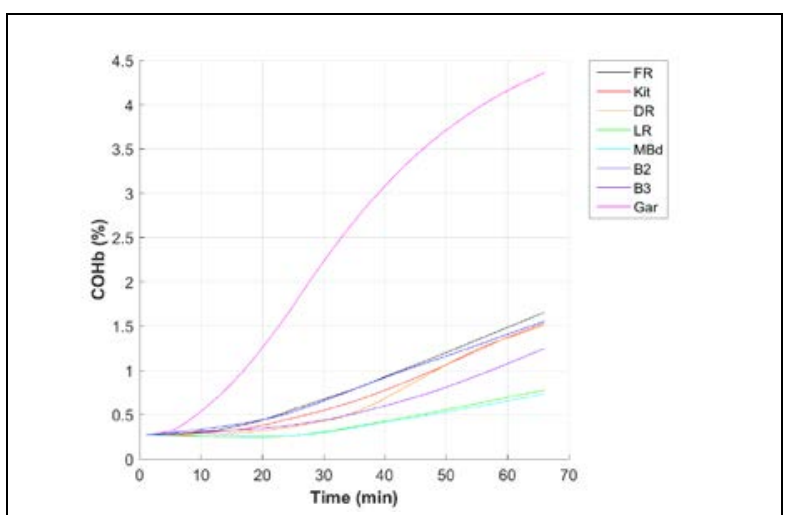

Figure 70d. COHb of house occupant during Case 26 Test 1 - G7a UL 2201. 


\section{Case 26 Test 2 - G7b UL 2201}

The following figures illustrate the results for Case 26 Test 2 applying the UL 2201 shutoff criteria. The G7b generator was fully fueled, positioned in the garage (near the interior person door from the garage to the utility room with the exhaust facing toward the utility room), and tested at $100 \%$ load $(7,000 \mathrm{~W})$. The interior person door was open $10 \mathrm{~cm}$ during the test. The shutoff sensor activated the algorithm to shut off the generator after approximately $11 \mathrm{~min}$ due to a sensor measurement of $150 \mu \mathrm{L} / \mathrm{L}$ for a 10 min average. Figure 71a shows the test house layout with generator location, generator exhaust direction, and sensor array location. Figure 71b shows the $\mathrm{CO}$ concentration measured in the garage (by the dedicated room sensor), by four sensors located in the garage (A1, A2, A3, and A4: height $91.0 \mathrm{~cm}$ ), and by the shutoff sensor located on the generator near the generator's electrical panel. The timeframe of the figure has been selected to show the degree of CO uniformity in the area around the generator between the shutoff sensor and the peak $\mathrm{CO}$ concentrations measured elsewhere in the garage near the time of shutoff. Figure 71c shows the $\mathrm{CO}$ concentration measured in each room of the house over the test. Figure 71d shows the calculated $\mathrm{COHb}$ profiles of simulated house occupants in each room of the house.

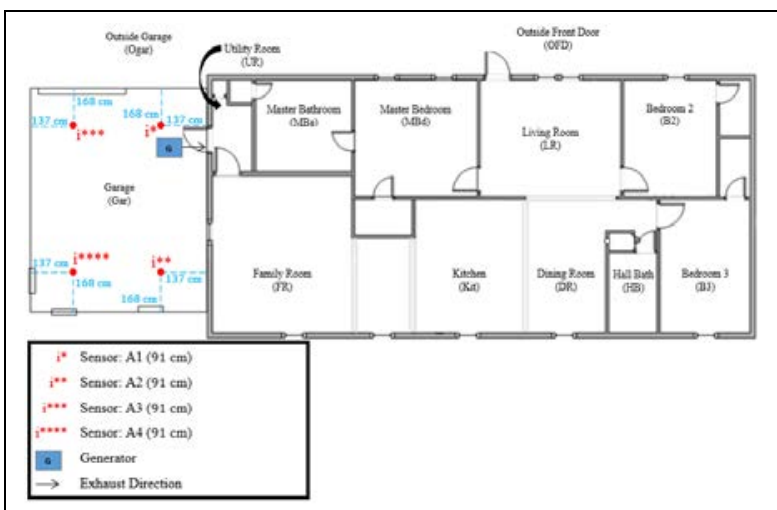

Figure 71a. Generator, exhaust direction, and sensor array layout during Case 26 Test 2 - G7b UL 2201.

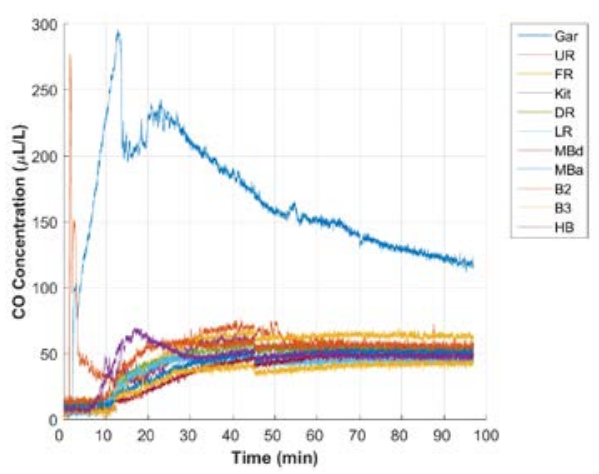

Figure 71c. $\mathrm{CO}$ concentration measured in each room of the house during Case 26 Test 2 - G7b UL 2201.

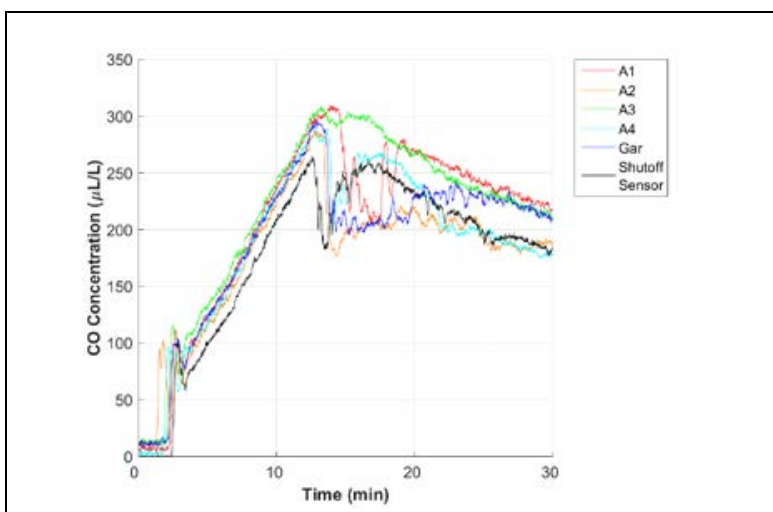

Figure 71b. CO concentration measured by the sensor array and the designated garage sensor during Case 26 Test 2 - G7b UL 2201.

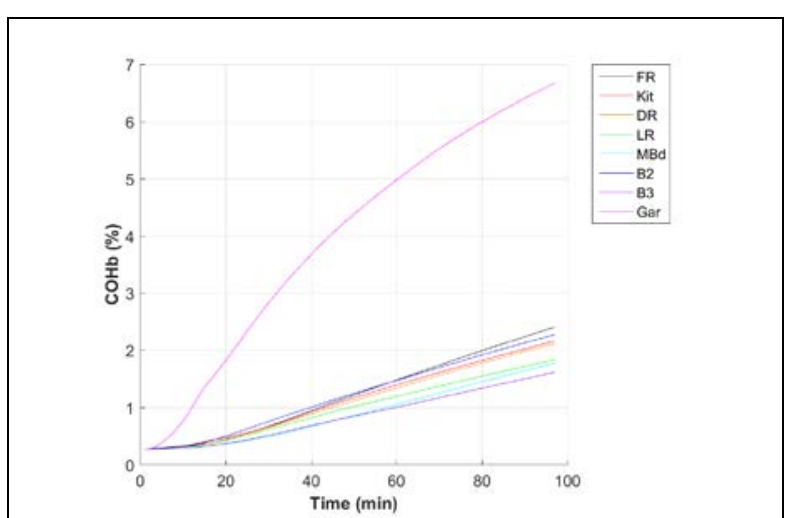

Figure 71d. COHb of house occupant during Case 26 Test 2 - G7b UL 2201. 


\section{Case 27 Test 1 - G15 G300}

The following figures illustrate the results for Case 27 Test 1 applying the G300 shutoff criteria. The G15 generator was fully fueled, positioned in the garage (30 $\mathrm{cm}$ from interior person door from the garage to the utility room with the exhaust facing away from the door), and tested at 90 $\%$ load $(13,500 \mathrm{~W})$. The interior person door (from garage to utility room) was open $10 \mathrm{~cm}$; the exterior person door (from garage to backyard) and the garage bay door were closed. The shutoff sensor activated the algorithm to shut off the generator after approximately 2 min due to an instantaneous sensor measurement of $800 \mu \mathrm{L} / \mathrm{L}$. Figure 72a shows the test house layout with generator location, exhaust direction, and sensor array location. Figure 72b shows the CO concentration measured in the garage (by the dedicated room sensor), by the sensor array placed in the garage located as shown in Figure 72a (A1: height $91 \mathrm{~cm}, \mathrm{A2}$ : height $91 \mathrm{~cm}$, A3: height 91 $\mathrm{cm}$, A4: height $91 \mathrm{~cm}$ ), and by the shutoff sensor located on the generator near the generator's electrical panel. The timeframe of the figure has been selected to show the degree of CO uniformity in the area around the generator between the shutoff sensor and the peak CO concentrations measured elsewhere in the garage near the time of shutoff. Figure 72c shows the CO concentration measured in each room of the house over the test. Figure 72d shows the CO concentration in the house with each room represented by a single line, which is composed of both a 'solid-line' pattern (showing measured CO concentration) and a 'dotted-line with a symbol' pattern (showing projected CO concentration). Figure 72e shows the calculated COHb profiles of simulated house occupants in each room of the house.

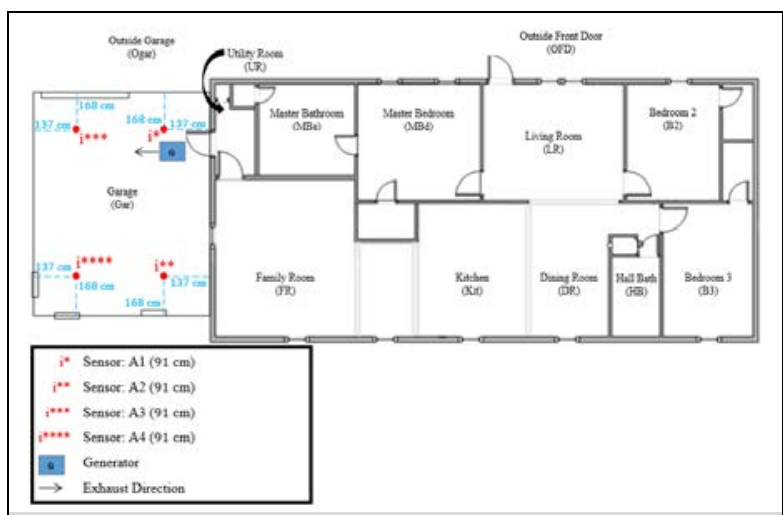

Figure 72a. Generator, exhaust direction, and sensor array layout during Case 27 Test 1 - G15 G300.

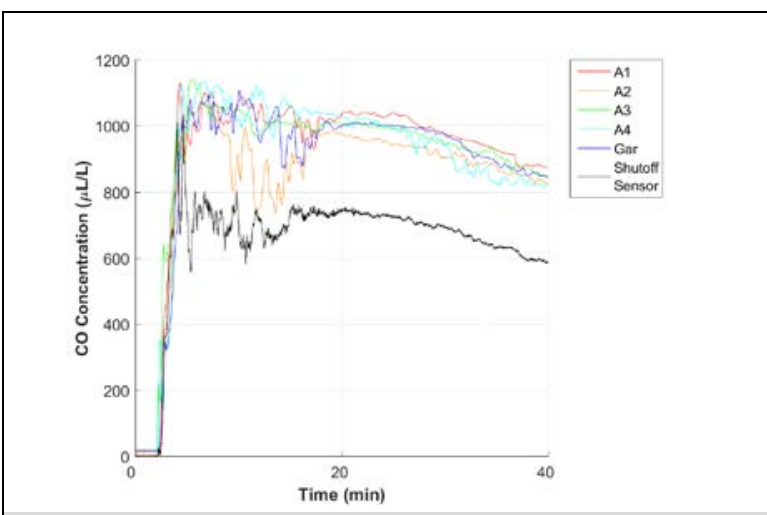

Figure 72b. CO concentration measured by the sensor array and the designated garage sensor during Case 27 Test 1 - G15 G300. 


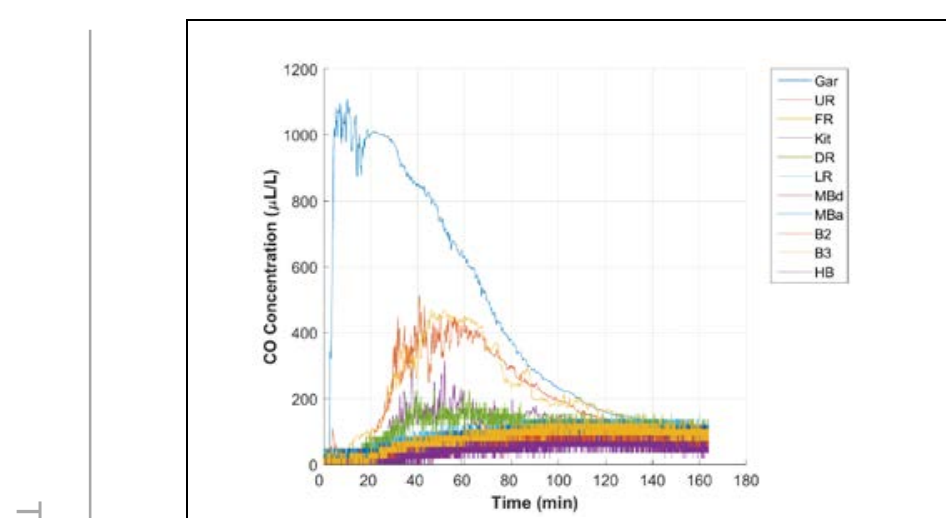

Figure 72c. CO concentration measured in each room of the house during Case 27 Test 1 - G15 G300.

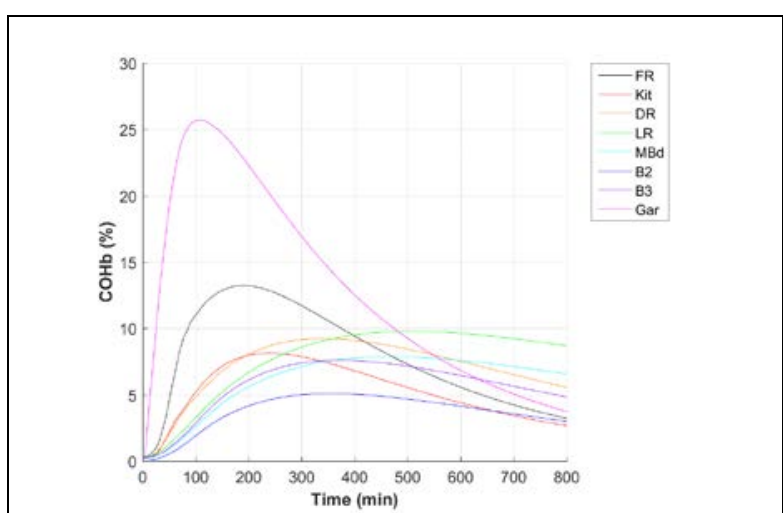

Figure 72e. $\mathrm{COHb}$ of house occupants during Case 27 Test 1 - G15 G300.

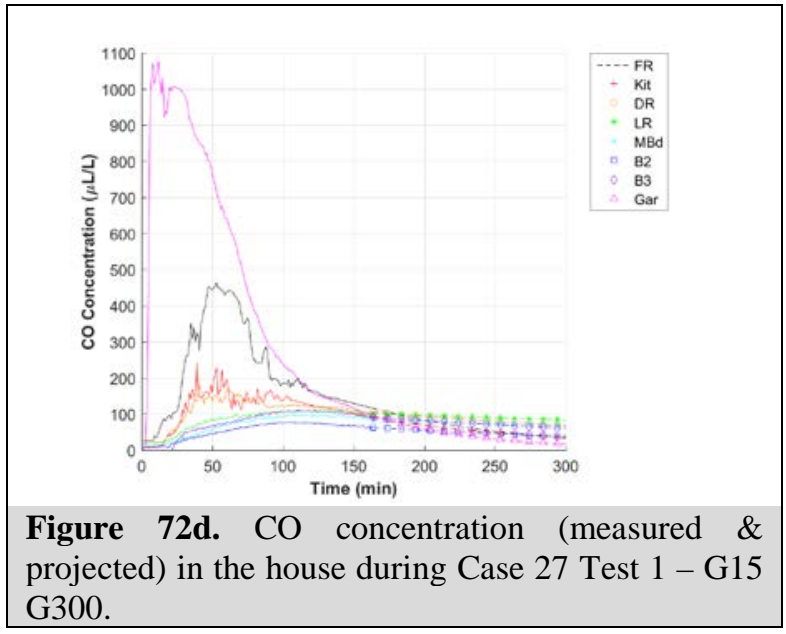

Figure 72d. CO concentration (measured \& projected) in the house during Case 27 Test 1 - G15 G300. 


\section{Case 28 Test 1 - G15 G300}

The following figures illustrate the results for Case 28 Test 1 applying the G300 shutoff criteria. The G15 generator was fully fueled, positioned in the garage (30 $\mathrm{cm}$ from interior person door from the garage to the utility room with the exhaust facing away from the door), and tested at 25 $\%$ load $(3,750 \mathrm{~W})$. The interior person door (from garage to utility room) was open $10 \mathrm{~cm}$; the exterior person door (from garage to backyard) and the garage bay door were closed. The shutoff sensor activated the algorithm to shut off the generator after approximately 3 min due to an instantaneous sensor measurement of $800 \mu \mathrm{L} / \mathrm{L}$. Figure 73a shows the test house layout with generator location, exhaust direction, and sensor array location. Figure 73b shows the CO concentration measured in the garage (by the dedicated room sensor), by the sensor array placed in the garage located as shown in Figure 73a (A1: height $91 \mathrm{~cm}, \mathrm{A2}$ : height $91 \mathrm{~cm}$, A3: height 91 $\mathrm{cm}$, A4: height $91 \mathrm{~cm}$ ), and by the shutoff sensor located on the generator near the generator's electrical panel. The timeframe of the figure has been selected to show the degree of CO uniformity in the area around the generator between the shutoff sensor and the peak CO concentrations measured elsewhere in the garage near the time of shutoff. Figure 73c shows the CO concentration measured in each room of the house over the test. Figure 73d shows the CO concentration in the house with each room represented by a single line, which is composed of both a 'solid-line' pattern (showing measured CO concentration) and a 'dotted-line with a symbol' pattern (showing projected CO concentration). Figure 73e shows the calculated COHb profiles of simulated house occupants in each room of the house.

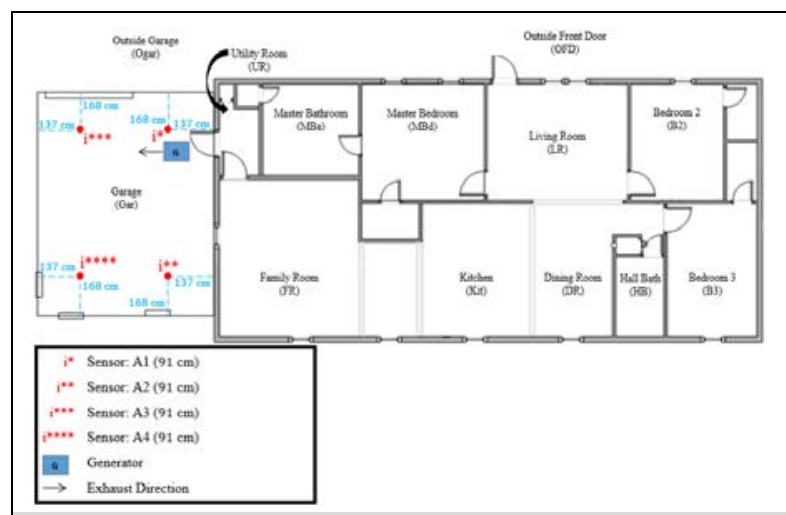

Figure 73a. Generator, exhaust direction, and sensor array layout during Case 28 Test 1 - G15 G300.

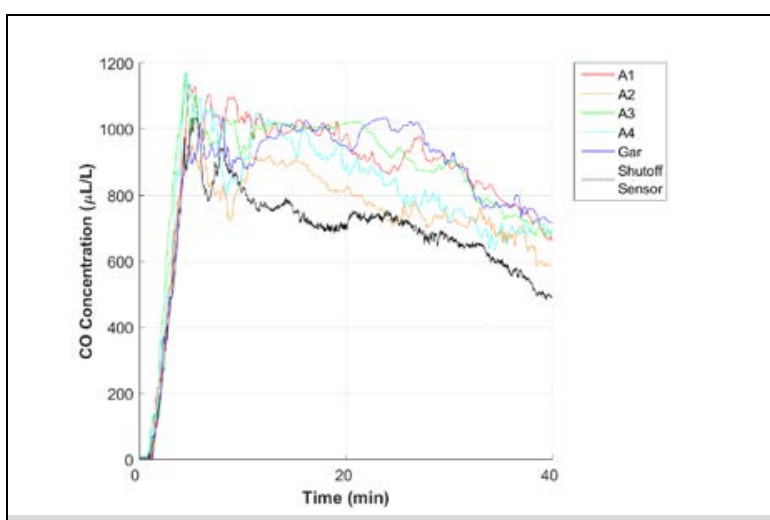

Figure 73b. CO concentration measured by the sensor array and the designated garage sensor during Case 28 Test 1 - G15 G300. 


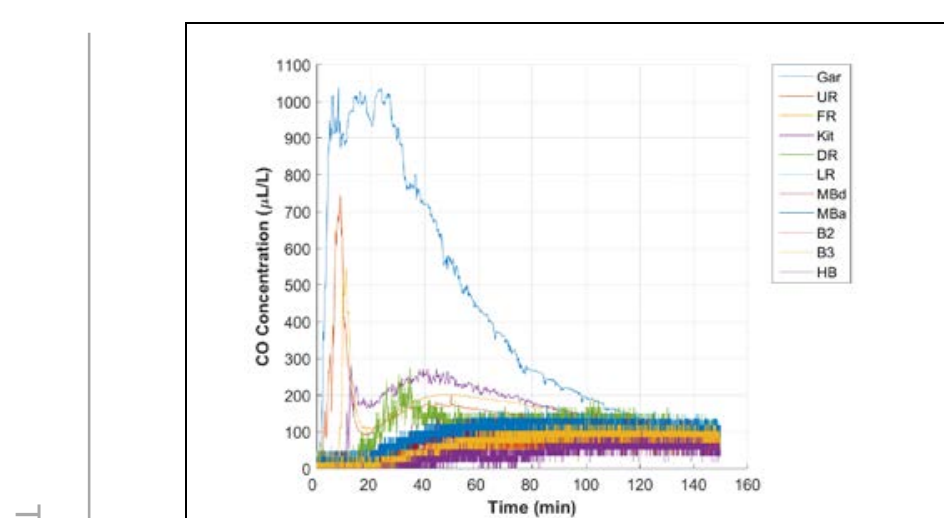

Figure 73c. CO concentration measured in each room of the house during Case 28 Test 1 - G15 G300.

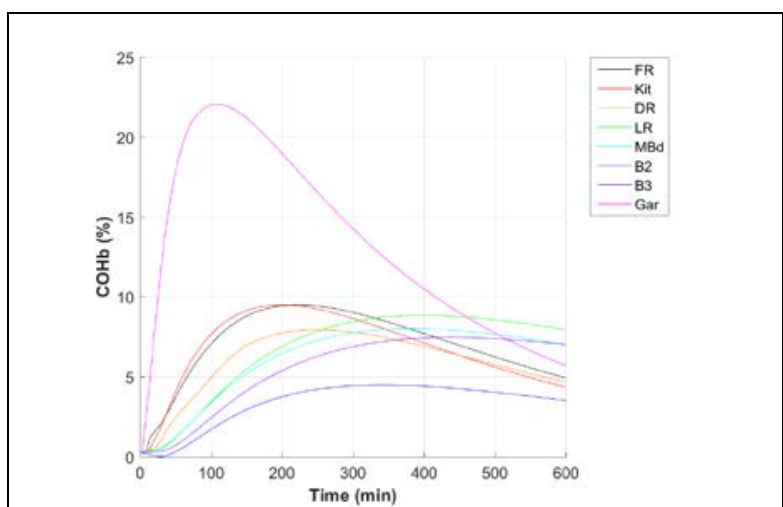

Figure 73e. $\mathrm{COHb}$ of house occupants during Case 28 Test $1-$ G15 G300.

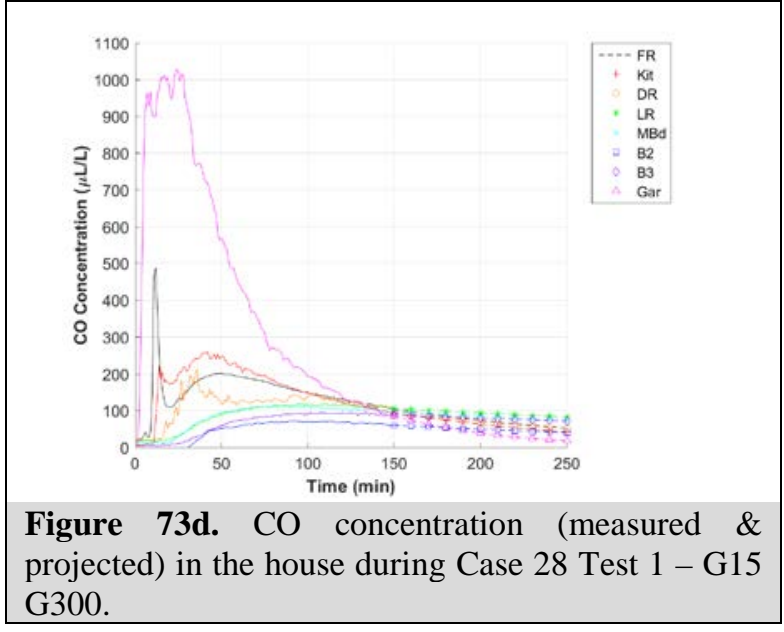

Figure 73d. CO concentration (measured \& projected) in the house during Case 28 Test $1-$ G15 G300. 


\section{Case 29 Test 1 - G6 G300}

The following figures illustrate the results for Case 29 Test 1 applying the G300 shutoff criteria. The G6 generator was fully fueled, positioned in the garage (30 cm from the interior person door from the garage to the utility room with the exhaust facing away from the door), and tested at 100 $\%(6,000 \mathrm{~W})$. The interior person door (from garage to utility room) was open $10 \mathrm{~cm}$ and exterior person door (from garage to backyard) was fully open; the garage bay door was closed. The shutoff sensor activated the algorithm to shut off the generator after approximately 10 min due to a sensor measurement of $400 \mu \mathrm{L} / \mathrm{L}$ for a $10 \mathrm{~min}$ average. Figure 74a shows the test house layout with generator location, exhaust direction, and sensor array location. Figure $\mathbf{7 4 b}$ shows the CO concentration measured in the garage (by the dedicated room sensor), by the sensor array placed in the garage located as shown in Figure 74a (A1: height $91 \mathrm{~cm}, \mathrm{A2}$ : height $91 \mathrm{~cm}$, A3: height 91 $\mathrm{cm}$, A4: height $91 \mathrm{~cm}$ ), and by the shutoff sensor located on the generator near the generator's electrical panel. The timeframe of the figure has been selected to show the degree of CO uniformity in the area around the generator between the shutoff sensor and the peak CO concentrations measured elsewhere in the garage near the time of shutoff. Figure 74c shows the CO concentration measured in each room of the house over the test. Figure 74d shows the calculated COHb profiles of simulated house occupants in each room of the house. The calculated $\mathrm{COHb}$ for simulated house occupants did not exceed $5 \%$ during the test.

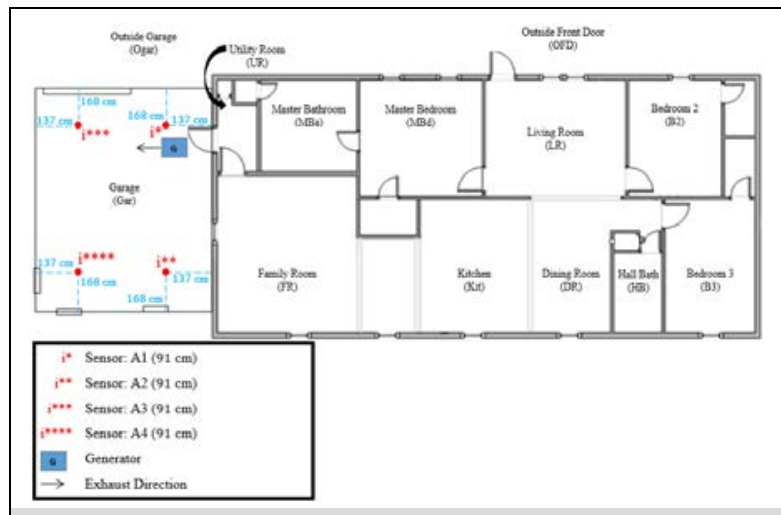

Figure 74a. Generator, exhaust direction, and sensor array layout during Case 29 Test 1 - G6 G300.

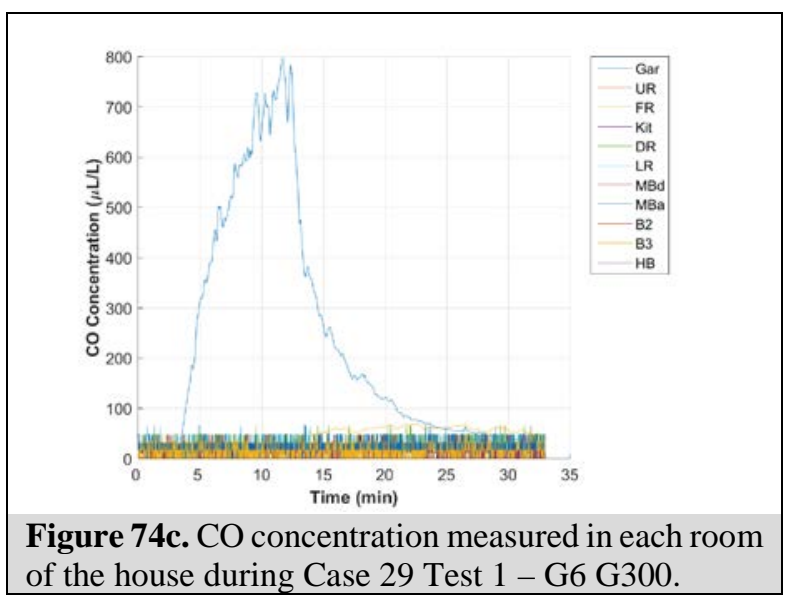

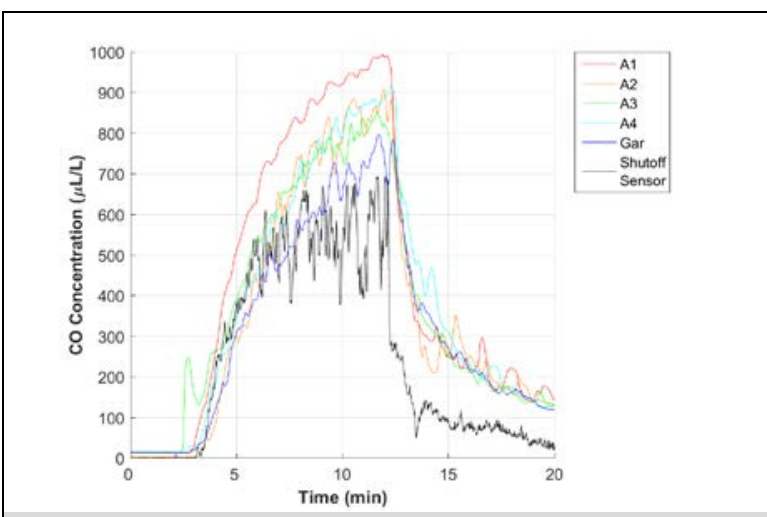

Figure 74b. CO concentration measured by the sensor array and the designated garage sensor during Case 29 Test 1 - G6 G300.

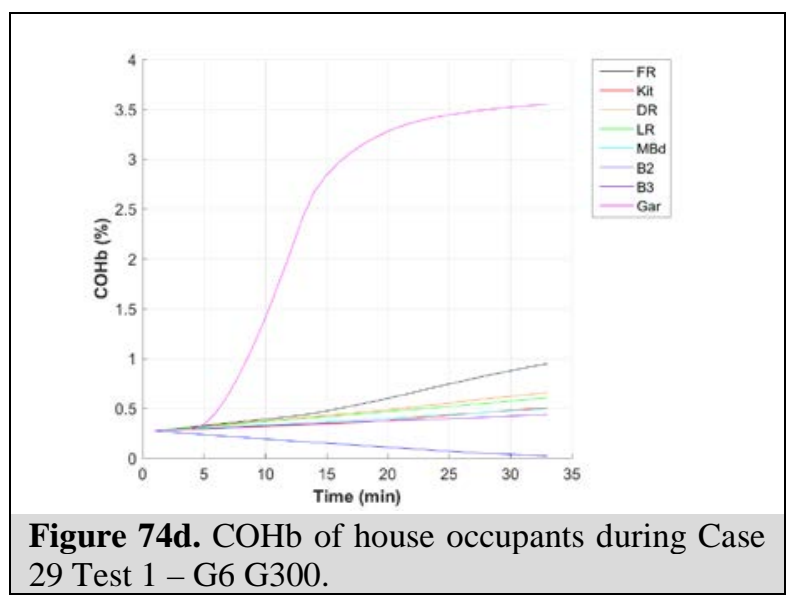




\section{Case 29 Test 1 - G6 G300 Restart}

The following figures illustrate the results for Restart Case 29 Test 1 applying the G300 shutoff criteria. The G6 generator was fully fueled, positioned in the garage (30 cm from the interior person door from the garage to the utility room with the exhaust facing away from the door), and tested at $100 \%(6,000 \mathrm{~W})$. The interior person door (from garage to utility room) was open $10 \mathrm{~cm}$ and exterior person door (from garage to backyard) was fully open and the garage bay door was closed. For the first generator run, the shutoff sensor activated the algorithm to shut off the generator after approximately 4 min due to an instantaneous sensor measurement of $800 \mu \mathrm{L} / \mathrm{L}$. The generator was restarted and the test load restored approximately $10 \mathrm{~min}$ after the first instance of shutoff initiated by the shutoff sensor. For the second generator run, the shutoff sensor activated the algorithm to shut off the generator after approximately 5 min due to an instantaneous sensor measurement of $800 \mu \mathrm{L} / \mathrm{L}$. Figure 75a shows the test house layout with generator location, exhaust direction, and sensor array location. Figure $\mathbf{7 5 b}$ shows the CO concentration measured in the garage (by the dedicated room sensor), by the sensor array placed in the garage located as shown in Figure 75a (A1: height $91 \mathrm{~cm}, \mathrm{A2}$ : height $91 \mathrm{~cm}, \mathrm{A3}$ : height $91 \mathrm{~cm}, \mathrm{~A} 4$ : height $91 \mathrm{~cm}$ ), and by the shutoff sensor located on the generator near the generator's electrical panel. The timeframe of the figure has been selected to show the degree of $\mathrm{CO}$ uniformity in the area around the generator between the shutoff sensor and the peak CO concentrations measured elsewhere in the garage near the time of shutoff. Figure 75c shows the CO concentration measured in each room of the house over the test. Figure 75d shows the calculated $\mathrm{COHb}$ profiles of simulated house occupants in each room of the house. The calculated $\mathrm{COHb}$ for simulated house occupants did not exceed $5 \%$ during the test.

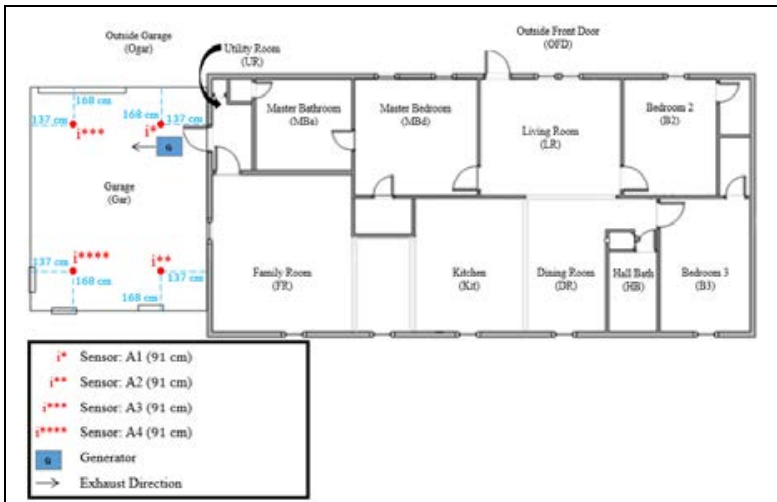

Figure 75a. Generator, exhaust direction, and sensor array layout during Restart Case 29 Test 1 - G6 G300.

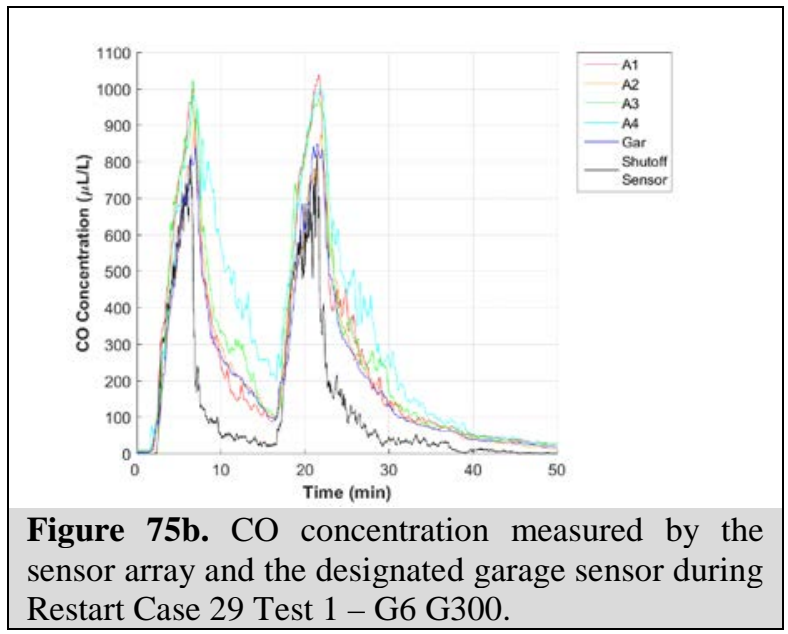




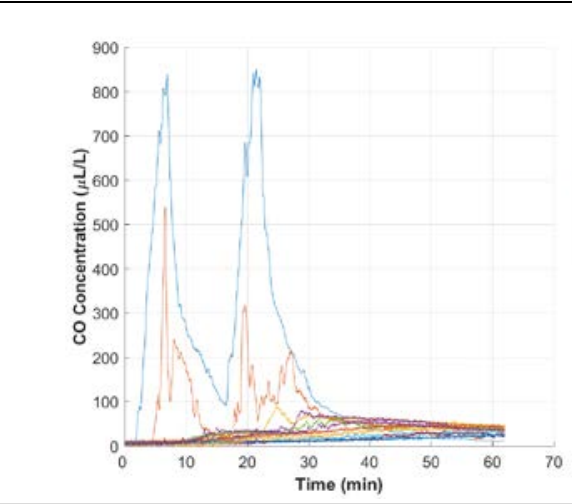

Figure 75c. CO concentration measured in each room of the house during Restart Case 29 Test 1 - G6 G300.

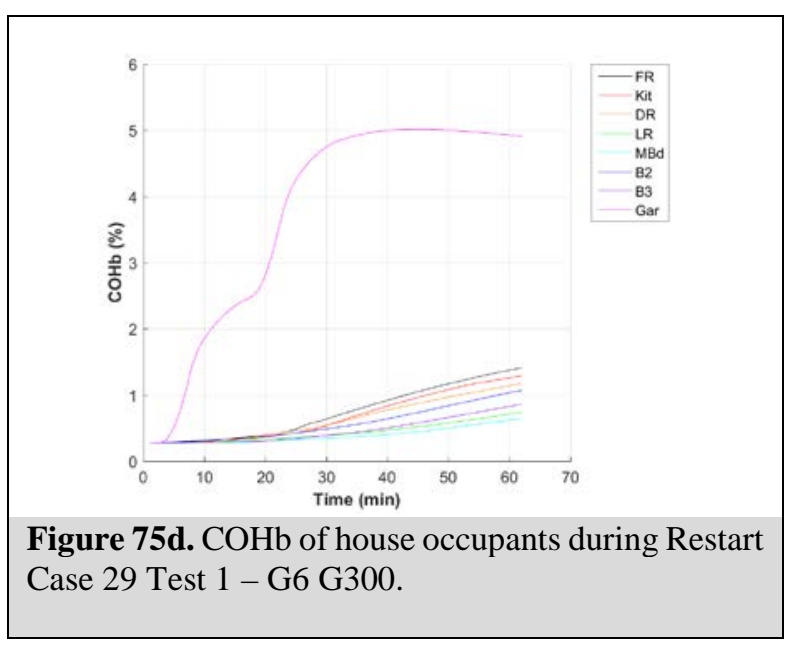

Figure 75d. $\mathrm{COHb}$ of house occupants during Restart Case 29 Test 1 - G6 G300. 


\section{Case 30 Test 1 - G15 G300}

The following figures illustrate the results for Case 30 Test 1 applying the G300 shutoff criteria. The G15 generator was fully fueled, positioned in the garage (centered, with the exhaust facing towards the family room), and tested at $90 \%$ load $(13,500 \mathrm{~W}$ ). The interior person door (from garage to utility room) was open $10 \mathrm{~cm}$, the garage bay door was fully open and the exterior person door (from garage to backyard) was closed. The shutoff sensor activated the algorithm to shut off the generator after approximately 3 min due to an instantaneous sensor measurement of $800 \mu \mathrm{L} / \mathrm{L}$. Figure 76a shows the test house layout with generator location, exhaust direction, and sensor array location. Figure 76b shows the CO concentration measured in the garage (by the dedicated room sensor), by the sensor array placed in the garage located as shown in Figure 76a (A1: height 91 $\mathrm{cm}$, A2: height $91 \mathrm{~cm}, \mathrm{A3}$ : height $91 \mathrm{~cm}, \mathrm{~A} 4$ : height $91 \mathrm{~cm}$ ), and by the shutoff sensor located on the generator near the generator's electrical panel. The timeframe of the figure has been selected to show the degree of CO uniformity in the area around the generator between the shutoff sensor and the peak CO concentrations measured elsewhere in the garage near the time of shutoff. Figure 76c shows the $\mathrm{CO}$ concentration measured in each room of the house over the test. Figure 76d shows the calculated $\mathrm{COHb}$ profiles of simulated house occupants in each room of the house. The calculated $\mathrm{COHb}$ for simulated house occupants did not exceed $5 \%$ during the test.

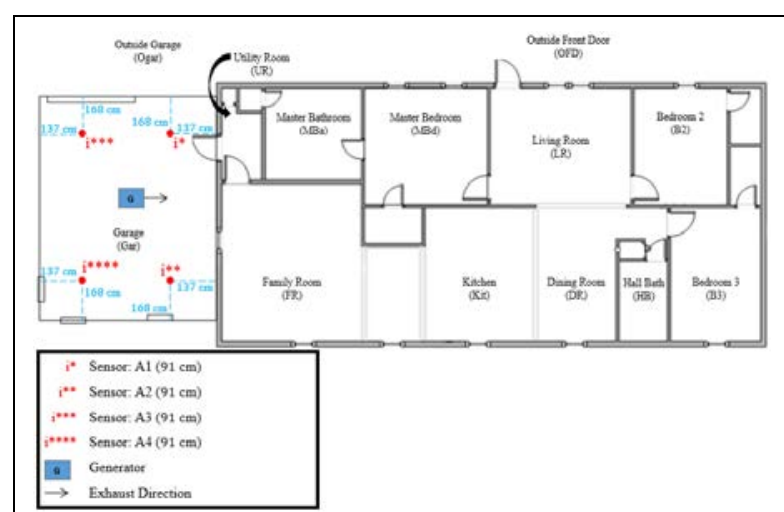

Figure 76a. Generator, exhaust direction, and sensor array layout during Case 30 Test 1 - G15 G300.

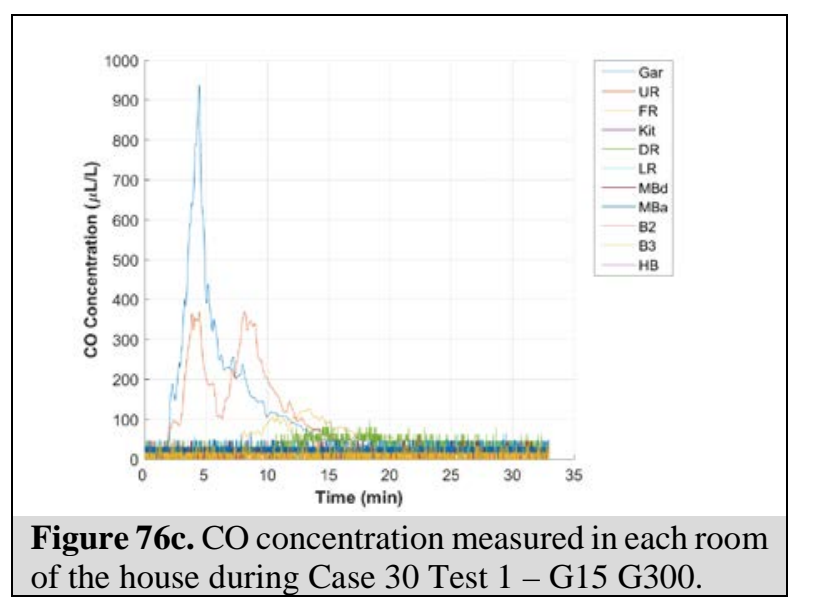

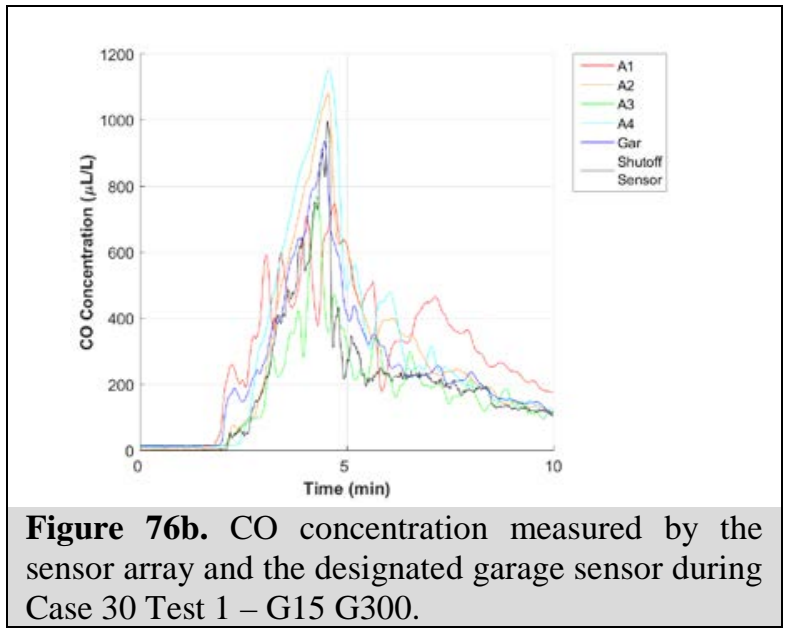

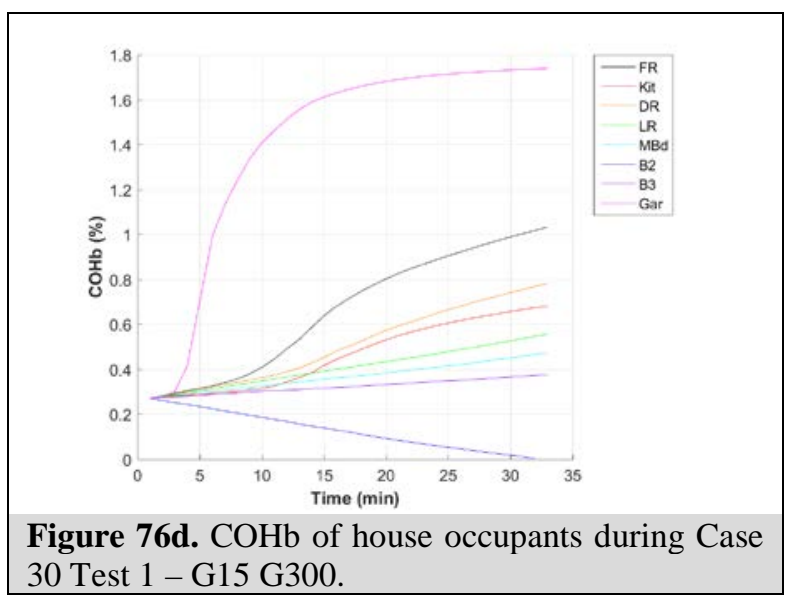




\section{Case 31 Test 1 - G15 G300}

The following figures illustrate the results for Case 31 Test 1 applying the G300 shutoff criteria. The G15 generator was fully fueled, positioned in the garage (centered, with the exhaust facing towards the family room), and tested at $25 \%$ load $(3,750 \mathrm{~W}$ ). The interior person door (from garage to utility room) was open $10 \mathrm{~cm}$, the garage bay door was fully open and the exterior person door (from garage to backyard) was closed. The shutoff sensor activated the algorithm to shut off the generator during startup, within 10 seconds of applying the load due to an instantaneous sensor measurement of $800 \mu \mathrm{L} / \mathrm{L}$. This was perceived as a startup issue and the generator was restarted within 2 min of the shutoff event. The test load was applied promptly and the generator eventually ran out of fuel after approximately $727 \mathrm{~min}$. Figure 77a shows the test house layout with generator location, exhaust direction, and sensor array location. Figure 77b shows the CO concentration measured in the garage (by the dedicated room sensor), by the sensor array placed in the garage located as shown in Figure 77a (A1: height $91 \mathrm{~cm}, \mathrm{~A} 2$ : height $91 \mathrm{~cm}$, A3: height $91 \mathrm{~cm}, \mathrm{~A} 4$ : height $91 \mathrm{~cm})$, and by the shutoff sensor located on the generator near the generator's electrical panel. The figure shows the degree of CO uniformity in the area around the generator between the shutoff sensor and the peak CO concentrations measured elsewhere in the garage. Figure 77c shows the $\mathrm{CO}$ concentration measured in each room of the house over the test. Figure 77d shows the CO concentration in the house with each room represented by a single line, which is composed of both a 'solid-line' pattern (showing measured CO concentration) and a 'dotted-line with a symbol' pattern (showing projected CO concentration). Figure 77e shows the calculated COHb profiles of simulated house occupants in each room of the house.

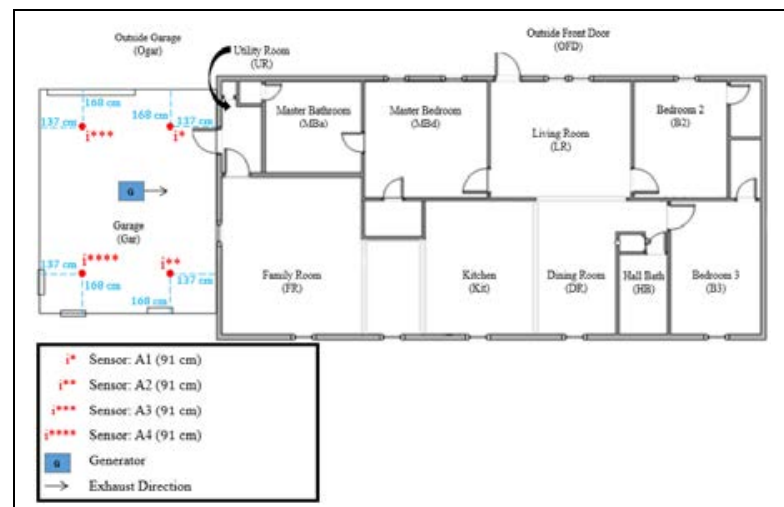

Figure 77a. Generator, exhaust direction, and sensor array layout during Case 31 Test 1 - G15 G300.

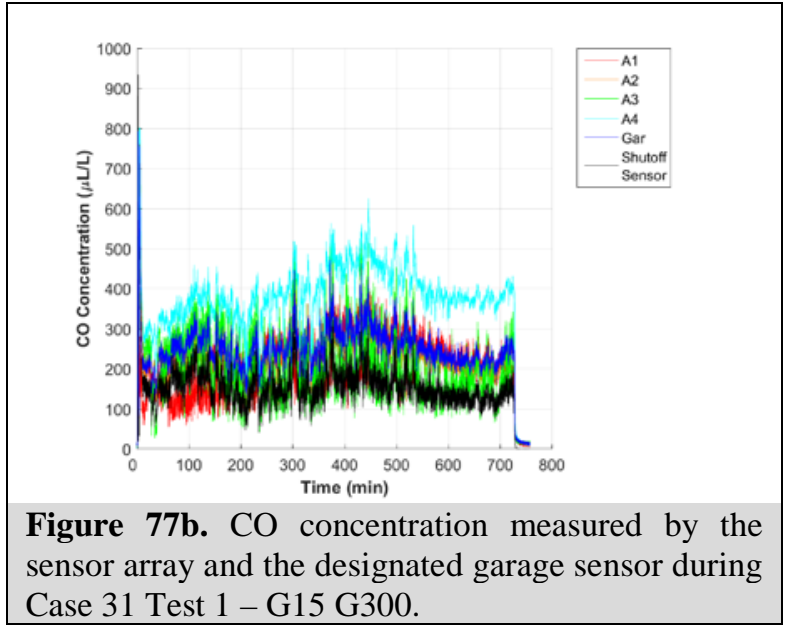
Case 31 Test 1 - G15 G300. 

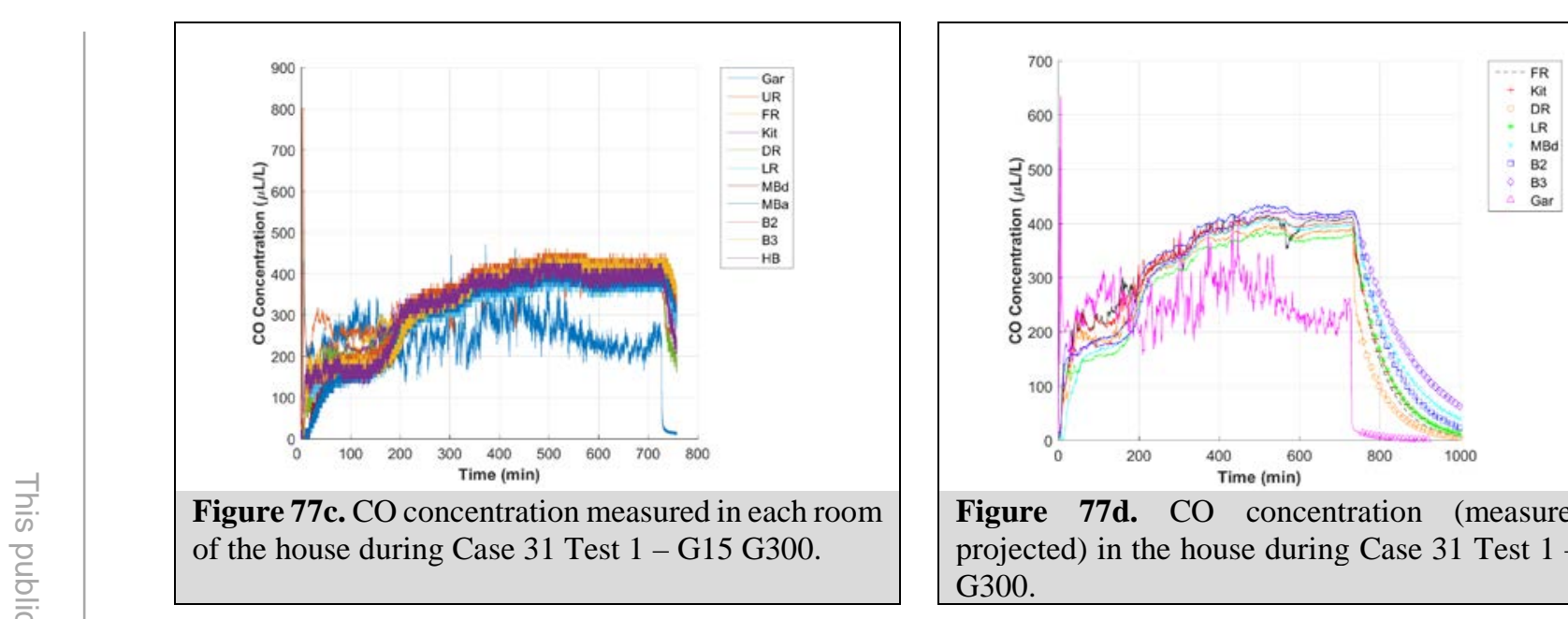

Figure 77d. CO concentration (measured \& projected) in the house during Case 31 Test 1 - G15 G300.

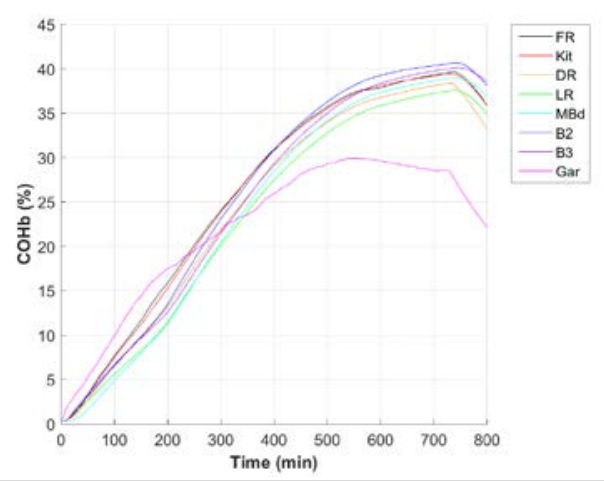

Figure 77e. $\mathrm{COHb}$ of house occupants during Case 31 Test 1 - G15 G300.

147 


\section{Case 32 Test 1 - G6 G300}

The following figures illustrate the results for Case 32 Test 1 applying the G300 shutoff criteria. The G6 generator was fully fueled, positioned in the garage (centered, with the exhaust facing towards the family room), and tested at $100 \%$ load $(6,000 \mathrm{~W}$ ). The interior person door (from garage to utility room) was open $10 \mathrm{~cm}$, the garage bay door was fully open and the exterior person door (from garage to backyard) was closed. The shutoff sensor was not activated and the generator was manually shutdown after approximately $264 \mathrm{~min}$ due to CO concentrations reaching approximate steady-state conditions. Figure 78a shows the test house layout with generator location, exhaust direction, and sensor array location. Figure $\mathbf{7 8 b}$ shows the CO concentration measured in the garage (by the dedicated room sensor), by the sensor array placed in the garage located as shown in Figure 78a (A1: height $91 \mathrm{~cm}, \mathrm{A2}$ : height $91 \mathrm{~cm}, \mathrm{A3}$ : height $91 \mathrm{~cm}, \mathrm{~A} 4$ : height $91 \mathrm{~cm})$, and by the shutoff sensor located on the generator near the generator's electrical panel. The figure shows the degree of CO uniformity in the area around the generator between the shutoff sensor and the peak CO concentrations measured elsewhere in the garage. Figure 78c shows the $\mathrm{CO}$ concentration measured in each room of the house over the test. Figure 78d shows the calculated $\mathrm{COHb}$ profiles of simulated house occupants in each room of the house.

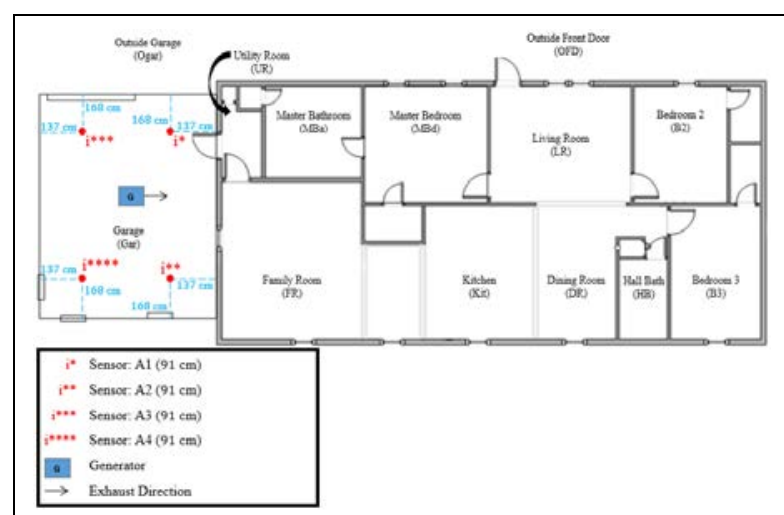

Figure 78a. Generator, exhaust direction, and sensor array layout during Case 32 Test 1 - G6 G300.

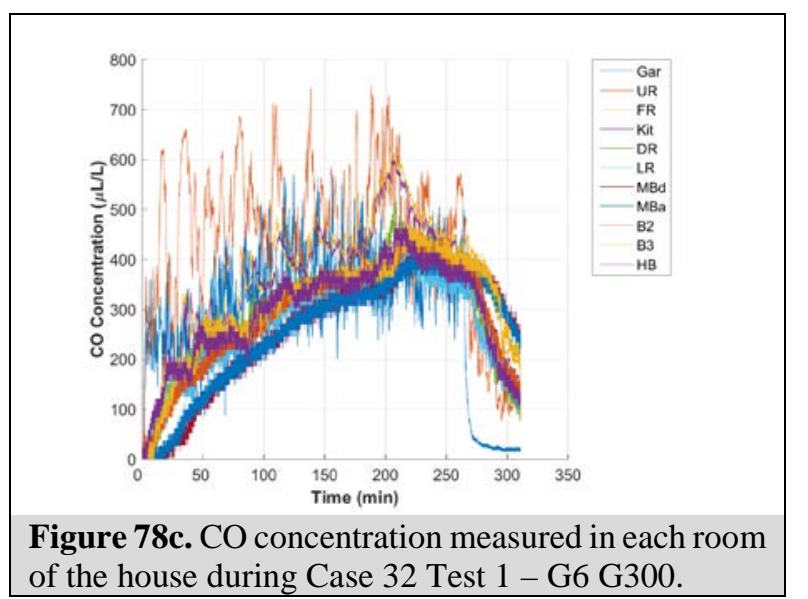

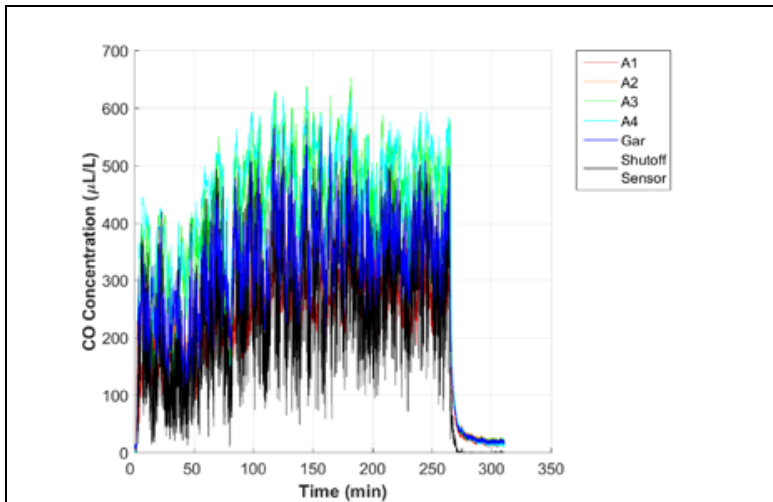

Figure 78b. CO concentration measured by the sensor array and the designated garage sensor during Case 32 Test 1 - G6 G300.

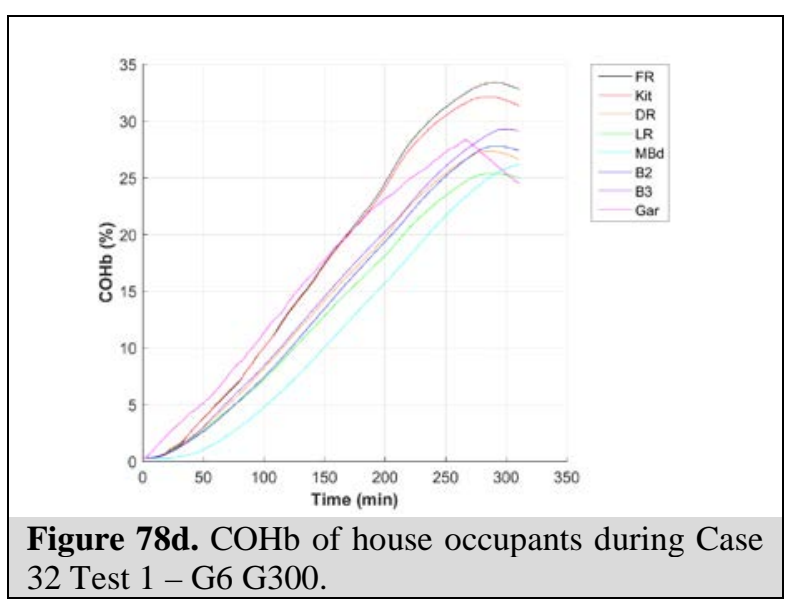




\section{Case 32 Test 2 - G6 G300}

The following figures illustrate the results for Case 32 Test 2 applying the G300 shutoff criteria. The G6 generator was fully fueled, positioned in the garage (centered, with the exhaust facing towards the family room), and tested at $100 \%$ load $(6,000 \mathrm{~W}$ ). The interior person door (from garage to utility room) was open $10 \mathrm{~cm}$, the garage bay door was fully open and the exterior person door (from garage to backyard) was closed. The shutoff sensor was not activated and the generator ran out of fuel after approximately $406 \mathrm{~min}$. Figure 79a shows the test house layout with generator location, exhaust direction, and sensor array location. Figure 79b shows the CO concentration measured in the garage (by the dedicated room sensor), by the sensor array placed in the garage located as shown in Figure 79a (A1: height $91 \mathrm{~cm}, \mathrm{A2}$ : height $91 \mathrm{~cm}, \mathrm{A3}$ : height $91 \mathrm{~cm}, \mathrm{~A} 4$ : height $91 \mathrm{~cm})$, and by the shutoff sensor located on the generator near the generator's electrical panel. The figure shows the degree of CO uniformity in the area around the generator between the shutoff sensor and the peak CO concentrations measured elsewhere in the garage. Figure 79c shows the $\mathrm{CO}$ concentration measured in each room of the house over the test. Figure 79d shows the CO concentration in the house - each room is represented by a single line, which is composed of both a 'solid-line' pattern (showing measured CO concentration) and a 'dotted-line with a symbol' pattern (showing projected CO concentration). Figure 79e shows the calculated COHb profiles of simulated house occupants in each room of the house.

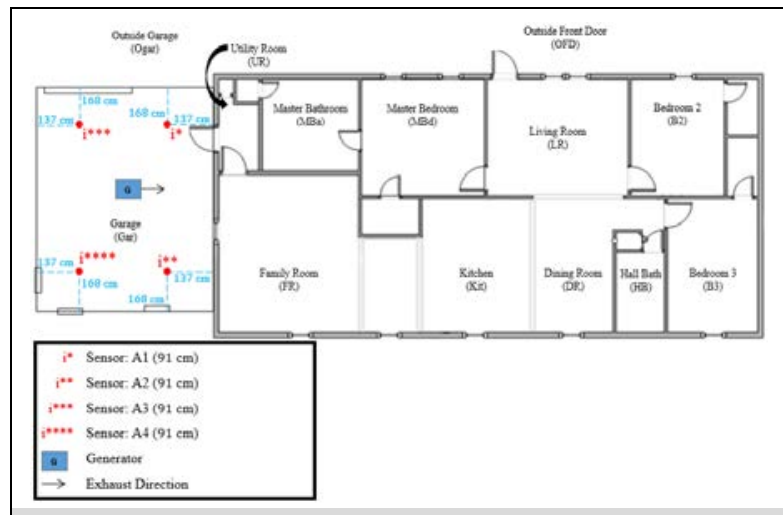

Figure 79a. Generator, exhaust direction, and sensor array layout during Case 32 Test 2 - G6 G300.

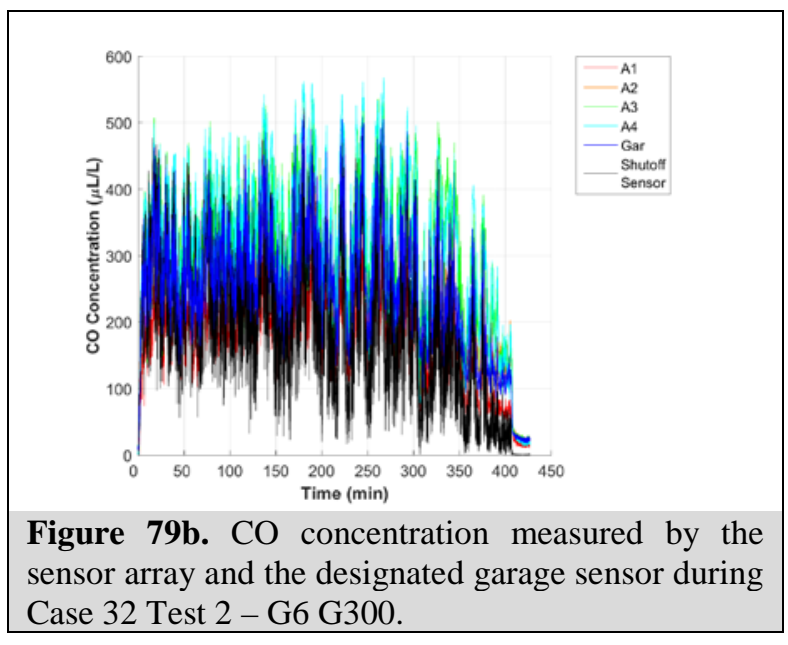




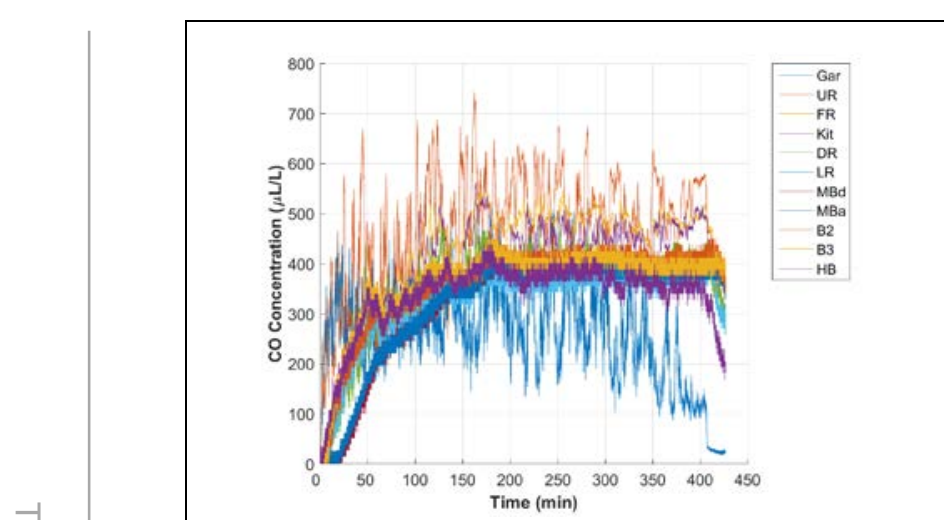

Figure 79c. $\mathrm{CO}$ concentration measured in each room of the house during Case 32 Test 2 - G6 G300.

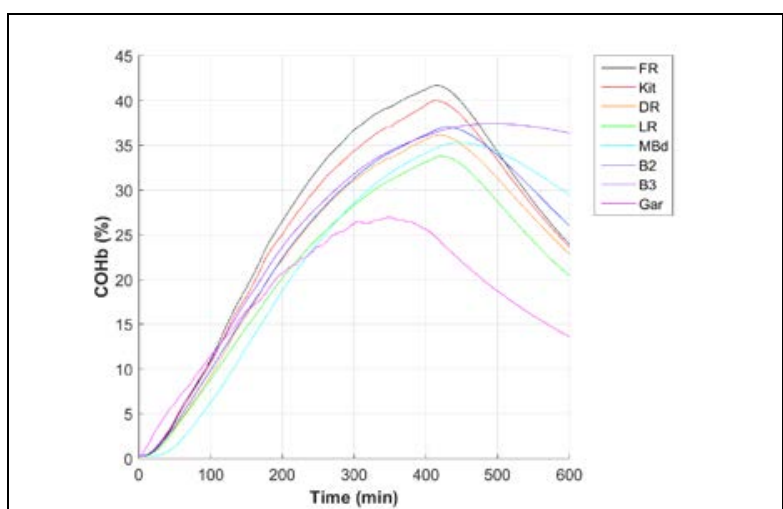

Figure 79e. $\mathrm{COHb}$ of house occupants during Case 32 Test 2 - G6 G300.

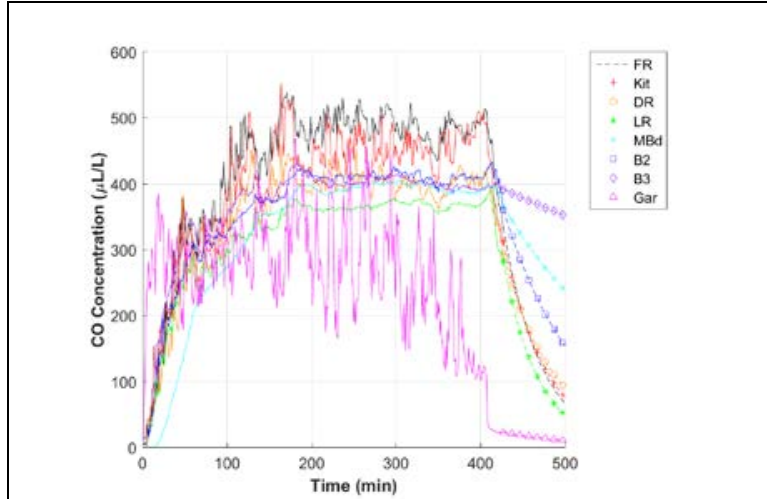

Figure 79d. CO concentration (measured \& projected) in the house during Case 32 Test 2 - G6 G300. 


\section{Case 32 Test 3 - G6 G300}

The following figures illustrate the results for Case 32 Test 3 applying the G300 shutoff criteria. The G6 generator was fully fueled, positioned in the garage (centered, with the exhaust facing towards the family room), and tested at $100 \%$ load $(6,000 \mathrm{~W}$ ). The interior person door (from garage to utility room) was open $10 \mathrm{~cm}$, the garage bay door was fully open and the exterior person door (from garage to backyard) was closed. The shutoff sensor was not activated and the generator ran out of fuel after approximately $414 \mathrm{~min}$. Figure 80a shows the test house layout with generator location, exhaust direction, and sensor array location. Figure 80b shows the CO concentration measured in the garage (by the dedicated room sensor), by the sensor array placed in the garage located as shown in Figure 80a (A1: height $91 \mathrm{~cm}, \mathrm{A2}$ : height $91 \mathrm{~cm}, \mathrm{A3}$ : height $91 \mathrm{~cm}, \mathrm{~A} 4$ : height $91 \mathrm{~cm})$, and by the shutoff sensor located on the generator near the generator's electrical panel. The figure shows the degree of CO uniformity in the area around the generator between the shutoff sensor and the peak CO concentrations measured elsewhere in the garage. Figure 80c shows the $\mathrm{CO}$ concentration measured in each room of the house over the test. Figure 80d shows the CO concentration in the house with each room represented by a single line, which is composed of both a 'solid-line' pattern (showing measured CO concentration) and a 'dotted-line with a symbol' pattern (showing projected CO concentration). Figure 80e shows the calculated COHb profiles of simulated house occupants in each room of the house.

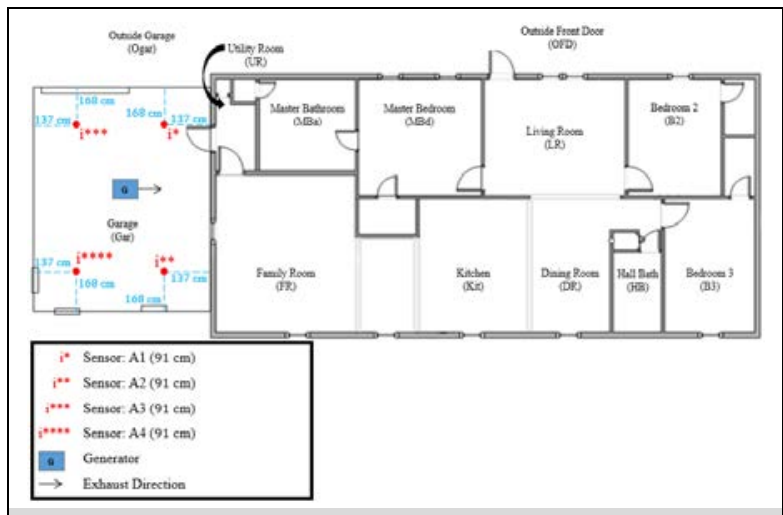

Figure 80a. Generator, exhaust direction, and sensor array layout during Case 32 Test 3 - G6 G300.

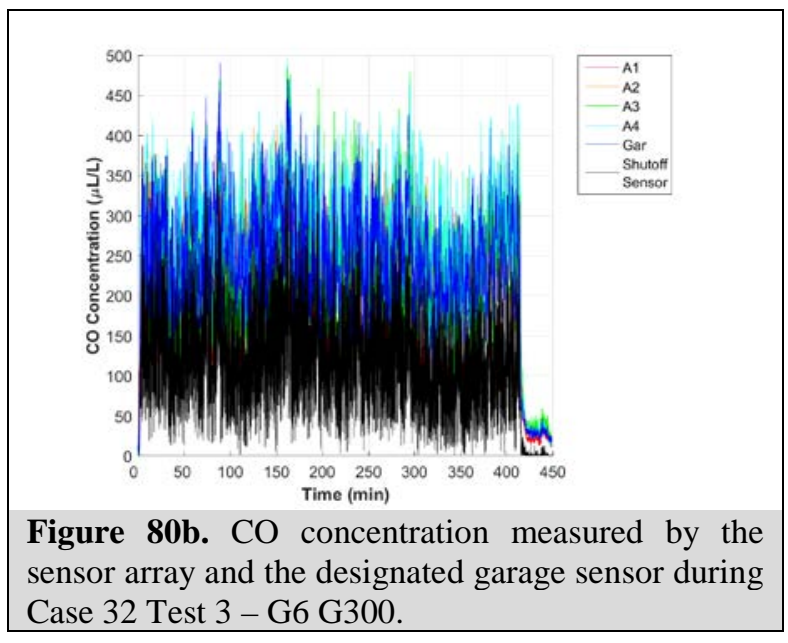



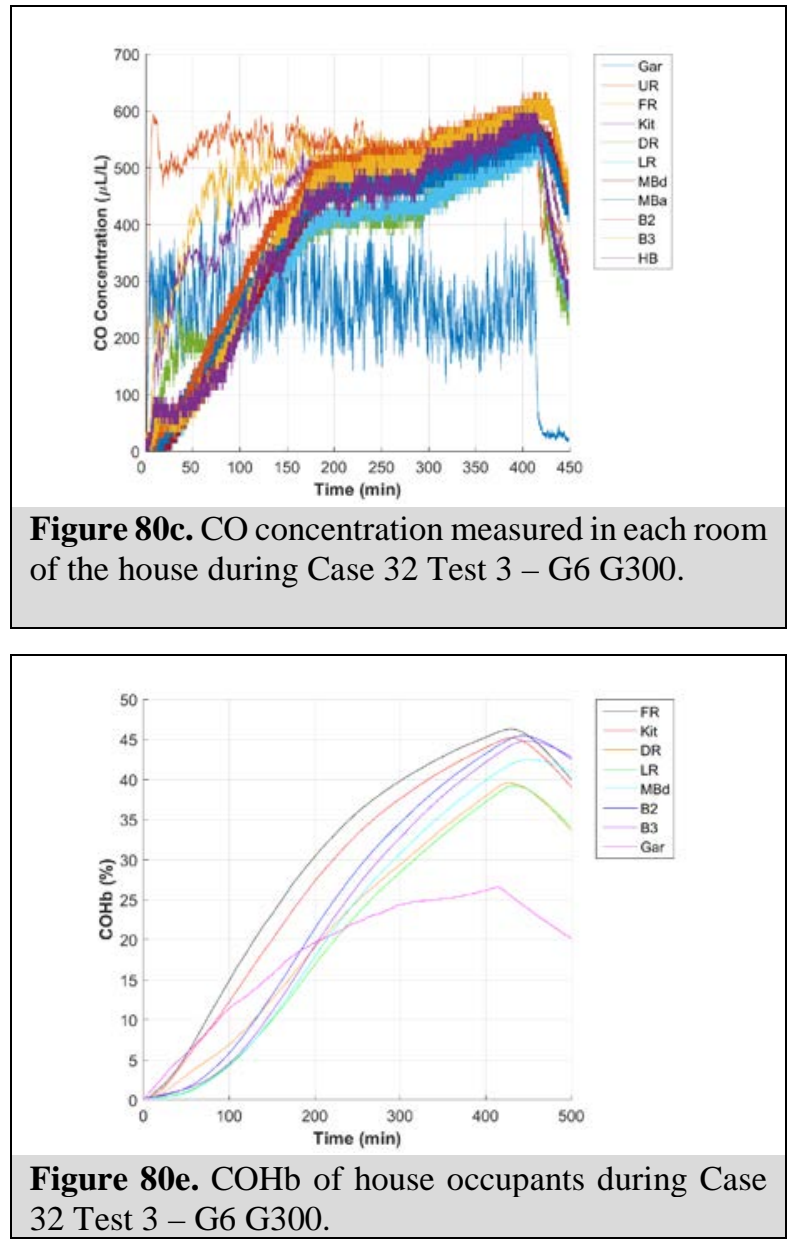

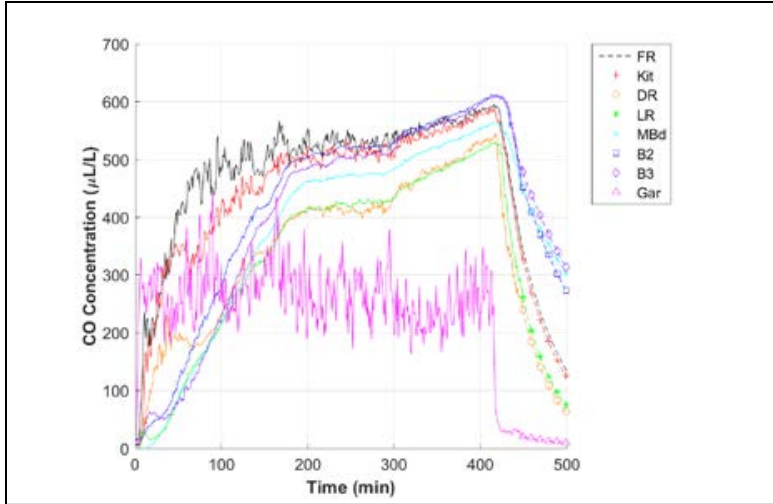

Figure 80d. CO concentration (measured \& projected) in the house during Case 32 Test 3 - G6 G300.

For the Case 32 tests that were run in triplicate (Tests 1, 2, and 3), in which the tests differed only by the environmental conditions, the results were very similar. In Tests 2 and 3, the generator ran for nominally 400 minutes before shutting off upon running out of fuel. The results produced $\mathrm{COHb}$ profiles within $4 \%$ to $5 \%$ of each other. For Test 1 , the generator was manually shutdown at 260 minutes after it appeared the $\mathrm{CO}$ in the house had reached steady state. The resulting $\mathrm{COHb}$ profile was lower than Tests 2 and 3; however, if the generator had been allowed to run as long as in Tests 2 and 3, the COHb profile may have reached approximately the same values. 


\section{Case 32 Test 1 - G7a UL 2201}

The following figures illustrate the results for Case 32 Test 1 applying the UL 2201 shutoff criteria. The G7a generator was fully fueled, positioned in the garage (near the center of the garage with the exhaust facing the wall shared with the family room), and tested at $100 \%$ load $(7,000 \mathrm{~W})$. The interior person door from the garage to the utility room was open $10 \mathrm{~cm}$ and the garage bay door was fully open during the test. The shutoff sensor did not meet the criteria to activate the algorithm that triggers the generator shutoff and the generator ran out of fuel after approximately $373 \mathrm{~min}$. Figure 81a shows the test house layout with generator location, generator exhaust direction, and sensor array location. Figure 81b shows the CO concentration measured in the garage (by the dedicated room sensor), by four sensors located in the garage (A1, A2, A3, and A4: height 91.0 $\mathrm{cm}$ ), and by the shutoff sensor located on the generator near the generator's electrical panel. The timeframe of the figure has been selected to show the degree of CO uniformity in the area around the generator between the shutoff sensor and the peak CO concentrations measured elsewhere in the garage near the time of shutoff. Figure 81c shows the $\mathrm{CO}$ concentration measured in each room of the house over the test. Figure 81d shows the CO concentration in the house with each room represented by a single line, which is composed of both a 'solid-line' pattern (showing measured CO concentration) and a 'dotted-line with a symbol' pattern (showing projected CO concentration). Figure 81e shows the calculated $\mathrm{COHb}$ profiles of simulated house occupants in each room of the house.

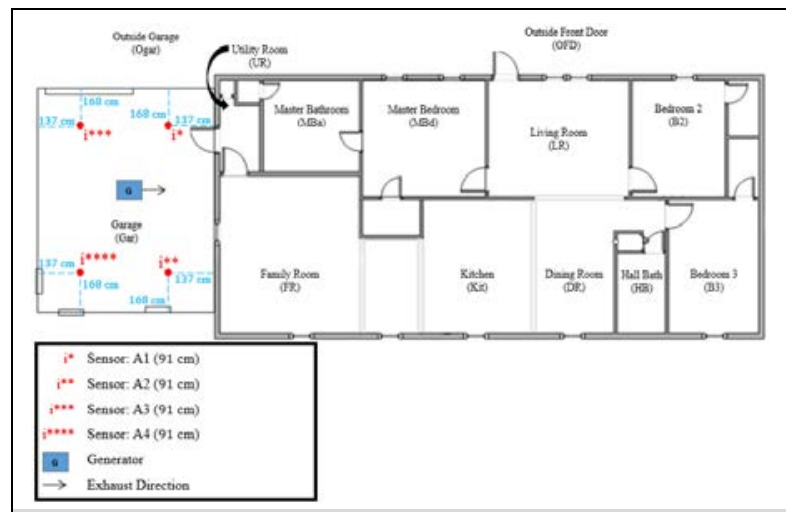

Figure 81a. Generator, exhaust direction, and sensor array layout during Case 32 Test 1 - G7a UL 2201.

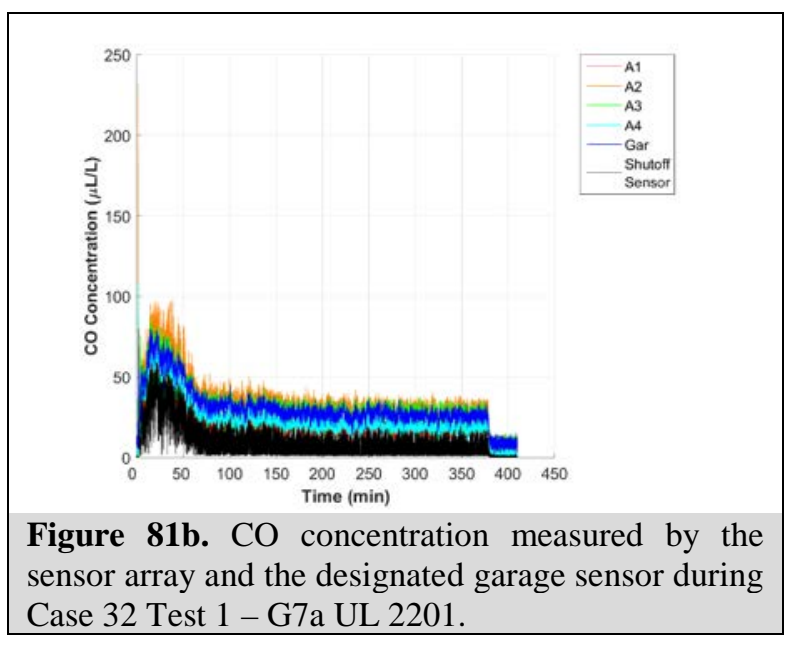

Figure 81b. CO concentration measured by the sensor array and the designated garage sensor during Case 32 Test 1 - G7a UL 2201 


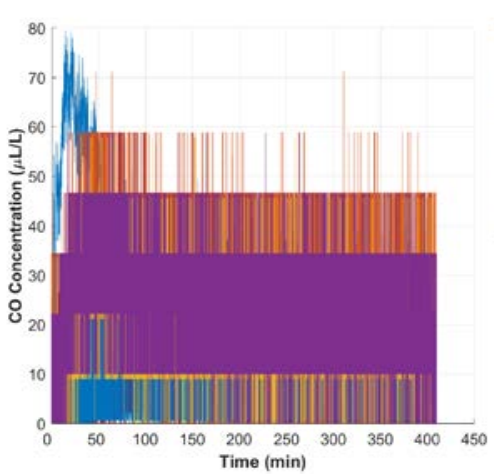

Figure 81c. CO concentration measured in each room of the house during Case 32 Test 1 - G7a UL 2201.

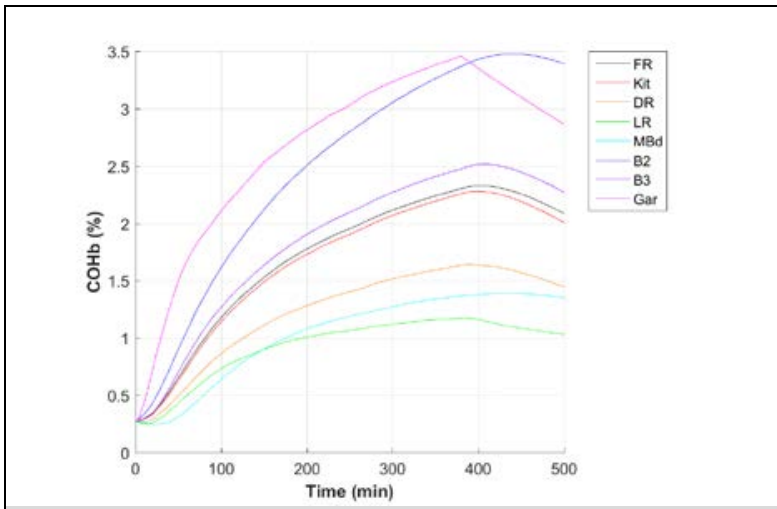

Figure 81e. $\mathrm{COHb}$ of house occupant during Case 32 Test 1 - G7a UL 2201.

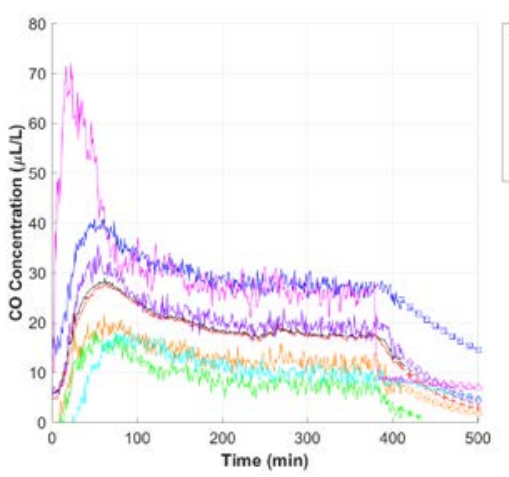

Figure 81d. CO concentration (measured \& projected) in the house during Case 32 Test 1 - G7a UL 2201. 


\section{Case 33 Test 1 - G2 G300}

The following figures illustrate the results for Case 33 Test 1 applying the G300 shutoff criteria. The G2 generator was fully fueled, positioned in the garage (centered, with the exhaust facing towards the family room), and tested at $10 \%$ load $(160 \mathrm{~W})$. The interior person door (from garage to utility room) was open $10 \mathrm{~cm}$ and both the exterior person door (from garage to backyard) and the garage bay door were closed. The shutoff sensor activated the algorithm to shut off the generator after approximately $15 \mathrm{~min}$ due to a sensor measurement of $400 \mu \mathrm{L} / \mathrm{L}$ for a $10 \mathrm{~min}$ average. Figure 82a shows the test house layout with generator location, exhaust direction, and sensor array location. Figure 82b shows the CO concentration measured in the garage (by the dedicated room sensor), by the sensor array placed in the garage located as shown in Figure 82a (A1: height $91 \mathrm{~cm}, \mathrm{A2}$ : height $91 \mathrm{~cm}, \mathrm{A3}$ : height $91 \mathrm{~cm}, \mathrm{~A} 4$ : height $91 \mathrm{~cm}$ ), and by the shutoff sensor located on the generator near the generator's electrical panel. The timeframe of the figure has been selected to show the degree of CO uniformity in the area around the generator between the shutoff sensor and the peak CO concentrations measured elsewhere in the garage near the time of shutoff. Figure 82c shows the CO concentration measured in each room of the house over the test. Figure 82d shows the calculated $\mathrm{COHb}$ profiles of simulated house occupants in each room of the house. The calculated COHb for simulated house occupants did not exceed $5 \%$ but did not reach a peak during the test.

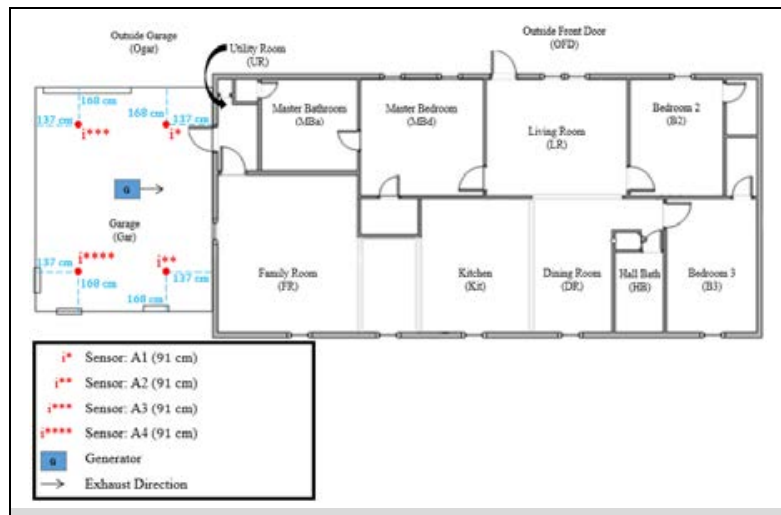

Figure 82a. Generator, exhaust direction, and sensor array layout during Case 33 Test 1 - G2 G300.

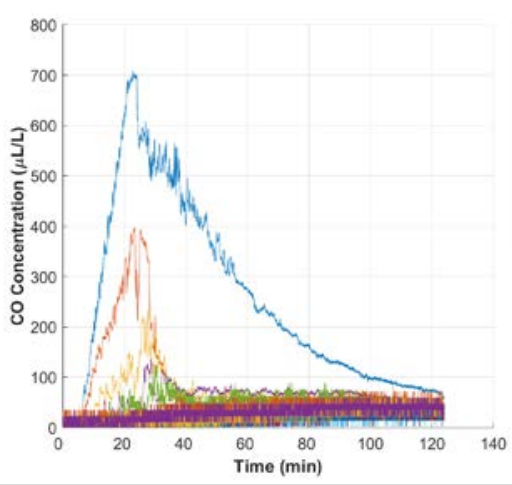

Figure 82c. CO concentration measured in each room of the house during Case 33 Test 1 - G2 G300.

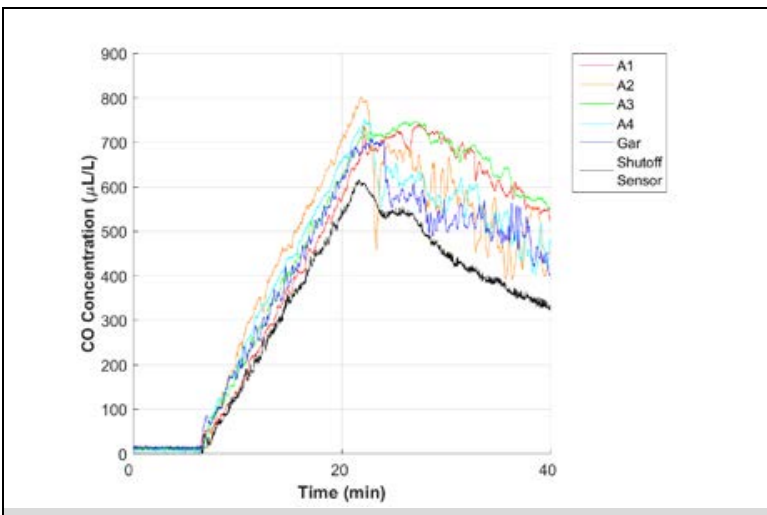

Figure 82b. CO concentration measured by the sensor array and the designated garage sensor during Case 33 Test 1 - G2 G300.

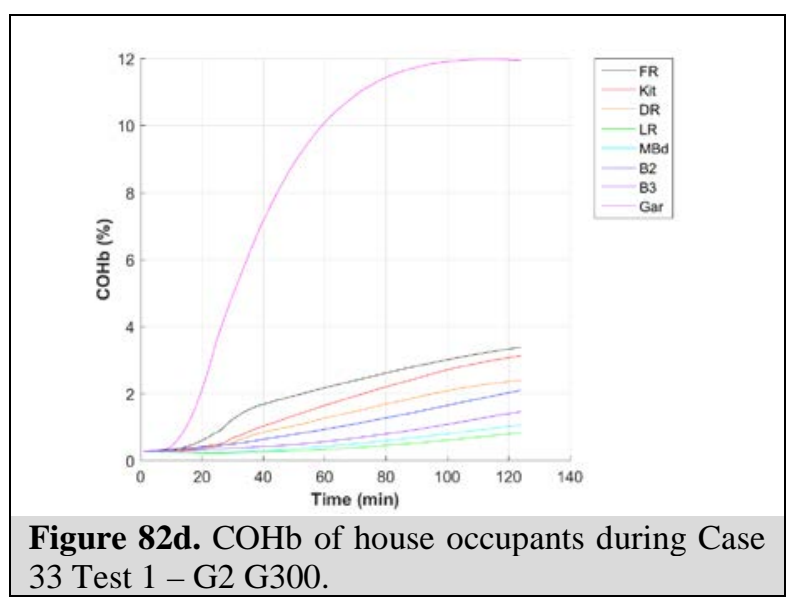




\section{Case 33 Test 1 - G2 UL 2201}

The following figures illustrate the results for Case 33 Test 1 applying the UL 2201 shutoff criteria. The G2 generator was fully fueled, positioned in the garage (centered, with the exhaust facing towards the family room), and tested at $10 \%$ load $(160 \mathrm{~W})$. The interior person door (from garage to utility room) was open $10 \mathrm{~cm}$ and both the exterior person door (from garage to backyard) and the garage bay door were closed. The shutoff sensor activated the algorithm to shut off the generator after approximately 9 min due to a sensor measurement of $150 \mu \mathrm{L} / \mathrm{L}$ for a $10 \mathrm{~min}$ average. Figure 83a shows the test house layout with generator location, exhaust direction, and sensor array location. Figure 83b shows the CO concentration measured in the garage (by the dedicated room sensor), by the sensor array placed in the garage located as shown in Figure 83a (A1: height $91 \mathrm{~cm}, \mathrm{A2}$ : height $91 \mathrm{~cm}$, A3: height $91 \mathrm{~cm}, \mathrm{~A} 4$ : height $91 \mathrm{~cm}$ ), and by the shutoff sensor located on the generator near the generator's electrical panel. The timeframe of the figure has been selected to show the degree of CO uniformity in the area around the generator between the shutoff sensor and the peak CO concentrations measured elsewhere in the garage near the time of shutoff. Figure 83c shows the $\mathrm{CO}$ concentration measured in each room of the house over the test. Figure 83d shows the calculated $\mathrm{COHb}$ profiles of simulated house occupants in each room of the house. The calculated COHb for simulated house occupants did not exceed $5 \%$ but did not peak during the test.

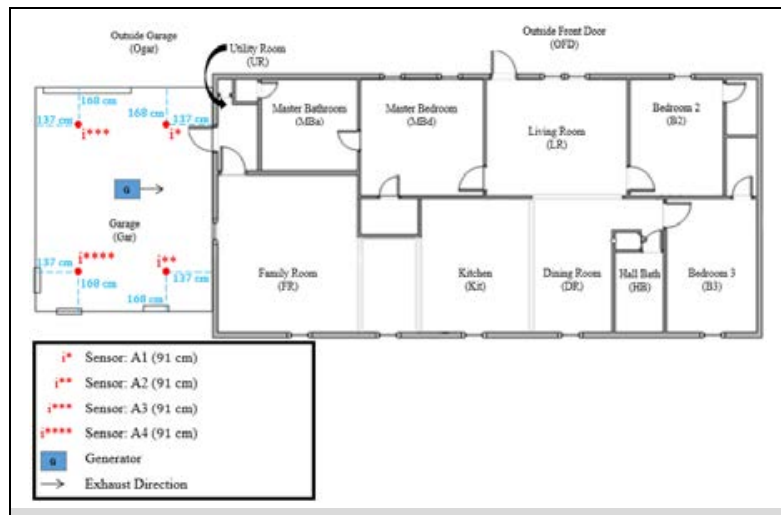

Figure 83a. Generator, exhaust direction, and sensor array layout during Case 33 Test 1 - G2 UL 2201.

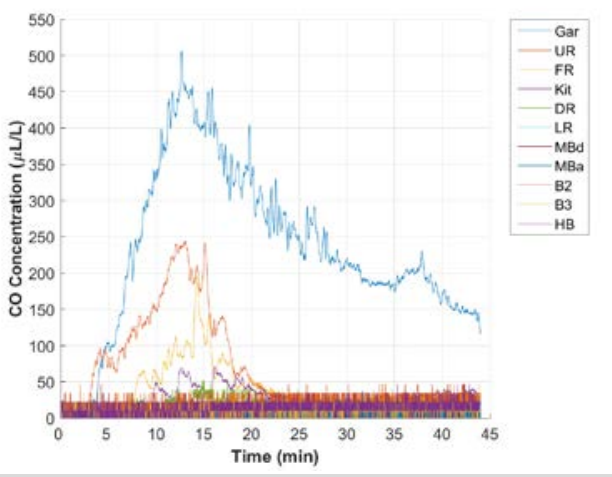

Figure 83c. CO concentration measured in each room of the house during Case 33 Test 1 - G2 UL 2201.

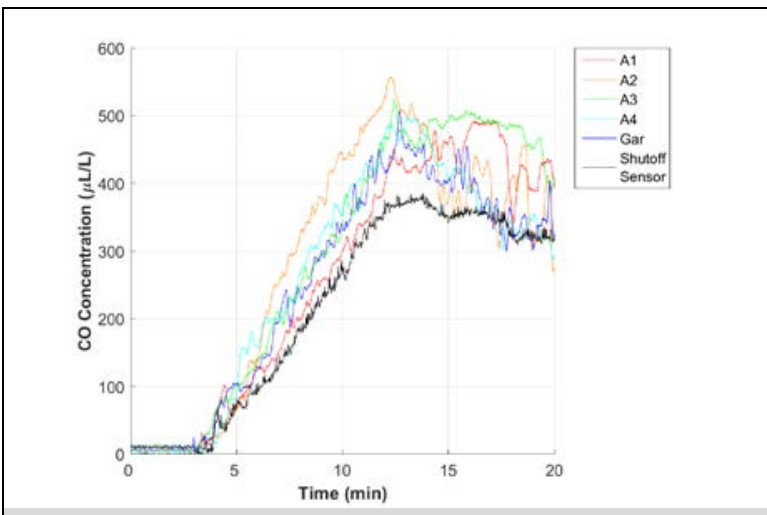

Figure 83b. CO concentration measured by the sensor array and the designated garage sensor during Case 33 Test 1 - G2 UL 2201.

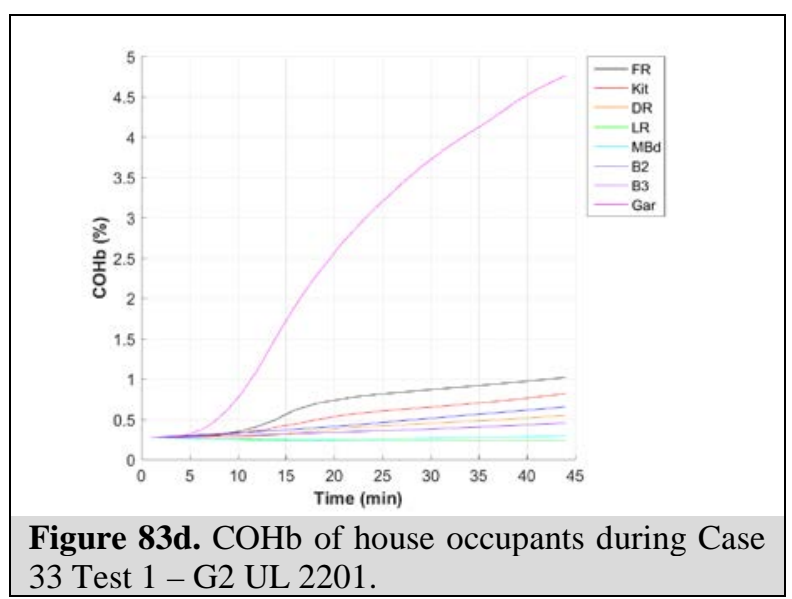




\section{Case 34 Test 1 - G15 G300}

The following figures illustrate the results for Case 34 Test 1 applying the G300 shutoff criteria. The G15 generator was fully fueled, positioned in the garage (centered, with the exhaust facing the rear wall), and tested at $90 \%$ load $(13,500 \mathrm{~W})$. The interior person door (from garage to utility room) was open $10 \mathrm{~cm}$, the garage bay door was fully open, and the exterior person door (from garage to backyard) was closed. The shutoff sensor activated the algorithm to shut off the generator after approximately 4 min due to an instantaneous sensor measurement of $800 \mu \mathrm{L} / \mathrm{L}$. Figure 84a shows the test house layout with generator location, exhaust direction, and sensor array location. Figure 84b shows the CO concentration measured in the garage (by the dedicated room sensor), by the sensor array placed in the garage located as shown in Figure 84a (A1: height $91 \mathrm{~cm}$, A2: height $91 \mathrm{~cm}$, A3: height $91 \mathrm{~cm}, \mathrm{~A} 4$ : height $91 \mathrm{~cm}$ ), and by the shutoff sensor located on the generator near the generator's electrical panel. The timeframe of the figure has been selected to show the degree of CO uniformity in the area around the generator between the shutoff sensor and the peak CO concentrations measured elsewhere in the garage near the time of shutoff. Figure 84c shows the $\mathrm{CO}$ concentration measured in each room of the house over the test. Figure 84d shows the calculated $\mathrm{COHb}$ profiles of simulated house occupants in each room of the house. The calculated $\mathrm{COHb}$ for simulated house occupants did not exceed $5 \%$ but did not reach a peak during the test.

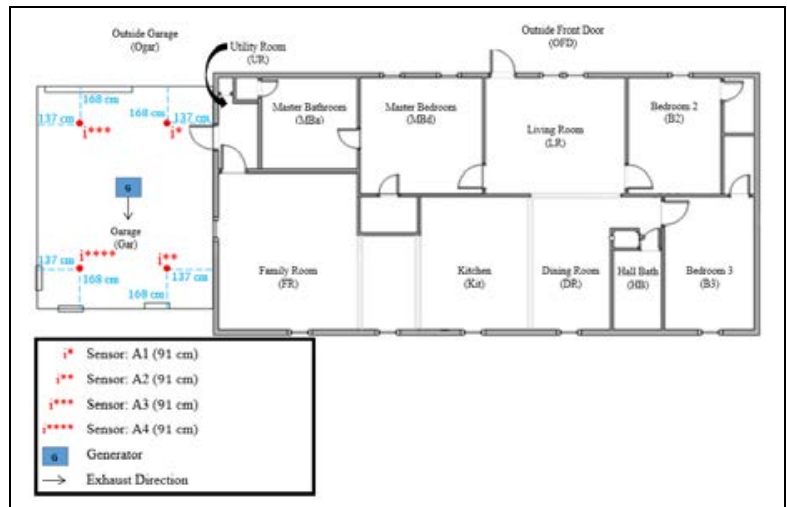

Figure 84a. Generator, exhaust direction, and sensor array layout during Case 34 Test 1 - G15 G300.

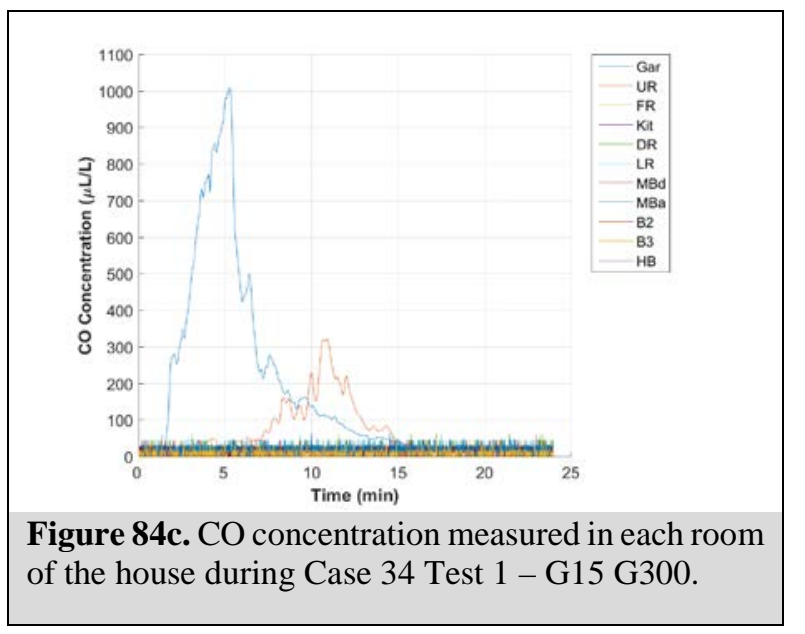

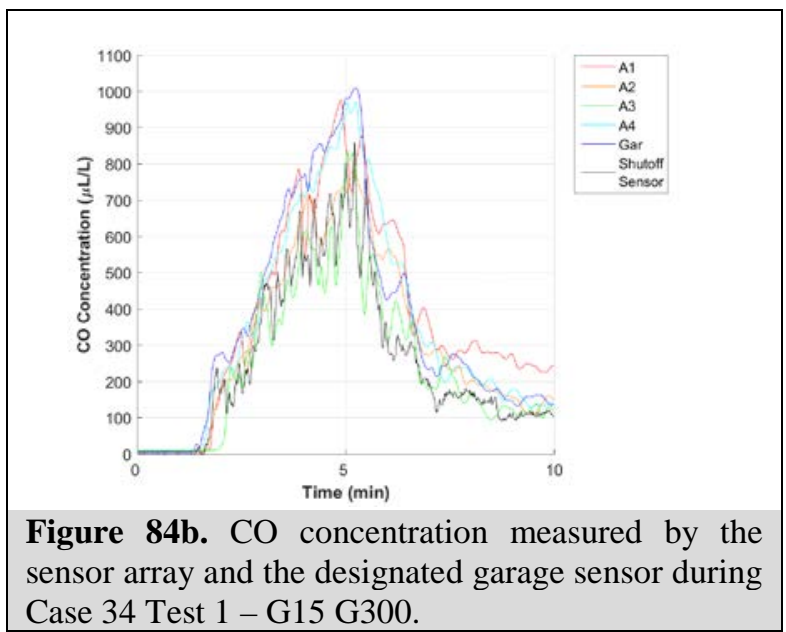

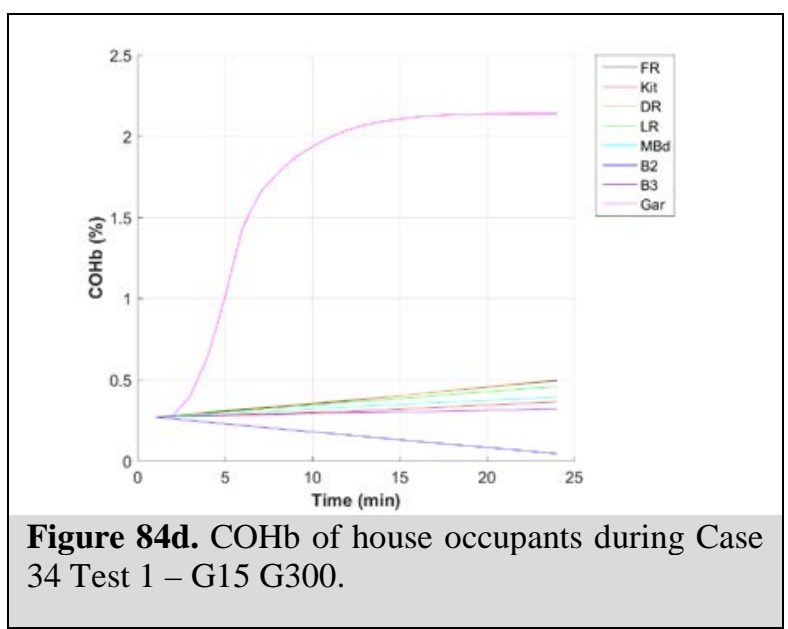




\section{Case 35 Test 1 - G15 G300}

The following figures illustrate the results for Case 35 Test 1 applying the G300 shutoff criteria. The G15 generator was fully fueled, positioned in the garage (centered, with the exhaust facing towards the rear wall), and tested at $25 \%$ load $(3,750 \mathrm{~W})$. The interior person door (from garage to utility room) was open $10 \mathrm{~cm}$ and the garage bay door was fully open; the exterior person door (from garage to backyard) was closed. The shutoff sensor activated the algorithm to shut off the generator after approximately $113 \mathrm{~min}$ due to a sensor measurement of $400 \mu \mathrm{L} / \mathrm{L}$ for a $10 \mathrm{~min}$ average. Figure 85a shows the test house layout with generator location, exhaust direction, and sensor array location. Figure 85b shows the CO concentration measured in the garage (by the dedicated room sensor), by the sensor array placed in the garage located as shown in Figure 85a (A1: height $91 \mathrm{~cm}, \mathrm{A2}$ : height $91 \mathrm{~cm}$, A3: height $91 \mathrm{~cm}, \mathrm{~A} 4$ : height $91 \mathrm{~cm}$ ), and by the shutoff sensor located on the generator near the generator's electrical panel. The timeframe of the figure has been selected to show the degree of CO uniformity in the area around the generator between the shutoff sensor and the peak CO concentrations measured elsewhere in the garage near the time of shutoff. Figure 85c shows the $\mathrm{CO}$ concentration measured in each room of the house over the test. Figure 85d shows the $\mathrm{CO}$ concentration in the house with each room represented by a single line, which is composed of both a 'solid-line' pattern (showing measured CO concentration) and a 'dotted-line with a symbol' pattern (showing projected CO concentration). Figure 85e shows the calculated $\mathrm{COHb}$ profiles of simulated house occupants in each room of the house.

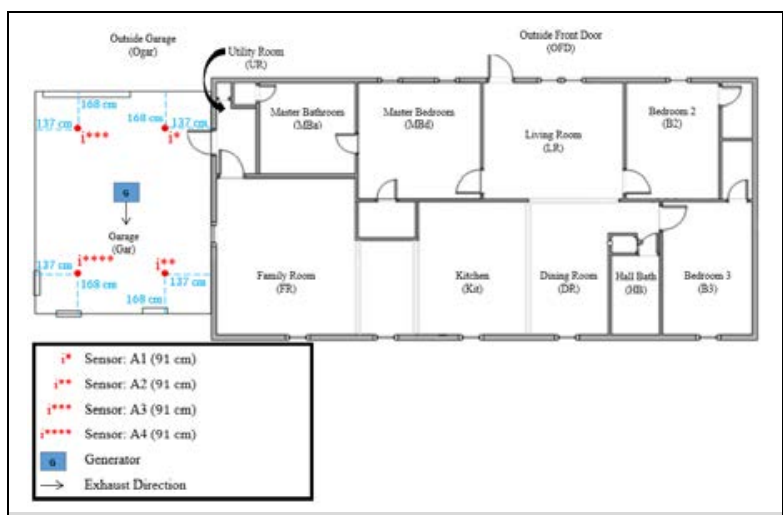

Figure 85a. Generator, exhaust direction, and sensor array layout during Case 35 Test 1 - G15 G300.

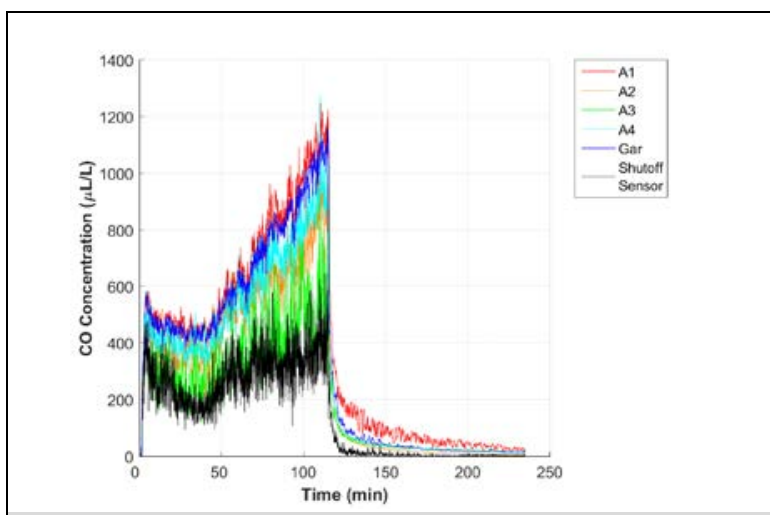

Figure 85b. CO concentration measured by the sensor array and the designated garage sensor during Case 35 Test 1 - G15 G300. 


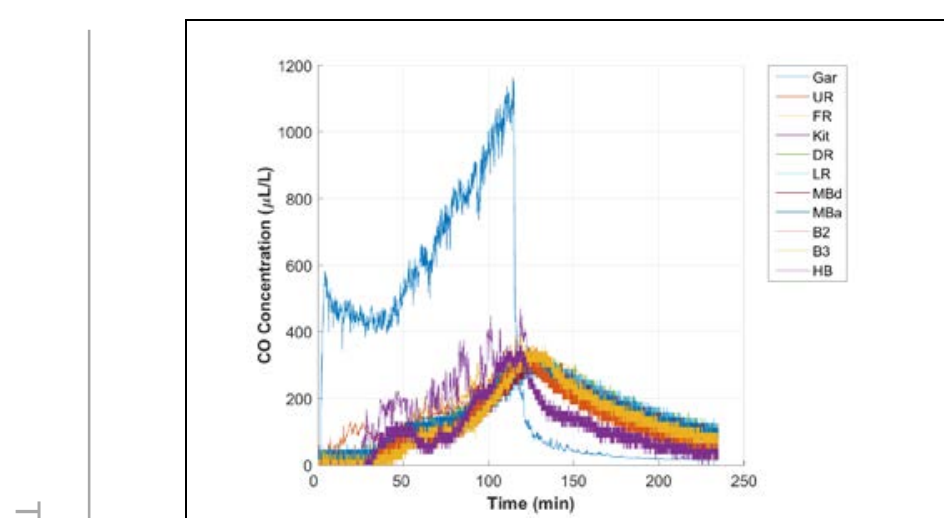

Figure 85c. CO concentration measured in each room of the house during Case 35 Test 1 - G15 G300.

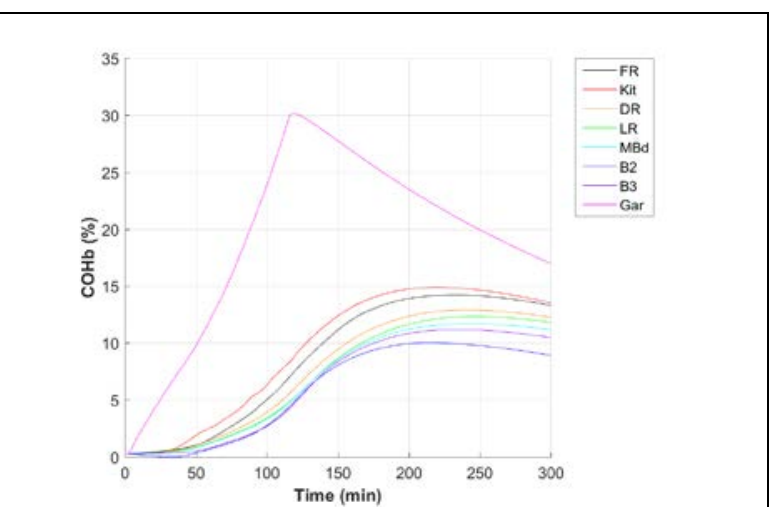

Figure 85e. $\mathrm{COHb}$ of house occupants during Case 35 Test 1 - G15 G300.

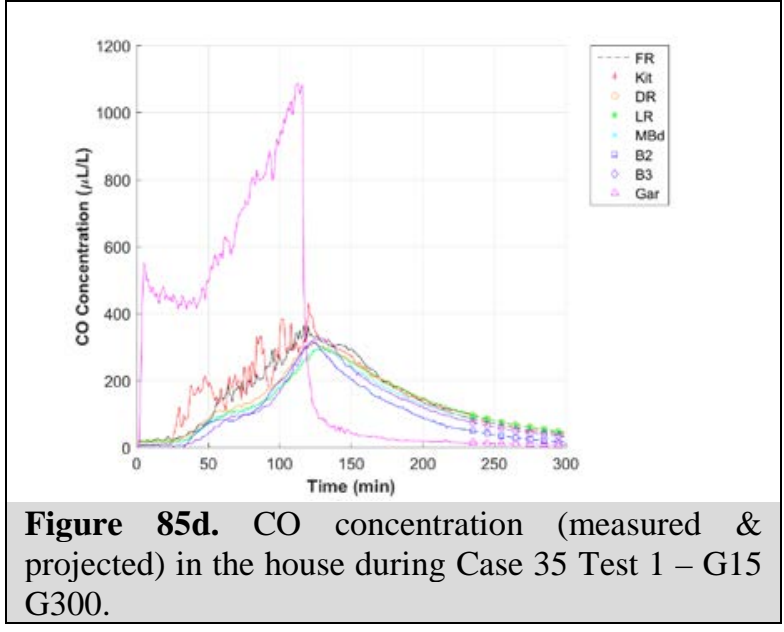

Figure 85d. CO concentration (measured \& G300. 


\section{Case 35 Test 1 - G15 G300 Restart}

The following figures illustrate the results for Restart Case 35 Test 1 applying the G300 shutoff criteria. The G15 generator was fully fueled, positioned in the garage (centered, with the exhaust facing towards the rear wall), and tested at $25 \%$ load $(3,750 \mathrm{~W}$ ). The interior person door (from garage to utility room) was open $10 \mathrm{~cm}$, the garage bay door was fully open and the exterior person door (from garage to backyard) was closed. The shutoff sensor was not activated, and the generator was manually shutdown after approximately 413 min due to CO concentration less than half the shutoff criteria at the shutoff sensor and the time of day. Figure 86a shows the test house layout with generator location, exhaust direction, and sensor array location. Figure 86b shows the CO concentration measured in the garage (by the dedicated room sensor), by the sensor array placed in the garage located as shown in Figure 86a (A1: height $91 \mathrm{~cm}, \mathrm{A2}$ : height $91 \mathrm{~cm}$, A3: height 91 $\mathrm{cm}$, A4: height $91 \mathrm{~cm}$ ), and by the shutoff sensor located on the generator near the generator's electrical panel. The timeframe of the figure has been selected to show the degree of CO uniformity in the area around the generator between the shutoff sensor and the peak CO concentrations measured elsewhere in the garage near the time of shutoff. Figure 86c shows the CO concentration measured in each room of the house over the test. Figure 86d shows the calculated COHb profiles of simulated house occupants in each room of the house.
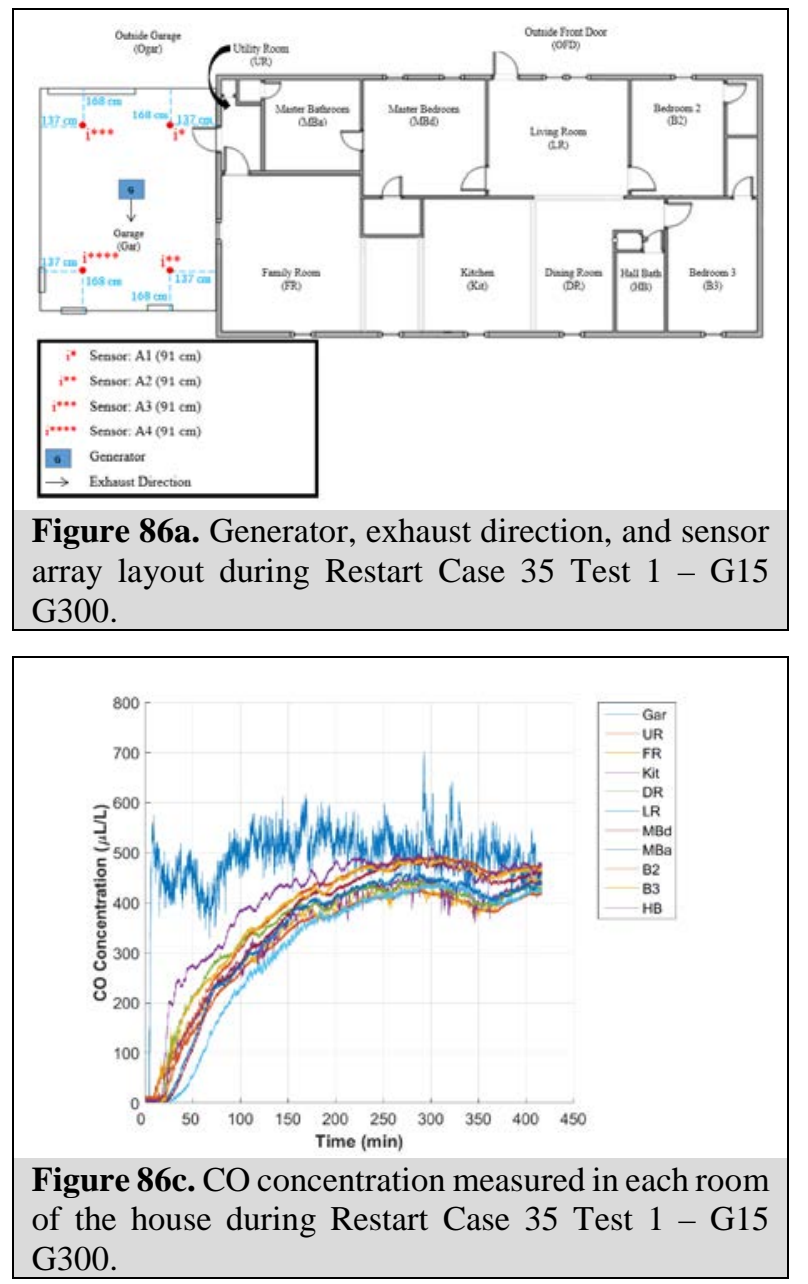
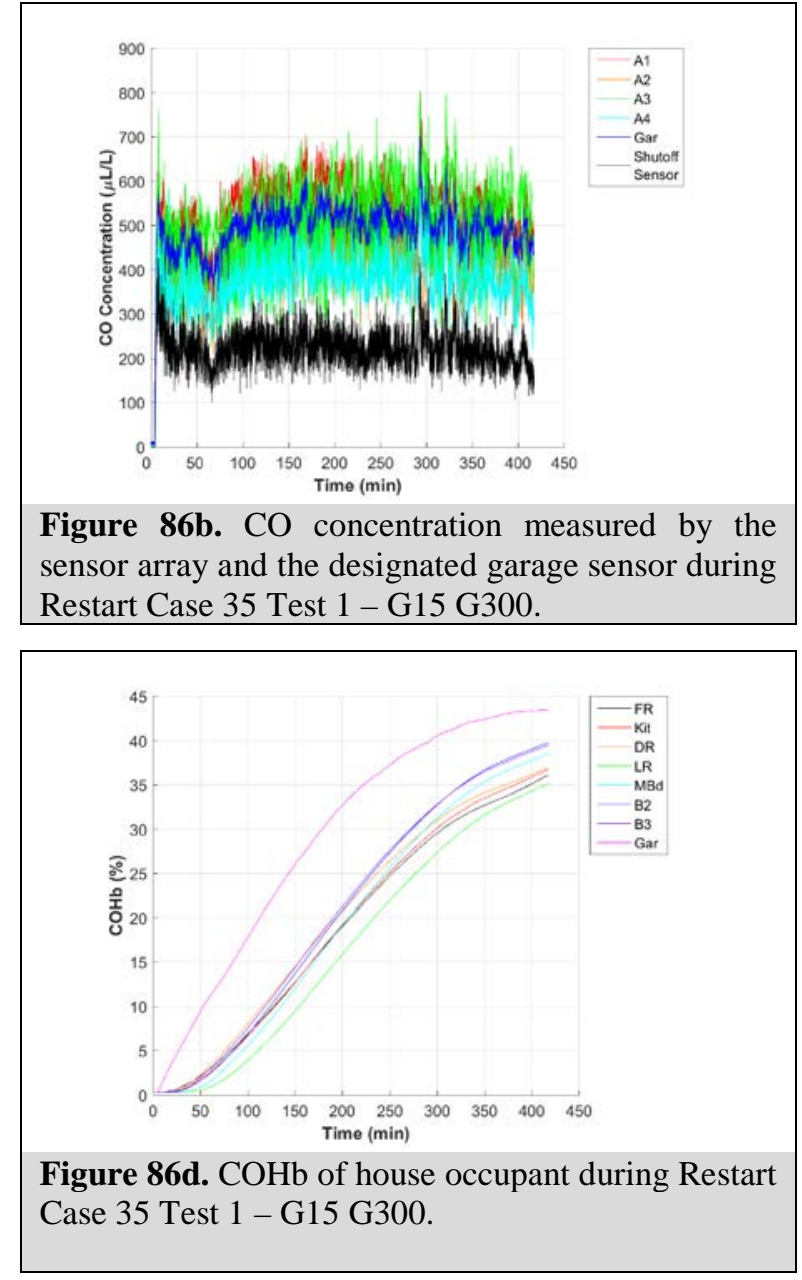


\section{Case 36 Test 1 - G6 G300}

The following figures illustrate the results for Case 36 Test 1 applying the G300 shutoff criteria. The G6 generator was fully fueled, positioned in the garage (centered, with the exhaust facing towards the rear wall), and tested at $100 \%$ load $(6,000 \mathrm{~W})$. The interior person door (from garage to utility room) was open $10 \mathrm{~cm}$, the garage bay door was fully open and the exterior person door (from garage to backyard) was closed. The shutoff sensor was not activated and the generator ran out of fuel after approximately $406 \mathrm{~min}$. Figure 87a shows the test house layout with generator location, exhaust direction, and sensor array location. Figure 87b shows the CO concentration measured in the garage (by the dedicated room sensor), by the sensor array placed in the garage located as shown in Figure 87a (A1: height $91 \mathrm{~cm}, \mathrm{A2}$ : height $91 \mathrm{~cm}, \mathrm{A3}$ : height $91 \mathrm{~cm}, \mathrm{~A} 4$ : height $91 \mathrm{~cm})$, and by the shutoff sensor located on the generator near the generator's electrical panel. The figure shows the degree of CO uniformity in the area around the generator between the shutoff sensor and the peak CO concentrations measured elsewhere in the garage Figure 87c shows the $\mathrm{CO}$ concentration measured in each room of the house over the test. Figure 87d shows the CO concentration in the house with each room represented by a single line, which is composed of both a 'solid-line' pattern (showing measured CO concentration) and a 'dotted-line with a symbol' pattern (showing projected CO concentration). Figure 87e shows the calculated COHb profiles of simulated house occupants in each room of the house.

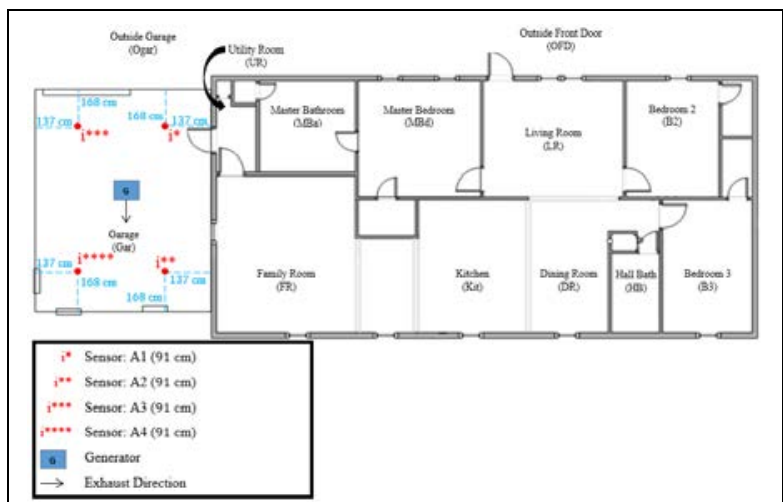

Figure 87a. Generator, exhaust direction, and sensor array layout during Case 36 Test 1 - G6 G300.

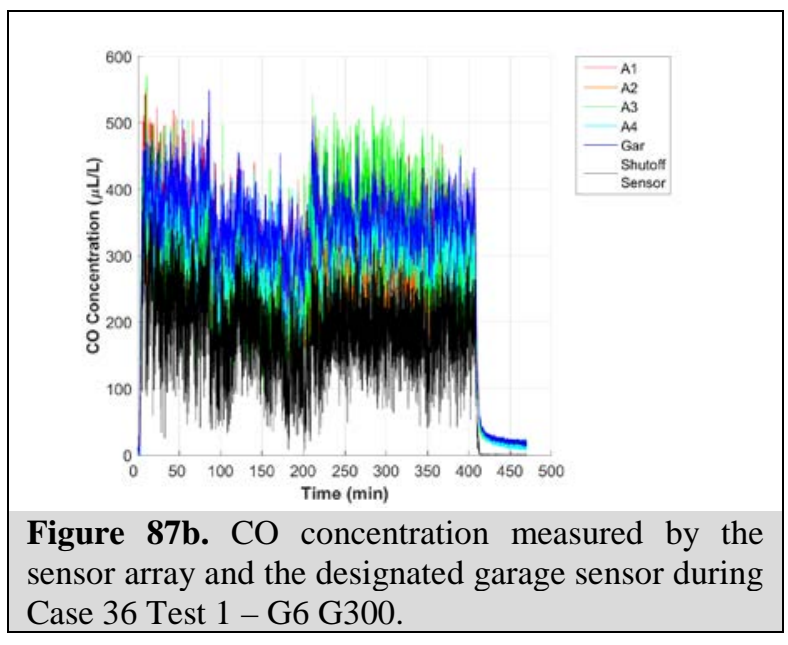




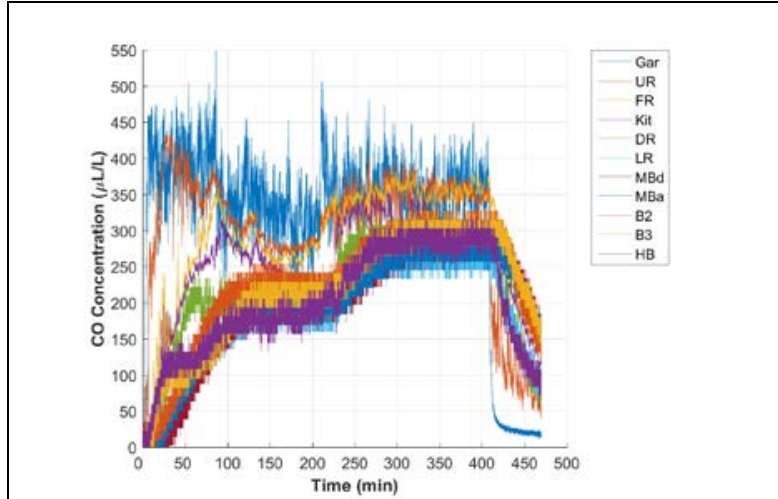

Figure 87c. CO concentration measured in each room of the house during Case 36 Test 1 - G6 G300.

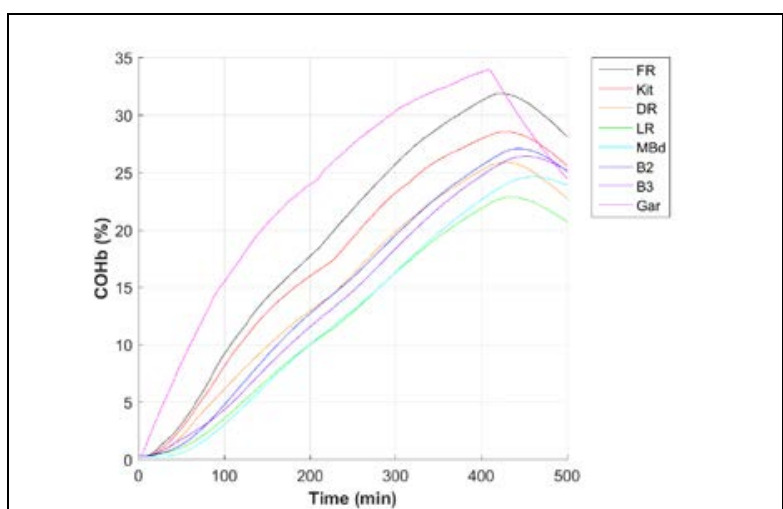

Figure 87e. $\mathrm{COHb}$ of house occupants during Case 36 Test $1-$ G6 G300.

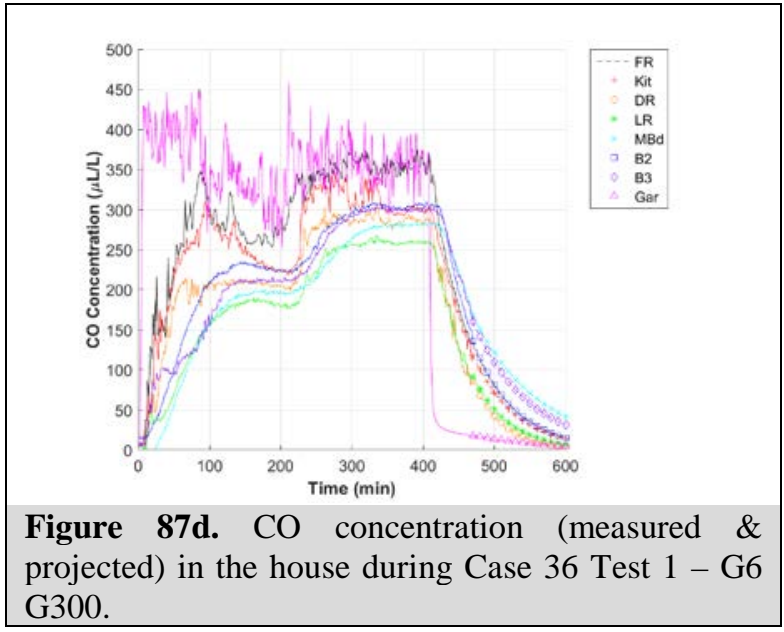

Figure 87d. CO concentration (measured \& G300. 


\section{Case 37 Test 1 - G15 G300}

The following figures illustrate the results for Case 37 Test 1 applying the G300 shutoff criteria. The G15 generator was fully fueled, positioned in the garage (15 cm inside the garage bay door, centered to the door with the exhaust facing towards the rear wall), and tested at $90 \%$ load (13,500 $\mathrm{W}$ ). The interior person door (from garage to utility room) was open $10 \mathrm{~cm}$, the garage bay door was open $61 \mathrm{~cm}$, and the exterior person door (from garage to backyard) was closed. The shutoff sensor activated the algorithm to shut off the generator after approximately 3 min due to an instantaneous sensor measurement of $800 \mu \mathrm{L} / \mathrm{L}$. Figure 88a shows the test house layout with generator location, exhaust direction, and sensor array location. Figure 88b shows the CO concentration measured in the garage (by the dedicated room sensor), by the sensor array placed in the garage located as shown in Figure 88a (A1: height $91 \mathrm{~cm}, \mathrm{A2}$ : height $91 \mathrm{~cm}$, A3: height 91 $\mathrm{cm}$, A4: height $91 \mathrm{~cm}$ ), and by the shutoff sensor located on the generator near the generator's electrical panel. The timeframe of the figure has been selected to show the degree of CO uniformity in the area around the generator between the shutoff sensor and the peak CO concentrations measured elsewhere in the garage near the time of shutoff. Figure 88c shows the CO concentration measured in each room of the house over the test. Figure 88d shows the calculated COHb profiles of simulated house occupants in each room of the house. The calculated $\mathrm{COHb}$ for simulated house occupants did not exceed $5 \%$ but did not reach a peak during the test.

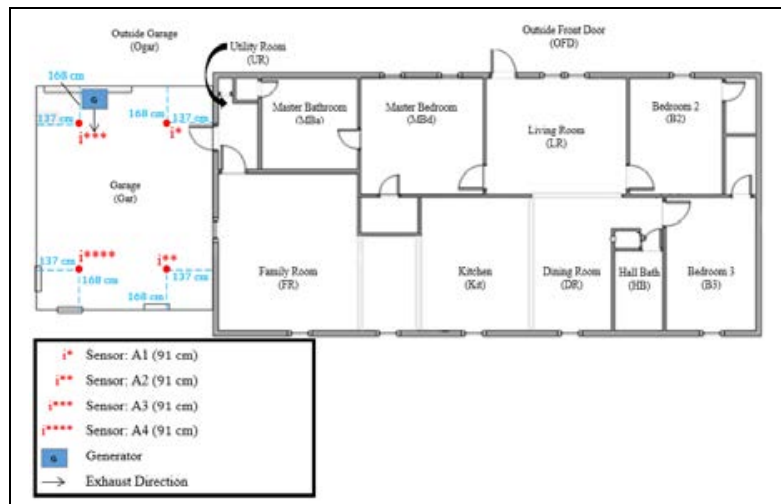

Figure 88a. Generator, exhaust direction, and sensor array layout during Case 37 Test 1 - G15 G300.

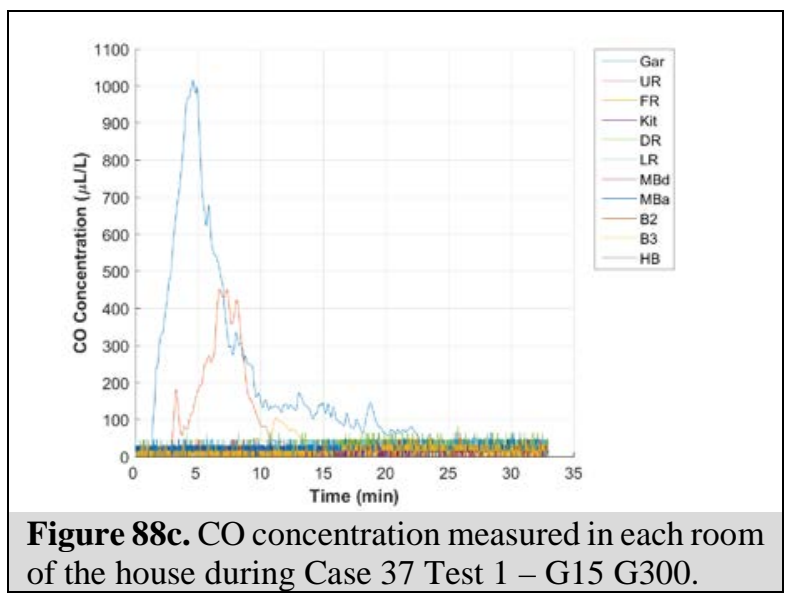

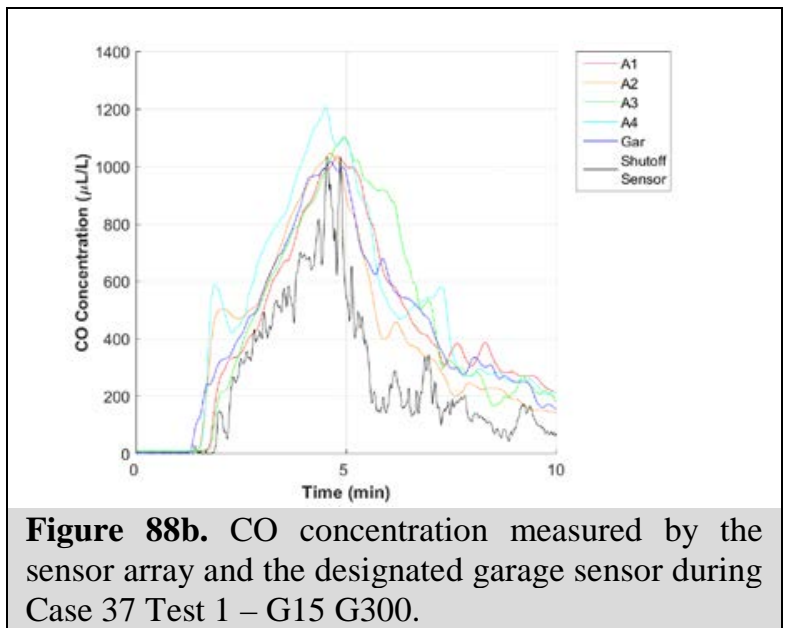

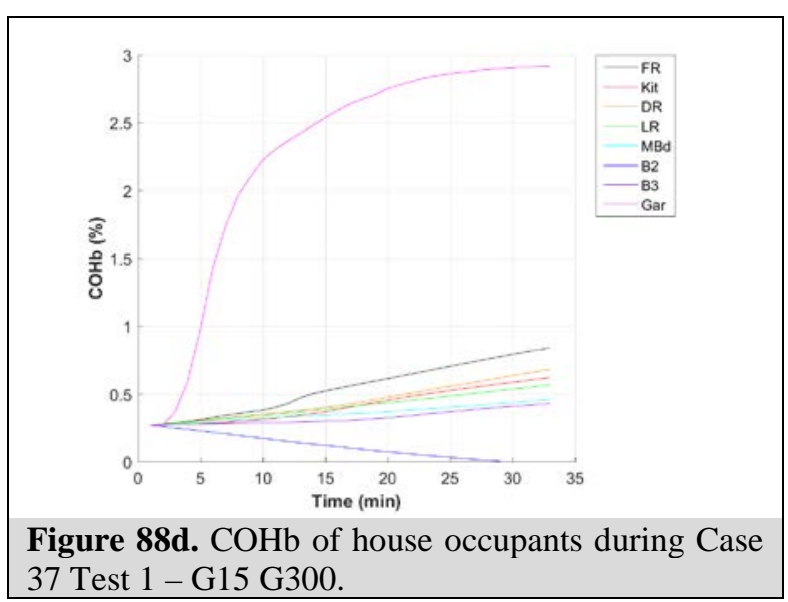




\section{Case 38 Test 1 - G15 G300}

The following figures illustrate the results for Case 38 Test 1 applying the G300 shutoff criteria. The G15 generator was fully fueled, positioned in the garage (15 cm inside the garage bay door, centered to the door with the exhaust facing towards the rear wall), and tested at $25 \%$ load (3,750 $\mathrm{W}$ ). The interior person door (from garage to utility room) was open $10 \mathrm{~cm}$, the garage bay door was open $61 \mathrm{~cm}$, and the exterior person door (from garage to backyard) was closed. The shutoff sensor activated the algorithm to shut off the generator after approximately 10 min due to a sensor measurement of $400 \mu \mathrm{L} / \mathrm{L}$ for a $10 \mathrm{~min}$ average. Figure 89a shows the test house layout with generator location, exhaust direction, and sensor array location. Figure 89b shows the CO concentration measured in the garage (by the dedicated room sensor), by the sensor array placed in the garage located as shown in Figure 89a (A1: height $91 \mathrm{~cm}, \mathrm{A2}$ : height $91 \mathrm{~cm}$, A3: height 91 $\mathrm{cm}$, A4: height $91 \mathrm{~cm}$ ), and by the shutoff sensor located on the generator near the generator's electrical panel. The timeframe of the figure has been selected to show the degree of CO uniformity in the area around the generator between the shutoff sensor and the peak CO concentrations measured elsewhere in the garage near the time of shutoff. Figure 89c shows the CO concentration measured in each room of the house over the test. Figure 89d shows the calculated COHb profiles of simulated house occupants in each room of the house. The calculated $\mathrm{COHb}$ for simulated house occupants did not exceed $5 \%$ but did not reach a peak during the test.

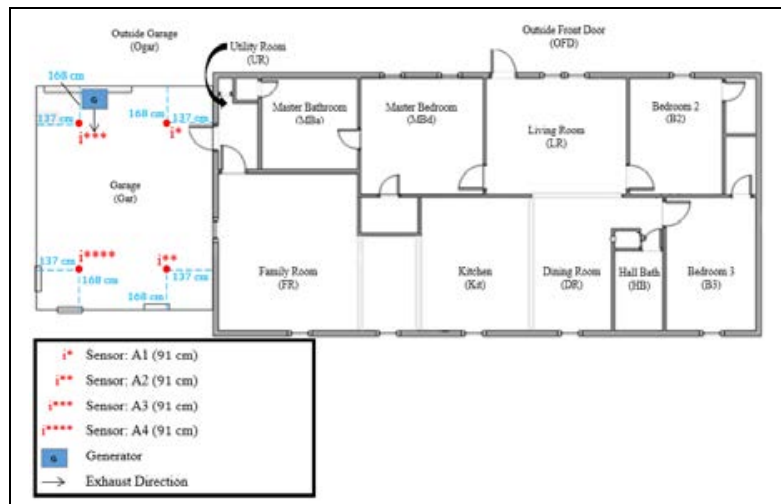

Figure 89a. Generator, exhaust direction, and sensor array layout during Case 38 Test 1 - G15 G300.

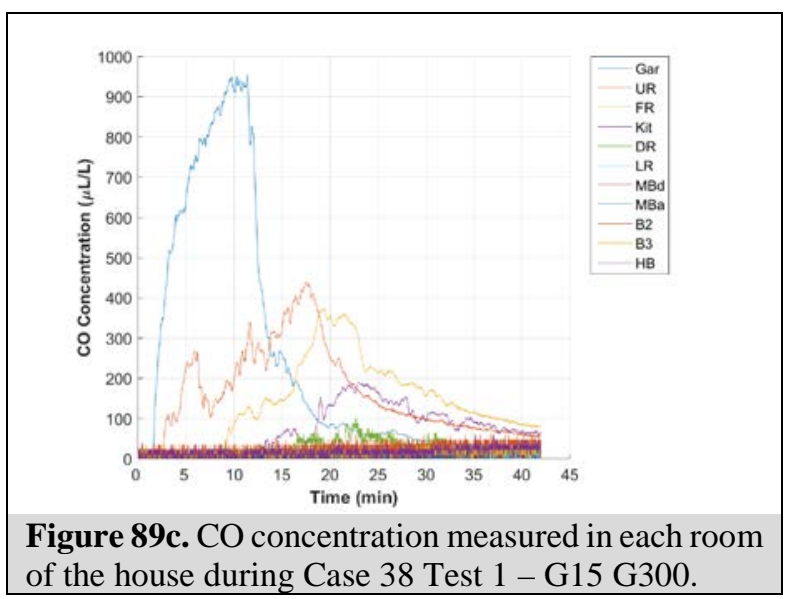

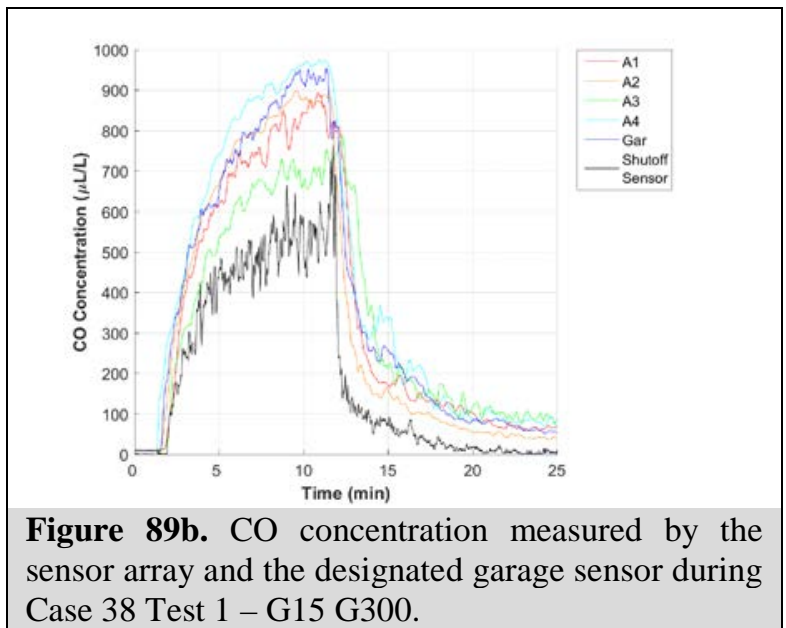

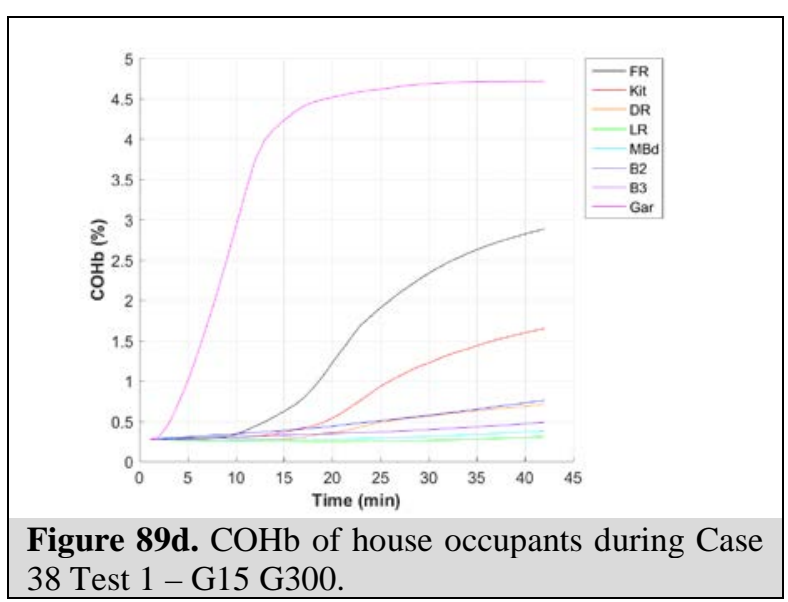




\section{Case 39 Test 1 - G6 G300}

The following figures illustrate the results for Case 39 Test 1 applying the G300 shutoff criteria. The G6 generator was fully fueled, positioned in the garage $(15 \mathrm{~cm}$ inside the garage bay door, centered to the door with the exhaust facing towards the rear wall), and tested at $100 \%$ load (6,000 $\mathrm{W}$ ). The interior person door (from garage to utility room) was open $10 \mathrm{~cm}$, the garage bay door was open $61 \mathrm{~cm}$, and the exterior person door (from garage to backyard) was closed. The shutoff sensor activated the algorithm to shut off the generator after approximately 12 min due to a sensor measurement of $400 \mu \mathrm{L} / \mathrm{L}$ for a $10 \mathrm{~min}$ average. Figure 90a shows the test house layout with generator location, exhaust direction, and sensor array location. Figure 90b shows the CO concentration measured in the garage (by the dedicated room sensor), by the sensor array placed in the garage located as shown in Figure 90a (A1: height $91 \mathrm{~cm}, \mathrm{A2}$ : height $91 \mathrm{~cm}$, A3: height 91 $\mathrm{cm}$, A4: height $91 \mathrm{~cm}$ ), and by the shutoff sensor located on the generator near the generator's electrical panel. The timeframe of the figure has been selected to show the degree of CO uniformity in the area around the generator between the shutoff sensor and the peak CO concentrations measured elsewhere in the garage near the time of shutoff. Figure 90c shows the CO concentration measured in each room of the house over the test. Figure 90d shows the calculated COHb profiles of simulated house occupants in each room of the house. The calculated $\mathrm{COHb}$ for simulated house occupants did not exceed $5 \%$ but did not reach a peak during the test.

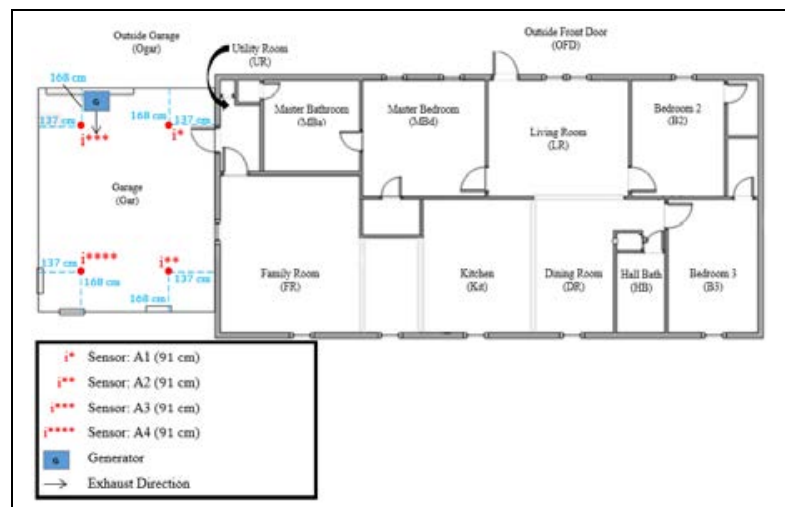

Figure 90a. Generator, exhaust direction, and sensor array layout during Case 39 Test 1 - G6 G300.

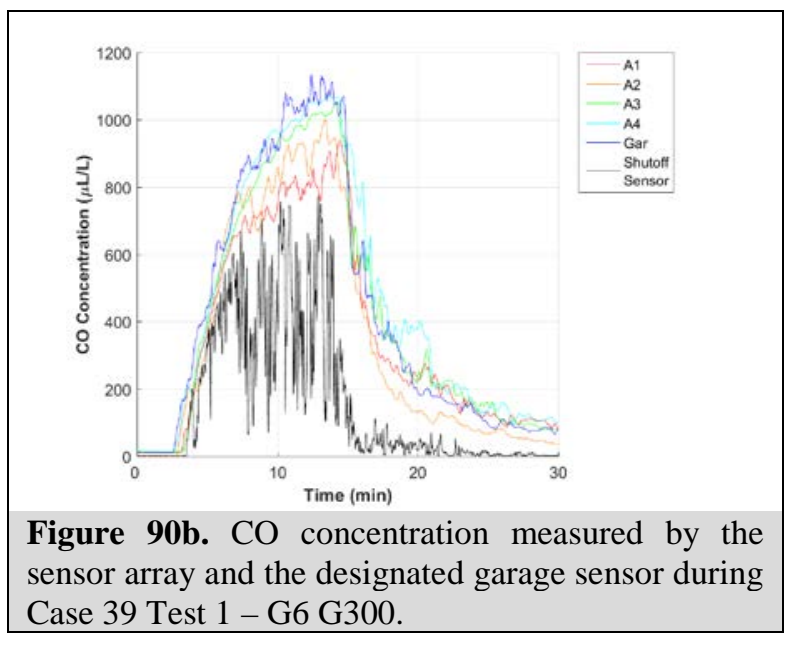




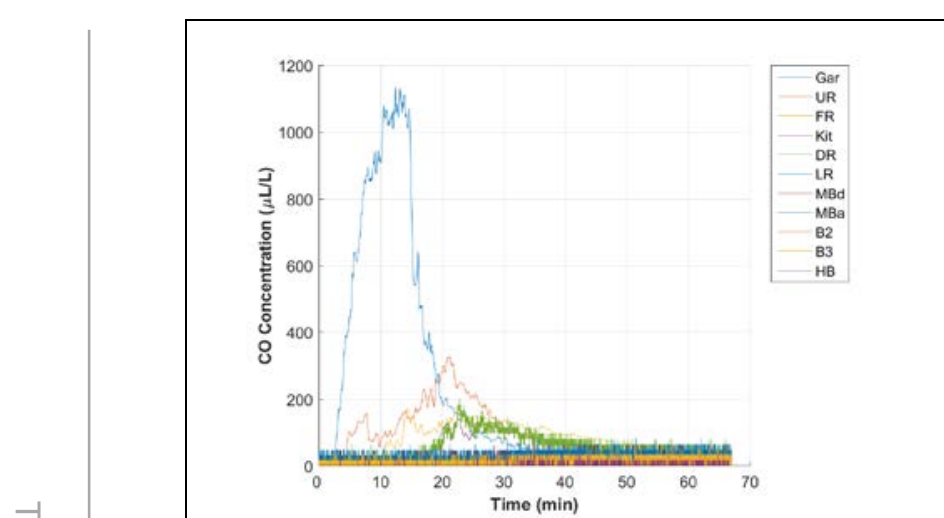

Figure 90c. CO concentration measured in each room of the house during Case 39 Test 1 - G6 G300.

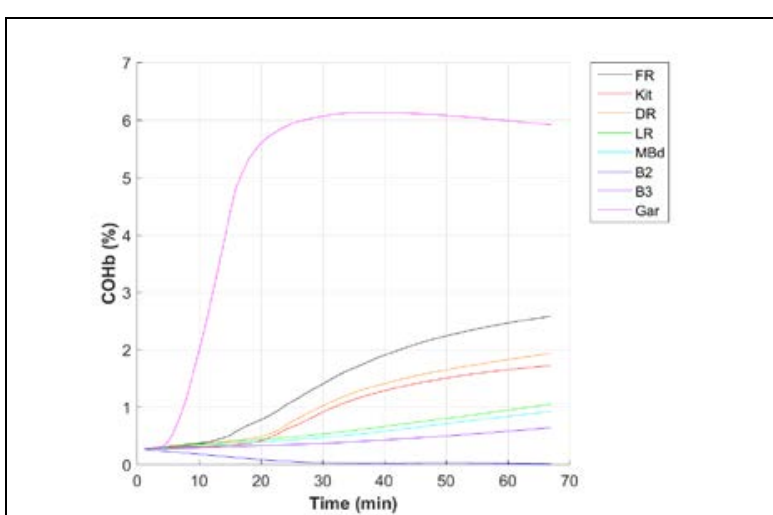

Figure 90d. $\mathrm{COHb}$ of house occupants during Case 39 Test 1 - G6 G300. 


\section{Case 39 Test 1 - G7b UL 2201}

The following figures illustrate the results for Case 39 Test 1 applying the UL 2201 shutoff criteria. The G7b generator was fully fueled, positioned in the garage $(15 \mathrm{~cm}$ inside the garage bay door, centered to the door with the exhaust facing towards the rear wall), and tested at $100 \%$ load (7,000 $\mathrm{W}$ ). The interior person door (from garage to utility room) was open $10 \mathrm{~cm}$, the garage bay door was open $61 \mathrm{~cm}$, and the exterior person door (from garage to backyard) was closed. The shutoff sensor was not activated, and the generator ran out of fuel after approximately 505 min. Figure 91a shows the test house layout with generator location, exhaust direction, and sensor array location. Figure 91b shows the $\mathrm{CO}$ concentration measured in the garage (by the dedicated room sensor), by the sensor array placed in the garage located as shown in Figure 91a (A1: height 91 $\mathrm{cm}, \mathrm{A} 2$ : height $91 \mathrm{~cm}, \mathrm{A3}$ : height $91 \mathrm{~cm}$, A4: height $91 \mathrm{~cm}$ ), and by the shutoff sensor located on the generator near the generator's electrical panel. The timeframe of the figure has been selected to show the degree of CO uniformity in the area around the generator between the shutoff sensor and the peak CO concentrations measured elsewhere in the garage near the time of shutoff. Figure 91c shows the $\mathrm{CO}$ concentration measured in each room of the house over the test. Figure 91d shows the calculated $\mathrm{COHb}$ profiles of simulated house occupants in each room of the house. The calculated $\mathrm{COHb}$ for simulated house occupants did not exceed $7 \%$ but did not reach a peak during the test.

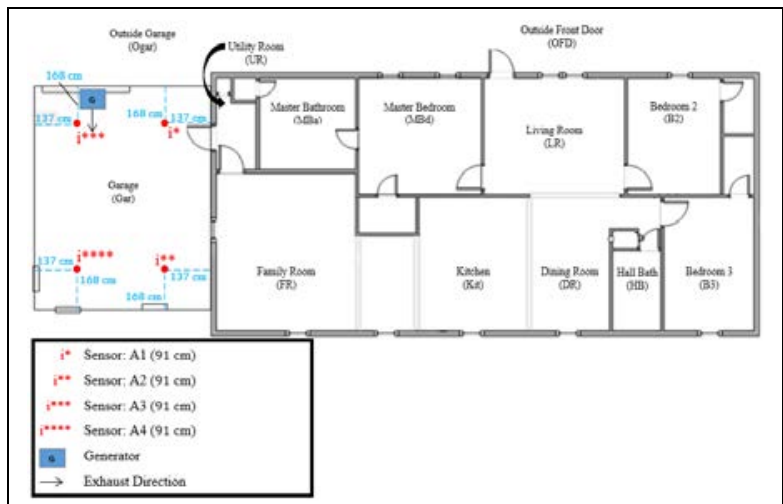

Figure 91a. Generator, exhaust direction, and sensor array layout during Case 39 Test 1 - G7b UL 2201.

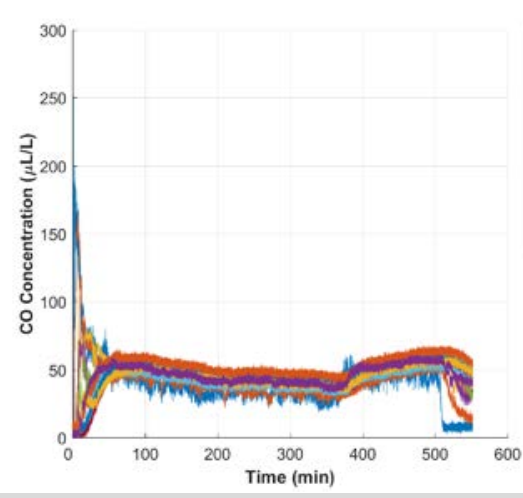

Figure 91c. CO concentration measured in each room of the house during Case 39 Test 1 - G7b UL 2201.

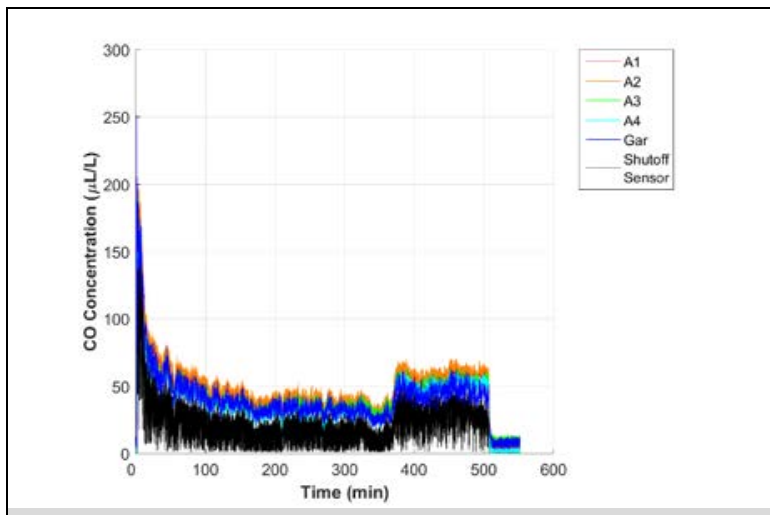

Figure 91b. CO concentration measured by the sensor array and the designated garage sensor during Case 39 Test 1 - G7b UL 2201.

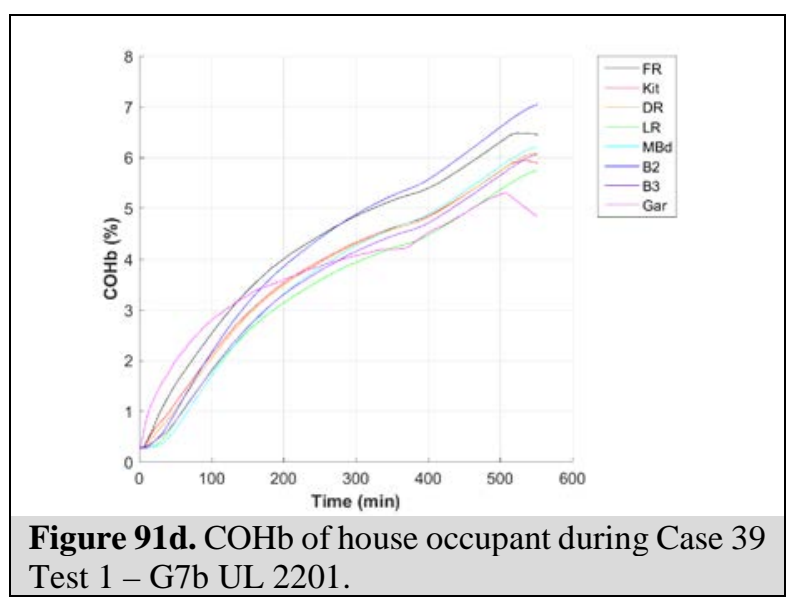




\section{Generator Location: Outside Garage}

Table 8 summarizes the results for all tests performed with a generator located outside the test house garage. The details are discussed below and shown in Figures 92 through 99.

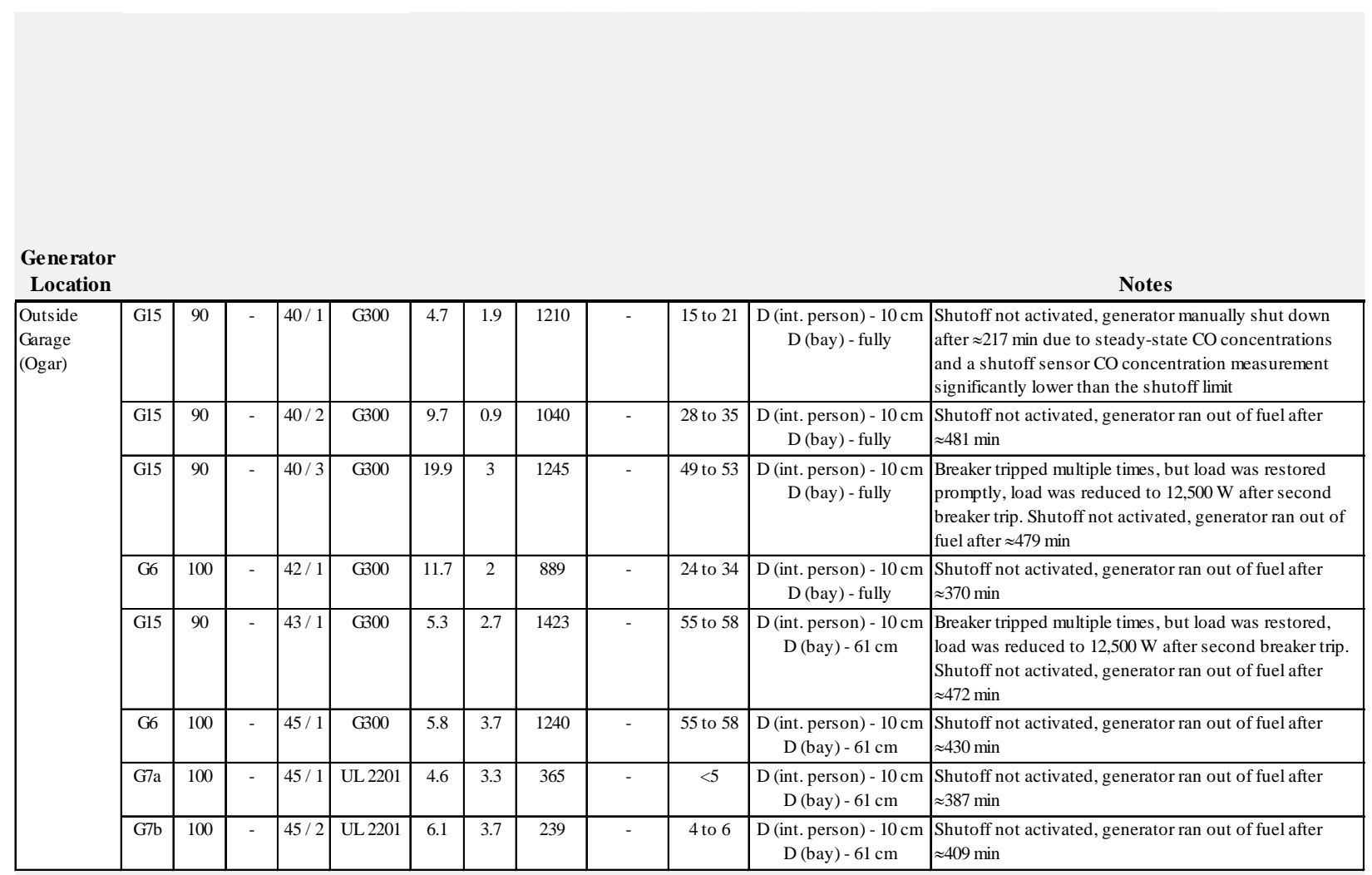

Table 8. Summary of test results for Outside Garage (Ogar) tests.

+Peak $\mathrm{COHb}$ Values are for house zones only.

HVentilation Note: Unless specified otherwise, during the test - all exterior doors are closed, all interior doors are fully open, and all windows are closed. 


\section{Case 40 Test 1 - G15 G300}

The following figures illustrate the results for Case 40 Test 1 applying the G300 shutoff criteria. The G15 generator was fully fueled, positioned outside the garage (centered with garage bay door, with the exhaust outlet in the plane of the bay door facing into garage), and tested at $90 \%$ load $(13,500 \mathrm{~W})$. The interior person door (from garage to utility room) was open $10 \mathrm{~cm}$, the garage bay door was fully open and the exterior person door (from garage to backyard) was closed. The shutoff sensor was not activated and the generator was manually shutdown after approximately 217 min due to approximately steady-state CO concentrations and a shutoff sensor CO concentration measurement significantly lower than the shutoff limit. Figure 92a shows the test house layout with generator location, exhaust direction, and sensor array location. Figure 92b shows the $\mathrm{CO}$ concentration measured in the garage, the sensor array placed in the garage (by the dedicated room sensor), by the sensor array placed in the garage located as shown in Figure 92a (A1: height $91 \mathrm{~cm}, \mathrm{A2}$ : height $91 \mathrm{~cm}, \mathrm{A3}$ : height $91 \mathrm{~cm}, \mathrm{~A} 4$ : height $91 \mathrm{~cm}$ ), and by the shutoff sensor located on the generator near the generator's electrical panel. The figure shows the degree of CO uniformity in the garage. Figure 92c shows the CO concentration measured in each room of the house over the test. Figure 92d shows the calculated $\mathrm{COHb}$ profiles of simulated house occupants in each room of the house. Note the $\mathrm{COHb}$ did not peak during the test as the generator was not out of fuel at test end.

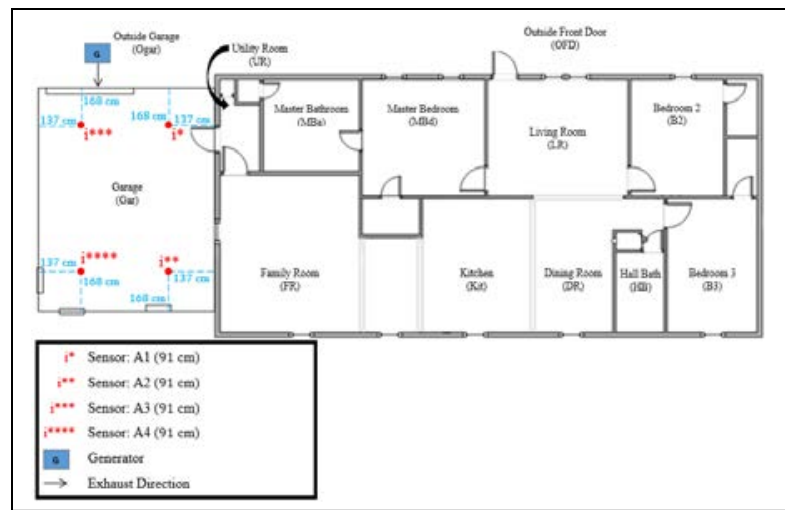

Figure 92a. Generator, exhaust direction, and sensor array layout during Case 40 Test 1 - G15 G300.

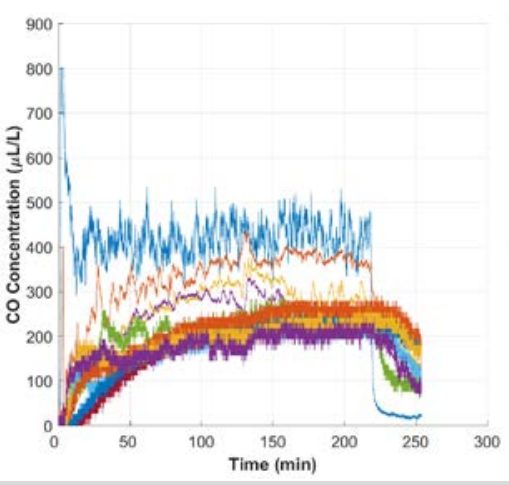

Figure 92c. CO concentration measured in each room of the house during Case 40 Test 1 - G15 G300.
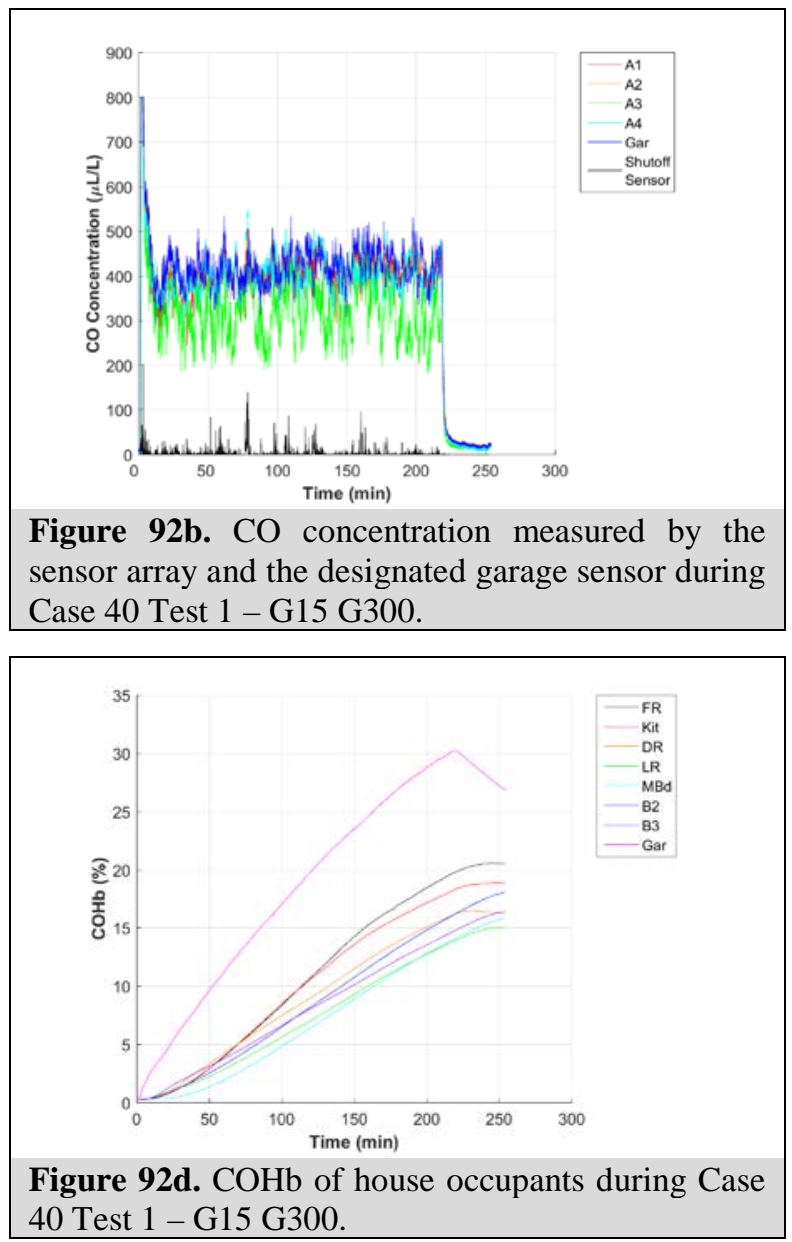


\section{Case 40 Test 2 - G15 G300}

The following figures illustrate the results for Case 40 Test 2 applying the G300 shutoff criteria. The G15 generator was fully fueled, positioned outside the garage (centered with garage bay door, with the exhaust outlet in the plane of the bay door facing into garage), and tested at $90 \%$ load $(13,500 \mathrm{~W})$. The interior person door (from garage to utility room) was open $10 \mathrm{~cm}$, the garage bay door was fully open and the exterior person door (from garage to backyard) was closed. The shutoff sensor was not activated and the generator ran out of fuel after approximately $481 \mathrm{~min}$. Figure 93a shows the test house layout with generator location, exhaust direction, and sensor array location. Figure 93b shows the CO concentration measured in the garage (by the dedicated room sensor), by the sensor array placed in the garage located as shown in Figure 93a (A1: height 91 $\mathrm{cm}, \mathrm{A2}$ : height $91 \mathrm{~cm}, \mathrm{A3}$ : height $91 \mathrm{~cm}, \mathrm{~A} 4$ : height $91 \mathrm{~cm}$ ), and by the shutoff sensor located on the generator near the generator's electrical panel. The figure shows the degree of CO uniformity in the garage. Figure 93c shows the CO concentration measured in each room of the house over the test. Figure 93d shows the CO concentration in the house with each room represented by a single line, which is composed of both a 'solid-line' pattern (showing measured CO concentration) and a 'dotted-line with a symbol' pattern (showing projected CO concentration). Figure 93e shows the calculated $\mathrm{COHb}$ profiles of simulated house occupants in each room of the house.

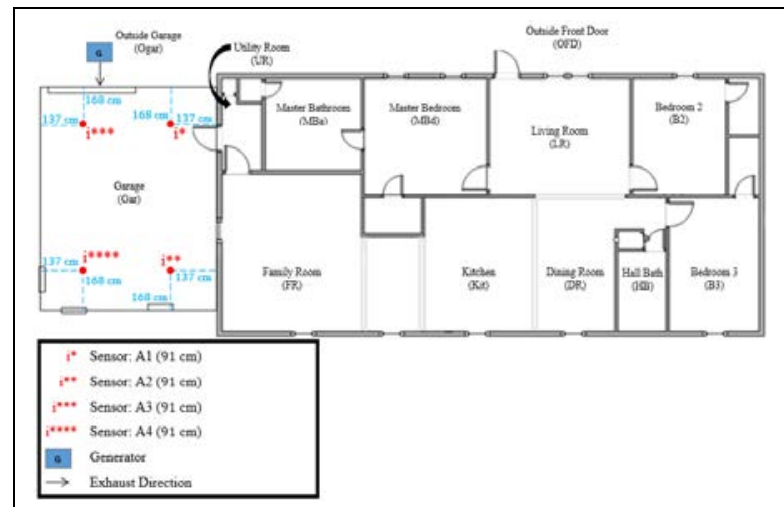

Figure 93a. Generator, exhaust direction, and sensor array layout during Case 40 Test 2 - G15 G300.

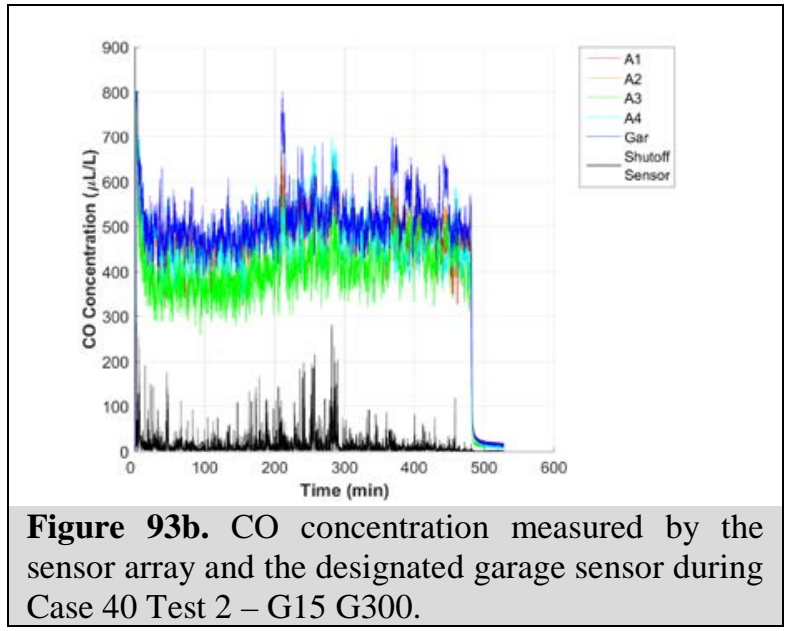




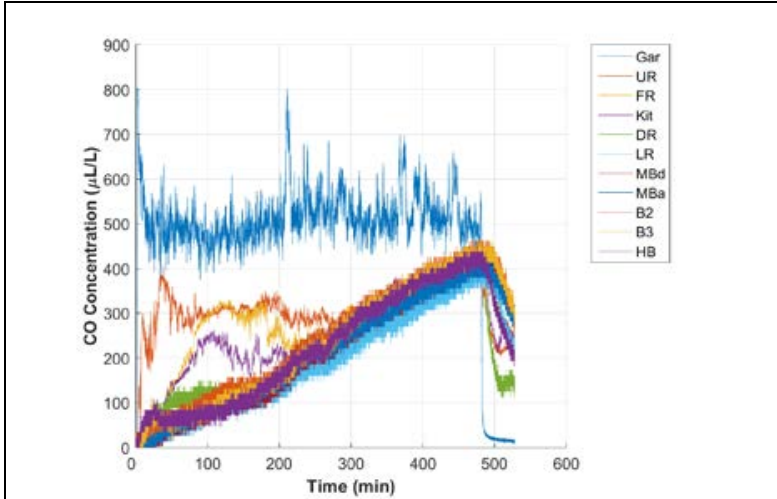

Figure 93c. CO concentration measured in each room of the house during Case 40 Test 2 - G15 G300.

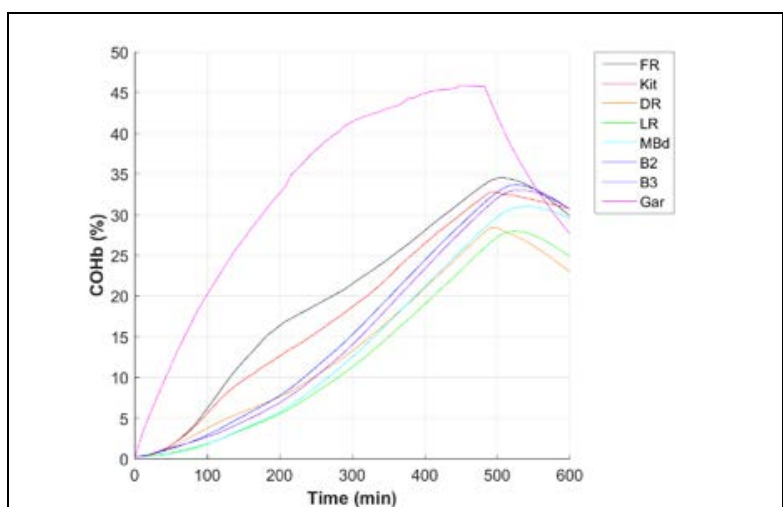

Figure 93e. $\mathrm{COHb}$ of house occupants during Case 40 Test 2 - G15 G300.

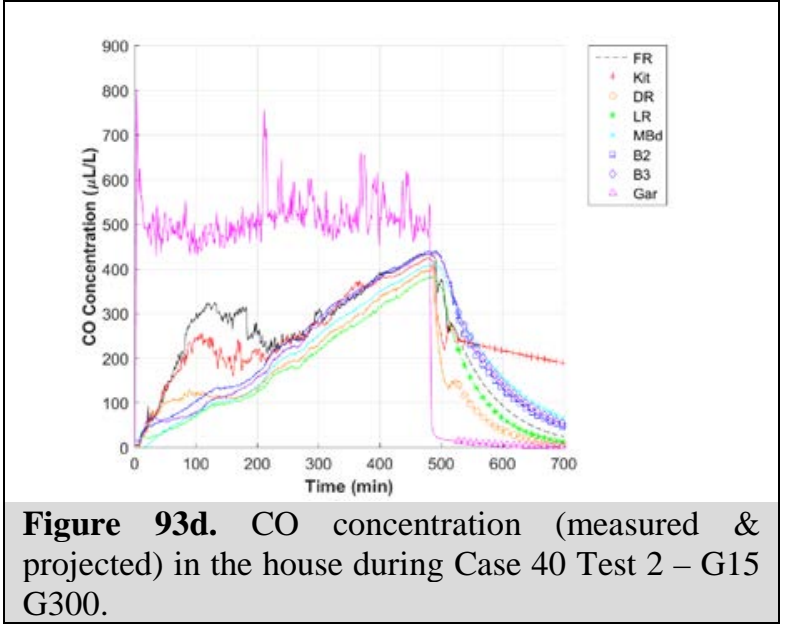

Figure 93d. CO concentration (measured \& projected) in the house during Case 40 Test 2 - G15 


\section{Case 40 Test 3 - G15 G300}

The following figures illustrate the results for Case 40 Test 3 applying the G300 shutoff criteria. The G15 generator was fully fueled, positioned outside the garage (centered with garage bay door, with the exhaust outlet in the plane of the bay door facing into garage), and tested at $90 \%$ load $(13,500 \mathrm{~W})$. The interior person door (from garage to utility room) was open $10 \mathrm{~cm}$, the garage bay door was fully open and the exterior person door (from garage to backyard) was closed. The breaker tripped three times during the test, but the load was restored promptly (visible as long, sharp dips in Figure 94b, Figure 94c, \& Figure 94d); the load was adjusted to 12,500 W following the second and third trips. The shutoff sensor was not activated and the generator ran out of fuel after approximately 479 min. Figure 94a shows the test house layout with generator location, exhaust direction, and sensor array location. Figure 94b shows the CO concentration measured in the garage, the sensor array placed in the garage (by the dedicated room sensor), by the sensor array placed in the garage located as shown in Figure 94a (A1: height $91 \mathrm{~cm}, \mathrm{A2}$ : height $91 \mathrm{~cm}$, A3: height $91 \mathrm{~cm}, \mathrm{~A} 4$ : height $91 \mathrm{~cm}$ ), and by the shutoff sensor located on the generator near the generator's electrical panel. The figure shows the degree of CO uniformity in the garage. Figure 94c shows the CO concentration measured in each room of the house over the test. Figure 94d shows the $\mathrm{CO}$ concentration in the house with each room represented by a single line, which is composed of both a 'solid-line' pattern (showing measured CO concentration) and a 'dotted-line with a symbol' pattern (showing projected CO concentration). Figure 94e shows the calculated $\mathrm{COHb}$ profiles of simulated house occupants in each room of the house.

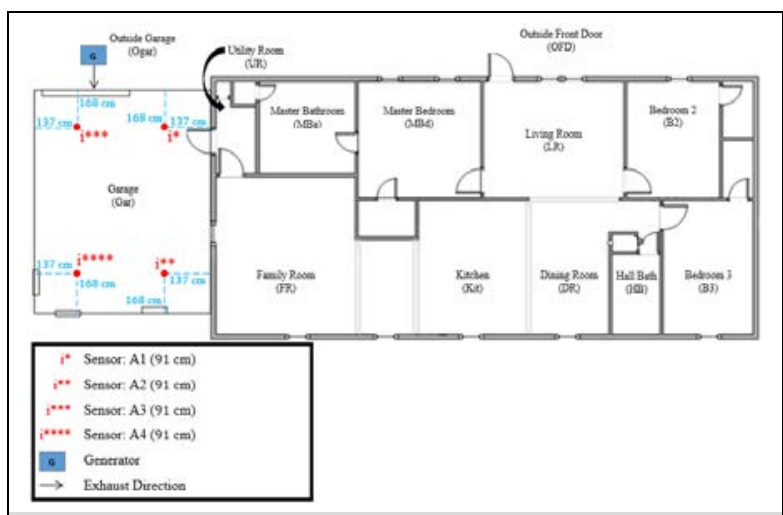

Figure 94a. Generator, exhaust direction, and sensor array layout during Case 40 Test 3 - G15 G300.

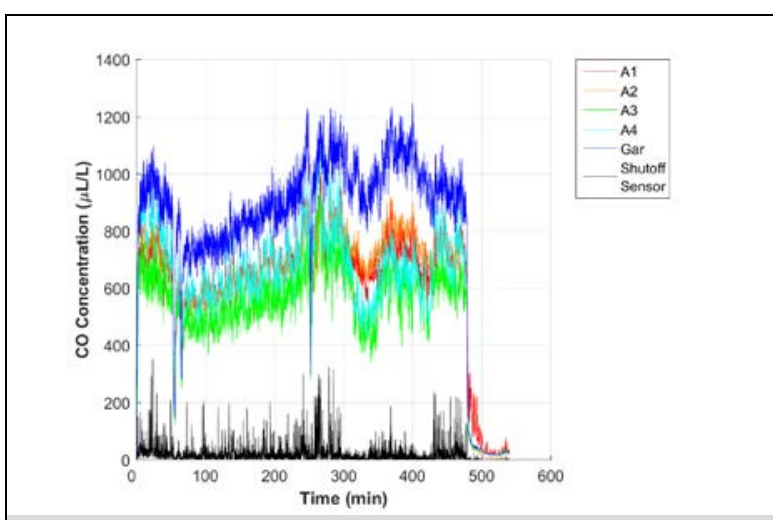

Figure 94b. CO concentration measured by the sensor array and the designated garage sensor during Case 40 Test 3 - G15 G300. 

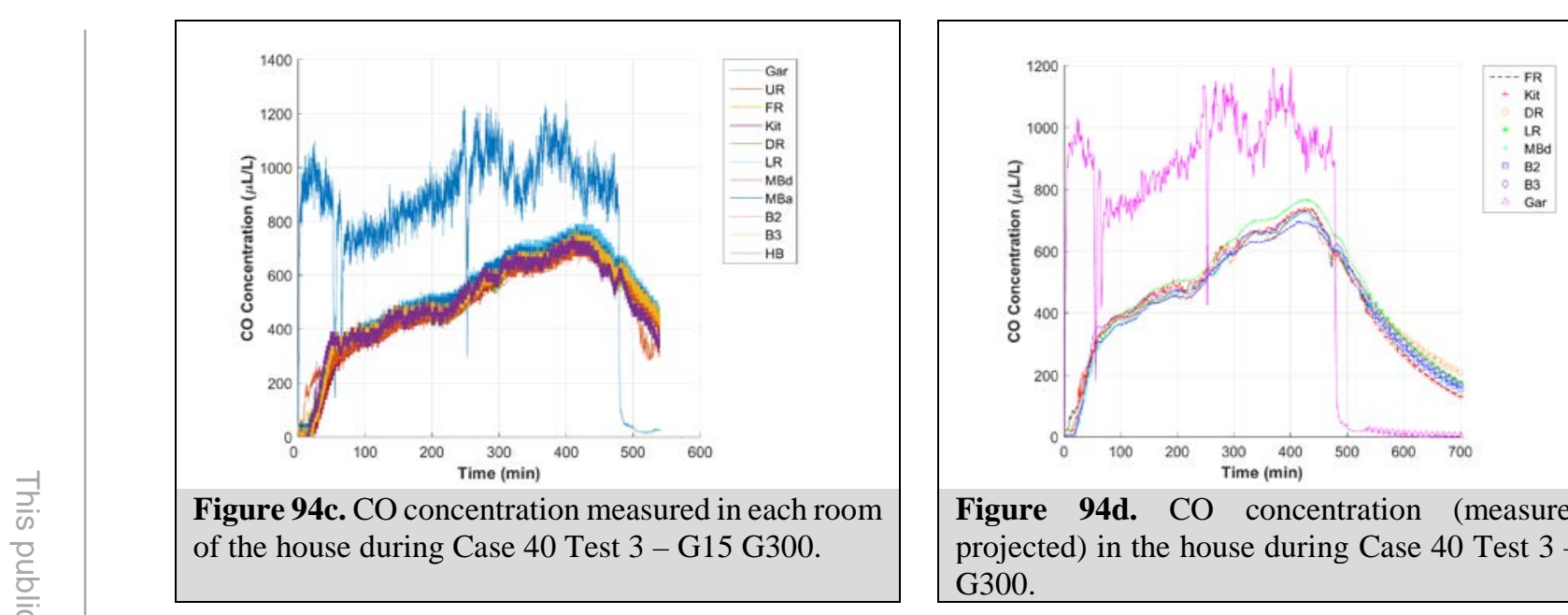

Figure 94d. CO concentration (measured \& projected) in the house during Case 40 Test 3-G15 G300.

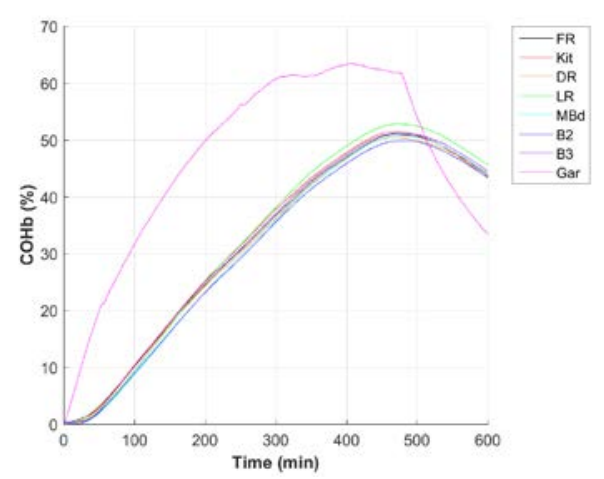

Figure 94e. $\mathrm{COHb}$ of house occupants during Case 40 Test $3-$ G15 G300.

173 


\section{Case 42 Test 1 - G6 G300}

The following figures illustrate the results for Case 42 Test 1 applying the G300 shutoff criteria. The G6 generator was fully fueled, positioned outside the garage (centered with garage bay door, with the exhaust outlet in the plane of the bay door facing into garage), and tested at $100 \%$ load $(6,000 \mathrm{~W})$. The interior person door (from garage to utility room) was open $10 \mathrm{~cm}$, the garage bay door was fully open and the exterior person door (from garage to backyard) was closed. The shutoff sensor was not activated and the generator ran out of fuel after approximately $370 \mathrm{~min}$. Figure 95a shows the test house layout with generator location, exhaust direction, and sensor array location. Figure 95b shows the CO concentration measured in the garage (by the dedicated room sensor), by the sensor array placed in the garage located as shown in Figure 95a (A1: height 91 $\mathrm{cm}$, A2: height $91 \mathrm{~cm}, \mathrm{A3}$ : height $91 \mathrm{~cm}, \mathrm{~A} 4$ : height $91 \mathrm{~cm}$ ), and by the shutoff sensor located on the generator near the generator's electrical panel. The figure shows the degree of CO uniformity in the garage. Figure 95c shows the CO concentration measured in each room of the house over the test. Figure 95d shows the CO concentration in the house with each room represented by a single line, which is composed of both a 'solid-line' pattern (showing measured CO concentration) and a 'dotted-line with a symbol' pattern (showing projected CO concentration). Figure 95e shows the calculated $\mathrm{COHb}$ profiles of simulated house occupants in each room of the house.

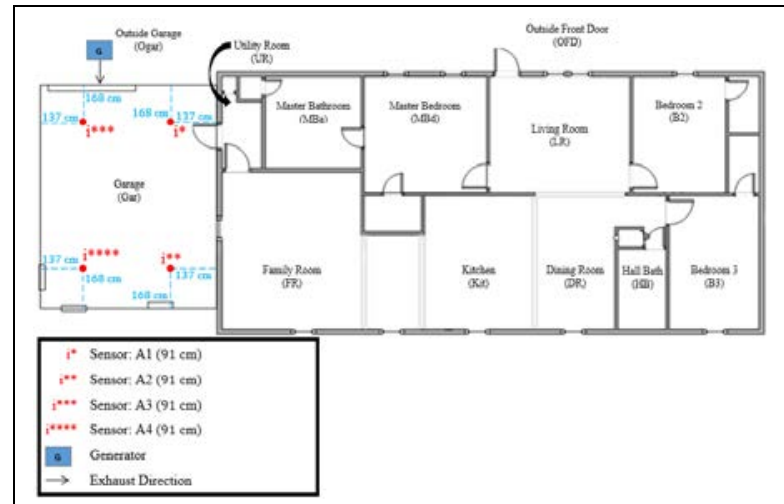

Figure 95a. Generator, exhaust direction, and sensor array layout during Case 42 Test 1 - G6 G300.

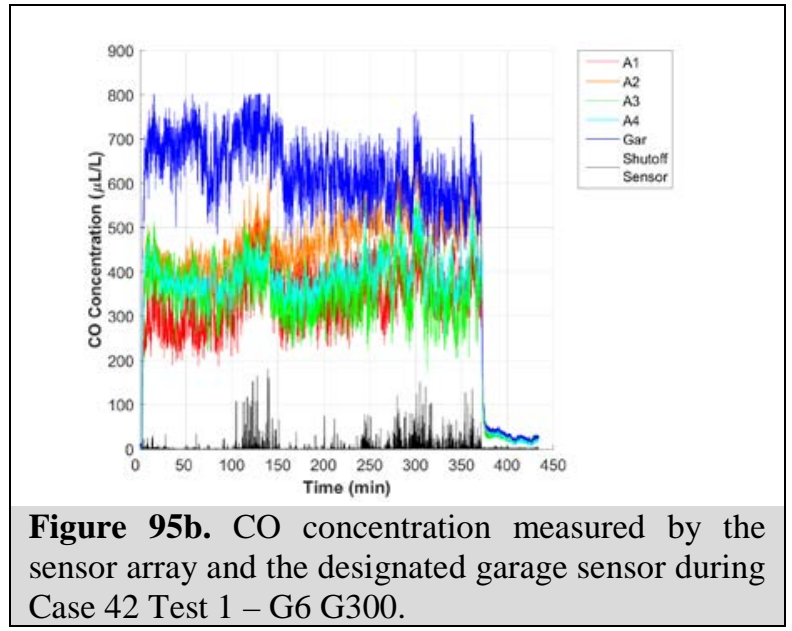




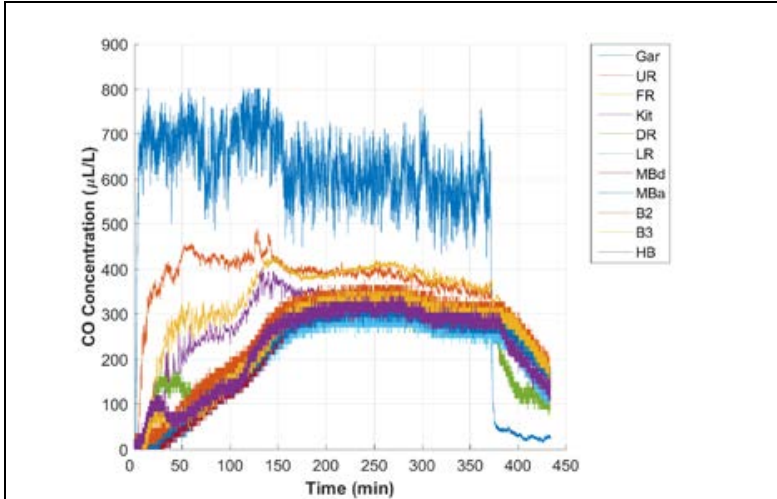

Figure 95c. CO concentration measured in each room of the house during Case 42 Test 1 - G6 G300.

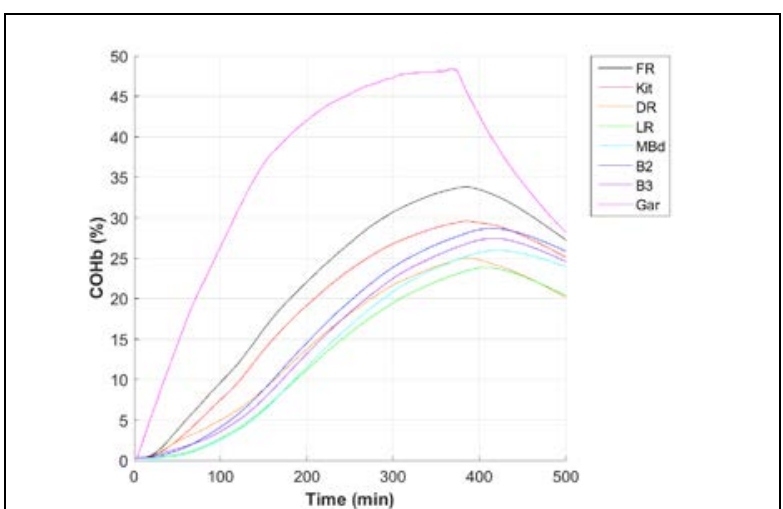

Figure 95e. $\mathrm{COHb}$ of house occupants during Case 42 Test 1 - G6 G300.

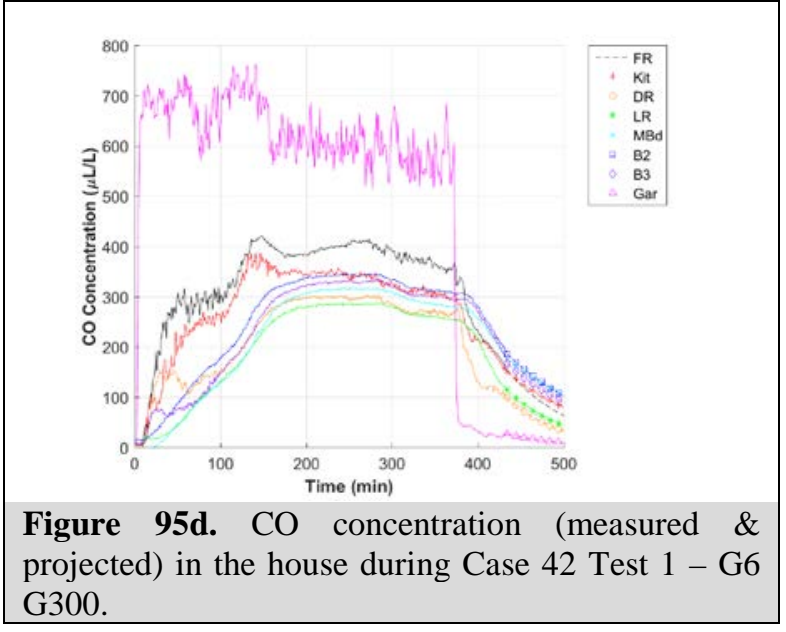

Figure 95d. CO concentration (measured \& projected) in the house during Case 42 Test 1 - G6 


\section{Case 43 Test 1 - G15 G300}

The following figures illustrate the results for Case 43 Test 1 applying the G300 shutoff criteria. The G15 generator was fully fueled, positioned outside the garage (centered with garage bay door, with the exhaust outlet in the plane of the bay door facing into garage), and tested at $90 \%$ load $(13,500 \mathrm{~W})$. The interior person door (from garage to utility room) was open $10 \mathrm{~cm}$, the garage bay door was open $61 \mathrm{~cm}$ and the exterior person door (from garage to backyard) was closed. The generator dropped the load three times during the test, but the load was restored each time (visible as long, sharp dips in Figure 96b, Figure 96c, \& Figure 96d); the load was adjusted to 12,500 W following the second and third drops. The shutoff sensor was not activated and the generator ran out of fuel after approximately $472 \mathrm{~min}$. Figure 96a shows the test house layout with generator location, exhaust direction, and sensor array location. Figure 96b shows the CO concentration measured in the garage (by the dedicated room sensor), by the sensor array placed in the garage located as shown in Figure 96a (A1: height $91 \mathrm{~cm}, \mathrm{A2}$ : height $91 \mathrm{~cm}, \mathrm{A3}$ : height $91 \mathrm{~cm}, \mathrm{~A} 4$ : height $91 \mathrm{~cm})$, and by the shutoff sensor located on the generator near the generator's electrical panel. The figure shows the degree of $\mathrm{CO}$ uniformity in the garage. Figure 96c shows the CO concentration measured in each room of the house over the test. Figure 96d shows the CO concentration in the house with each room represented by a single line, which is composed of both a 'solid-line' pattern (showing measured CO concentration) and a 'dotted-line with a symbol' pattern (showing projected CO concentration). Figure 96e shows the calculated COHb profiles of simulated house occupants in each room of the house.

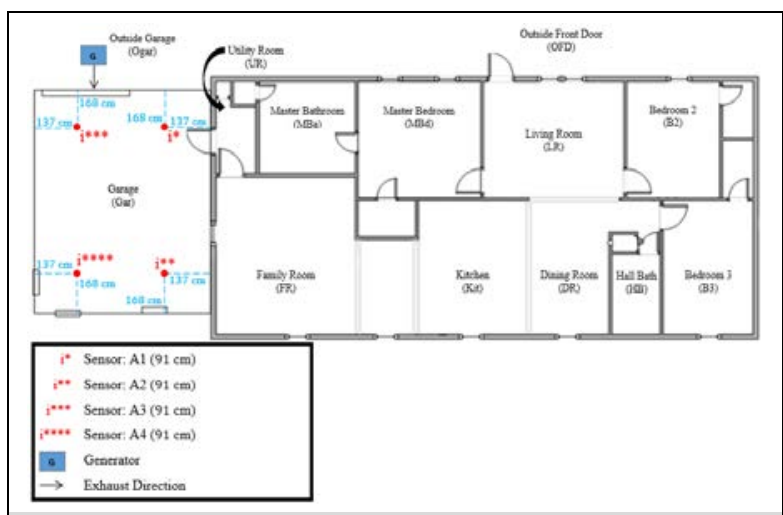

Figure 96a. Generator, exhaust direction, and sensor array layout during Case 43 Test 1 - G15 G300.

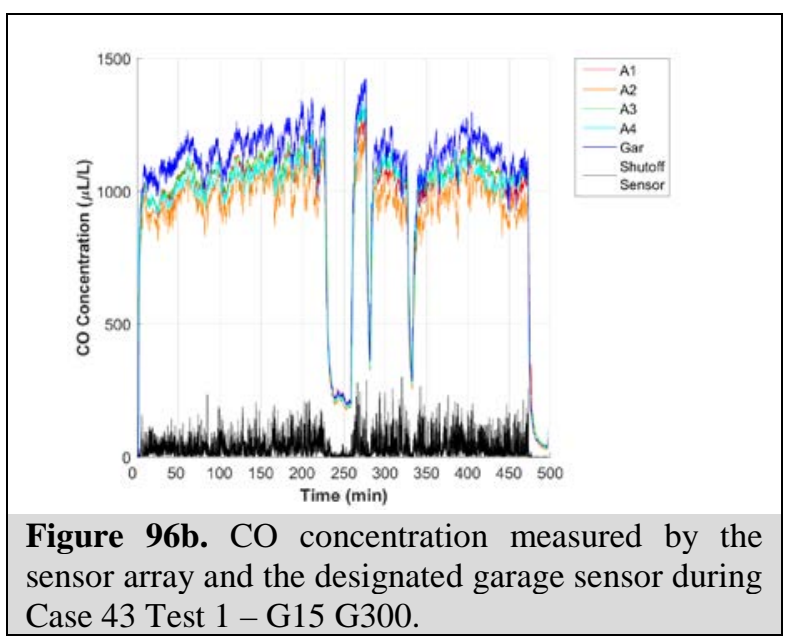




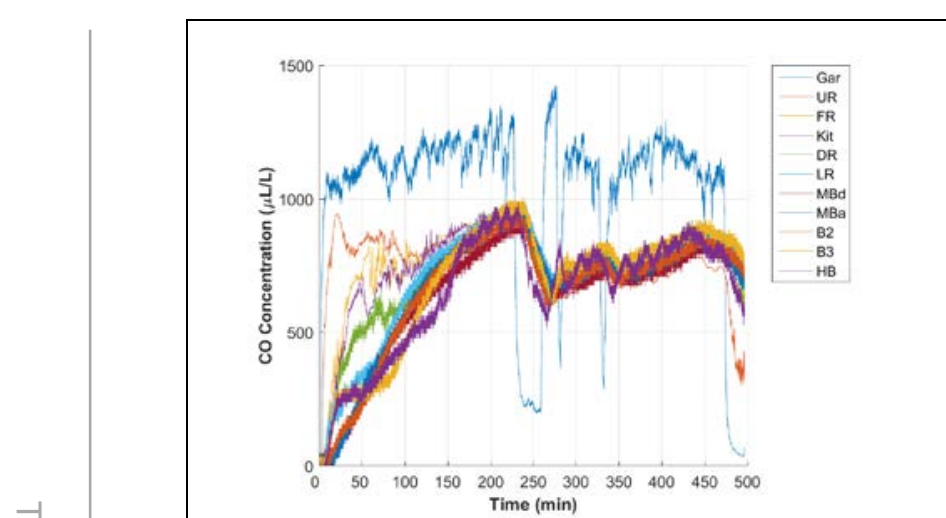

Figure 96c. CO concentration measured in each room of the house during Case 43 Test 1 - G15 G300.

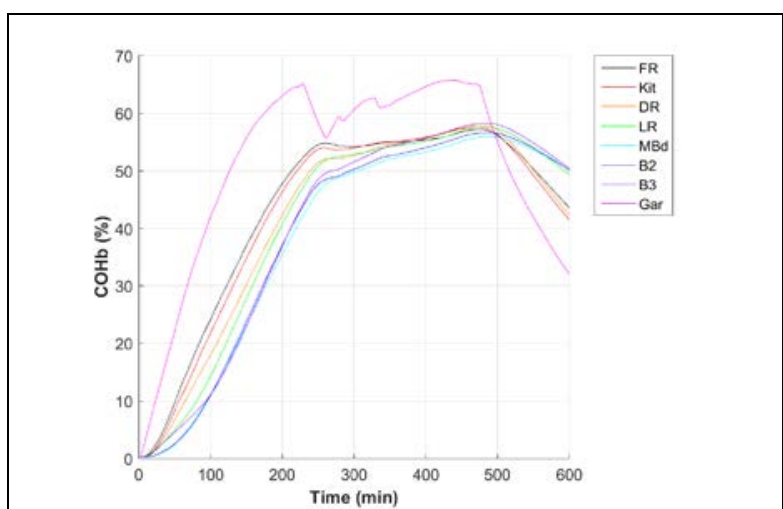

Figure 96e. $\mathrm{COHb}$ of house occupants during Case 43 Test 1 - G15 G300.

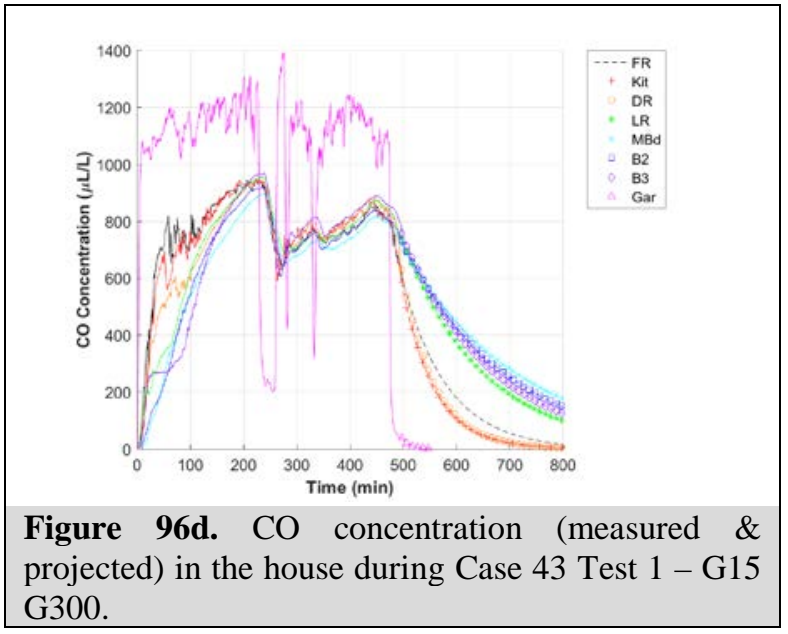

Figure 96d. CO concentration (measured \& G300. 


\section{Case 45 Test 1 - G6 G300}

The following figures illustrate the results for Case 45 Test 1 applying the G300 shutoff criteria. The G6 generator was fully fueled, positioned outside the garage (centered with garage bay door, with the exhaust outlet in the plane of the bay door facing into garage), and tested at $100 \%$ load $(6,000 \mathrm{~W})$. The interior person door (from garage to utility room) was open $10 \mathrm{~cm}$, the garage bay door was open $61 \mathrm{~cm}$ and the exterior person door (from garage to backyard) was closed. The shutoff sensor was not activated and the generator ran out of fuel after approximately $430 \mathrm{~min}$. Figure 97a shows the test house layout with generator location, exhaust direction, and sensor array location. Figure 97b shows the CO concentration measured in the garage (by the dedicated room sensor), by the sensor array placed in the garage located as shown in Figure 97a (A1: height 91 $\mathrm{cm}, \mathrm{A} 2$ : height $91 \mathrm{~cm}, \mathrm{A3}$ : height $91 \mathrm{~cm}$, A4: height $91 \mathrm{~cm}$ ), and by the shutoff sensor located on the generator near the generator's electrical panel. The figure shows the degree of CO uniformity in the garage. Figure 97c shows the CO concentration measured in each room of the house over the test. Figure 97d shows the CO concentration in the house with each room represented by a single line, which is composed of both a 'solid-line' pattern (showing measured CO concentration) and a 'dotted-line with a symbol' pattern (showing projected CO concentration). Figure 97e shows the calculated $\mathrm{COHb}$ profiles of simulated house occupants in each room of the house.

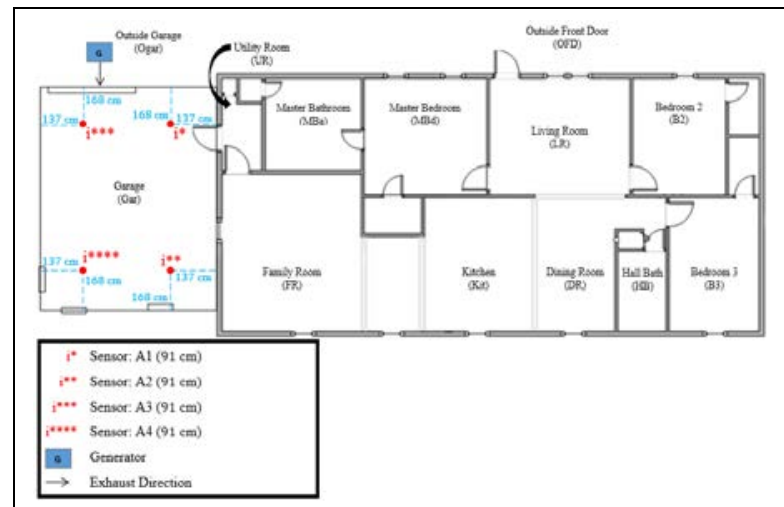

Figure 97a. Generator, exhaust direction, and sensor array layout during Case 45 Test 1 - G6 G300.

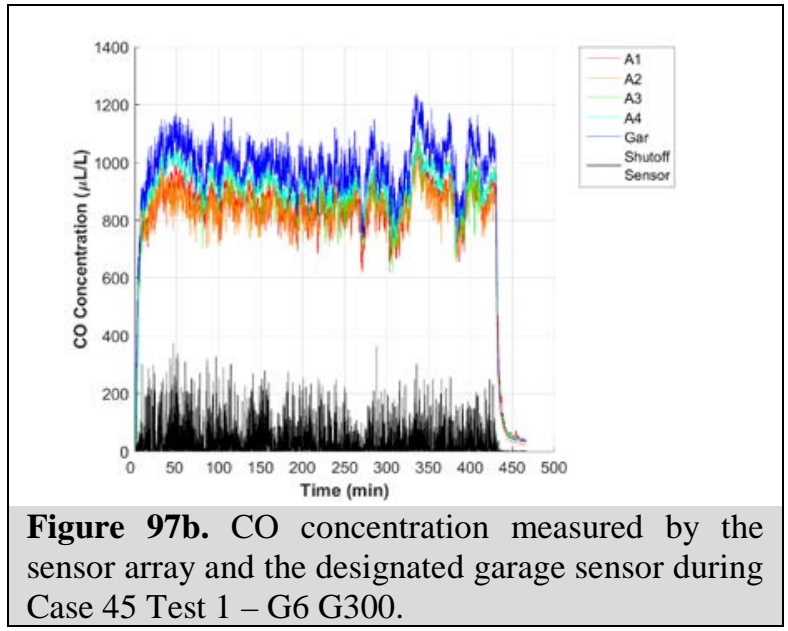




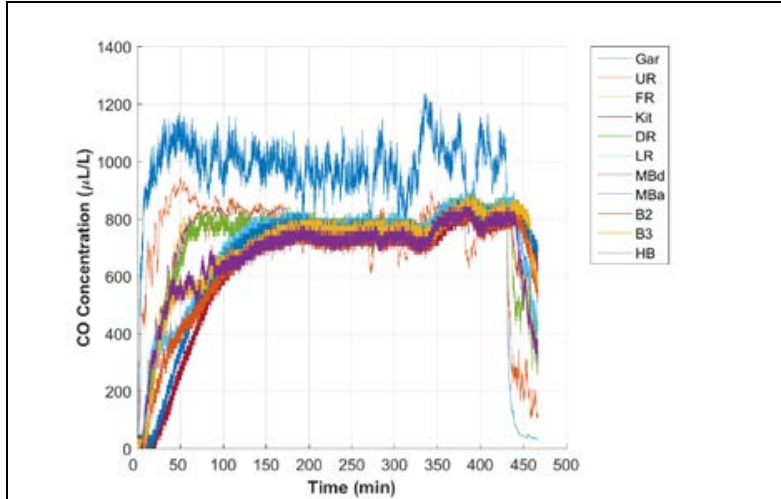

Figure 97c. CO concentration measured in each room of the house during Case 45 Test 1 - G6 G300.

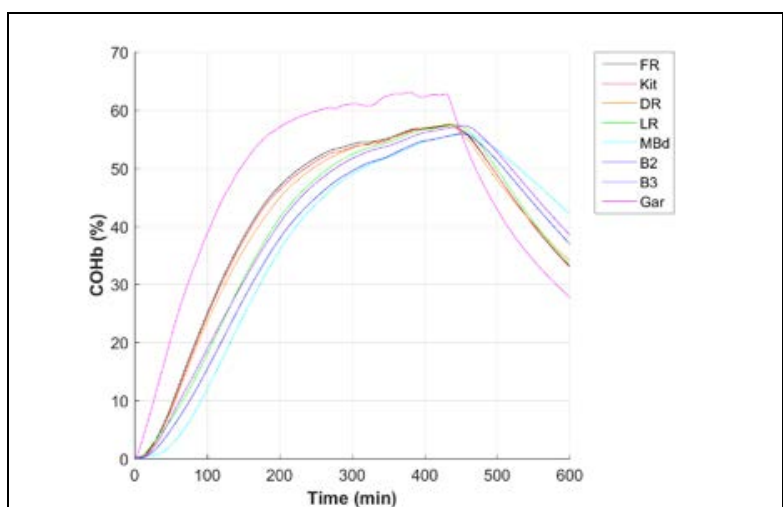

Figure 97e. $\mathrm{COHb}$ of house occupants during Case 45 Test 1 - G6 G300.

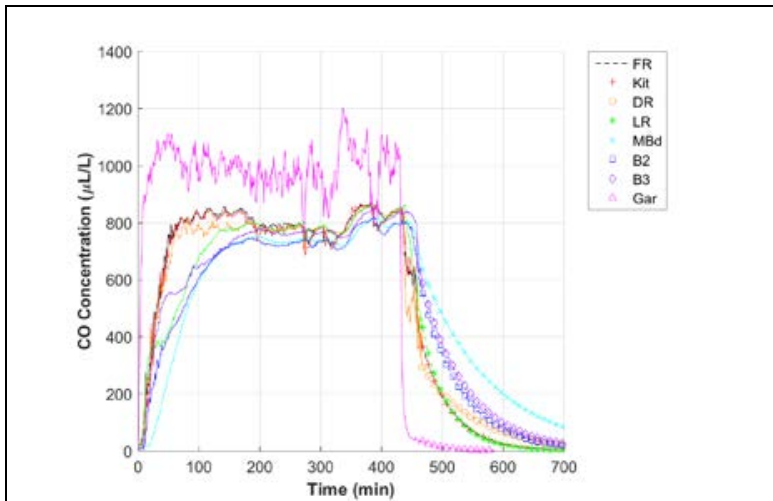

Figure 97d. CO concentration (measured \& projected) in the house during Case 45 Test 1 - G6 G300. 


\section{Case 45 Test 1 - G7a UL 2201}

The following figures illustrate the results for Case 45 Test 1 applying the UL 2201 shutoff criteria. The G7a generator was fully fueled, positioned outside the garage (centered with garage bay door, with the exhaust outlet in the plane of the bay door facing into garage), and tested at $100 \%$ load $(7,000 \mathrm{~W})$. The interior person door was open $10 \mathrm{~cm}$ and the garage bay door was open $61 \mathrm{~cm}$ during the test. The shutoff sensor did not meet the criteria to activate the algorithm that triggers the generator shutoff and the generator ran out of fuel after approximately $387 \mathrm{~min}$. Figure 98a shows the test house layout with generator location, generator exhaust direction, and sensor array location. Figure 98b shows the CO concentration measured in the garage (by the dedicated room sensor), by four sensors located in the garage (A1, A2, A3, and A4: height $91 \mathrm{~cm}$ ), and by the shutoff sensor located on the generator near the generator's electrical panel. The timeframe of the figure has been selected to show the degree of CO uniformity in the area around the generator between the shutoff sensor and the peak CO concentrations measured elsewhere in the garage near the time of shutoff. Figure 98c shows the CO concentration measured in each room of the house over the test. Figure 98d shows the $\mathrm{CO}$ concentration in the house with each room represented by a single line, which is composed of both a 'solid-line' pattern (showing measured CO concentration) and a 'dotted-line with a symbol' pattern (showing projected CO concentration). Figure 98e shows the calculated $\mathrm{COHb}$ profiles of simulated house occupants in each room of the house.

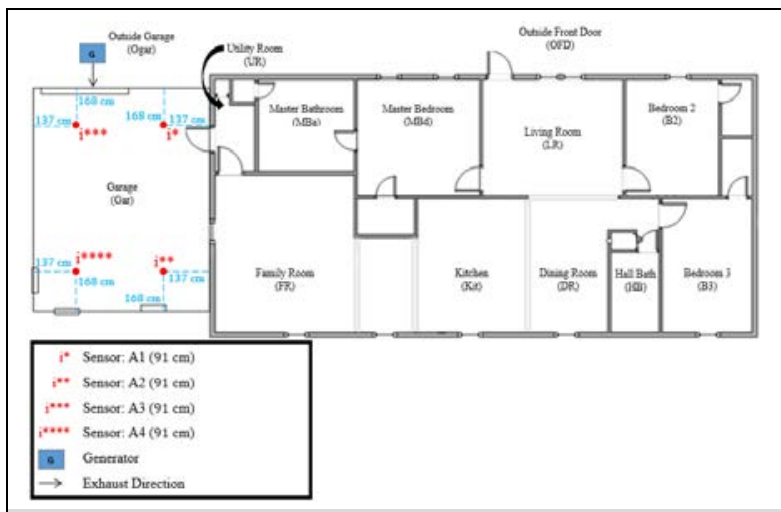

Figure 98a. Generator, exhaust direction, and sensor array layout during Case 45 Test 1 - G7a UL 2201.

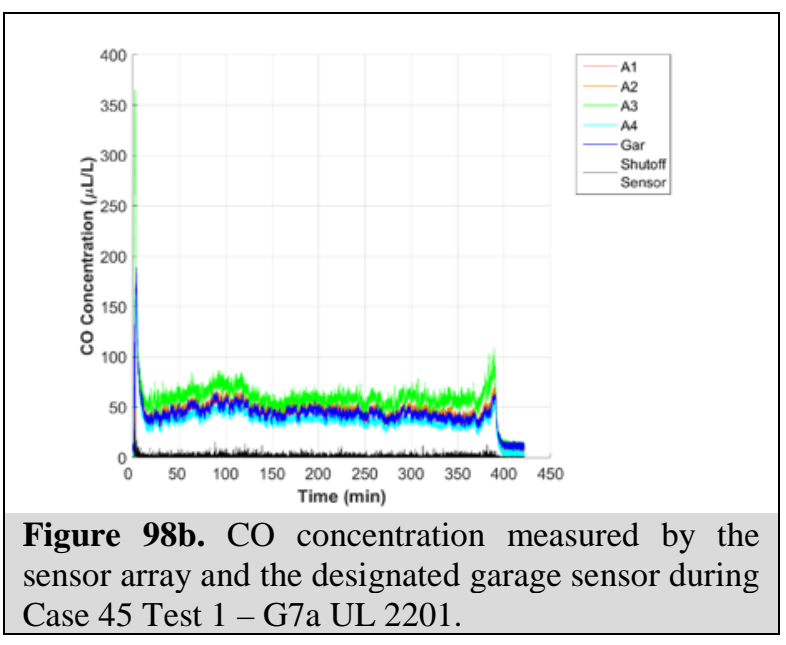

Figure 98b. CO concentration measured by the sensor array and the designated garage sensor during Case 45 Test 1 - G7a UL 2201. 


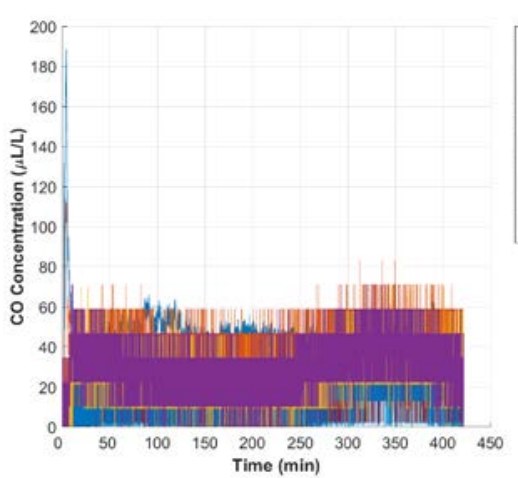

Figure 98c. CO concentration measured in each room of the house during Case 45 Test 1 - G7a UL 2201.

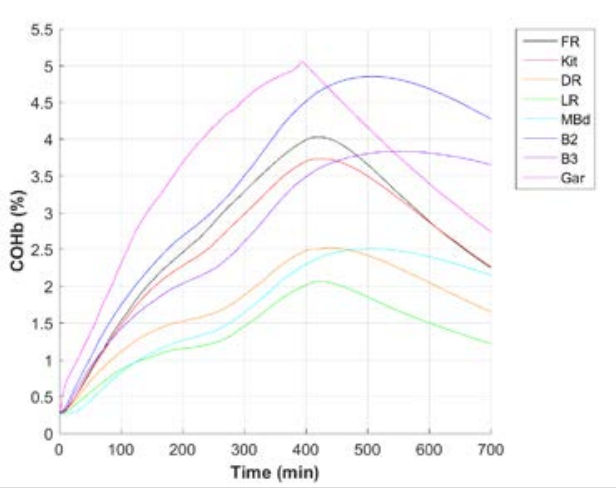

Figure 98e. $\mathrm{COHb}$ of house occupant during Case 45 Test 1 - G7a UL 2201.

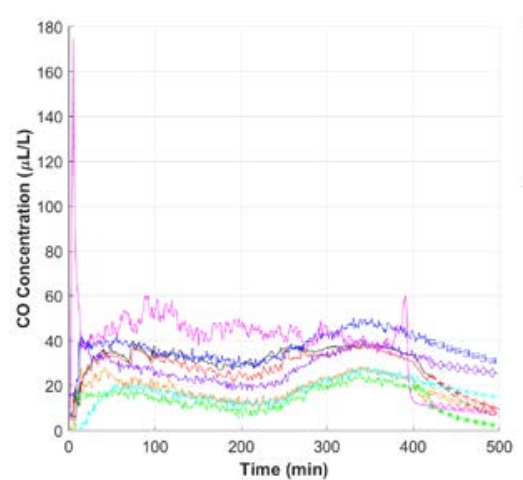

Figure 98d. CO concentration (measured \& projected) in the house during Case 45 Test 1 - G7a UL 2201. 


\section{Case 45 Test 2 - G7b UL 2201}

The following figures illustrate the results for Case 45 Test 2 applying the UL 2201 shutoff criteria. The G7b generator was fully fueled, positioned outside the garage (centered with garage bay door, with the exhaust outlet in the plane of the bay door facing into garage) and tested at $100 \%$ load $(7,000 \mathrm{~W})$. The interior person door was open $10 \mathrm{~cm}$ and the garage bay door was open $61 \mathrm{~cm}$ during the test. The shutoff sensor did not meet the criteria to activate the algorithm that triggers the generator shutoff and the generator ran out of fuel after approximately $409 \mathrm{~min}$. Figure 99a shows the test house layout with generator location, generator exhaust direction, and sensor array location. Figure 99b shows the CO concentration measured in the garage (by the dedicated room sensor), by four sensors located in the garage (A1, A2, A3, and A4: height $91 \mathrm{~cm}$ ), and by the shutoff sensor located on the generator near the generator's electrical panel. The timeframe of the figure has been selected to show the degree of $\mathrm{CO}$ uniformity in the area around the generator between the shutoff sensor and the peak CO concentrations measured elsewhere in the garage near the time of shutoff. Figure 99c shows the CO concentration measured in each room of the house over the test. Figure 99d shows the calculated $\mathrm{COHb}$ profiles of simulated house occupants in each room of the house.

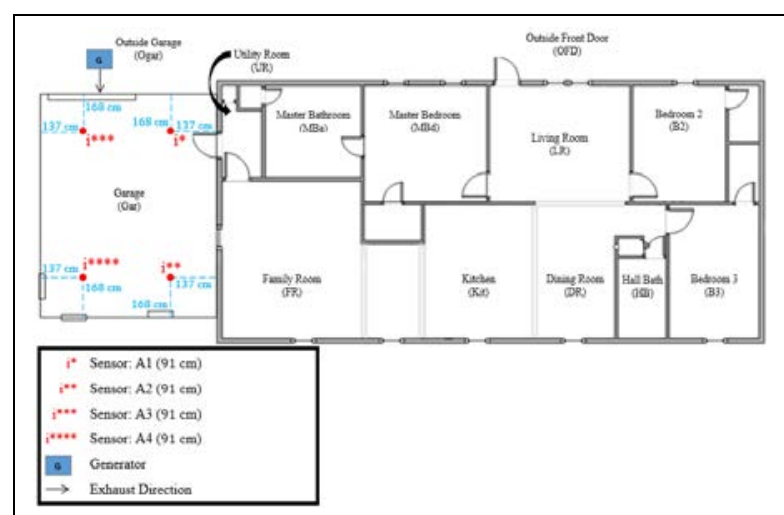

Figure 99a. Generator, exhaust direction, and sensor array layout during Case 45 Test 2 - G7b UL 2201.

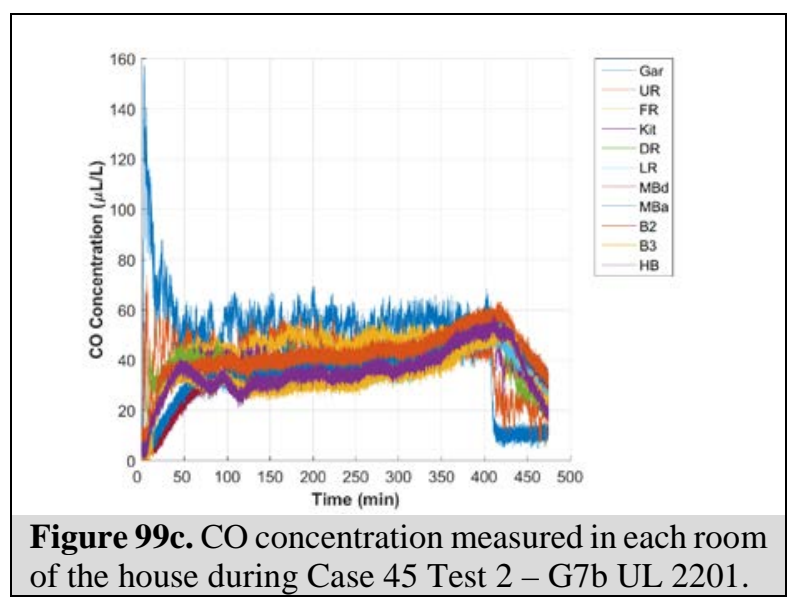

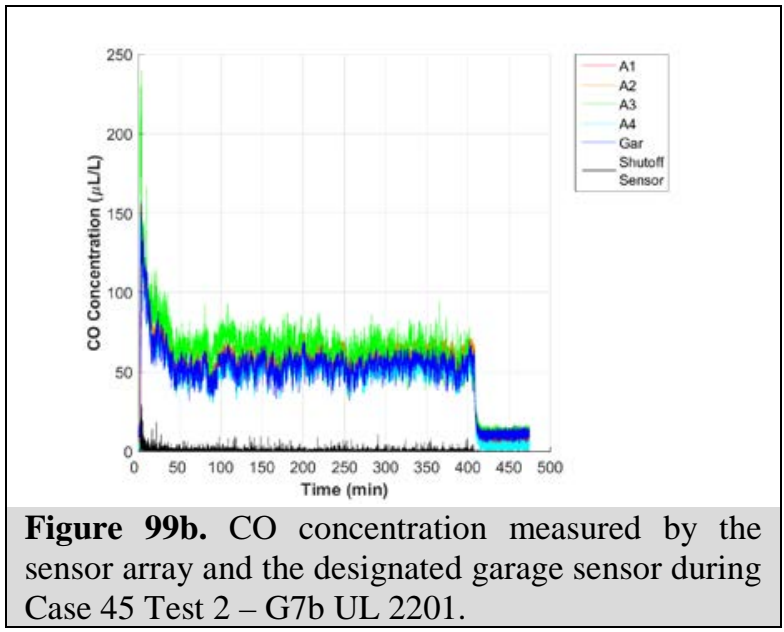

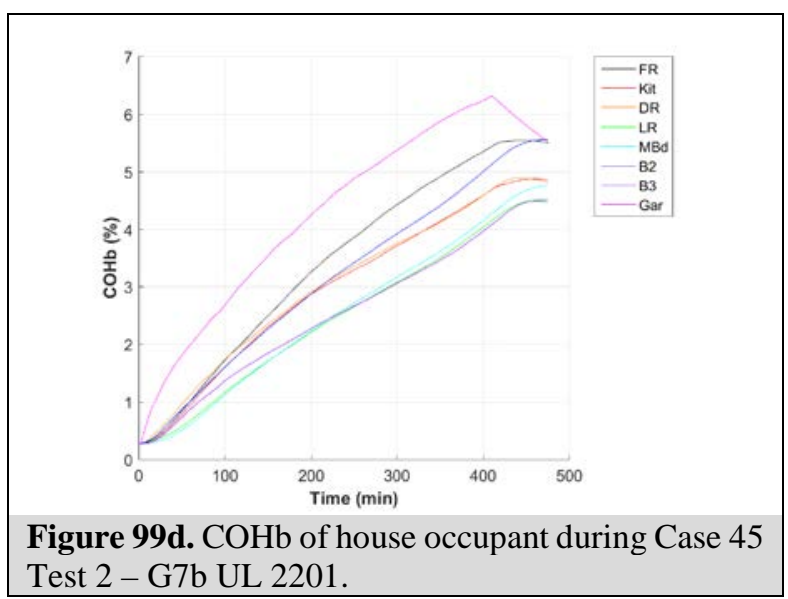




\section{Generator Location: Outside Front Door}

Table 9 summarizes the results for all tests performed with a generator located outside the test house front door. The details are discussed below and shown in Figures 100 through 110.

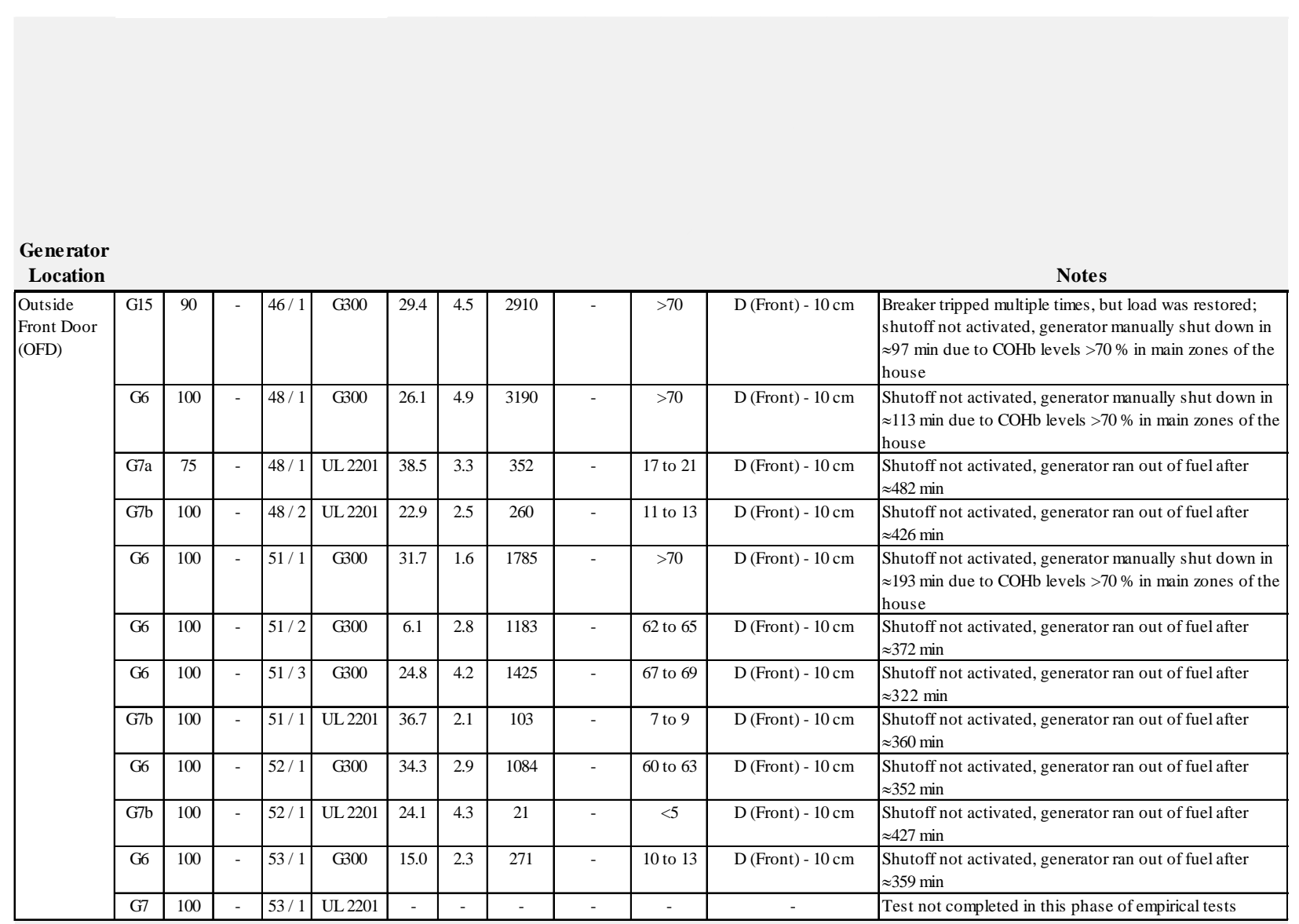

Table 9. Summary of test results for Outside Front Door (OFD) tests.

+Peak COHb Values are for house zones only.

+Ventilation Note: Unless specified otherwise, during the test - all exterior doors are closed, all interior doors are fully open, and all windows are closed. 


\section{Case 46 Test 1 - G15 G300}

The following figures illustrate the results for Case 46 Test 1 applying the G300 shutoff criteria. The G15 generator was fully fueled, positioned outside the front door (centered, $30 \mathrm{~cm}$ away from plane of the door, with the exhaust facing the door), and tested at $90 \%$ load $(13,500 \mathrm{~W})$. The front door was open $10 \mathrm{~cm}$. The breaker tripped five times during the test, but the load was restored promptly (visible as short, sharp dips in Figure 100b, Figure 100c, \& Figure 100d); each time the load was restored, it was set to a level lower than the original $(13,000 \mathrm{~W}, 12,500 \mathrm{~W}, 12,000$ $\mathrm{W}, 12,000 \mathrm{~W}, \& 11,500 \mathrm{~W})$. The shutoff sensor was not activated and the generator was manually shutdown after approximately $97 \mathrm{~min}$ due to $\mathrm{COHb}$ levels $>70 \%$ in main zones of the house. Figure 100a shows the test house layout with generator location, exhaust direction, and sensor array location. Figure 100b shows the $\mathrm{CO}$ concentration measured in the living room (by the dedicated room sensor), by the sensor array placed in the living room located as shown in Figure 100a (A1: height $183 \mathrm{~cm}, \mathrm{~A} 2$ : height $61 \mathrm{~cm}, \mathrm{A3}$ : height $183 \mathrm{~cm}, \mathrm{~A} 4$ : height $61 \mathrm{~cm}$ ), and by the shutoff sensor located on the generator near the generator's electrical panel. Figure 100c shows the CO concentration measured in each room of the house over the test. Figure 100d shows the calculated $\mathrm{COHb}$ profiles of simulated house occupants in each room of the house.

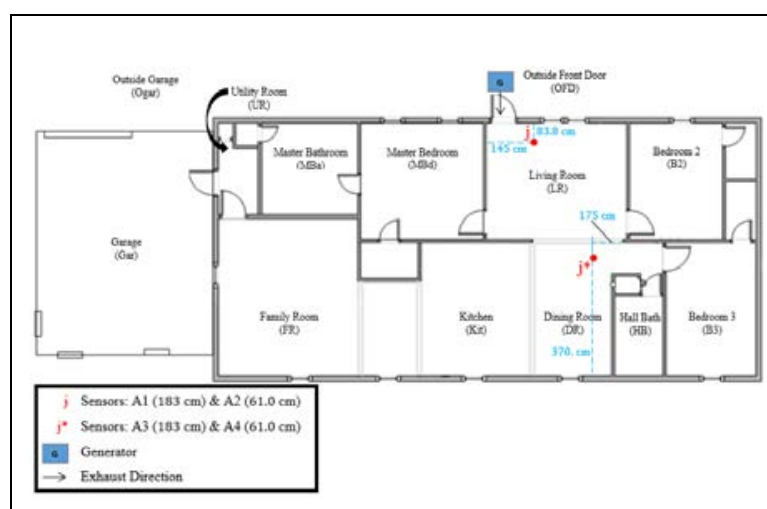

Figure 100a. Generator, exhaust direction, and sensor array layout during Case 46 Test 1 - G15 G300.

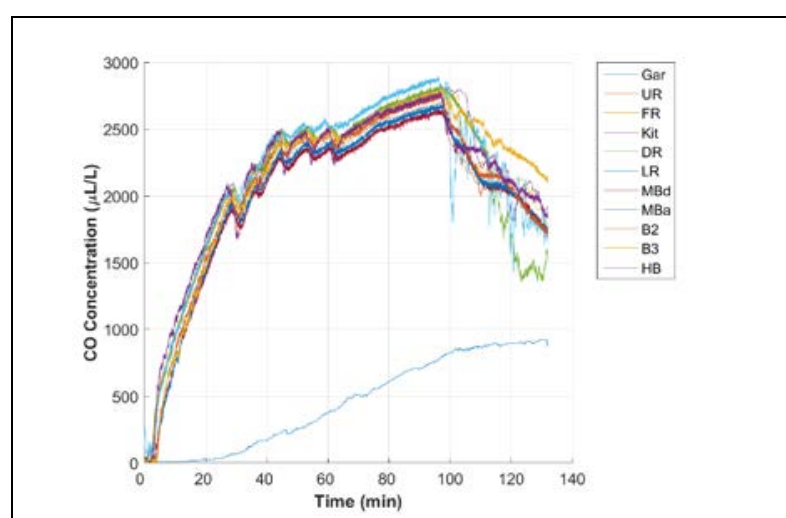

Figure 100c. CO concentration measured in each room of the house during Case 46 Test 1 - G15 G300.

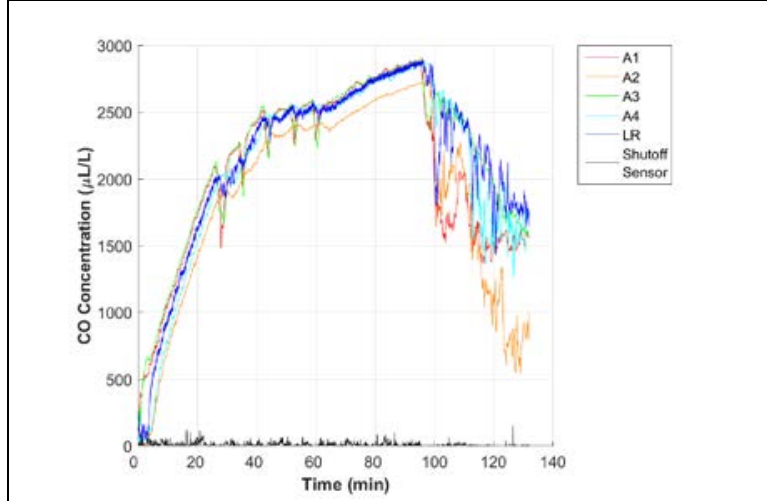

Figure 100b. CO concentration measured by the sensor array and the designated living room sensor during Case 46 Test 1 - G15 G300.

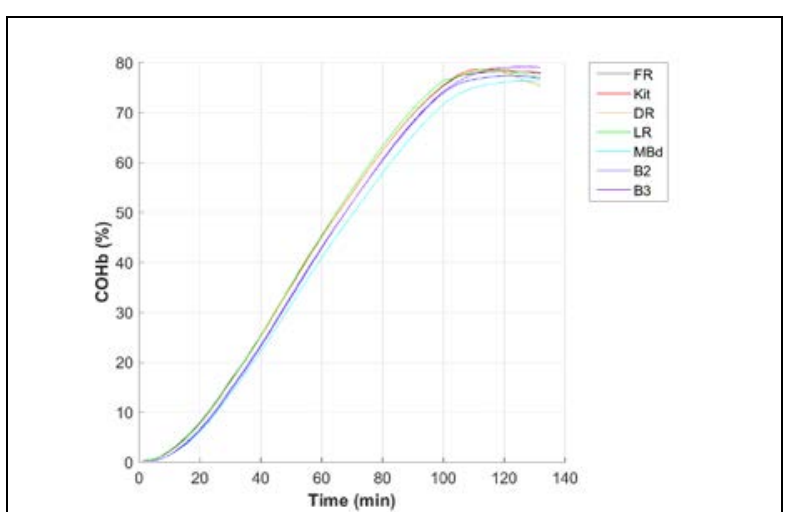

Figure 100d. COHb of house occupants during Case 46 Test $1-$ G15 G300. 


\section{Case 48 Test 1 - G6 G300}

The following figures illustrate the results for Case 48 Test 1 applying the G300 shutoff criteria. The G6 generator was fully fueled, positioned outside the front door (centered, $30 \mathrm{~cm}$ away from plane of the door, with the exhaust facing the door), and tested at $100 \%$ load (6,000 W). The front door was open $10 \mathrm{~cm}$. The shutoff sensor was not activated and the generator was manually shutdown after approximately 113 min due to $\mathrm{COHb}$ levels $>70 \%$ in main zones of the house. Figure 101a shows the test house layout with generator location, exhaust direction, and sensor array location. Figure 101b shows the CO concentration measured in the living room (by the dedicated room sensor), by the sensor array placed in the living room located as shown in Figure 101a (A1: height $183 \mathrm{~cm}, A 2$ : height $61 \mathrm{~cm}$, A3: height $183 \mathrm{~cm}, A 4$ : height $61 \mathrm{~cm}$ ), and by the shutoff sensor located on the generator near the generator's electrical panel. Figure 101c shows the $\mathrm{CO}$ concentration measured in each room of the house over the test. Figure 101d shows the calculated $\mathrm{COHb}$ profiles of simulated house occupants in each room of the house.

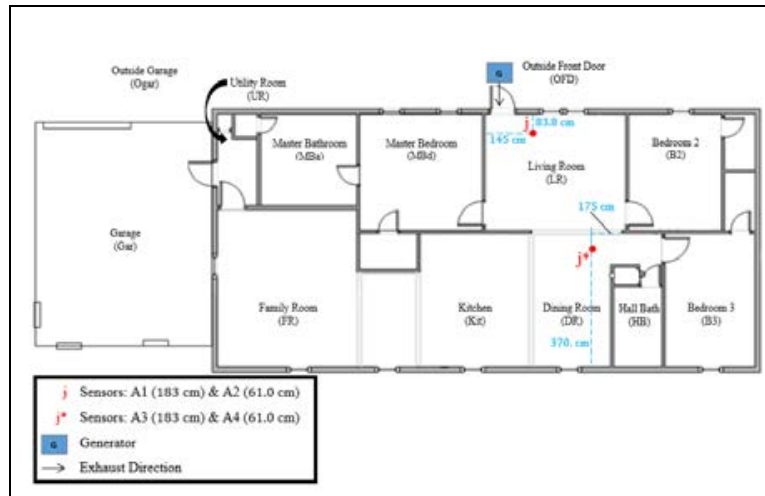

Figure 101a. Generator, exhaust direction, and sensor array layout during Case 48 Test 1 - G6 G300.

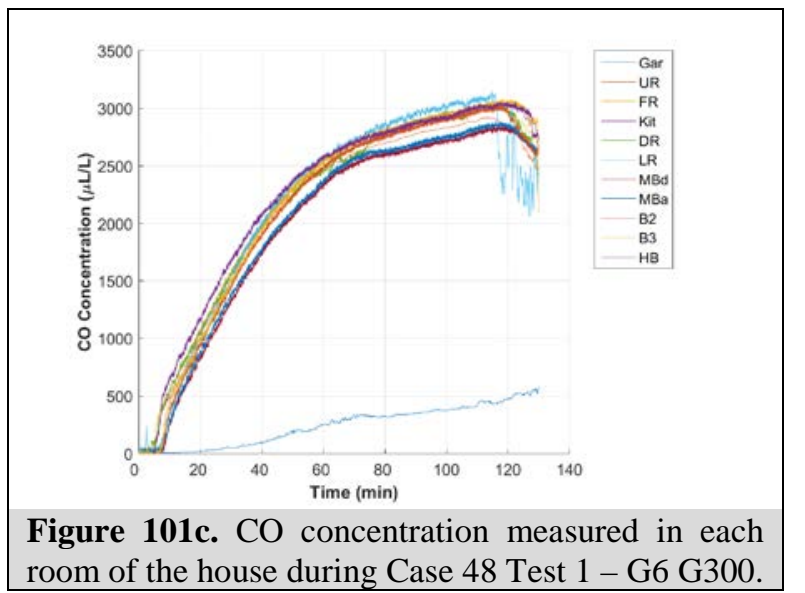

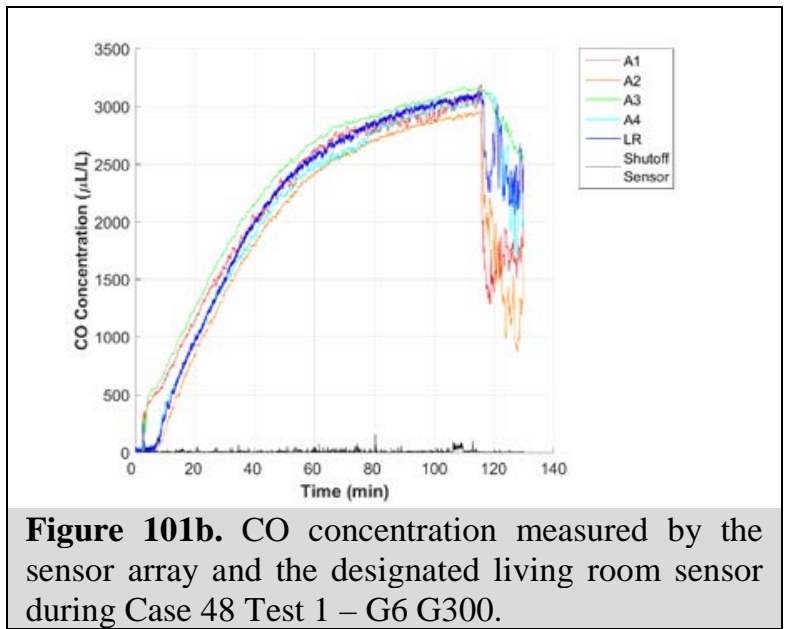

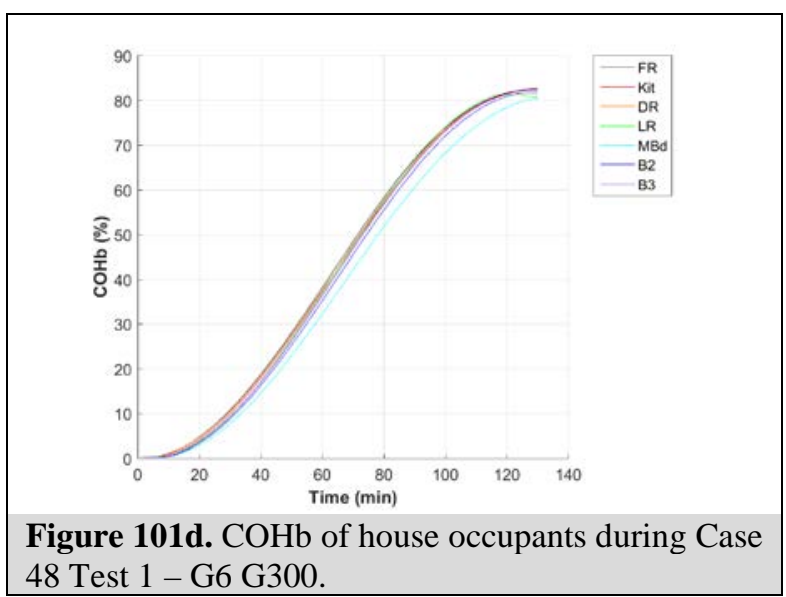




\section{Case 48 Test 1 - G7a UL 2201}

The following figures illustrate the results for Case 48 Test 1 applying the UL 2201 shutoff criteria. The G7a generator was fully fueled, positioned outside the front door (centered, $30 \mathrm{~cm}$ away from plane of the door, with the exhaust facing the door), and tested at $75 \%$ load $(5,250 \mathrm{~W})$ due to problems maintaining the load during previous tests. The front door was open $10 \mathrm{~cm}$ during the test. The shutoff sensor did not meet the criteria to activate the algorithm that triggers the generator shutoff and the generator ran out of fuel after approximately $482 \mathrm{~min}$. Figure 102a shows the test house layout with generator location, generator exhaust direction, and sensor array location. Figure 102b shows the CO concentration measured in the living room (by the dedicated room sensor), by two sensors located in the living room near the front door (A1: height $183 \mathrm{~cm}, \mathrm{~A} 2$ : height $61 \mathrm{~cm}$ ), by the other two sensors located on the living room-dining room boundary (A3: height $183 \mathrm{~cm}$, A4: height $61 \mathrm{~cm}$ ), and by the shutoff sensor located on the generator near the generator's electrical panel. The timeframe of the figure has been selected to show the degree of $\mathrm{CO}$ uniformity in the area around the generator between the shutoff sensor and the peak CO concentrations measured elsewhere in the living room near the time of shutoff. Figure 102c shows the $\mathrm{CO}$ concentration measured in each room of the house over the test. Figure 102d shows the calculated $\mathrm{COHb}$ profiles of simulated house occupants in each room of the house.

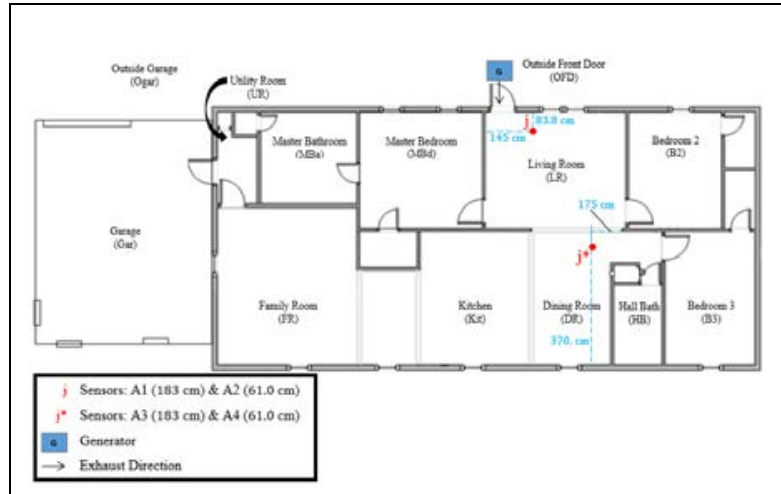

Figure 102a. Generator, exhaust direction, and sensor array layout during Case 48 Test 1 - G7a UL 2201.

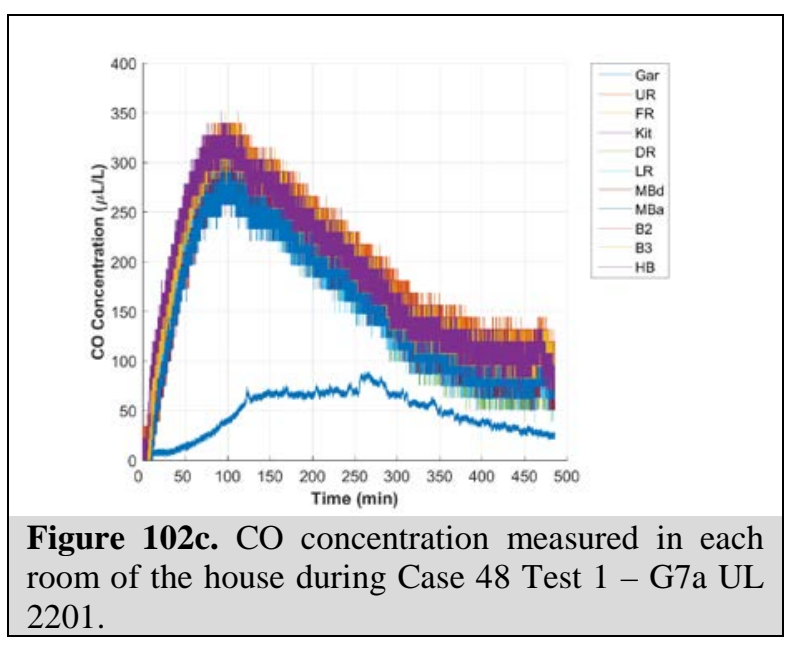

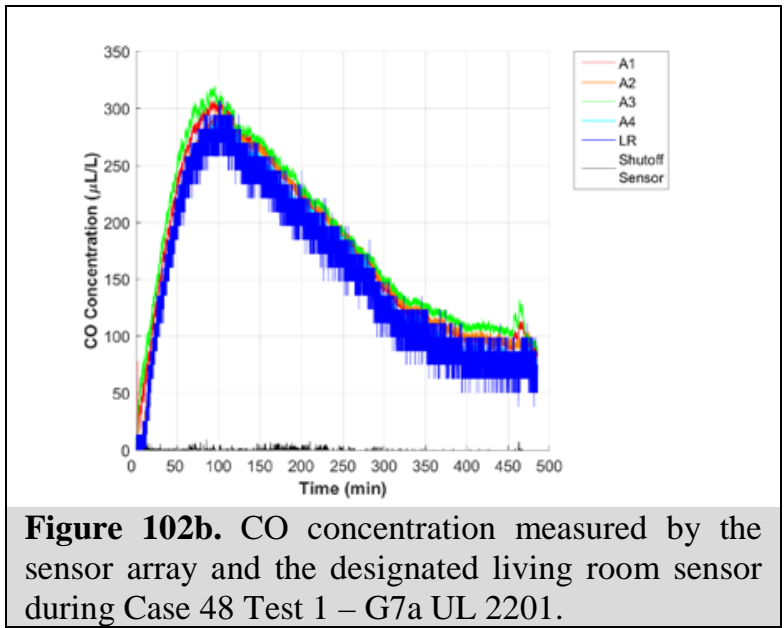

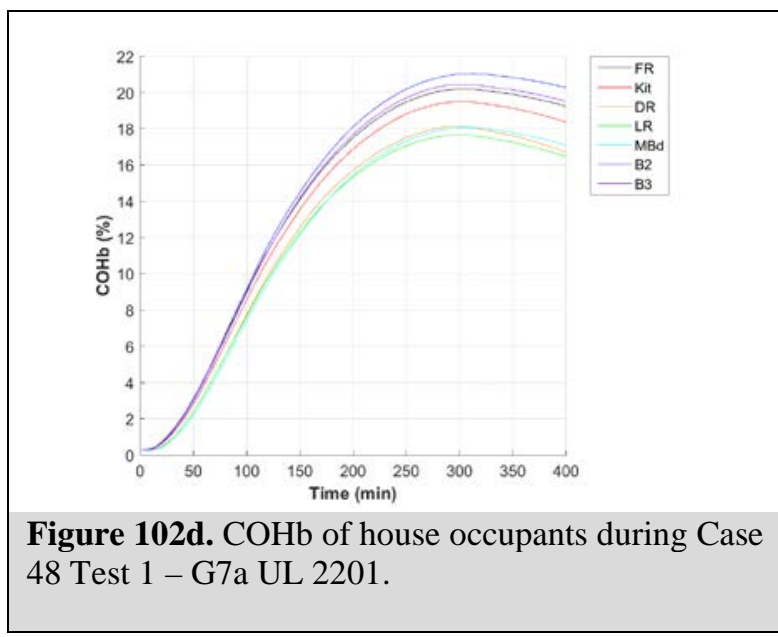




\section{Case 48 Test 2 - G7b UL 2201}

The following figures illustrate the results for Case 48 Test 2 applying the UL 2201 shutoff criteria. The G7b generator was fully fueled, positioned outside the front door (centered, $30 \mathrm{~cm}$ away from plane of the door, with the exhaust facing the door) and tested at $100 \%$ load (7,000 W). The front door was open $10 \mathrm{~cm}$ during the test. The shutoff sensor did not meet the criteria to activate the algorithm that triggers the generator shutoff and the generator ran out of fuel after approximately 426 min. Figure 103a shows the test house layout with generator location, generator exhaust direction, and sensor array location. Figure 103b shows the CO concentration measured in the living room (by the dedicated room sensor), by two sensors located in the living room near the front door (A1: height $183 \mathrm{~cm}, \mathrm{~A} 2$ : height $61 \mathrm{~cm}$ ), by the other two sensors located on the living room-dining room boundary (A3: height $183 \mathrm{~cm}$, A4: height $61 \mathrm{~cm}$ ), and by the shutoff sensor located on the generator near the generator's electrical panel. Figure 103c shows the CO concentration measured in each room of the house over the test. Figure 103d shows the CO concentration in the house with each room represented by a single line, which is composed of both a 'solid-line' pattern (showing measured CO concentration) and a 'dotted-line with a symbol' pattern (showing projected CO concentration). Figure 103e shows the calculated COHb profiles of simulated house occupants in each room of the house.

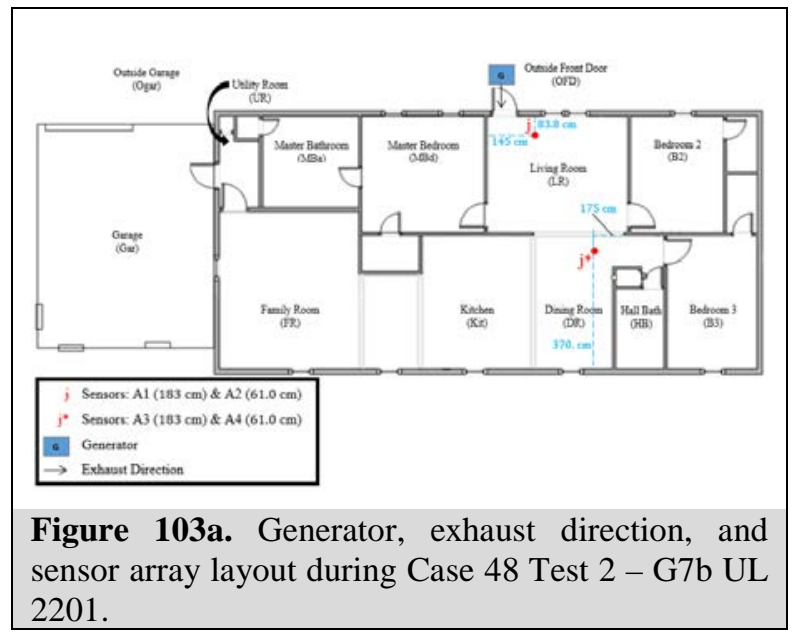

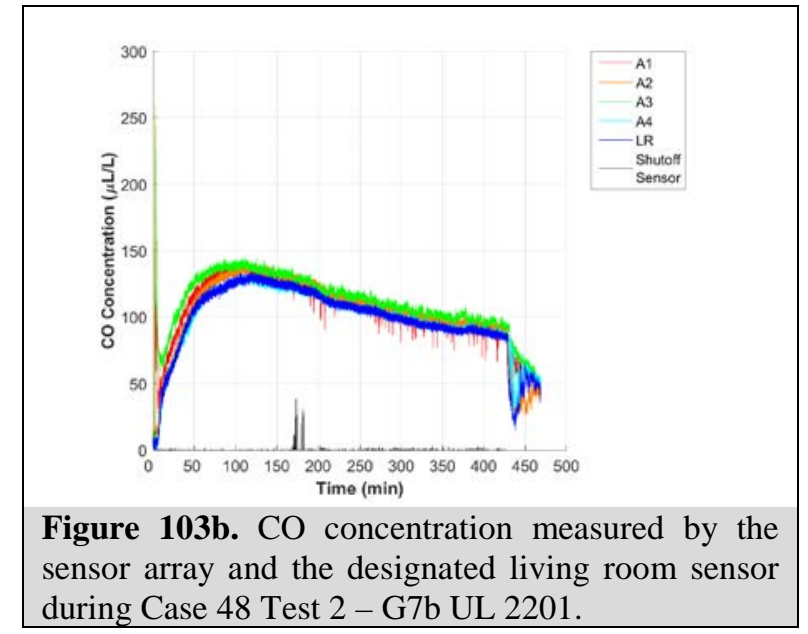




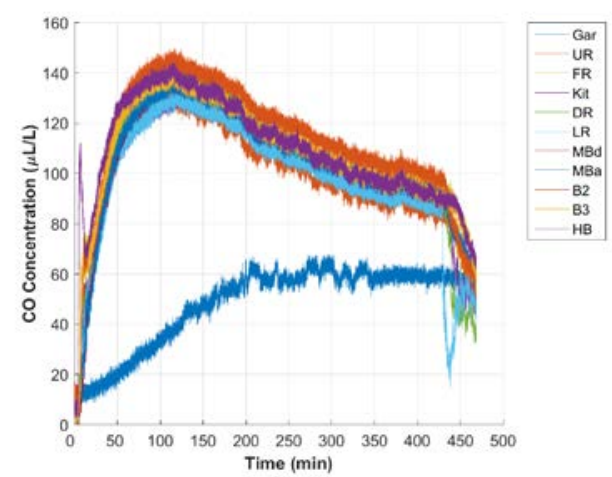

Figure 103c. CO concentration measured in each room of the house during Case 48 Test 2 - G7b UL 2201.

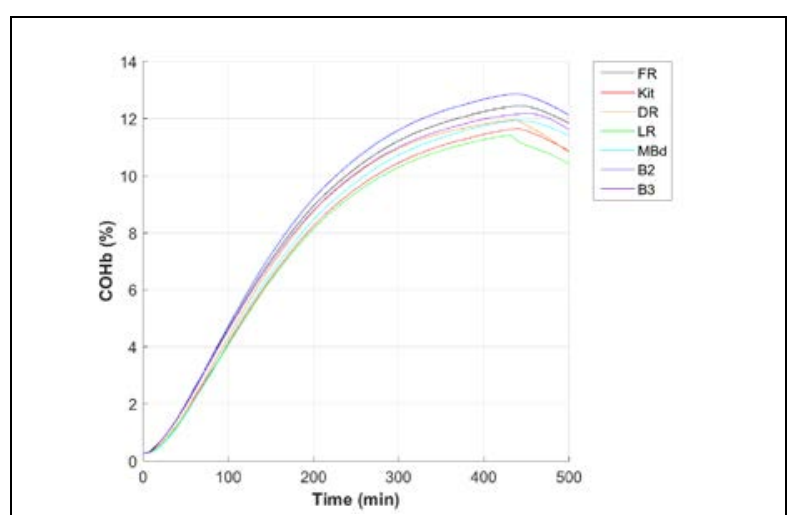

Figure 103e. $\mathrm{COHb}$ of house occupants during Case 48 Test 2 - G7b UL 2201.

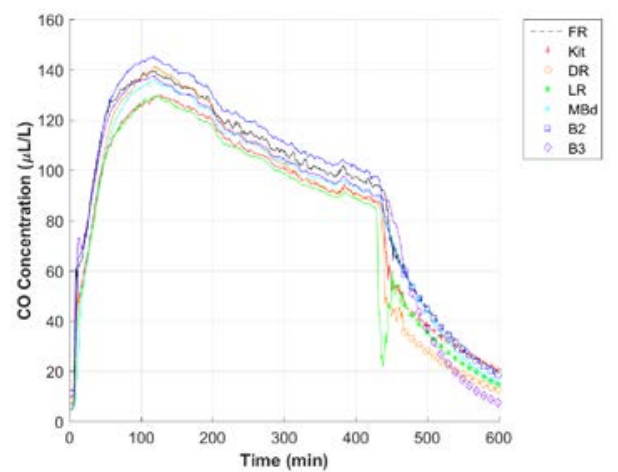

Figure 103d. CO concentration (measured \& projected) in the house during Case 48 Test 2 - G7b UL 2201. 


\section{Case 51 Test 1 - G6 G300}

The following figures illustrate the results for Case 51 Test 1 applying the G300 shutoff criteria. The G6 generator was fully fueled, positioned outside the front door (155 cm away from plane of the door, with the exhaust facing the door), and tested at $100 \%$ load $(6,000 \mathrm{~W})$. The front door was open $10 \mathrm{~cm}$ during the test. The shutoff sensor was not activated, and the generator was manually shutdown after approximately 193 min due to COHb levels $>70 \%$ in main zones of the house. Due to signal interference, the data for Bedroom 2 has not been included in the figures (Figure 104c and 104d) Figure 104a shows the test house layout with generator location, generator exhaust direction, and sensor array location. Figure 104b shows the CO concentration measured in the living room (by the dedicated room sensor), by two sensors located in the living room near the front door (A1: height $183 \mathrm{~cm}, \mathrm{A2}$ : height $61 \mathrm{~cm}$ ), by the other two sensors located outside the front door, halfway between the generator and the door (A3: height $183 \mathrm{~cm}$, A4: height $61 \mathrm{~cm}$ ), and by the shutoff sensor located on the generator near the generator's electrical panel. Figure 104c shows the $\mathrm{CO}$ concentration measured in each room of the house over the test. Figure 104d shows the calculated COHb profiles of simulated house occupants in each room of the house.

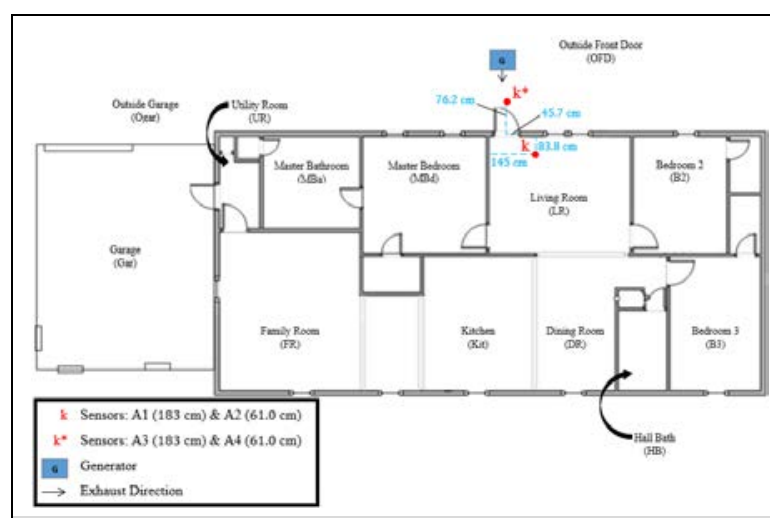

Figure 104a. Generator, exhaust direction, and sensor array layout during Case 51 Test 1 - G6 G300.

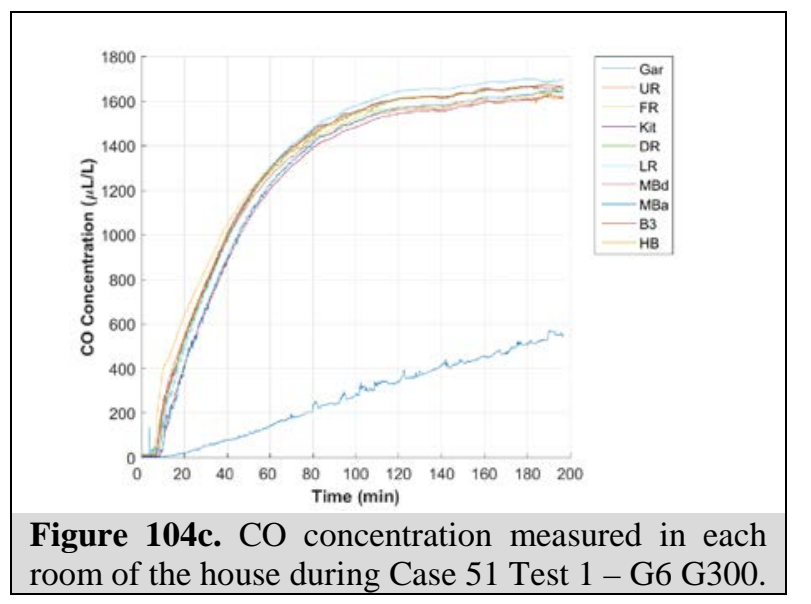

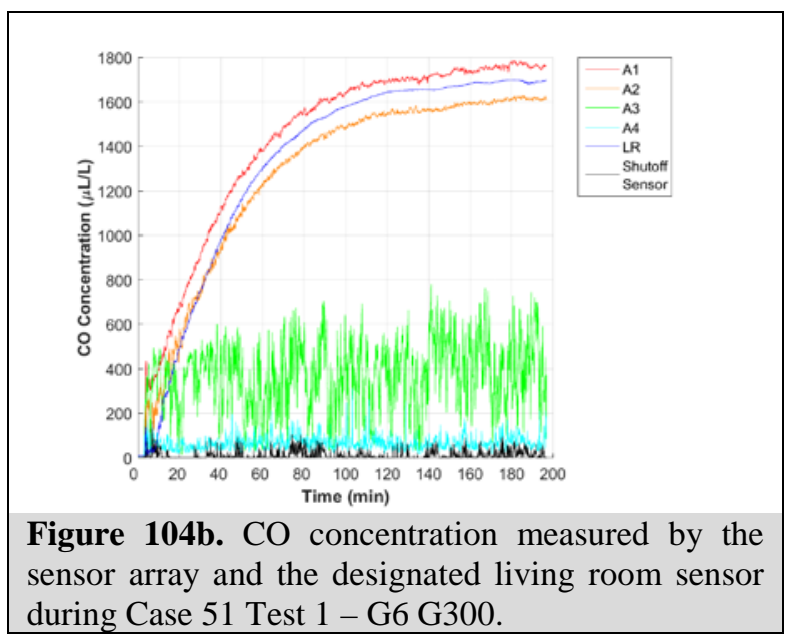

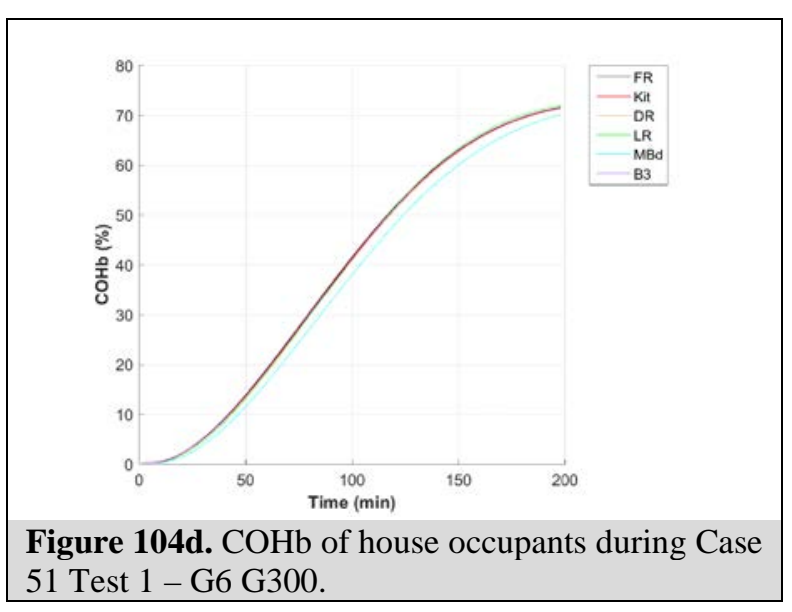




\section{Case 51 Test 2 - G6 G300}

The following figures illustrate the results for Case 51 Test 2 applying the G300 shutoff criteria. The G6 generator was fully fueled, positioned outside the front door (155 cm away from plane of the door, with the exhaust facing the door), and tested at $100 \%$ load $(6,000 \mathrm{~W})$. The front door was open $10 \mathrm{~cm}$ during the test. The shutoff sensor did not meet the criteria to activate the algorithm that triggers the generator shutoff and the generator ran out of fuel after approximately $372 \mathrm{~min}$. Figure 105a shows the test house layout with generator location, generator exhaust direction, and sensor array location. Figure 105b shows the CO concentration measured in the living room (by the dedicated room sensor), by two sensors located in the living room near the front door (A1: height $183 \mathrm{~cm}, \mathrm{A2}$ : height $61 \mathrm{~cm}$ ), and by the other two sensors located outside the front door, halfway between the generator and the door (A3: height $183 \mathrm{~cm}$, A4: height $61 \mathrm{~cm}$ ). The "Shutoff Sensor" data is not shown due to maintenance of the equipment for that measurement at the time of the test. Figure 105c shows the CO concentration measured in each room of the house over the test. Figure 105d shows the calculated COHb profiles of simulated house occupants in each room of the house.

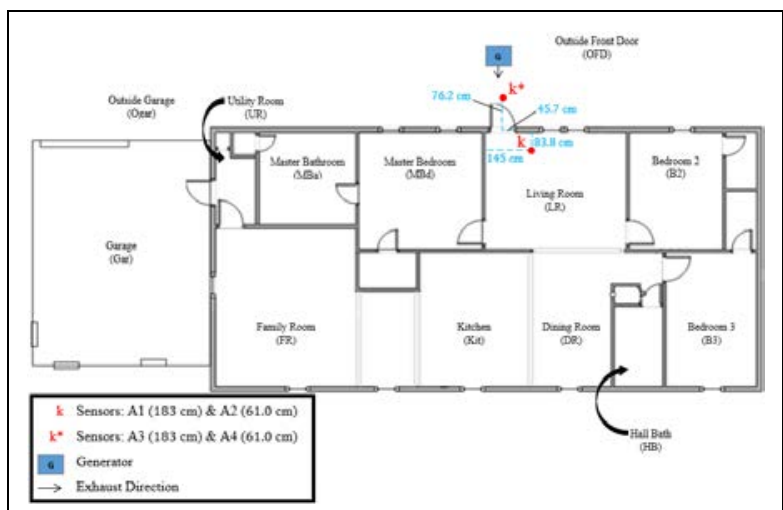

Figure 105a. Generator, exhaust direction, and sensor array layout during Case 51 Test 2 - G6 G300.

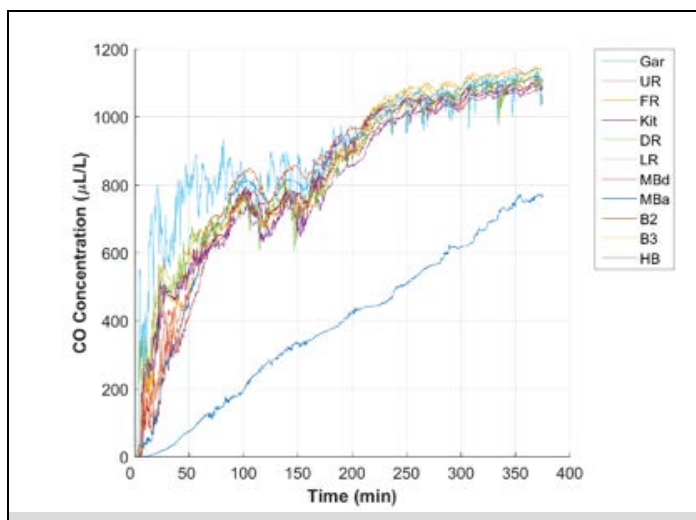

Figure 105c. CO concentration measured in each room of the house during Case 51 Test 2 - G6 G300.

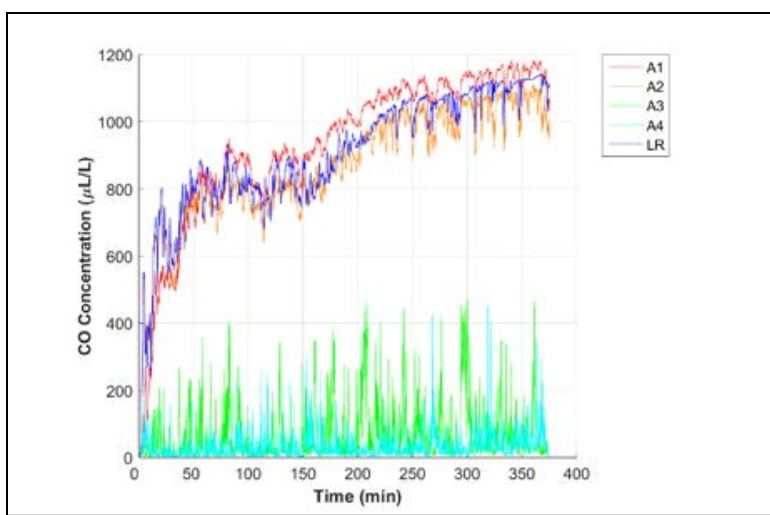

Figure 105b. CO concentration measured by the sensor array and the designated living room sensor during Case 51 Test 2 - G6 G300.

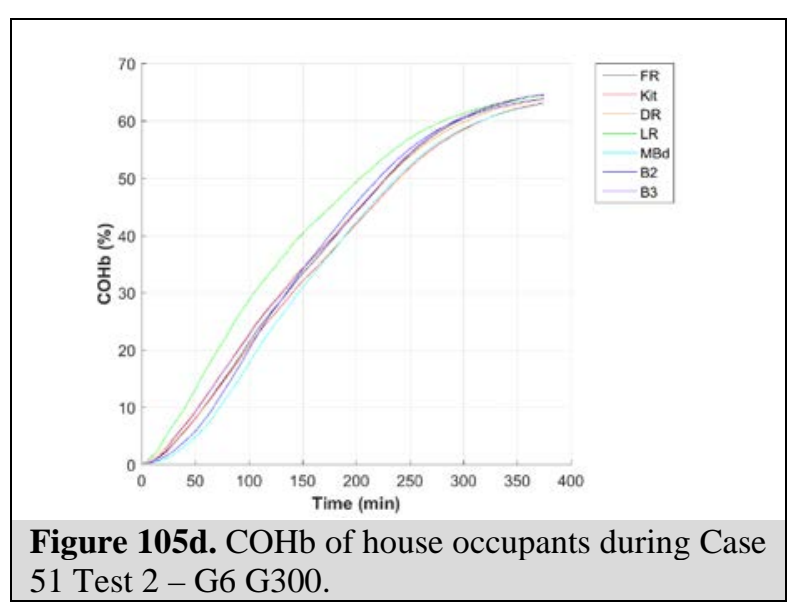




\section{Case 51 Test 3 - G6 G300}

The following figures illustrate the results for Case 51 Test 3 applying the G300 shutoff criteria. The G6 generator was fully fueled, positioned outside the front $(155 \mathrm{~cm}$ away from plane of the door, with the exhaust facing the door), and tested at $100 \%$ load $(6,000 \mathrm{~W})$. The front door was open $10 \mathrm{~cm}$ during the test. The shutoff sensor did not meet the criteria to activate the algorithm that triggers the generator shutoff; the generator ran out of fuel after approximately 322 min. Figure 106a shows the test house layout with generator location, generator exhaust direction, and sensor array location. Figure 106b shows the CO concentration measured in the living room (by the dedicated room sensor), by two sensors located in the living room near the front door (A1: height $183 \mathrm{~cm}, \mathrm{~A} 2$ : height $61 \mathrm{~cm}$ ), and by the other two sensors located outside the front door, halfway between the generator and the door (A3: height $183 \mathrm{~cm}$, A4: height $61 \mathrm{~cm}$ ). The "Shutoff Sensor" data is not shown due to maintenance of the equipment for that measurement at the time of the test. Figure 106c shows the CO concentration measured in each room of the house over the test. Figure 106d shows the calculated $\mathrm{COHb}$ profiles of simulated house occupants in each room of the house.

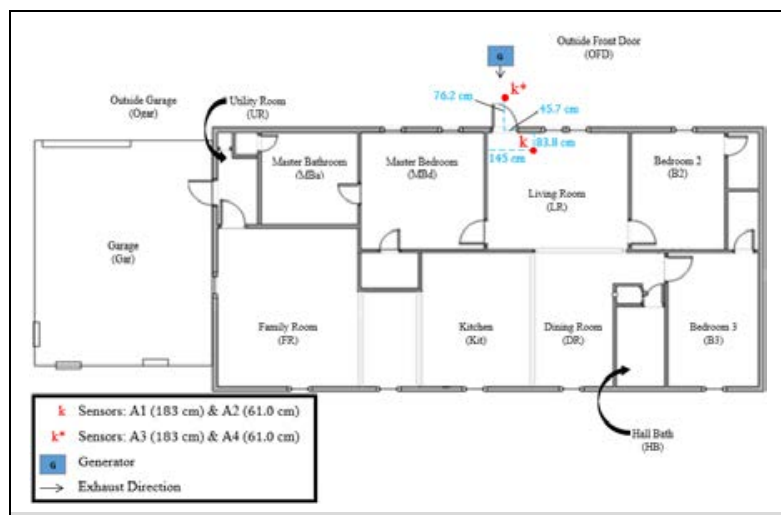

Figure 106a. Generator, exhaust direction, and sensor array layout during Case 51 Test 3 - G6 G300.

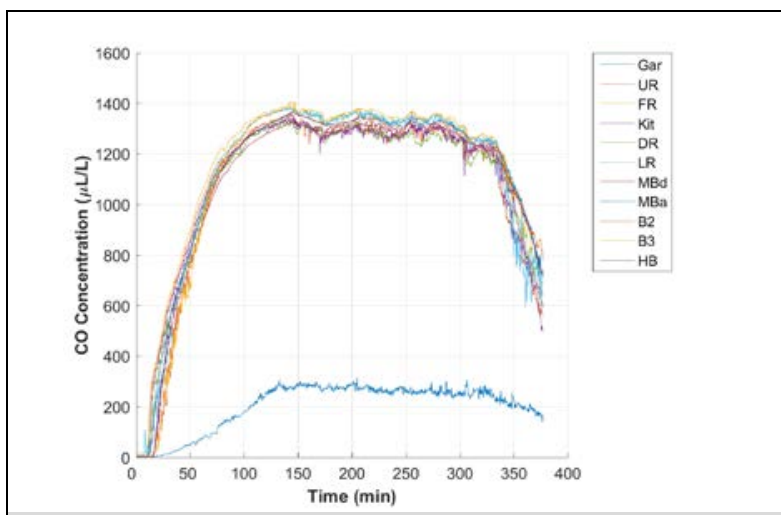

Figure 106c. $\mathrm{CO}$ concentration measured in each room of the house during Case 51 Test 3 - G6 G300.

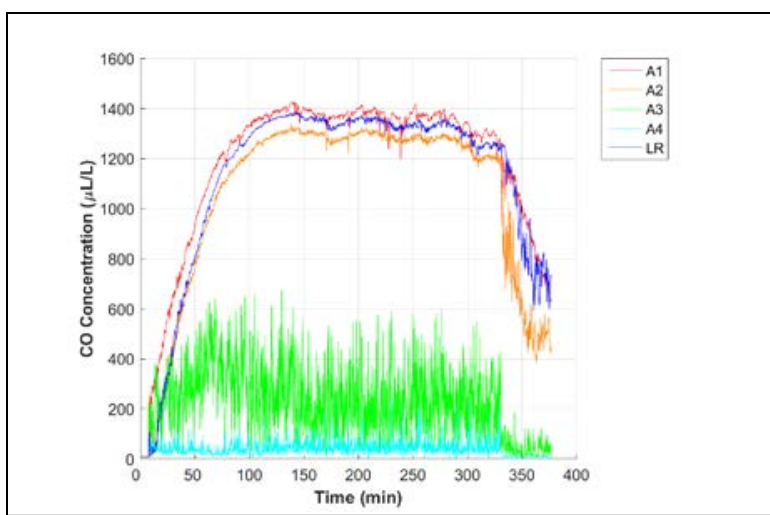

Figure 106b. CO concentration measured by the sensor array and the designated living room sensor during Case 51 Test 3 - G6 G300.

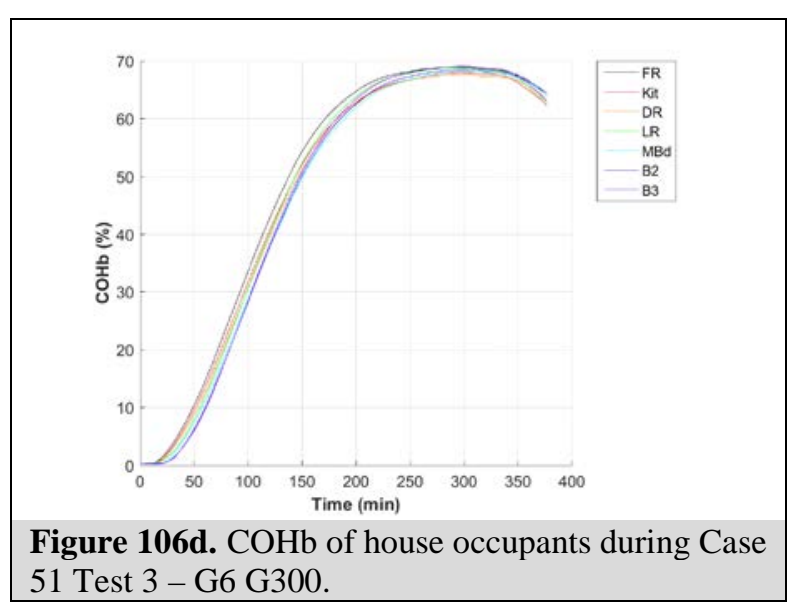




\section{Case 51 Test 1 - G7b UL 2201}

The following figures illustrate the results for Case 51 Test 1 applying the UL 2201 shutoff criteria. The G7b generator was fully fueled, positioned outside the front door (155 cm away from plane of the door, with the exhaust facing the door), and tested at $100 \%$ load (7,000 W). The front door was open $10 \mathrm{~cm}$ during the test. The shutoff sensor did not meet the criteria to activate the algorithm that triggers the generator shutoff and the generator ran out of fuel after approximately $360 \mathrm{~min}$. Figure 107a shows the test house layout with generator location, generator exhaust direction, and sensor array location. Figure 107b shows the CO concentration measured in the living room (by the dedicated room sensor), by two sensors located in the living room near the front door (A1: height $183 \mathrm{~cm}, \mathrm{~A} 2$ : height $61 \mathrm{~cm}$ ), by the other two sensors located outside the front door, halfway between the generator and the door (A3: height $183 \mathrm{~cm}, \mathrm{~A} 4$ : height $61 \mathrm{~cm}$ ), and by the shutoff sensor located on the generator near the generator's electrical panel. Figure 107c shows the CO concentration measured in each room of the house over the test. Figure 107d shows the $\mathrm{CO}$ concentration in the house with each room represented by a single line, which is composed of both a 'solid-line' pattern (showing measured CO concentration) and a 'dotted-line with a symbol' pattern (showing projected CO concentration). Figure 107e shows the calculated $\mathrm{COHb}$ profiles of simulated house occupants in each room of the house.

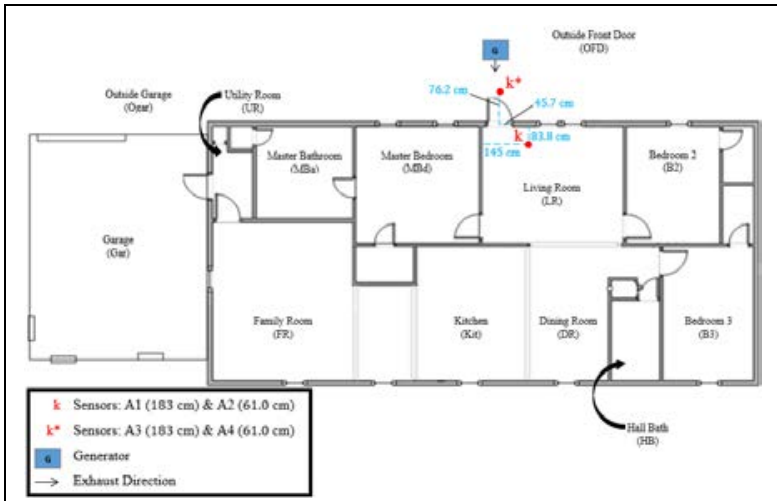

Figure 107a. Generator, exhaust direction, and sensor array layout during Case 51 Test 1 - G7b UL 2201.

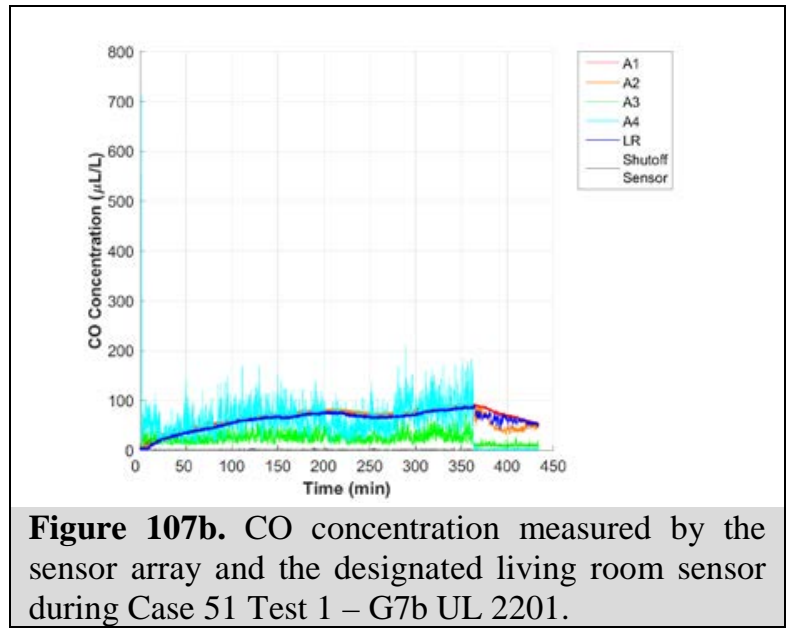




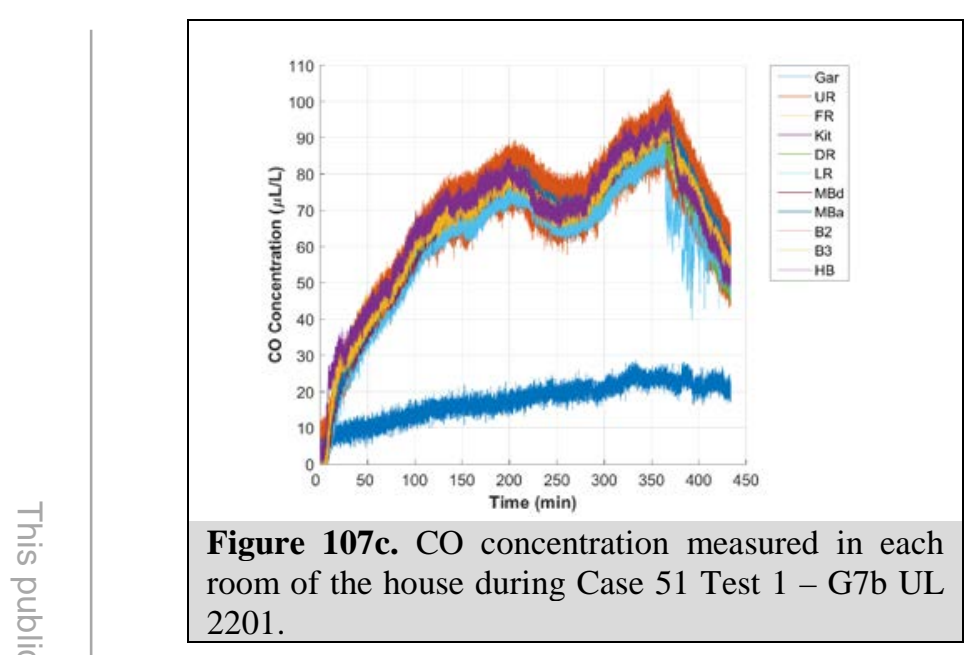

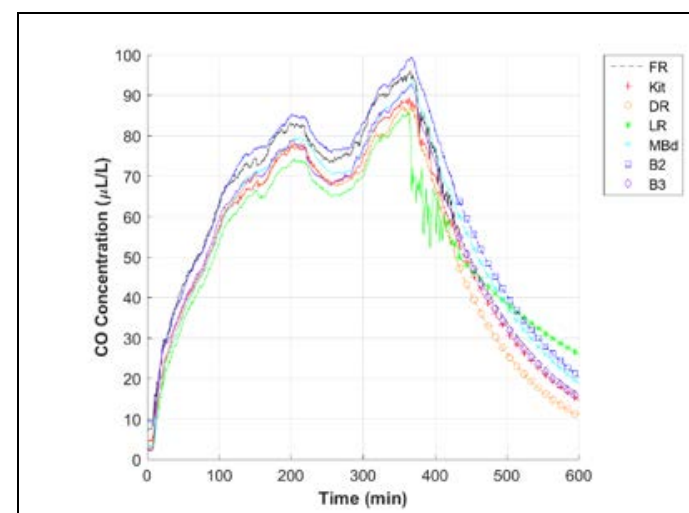

Figure 107d. CO concentration (measured \& projected) in the house during Case 51 Test $1-\mathrm{G} 7 \mathrm{~b}$ UL 2201.

193 


\section{Case 52 Test 1 - G6 G300}

The following figures illustrate the results for Case 52 Test 1 applying the G300 shutoff criteria. The G6 generator was fully fueled, positioned outside the front door (305 cm away from plane of the door, with the exhaust facing the door), and tested at $100 \%$ load $(6,000 \mathrm{~W})$. The front door was open $10 \mathrm{~cm}$ during the test. The shutoff sensor did not meet the criteria to activate the algorithm that triggers the generator shutoff and the generator ran out of fuel after approximately 352 min. Figure 108a shows the test house layout with generator location, generator exhaust direction, and sensor array location. Figure 108b shows the CO concentration measured in the living room (by the dedicated room sensor), by two sensors located in the living room near the front door (A1: height $183 \mathrm{~cm}, \mathrm{~A} 2$ : height $61 \mathrm{~cm}$ ), by the other two sensors located outside the front door, halfway between the generator and the door (A3: height $183 \mathrm{~cm}$, A4: height $61 \mathrm{~cm}$ ), and by the shutoff sensor located on the generator near the generator's electrical panel. Figure 108c shows the CO concentration measured in each room of the house over the test. Figure 108d shows the calculated $\mathrm{COHb}$ profiles of simulated house occupants in each room of the house.

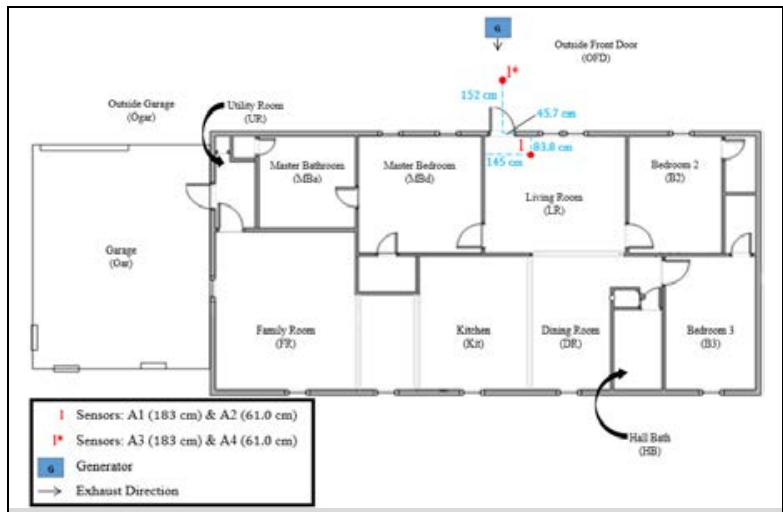

Figure 108a. Generator, exhaust direction, and sensor array layout during Case 52 Test 1 - G6 G300.

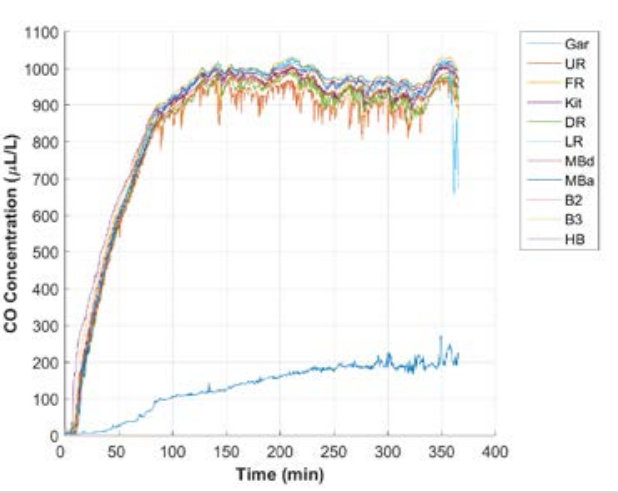

Figure 108c. CO concentration measured in each room of the house during Case 52 Test 1 - G6 G300.

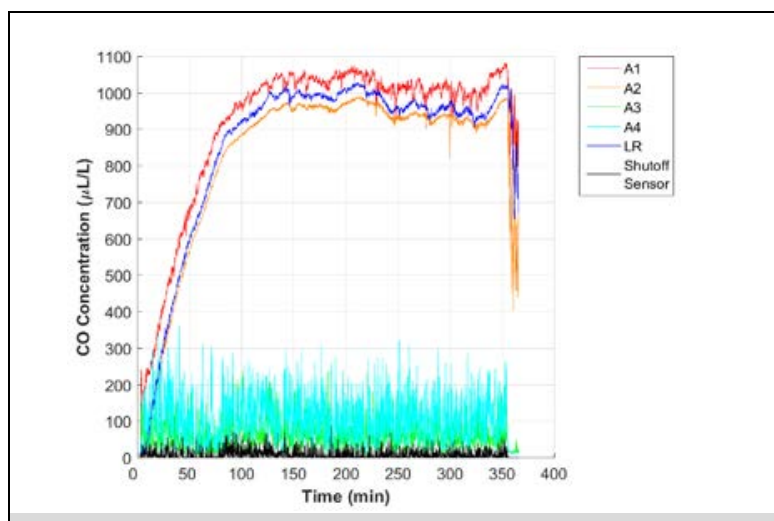

Figure 108b. CO concentration measured by the sensor array and the designated living room sensor during Case 52 Test 1 - G6 G300.

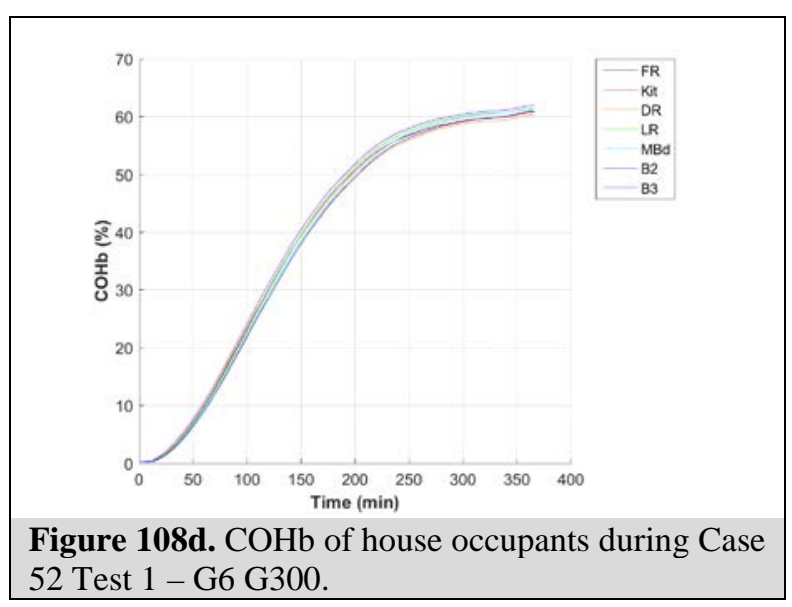




\section{Case 52 Test 1 - G7b UL 2201}

The following figures illustrate the results for Case 52 Test 1 applying the UL 2201 shutoff criteria. The G7b generator was fully fueled, positioned outside the front door $(305 \mathrm{~cm}$ away from plane of the door, with the exhaust facing the door), and tested at $100 \%$ load (7,000 W). The front door was open $10 \mathrm{~cm}$ during the test. The shutoff sensor did not meet the criteria to activate the algorithm that triggers the generator shutoff and the generator ran out of fuel after approximately $427 \mathrm{~min}$. Figure 109a shows the test house layout with generator location, generator exhaust direction, and sensor array location. Figure 109b shows the CO concentration measured in the living room (by the dedicated room sensor), by two sensors located in the living room near the front door (A1: height $183 \mathrm{~cm}, \mathrm{~A} 2$ : height $61 \mathrm{~cm}$ ), by the other two sensors located outside the front door, halfway between the generator and the door (A3: height $183 \mathrm{~cm}$, A4: height $61 \mathrm{~cm}$ ), and by the shutoff sensor located on the generator near the generator's electrical panel. Figure 109c shows the CO concentration measured in each room of the house over the test. Figure 109d shows the calculated $\mathrm{COHb}$ profiles of simulated house occupants in each room of the house.

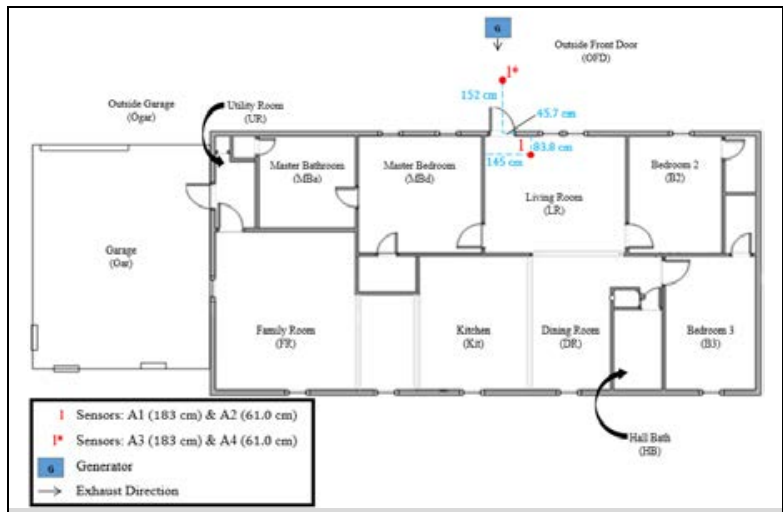

Figure 109a. Generator, exhaust direction, and sensor array layout during Case 52 Test 1 - G7b UL 2201.

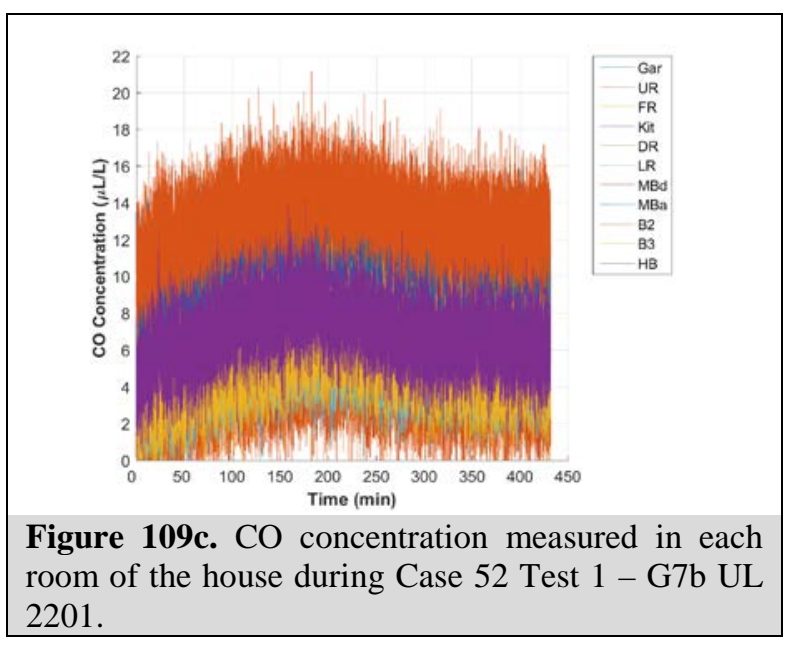

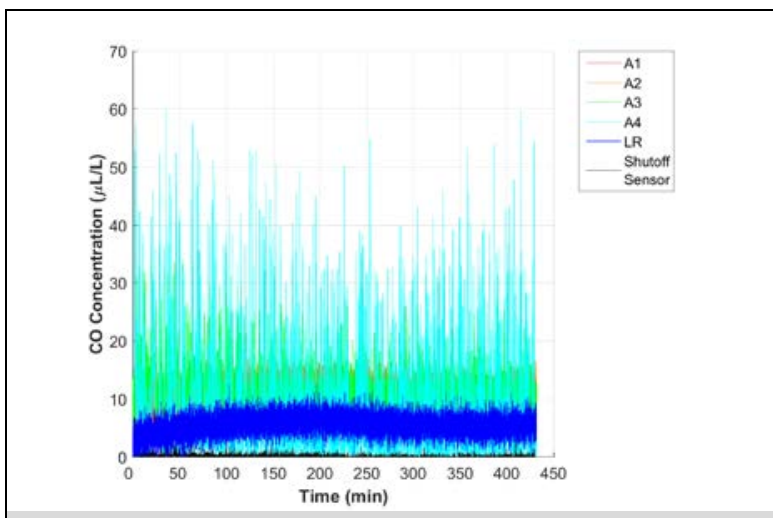

Figure 109b. CO concentration measured by the sensor array and the designated living room sensor during Case 52 Test 1 - G7b UL 2201.

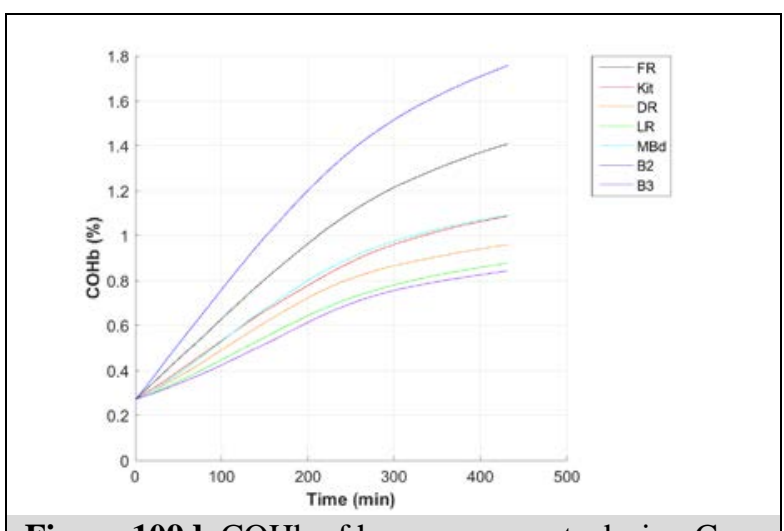

Figure 109d. COHb of house occupants during Case 52 Test 1 - G7b UL 2201. 


\section{Case 53 Test 1 - G6 G300}

The following figures illustrate the results for Case 53 Test 1 applying the G300 shutoff criteria. The G6 generator was fully fueled, positioned outside the front door (457 cm away from plane of the door, with the exhaust facing the door), and tested at $100 \%$ load $(6,000 \mathrm{~W})$. The front door was open $10 \mathrm{~cm}$ during the test. The shutoff sensor did not meet the criteria to activate the algorithm that triggers the generator shutoff; the generator ran out of fuel after approximately 359 min. Figure 110a shows the test house layout with generator location, generator exhaust direction, and sensor array location. Figure 110b shows the CO concentration measured in the living room (by the dedicated room sensor), by two sensors located in the living room near the front door (A1: height $183 \mathrm{~cm}, \mathrm{~A} 2$ : height $61.0 \mathrm{~cm}$ ), by the other two sensors located outside the front door, halfway between the generator and the door (A3: height $183 \mathrm{~cm}$, A4: height $61.0 \mathrm{~cm}$ ), and by the shutoff sensor located on the generator near the generator's electrical panel. Figure 110c shows the CO concentration measured in each room of the house over the test. Figure 110d shows the $\mathrm{CO}$ concentration in the house with each room represented by a single line, which is composed of both a 'solid-line' pattern (showing measured CO concentration) and a 'dotted-line with a symbol' pattern (showing projected CO concentration). Figure 110e shows the calculated COHb profiles of simulated house occupants in each room of the house.

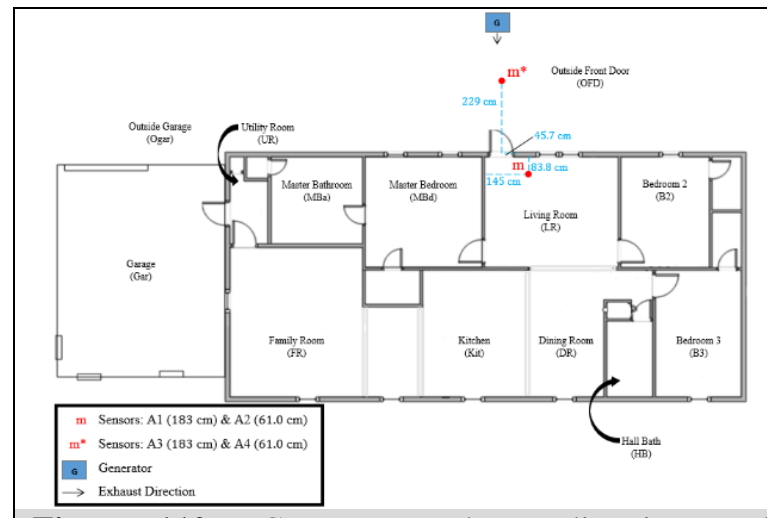

Figure 110a. Generator, exhaust direction, and sensor array layout during Case 53 Test 1 - G6 G300.

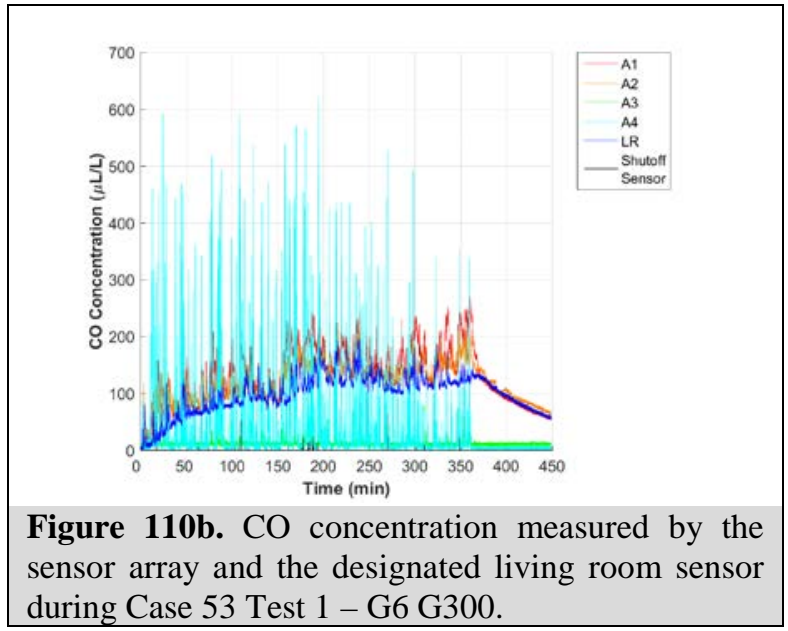




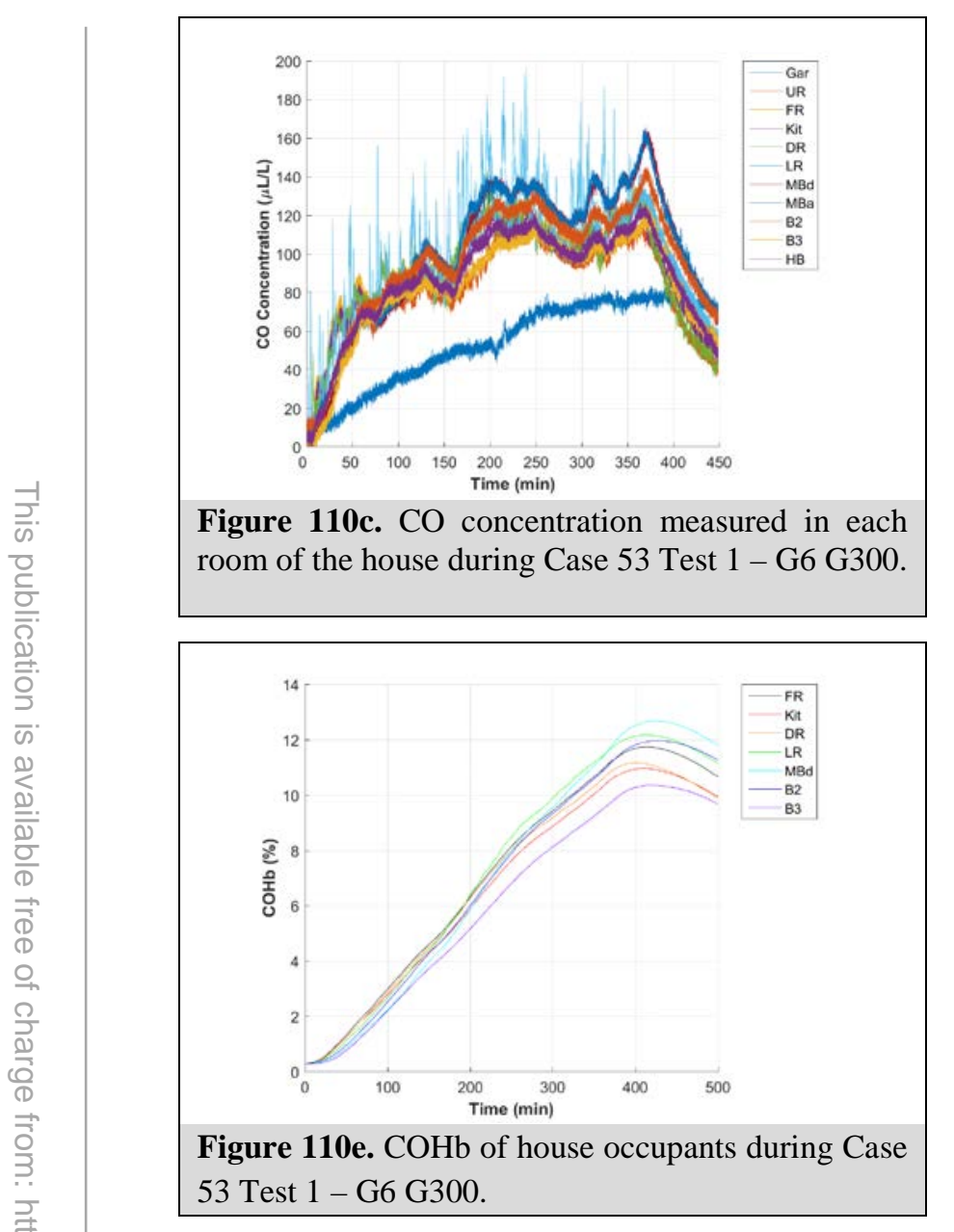

197 


\section{CONTAM Model Validation}

As discussed in the Introduction section of this report, a future study is planned to evaluate the impact of the generator shutoff requirements in the PGMA and UL voluntary standards on indoor CO exposure. Use of CONTAM for the planned study, however, will differ from how CONTAM was used to evaluate the impact of the reduced CO emissions rates in the NPR. As noted earlier, CONTAM generally assumes that the $\mathrm{CO}$ concentration in a zone is uniform within each zone, which was a reasonable assumption for the modeling effort for the NPR given that the generator run time was dependent only on the fuel consumption and capacity rather than being linked a single-point value of $\mathrm{CO}$ in a building zone. For the shutoff analysis, however, the assumption of uniform concentration may not be valid because the distribution of $\mathrm{CO}$ in the space where the generator is operating, particularly near the generator-mounted shutoff sensor, can be dependent on multiple factors. These factors include where the generator is located within the space, how the exhaust is oriented relative to nearby surfaces, and the velocity and temperature of the exhaust jet exiting the exhaust pipe. The non-uniformity of CO concentration is a critical factor because generators equipped with a CO safety shutoff device are intended to shut off in a timely manner when operated in an enclosed space. Extended run-times will result in increased CO mass emitted, which for a given set of ventilation and air leakage conditions in a building, will create higher $\mathrm{COHb}$ levels compared to cases where the generator shuts off more quickly. Therefore, the simulations that will be performed in the planned study to evaluate the impacts of shutoff requirements must account for the non-uniformity of CO observed in the testing as documented in the Test Results section.

To support these planned simulations, this section describes model validation efforts that were applied to some of the experiments described earlier in this report to establish how well the CONTAM model predicts CO levels in the garage and the house compared and to evaluate the impact of any differences between the predictions and measurements on calculated COHB profiles of simulated occupants. Based on these comparisons, options are examined to reduce the uncertainty in the CO levels predicted by CONTAM, such that they will be more reliable for estimating $\mathrm{COHb}$ profiles.

CONTAM (Dols and Polidoro 2015) is a multizone indoor air quality (IAQ) and airflow model developed in the Engineering Laboratory (EL) at NIST. The multizone modeling approach is implemented by constructing a building model as a network of elements describing the flow paths (HVAC ducts, doors, windows, cracks, etc.) between the zones (primarily rooms) of a building. The network nodes represent the zones, which are modeled with a hydrostatically varying pressure, and uniform temperature and pollutant concentration within each zone. After calculating the airflow between zones and the outdoors, zone pollutant concentrations are calculated by applying pollutant mass balance equations. CONTAM has been used to study a variety of residential IAQ issues in the past (e.g., Emmerich and Persily 1996, Emmerich et al. 2005). For more detail on the CONTAM model, see the user's manual which is available online along with the CONTAM program (https://www.nist.gov/el/energy-and-environment-division-73200/nist-multizonemodeling/software-tools/contam).

An absolute validation of a complex model, such as CONTAM, is impossible as there are infinite possible building models. However, several past studies (e.g., Emmerich and Nabinger (2001) and Emmerich et al. (2004)) have examined multizone IAQ model validation as reviewed in Emmerich 
(2001). Additionally, previous publications addressed the validation of the ability of the CONTAM model to predict CO levels due to operation of portable generators in the same NIST test house used in this study (Emmerich et al. 2013, Emmerich and Dols 2016).

The test house model was described in detail in NIST Technical Note 1781 (Emmerich et al. 2013). A graphical representation of the main floor of the test house as it appears in CONTAM is shown in Figure 111. The test house crawlspace and attic were included in the model but are not shown. The layout of the test house within CONTAM and the division of the zones were defined to represent the actual floorplan of the test house, therefore each zone typically represents one room although the kitchen, living, dining and family room spaces are combined into a single CONTAM zone, referred to as zone LFK, due to the lack of any partitions separating them.

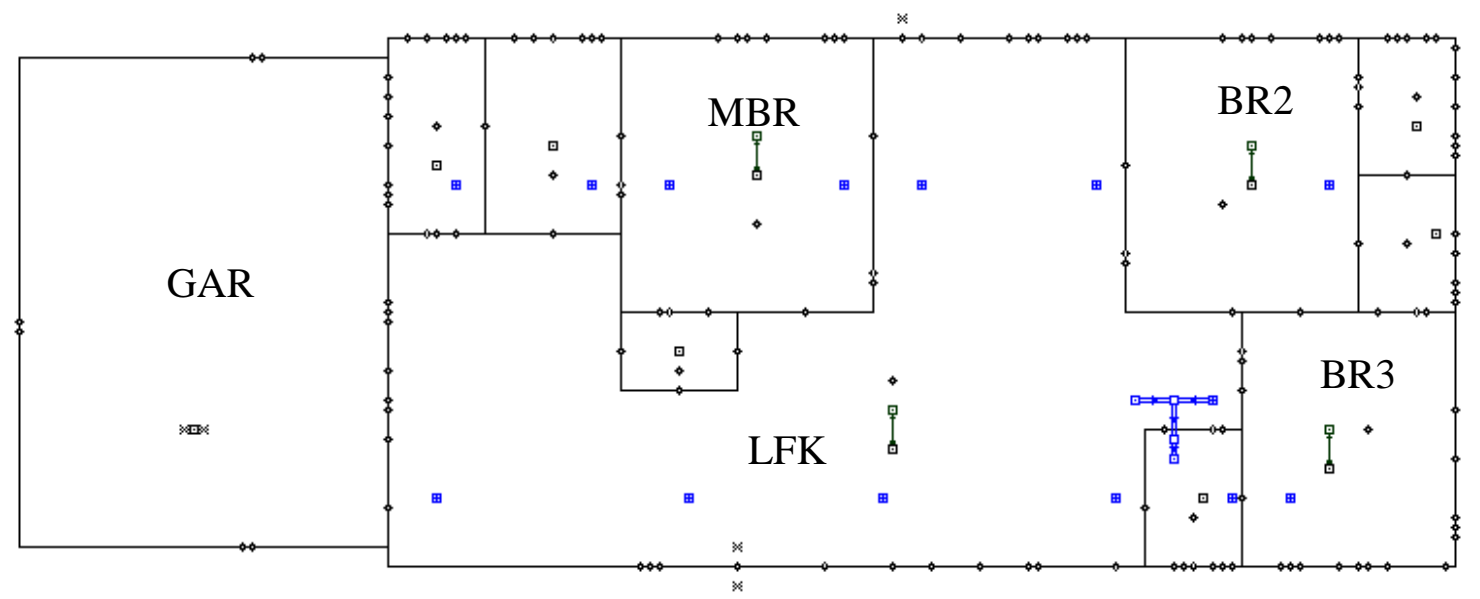

Figure 111 CONTAM Sketchpad Representation of Test House

Table 10 includes a summary of the validation tests conducted, including information on the generator tested and its applied electrical load (as \% of capacity), the generator location, the test house configuration (door and window positions), the average outdoor temperature and wind speed, and the date of the test. As shown in Table 10, thirteen validation cases were simulated under a variety of conditions including three that were repeated multiple times. The tests consisted of operating one of the generators in the house, garage or outside, and measuring the CO concentrations in the house and garage for up to 8 hours. The case and test numbers correspond to their numbers in Table 1 through Table 9. All the validation cases used the G300 shutoff criteria. Control networks, a feature of the CONTAM program to enable a variety of system control and operation functions, were used in the CONTAM models to simulate the generator shutoff sensor concentration and predict generator shutoff. Note that $\mathrm{COHb}$ values from the experiments and simulations (calculated per the equations in Appendix 2 of this report) were compared in addition to $\mathrm{CO}$ concentrations, as $\mathrm{COHb}$ is the key variable of interest in quantifying exposure. 
Table 10 Model Validation Tests Conducted

\begin{tabular}{|c|c|c|c|c|c|c|}
\hline $\begin{array}{l}\text { Case/Test } \\
\text { Number }\end{array}$ & Generator/load & $\begin{array}{l}\text { Test } \\
\text { Date }\end{array}$ & $\begin{array}{l}\text { Generator } \\
\text { Location }\end{array}$ & House setup notes & $\begin{array}{c}\text { Average } \\
\text { Outdoor } \\
\text { Temp } \\
\left({ }^{\circ} \mathrm{C}\right) \\
\end{array}$ & $\begin{array}{c}\text { Average } \\
\text { Wind } \\
\text { Speed } \\
\text { (m/s) } \\
\end{array}$ \\
\hline $5 / 2$ & G2/50 \% & $02 / 01 / 18$ & Kitchen & $\begin{array}{l}\text { All exterior doors closed, all windows closed, interior doors } \\
\text { open } 10 \mathrm{~cm}\end{array}$ & 8.2 & 2.6 \\
\hline $5 / 3$ & G2/50 \% & $02 / 02 / 18$ & Kitchen & $\begin{array}{l}\text { All exterior doors closed, all windows closed, interior doors } \\
\text { open } 10 \mathrm{~cm}\end{array}$ & -3.8 & 6.1 \\
\hline $5 / 4$ & G2/50 \% & $02 / 02 / 18$ & Kitchen & $\begin{array}{l}\text { All exterior doors closed, all windows closed, interior doors } \\
\text { open } 10 \mathrm{~cm}\end{array}$ & -4.4 & 5.9 \\
\hline $17 / 1$ & G6/100 \% & $04 / 11 / 18$ & Bedroom3 & $\begin{array}{l}\text { All exterior doors closed, Bed3 window open } 10 \mathrm{~cm} \text {, interior } \\
\text { doors fully open }\end{array}$ & 12.0 & 2.2 \\
\hline $23 / 1$ & G2/50 \% & 05/03/18 & $\begin{array}{l}\text { Utility } \\
\text { room }\end{array}$ & $\begin{array}{l}\text { Garage person door fully open, all windows closed, interior } \\
\text { doors fully open }\end{array}$ & 30.1 & 5.9 \\
\hline $32 / 1$ & G6/100 \% & 02/06/18 & Garage & $\begin{array}{l}\text { Garage bay door fully open, all windows closed, door from } \\
\text { garage to utility open } 10 \mathrm{~cm} \text {, interior doors fully open }\end{array}$ & 2.8 & 2.8 \\
\hline $32 / 2$ & G6/100 \% & 02/08/18 & Garage & $\begin{array}{l}\text { Garage bay door fully open, all windows closed, door from } \\
\text { garage to utility open } 10 \mathrm{~cm} \text {, interior doors fully open }\end{array}$ & -0.3 & 2.5 \\
\hline $32 / 3$ & G6/100 \% & 02/09/18 & Garage & $\begin{array}{l}\text { Garage bay door fully open, all windows closed, door from } \\
\text { garage to utility open } 10 \mathrm{~cm} \text {, interior doors fully open }\end{array}$ & 6.2 & 1.7 \\
\hline $40 / 1$ & G15/90 \% & $02 / 13 / 18$ & $\begin{array}{l}\text { Outside } \\
\text { garage }\end{array}$ & $\begin{array}{l}\text { Garage bay door fully open, all windows closed, door from } \\
\text { garage to utility open } 10 \mathrm{~cm} \text {, interior doors fully open }\end{array}$ & 4.7 & 1.9 \\
\hline $40 / 2$ & G15/90 \% & $02 / 14 / 18$ & $\begin{array}{c}\text { Outside } \\
\text { garage }\end{array}$ & $\begin{array}{l}\text { Garage bay door fully open, all windows closed, door from } \\
\text { garage to utility open } 10 \mathrm{~cm} \text {, interior doors fully open }\end{array}$ & 9.7 & 0.9 \\
\hline $51 / 1$ & G6/100 \% & 09/20/18 & $\begin{array}{c}\text { Outside } \\
\text { front door }\end{array}$ & $\begin{array}{l}\text { Front door open } 10 \mathrm{~cm} \text {, all windows closed, interior doors fully } \\
\text { open }\end{array}$ & 31.7 & 1.6 \\
\hline $51 / 2$ & G6/100 \% & 2/19/19 & $\begin{array}{l}\text { Outside } \\
\text { front door }\end{array}$ & $\begin{array}{l}\text { Front door open } 10 \mathrm{~cm} \text {, all windows closed, interior doors fully } \\
\text { open }\end{array}$ & 6.1 & 2.8 \\
\hline $51 / 3$ & G6/100 \% & $3 / 14 / 19$ & $\begin{array}{c}\text { Outside } \\
\text { front door }\end{array}$ & $\begin{array}{l}\text { Front door open } 10 \mathrm{~cm} \text {, all windows closed, interior doors fully } \\
\text { open }\end{array}$ & 24.8 & 4.2 \\
\hline
\end{tabular}




\section{Case \#5}

The first three validation cases were Case 5, Tests 2, 3 and 4. The G2 generator was positioned in the kitchen (30 cm from the master bedroom closet wall with the exhaust facing the dining room) and tested at $50 \%$ load. All exterior doors and windows were closed during these tests. Interior doors connected to the main living space were open $10 \mathrm{~cm}$. During Case 5 Test 2, the person door from the garage to the outdoors was unintentionally left open. However, since the COHb validation analysis did not include garage concentrations, this test was included because the $\mathrm{COHb}$ in the garage is not of particular interest. Based on generator characterization tests conducted at CPSC (Brookman 2018, included in Appendix 1), a constant CO emission rate of $345.0 \mathrm{~g} / \mathrm{h}$ was simulated in this case.

The observed and predicted CO concentrations for the main living space zones in the house are shown in Figures 112a, 112b and 112c for Case 5, Tests 2, 3, and 4 respectively (lines for the predictions and symbols for the measurements). For all the validation cases, the measured concentrations in the family room, dining room, kitchen and living room were averaged for comparison to the model predictions, which included a single well-mixed zone to represent those rooms. As seen in Figures 112a, 112b, and 112c, the results of the Case 5 tests were similar with peak CO concentrations in the main living zones typically ranging from around $200 \mu \mathrm{L} / \mathrm{L}$ to $500 \mu \mathrm{L} / \mathrm{L}$. Due to non-uniform mixing in the source zone, the simulated cases predicted generator shutoff 5 minutes to 10 minutes earlier than observed, resulting in underpredicted $\mathrm{CO}$ concentrations.

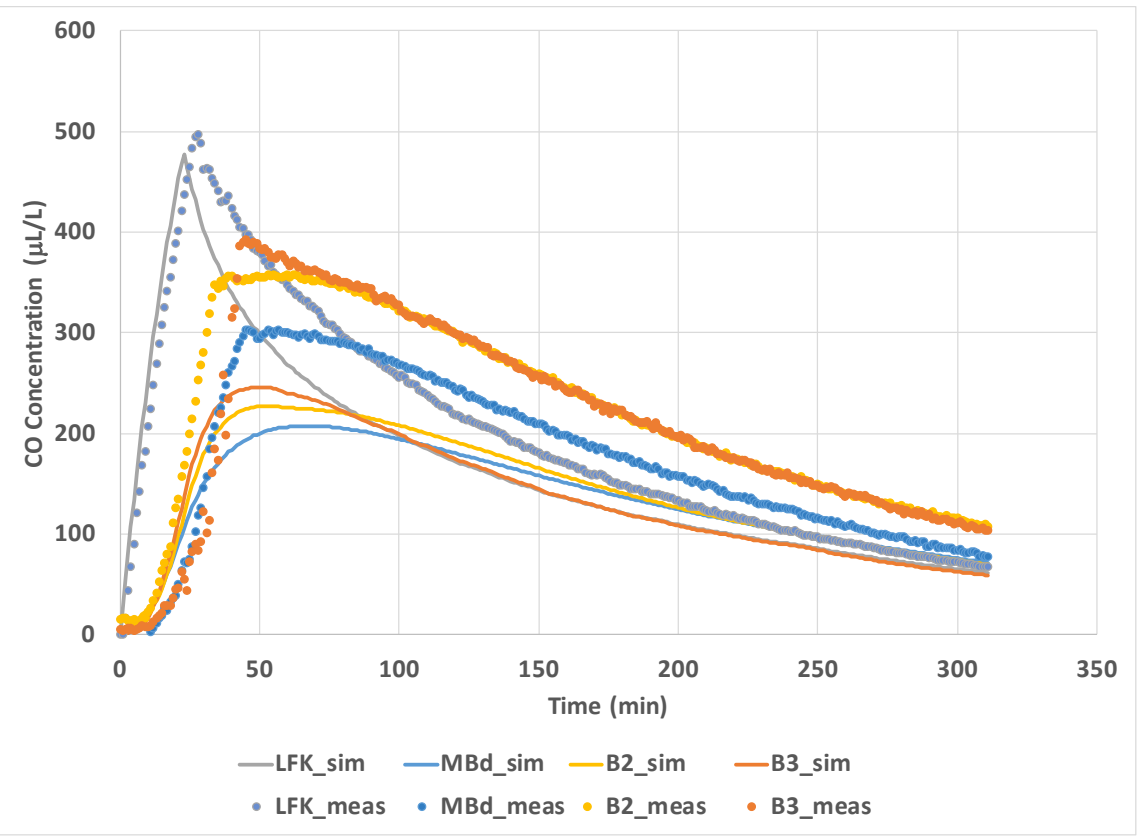

Figure 112a Observed and predicted CO concentrations in main living space zones for Case 5 Test 2 


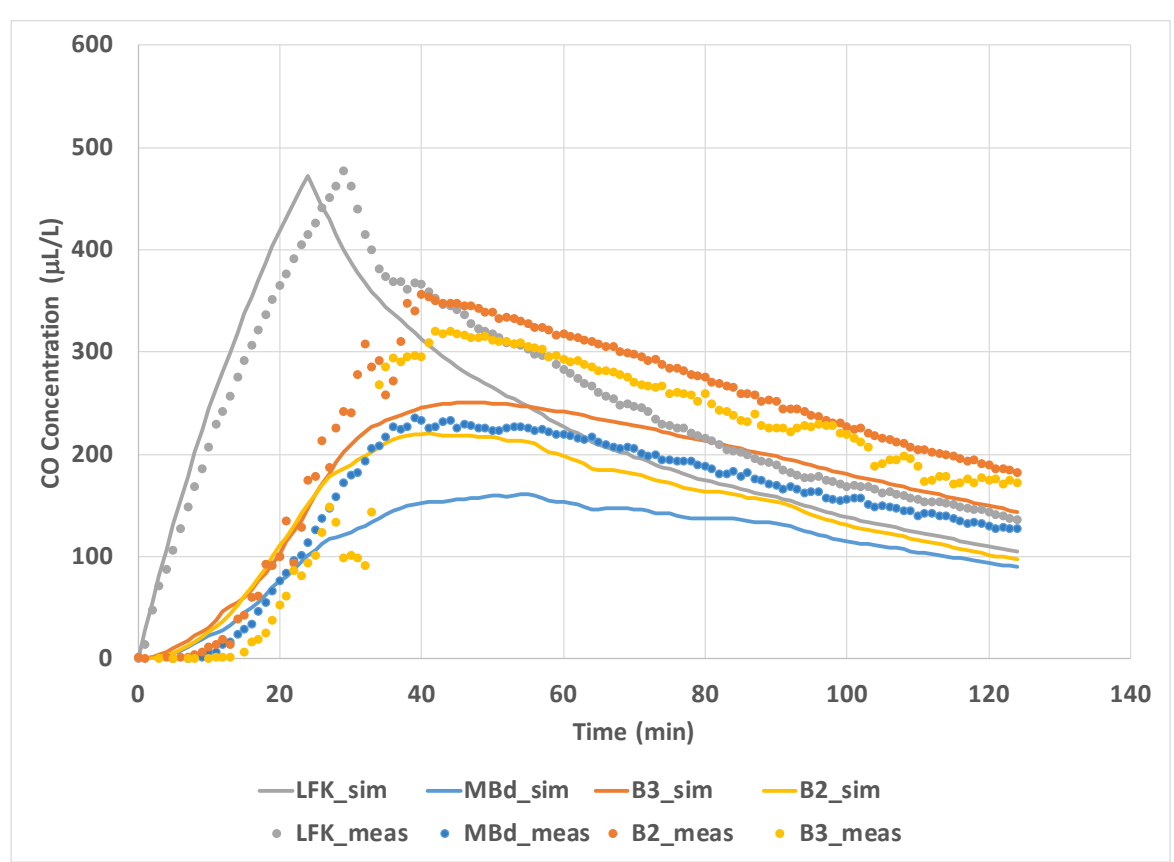

Figure 112b Observed and predicted CO concentrations in main living space zones for Case 5 Test 3

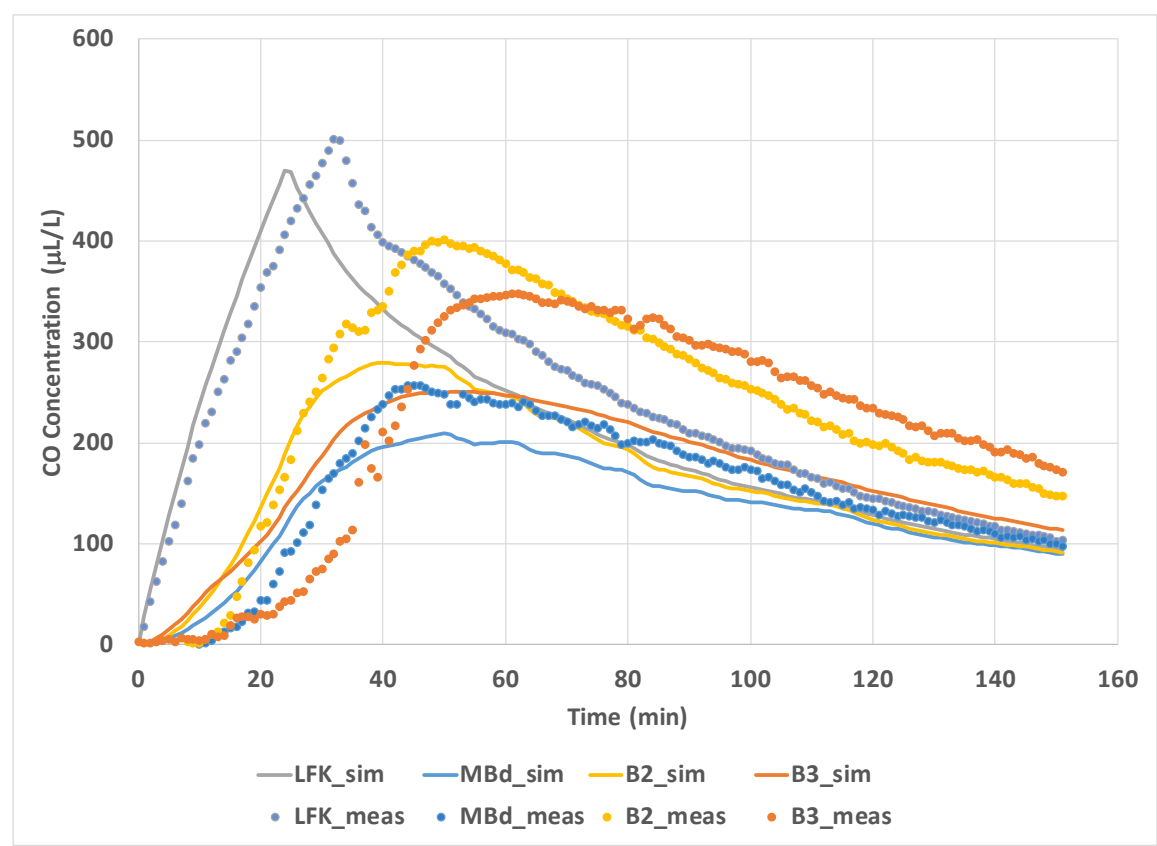

Figure 112c Observed and predicted CO concentrations in main living space zones for Case 5 Test 4

Since the objective of the planned CONTAM simulation study will be to predict $\mathrm{COHb}$ values for simulated occupants in the living spaces of houses during generator operation following the methodology of NIST Technical Note 1925 (Emmerich et al. 2016), \% COHb values were calculated from the observed and predicted CO concentrations for the main living space zones in the test house. The \% COHb values are shown in Figures 113a, 113b, and 113c for Case 5, Tests 2 , 3, and 4 respectively. As seen in the figures, the $\mathrm{COHb}$ results of all the Case 5 tests were similar, with values ranging between $5 \%$ to $15 \%$ at the 120 -minute mark (note that the tests were not all 
the same length, with Test 2 being much longer). Since the simulated cases predicted generator shutoff 5 minutes to 10 minutes earlier than observed resulting in underpredicted $\mathrm{CO}$ concentrations, the calculated $\mathrm{COHb}$ for the predictions (solid lines in the figures, referred to as "predicted COHb") were also lower than for the measurements (dashed lines in the figures, referred to "observed COHb").

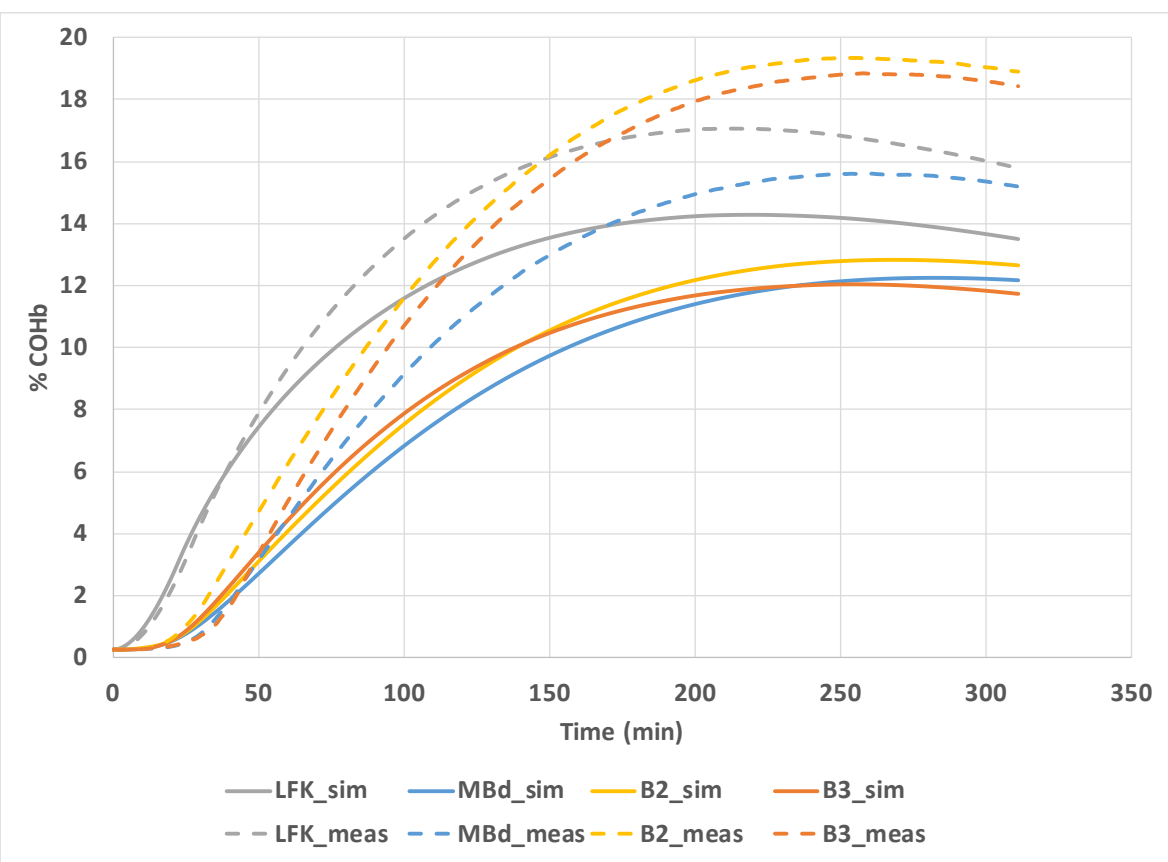

Figure 113a Observed and predicted COHb in main living space zones for Case 5 Test 2

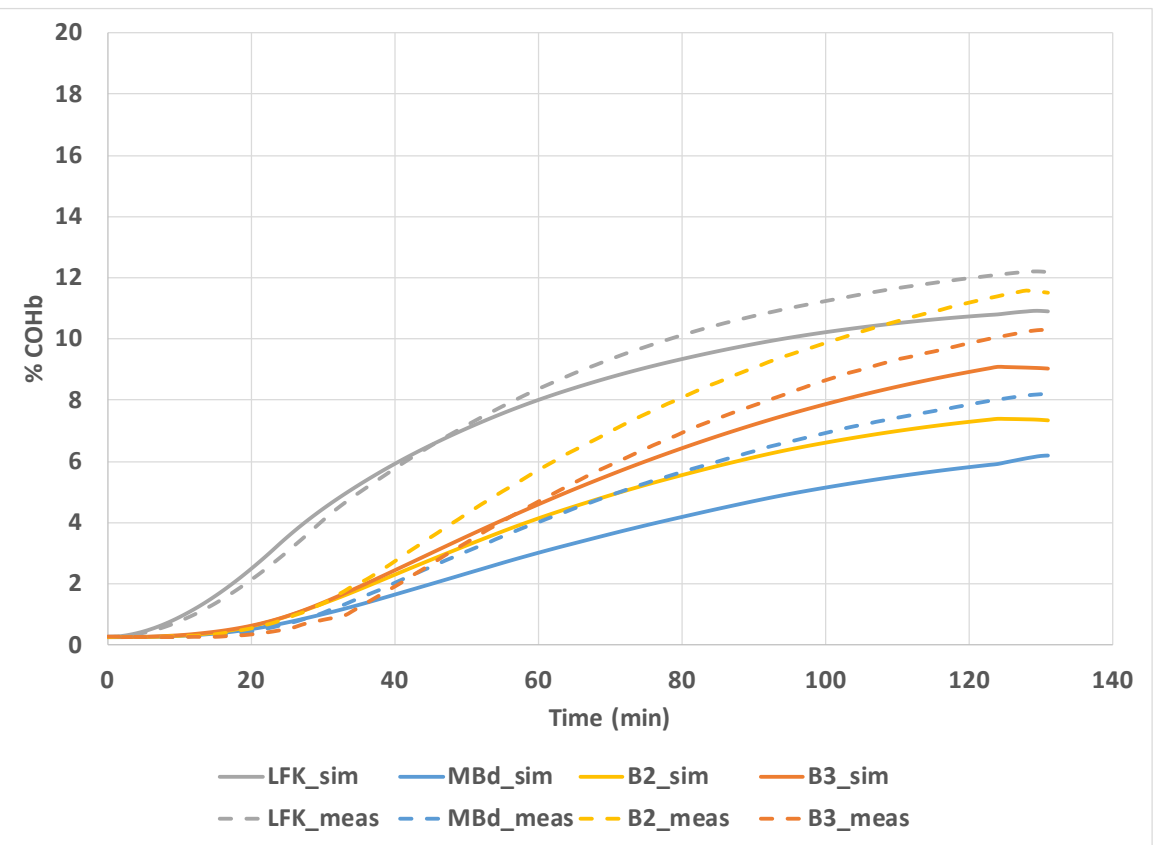

Figure 113b Observed and predicted COHb in main living space zones for Case 5 Test 3 


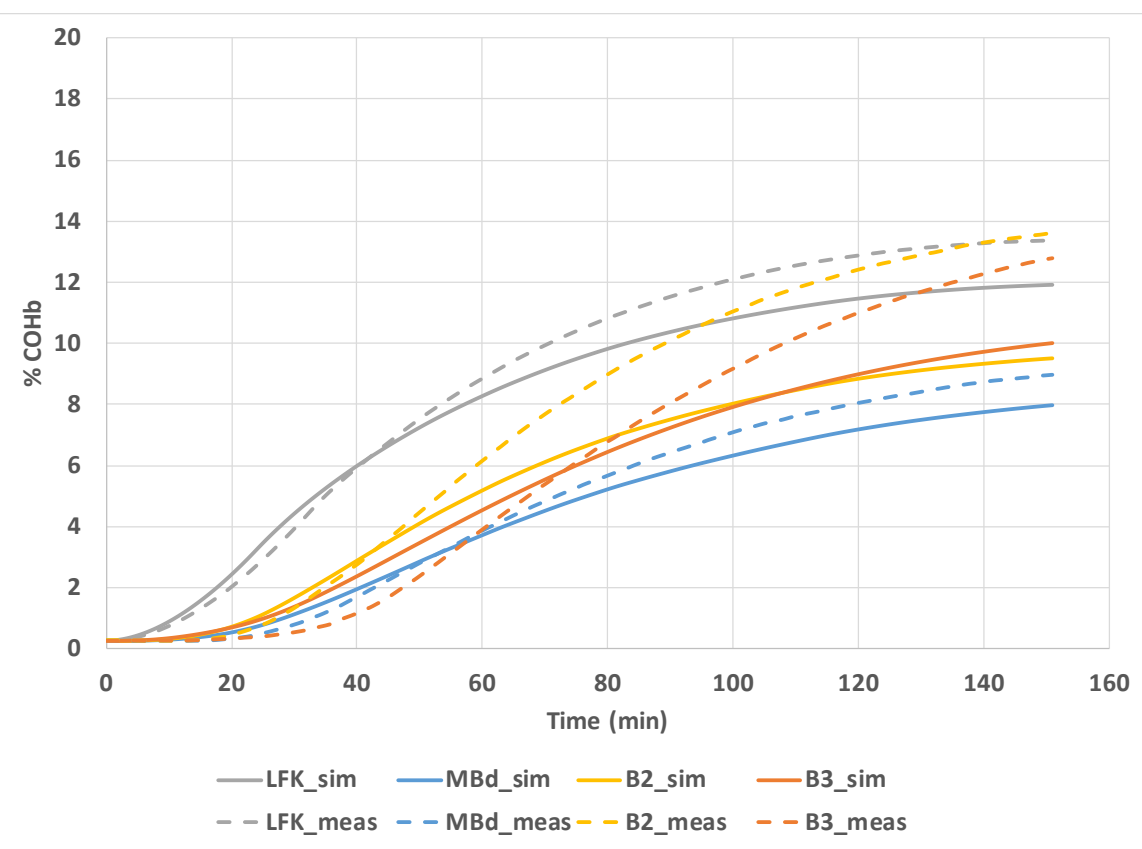

Figure 113c Observed and predicted $\mathrm{COHb}$ in main living space zones for Case 5 Test 4

Table 11 summarizes the maximum $\mathrm{COHb}$ values for the observed and predicted zone $\mathrm{CO}$ concentrations, along with the percent differences and the absolute average percent difference. For the Case 5 tests, the calculated maximum \% COHb was consistently underpredicted with an average percent difference of $22.0 \%$. However, the consistent underprediction indicates the need to examine modeling options to improve the agreement. 
Table 11 Comparison of maximum $\mathrm{COHb}$ for predictions and measurements for Case 5, Tests 2, 3, and 4

\begin{tabular}{|c|c|c|c|}
\hline Zone & COHb (\% predicted) & COHb (\% observed) & $\%$ diff \\
\hline \multicolumn{4}{|c|}{ Test 2} \\
\hline B3 & 12.1 & 18.8 & -36.0 \\
\hline LFK & 14.3 & 17.1 & -16.2 \\
\hline B2 & 12.8 & 19.4 & -33.6 \\
\hline MBd & 12.3 & 15.6 & -21.5 \\
\hline \multicolumn{4}{|c|}{ Test 3} \\
\hline B3 & 9.1 & 10.3 & -11.8 \\
\hline LFK & 10.9 & 12.2 & -10.4 \\
\hline B2 & 7.4 & 11.6 & -36.0 \\
\hline MBd & 6.2 & 8.2 & -24.7 \\
\hline \multicolumn{4}{|c|}{ Test 4} \\
\hline B3 & 10.0 & 12.8 & -21.8 \\
\hline LFK & 11.9 & 13.4 & -10.9 \\
\hline B2 & 9.5 & 13.6 & -30.3 \\
\hline MBd & 8.0 & 9.0 & -11.2 \\
\hline Average $\mid \%$ difference $\mid$ & & & 22.0 \\
\hline
\end{tabular}

Due to the consistent underprediction of $\mathrm{CO}$ and $\mathrm{COHb}$ in all three Case 5 tests, a modification was developed to account for the lack of uniform mixing in the zone with the generator (see Case 5 , Figures 22b, 23b and 24b that show lower concentrations at the shutoff sensor than the room average). The ratio of the zone average CO to the shutoff sensor CO measurement was calculated for the 12 tests, which were available at the time of this validation study. These ratios were calculated for tests without open doors or windows to the outdoors (which could be expected to affect mixing) for which shutoff occurred due to the 10-minute average concentration reaching 400 ppmv. The zone average CO was calculated as the average of the dedicated room sensor and any of the array sensors that were in the source zone. The ratio was calculated to be $1.2 \pm 0.08$, which means that the average $\mathrm{CO}$ concentration at the shutoff sensor for these 12 tests was approximately $20 \%$ below the average CO concentration in the source zone. The Case 5 simulations were repeated with this shutoff ratio applied to the shutoff limit in the model (i.e., the shutoff limit in the CONTAM control network was increased by a factor of 1.2 for this case).

The CO concentrations and \% COHb values for modified Case 5, Tests 2, 3 and 4 with the shutoff ratio are shown in Figures 114a to 114c and 115a to 115c, respectively. Table 12 summarizes the maximum COHb values for the observed and predicted zone CO concentrations for the Case 5 tests with this modification. This modification reduced the average percent difference to just $11.1 \%$ and eliminated the consistent underprediction bias. 


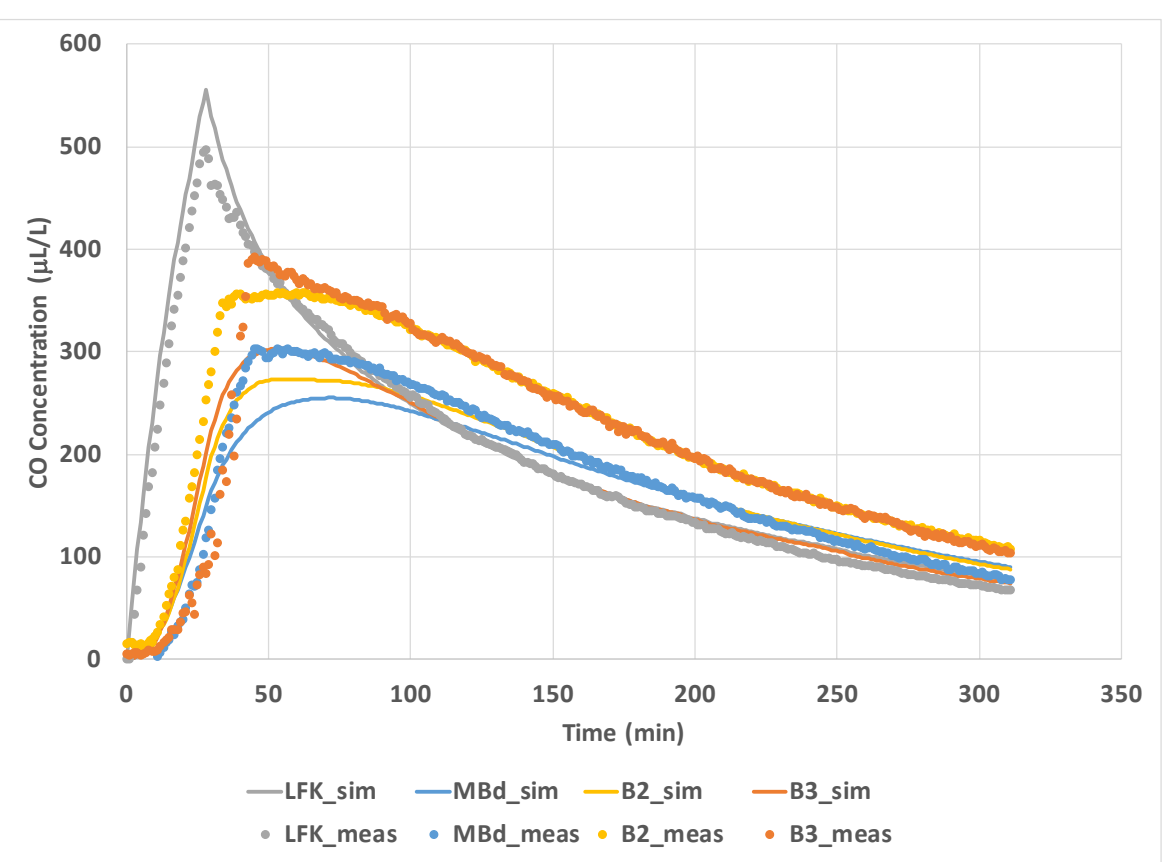

Figure 114a Observed and predicted CO concentrations in main living space zones for Case 5 Test 2 with modeling factor

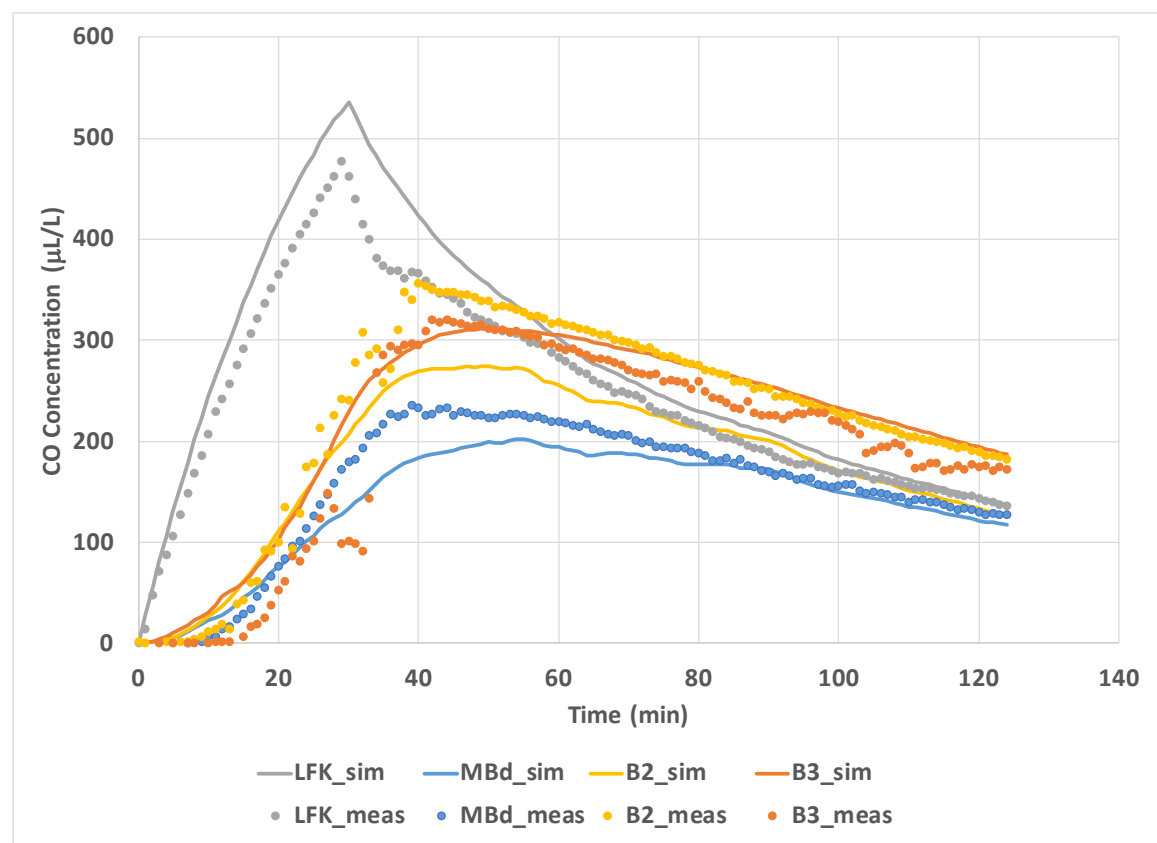

Figure 114b Observed and predicted CO concentrations in main living space zones for Case 5 Test 3 with modeling factor 


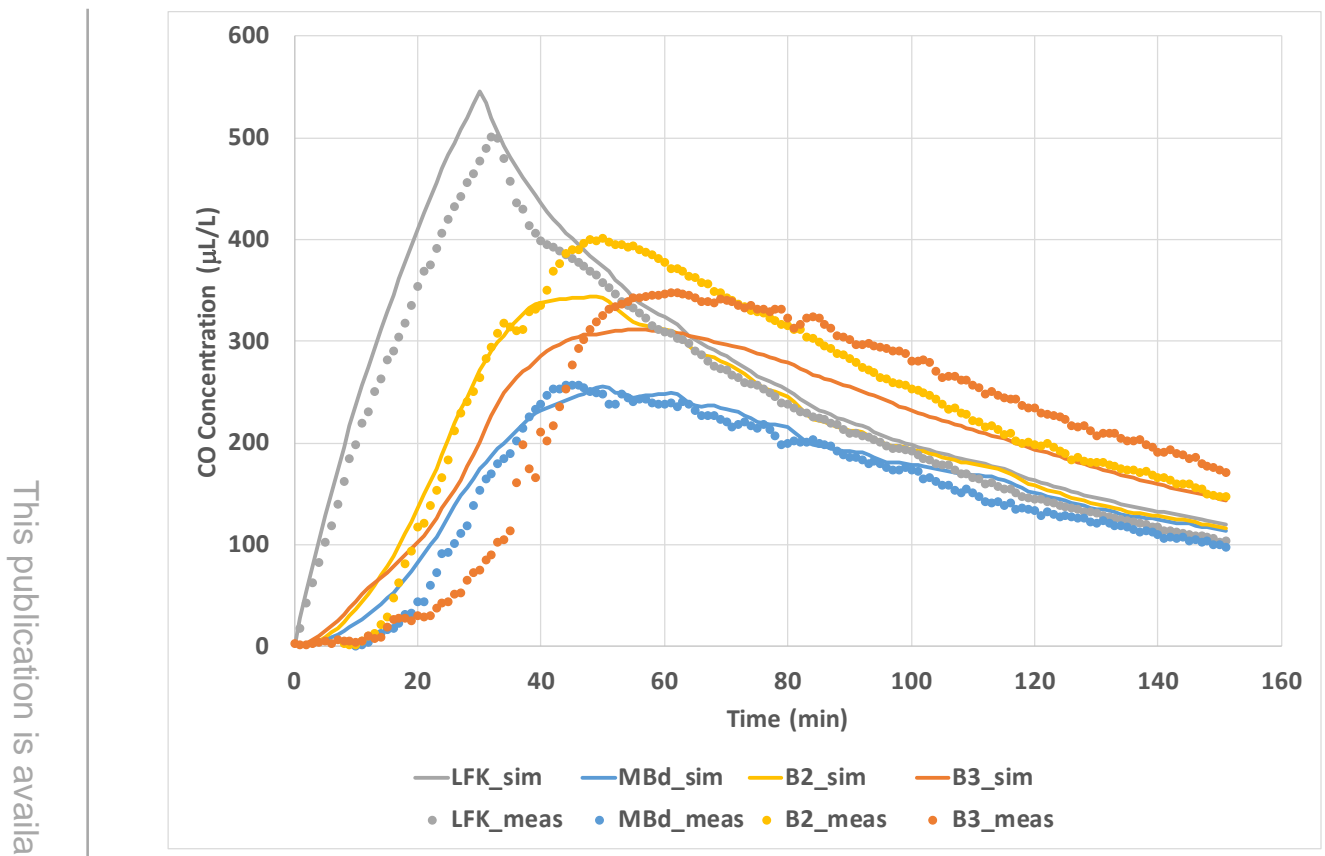

Figure 114c Observed and predicted CO concentrations in main living space zones for Case 5 Test 4 with modeling factor

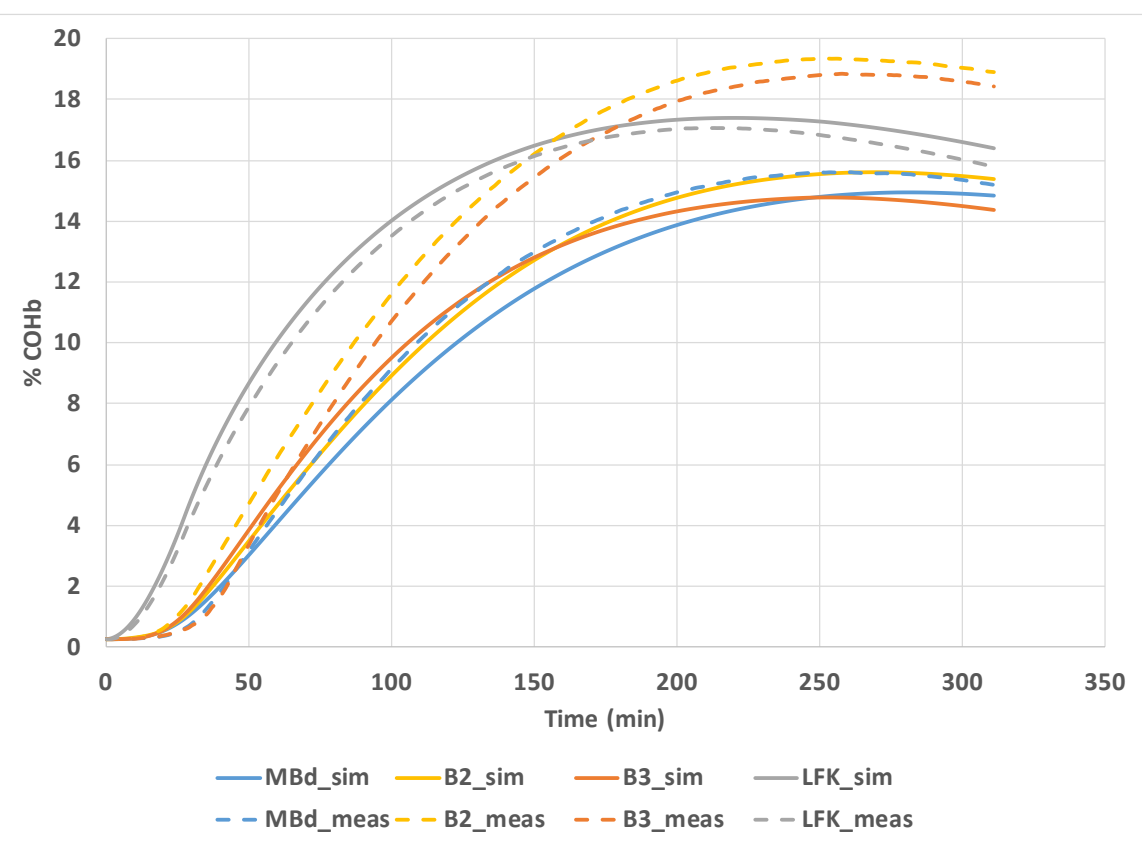

Figure 115a Observed and predicted $\mathrm{COHb}$ in main living space zones for Case 5 Test 2 with modeling factor 


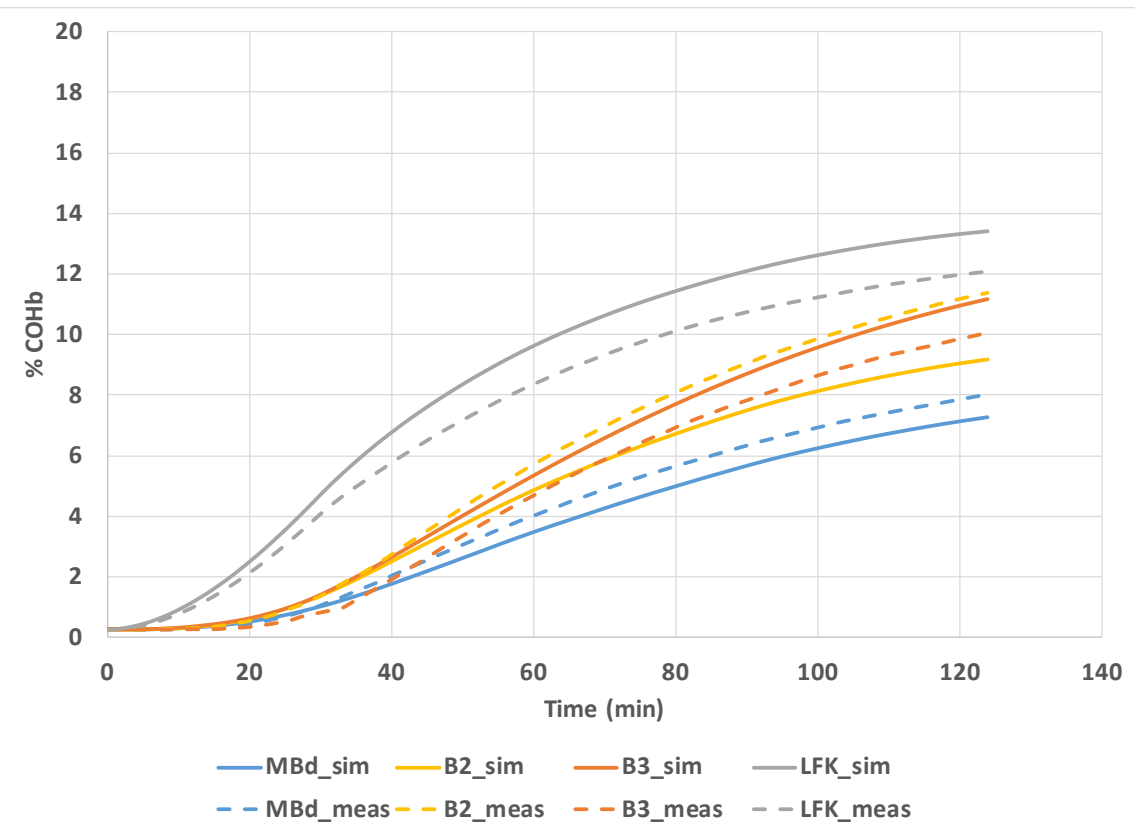

Figure 115b Observed and predicted COHb in main living space zones for Case 5 Test 3 with modeling factor

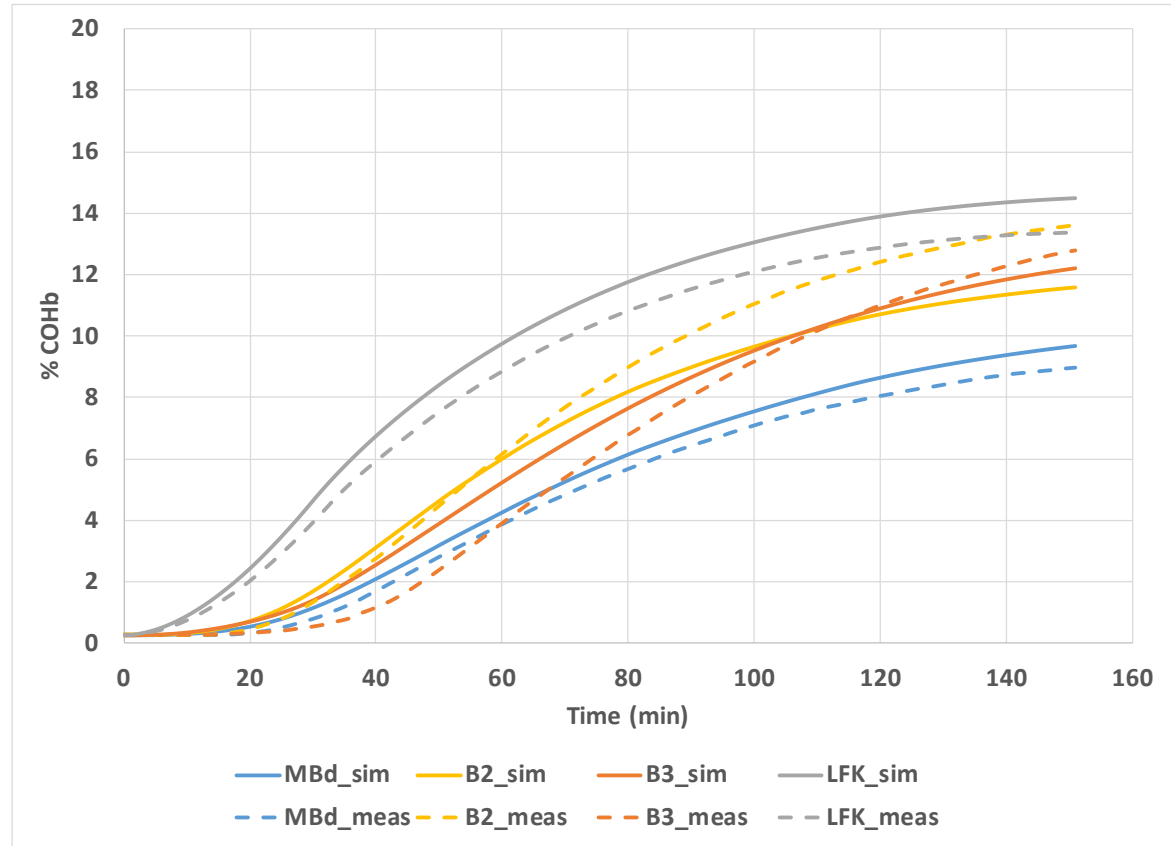

Figure 115c Observed and predicted $\mathrm{COHb}$ in main living space zones for Case 5 Test 4 with modeling factor 
Table 12 Comparison of maximum COHb for predictions and measurements for Case 5, Tests 2, 3, and 4 with modification

\begin{tabular}{|c|c|c|c|}
\hline Zone & COHb (\% predicted) & COHb (\% observed) & $\%$ diff \\
\hline \multicolumn{4}{|c|}{ Test 2} \\
\hline B3 & 14.8 & 18.8 & -21.6 \\
\hline LFK & 17.4 & 17.1 & 2.0 \\
\hline B2 & 15.6 & 19.4 & -19.4 \\
\hline MBd & 15.0 & 15.6 & -4.2 \\
\hline \multicolumn{4}{|c|}{ Test 3} \\
\hline B3 & 11.2 & 10.1 & 11.1 \\
\hline LFK & 13.4 & 12.1 & 10.8 \\
\hline B2 & 9.2 & 11.4 & -19.5 \\
\hline MBd & 7.3 & 8.0 & -9.2 \\
\hline \multicolumn{4}{|c|}{ Test 4} \\
\hline B3 & 12.2 & 12.8 & -4.4 \\
\hline LFK & 14.5 & 13.4 & 8.4 \\
\hline B2 & 11.6 & 13.6 & -15.1 \\
\hline MBd & 9.7 & 9.0 & 7.8 \\
\hline Average $\mid \%$ difference $\mid$ & & & 11.1 \\
\hline
\end{tabular}

\section{Case \# 17}

The next validation case was Case 17 Test 1, in which the G6 generator was positioned in Bedroom 3 (near the door with the exhaust facing the hallway and in the plane of the door) and tested at 100 $\%$ load. The door leading from the bedroom to the hallway was open; the bedroom window was open $10 \mathrm{~cm}$. All exterior doors and other windows were closed during these tests. All other interior doors connected to the main living space were fully open. Based on generator characterization tests conducted at CPSC (Brookman 2018), a constant CO emission rate of $1841.6 \mathrm{~g} / \mathrm{h}$ was applied in the simulations of this case. Initially, the source was modeled in bedroom 3.

The observed and predicted CO concentrations for the main living space zones in the house are shown in Figure 116 for Case 17 Test 1. As seen in Figure 116, the model predicted shutoff in about one minute due to exceeding the $800 \mathrm{ppmv}$ instantaneous criterion when the generator actually shutoff after about $12 \mathrm{~min}$. The resulting strong underprediction of CO concentrations in the test house living spaces occurred because including the source in the bedroom did not account for the jet of the exhaust which flowed down the hallway into the dining room. 


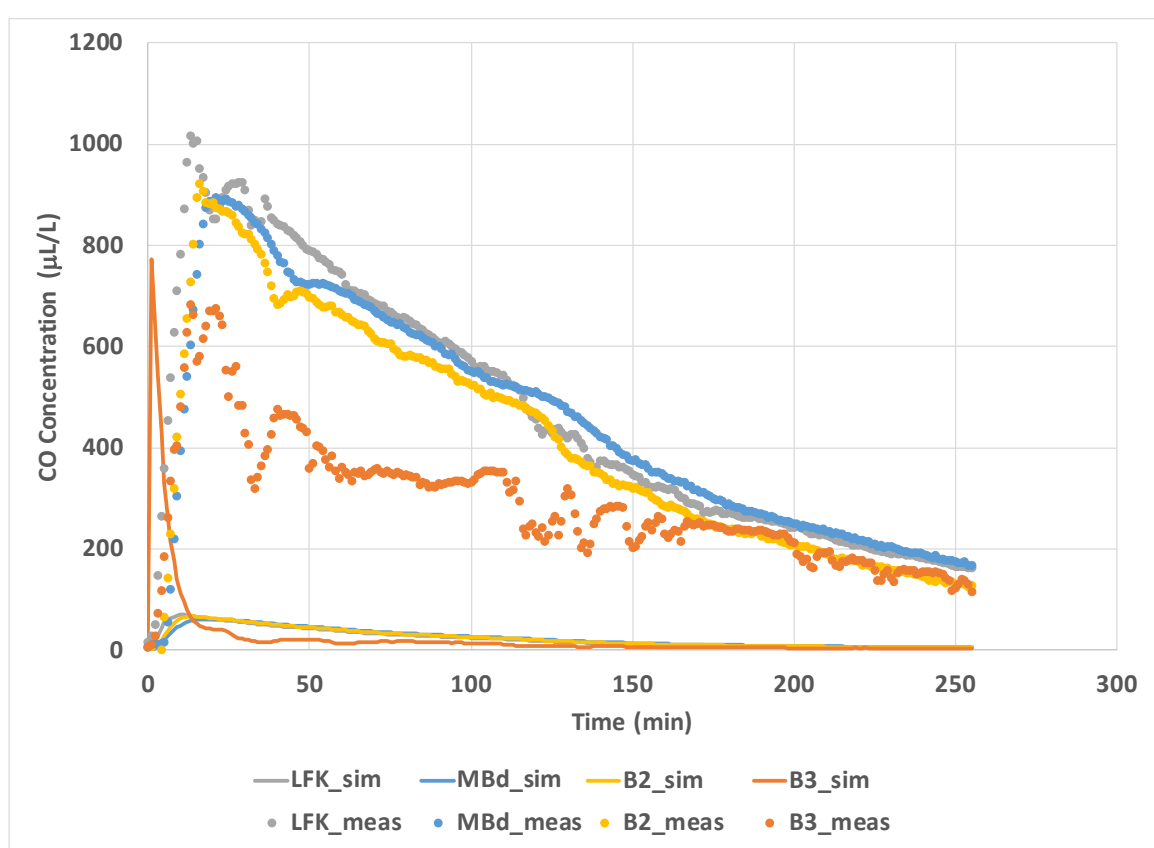

Figure 116 Observed and predicted CO concentrations in main living space zones for Case 17 Test 1 with source in Bedroom 3

$\mathrm{COHb}$ values calculated for the measurement and predictions are shown in Figure $\mathbf{1 1 7}$ for Case 17 Test 1 . Due to the strong underprediction of CO as seen in Figure 116, the COHb for the simulation was also underpredicted compared to the measurements.

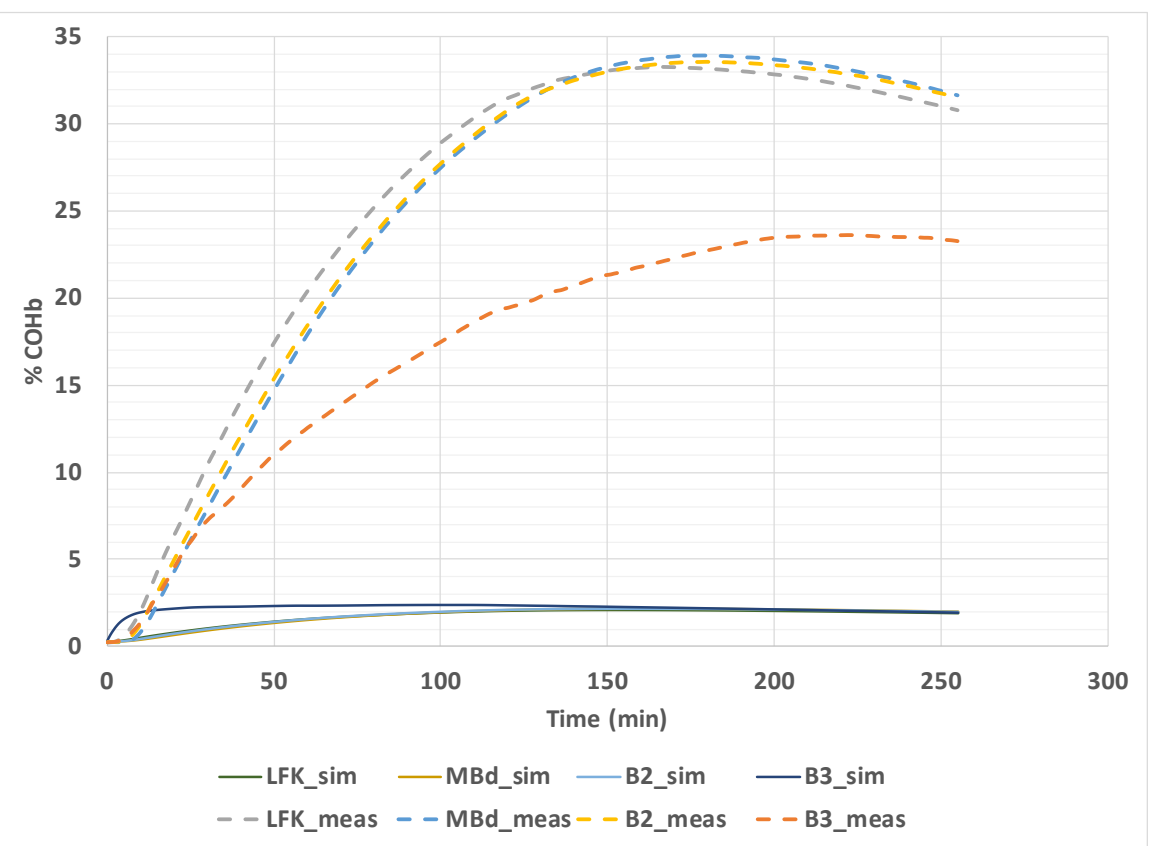

Figure 117 Observed and predicted $\mathrm{COHb}$ in main living space zones for Case 17 Test 1 with source in Bedroom 3

Table 13 summarizes the maximum $\mathrm{COHb}$ values for the observed and predicted zone $\mathrm{CO}$ concentrations along with the percent differences and the absolute average percent difference for 
Case 17 Test 1 with the CO source modeled in Bedroom 3. Not surprisingly, the calculated maximum $\mathrm{COHb}$ was strongly underpredicted with an average percent difference of over $90 \%$.

\section{Table 13 Comparison of maximum COHb for predictions and measurements for} Case17 Test 1 - Bedroom 3 source

\begin{tabular}{|l|r|r|c|}
\hline Zone & $\begin{array}{r}\text { COHb (\% } \\
\text { predicted) }\end{array}$ & $\begin{array}{c}\text { COHb (\% } \\
\text { observed) }\end{array}$ & $\begin{array}{c}\text { \% } \\
\text { diff }\end{array}$ \\
\hline B3 & 2.4 & 21.7 & -89.0 \\
\hline LFK & 2.1 & 33.3 & -93.6 \\
\hline B2 & 2.2 & 30.3 & -92.9 \\
\hline MBd & 2.2 & 32.5 & -93.3 \\
\hline Average |\% difference| & & & $\mathbf{9 2 . 2}$ \\
\hline
\end{tabular}

Case 17 Test 1 was modeled a second time with the source located in the LFK zone to account for the fact that the generator exhaust jet was directly pointed out of Bedroom 3 into the dining room. The CO concentrations and $\mathrm{COHb}$ values for the modified Case 17 Test 1 with the source located in the LFK zone are shown in Figures 118 and 119, respectively. This modification came much closer to predicting the actual results of the measurements. As seen in Table 14, the average percent difference was only $8.9 \%$.

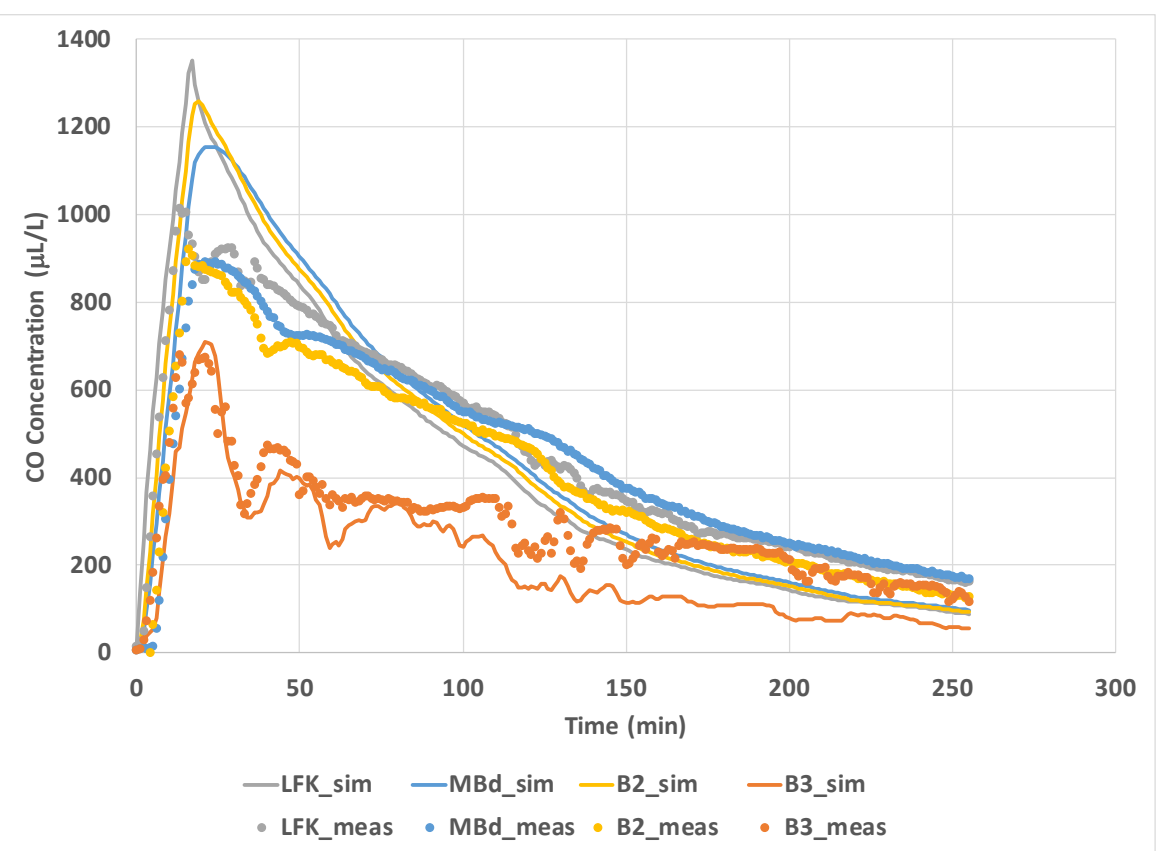

Figure 118 Observed and predicted CO concentrations in main living space zones for Case 17 Test 1 with source in LFK zone 


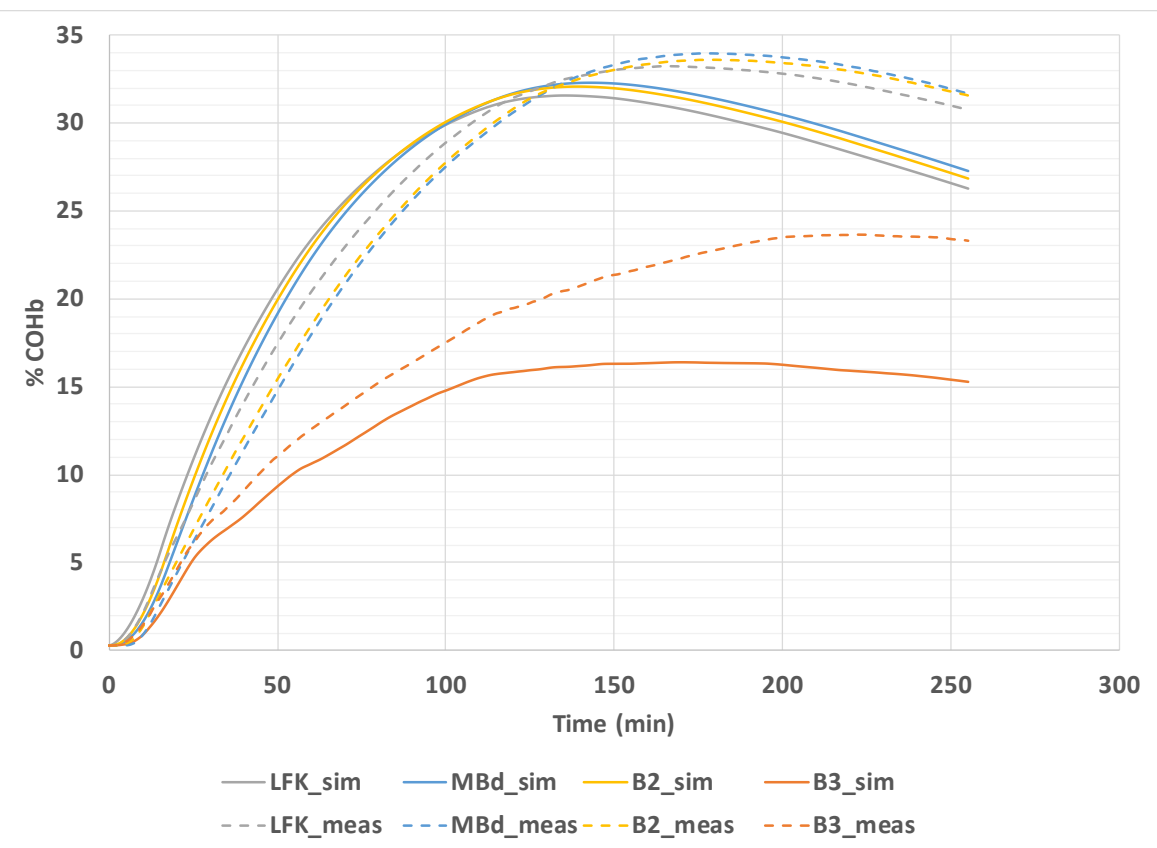

Figure 119 Observed and predicted $\mathrm{COHb}$ in main living space zones for Case 17 Test 1 with source in LFK zone

Table 14 Comparison of maximum COHb for predictions and measurements for Case 17 Test 1 - LFK source

\begin{tabular}{|l|r|r|r|}
\hline Zone & COHb (\% predicted) & COHb (\% observed) & \% diff \\
\hline B3 & 16.4 & 21.7 & -24.4 \\
\hline LFK & 31.6 & 33.3 & -5.1 \\
\hline B2 & 32.0 & 30.3 & 5.7 \\
\hline MBd & 32.3 & 32.5 & -0.6 \\
\hline Average |\% difference| & & & $\mathbf{8 . 9}$ \\
\hline
\end{tabular}




\section{Case \# 23}

The next validation case was Case 23 Test 1 . The G2 generator was positioned in the utility room (centered, with the exhaust facing into the family room) and tested at $50 \%$ load. The interior person door (from utility room to garage) and exterior person door (from garage to outdoors) were fully open. The garage bay door, all other exterior doors, and all windows were closed during this test. All other interior doors connected to the main living space were fully open. Based on generator characterization tests conducted at CPSC (Brookman 2018), a constant CO emission rate of $345.0 \mathrm{~g} / \mathrm{h}$ was applied in these simulations, with the source modeled in the utility room.

The observed and predicted CO concentrations for the main living space zones in the house are shown in Figure 120 for Case 23 Test 1 . The model predicted shutoff in under 20 min, while the generator actually shutoff after about 94 min after reaching the 400 ppmv for 10 minute criterion. As in Case 17, the resulting strong underprediction of CO concentrations in the test house living spaces occurred because including the source in the utility room did not account for the jet of the exhaust which flowed into the family room.

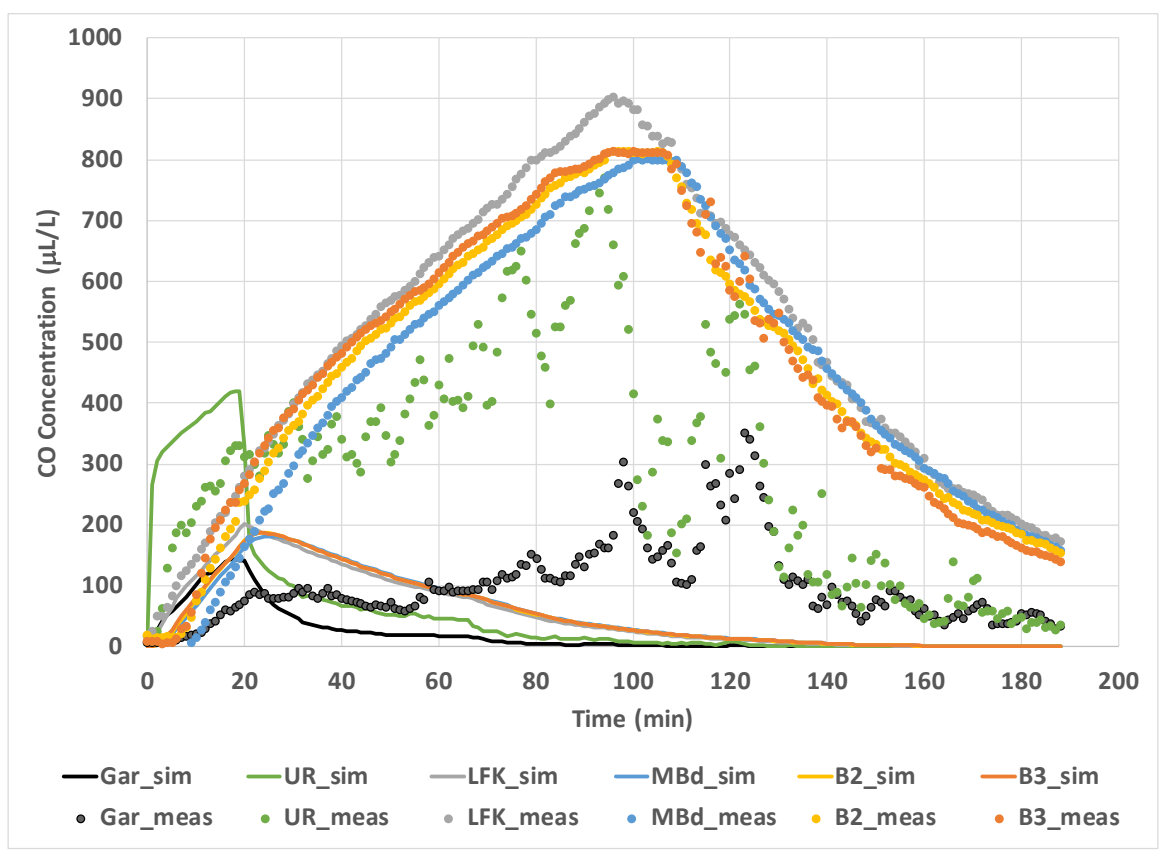

Figure 120 Observed and predicted CO concentrations in main living space zones for Case 23 Test 1 with source in Utility Room

The COHb values calculated for the measurement and predictions are shown in Figure $\mathbf{1 2 1}$ for Case 23 Test 1. Due to the strong underprediction of $\mathrm{CO}$ as seen in Figure 120, the COHb for the simulation was also underpredicted compared to the measurements. 


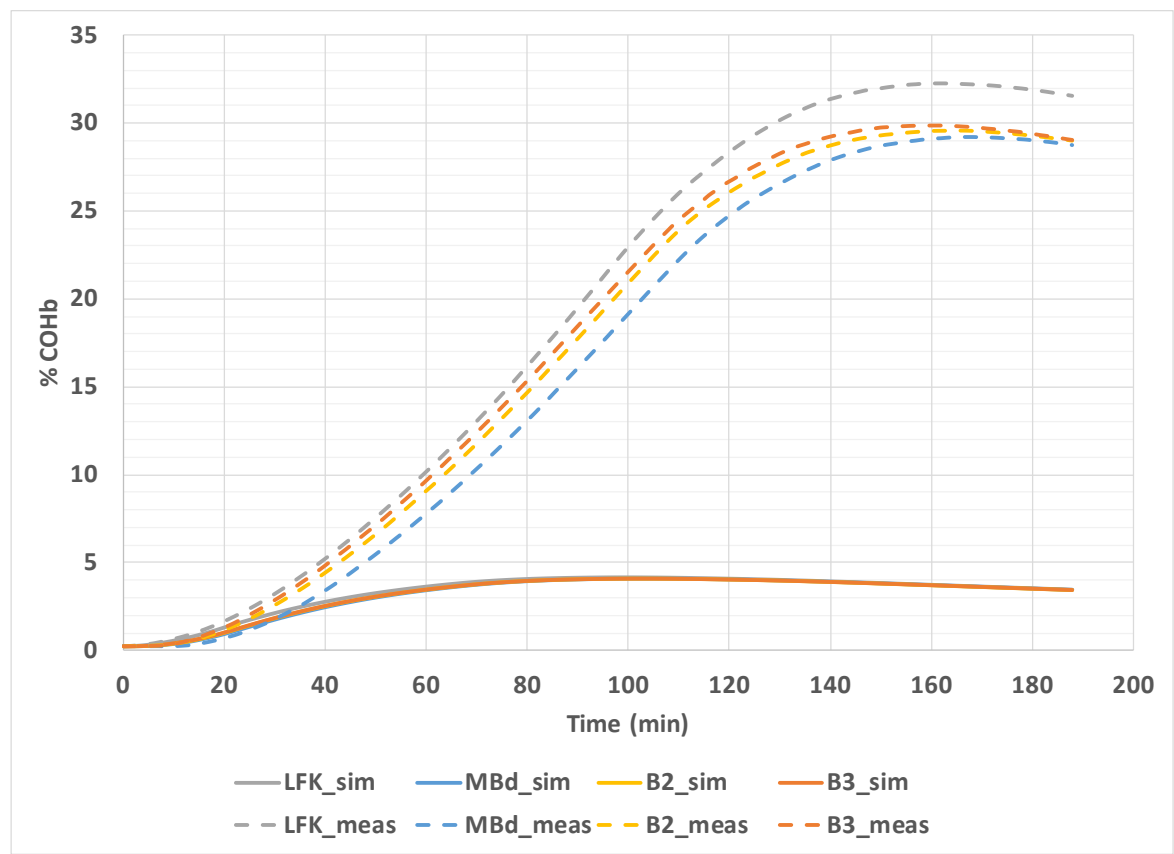

Figure 121 Observed and predicted $\mathrm{COHb}$ in main living space zones for Case 23 Test 1 with source in Utility Room

Table 15 summarizes the maximum $\mathrm{COHb}$ values for the observed and predicted zone $\mathrm{CO}$ concentrations along with the percent differences and the absolute average percent difference for Case 23 Test 1 with the CO source modeled in the Utility room. Not surprisingly, the calculated maximum $\mathrm{COHb}$ was strongly underpredicted, with an average percent difference of $86.0 \%$.

\section{Table 15 Comparison of maximum COHb for predictions and measurements for} Case 23 Test 1 - Utility room source

\begin{tabular}{|l|r|r|c|}
\hline Zone & $\begin{array}{r}\text { COHb (\% } \\
\text { predicted) }\end{array}$ & $\begin{array}{c}\text { COHb (\% } \\
\text { observed) }\end{array}$ & $\begin{array}{c}\text { \% } \\
\text { diff }\end{array}$ \\
\hline B3 & 4.1 & 29.9 & -86.2 \\
\hline LFK & 4.2 & 32.2 & -87.0 \\
\hline B2 & 4.1 & 29.6 & -86.1 \\
\hline MBd & 4.1 & 29.2 & -86.0 \\
\hline Average $\mid \%$ difference & & & 86.3 \\
\hline
\end{tabular}

As with Case 17, Case 23 Test 1 was modeled a second time with the source located in the LFK zone to account for the fact that the generator exhaust jet was directly pointed out of the Utility room into the family room. The $\mathrm{CO}$ concentrations and $\mathrm{COHb}$ values for the modified Case 23 Test 1 with the source located in the LFK zone are shown in Figures 122 and 123, respectively. Once again, this modification came much closer to predicting the actual results of the measurements. As seen in Table 16, the average percent difference was only $5.6 \%$. 


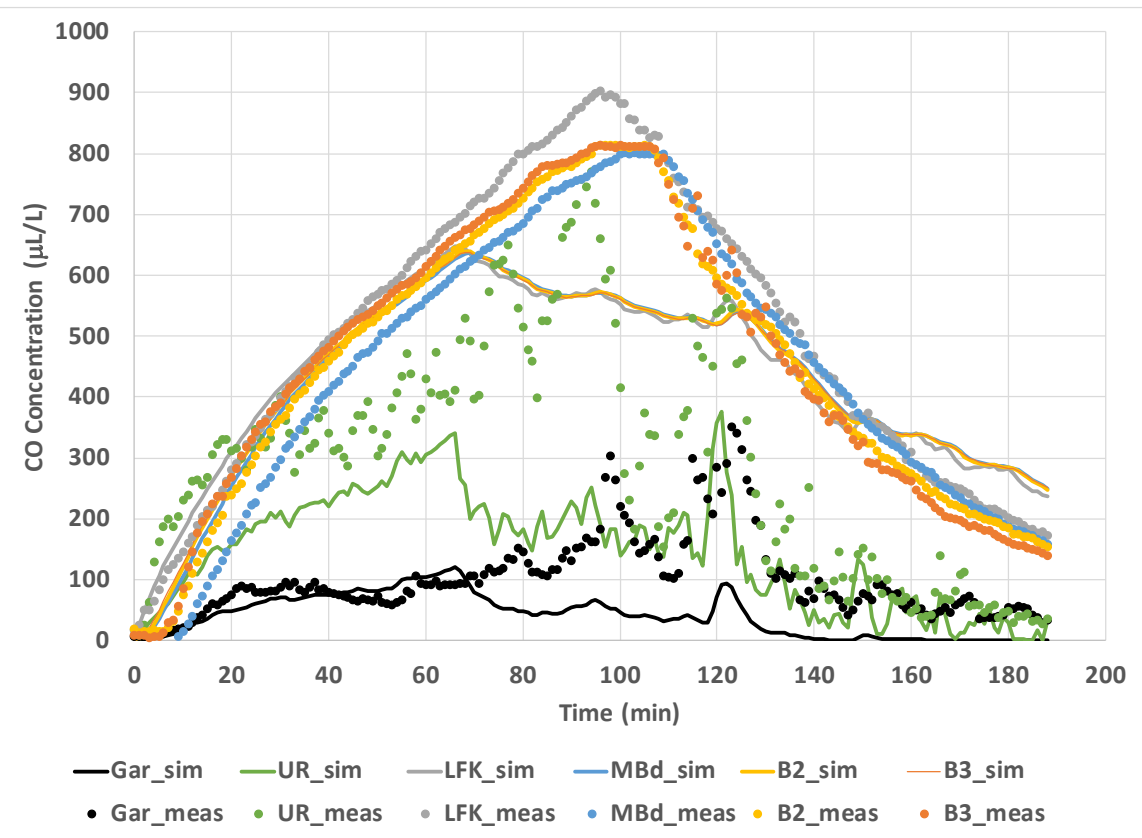

Figure 122 Observed and predicted CO concentrations in main living space zones for Case 23 Test 1 with source in LFK zone

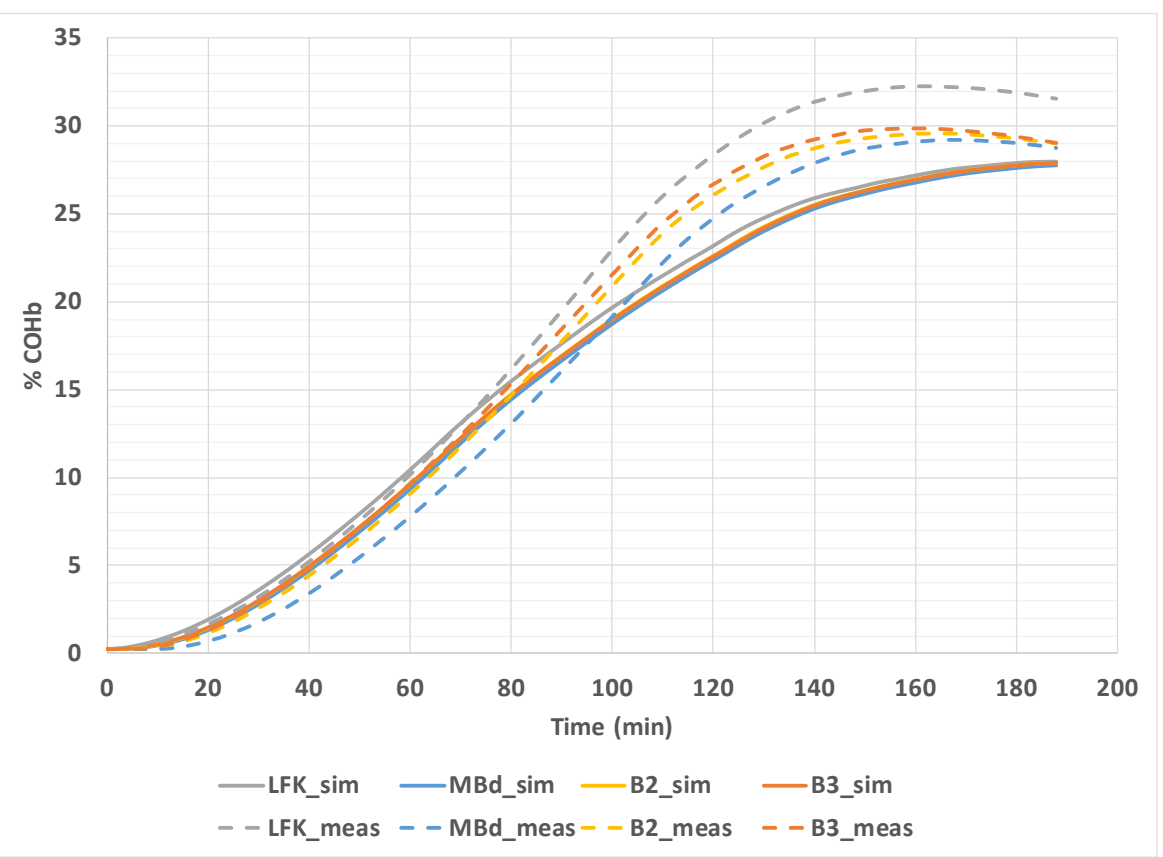

Figure 123 Observed and predicted COHb in main living space zones for Case 23 Test 1 with source in LFK zone 
Table 16 Comparison of maximum $\mathrm{COHb}$ for predictions and measurements for Case 23 Test 1 - LFK source

\begin{tabular}{|l|r|r|r|}
\hline Zone & COHb (\% predicted) & COHb (\% observed) & \% diff \\
\hline B3 & 27.9 & 29.9 & -6.7 \\
\hline LFK & 27.9 & 32.3 & -13.4 \\
\hline B2 & 27.9 & 29.6 & -5.7 \\
\hline MBd & 27.8 & 29.2 & -4.8 \\
\hline Average |\% difference| & & & $\mathbf{7 . 6}$ \\
\hline
\end{tabular}




\section{Case \# 32}

The next three validation cases were Case 32 Tests 1, 2, and 3. The G6 generator was positioned in the garage (centered, with the exhaust facing towards the family room) and tested at $100 \%$ load. The interior person door (from utility room to garage) was open $10 \mathrm{~cm}$, and the garage bay door was fully open; the exterior person door (from garage to backyard) was closed. All interior doors connected to the living space were fully open. Based on generator characterization tests conducted at CPSC (Brookman 2018), a constant CO emission rate of $1841.6 \mathrm{~g} / \mathrm{h}$ was applied in this case.

The observed and predicted CO concentrations for the main living space zones in the house and the garage are shown in Figures 124a, 124b and 124c for Case 32 Tests 1, 2, and 3 respectively (lines for the predictions and symbols for the measurements). The shutoff sensor was not activated during any of these tests. The generator was manually shutdown after about $260 \mathrm{~min}$ for Test 1, but ran until using a full tank of fuel for Tests 2 and 3. As seen in the figures, the results of the Case 32 tests were similar with measured peak $\mathrm{CO}$ concentrations in the main living zones ranging from around $400 \mu \mathrm{L} / \mathrm{L}$ to $600 \mu \mathrm{L} / \mathrm{L}$, while predicted peak CO concentrations were lower at around 200 $\mu \mathrm{L} / \mathrm{L}$ to $300 \mu \mathrm{L} / \mathrm{L}$. Figures 124a through $124 \mathrm{c}$ all show that the Utility Room concentrations were substantially higher than the garage average concentrations, indicating that the model did not capture the transport of CO from the generator exhaust jet to the Utility Room even though the generator was in the middle of the garage and not directly adjacent to the Utility Room.

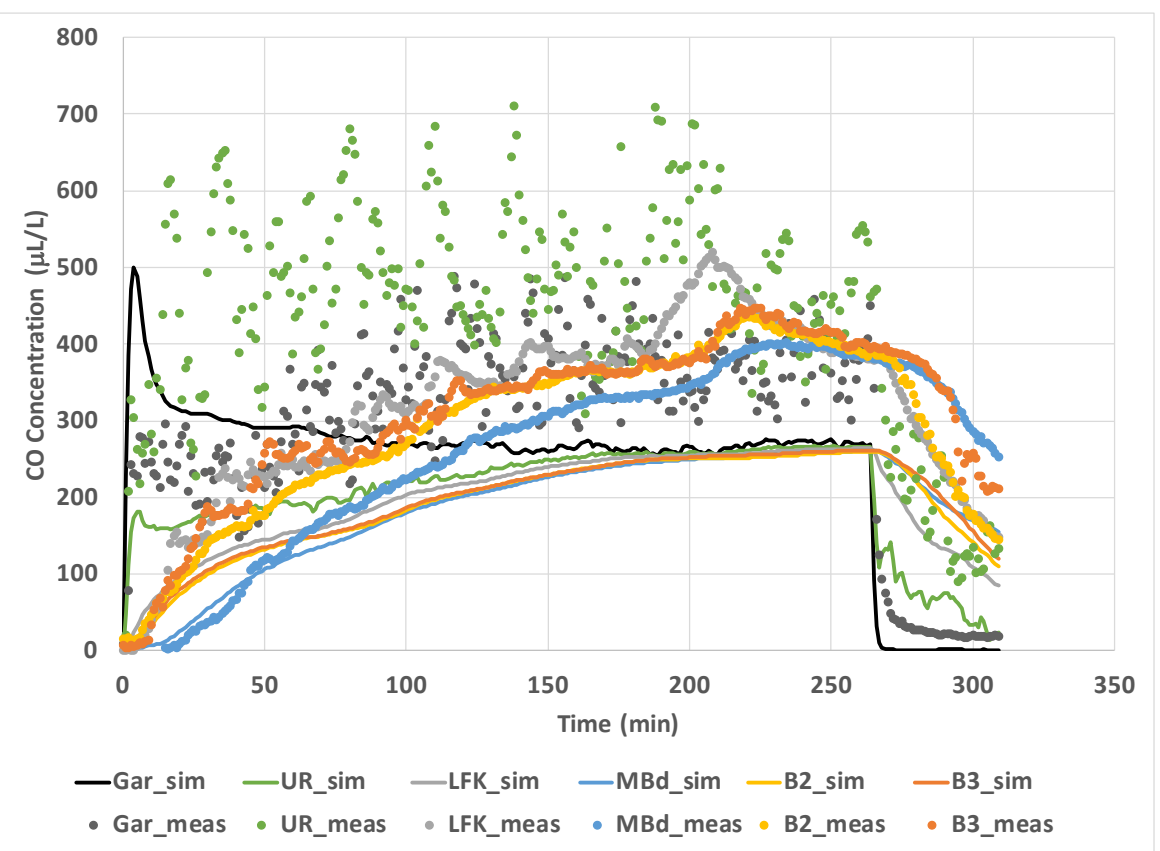

Figure 124a Observed and predicted CO concentrations in main living space zones for Case 32 Test 1 


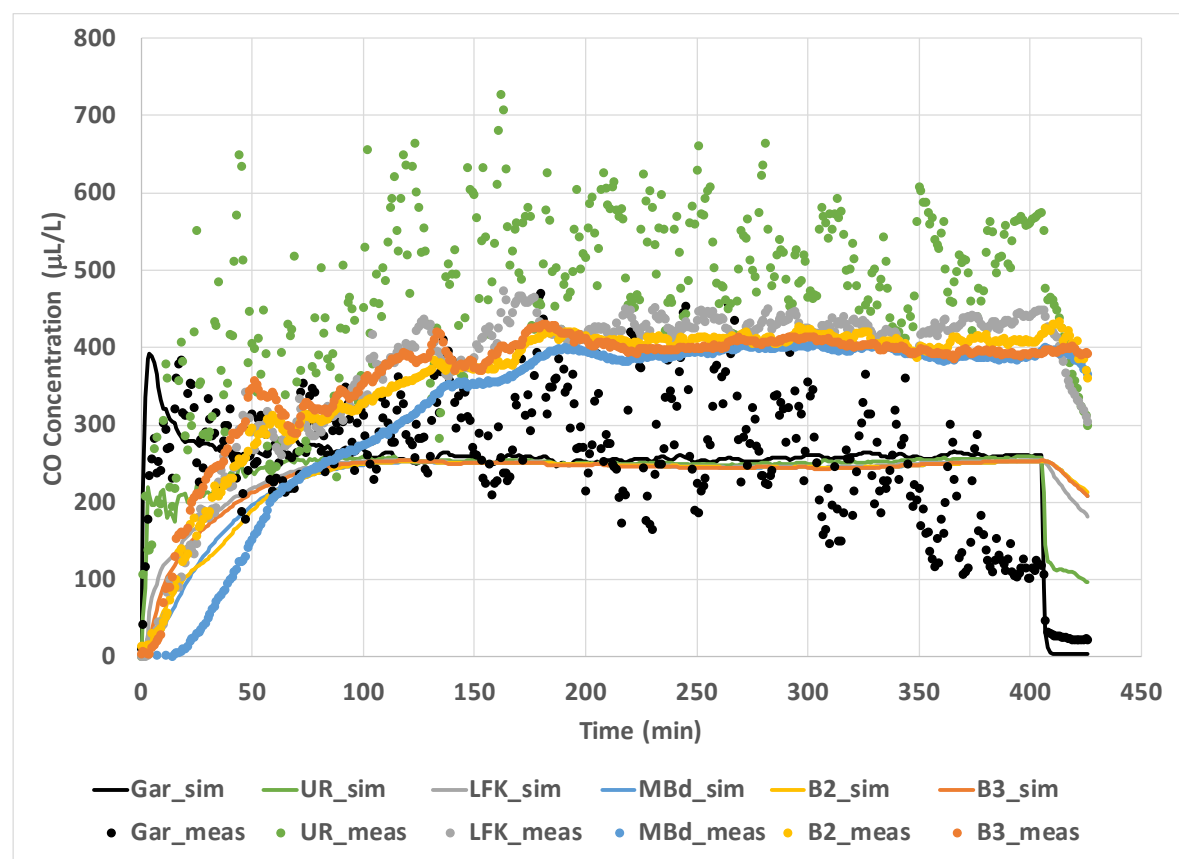

Figure 124b Observed and predicted CO concentrations in main living space zones and garage for Case 32 Test 2

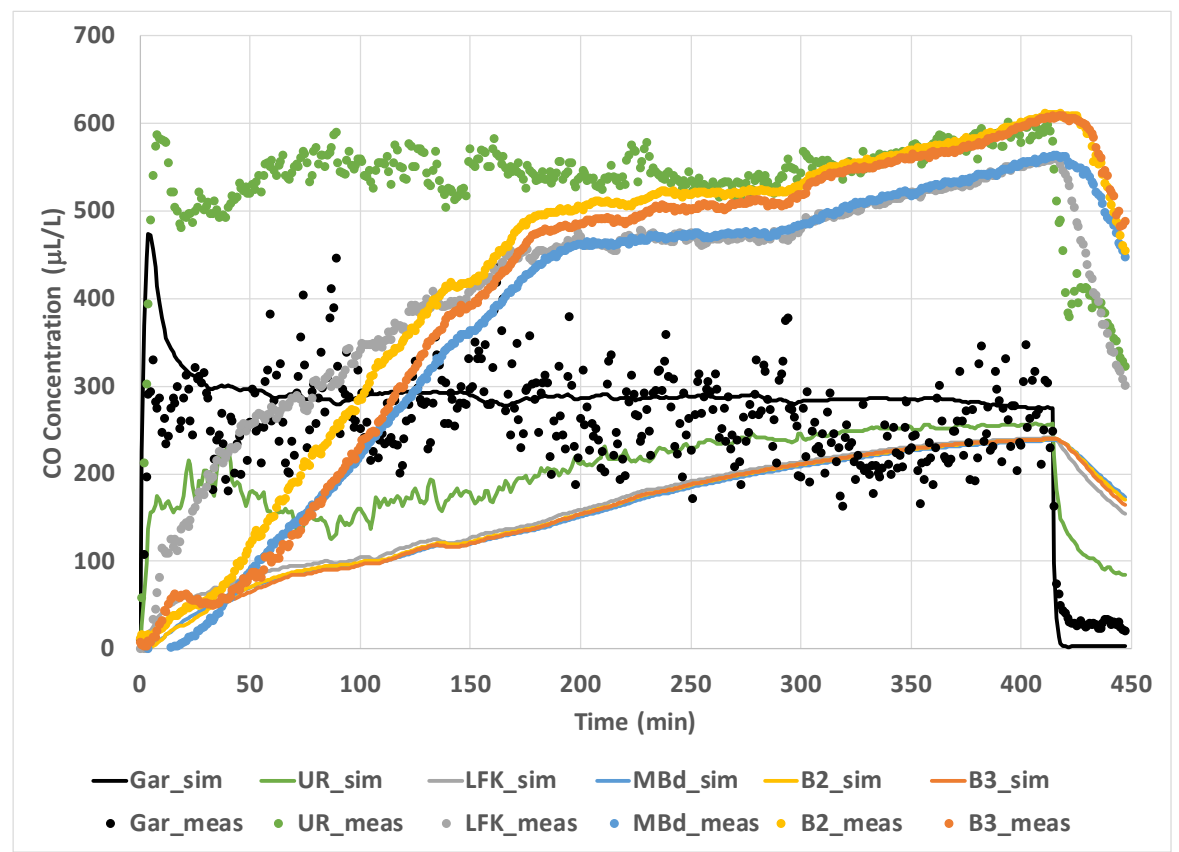

Figure 124c Observed and predicted CO concentrations in main living space zones and garage for Case 32 Test 3

$\mathrm{COHb}$ values calculated for the observed and predicted $\mathrm{CO}$ concentrations for the main living space zones in the test house are shown in Figures 125a, 125b and 125c for Case 32 Tests 1, 2, and 3, respectively. As seen in the figures, the $\mathrm{COHb}$ results of the Case 32 tests were similar, with values 
reaching over $30 \%$ at the 250-minute mark (note that the tests were not all the same length, with Test 1 being much shorter and including a CO decay period after $260 \mathrm{~min}$ ). Since the CO concentration is significantly underpredicted for these tests, the calculated $\mathrm{COHb}$ for the predictions were also significantly lower than for the measurements.

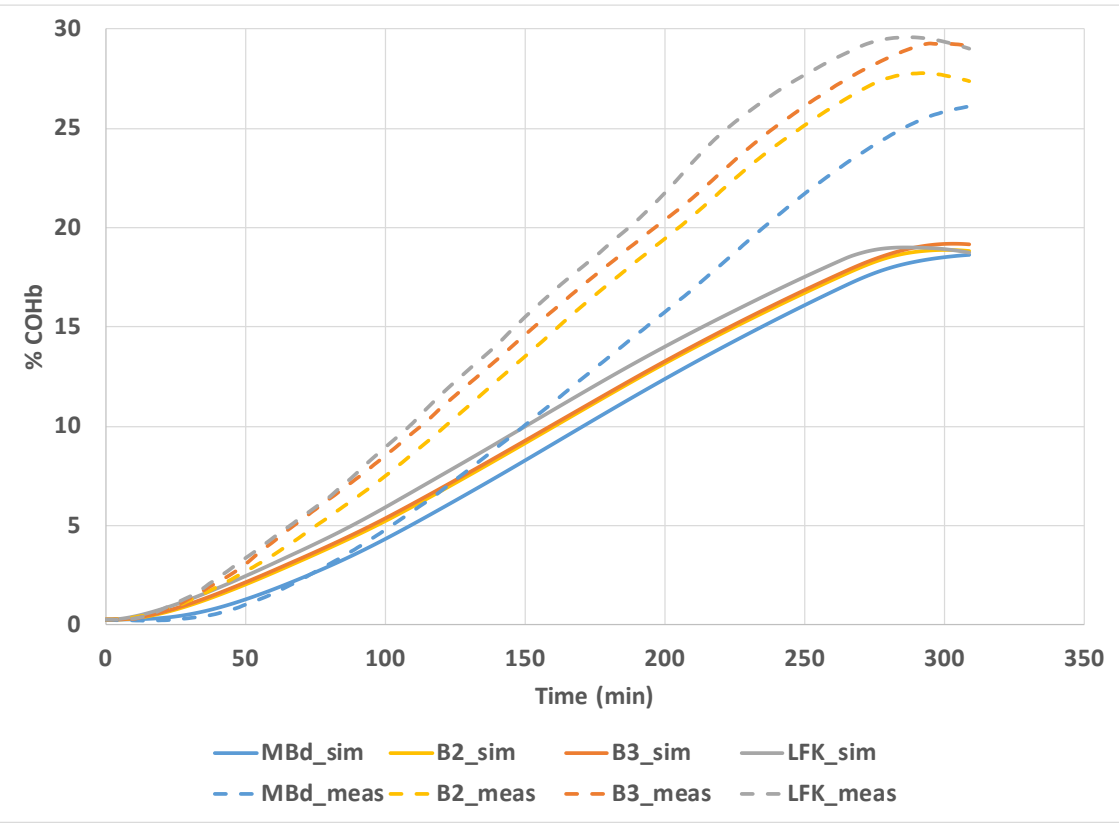

Figure 125a Observed and predicted $\mathrm{COHb}$ in main living space zones for Case 32 Test 1

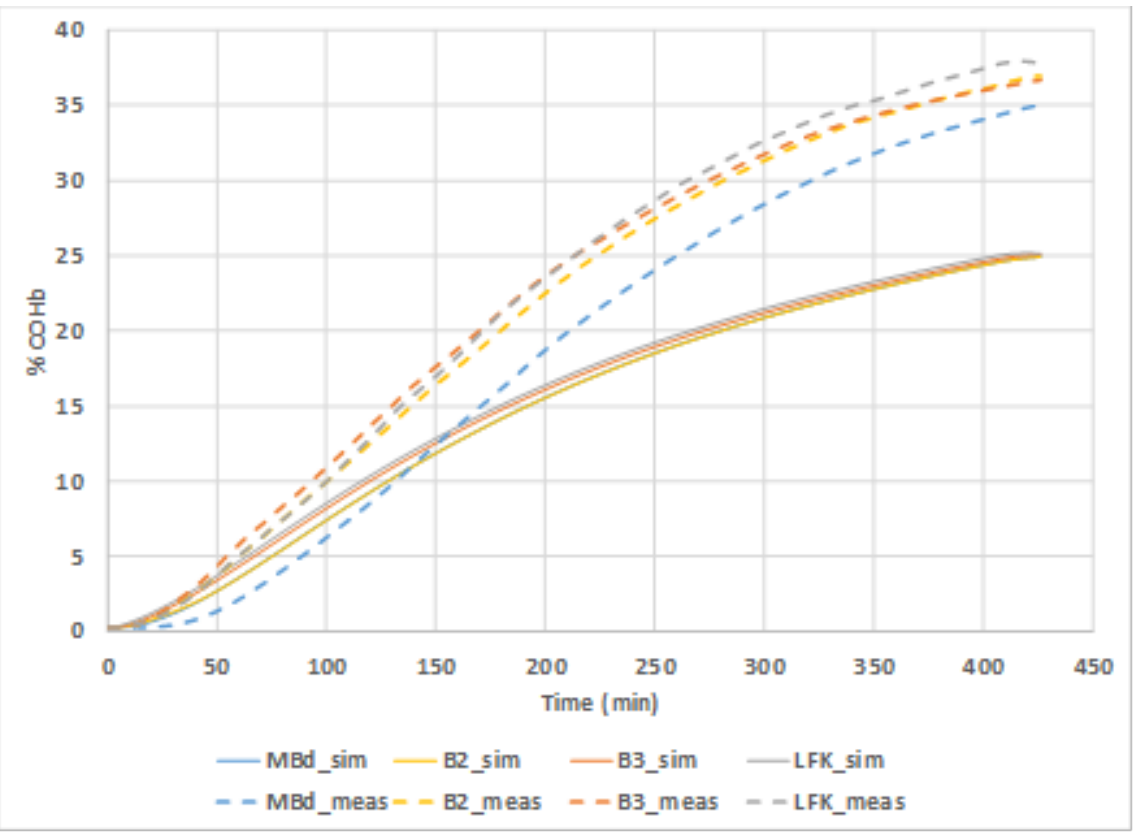

Figure 125b Observed and predicted COHb in main living space zones for Case 32 Test 2 


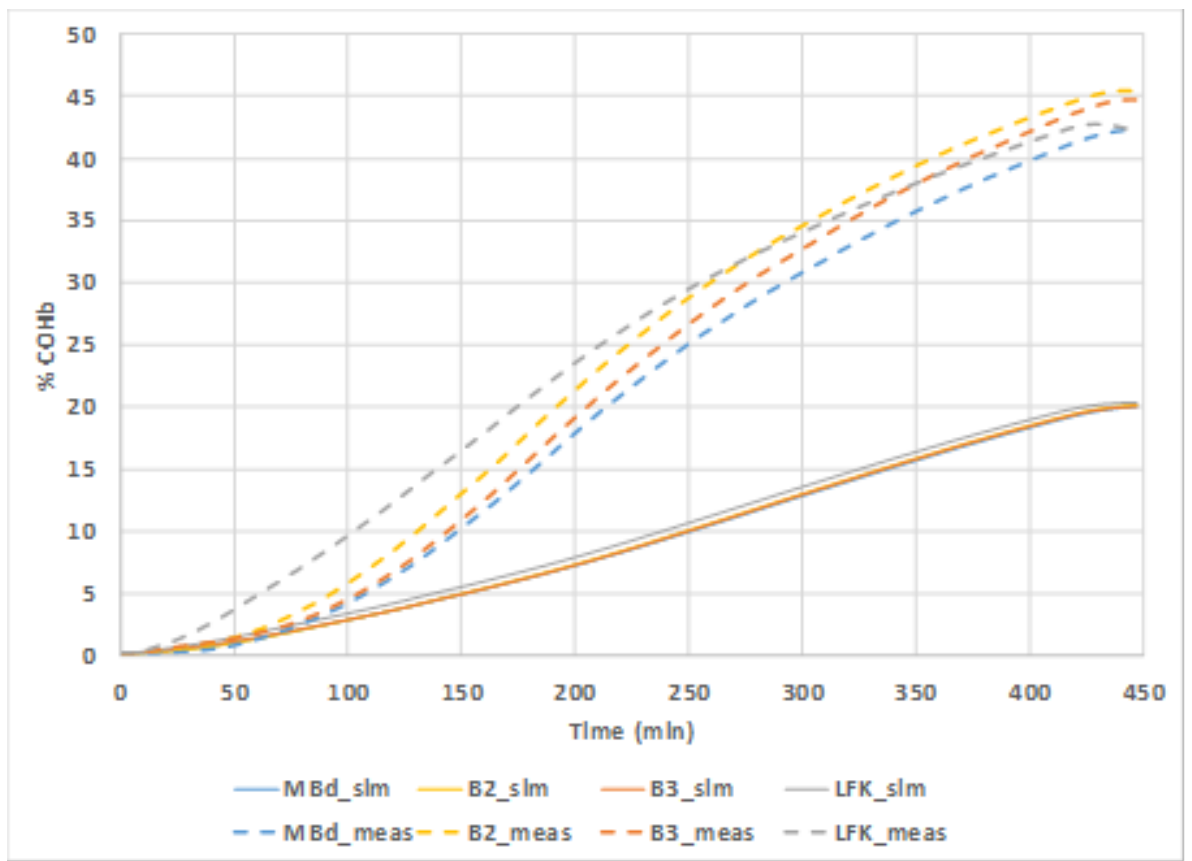

Figure 125c Observed and predicted COHb in main living space zones for Case 32 Test 3

Table 17 summarizes the maximum $\mathrm{COHb}$ values for the observed and predicted zone $\mathrm{CO}$ concentrations, along with the percent differences and the absolute average percent difference. For the Case 32 tests, the calculated maximum $\mathrm{COHb}$ was consistently underpredicted with an average percent difference of $39.5 \%$. The consistent and significant underprediction of $\mathrm{CO}$ and $\mathrm{COHb}$ in all three Case 32 tests indicates the need for modification of the model to account for the lack of uniform mixing in the garage and the transport of CO from the garage to the Utility Room. 
Table 17 Comparison of maximum COHb for predictions and measurements for Case 32 Tests 1, 2, and 3

\begin{tabular}{|c|c|c|c|}
\hline Zone & COHb (\% predicted) & COHb (\% observed) & $\%$ diff \\
\hline \multicolumn{4}{|c|}{ Test 1} \\
\hline B3 & 19.2 & 29.3 & -34.5 \\
\hline LFK & 19.0 & 29.6 & -35.9 \\
\hline B2 & 18.8 & 27.8 & -32.2 \\
\hline MBd & 18.6 & 26.1 & -28.6 \\
\hline \multicolumn{4}{|c|}{ Test 2} \\
\hline B3 & 25.1 & 36.7 & -31.5 \\
\hline LFK & 25.1 & 37.9 & -33.8 \\
\hline B2 & 24.9 & 36.9 & -32.5 \\
\hline MBd & 24.9 & 35.0 & -28.8 \\
\hline \multicolumn{4}{|c|}{ Test 3} \\
\hline B3 & 20.1 & 44.8 & -55.1 \\
\hline LFK & 20.3 & 42.8 & -52.5 \\
\hline B2 & 20.1 & 45.5 & -55.7 \\
\hline MBd & 20.0 & 42.4 & -52.8 \\
\hline Average $\mid \%$ difference $\mid$ & & & 39.5 \\
\hline
\end{tabular}

Due to the consistent underprediction of $\mathrm{CO}$ and $\mathrm{COHb}$ in all three Case 32 tests, a modification was made to account for the exhaust jet directly transporting $\mathrm{CO}$ into the adjacent utility room by including a CO source in the utility room of the model. An iterative simulation process was used to estimate that a utility room source term with $5 \%$ of the CO generated could accurately represent the fraction of CO directly entering the house (the other $95 \%$ of the source remained in the garage) for Test 1 . All Case 32 simulations were then repeated with this source term modification. The CO concentrations and \% COHb values for modified Case 32, Tests 1, 2, and 3 with a $5 \%$ source in the utility room are shown in Figures 126a to 126c and 127a to 127c. Table 18 summarizes the maximum $\mathrm{COHb}$ values for the observed and predicted zone $\mathrm{CO}$ concentrations for Case 32 tests with this modification, which reduced the average percent difference to $9.5 \%$. 


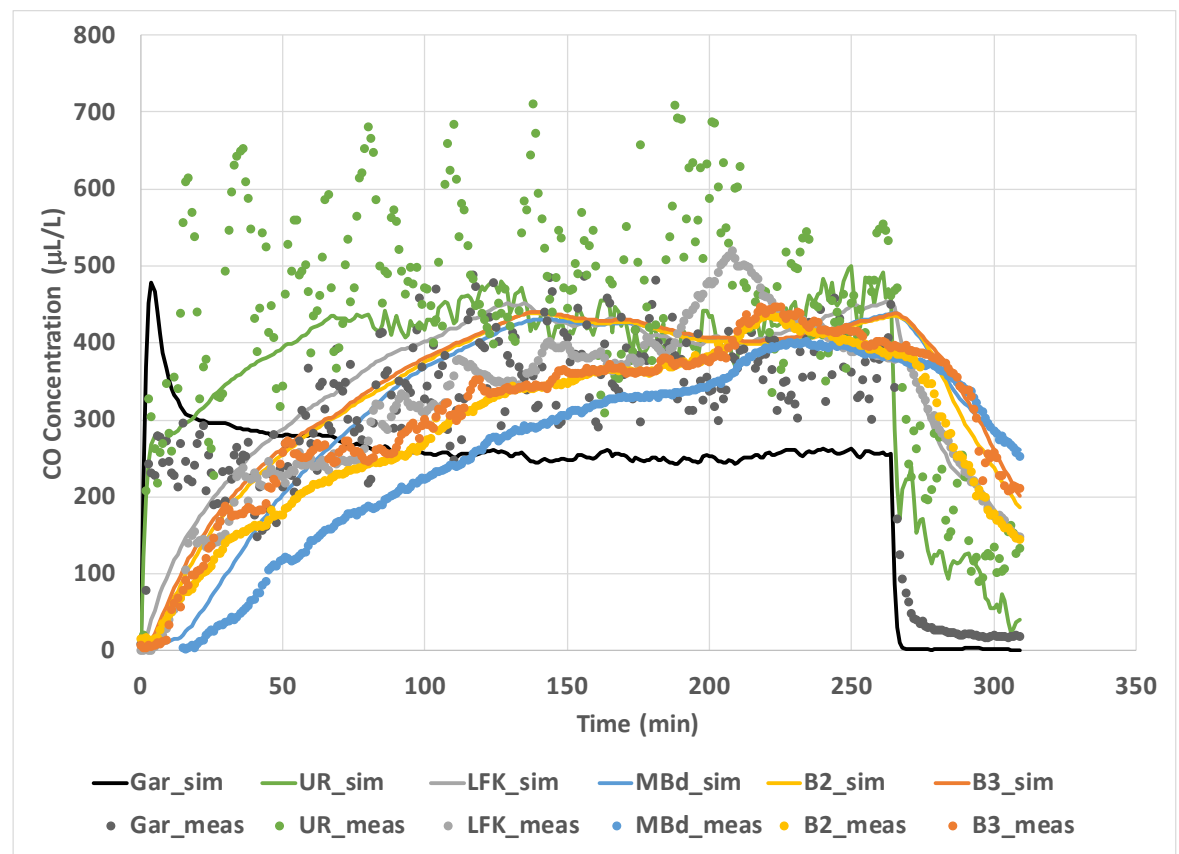

Figure 126a Observed and predicted CO concentrations in main living space zones for Case 32 Test 1 with utility room source

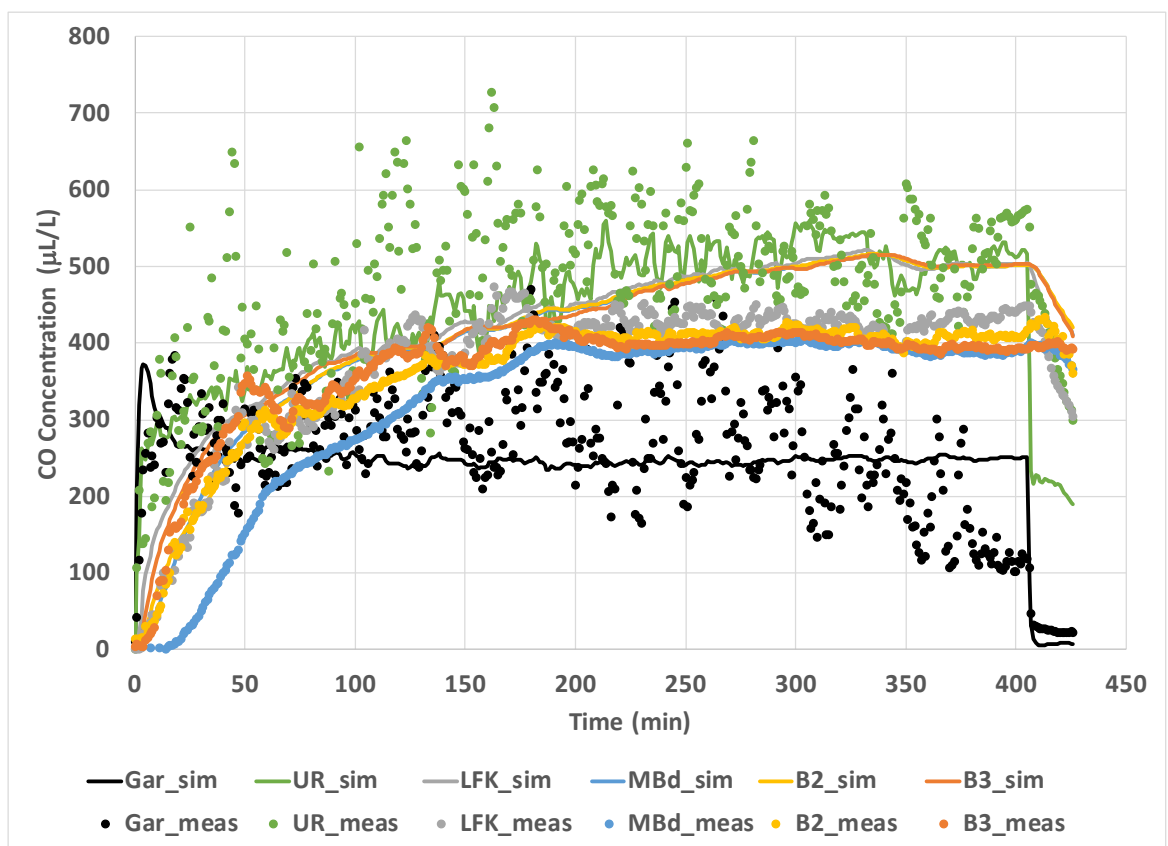

Figure 126b Observed and predicted CO concentrations in main living space zones and garage for Case 32 Test 2 with utility room source 


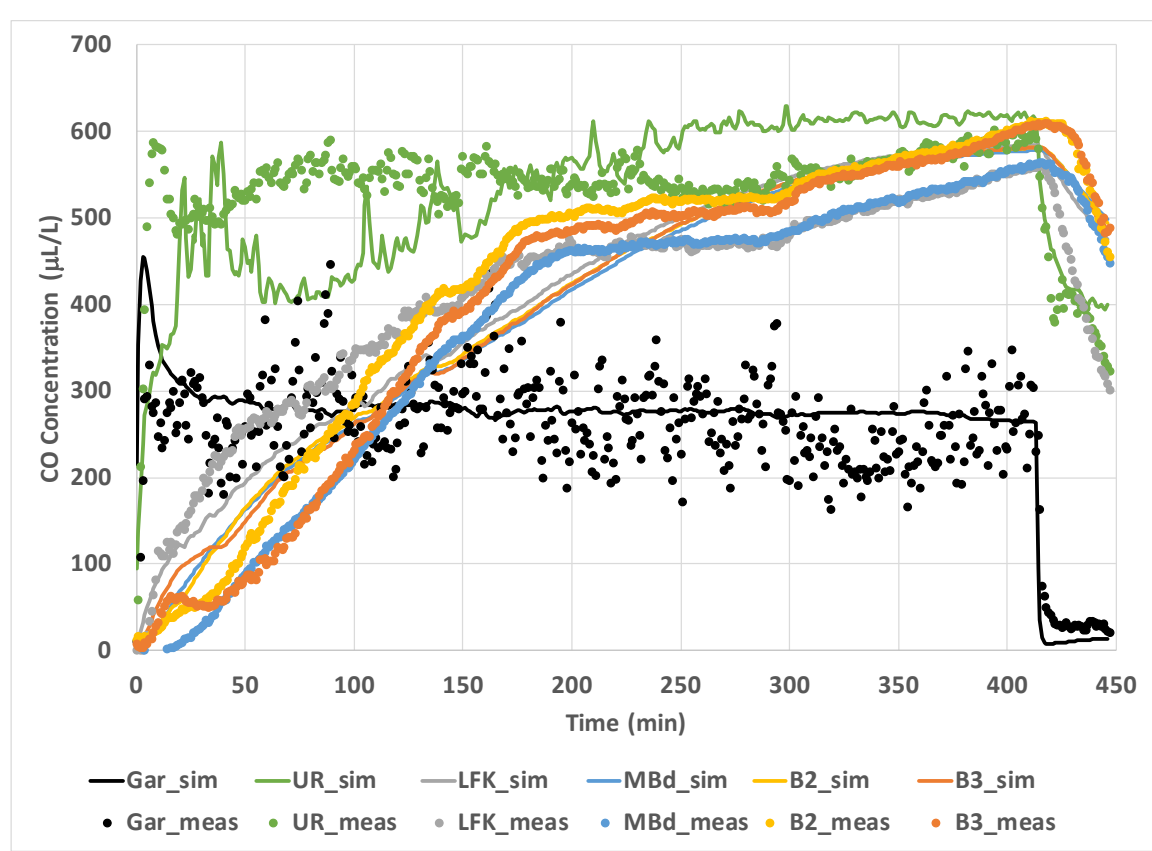

Figure 126c Observed and predicted CO concentrations in main living space zones and garage for Case 32 Test 3 with utility room source

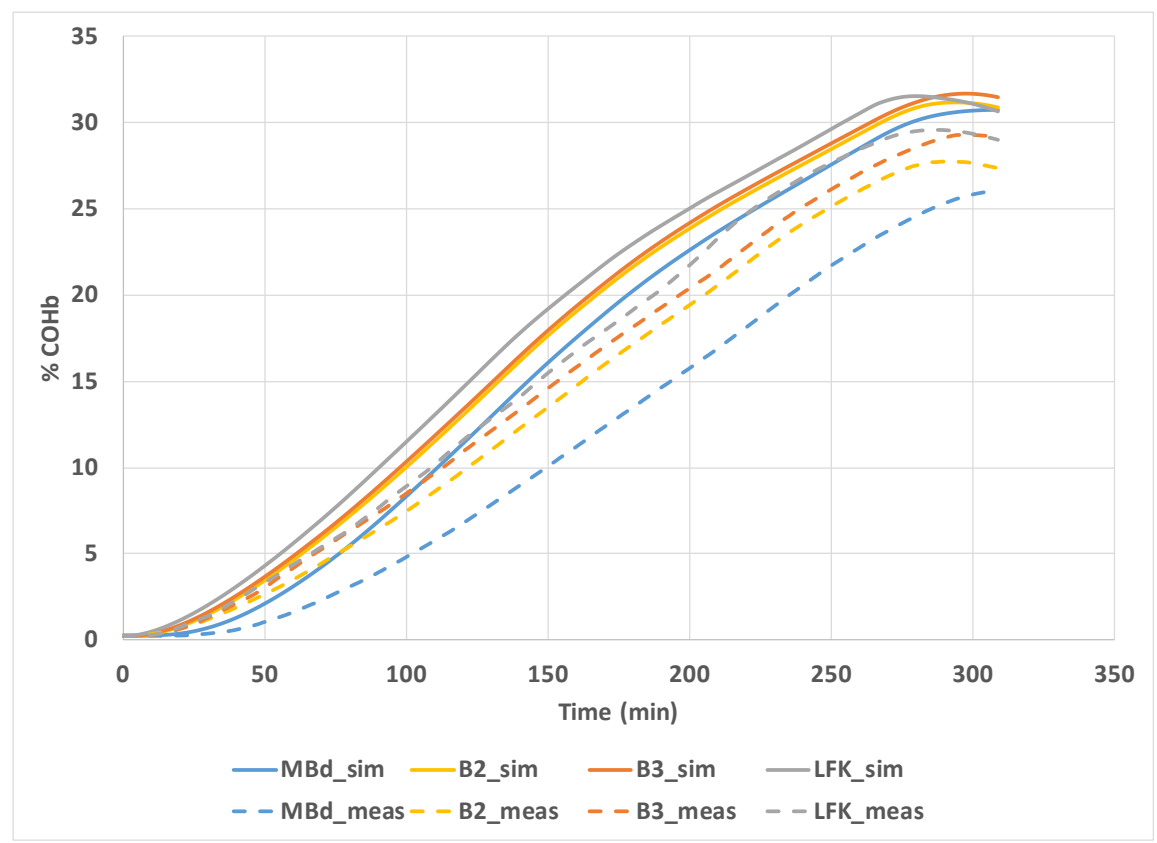

Figure 127a Observed and predicted $\mathrm{COHb}$ in main living space zones for Case 32 Test 1 with utility room source 


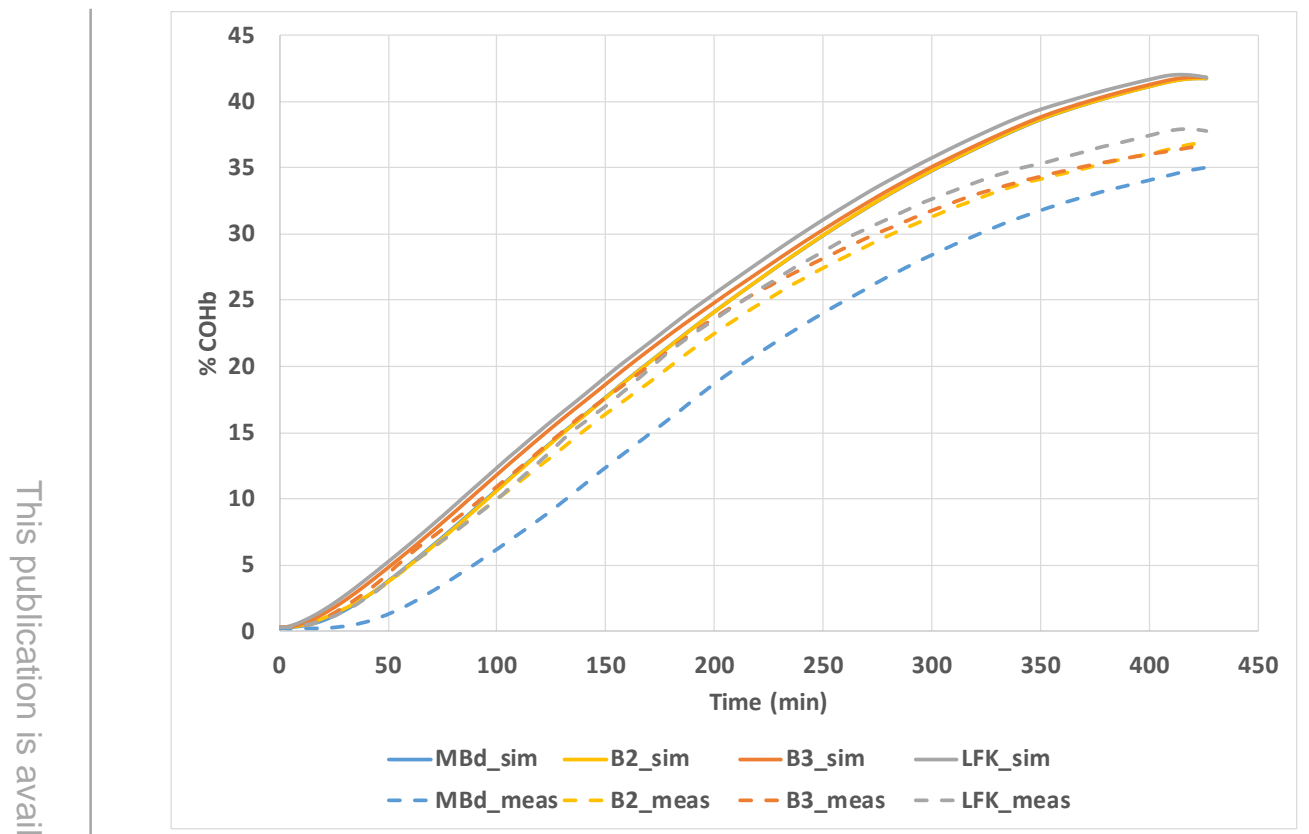

Figure 127b Observed and predicted COHb in main living space zones for Case 32 Test 2 with utility room source

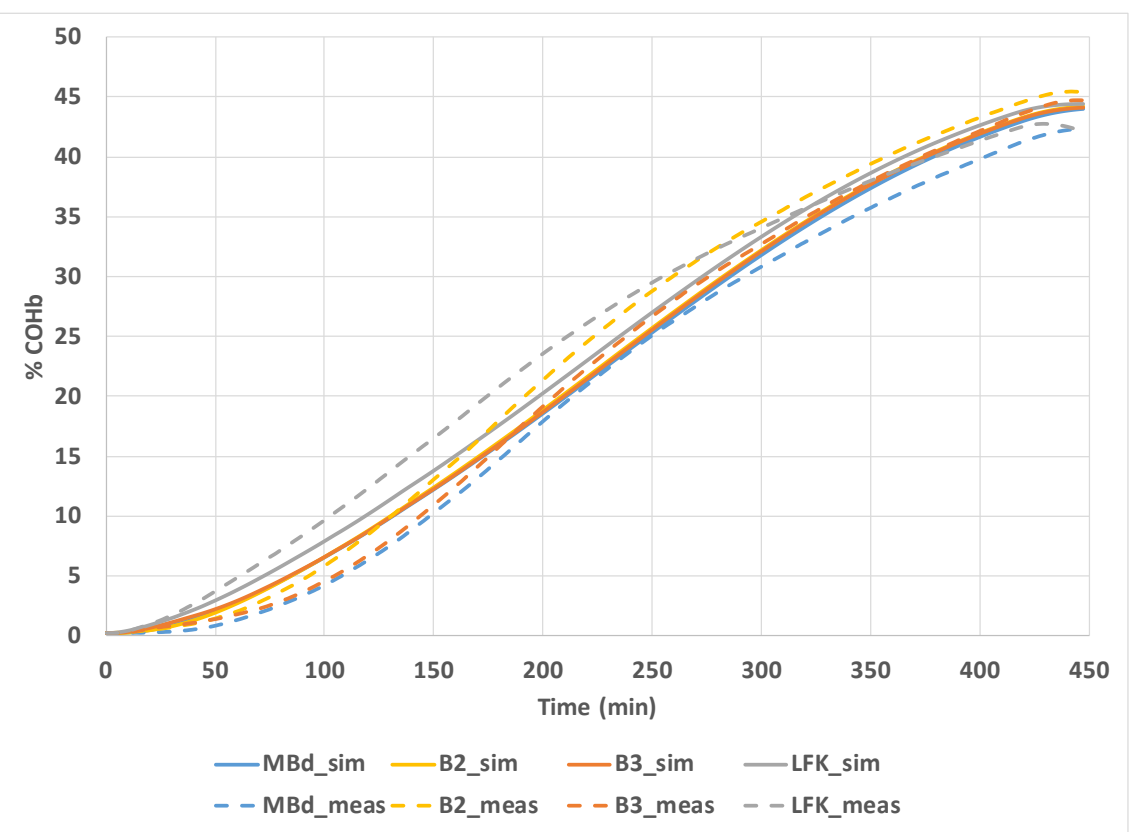

Figure 127c Observed and predicted COHb in main living space zones for Case 32 Test 3 with utility room source 
Table 18 Comparison of maximum COHb for predictions and measurements for Case 32 Tests 1, 2, and 3 with utility room source

\begin{tabular}{|c|c|c|c|}
\hline Zone & COHb (\% predicted) & COHb (\% observed) & $\%$ diff \\
\hline \multicolumn{4}{|c|}{ Test 1} \\
\hline B3 & 31.7 & 29.3 & 8.3 \\
\hline LFK & 31.5 & 29.6 & 6.4 \\
\hline B2 & 31.2 & 27.8 & 12.3 \\
\hline MBd & 30.7 & 26.1 & 17.9 \\
\hline \multicolumn{4}{|c|}{ Test 2} \\
\hline B3 & 41.9 & 36.7 & 14.2 \\
\hline LFK & 42.0 & 37.9 & 10.7 \\
\hline B2 & 41.8 & 36.9 & 13.1 \\
\hline MBd & 41.7 & 35.0 & 19.1 \\
\hline \multicolumn{4}{|c|}{ Test 3} \\
\hline B3 & 44.4 & 44.8 & -1.4 \\
\hline LFK & 44.4 & 42.8 & 3.9 \\
\hline B2 & 44.2 & 45.5 & -2.9 \\
\hline MBd & 44.0 & 42.4 & 3.7 \\
\hline Average $\mid \%$ difference $\mid$ & & & 9.5 \\
\hline
\end{tabular}




\section{Case \# 40}

The next two validation cases were Case 40 Tests 1 and 2. The G15 generator was positioned outside the garage (centered with the garage bay door, with the termination of its exhaust pipe in the plane of the bay door facing into garage) and tested at $90 \%$ load. The interior person door (from utility room to garage) was open $10 \mathrm{~cm}$ and the garage bay door was fully open; the exterior person door (from garage to backyard) was closed. All interior doors connected to the main living space were fully open. Based on generator characterization tests conducted at CPSC (Brookman 2018), a constant CO emission rate of $3083.8 \mathrm{~g} / \mathrm{h}$ was applied in this case. Although the generator was located outside the garage, the source was modeled as inside the garage since the generator exhaust jet was pointed directly into the garage.

The observed and predicted CO concentrations for the main living space zones in the house and the garage are shown in Figures 128a and 128b for Case 40 Tests 1 and 2 respectively (lines for the predictions and symbols for the measurements). The shutoff sensor was not activated during either of these tests. The generator was manually shut down after about 210 min for Test 1 , but ran until using a full tank of fuel for Test 2. As seen in the figures, the results of the Case 40 tests were similar with higher concentrations in Test 1 . In contrast to Case 32, Figures 128a and 128b both show that the utility room concentrations were lower than the garage average concentrations, indicating that the $\mathrm{CO}$ mixed more fully into the garage rather than higher non-uniform concentrations entering the utility room.

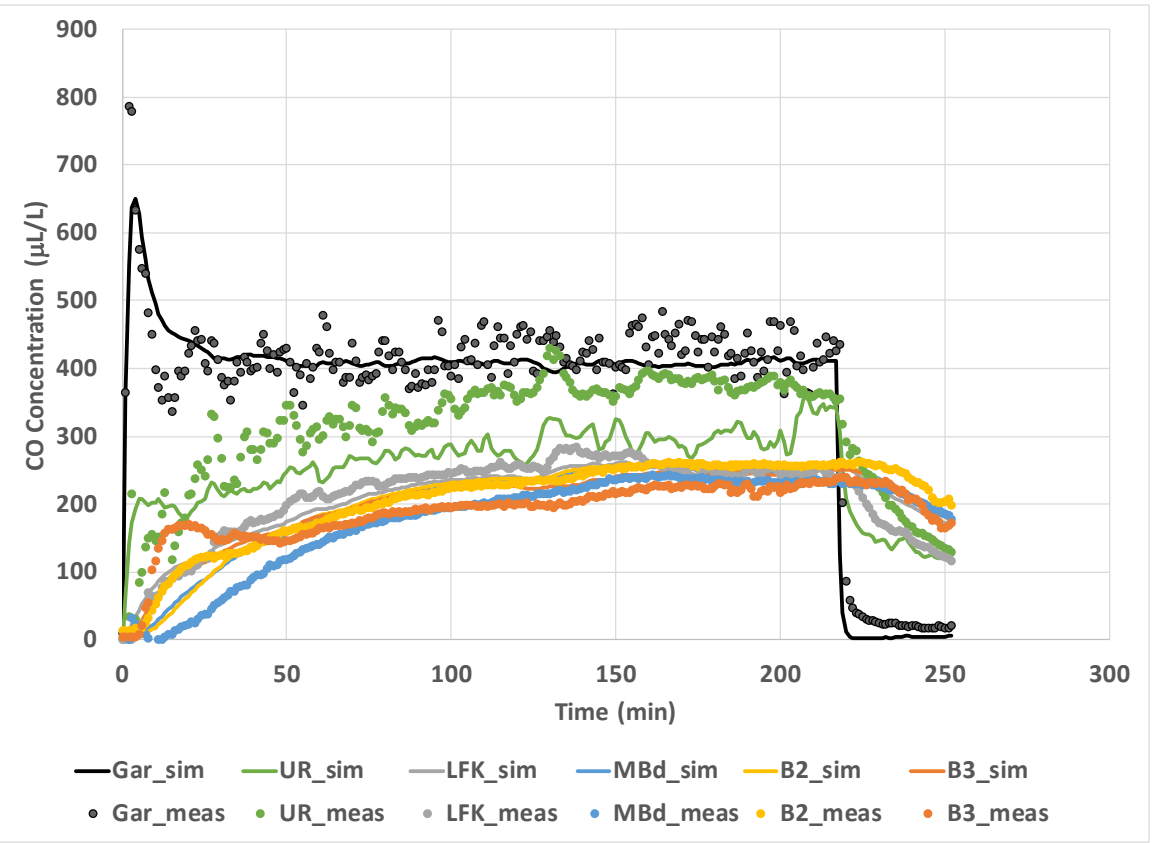

Figure 128a Observed and predicted CO concentrations in main living space zones and garage for Case 40 Test 1 


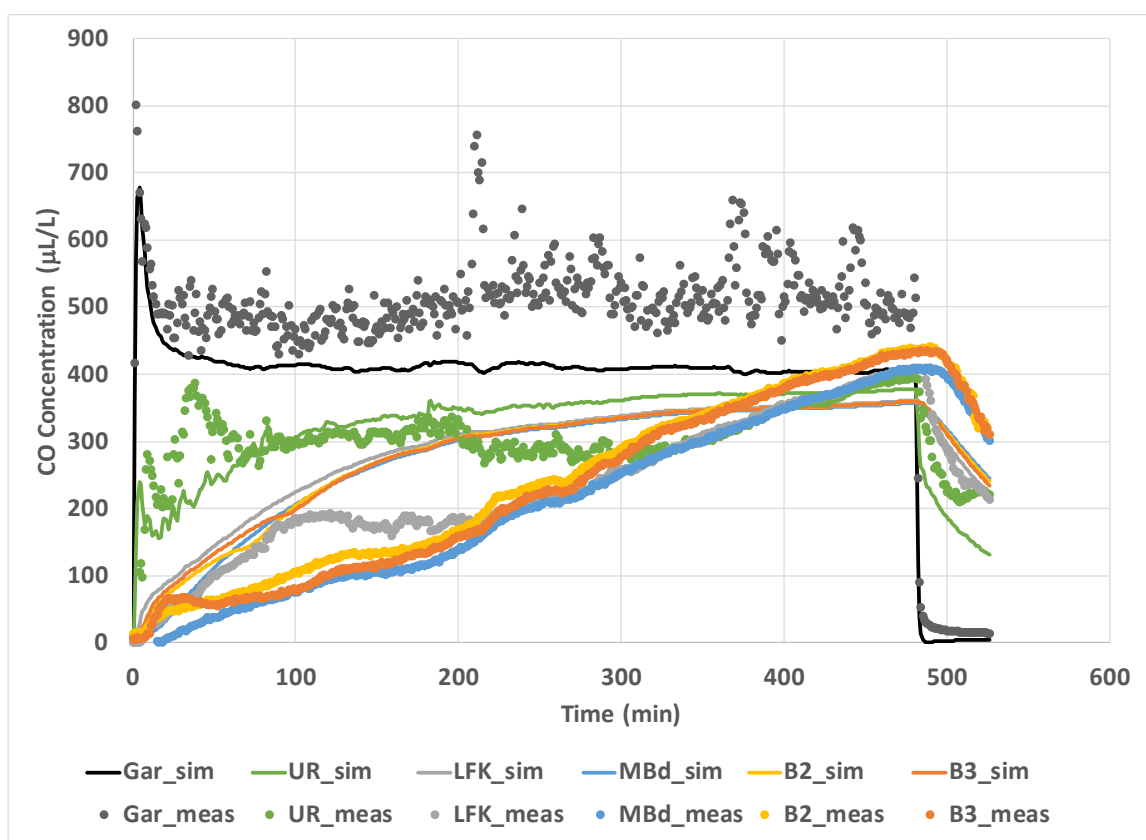

Figure 128b Observed and predicted CO concentrations in main living space zones and garage for Case 40 Test 2

$\mathrm{COHb}$ values calculated for the observed and predicted $\mathrm{CO}$ concentrations for the main living space zones in the test house are shown in Figures 129a and 129b for Case 40 Tests 1 and 2, respectively. As seen in the figures, the $\mathrm{COHb}$ results of the Case 40 tests were fairly similar with values reaching over $15 \%$ at the 200-minute mark (note that the tests were not the same length, with Test 1 being much shorter and including a CO decay period after $210 \mathrm{~min}$ ).

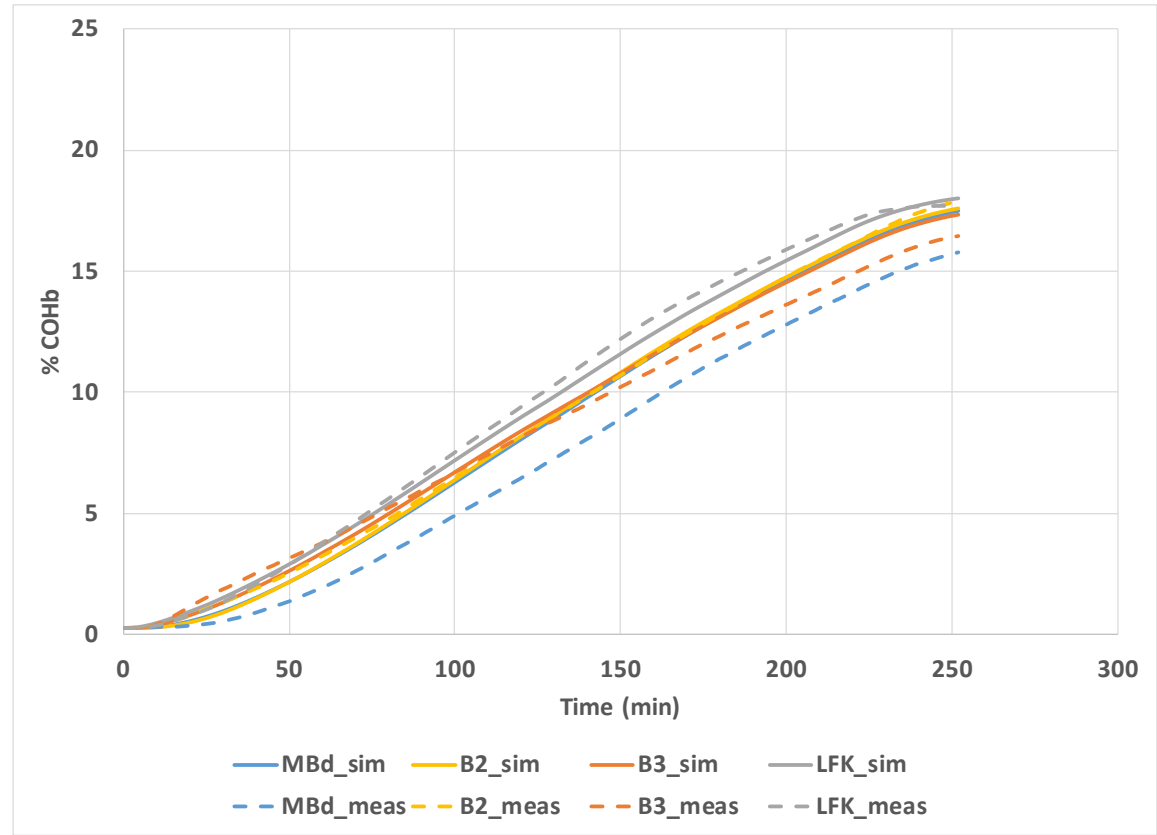

Figure 129a Observed and predicted COHb in main living space zones for Case 40 Test 1 


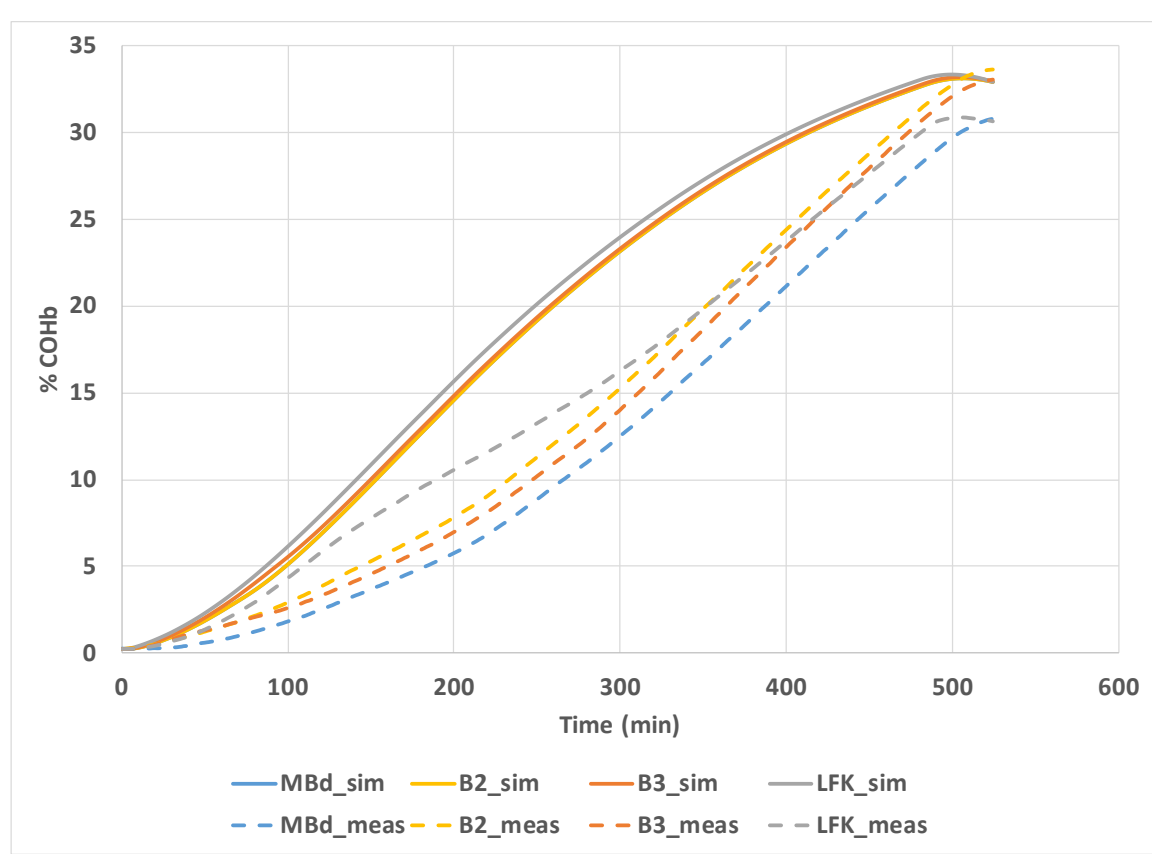

Figure 129b Observed and predicted COHb in main living space zones for Case 40 Test 2

Table 19 summarizes the maximum $\mathrm{COHb}$ values for the observed and predicted zone $\mathrm{CO}$ concentrations along with the percent differences and the absolute average percent difference. For the Case 40 tests, the average percent difference of the calculated maximum COHb was just $4.6 \%$, indicating no need for other modifications to the model for these cases.

Table 19 Comparison of maximum COHb for predictions and measurements for Case 40 Tests 1 and 2

\begin{tabular}{|c|c|c|c|}
\hline Zone & COHb (\% predicted) & COHb (\% observed) & $\%$ diff \\
\hline \multicolumn{4}{|c|}{ Test 1} \\
\hline B3 & 17.3 & 16.4 & 5.5 \\
\hline LFK & 18.0 & 17.7 & 1.7 \\
\hline B2 & 17.6 & 17.9 & -1.7 \\
\hline MBd & 17.5 & 15.8 & 10.9 \\
\hline \multicolumn{4}{|c|}{ Test 2} \\
\hline B3 & 33.2 & 33.1 & 0.2 \\
\hline LFK & 33.4 & 30.9 & 8.1 \\
\hline B2 & 33.2 & 33.6 & -1.5 \\
\hline MBd & 33.2 & 30.8 & 7.5 \\
\hline Average $\mid \%$ difference $\mid$ & & & 4.6 \\
\hline
\end{tabular}




\section{Case \# 51}

The final three validation cases were Case 51 Tests 1, 2 and 3. The G6 generator was fully fueled, positioned outside the front door $(155 \mathrm{~cm}$ away from plane of the door, with the exhaust facing the door), and tested at $100 \%$ load $(6,000 \mathrm{~W})$. The front door was open $10 \mathrm{~cm}$ during the test. All interior doors connected to the main living space were fully open. Although the generator was located outside the front door of the house, the source was modeled as inside the living room since the generator exhaust jet was pointed toward the partially open front door. Based on generator characterization tests conducted at CPSC (Brookman 2018), a constant CO emission rate of $1846.1 \mathrm{~g} / \mathrm{h}$ was initially applied in this case. However, the model significantly overpredicted the indoor CO concentrations (results not shown here), indicating that not all the generator exhaust entered the house. An iterative simulation process was used to estimate that around $400 \mathrm{~g} / \mathrm{h}$ of CO actually entered the house, and this value was then used as the source term for these tests

The observed and predicted $\mathrm{CO}$ concentrations for the main living space zones and the garage are shown in Figures 130a, 130b and 130c for Case 51 Tests 1, 2 and 3 respectively (lines for the predictions and symbols for the measurements). The shutoff sensor was not activated during any of these tests. The generator was manually shut down at around 193 min for Test 1 when calculated $\mathrm{COHb}$ levels for simulated house occupants exceeded $70 \%$. The generator ran until using a full tank of fuel for the other tests. As seen in the figures, the results of the Case 40 tests varied due to weather conditions, with the highest concentrations in Test 1.

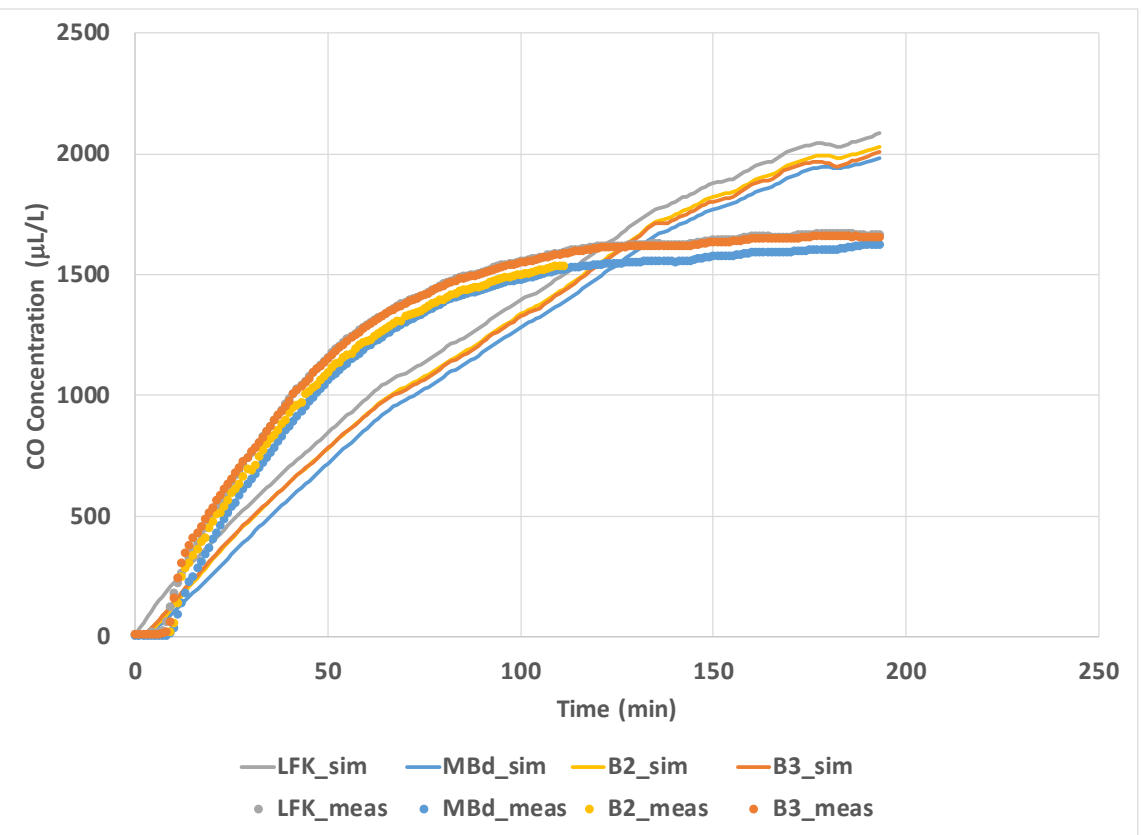

Figure 130a Observed and predicted CO concentrations in main living space zones for Case 51 Test 1 (Note the Bedroom 2 hi sensor failed halfway through the test.) 


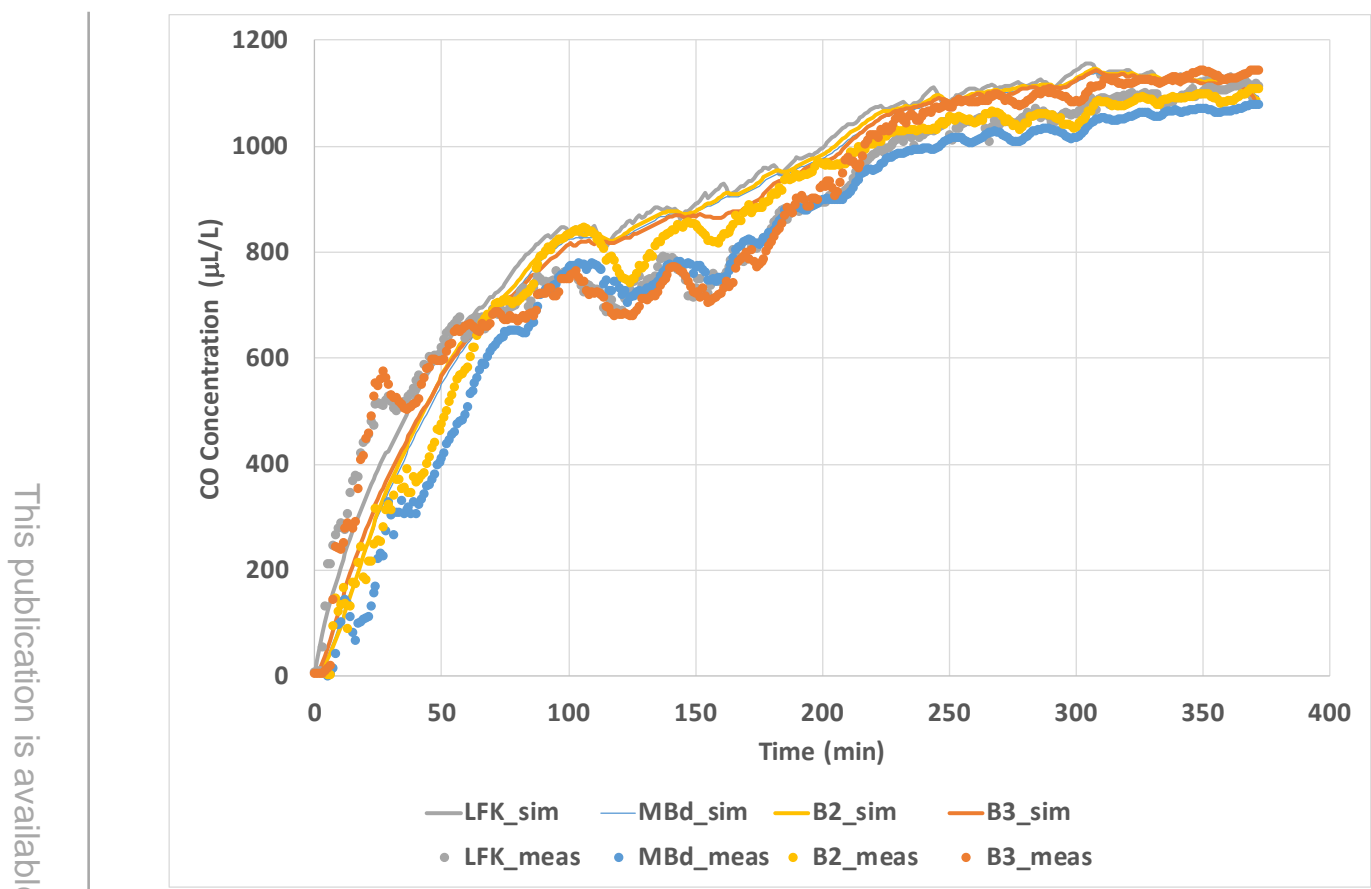

Figure 130b Observed and predicted CO concentrations in main living space zones for Case 51 Test 2

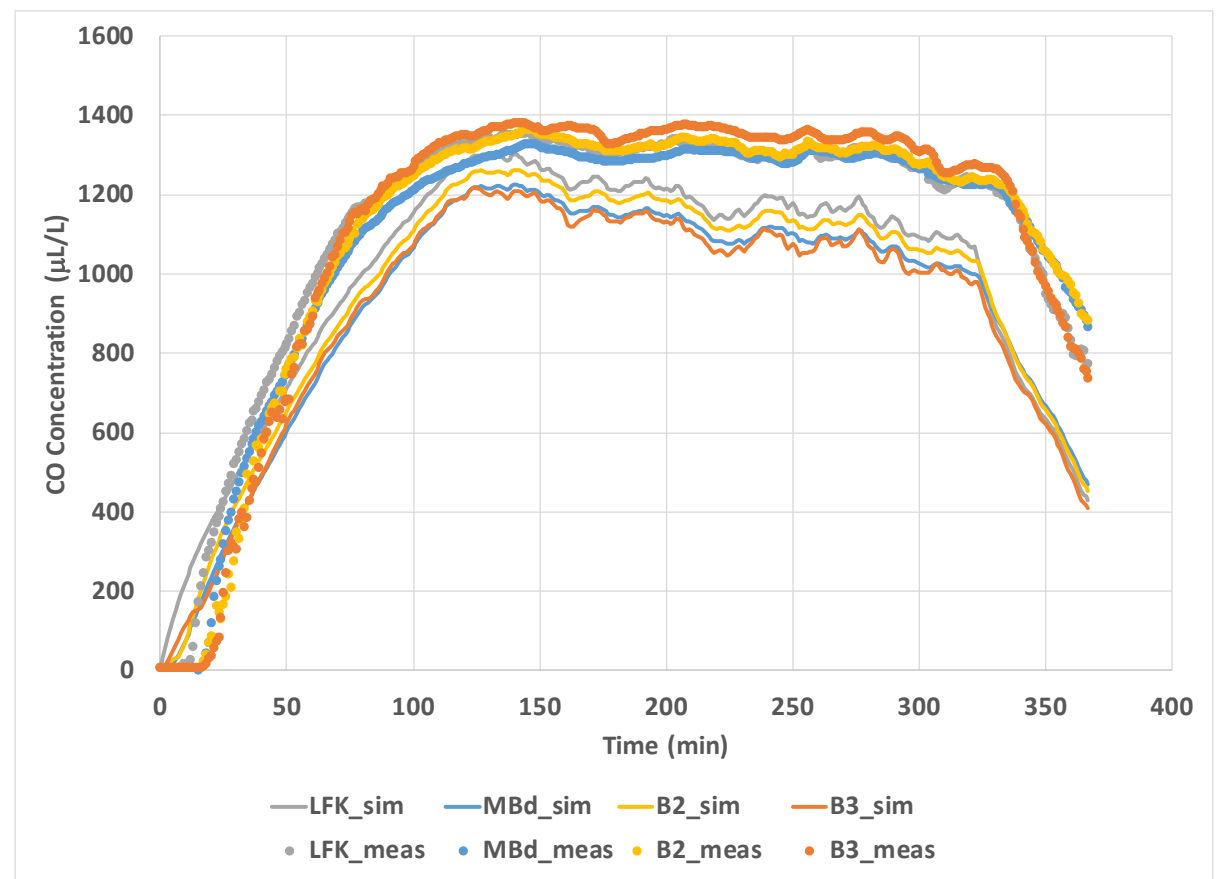

Figure 130c Observed and predicted CO concentrations in main living space zones for Case 51 Test 3 
$\mathrm{COHb}$ values calculated for the observed and predicted $\mathrm{CO}$ concentrations for the main living space zones in the test house are shown in Figures 131a, 131b and 131c for Case 51 Tests 1, 2 and 3, respectively. As seen in the figures, the $\mathrm{COHb}$ results of the Case 51 tests were fairly similar with the value reaching $70 \%$ at the 200-minute mark for Test 1 and reaching between $60 \%$ and $70 \%$ for the other two tests.

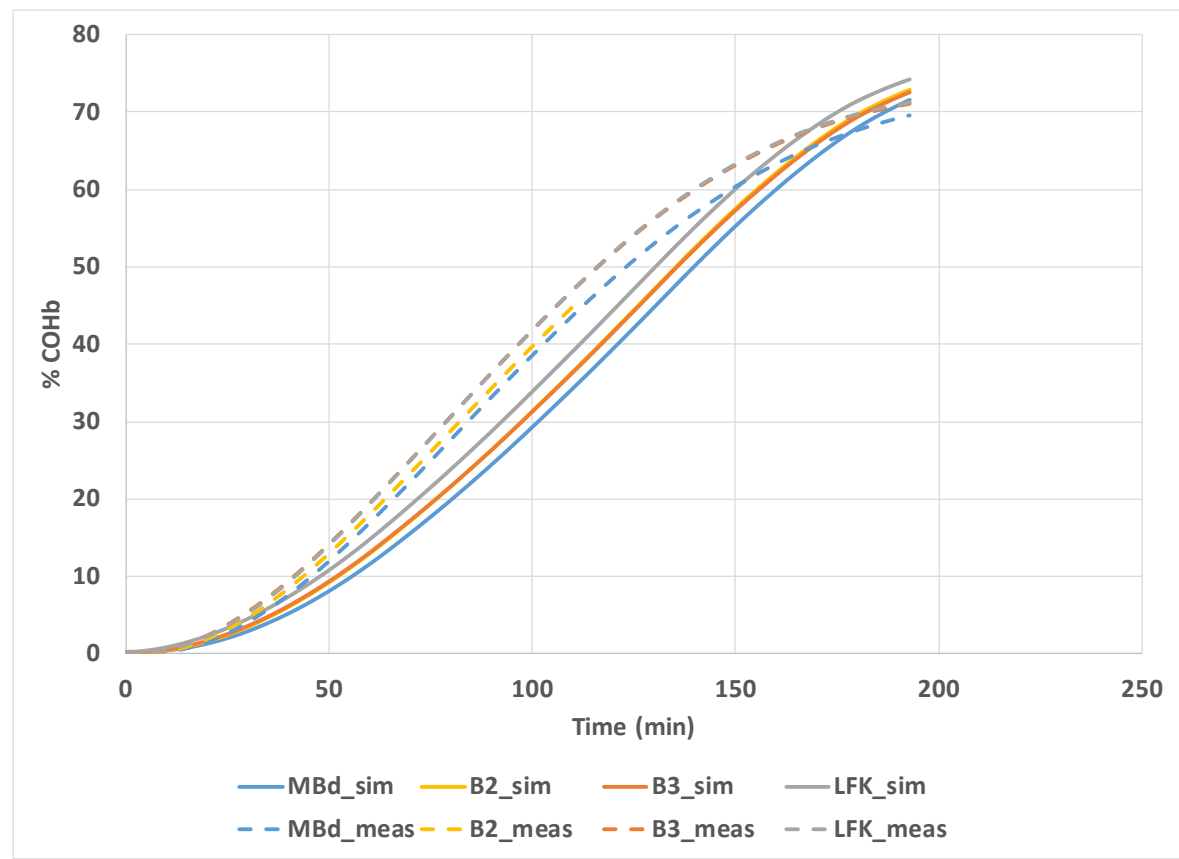

Figure 131a Observed and predicted COHb in main living space zones for Case 51 Test 1 (Note the Bedroom 2 hi sensor failed halfway through the test.)

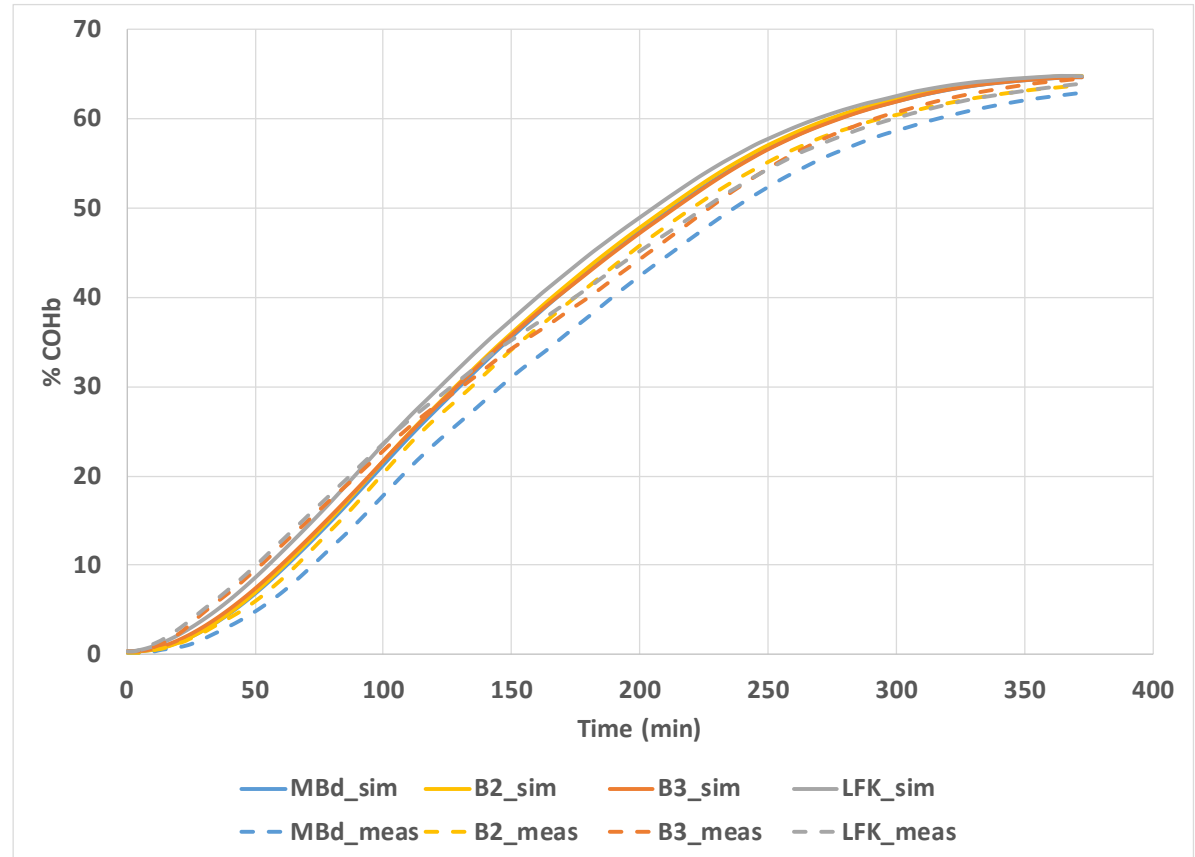

Figure 131b Observed and predicted $\mathrm{COHb}$ in main living space zones for Case 51 Test 2 


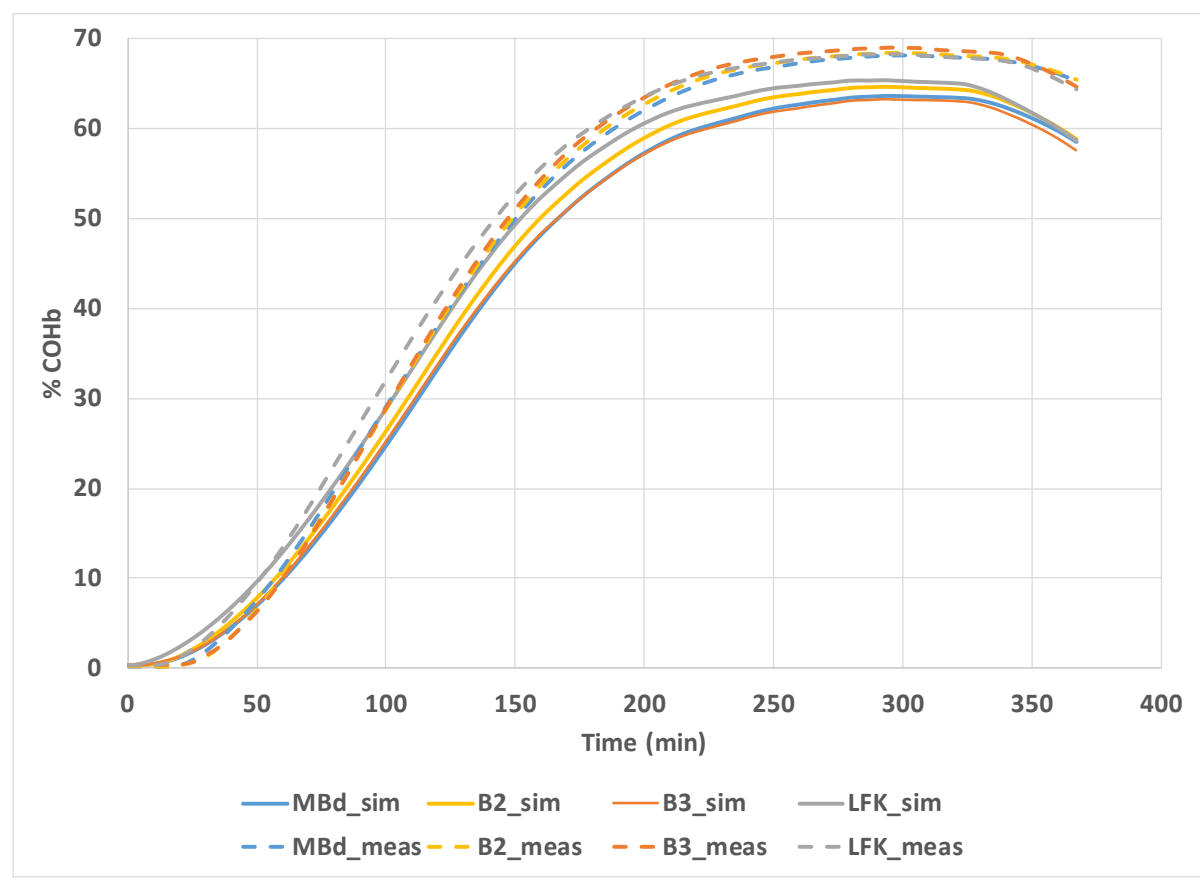

Figure 131c Observed and predicted COHb in main living space zones for Case 51 Test 3

Table 20 summarizes the maximum $\mathrm{COHb}$ values for the observed and predicted zone $\mathrm{CO}$ concentrations along with the percent differences and the absolute average percent difference. For the Case 51 tests, the average percent difference of the calculated maximum $\mathrm{COHb}$ was just $3.3 \%$ indicating no need to modify the model for this case. 
Table 20 Comparison of maximum $\mathrm{COHb}$ for predictions and measurements for Case 51 Tests 1, 2 and 3

\begin{tabular}{|c|c|c|c|}
\hline Zone & COHb (\% predicted) & COHb (\% observed) & $\%$ diff \\
\hline \multicolumn{4}{|c|}{ Test 1} \\
\hline B3 & 72.5 & 71.1 & 2.0 \\
\hline LFK & 74.2 & 71.2 & 4.2 \\
\hline B2 & $*$ & * & * \\
\hline MBd & 71.5 & 69.6 & 2.7 \\
\hline \multicolumn{4}{|c|}{ Test 2} \\
\hline B3 & 64.6 & 64.6 & 0 \\
\hline LFK & 64.8 & 63.9 & 1.5 \\
\hline B2 & 64.7 & 63.8 & 1.5 \\
\hline MBd & 64.6 & 62.9 & 2.8 \\
\hline B3 & 63.3 & 69.0 & -8.3 \\
\hline \multicolumn{4}{|c|}{ Test 3} \\
\hline LFK & 65.4 & 68.3 & -4.2 \\
\hline B2 & 64.6 & 68.4 & -5.6 \\
\hline MBd & 63.6 & 68.1 & -6.5 \\
\hline Average $\mid \%$ difference $\mid$ & & & 3.6 \\
\hline
\end{tabular}

*The B2 sensor failed during Test 1. 


\section{Summary}

This report presents measured $\mathrm{CO}$ concentrations, and calculated $\mathrm{COHb}$ profiles based on those concentrations, from tests conducted with five different portable generators under a wide range of operational and environmental conditions to characterize the indoor CO concentrations resulting from generators operating in or near a test house. The tests are intended to provide CPSC staff with information to enable comparisons of residential $\mathrm{CO}$ exposures reflecting operation of current designs of portable engine-driven electric generators, inside or near homes or in attached garages, versus operation of portable generators equipped with a CO safety shutoff device. CO concentrations varied widely with peak living space CO concentrations ranging from under 100 $\mu \mathrm{L} / \mathrm{L}$ to over $3500 \mu \mathrm{L} / \mathrm{L}$ and peak living space COHb levels ranging from less than $5 \%$ to over $80 \%$. These results apply to the specific units and conditions tested; other units and test conditions may produce different results.

Those measured data were then compared to CONTAM predictions of CO levels and $\mathrm{COHb}$ profiles of simulated occupants calculated from CONTAM CO predictions. NIST also examined modeling options to reduce the uncertainty in the CO levels predicted by CONTAM, such that they can be more reliable when estimating $\mathrm{COHb}$ profiles in a planned simulation study of the impact of the shutoff requirements in the PGMA and UL voluntary standards on indoor CO exposure. The average difference between peak \% COHb based on predicted and measured CO concentrations in the main test house living space zones for a given test case ranged from less than $5 \%$ to over $90 \%$. In general, the poorest agreement was found for cases in which the generator exhaust was pointed toward a doorway without accounting for the exhaust jet driven transport of $\mathrm{CO}$ to the adjoining zone. Additionally, closer agreement could be achieved by using a "shutoff ratio" to account for the difference between local CO concentration at the shutoff sensor compared to the room average concentration. The model validation results indicate the need to account for non-uniform zone CO concentrations when modeling portable generators with onboard CO shutoff sensors for many operating conditions.

\section{Acknowledgements}

NIST's participation in this study was supported by an interagency agreement between the National Institute for Standards and Technology and the Consumer Product Safety Commission (CPSC-I17-0023). The content of this publication does not necessarily reflect the views of the Commission, nor does mention of trade names, commercial products, or organizations imply endorsement by the Commission. The authors would like to acknowledge the contributions of Janet Buyer, Matthew Hnatov, Sandy Inkster, and Andrew Lock from CPSC. 


\section{References}

ASTM. 2011. Standard test method for determining air change in a single zone by means of a tracer gas dilution. E741-06 American Society for Testing and Materials.

Bassett, M. Infiltration and Leakage Paths in Single Family Houses - a Multizone Infiltration Case Study. 1990. AIVC Technical Note 27, Air Infiltration and Ventilation Centre.

Brookman, MJ. 2018. Memo on Portable Generator Characterization for Evaluation of Carbon Monoxide Shutoff Concepts.

Dols WS and Polidoro BJ. 2015. CONTAM User Guide and Program Documentation Version 3.2. NIST Technical Note 1887, National Institute of Standards and Technology.

Emmerich SJ and SJ Nabinger. 2001. Measurement and Simulation of the IAQ Impact of Particle Air Cleaners in a Single-Zone Building. International Journal of HVAC\&R Research Vol. 1, No. 7, ASHRAE.

Emmerich SJ. 2001. Validation of Multizone IAQ Modeling of Residential-scale Buildings: A Review. ASHRAE Transactions Vol. 107.2. 49.

Emmerich SJ, and AK Persily. 1996. Multizone modeling of three residential indoor air quality control options. Building Simulation '95.

Emmerich SJ, Persily AK, and SJ Nabinger. 2002. Modeling moisture in residential buildings with a multizone IAQ program. Proceedings of Indoor Air 2002.

Emmerich SJ, Reed CH, and A Gupta. 2005. Modeling the IAQ impact of HHI interventions in inner-city housing. NISTIR 5212, National Institute of Standards and Technology.

Emmerich SJ, Howard-Reed C, and SJ Nabinger. 2004. Validation of Multizone IAQ Model Predictions for Tracer Gas in a Townhouse. Building Services Engineering Research and Technology, Vol. 25 (4).

Emmerich SJ, Persily AK, and L Wang. 2013. Modeling and Measuring the Effects of Portable Gasoline Powered Generator Exhaust on Indoor Carbon Monoxide Level. NIST Technical Note 1781.

Emmerich SJ and WS Dols. 2016. Model Validation Study of Carbon Monoxide Transport due to Portable Generator Operation in an Attached Garage. Journal of Building Performance Simulation 9 (4), 397-410. 10.

Emmerich SJ, Polidoro B, and WS Dols. 2016. Simulation of Residential Carbon Monoxide Exposure Due to Generator Operation in Enclosed Spaces. NIST Technical Note 1925.

Hnatov MV. 2018. Incidents, Deaths, and In-Depth Investigations Associated with Non-Fire Carbon Monoxide from Engine-Driven Generators and Other Engine-Driven Tools, 2005-2017; U.S. Consumer Product Safety Commission: Bethesda, MD.

Hnatov MV. 2017. Non-Fire Carbon Monoxide Deaths Associated with the Use of Consumer Products, 2014 Annual Estimates, U.S. Consumer Product Safety Commission, Bethesda, MD. 
Nabinger SJ, AK Persily, and WS Dols. 2010. Impacts of Airtightening Retrofits on Ventilation Rates and Energy Consumption in a Manufactured Home. NIST Technical Note 1673.

Nabinger SJ and AK Persily. 2008. Airtightness, Ventilation and Energy Consumption in a Manufactured House: Pre-Retrofit Results. NISTIR 7478.

Persily AK, A Musser, and D Leber. 2006. A Collection of Homes to Represent the U.S. Housing Stock. NISTIR 7330. National Institute of Standards and Technology.

Stewart RD. 1975. The effect of carbon monoxide on humans. Annual review of pharmacology. 15, 409-23.

Wang LL, Dols WS, and Q Chen. 2010. Using CFD capabilities of CONTAM 3.0 for simulating airflow and contaminant transport in and around buildings. HVAC\&R Research. 2010 Nov 1;16(6):749-63. 


\section{Appendix 1 - CPSC Letter to NIST on Portable Generator Characterization for Evaluation of Carbon Monoxide Shutoff Concepts}

This appendix contains a letter written by CPSC staff to NIST to provide data on the generators included in this report. These data were for potential use as input to the model validation effort and include $\mathrm{CO}$ emission rate, exhaust temperature and velocity, block temperature, and applied load. 


\title{
U.S. CONSUMER PRODUCT SAFETY COMMISSION \\ 5 RESEARCH PLACE \\ ROCKVILLE, MD 20850
}

November 1, 2018

\begin{abstract}
Steven J. Emmerich
Indoor Air Quality \& Ventilation Group Leader

Energy and Environment Division

Engineering Laboratory

National Institute of Standards and Technology

100 Bureau Dr. - Mail Stop 8633

Gaithersburg, MD 20899-8633

301-975-6459
\end{abstract}

Subject: Portable Generator Characterization for Evaluation of Carbon Monoxide Shutoff Concepts

Dear Mr. Emmerich:

This letter provides data on the generators used for ongoing portable generator testing in the NIST Indoor Air Quality (IAQ) Test House. The data are for the modeling team to validate simulation methodology and develop input values for the house modeling program. ${ }^{1}$ These models will be used to estimate carbon monoxide (CO) exposure profiles for occupants of the suite of structures used in the NIST Technical Note 1925 modeling effort.

U.S. Consumer Product Safety Commission (CPSC) staff characterized the generators to be used for empirical testing under Interagency Agreement (IAA) CPSC-I-17-0023. Staff plans to use the data to develop inputs for modeling portable generators equipped with shutoff devices.

The generators chosen for this evaluation represent the range of available power outputs from portable generators. Currently, CPSC staff selected and characterized five generators to develop modeling input values. Staff identified these generators as G2, G2P, G6, G7, and G15.

G2: $\quad 2 \mathrm{~kW}$ gasoline-powered generator

G2P: $\quad 2 \mathrm{~kW}$ propane-powered generator

G6: $\quad 6 \mathrm{~kW}$ gasoline-powered generator

G7: $\quad 7 \mathrm{~kW}$ gasoline-powered generator

\footnotetext{
1 These comments are those of CPSC staff, and they have not been reviewed or approved by, and may not necessarily reflect the views of, the Commission.
} 


\section{G15: $\quad 15 \mathrm{~kW}$ gasoline-powered generator}

To reproduce the effects of each generator in a computer simulation, staff measured the $\mathrm{CO}$ emission rate, exhaust temperature and velocity, block temperature, and applied load. CPSC staff measured the $\mathrm{CO}$ emission rate using a constant-volume sampling (CVS) tunnel and an AVL AMA i60 emissions test bench. Each generator test began with cold start conditions, with the block temperature near an ambient lab temperature of $21^{\circ} \mathrm{C}$. Staff applied the desired load as soon as the engine speed stabilized. Staff chose the loads applied to each generator to provide a range of emission rates representative of the rates produced by portable generators available on the market as of September 2017. Emissions were measured during cold start, warm-up under load, and for a period of approximately $30 \mathrm{~min}$ after the emission rate became steady. Staff performed emission testing in triplicate for each generator. Staff recorded the applied load, block temperature, and exhaust temperature, in addition to the $\mathrm{CO}$ emissions during emissions testing. Staff recorded the exhaust velocity during a separate test using a vane-type anemometer, where staff warmed the generator to operating temperature for the desired load. The exhaust outlet diameter was also measured.

The data provided in the tables below represent average values during the period when the generator block temperature and emissions were steady. Staff performed a comparison test between hot-start and cold-start emissions on the G2 and G15 generators. 


\section{G2 Data Summary}

Table 1. G2 @ 50\% Load

\begin{tabular}{|l|l|l|}
\hline G2 @ 50\% Load & Value & Units \\
\hline Avg. CO Emission Rate & 345.0 & $\mathrm{~g} / \mathrm{hr}$ \\
\hline Avg. Block Temp & 75.5 & ${ }^{\circ} \mathrm{C}$ \\
\hline Avg. Exhaust Temp & 152.3 & ${ }^{\circ} \mathrm{C}$ \\
\hline
\end{tabular}

Table 2. G2 @ 10\% Load

\begin{tabular}{|l|l|l|}
\hline G2 @ 10\% Load & Value & Units \\
\hline Avg. CO Emission Rate & 197.3 & $\mathrm{~g} / \mathrm{hr}$ \\
\hline Avg. Block Temp & 66.6 & ${ }^{\circ} \mathrm{C}$ \\
\hline Avg. Exhaust Temp & 102.2 & ${ }^{\circ} \mathrm{C}$ \\
\hline
\end{tabular}

Table 3. G2 Exhaust Parameters

\begin{tabular}{|l|l|l|}
\hline G2 Exhaust Parameters & Value & Unit \\
\hline Exhaust Exit Diameter & 1.16 & $\mathrm{~cm}$ (I.D.) \\
\hline Exhaust Velocity @ 50\% Load & 11.3 & $\mathrm{~m} / \mathrm{s}$ \\
\hline Exhaust Velocity @ 10\% Load & 10.0 & $\mathrm{~m} / \mathrm{s}$ \\
\hline
\end{tabular}

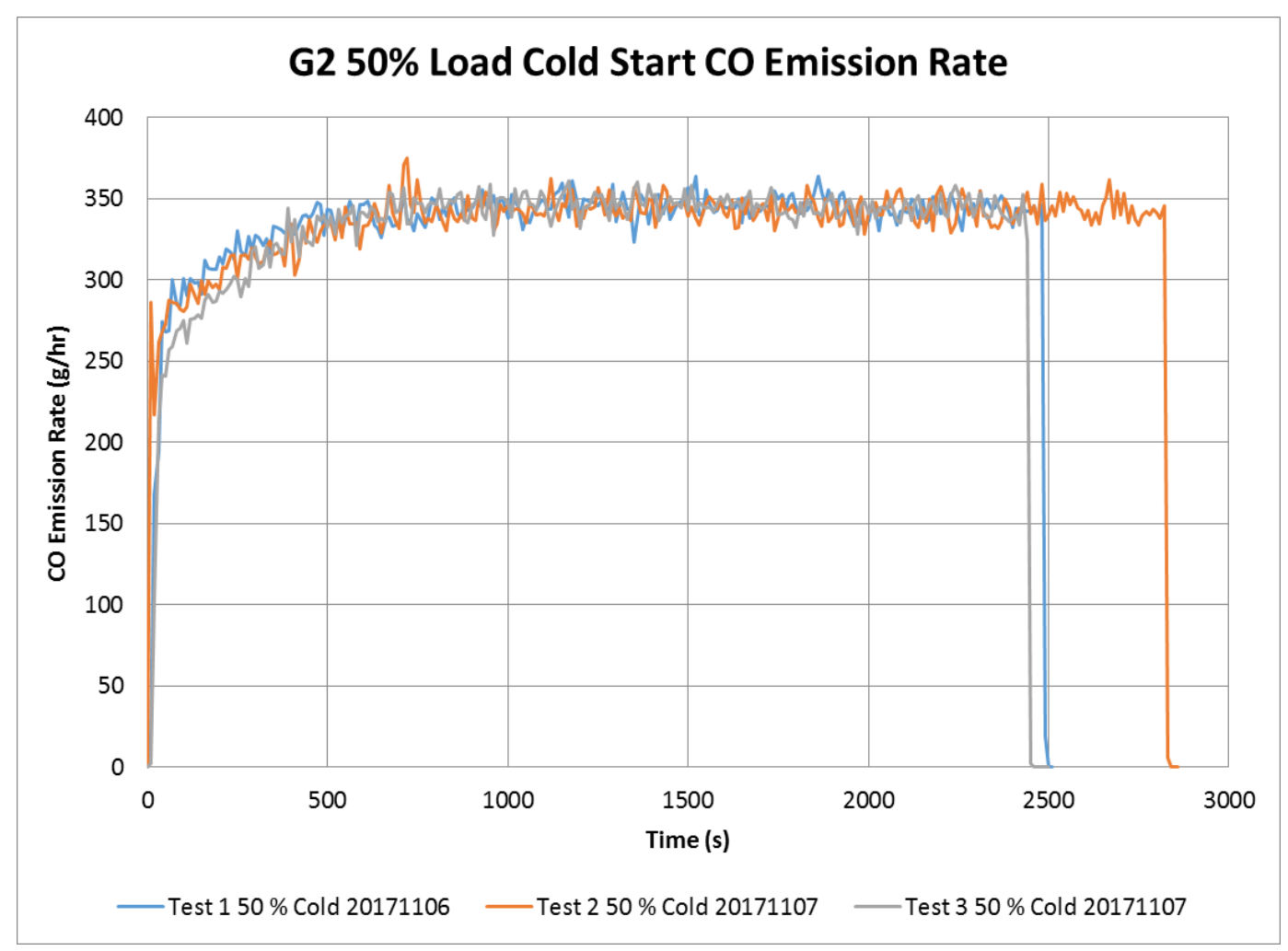

Figure 1. G2 50\% Load Cold Start Emission Rate 


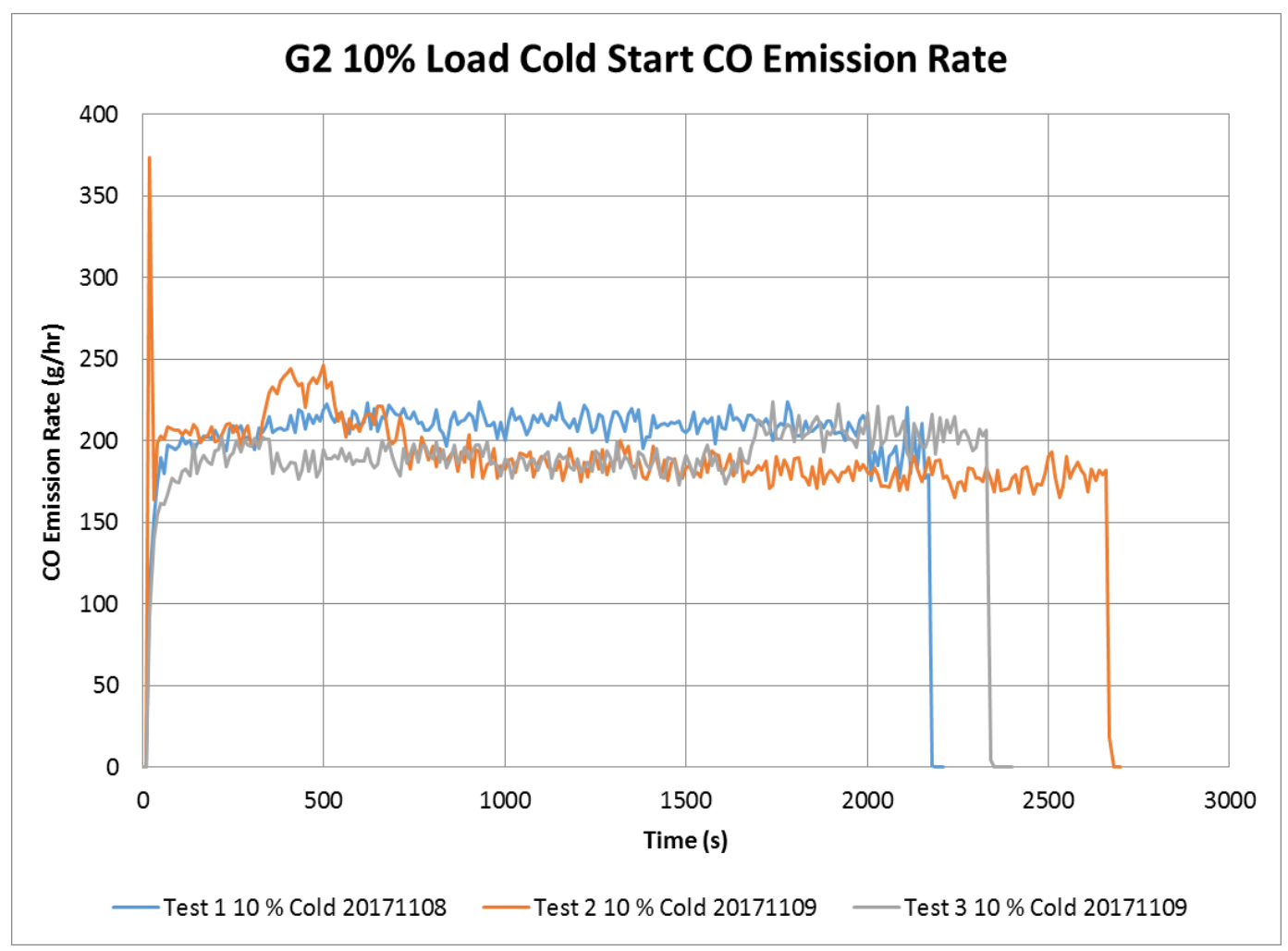

Figure 2. G2 10\% Load Cold Start Emission Rate

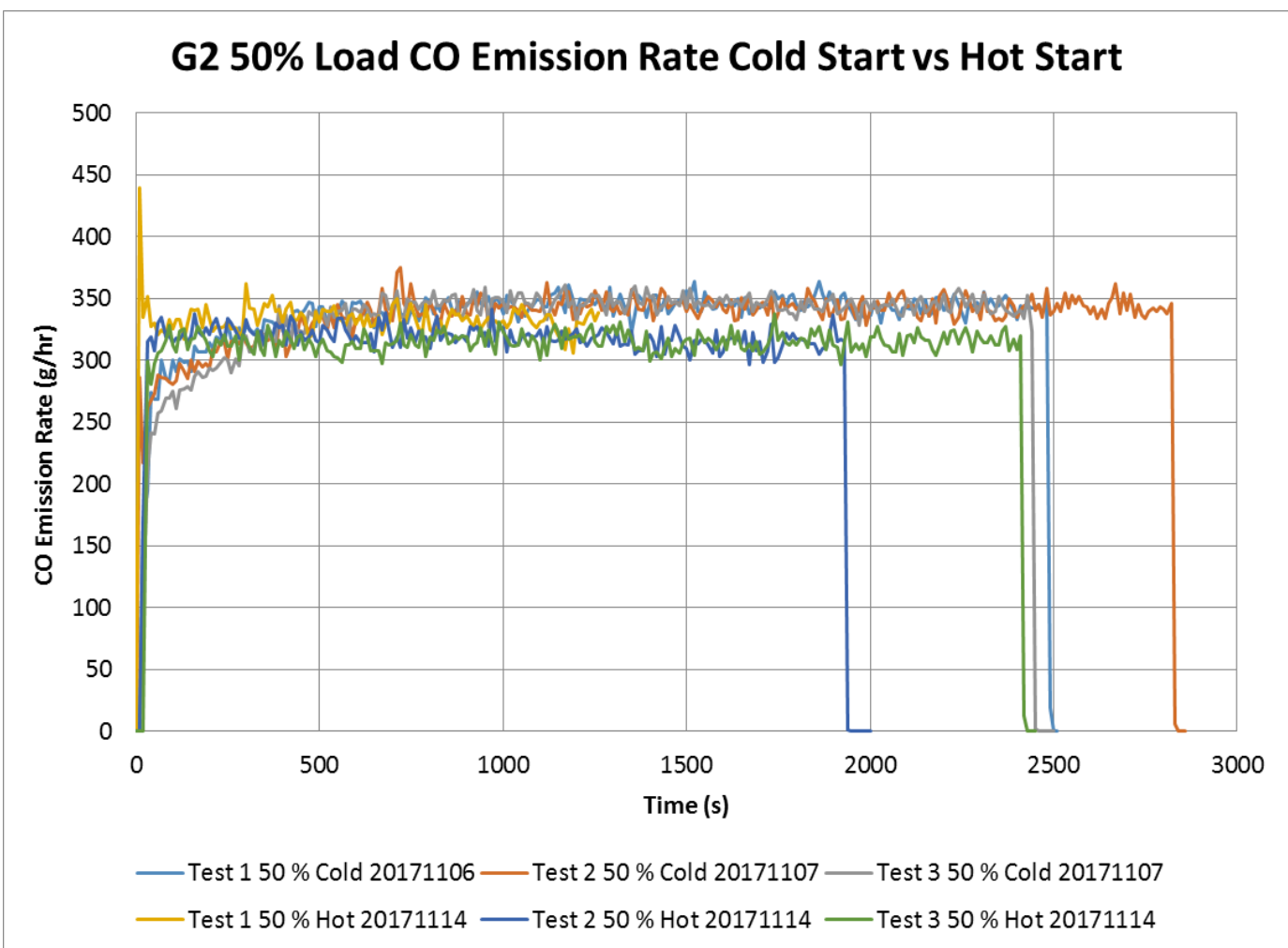

Figure 3. G2 50\% load CO Emission Rate Cold start vs Hot Start 


\section{G2P Data Summary}

Staff tested the G2P at 10 percent and 50 percent of rated load. The emissions from this generator at 10 percent rated load were too low to be measured accurately by the emissions test system. The emission rate is estimated to be approximately $10 \mathrm{~g} / \mathrm{hr}$ at 10 percent load, based on the measured CO concentration within the CVS tunnel before and during testing of the generator. The emissions data at 50 percent load varied between tests. While tests 2 and 3 exhibited the same emission rate profile, test 1 produced a different profile. This variability may be a characteristic of this generator.

Table 4. G2P @ 50\% Load

\begin{tabular}{|l|l|l|}
\hline G2P @ 50\% Load & Value & Unit \\
\hline Avg. CO Emission Rate & 68.0 & $\mathrm{~g} / \mathrm{hr}$ \\
\hline Avg. Block Temp & 90.9 & ${ }^{\circ} \mathrm{C}$ \\
\hline Avg. Exhaust Temp & 193.9 & ${ }^{\circ} \mathrm{C}$ \\
\hline
\end{tabular}

Table 5. G2P 10\% Load

\begin{tabular}{|l|l|l|}
\hline G2P @ 10\% Load & Value & Unit \\
\hline Avg. CO Emission Rate & $\sim 10.0$ & g/hr (estimate) \\
\hline Avg. Block Temp & 74.4 & ${ }^{\circ} \mathrm{C}$ \\
\hline Avg. Exhaust Temp & 58.7 & ${ }^{\circ} \mathrm{C}$ \\
\hline
\end{tabular}

Table 6. G2P Exhaust Parameters

\begin{tabular}{|l|l|l|}
\hline G2P Exhaust Parameters & Value & Unit \\
\hline Exhaust Exit Diameter & 1.16 & $\mathrm{~cm}$ (I.D.) \\
\hline Exhaust Velocity @ 50\% Load & 10.5 & $\mathrm{~m} / \mathrm{s}$ \\
\hline Exhaust Velocity @ 10\% Load & 10.0 & $\mathrm{~m} / \mathrm{s}$ \\
\hline
\end{tabular}




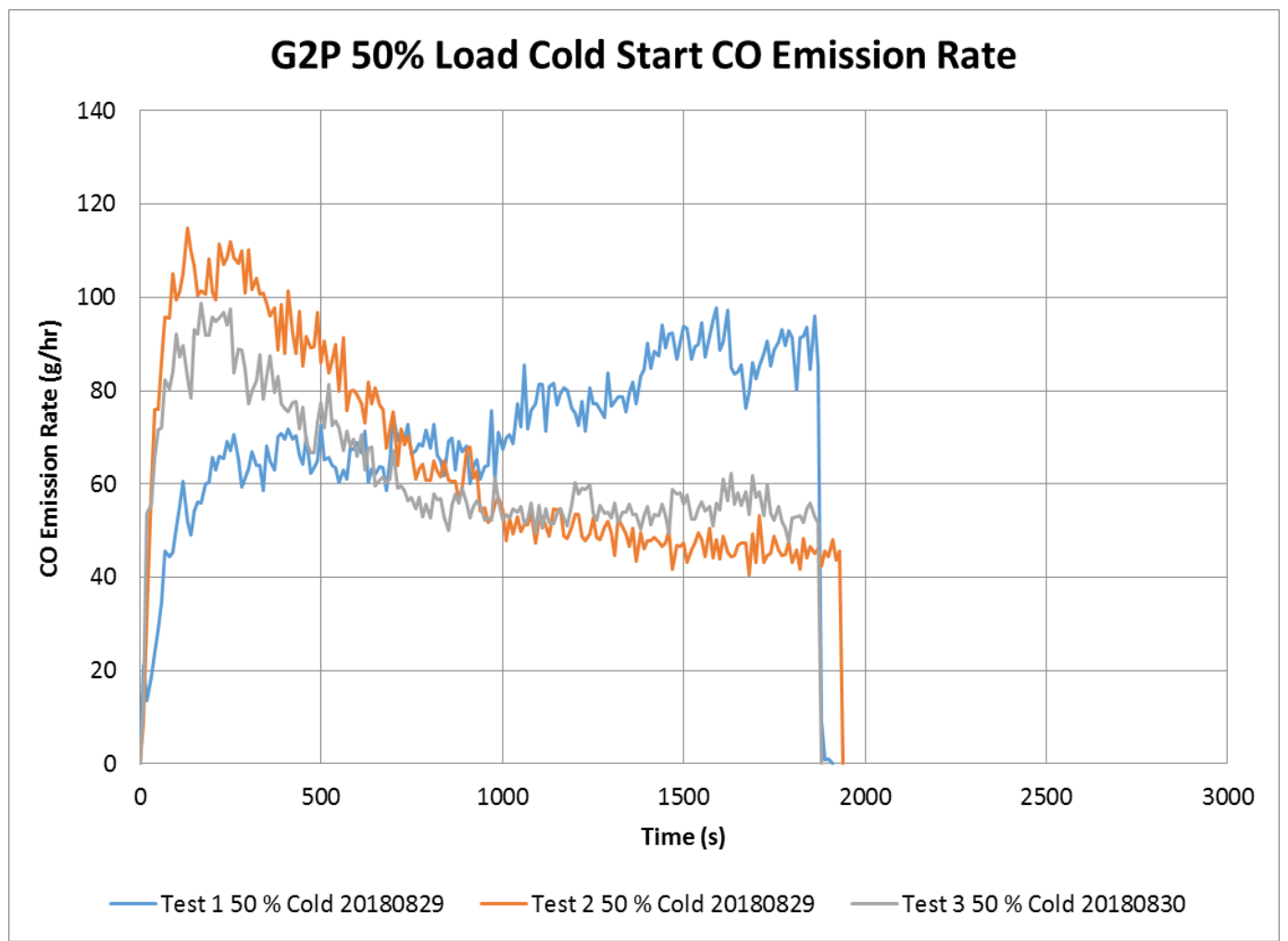

Figure 4. G2P 50\% Load Cold Start Emission Rate 


\section{G6 Data Summary}

Table 7. G6 @ 100\% Load

\begin{tabular}{|l|l|l|}
\hline G6 @ 100\% Load & Value & Unit \\
\hline Avg. CO Emission Rate & 1841.6 & $\mathrm{~g} / \mathrm{hr}$ \\
\hline Avg. Block Temp & 85.6 & ${ }^{\circ} \mathrm{C}$ \\
\hline Avg. Exhaust Temp & 532.9 & ${ }^{\circ} \mathrm{C}$ \\
\hline
\end{tabular}

Table 8. G6 @ 50\% Load

\begin{tabular}{|l|l|l|}
\hline G6 @ 50\% Load & Value & Unit \\
\hline Avg. CO Emission Rate & 749.6 & $\mathrm{~g} / \mathrm{hr}$ \\
\hline Avg. Block Temp & 68.5 & ${ }^{\circ} \mathrm{C}$ \\
\hline Avg. Exhaust Temp & 398.8 & ${ }^{\circ} \mathrm{C}$ \\
\hline
\end{tabular}

Table 9. G6 Exhaust Parameters

\begin{tabular}{|l|l|l|}
\hline G6 Exhaust Parameters & Value & Unit \\
\hline Exhaust Exit Diameter & 1.91 & $\mathrm{~cm}$ (I.D.) \\
\hline Exhaust Velocity @ 100\% Load & 22.0 & $\mathrm{~m} / \mathrm{s}$ \\
\hline Exhaust Velocity @ 50\% Load & 15.0 & $\mathrm{~m} / \mathrm{s}$ \\
\hline
\end{tabular}




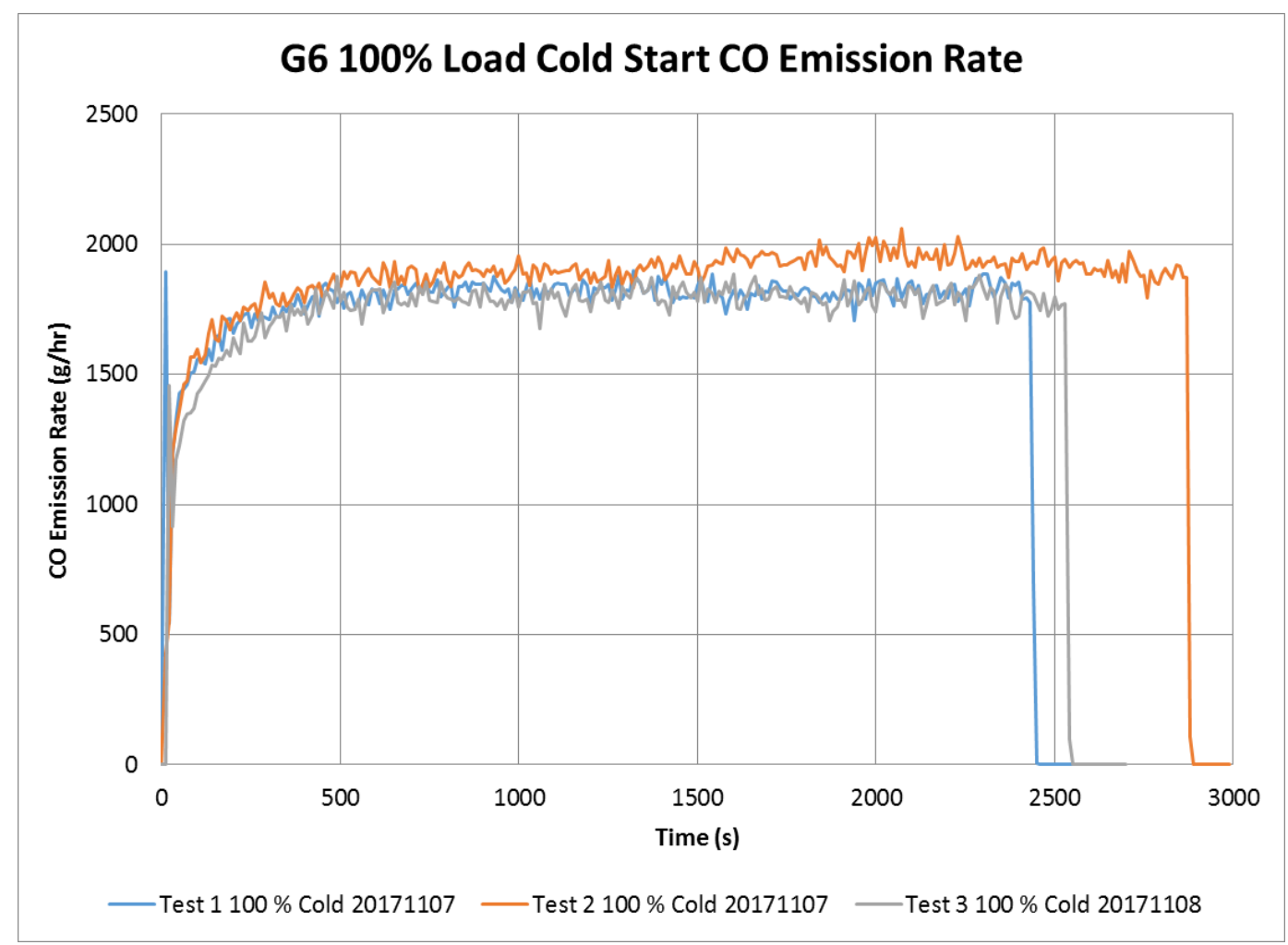

Figure 5. G6 100\% Load Cold Start CO Emission Rate

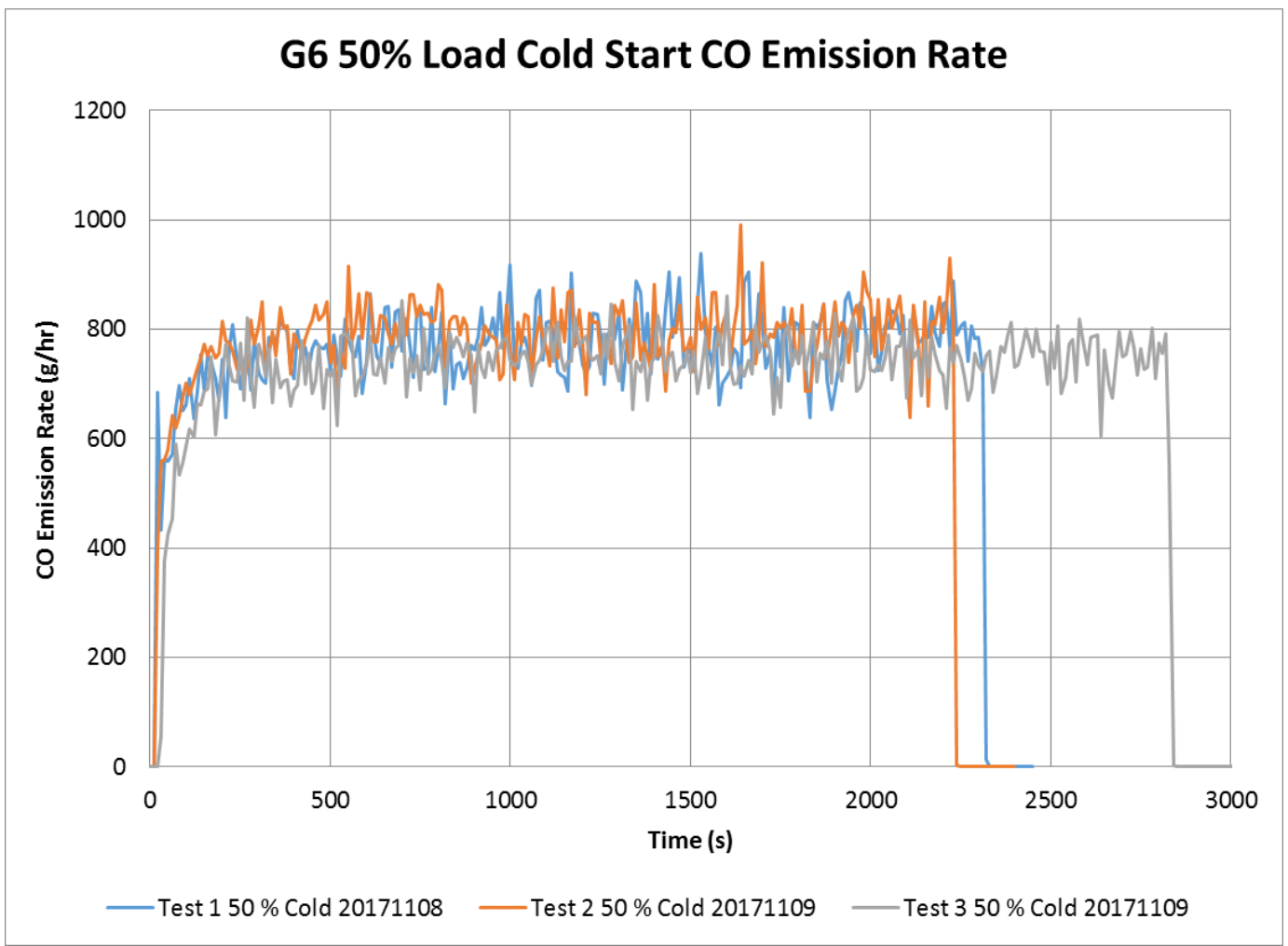

Figure 6. G6 50\% Load Cold Start CO Emission Rate 


\section{G7 Data Summary}

Table 10. G7 100\% Load

\begin{tabular}{|l|l|l|}
\hline G7 @ 100\% Load & Value & Unit \\
\hline Avg. CO Emission Rate & 215.1 & $\mathrm{~g} / \mathrm{hr}$ \\
\hline Avg. Block Temp & 116.7 & ${ }^{\circ} \mathrm{C}$ \\
\hline Avg. Exhaust Temp & 457.6 & ${ }^{\circ} \mathrm{C}$ \\
\hline
\end{tabular}

Table 11. G7 @ 50\% Load

\begin{tabular}{|l|l|l|}
\hline G7 @ 50\% Load & Value & Unit \\
\hline Avg. CO Emission Rate & 273.8 & $\mathrm{~g} / \mathrm{hr}$ \\
\hline Avg. Block Temp & 88.3 & ${ }^{\circ} \mathrm{C}$ \\
\hline Avg. Exhaust Temp & 294.1 & ${ }^{\circ} \mathrm{C}$ \\
\hline
\end{tabular}

Table 12. G7 Exhaust Parameters

\begin{tabular}{|l|l|l|}
\hline G7 Exhaust Parameters & Value & Unit \\
\hline Exhaust Exit Diameter & 0.93 & $\mathrm{~cm}$ (I.D.) \\
\hline Exhaust Velocity @ 100\% Load & 20.5 & $\mathrm{~m} / \mathrm{s}$ \\
\hline Exhaust Velocity @ 50\% Load & 13.0 & $\mathrm{~m} / \mathrm{s}$ \\
\hline
\end{tabular}




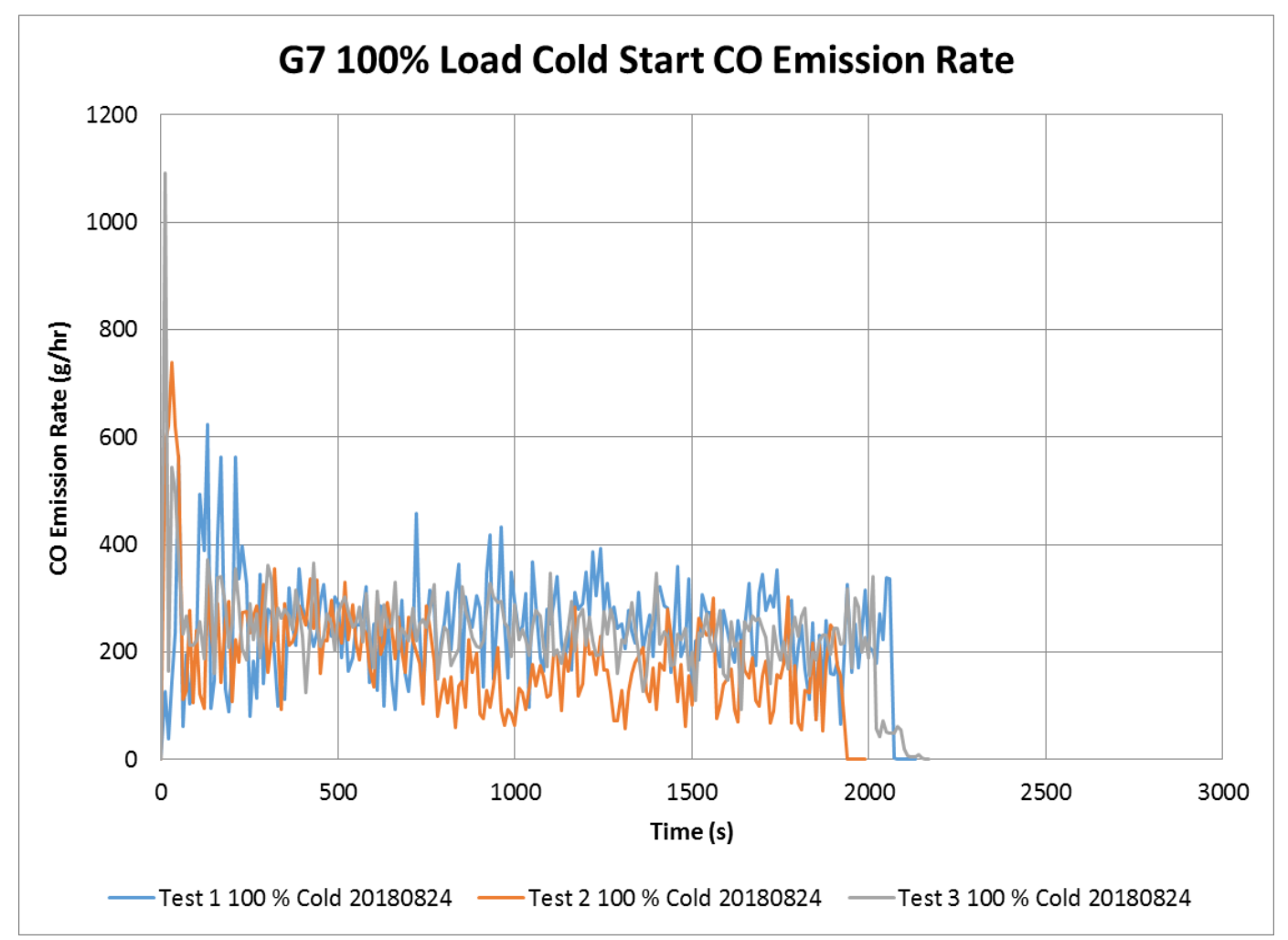

Figure 7. G7 100\% Load Cold Start CO Emission Rate

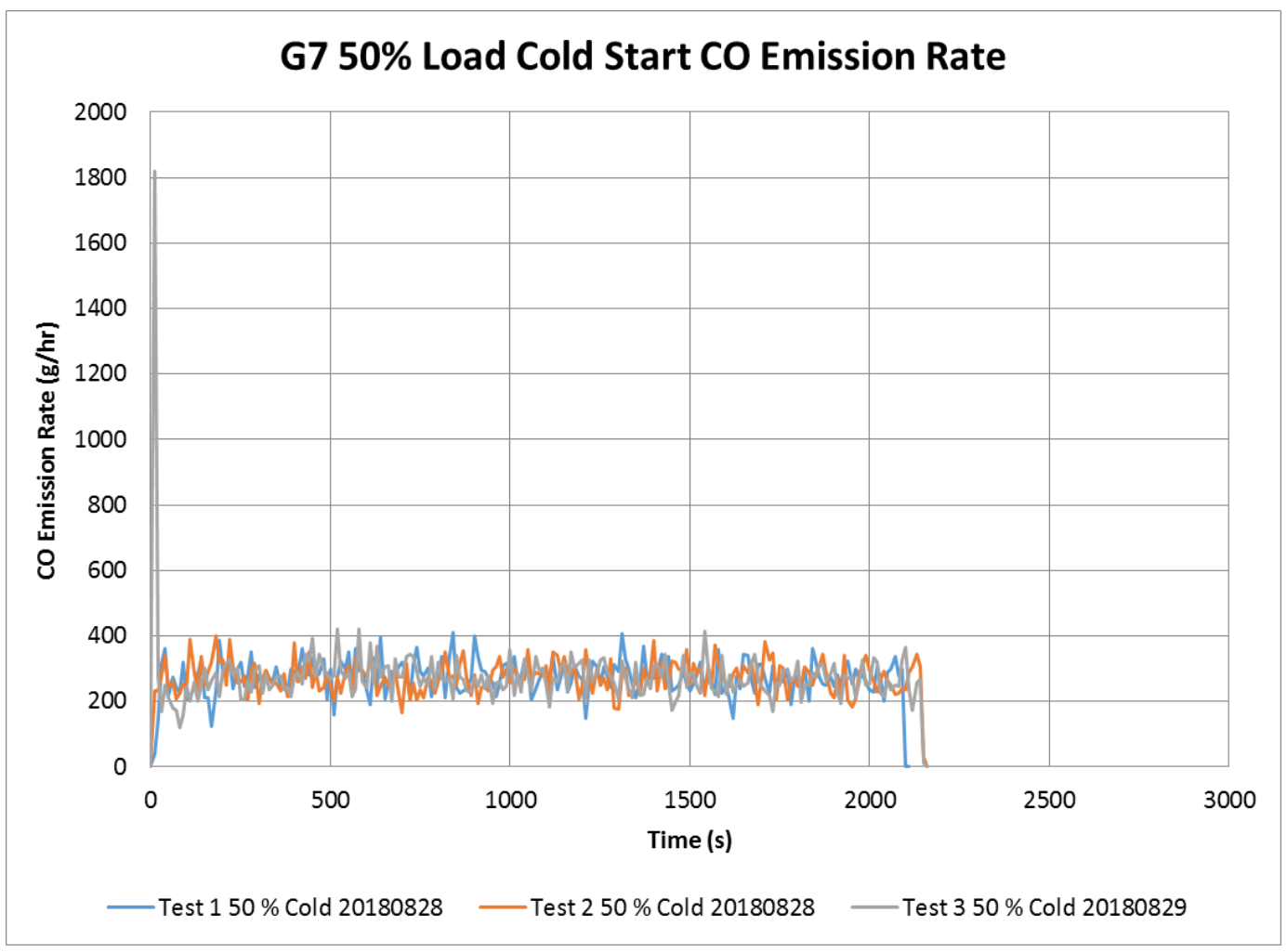

Figure 8. G7 50\% Load Cold Start CO Emission Rate 


\section{G15 Data Summary}

Table 13. G15 @ 90\% Load

\begin{tabular}{|l|l|l|}
\hline G15 @ 90\% Load & Value & Unit \\
\hline Avg. CO Emission Rate & 3083.8 & $\mathrm{~g} / \mathrm{hr}$ \\
\hline Avg. Block Temp & 79.9 & ${ }^{\circ} \mathrm{C}$ \\
\hline Avg. Exhaust Temp & 566.8 & ${ }^{\circ} \mathrm{C}$ \\
\hline
\end{tabular}

Table 14. G15 @ 25\% Load

\begin{tabular}{|l|l|l|}
\hline G15 @ 25\% Load & Value & Unit \\
\hline Avg. CO Emission Rate & 1157.5 & $\mathrm{~g} / \mathrm{hr}$ \\
\hline Avg. Block Temp & 64.0 & ${ }^{\circ} \mathrm{C}$ \\
\hline Avg. Exhaust Temp & 546.0 & ${ }^{\circ} \mathrm{C}$ \\
\hline
\end{tabular}

Table 15. G15 Exhaust Parameters

\begin{tabular}{|l|l|l|}
\hline G15 Exhaust Parameters & Value & Unit \\
\hline Exhaust Exit Diameter & 2.98 & $\mathrm{~cm}$ (I.D.) \\
\hline Exhaust Velocity @ 90\% Load & 34.0 & $\mathrm{~m} / \mathrm{s}$ \\
\hline Exhaust Velocity @ 25\% Load & 29.0 & $\mathrm{~m} / \mathrm{s}$ \\
\hline
\end{tabular}




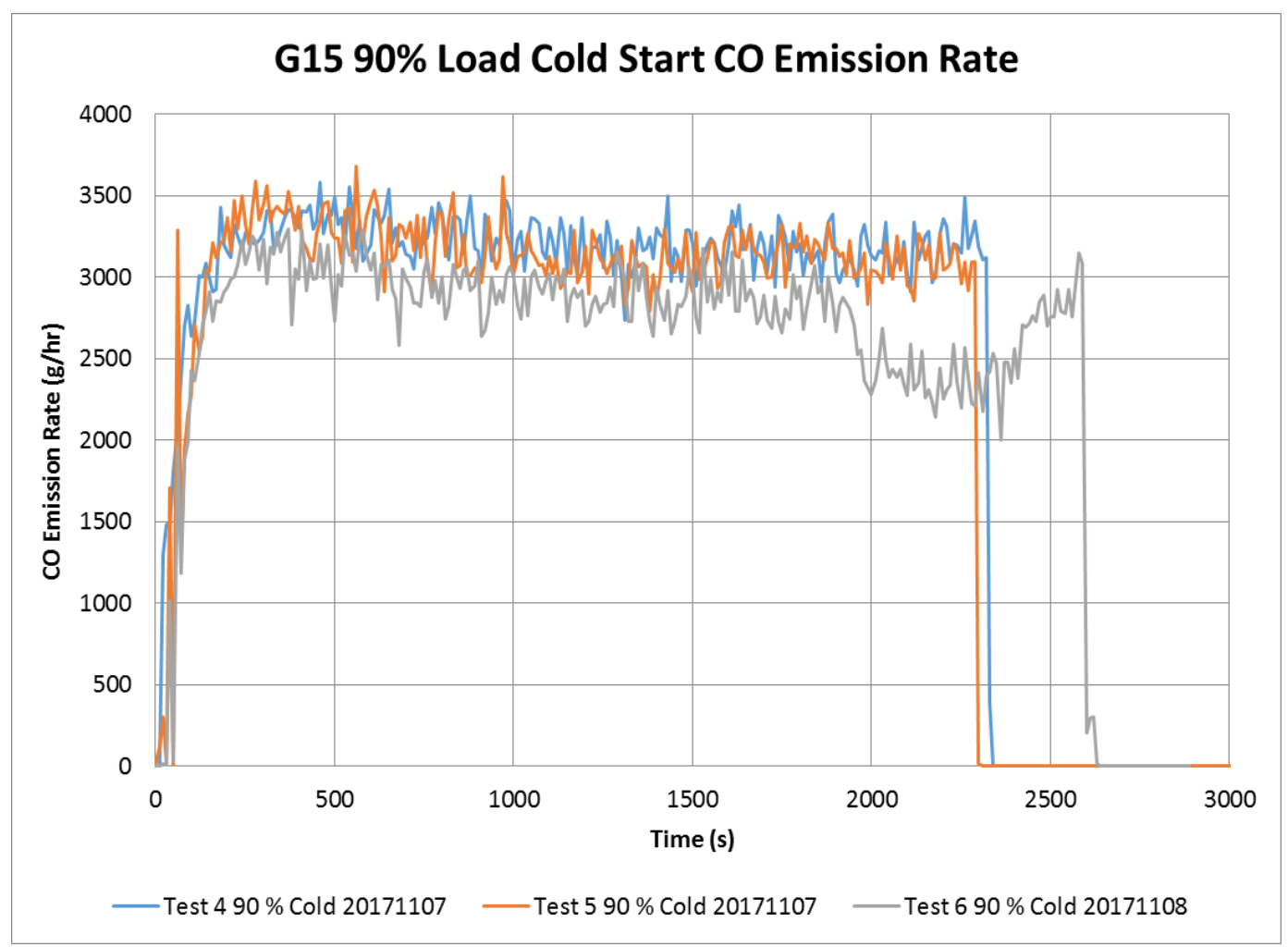

Figure 9. G15 90\% Load Cold Start CO Emission Rate

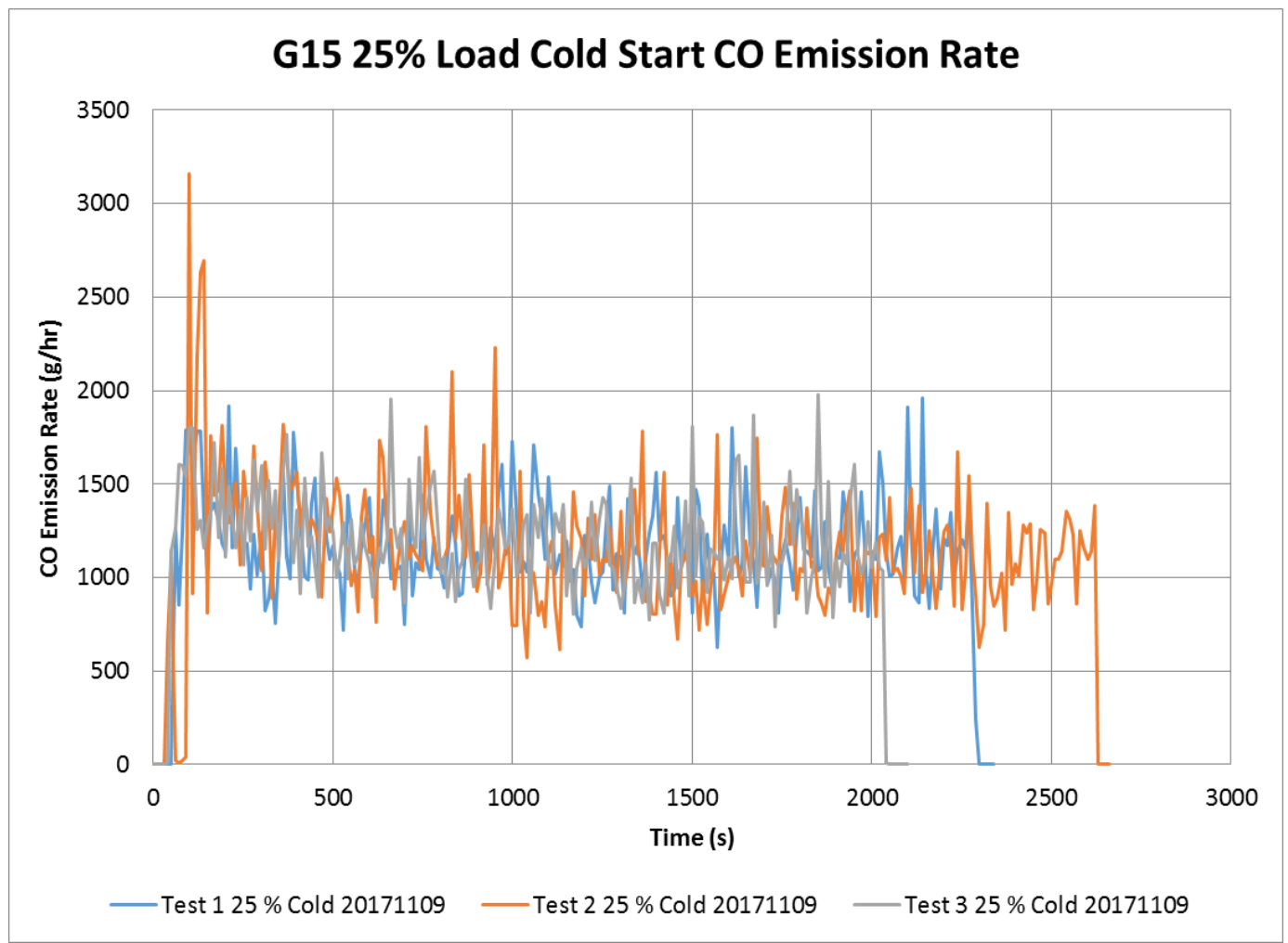

Figure 10. G15 25\% Load Cold Start CO Emission Rate 


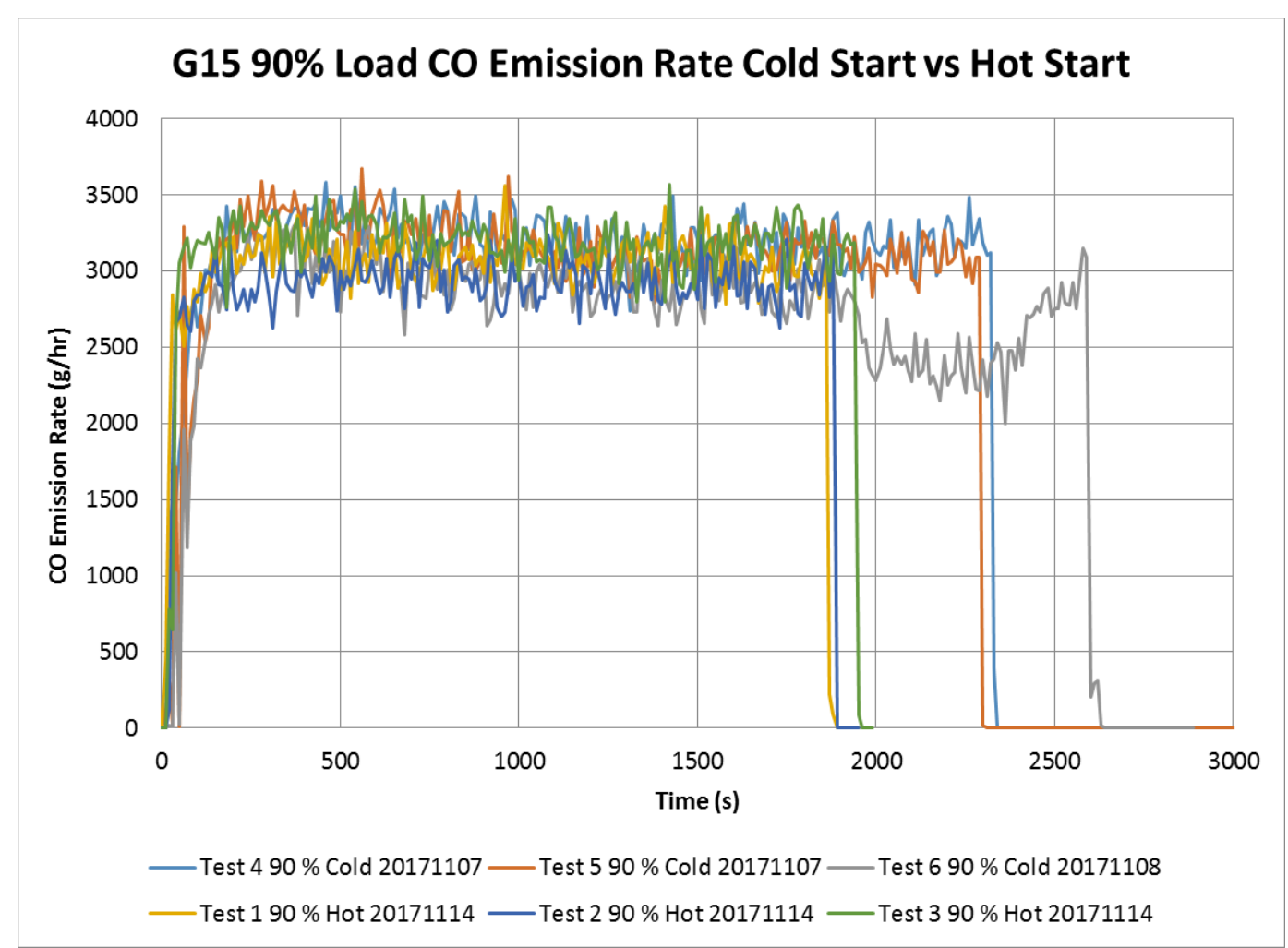

Figure 11. G15 90\% Load CO Emission Rate Cold Start vs Hot Start

Staff expects that other generators may be used for empirical testing as they become available. These generators will be characterized similarly to the generators in this memorandum, as needed.

Sincerely,

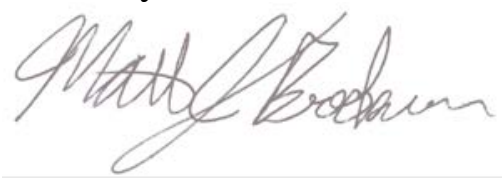

Matthew J. Brookman, P.E. PMP

Mechanical/ Fire Protection Engineer

U.S. Consumer Product Safety Commission

National Product Testing and Evaluation Center

5 Research Place

Rockville, MD, 20850

301-987-2467 


\section{Appendix 2 - COHb Analysis Method}

In 1965, Coburn, Forster and Kane developed a differential equation (CFK model) to describe the major physiological variables that determine the $\mathrm{COHb}$ in blood using data from patients with increased endogenous production of $\mathrm{CO}$ due to anemia (Coburn et al., $1965^{2}$ ). The CFK model is represented by the following equation:

$$
\frac{d(\mathrm{COHb})_{t}}{d t}=\frac{V_{\mathrm{CO}}}{V_{b}}-\frac{C O H b_{t} * P_{\mathrm{O}_{2}}}{M^{*} B^{*} V b^{*} \mathrm{OHb}}+\frac{P_{\mathrm{CO}}}{B^{*} V b}
$$

where

$$
\begin{aligned}
& B=1 / D_{L}+P_{L} / V_{A} \\
& M=\text { Ratio of affinity of blood for } \mathrm{CO} \text { to that for } \mathrm{O}_{2}, M=218 \\
& O H b=\mathrm{ml} \text { of } \mathrm{O}_{2} \text { per ml blood, } \mathrm{OHb}=0.2 \\
& C O H b_{t}=\mathrm{ml} \text { of } \mathrm{CO} \text { per ml blood at time } \\
& P_{\mathrm{O}_{2}}=\text { average partial pressure of oxygen in the lung capillaries, } P_{O_{2}}=100 \mathrm{~mm} \mathrm{Hg} \\
& V_{C O}=\text { rate of endogenous CO production, } V_{C O}=0.007 \mathrm{ml} / \mathrm{min} \\
& D_{L}=\text { diffusivity of the lung for } \mathrm{CO}, D_{L}=30 \mathrm{ml} / \mathrm{min} \mathrm{mm} \mathrm{Hg} \\
& P_{L}=\text { barometric pressure minus the vapor pressure of water at body temperature, } 713 \\
& \mathrm{~mm} \text { Hg } \\
& V b=\text { blood volume, } \mathrm{Vb}=5500 \mathrm{ml} \\
& P_{C O}=\text { partial pressure of } \mathrm{CO} \text { in the air inhaled, mm Hg } \\
& V_{A}=\text { alveolar ventilation rate, } V_{A}=6000 \mathrm{ml} / \mathrm{min} \text { (awake), } 4000 \mathrm{ml} \text { (sleeping) } \\
& t=\text { exposure duration, min }
\end{aligned}
$$

In another study by Peterson and Stewart (1975), data from a series of human exposures to CO were analyzed to determine the fit to the theoretical CFK equation. 19 men and 3 women were exposed to concentrations of $50 \mathrm{ppm}, 100 \mathrm{ppm}$ or $200 \mathrm{ppm}$ for $0.33 \mathrm{~h}$ to $5.25 \mathrm{~h}$. Three exercise levels from sedentary to $0 \mathrm{kpm} / \mathrm{min}, 150 \mathrm{kpm} / \mathrm{min}$ or $300 \mathrm{kpm} / \mathrm{min}$ on an ergometer were used (15 subjects in total). These resulted in mean ventilation rates of $10.1 \mathrm{l} / \mathrm{min}$ (9.1 l/min for women), $14.0 \mathrm{l} / \mathrm{min}, 24.0 \mathrm{l} / \mathrm{min}$ (19.7 l/min for women) and $29.7 \mathrm{l} / \mathrm{min}$, respectively. The CFK model predicted $\mathrm{COHb}$ for both men and women as well as for resting and exercising subjects within a standard error of about $2 \%$. In contrast to the original model, which assumes all variables to be constant except $t, P_{L}$, $\mathrm{COHb}_{t}$ and $\mathrm{P}_{\mathrm{CO}}$, the following parameter alterations were introduced:

$\mathrm{P}_{\mathrm{O}_{2}}$ : When the partial pressure of oxygen in inspired air $\left(\mathrm{Pi}_{\mathrm{O}_{2}}\right)$ is less than the $149 \mathrm{mmHg}$ found under normal conditions, the partial pressure of oxygen in the lung capillaries will be less than the value of $100 \mathrm{mmHg}$ assumed by Coburn and coworkers. From measurements of oxygen partial pressure in arterial blood, which is assumed to be the same as the oxygen partial pressure in lung capillaries, the following equation was derived:

\footnotetext{
2 Coburn, R.F., Forster, R.E. and Kane, P.B. 1965. Considerations of the physiological variables that determine the blood carboxyhemoglobin concentration in man. Journal of Clinical Investigation, 44 (11), 1899-1910.
} 


$$
\begin{aligned}
& P_{\mathrm{O}_{2}}=1 /\left(0.072-0.00079 \mathrm{Pi}_{\mathrm{O}_{2}}+0.000002515\left(\mathrm{Pi}_{\mathrm{O}_{2}}\right)^{2}\right) \text { and } \\
& \mathrm{Pi}_{\mathrm{O}_{2}}=\mathrm{Fi}_{\mathrm{O}_{2}}\left(P_{\mathrm{B}}-47-\mathrm{Pi}_{\mathrm{CO}}\right) \text { with } \mathrm{Fi}_{\mathrm{O}_{2}}=\text { fraction of oxygen in inspired air, } \\
& P_{\mathrm{B}}=\text { barometric pressure (mm Hg), } \mathrm{Pi} \mathrm{i}_{\mathrm{CO}}=\text { partial pressure of CO in inspired air }
\end{aligned}
$$

$D_{L}$ : Body size effects on diffusivity at rest were was calculated from published data as:

$$
D_{L}=1 /(-0.0287+0.1188 / A) \text { with } A=\text { body surface in } \mathrm{m}^{2}
$$

$\mathrm{Vb}$ : the published blood volume relationship of $74 \mathrm{mg} / \mathrm{kg}$ of body weight for men and 73 $\mathrm{ml} / \mathrm{kg}$ for women was used.

$V_{A}$ : The alveolar ventilation rate was expressed as: $V_{A}=V_{E}-f V_{D}$; with $V_{E}=$ total rate of ventilation $(\mathrm{ml} / \mathrm{min}), f=$ respiration rate $\left(\mathrm{min}^{-1}\right)$ and $V_{D}=$ dead space $(\mathrm{ml})$

$\mathrm{OHb}_{t}$ : At standard concentrations, $1 \mathrm{~g}$ of hemoglobin will hold $1.38 \mathrm{ml}$ of oxygen and thus $O H b_{\text {max }}=1.38[\mathrm{Hb}] / 100$, with [2] being the hemoglobin concentration in blood $(\mathrm{g} / 100 \mathrm{ml})$. During and after $\mathrm{CO}$ exposure, the value of $\mathrm{OH} b_{t}$ that must be used is actually $O H b_{t}=O H b_{\text {max }}-\mathrm{COH}_{t}$. In this case, the CFK equation can only be solved by iterative procedures.

$\mathrm{COHb}$ : This value can be converted to the more conventional, percentage saturation by:

$$
\% \mathrm{COHb}=\mathrm{COHb} * 100 / \mathrm{OH}_{\max }
$$

For the calculation of concentration-time combinations that result in a certain $\mathrm{COHb}$, the model of Coburn, Forster and Kane (CFK model) was used. Since this model in the formulation of Peterson and Stewart (1975) calculates COHb larger than $100 \%$ at high exposure concentrations, the following correction proposed by Peterson and Stewart (1975) was used: the amount of bound oxygen is actually not constant, but is dependent on the $\mathrm{COHb}$, therefore:

$$
O H b_{t}=O H b_{\text {max }}-\mathrm{COHb}_{t}
$$

Since in this case, the CFK equation can only be solved iteratively (EPA, 2006), calculations were done using time steps $(\Delta t)$ of 1 minute for the period of 24 hours. In each step, the $\mathrm{COHb}$ of the step before was used to calculate $O H b_{t}$. For the first step, a background $\mathrm{COHb}$ of $0.24 \%$ was assumed.

Calculations using the following equation were carried out in a NIST-developed computer program:

$$
\begin{aligned}
& \Delta(\mathrm{COHb})_{t}=\left(\frac{V_{\mathrm{CO}}}{V_{b}}-\frac{(\mathrm{COHb})_{t-1} * P_{O 2}}{M * B * V b\left(O H b_{\text {max }}-(\mathrm{COHb})_{t-1}\right)}+\frac{P_{\mathrm{CO}}}{B^{*} V b}\right) \Delta t \\
& (\mathrm{COHb})_{t}=(\mathrm{COHb})_{t-1}+\Delta(\mathrm{COHb})_{t}
\end{aligned}
$$

where $(\mathrm{COHb})_{t}=\mathrm{ml}$ of $\mathrm{CO}$ per $\mathrm{ml}$ blood at time $\mathrm{t}$, min Conversion: $\% \mathrm{COHb}=(\mathrm{COHb})_{t} * 100 / \mathrm{OHb}_{\max }$ $(\mathrm{COHb})_{0}=$ initial level, background $=0.0024$ for non-smokers $O H b_{\max }=\mathrm{ml}$ of $\mathrm{O}_{2}$ per ml blood under normal conditions, $\mathrm{OH} b_{\max }=1.38[\mathrm{Hb}] / 100$ 
[2] = hemoglobin concentration, $\mathrm{g} / 100 \mathrm{ml}$ blood, $[\mathrm{Hb}]=15$ for healthy adults

$V_{C O}=$ rate of endogenous $C O$ production, $V_{C O}=0.007 \mathrm{ml} / \mathrm{min}$

$\mathrm{Vb}=$ blood volume, $\mathrm{ml} ; \mathrm{Vb}(70-\mathrm{kg}$ man $)=5500 \mathrm{ml}$

$M=$ Ratio of affinity of blood for CO to that for $\mathrm{O}_{2} ; M=218$ (newborn: $M=240$ )

$B=1 / D_{L}+P_{L} / V_{A}$ with: $D_{L}=$ diffusivity of the lung for CO, $D_{L}=35 * V_{O_{2}} * \exp (0.33)$

$P_{L}=$ barometric pressure minus the vapor pressure of water at body temperature,

$P_{L}=P b-43 \mathrm{mmHg}$

$V_{\mathrm{O}_{2}}=$ rate of oxygen consumption, $\mathrm{L} / \mathrm{min} . V_{\mathrm{O}_{2}}=R M V / 22.274-0.0309$

$V_{A}=$ alveolar ventilation rate, $\mathrm{ml} / \mathrm{min}, V_{A}=0.933 V_{e}-132 f$

$f=$ respiration rate, $1 / \mathrm{min} . f=\exp [0.0165 R M V+2.3293]$

$R M V=$ respiratory minute volume, $1 / \mathrm{min} . R M V=15 \mathrm{l} / \mathrm{min}$ for the person between light activity (10 l/min) and moderate activity (20 l/min)

$\mathrm{P}_{\mathrm{HO}_{2}}=$ partial pressure of inhaled $\mathrm{O}_{2}$, torr.

$\mathrm{Pi}_{\mathrm{O}_{2}}=148.304-0.0208 P_{\mathrm{CO}}=\mathrm{Fi}_{\mathrm{O}_{2}}\left(\mathrm{~Pb}-47-\mathrm{P}_{\mathrm{CO}}\right)$

$\mathrm{P}_{\mathrm{O}_{2}}=$ average partial pressure of oxygen in the lung capillaries; torr

$P_{\mathrm{O}_{2}}=\mathrm{Pi}_{\mathrm{O}_{2}}-49$ for $\mathrm{Pi}_{\mathrm{O}_{2}} \geq 149$ torr

$P_{\mathrm{O}_{2}}=1 /\left[0.072-0.00079 \mathrm{Pi}_{\mathrm{O}_{2}}+0.000002515\left(\mathrm{Pi}_{\mathrm{O}_{2}}\right)^{2}\right]$, for $\mathrm{Pi}_{\mathrm{O}_{2}}<149$ torr

$\mathrm{Fi}_{\mathrm{O}_{2}}=$ fraction of inhaled $\mathrm{O}_{2}$, normally $\mathrm{Fi}_{\mathrm{O}_{2}}=0.209$

$\mathrm{Pb}=$ barometric pressure, torr, 760 at sea level.

$P_{C O}=$ partial pressure of $\mathrm{CO}$ in the air inhaled, $\mathrm{mm} \mathrm{Hg}$,

Conversion: $P_{C O}(m m H g)=P_{C O}(p p m) / 1316$

$\Delta t=$ exposure time interval, min; $\Delta t=1 \mathrm{~min}$. 